Nicolaus Müller

\title{
Marketingstrategien in High-Tech-Märkten
}




\section{Nicolaus Müller}

\section{Marketingstrategien in High-Tech-Märkten}

In den sich dynamisch entwickelnden High-Tech-Märkten gewinnt die Beobachtung und Analyse des wettbewerbsstrategischen Verhaltens von Unternehmen zunehmend an Bedeutung. Aus Sicht der Praxis und der betriebswirtschaftlichen Forschung ist es von besonderem Interesse zu erfahren, welche Marketingstrategien und -maßnahmen zum Aufbau von Wettbewerbsvorteilen und zur Sicherung einer dauerhaften Erfolgsposition besonders geeignet sind. Vor diesem Hintergrund wird in der Arbeit das Verhalten von 385 High-Tech-Anbietern analysiert. Auf der Grundlage des Konzeptes der strategischen Gruppen werden das strategische und operative Unternehmensverhalten, die Bedeutung ziel- und kulturbezogener Einflußfaktoren sowie die Erfolgswirkung von Marketingstrategien in High-TechMärkten untersucht.

Nicolaus Müller wurde 1963 in Köln geboren. Er studierte von 1984 bis 1989 Betriebswirtschaftlehre an der Universität Münster. Nach Abschluß des Studiums Projekttätigkeit am Institut für Marketing bis Mitte 1990. Seitdem wissenschaftlicher Mitarbeiter an diesem Institut; 1994 Promotion. 
Marketingstrategien in High-Tech-Märkten

Typologisierung, Ausgestaltungsformen und Einflußfaktoren auf der Grundlage strategischer Gruppen 


\section{Schriften zu Marketing und Manasement}

Herausgegeben von Prof.Dr. Dr.h.c. Heribert Meffert

Band 25

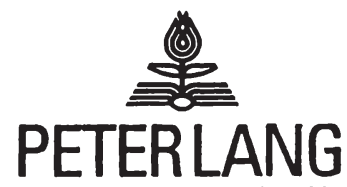

Frankfurtam Main · Berlin · Bern. New York. Paris - Wien

Nikolaus Müller and Universität Münster - 978-3-631-75075-9 Downloaded from PubFactory at 01/11/2019 08:36:03AM 


\section{Nicolaus Müller}

\section{Marketingstrategien in High-Tech-Märkten}

Typologisierung, Ausgestaltungsformen und Einflußfaktoren aufder Grundlage strategischerGruppen

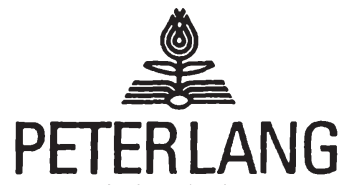


Die Deutsche Bibliothek - CIP-Einheitsaufnahme

Müller, Nicolaus:

Marketingstrategien in High-Tech-Märkten : Typologisierung, Ausgestaltungsformen und Einflußfaktoren auf der Grundlage strategischer Gruppen / Nicolaus Müller. - Frankfurt am Main ; Berlin ; Bern ; New York ; Paris ; Wien : Lang, 1995

(Schriften zu Marketing und Management ; Bd. 25)

Zugl: Münster (Westfalen), Univ., Diss., 1994

ISBN 3-631-48496-8

NE: GT

Open Access: The online version of this publication is published on www.peterlang.com and www.econstor.eu under the international Creative Commons License CC-BY 4.0. Learn more on how you can use and share this work: http://creativecommons. org/licenses/by/4.0.

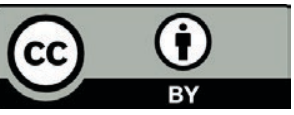

This book is available Open Access thanks to the kind support of ZBW - Leibniz-Informationszentrum Wirtschaft.

\author{
D 6 \\ ISSN 0176-2729 \\ ISBN 3-631-48496-8 \\ ISBN 978-3-631-75075-9 (eBook) \\ (c) Peter Lang $\mathrm{GmbH}$ \\ Europäischer Verlag der Wissenschaften \\ Frankfurt am Main 1995 \\ Alle Rechte vorbehalten.
}

Das Werk einschließlich aller seiner Teile ist urheberrechtlich geschützt. Jede Verwertung außerhalb der engen Grenzen des Urheberrechtsgesetzes ist ohne Zustimmung des Verlages unzulässig und strafbar. Das gilt insbesondere für

Vervielfältigungen, Übersetzungen, Mikroverfilmungen und die Einspeicherung und Verarbeitung in elektronischen Systemen. 


\section{Vorwort des Herausgebers}

Lange Zeit wurden in der wissenschaftlichen Forschung zum High-TechMarketing Fragestellungen zum strategischen Unternehmensverhalten stiefmütterlich behandelt oder allein aus angebotsbezogener Sicht aufgearbeitet. Nachfrager- bzw. wettbewerbsbezogene Aspekte wurden erst ab Mitte der 80er Jahre verstärkt in der Strategiediskussion aufgegriffen. Trotz eines wachsenden Interesses von Wissenschaft und Praxis an einer umfassenderen Analyse der Marketingstrategien von High-Tech-Unternehmen und einer näheren Betrachtung der Einflußfaktoren und Ausgestaltungsformen von Marketingstrategien ist das Verhalten strategischer Gruppen in diesen Märkten bislang wenig untersucht worden.

Vor diesem Hintergrund wird mit der vorliegenden Arbeit die Zielsetzung verfolgt, Informationen darüber bereitzustellen, welche Wettbewerbsverhältnisse in High-Tech-Märkten herrschen und welches Verhalten zur Erzielung von Wettbewerbsvorteilen und zur Sicherung einer dauerhaften Erfolgsposition geeignet ist. Auf der Grundlage des Konzeptes der strategischen Gruppen werden im Rahmen eines situativen Ansatzes das strategische und operative Verhalten von High-Tech-Unternehmen, der Einfluß der Unternehmensziele und -kultur auf das strategische Verhalten sowie die Erfolgswirkung von Marketingstrategien in High-Tech-Märkten analysiert.

Nach einer Betrachtung der theoretischen Grundlagen zum Konzept der strategischen Gruppen und aktueller Forschungen wird ein zweistufiger Operationalisierungsansatz für eine Typologisierung von Marketingstrategien in High-Tech-Märkten entwickelt. Zur Identifikation homogener Umfeldsituationen werden theoriegestützt geeignete Kontextvariablen und zur Bildung strategischer Gruppen geeignete Strategievariablen abgeleitet. Dieser Operationalisierungsansatz wird im Rahmen einer empirischen Analyse, die auf der Befragung von 385 High-Tech-Anbietern beruht, validiert.

Zunächst werden über eine explorative Faktorenanalyse vier grundlegende Marketingstrategien ermittelt. Die Untersuchung der Basisstrategien verdeutlicht, daß in High-Tech-Märkten einerseits eigenständige strategische Grund- 
haltungen existieren, während andererseits bestimmte Basisstrategien mit den Wettbewerbsstrategien von Porter korrespondieren. Anschließend werden vom Verfasser jeweils drei strategische Verhaltensgruppen in den vorab gebildeten homogenenen Umfeldsituationen identifiziert, die signifikante Unterschiede in ihren Strategieschwerpunkten aufweisen. Eine Untersuchung des Unternehmensverhaltens in den unterschiedlichen Situationen macht deutlich, daß die strategischen Gruppen ähnliche Strategieschwerpunkte besitzen und nur geringe Strategieunterschiede bestehen. Diese Ergebnisse unterstreichen die hohe Validität der identifizierten strategischen Gruppen und weisen darauf hin, daß das strategische Unternehmenverhalten nur bedingt durch die Umfeldsituation beeinflußt wird.

In der weiterfuihrenden Untersuchung werden zum einen die Ausgestaltungsformen der High-Tech-Unternehmen analysiert. Hierbei wird deutlich, daß sich die Strategieschwerpunkte der strategischen Gruppen auch im operativen Verhalten niederschlagen. Zum anderen wird betrachtet, ob bestimmte Zielsysteme und kulturelle Grundorientierungen das Enstehen von strategischen Gruppen beeinflussen. Der Verfasser stellt dabei heraus, daß ein Zusammenhang zwischen den Zielen und Marketingstrategien der High-Tech-Anbieter besteht und $\mathrm{da} \beta$ die einzelnen strategischen Gruppen tendenziell eine andere Unternehmenskultur besitzen. In einem weiteren Analyseschritt wird darüber hinaus dargelegt, daß die Marketingstrategien der strategischen High-TechGruppen unterschiedlich erfolgreich sind.

Die vorliegende Untersuchung stellt mit einer umfassenden Bestandsaufnahme des strategischen und operativen Verhaltens deutscher High-Tech-Anbieter einen beachtlichen Beitrag zum High-Tech-Marketing dar. Es werden interessante Erkenntnisse über den Einfluß der Unternehmensziele und Unternehmenskultur auf das Unternehmensverhalten sowie die Erfolgswirkung unterschiedlicher Marketingstrategien in High-Tech-Märkten aufgezeigt. Es bleibt zu wünschen, daß die Veröffentlichung in Wissenschaft und Praxis auf eine entsprechende Resonanz stößt. 


\section{Vorwort des Verfassers}

Seit Beginn der $80 \mathrm{er}$ Jahre versucht eine Vielzahl von Unternehmen, am Wachstumspotential von High-Tech-Märkten zu partizipieren. Es ist festzustellen, daß der Erfolg von High-Tech-Produkten nicht mehr allein vom technologischen Know-how und dem Wissen um die Kundenbedürfnisse abhängt, sondern zunehmend von der Kenntnis der spezifischen Wettbewerbsbedingungen in High-Tech-Märkten bestimmt wird.

Traditionell wird die Diskussion in der Strategieforschung aus zeit-/technologie- oder aus wettbewerbsbezogener Sicht geführt. Eine stärker integrierte Betrachtung von Marketingstrategien in High-Tech-Märkten, die zudem den Einfluß der Unternehmensumwelt auf die Strategiegestaltung berücksichtigt, wird bislang vernachlässigt. Darüber hinaus fehlt in der wissenschaftlichen Forschung eine differenzierte Analyse der Unternehmensziele und -kultur von High-Tech-Anbietern und ihrer Auswirkung auf die Gestaltung von Marketingstrategien.

Vor dem Hintergrund der bestehenden Erkenntnisdefizite wird in der vorliegenden Arbeit eine theoretische Darlegung und empirische Fundierung des Untersuchungskomplexes geleistet. Hierzu werden auf Basis des Konzeptes der strategischen Gruppen im Rahmen eines situativen Ansatzes strategische Verhaltensmuster und deren maßnahmenorientierte Umsetzung in High-TechMärkten untersucht. Weiterhin werden ziel- und kulturbezogene Unterschiede zwischen den strategischen Verhaltensgruppen aufgezeigt und ihr Einfluß auf die Gruppenbildung erforscht. Abschließend wird eine Bewertung der strategischen Gruppen im Hinblick auf ihren Markterfolg vorgenommen.

Die Erstellung der Arbeit war nur mit der Unterstützung zahlreicher Personen möglich. Mein besonderer Dank gilt an erster Stelle meinem akademischen Lehrer, Herrn Professor Dr. Dr. h.c. Heribert Meffert, der die Themenstellung anregte und die Fertigstellung der Arbeit förderte. Herrn Professor Klaus Backhaus sei für die Übernahme des Zweitgutachtens gedankt. 
Dank schulde ich auch allen Kolleginnen und Kollegen am Institut für Marketing, die mich während der Erstellung der Arbeit entlastet und vielfältig unterstützt haben. Hervorheben möchte ich Herrn Dr. Manfred Kirchgeorg, der mir während der Assistentenzeit stets ein geduldiger Ansprechpartner war und mir im konzeptionellen Stadium der Arbeit wertvolle fachliche Anregungen gab. Mein Dank gilt ferner Herrn Dr. Clemens Pues und Herrn Dipl.-Kfm. Michael Szeliga für ihre konstrutiv-kritischen Hinweise bei der Abfassung und für ihre Unterstützung bei der Fertigstellung der Arbeit. Frau Dipl.-Kffr. Karin Hillebrand und Frau Sabine Voß verdanke ich die vorbildliche und sorgfältige Durchsicht des Manuskriptes, Herrn Oliver Voß die exzellente und flexible Erstellung der Abbildungen und Herrn Michael zur Mühlen die perfekte und engagierte Ab-wicklung technischer Aufgaben bei der Manuskripterstellung.

Darüber hinaus möchte ich meinen Eltern danken, die das Interesse an betriebswirtschaftlichen Fragestellungen bereits früh in mir geweckt haben und mich seit langem in umfassender Weise fördern. Sie haben mich nachhaltig geprägt und zusammen mit meinen Geschwistern mir in allen Phasen meiner beruflichen und privaten Entwicklung ihr Verständnis und ihre Hilfe entgegengebracht. Mein ganz besonderer Dank gilt meiner großartigen Beate, die mir während der Zeit am Institut stets verständnisvoll, geduldig und motivierend zur Seite stand. 


\section{Inhaltsverzeichnis}

Seite

A. Stellenwert von Marketingstrategien für die strategische

Unternehmensführung in High-Tech-Märkten

1. Besonderheiten von High-Tech-Märkten aus wettbewerbsstrategischer Sicht

2. Gegenstand und Bedeutung von Marketingstrategien in High-

Tech-Märkten

3. Erklärungsansätze zu strategischen Gruppen in High-TechMärkten 16

4. Zielsetzung und Gang der Untersuchung 27

B. Typologisierung von Marketingstrategien in High-Tech-Märkten auf der Grundlage strategischer Gruppen

1. Theoriegeleitete Analyse strategischer Gruppen in High-TechMärkten

1.1 Grundlagen zum Konzept der strategischen Gruppen .32

1.2 Übertragung des Konzeptes der strategischen Gruppen auf High-Tech-Märkte

2. Theoriegeleitete Analyse von Determinanten zur Bildung strategischer Gruppen in High-Tech-Märkten

2.1 Kennzeichnung der Unternehmensumwelt und Ableitung zentraler Kontextdimensionen zur Bildung strategischer Gruppen in High-Tech-Märkten 52

2.11 Technologiebezogene Umweltsituation .53

2.12 Marktbezogene Umweltsituation .58

2.13 Abnehmerbezogene Umweltsituation 60

2.14 Konkurrenzbezogene Umweltsituation 
Seite

2.2 Ansätze zur Systematisierung von Marketingstrategien in

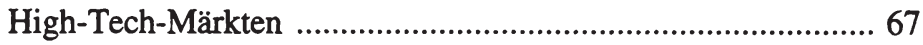

2.21 Zeit- und technologieorientierte Strategieansätze ............. 69

2.22 Wettbewerbsorientierte Strategieansätze ......................... 72

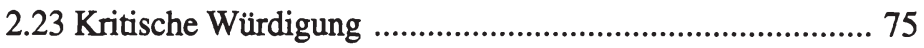

2.3 Ableitung zentraler Strategiedimensionen zur Bildung strategischer Gruppen in High-Tech-Märkten ......................... 76

2.31 Marktfeldbezogene Strategiedimensionen ....................... 76

2.32 Wettbewerbsbezogene Strategiedimensionen ................... 84

2.33 Timingbezogene Strategiedimensionen ........................... 99

3. Empirische Analyse strategischer Gruppen in High-Tech-Märkten .. 106 3.1 Design der empirischen Untersuchung ...................................107

3.2 Identifikation von Basisstrategien in High-Tech-Märkten ........ 109

3.3 Identifikation homogener Situationscluster in High-Tech-

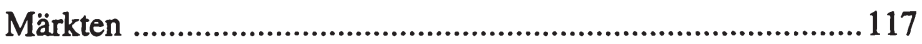

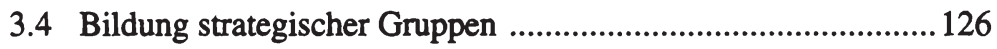

C. Ausgestaltungsformen und Einflußfaktoren der Marketingstrategien von strategischen Gruppen in High-Tech-Märkten

1. Instrumentelle Ausrichtung der Marketingstrategien von strategischen Gruppen in High-Tech-Märkten 138

1.1 Unternehmensaktivitäten im Marketing-Mix-Bereich 138

1.11 Ansatzpunkte zur Ausgestaltung von Marketingstrategien im Marketing-Mix-Bereich

1.12 Empirische Analyse der Marketingmaßnahmen von strategischen Gruppen 
1.2 Unternehmensaktivitäten zur Verkürzung der Entwicklungszeiten und Beschleunigung der Vermarktung von High-TechProdukten

1.21 Ansatzpunkte zur Verkürzung der Entwicklungszeiten und Beschleunigung der Vermarktung von High-TechProdukten

1.22 Empirische Analyse der Maßnahmen zur Verkürzung der Entwicklungszeiten und Beschleunigung der Vermarktung von High-Tech-Produkten von strategischen Gruppen

2. Unternehmensbezogene Einflußfaktoren der Marketingstrategien von strategischen Gruppen in High-Tech-Märkten

2.1 Unternehmensziele als Einflußfaktoren der strategischen Gruppen

2.11 Theoretische Grundlagen zum Zusammenhang zwischen Unternehmenszielen und Marketingstrategien von strategischen Gruppen

2.12 Empirische Analyse der Unternehmensziele von strategischen Gruppen

2.2 Unternehmenskultur als Einflußfaktor der strategischen Gruppen

2.21 Theoretische Grundlagen zum Zusammenhang zwischen Unternehmenskultur und Marketingstrategien von strategischen Gruppen

2.22 Empirische Analyse der Unternehmenskultur von strategischen Gruppen 
3. Analyse des Markterfolges der Marketingstrategien von strategischen Gruppen in High-Tech-Märkten

3.1 Theoretische Grundlagen zum Markterfolg der Marketingstrategien von strategischen Gruppen

3.2 Empirische Analyse des Markterfolges von strategischen Gruppen

D. Zusammenfassung und Implikationen

1. Zusammenfassende Würdigung der Untersuchungsergebnisse

2. Implikationen für das Management in High-Tech-Unternehmen ......210

3. Implikationen für die weiterführende Forschung zum Konzept der strategischen Gruppen

Anhang I: Studien zum Untersuchungsgegenstand ..................................217

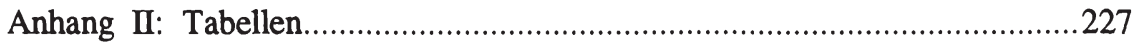

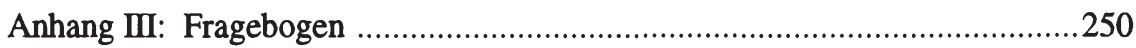

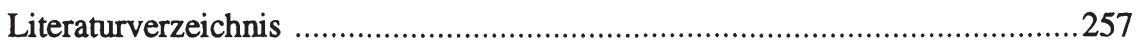




\section{Abbildungsverzeichnis}

Seite

Abb. 1: Strategische Herausforderungen in High-Tech Märkten 4

Abb. 2: Allgemeiner Bezugsrahmen zur Systematisierung der Analyseschritte des Forschungsvorhabens .28

Abb. 3: Bezugsrahmen der empirischen Untersuchung ................................30

Abb. 4: Dreidimensionale Abgrenzung ausgewählter High-Tech-Märkte .....38

Abb. 5: Übersicht ausgewählter empirischer Studien zur Bildung strategischer Gruppen

Abb. 6: Übersicht ausgewählter empirischer Studien zur Bildung strategischer Gruppen (Fortsetzung)

Abb. 7: Übersicht ausgewählter empirischer Studien zur Bildung strategischer Gruppen (Fortsetzung)

Abb. 8: Technologiedynamik in High-Tech-Märkten .................................55

Abb. 9: Ausgewählte High-Tech-Märkte im Marktlebenszyklus .................59

Abb. 10: Typische Wettbewerbssituationen in High-Tech-Märkten ...............65

Abb. 11: Determinanten der spezifischen Unternehmenssituation in HighTech-Märkten

Abb. 12: Zentrale zeit- und technologieorientierte Ansätze zur Systematisierung von Marketingstrategien in High-Tech-Märkten

Abb. 13: Zentrale wettbewerbsorientierte Ansätze zur Systematisierung von Marketingstrategien in High-Tech-Märkten

Abb. 14: Entwicklungsdynamik bei Mikroprozessoren 
Abb. 15: Prognose der Entwicklung ausgewählter Produkte und Anwendungen aus dem Bereich der Informations- und Kommunikationstechnologie 89

Abb. 16: Abgrenzung strategischer Timingoptionen 101

Abb. 17: Übersicht wettbewerbsrelevanter Strategiedimensionen in HighTech-Märkten 105

Abb. 18: Verteilung der Unternehmen auf die High-Tech-Branchen 108

Abb. 19: Verteilung der High-Tech-Unternehmen nach der Unternehmensgröße 108

Abb. 20: Faktoranalytisch verdichtete Basisstrategien in High-TechMärkten

Abb. 21: Stabilitätstest für die Basisstrategien in High-Tech-Märkten 116

Abb. 22: Beschreibung der Situationscluster in High-Tech-Märkten

Abb. 23: Marktwachstum in den Situationsclustern

Abb. 24: Produktlebenszyklen in den Situationsclustern

Abb. 25: Verteilung der Marktwachstumsphasen in den Situationsclustern .. 123

Abb. 26: Verteilung der High-Tech-Branchen in den Situationsclustern

Abb. 27: Übersicht der strategischen High-Tech-Gruppen in den einzelnen Umfeldsituationen 128

Abb. 28: Beschreibung der strategischen High-Tech-Gruppen in den Umfeldsituationen

Abb. 29: Beurteilung der Trennschärfe der strategischen High-TechGruppen anhand ausgewählter Prüfkriterien 
Abb. 30: Darstellung der High-Tech-Gruppen im dreidimensionalen Raum.. 136

Abb. 31: Bedeutung der Marketinginstrumente für die strategischen High-

Tech-Gruppen in den Umfeldsituationen

Abb. 32: Bedeutung der Maßnahmen zur Verkürzung der Entwicklungszeiten und Beschleunigung der Vermarktung für die strategischen High-Tech-Gruppen in den Umfeldsituationen

Abb. 33: Bedeutung der Unternehmensziele für die strategischen HighTech-Gruppen in den Umfeldsituationen

Abb. 34: Bedeutung der Kulturdimensionen für die strategischen HighTech-Gruppen in den Umfeldsituationen

Abb. 35: Durchschnittliche Umsatzveränderung der strategischen HighTech-Gruppen innerhalb der Umfeldsituationen in den Jahren 1988-1990

Abb. 36: Durchschnittliche Marktanteile der strategischen High-TechGruppen innerhalb der Umfeldsituationen im Jahr 1990

Abb. 37: Relative durchschnittliche Umsatzveränderung der strategischen High-Tech-Gruppen innerhalb der Umfeldsituationen in den Jahren 1988-1990

Abb. 38: Relative durchschnittliche Marktanteile der strategischen HighTech-Gruppen innerhalb der Umfeldsituationen im Jahr 1990

Abb. 39: Mittelwertvergleichstest der relativen Erfolgsgrößen 196

Abb. 40: Situationsübergreifende Gemeinsamkeiten der strategischen High-Tech-Gruppen 205

Abb. 41: Situationsübergreifende Unterschiede der strategischen HighTech-Gruppen .206 


\section{Abkürzungsverzeichnis}

a.a.O.

Abb.

a.L.

a.M.

asw

Aufl.

bit

Bd.

BddW

BMFT

BMW

BTX

bzw.

ca.

CIM

CIO

Conn.

DBW

DEC

DM

d.h.

Diss.

DOB

DRAM

EDV

EPA

erw. Aufl.

et al.

etc.

e.V.

f., ff.

FAH

FAZ

F\&E am angegebenen Ort

Abbildung

am Lech

am Main

Absatzwirtschaft

Auflage

binary digit

Band

Blick durch die Wirtschaft

Bundesministerium für Forschung und Technologie

Bayerische Motoren Werke

Bildschirmtext

beziehungsweise

circa

Computer Integrated Manufacturing

Computer Integrated Office

Connecticut

Die Betriebswirtschaft

Digital Equipment Corporation

Deutsche Mark

das heißt

Dissertation

Damenoberbekleidung

Dynamic random access memory

Elektronische Datenverarbeitung

Economic Planning Institut

erweiterte Auflage

et alteri

et cetera

eingetragener Verein

folgende, fortfolgende

Forschungsinstitut für Absatz und Handel

Frankfurter Allgemeine Zeitung

Forschung und Entwicklung 
ggf.

ggü.

GMD

$\mathrm{GmbH}$

$\mathrm{H}$

Habil.

Hrsg.

i.d.R.

i.e.S.

Ifo

III.

IMEDE

Inc.

i.S.

ISI

IT

I+T

i.w.S.

Jg.

Kap.

Mass.

Mio.

MIT

Mrd.

MSA

N.J.

N.Y.

No.

Nr.

n.s.

o.g.

o. Jg.

$\mathrm{OH}$

o.O.

o.S. gegebenenfalls

gegenüber

Gesellschaft für Mathematik und Daten-

verarbeitung

Gesellschaft mit beschränkter Haftung

Hypothese

Habilitation

Herausgeber

in der Regel

im engeren Sinne

Institut für Wirtschaftsforschung

Illinois

International Management Development Institute

Incorporation

im Sinne

Fraunhofer Institut für Systemtechnik und

Innovationsforschung

Informationstechnologien

Investitionsgüter und Technologie

im weiteren Sinne

Jahrgang

Kapitel

Massachusetts

Millionen

Massachusetts Institute of Technology

Milliarden

Measure of sampling adequacy

New Jersey

New York

Number

Nummer

nicht signifikant

oben genannt

ohne Jahrgang

Ohio

ohne Ortsangabe

ohne Seitenangabe 
o.V.

PC

PIMS

$\mathrm{R}+\mathrm{D}$

ROI

S.

sog.

Sp.

SPSS

Stck.

Tab.

Tsd.

TV

u.a.

überarb.

unver.

USA

usw.

u.U.

vgl.

vollst.

Vol.

vs.

WiSt

WISU

W\&V

z.B.

ZfB

$\mathrm{ZfbF}$

ZFO

ZFP

z.T. ohne Verfasser

Personal Computer

Profit Impact of Market Strategies

Research and Development

Return on Investment

Seite

sogenannt

Spalte

Statistical Package for the Social Sciences

Stuick

Tabelle

Tausend

Television

und andere, unter anderem

überarbeitet

unveröffentlicht

United States of America

und so weiter

unter Umständen

vergleiche

vollständig

Volume

versus

Wirtschaftswissenschaftliches Studium

Das Wirtschaftsstudium

Werben und Verkaufen

zum Beispiel

Zeitschrift für Betriebswirtschaft

Zeitschrift für betriebswirtschaftliche

Forschung

Zeitschrift für Organisation

Zeitschrift für Forschung und Praxis

zum Teil 


\section{A. Stellenwert von Marketingstrategien für die strategische Unternehmensführung in High-Tech-Märkten}

\section{Besonderheiten von High-Tech-Märkten aus wettbewerbsstrategischer Sicht}

Zur Erschließung neuer Wachstumschancen treten seit Beginn der 80er Jahre immer mehr Unternehmen mit technologischen Innovationen in sog. High-TechMärkte ein. ${ }^{1}$ Die Nettoproduktion von High-Tech-Produkten ist dabei in der Bundesrepublik Deutschland im Zeitraum 1980 (Index 100) bis 1992 um ca. $70 \%$ gestiegen, während der Produktionszuwachs bei Low-Tech-Produkten lediglich $20 \%$ betrug. ${ }^{2}$ Im Jahr 1997 wird für den schnell wachsenden Bereich der Mikroelektronik ein Marktvolumen von $22 \mathrm{Mrd}$. DM prognostiziert. ${ }^{3}$ Neben dem Chancenaspekt werden in zunehmendem Maße die besonderen Herausforderungen und Probleme bei der Sicherung von Wettbewerbsvorteilen in den sich dynamisch entwickelnden High-Tech-Märkten zum Gegenstand der praxisbezogenen und betriebswirtschaftlichen Diskussion. ${ }^{4}$

1 Vgl. Meffert, H., Marketing-Management, Analyse, Strategie, Implementierung, Wiesbaden 1994, S. 205; Meffert, H., Entwicklungslinien des Marketing, Akzente der marktorientierten Unternehmensfuhrung in den 90er Jahren, in: Jahrbuch Marketing, Hrsg.: Schöttle, K.M., 5. Aufl., Wiesbaden 1990, S. 13.

Vgl. Böndel, B., Innovationsklima: Ära der Systeme, in: Wirtschaftswoche, 1994, Nr. 3, S. 71.

3 Arbeitsgruppe der Industrie des IT-Gesprächskreises "Mikroelektronik", Konsenspapier der Industrie zum Stand der Mikroelektronik in Deutschland mit Vorschlägen zur Sicherung der Wettbewerbsfähigkeit der deutschen Halbleiterunternehmen, deren Kunden und Zulieferer, o.O. 1993, o.S.

Vgl. Milling, P., Schwierigkeiten bei der Umsetzung des neuen Wissens, Serie: Standort Deutschland (2), in: BddW, 1993, Nr. 147, S. 7; Benkenstein, M., Strategisches Marketing-Management in High-Tech-Branchen, in: Thexis, 1992, Nr. 1, S. 9 ff.; Meffert, H., Lamnek, S., Maisberger, P., High-Tech-Marketing, Branchenspezifische Trends und Strategien für die 90er Jahre, Düsseldorf 1991, S. 6 ff.; Perillieux, R., Wittkemper, G., Ziele und Module eines integrierten Technologie- und Innovationsmanagements, in: Integriertes Technologie- und Innovationsmanagement, Konzepte zur Stärkung der Wettbewerbskraft von High-Tech-Unternehmen, Hrsg.: Booz, Allen \& Hamilton, Berlin 1991, S. 13 ff. 
Für eine Analyse der Besonderheiten, mit denen sich Unternehmen in diesen Märkten auseinandersetzen müssen, wird zunächst eine Konkretisierung von High-Tech-Märkten über eine Präzisierung des Begriffs "High-Technology" vorgenommen. In der Literatur sind hierbei unterschiedliche Definitionsansätze zu finden, die insbesondere drei Schwerpunkte erkennen lassen:

Deskriptive, produktbezogene Ansätze umschreiben High-Tech-Märkte durch eine Aufzählung von Technologien bzw. Produkten. Ein Defizit dieser Ansätze ist darin zu sehen, daß die Technologiedynamik zu einer Erweiterung oder Einengung des Begriffsinhaltes im Zeitablauf führt. ${ }^{5}$

An zweiter Stelle sind Definitionsansätze zu nennen, die sich an der Charakteristik von Technologien orientieren. ${ }^{6}$ Als High-Technologie werden solche Technologien eingeordnet, deren Leistungsdaten sich in kurzen Zeitabständen massiv verändern und dadurch traditionelle Technologien in schneller Folge substituieren. ${ }^{7}$ Diese Kennzeichnung ist insbesondere aus wettbewerbsstrategischer Sicht interessant, da bereits auf Marktwirkungen in Form von Substitutionsprozessen Bezug genommen wird.

Eine dritte Konkretisierung erfolgt auf der Basis angebotsbezogener Merkmale von Unternehmen. In diesem Zusammenhang hat das amerikanische Bureau of Labor Statistics drei graduell abgestufte Definitionen zur Kennzeichnung von High-Tech-Unternehmen erstellt. ${ }^{8}$ Die erste Definition erfolgt unter Bezugnahme auf die Forschungsintensität, gemessen am Aufwand für F\&E-Vorhaben. Hiernach werden Unternehmen aus den Bereichen Luft- und Raumfahrt, elektrotechnische Komponenten, Computer und Informationsübermittlung dem High-Tech-

5 Vgl. Rüdiger, B., Schlüsseltechnologien: Die Herausforderungen für die 90er Jahre, in: Technologie-Marketing, Hrsg.: Töpfer, A., Sommerlatte, T., Landsberg a.L. 1991, S. 37 ff.; Sommerlatte, T., Deschamps, J.-Ph., Der strategische Einsatz von Technologien, in: Management im Zeitalter der strategischen Führung, Hrsg.: Little, A.D., Wiesbaden 1985, S. $49 \mathrm{ff}$.

6 In diesem Zusammenhang wird unter einer Technologie die Kenntnis naturwissenschaftlich-technischer Zusammenhänge verstanden, durch die technisch bedingte Innovationsprobleme gelöst werden. Vgl. Dussauge, P., Hart, St., Ramanantsoa, B., Strategic technology management, Chichester u.a. 1992, S. 6 ff.; Perillieux, R., Der Zeitfaktor im strategischen Technologie-Management, Berlin 1987, S. 12.

7 Vgl. Wolfrum, B., Technologiestrategien im strategischen Management, in: Marketing ZFP, 1992, Nr. 1, S. 24; Shanklin, W.L., Ryans, J.K., Essentials of marketing high technology, Lexington, Mass., Toronto 1987, S. 164; Zörgiebel, W.W., Technologie in der Wettbewerbsstrategie, Berlin 1983, S. 11 f.; Davis, R.T., Smith, F.G., Marketing in emerging companies, Reading, Mass. 1984, S. 90.

Vgl. Bleicher, K., Chancen für Europas Zukunft, Wiesbaden 1989, S. 342 f.; o.V., High Tech, High tech can be anywhere you define it, in: Wall Street Journal, 4th of January 1984, S. 3. 
Bereich zugerechnet. In zwei erweiterten Abgrenzungen werden alle Unternehmen berücksichtigt, deren F\&E-Aufwendungen im Verhältnis zum Umsatz bzw. deren Anzahl wissenschaftlicher Mitarbeiter doppelt so hoch sind wie der Industriedurchschnitt. ${ }^{9}$

Zusammenfassend läßt sich festhalten, daß die Unternehmen aus einer primär angebotsorientierten Sicht über eine prozeß- und ergebnisbezogene Charakteristik den High-Tech-Märkten zugeordnet werden. Der F\&E-Prozeß von High-TechUnternehmen weist einerseits eine hohe Intensität auf. Andererseits substituieren die aus dem Innovationsprozeß resultierenden Technologien aufgrund ihres Leistungsvorsprungs traditionelle Technologien.

Ausgehend von dieser angebotsbezogenen Betrachtung besitzt die Analyse der Besonderheiten und Herausforderungen in High-Tech-Märkten auch unter Einbeziehung nachfrager- und konkurrenzbezogener Merkmale eine große Bedeutung. Dies liegt darin begründet, daß Wettbewerbsvorteile in High-TechMärkten nur bei einer hinreichenden Kenntnis der spezifischen technologie- und marktteilnehmerbezogenen Gegebenheiten erzielt werden können. Hierzu gilt es, wesentliche strategische Herausforderungen zu kennzeichnen, denen Unternehmen in High-Tech-Märkten bei der Identifikation und dem Aufbau von Wettbewerbsvorteilen gegenüberstehen (vgl. Abbildung 1). ${ }^{10}$

Vgl. Moriarty, R.T., Kosnik, Th.J., High-Tech marketing: Concepts, continuity and change, in: Sloan Management Review, 1989, S. 8; Remmerbach, K.U., Markteintrittsentscheidungen, Wiesbaden 1988, S. 134 f.; Beckurts, K.H., Chancen für einen zweiten Aufbruch, in: Manager Magazin, 1984, Nr. 9, S. 154 ff.

Vgl. Baaken, Th., Technologie-Marketing, in: Investitionsgütermarketing, Hrsg.: Kliche, M., Wiesbaden 1990, S. 293 ff.; Moriarty, R.T., Kosnik, Th.J., High-Tech marketing: Concepts, continuity, and change, a.a.O., S. 8 ff.; Meffert, H., Remmerbach, K.U., Marketingstrategien in jungen Märkten, in: DBW, 1988, Nr. 3, S. 332 ff. 


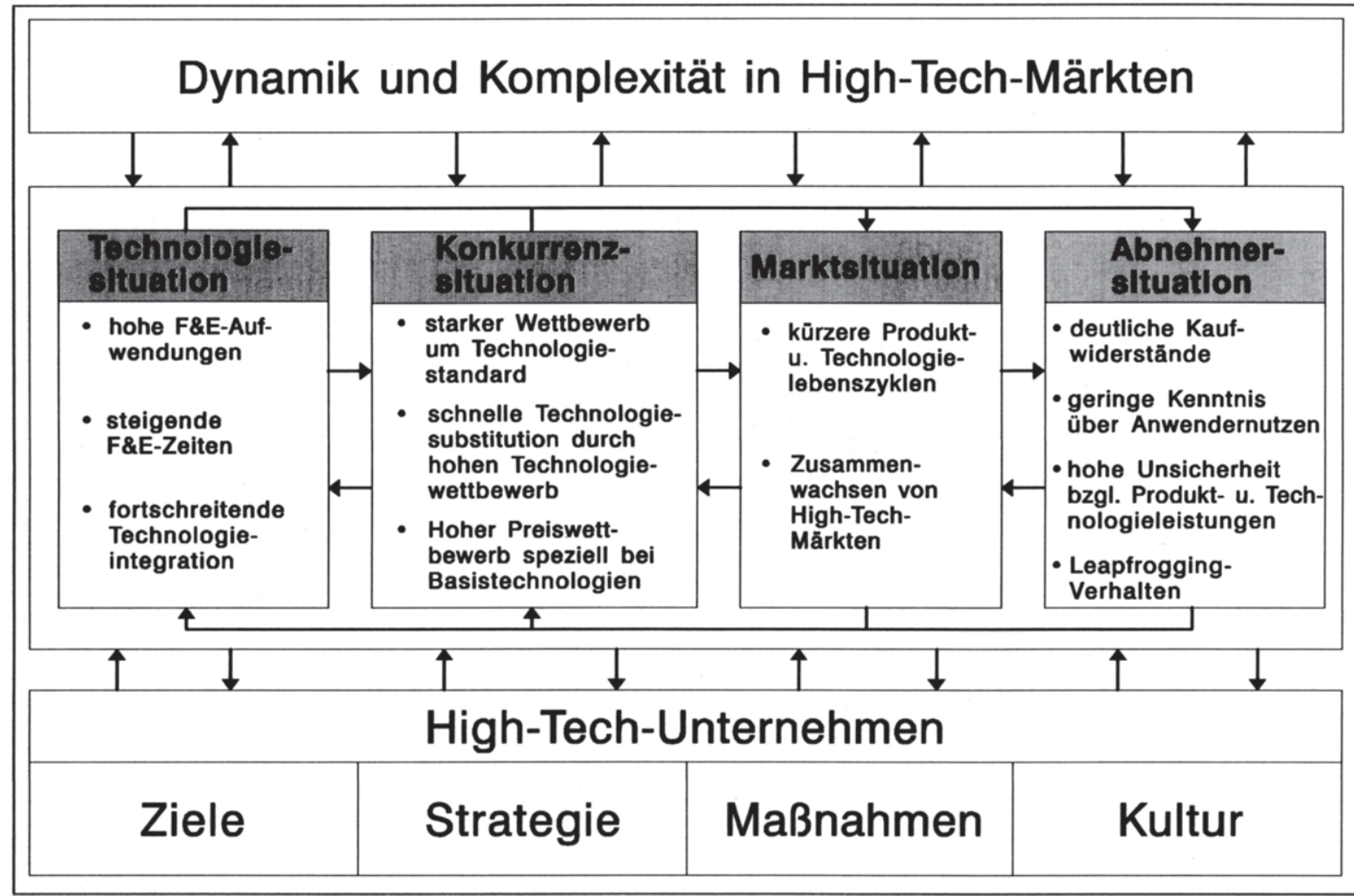

Abb 1: Strategische Herausforderungen in High-Tech-Mårkten 
Aus angebotsorientierter Sicht ist zunächst hervorzuheben, daß die Erzielung von Wettbewerbsvorteilen in High-Tech-Märkten nur mit Hilfe einer überdurchschnittlichen F\&E-Intensität möglich ist. ${ }^{11}$ Aus dem hohen Investitionsvolumen und dem starken Zeitdruck zur Hervorbringung von Technologieinnovationen resultiert ein erhebliches Entwicklungsrisiko. Da alle Unternehmen in HighTech-Märkten intensive F\&E-Aktivitäten betreiben, konkurrieren i.d.R. alternative Technologien zu ähnlichen Zeitpunkten um die Durchsetzung als Standard und unterliegen durch die laufende Entwicklung neuer bzw. verbesserter Technologien einer schnellen Substitution. ${ }^{12}$ Die Möglichkeit der Erreichung einer dauerhaften Vorteilsposition gegenüber der Konkurrenz ist deshalb als besonders schwierig einzustufen.

Aufgrund der Technologiekomplexität sind neben höheren Investitionen häufig auch längere Zeiten zur Entwicklung von High-Tech-Produkten erforderlich. Gleichzeitig haben sich die Produkt- und Technologielebenszyklen stark verkürzt, wie z.B. im Kommunikations- und Mikroelektronikbereich. ${ }^{13}$ Für die High-TechUnternehmen resultiert hieraus neben dem hohen Entwicklungsrisiko auch ein hohes Vermarktungsrisiko, da sie gezwungen sind, die hohen F\&EAufwendungen in immer kürzeren Zeiten zu amortisieren. Um technologische Innovationen in kürzeren F\&E-Zyklen hervorzubringen, sind oftmals technologische Quantensprünge notwendig, die nicht nur marginale, sondern grundlegende Veränderungen der Leistungsdaten bewirken. ${ }^{14}$

11 Vgl. Knorr, J., High-Tech-Marketing in der Mikroelektronik: Erfahrungen aus dem Mega-Projekt, in: Technologie-Marketing, Hrsg.: Töpfer, A., Sommerlatte, T., Landsberg a.L. 1991, S. 342; McKenna, R., Market positioning in high technology, in: California Management Review, Vol. 27 (1985), No. 3, S. 93.

Vgl. Pfeiffer, St., Technologie-Frühaufklärung, Hamburg 1992, S. 79 ff.; Porter, M., Wettbewerbsstrategie, Frankfurt a.M., New York 1988, S. 275; Walters, M., Marktwiderstände und Marketingplanung, Wiesbaden 1984, S. $15 \mathrm{ff}$.

Vgl. Backhaus, K, Auswirkungen kurzer Lebenszyklen bei High-Tech-Produkten, in: Thexis, 1991, Nr. 6, S. 11 f.; Benkenstein, M., Modelle technologischer Entwicklungen als Grundlage für das Technologiemanagement, in: DBW, 1989, Nr. 4, S. $498 \mathrm{ff}$.

Vgl. Henzler, H., Europreneurs: Europas Unternehmen melden sich zurück, Frankfurt a.M. 1992, S. 231 ff.; Backhaus, K., Investitionsgütermarketing, 3. Aufl., München 1992, S. 12. 
Als angebotsbezogene Besonderheit in High-Tech-Märkten ist auch die aus der Entwicklungsdynamik resultierende Technologieintegration hervorzuheben, die zur Fusion bisher getrennter Technologien führte. So hat sich beispielsweise im Bereich der Informationstechnologien eine Entwicklung von zentralen über dezentralen zu vernetzten Informationssystemen vollzogen, die auf der Anbieterund Nachfragerseite eine erhebliche Ausweitung des Angebots- und Anwendungsfeldes bewirkte. ${ }^{15}$

Durch die ständige Ausweitung des Produktnutzens i.d.S., daß heute ein einzelnes High-Tech-Produkt mehrere Funktionen erfullt, verschwimmen traditionelle Marktgrenzen. Hiermit sind gravierende Auswirkungen auf die Abgrenzung des relevanten Absatzmarktes verbunden. Einerseits entstehen durch neue Anwendungspotentiale synthetische Märkte, z.B. Automatisierungstechnik, Mikroelektronik, Biotechnologie. ${ }^{16}$ Andererseits verändern sich etablierte Marktstrukturen, und Unternehmen stehen häufig mit neuen Konkurrenten im Wettbewerb, die bestehende Wettbewerbsvorteile schnell erodieren lassen. Als Konsequenz ist eine zunehmende Wettbewerbsintensität in High-Tech-Märkten zu beobachten. So wurden beispielsweise die ursprünglich nicht in einem direkten Wettbewerb stehenden Elektronikunternehmen Bosch und Siemens durch Marktveränderungen in der Automobilelektronik und Kommunikationstechnik zu direkten Konkurrenten. $^{17}$

Vgl. ONeil, P., Proprietäre Systeme und isolierte PC-Stationen als Herausforderung: Im Büro bleibt für den Generalunternehmer noch viel zu tun, in: Computer Zeitung, 1993, Nr. 29, S. 19; Backhaus, K., Weiber, R., Technologieintegration und Marketing, Arbeitspapier Nr. 10 des Betriebswirtschaftlichen Instituts für Anlagen und Systemtechnologien, Münster 1988, S. $10 \mathrm{ff}$.

16 Vgl. ISI (Hrsg.), Technologie am Beginn des 21. Jahrhunderts, Diskussionspapier, Karlsruhe 1993, S. 25 ff.; Green, K., Creating demand for biotechnology, in: Technological change and company strategies, Hrsg.: Coombs, R., Saviotti, P., Walsh, V., London u.a. 1992, S. $172 \mathrm{ff}$.

17 Vgl. Meffert, H., Strategische Unternehmensfuihrung und Marketing, Wiesbaden 1988, S. 59; Bühner, R., Technologieorientierung als Wettbewerbsstrategie, in: ZfbF, 1988, Nr. 5, S. 394 f. 
Aufgrund spezifischer nachfragebezogener Faktoren lassen sich weitere wettbewerbsstrategische Besonderheiten in High-Tech-Märkten herausstellen. ${ }^{18}$ Generell bildet die Prognose des Nachfrageverhaltens ein besonderes Problem. Aufgrund der Entwicklungsdynamik der High-Tech-Märkte, der Technologiekomplexität und der Verfügbarkeit alternativer Technologien ist das Nachfrageverhalten durch eine hohe Verunsicherung gekennzeichnet, die sich oftmals in Kaufwiderständen niederschlägt. ${ }^{19}$

Die Ursachen für die mangelnde Akzeptanz von High-Tech-Produkten sind einerseits in einem noch nicht klar definierten Anwendernutzen begründet, d.h. Wichtigkeit und Wahrnehmbarkeit des Leistungsvorteils neuer Technologien sind aus Kundensicht nicht immer gegeben. Indem die High-Tech-Unternehmen häufig sog. "Technology Push"-Innovationen ohne genügende Berücksichtigung nachfragebezogener Anforderungen im Markt einführen, sind zahlreiche Produktmodifikationen und anwenderbezogene Applikationen zur Erreichung einer hinreichenden Marktakzeptanz notwendig. ${ }^{20}$

Durch die zunehmende Technologieintegration werden andererseits ganze Systemtechnologien angeboten, die modulweise aufgebaut sind. So setzen sich z.B. Computer Integrated Manufacturing-Systeme (CIM) aus planungs-, (Computer Aided Design, Planning und Engineering) produktions- (Computer Aided Manufacturing und flexible Fertigungssysteme) und kontrollbezogenen

18 Vgl. Meldrum, M.J., Millmann, A.F., Ten risks in marketing high-technology products, in: Industrial Marketing Management, Vol. 20 (1991), S. 43 ff.; Schaible, J., Hönig, A., High-Tech-Marketing in der Praxis, München 1991, S. 8 ff.; MacInnis, M., Heslop, L.A., Market planning in a high-tech environment, in: Industrial Marketing Management, Vol. 19 (1990), S. 114

Vgl. Weiss, P., Die Kompetenz von Systemanbietern: Ein neuer Ansatz im Marketing für Systemtechnologien, Berlin 1992, S. 32 ff.; Töpfer, A., Marketing für Start-upGeschäfte mit Technologieprodukten, in: Technologie-Marketing, Hrsg.: Töpfer, A., Sommerlatte, T., Landsberg a.L. 1991, S. 171 ff.; Walters unterscheidet zwischen produkt- und herstellerbezogenen Kaufwiderständen. Vgl. Walters, M., Marktwiderstände und Marketingplanung, a.a.O., S. $51 \mathrm{ff}$. u. $155 \mathrm{ff}$. 
(Computer Aided Quality) Teilsystemen zusammen. ${ }^{21}$ Vielfach befinden sich einzelne Systemmodule noch in der Entwicklungsphase, so daß ein Nachfrager beim Einstieg in ein entsprechendes System noch nicht in der Lage ist, die Leistungsvorteile des Gesamtsystems zu überprüfen. In diesem Fall kann ein Nachfrager lediglich auf die Leistungsfähigkeit des High-Tech-Anbieters vertrauen. ${ }^{22}$ Hieraus resultiert eine hohe leistungsbezogene Unsicherheit, inwieweit der Anbieter die weiteren Systemkomponenten qualifiziert entwickeln kann und welchen Beitrag die Systemtechnologien zur Problemlösung tatsächlich leisten. ${ }^{23}$

Die vorhandenen Kaufwiderstände führen teilweise zu einem sog. LeapfroggingVerhalten in High-Tech-Märkten. Nachfrager, auf die ein LeapfroggingVerhalten zutrifft, überspringen bewußt eine Technologiegeneration und verlagern die Kaufentscheidung auf zukünftig erwartete Technologiegenerationen. ${ }^{24}$ Dieses Kaufverhalten kann zudem durch den rapiden Preisverfall von High-TechProdukten verstärkt werden, der gegenwärtig bei Personal Computern, Anrufbeantwortern und mobilen Telefonen festzustellen ist. ${ }^{25}$

Die aufgezeigten Besonderheiten von High-Tech-Märkten machen deutlich, daß in diesen Märkten eine hohe Unsicherheit bezüglich der markt-, technologie-,

21 Vgl. Meffert, J., Standards als Integrationsinstrument in der Computer- und Kommunikationsindustrie, Konstanz 1994, S. 67 ff.; Backhaus, K., Investitionsgütermarketing, a.a.O., S. 350 f.

22 Zur Unterscheidung zwischen Vertrauens-, Erfahrungs- und Inspektionsgütern siehe z.B. Backhaus, K., Systemgeschäfte verändern Marktprozesse, in: asw, 1992, Nr. 8, S. 61; Weiss, P., Die Kompetenz von Systemanbietern: Ein neuer Ansatz im Marketing für Systemtechnologien, a.a.O., S. 57 f.

23 Vgl. Wieandt, A., Zum Marketing von Innovationen, in: Marketing ZFP, 1994, Nr. 1, S. 22 ff.; Weiss, P., Die Kompetenz von Systemanbietern: Ein neuer Ansatz im Marketing für Systemtechnologien, a.a.O., S. 40 ff.; Backhaus, K., Weiber, R., Systemtechnologien - Herausforderung des Investitionsgütermarketing, in: Harvard Manager, 1987, Nr. 4, S. $78 \mathrm{f}$.

24 Vgl. Weiber, R., Leapfrogging-Behavior: Herausforderung für das MarketingManagement neuer Technologien, Beitrag zur Hochschullehrertagung, Stuttgart 1993, o.S.

25 Vgl. Meffert, J., Standards als Integrationsinstrument in der Computer- und Kommunikationsindustrie, a.a.O., S. 103 ff.; Maringer, A., Preisverfall mikroelektronischer Bauelemente am Beispiel DRAM-Speicherbauelemente, in: ZfbF, 1990, Nr. 4, S. $423 \mathrm{ff}$. 
abnehmer- und konkurrenzbezogenen Entwicklung besteht. Für High-TechUnternehmen ergeben sich hieraus besondere Herausforderungen im Hinblick auf den Aufbau und die Sicherung einer strategischen Wettbewerbsposition, die insbesondere bei der Entwicklung von Marketingstrategien in High-TechMärkten zu berücksichtigen sind. Vor diesem Hintergrund werden im folgenden Abschnitt Stellenwert und Inhalte von Marketingstrategien in High-Tech-Märkten näher untersucht.

\section{Gegenstand und Bedeutung von Marketingstrategien in High-Tech- Märkten}

Seit Anfang der 80er Jahre werden in der wissenschaftlichen Forschung und Unternehmenspraxis verstärkt Fragestellungen bezüglich der Planung und Festlegung von Marketingstrategien diskutiert. ${ }^{26}$ Angesichts der Tatsache, daß in der Literatur ein weit auseinandergehendes Verständnis zum Begriff der Marketingstrategie vorherrscht, ist für eine Analyse von Marketingstrategien in High-TechMärkten eine Präzisierung und Eingrenzung des Strategiebegriffs erforderlich. ${ }^{27}$ In diesem Zusammenhang bietet es sich aus managementorientierter Sicht an, Marketingstrategien hinsichtlich ihrer Planungsebene einer näheren Betrachtung zu unterziehen. ${ }^{28}$

Vgl. z.B. Becker, J., Marketing-Konzeption, Grundlagen des strategischen MarketingManagement, 5. Aufl., München 1992, S. 111 ff.; Meffert, H., Marketing, Grundlagen der Absatzpolitik, 7. Aufl., Wiesbaden 1986, S. 90 ff. Neben der Strategieformulierung bilden die Strategieimplementierung und methodische Aspekte der Strategieplanung weitere Betrachtungsschwerpunkte. Siehe hierzu u.a. Meffert, H., MarketingManagement, a.a.O., S. 17 f.; Hilker, J., Marketingimplementierung, Konzeptionelle Analysen und Umsetzung am Beispiel ostdeutscher Unternehmen, Diss., Münster 1993, S. $7 \mathrm{ff}$.

Vgl. Raffée, H., Effenberger, J., Fritz, W., Strategieprofile als Faktoren des Unternehmenserfolges, Arbeitspapier Nr. 93 des Instituts für Marketing, Hrsg.: Dichtl, E., Geist, M., Raffée, H., Mannheim 1992, S. 16; Wüthrich, H.A., Neuland des strategischen Denkens, Wiesbaden 1991, S. 12 ff.; Steffenhagen, H., Der Strategiebegriff in der Marketingplanung, Arbeitspapier Nr. 29 des Instituts für Marketing der Universität Münster, Hrsg.: Meffert, H., Münster 1982, S. 5 ff.

Vgl. zu dieser Einschätzung auch Meffert, H., Marketing-Management, a.a.O., S. 24 f.; Westphal, J., Vertikale Wettbewerbsstrategien in der Konsumgüterindustrie, Wiesbaden 1991, S. 72 ff.; Steffenhagen, H., Der Strategiebegriff in der Marketingplanung, a.a.O., S. 57 ff.; Bourgeois, L.J., Strategy and environment - A conceptional integration, in: The 
Bei einem engeren Strategieverständnis lassen sich auf einer eher globalen Planungsebene die Unternehmensstrategie und Geschäftsfeldstrategien unterscheiden. ${ }^{29}$ In der Unternehmensstrategie kommt die Grundsatzentscheidung zum Ausdruck, in welchen Geschäftsfeldern ${ }^{30}$ das Unternehmen vertreten sein wird und wie die vorhandenen Unternehmensressourcen auf diese Geschäftsfelder zu verteilen sind. Unter Berücksichtigung der Marktattraktivität und der relativen Wettbewerbsstärke kommen für die Geschäftsfelder als Entwicklungsrichtungen primär die Wachstums-, die Behauptungs- und die Rückzugsstrategie in Betracht. ${ }^{31}$ Für Unternehmen, die in High-Tech-Märkten agieren, sind aufgrund des hohen Marktwachstums insbesondere wachstumsorientierte Strategien wie die Produktentwicklung, Marktentwicklung oder Diversifikation von Interesse. ${ }^{32}$

Durch Geschäftsfeldstrategien werden der Umfang der Marktabdeckung, die angestrebten Wettbewerbsvorteile und das Timing von Innovationen bestimmt. ${ }^{33}$ Im Hinblick auf die Marktabdeckung legt ein High-Tech-Unternehmen fest, ob

Academy of Management Review, Vol. 5 (1980), No. 1, S. 26 ff. Eine Systematisierung von Marketingstrategien kann auch über den Umfang der berücksichtigten Strategiedimensionen (partielle vs. integrative Ansätze), über den Zeitbezug (statische vs. dynamische Ansätze) und über den Kontextbezug (generische vs. situative Ansätze) erfolgen. Siehe hierzu z.B. Meffert, H., Marketing-Management, a.a.O., S. $107 \mathrm{ff}$;; Meffert, H., Marketing und strategische Unternehmensführung - ein wettbewerbsorientierter Kontingenzansatz, in: Strategische Unternehmensplanung, Hrsg.: Hahn, D., Taylor, B., Wien 1986, S. 675 ff.; Porter, M., Wettbewerbsstrategie, a.a.O., S. 62 ff. u. $247 \mathrm{ff}$. Hierauf wird in der vorliegenden Arbeit jedoch nicht weiter eingegangen.

Vgl. Bolz, J., Wettbewerbsorientierte Standardisierung der internationalen Marktbearbeitung, Darmstadt 1992, S. 24 f.; Hinterhuber, H., Wettbewerbsstrategie, 2. Aufl., Berlin, New York 1990, S. 54 ff.; Jain, S.C., Marketing planning and strategy, Cincinnati, OH 1985, S. $11 \mathrm{f}$.

30 Zur Kennzeichnung von Geschäftsfeldern und Abgrenzung ggü. Geschäftseinheiten siehe Meffert, H., Marketing-Management, a.a.O., S. $41 \mathrm{ff}$.

31 Vgl. z.B. Westphal, J., Vertikale Wettbewerbsstrategien in der Konsumgüterindustrie, a.a.O., S. 73 f.; Meffert, H., Marketing, Grundlagen der Absatzpolitik, a.a.O., S. 93 ff.

Vgl. o.V., PC-Markt wächst weltweit um 16 Prozent, in: Inside Multimedia, 1994, Nr. 4, S. 9. Das durchschnittliche Marktwachstum der untersuchten High-Tech-Märkte liegt bei ca. $14 \%$. Vgl. Meffert, H., Lamnek, S., Maisberger, P., High-Tech-Marketing, Branchenspezifische Trends und Strategien für die 90er Jahre, a.a.O., S. 23.

Vgl. Meffert, H., Marketing-Management, a.a.O., S. 123 f.; Bolz, J., Wettbewerbsorientierte Standardisierung der internationalen Marktbearbeitung, a.a.O., S. 25; Czepiel, J.A., Competitive marketing strategy, Englewood Cliffs, N.J. 1992, S. 32 ff.; Porter, M., Wettbewerbsvorteile, Frankfurt a.M., New York 1989, S. 31 ff. 
es im Gesamtmarkt oder lediglich in einem Teilmarkt tätig sein wird. ${ }^{34}$ Die Konzentration auf eine Nische stellt für High-Tech-Anbieter ein hohes Unternehmensrisiko dar. Das dynamische Wachstum ursprünglich kleinerer High-TechNischen, wie z.B. der Markt für CD-ROM-Laufwerke ${ }^{35}$ und das häufige Zusammenwachsen traditionell getrennter High-Tech-Märkte kann durch den schnellen Eintritt neuer Konkurrenten zu einer abrupten Veränderung der Wettbewerbsbedingungen führen. ${ }^{36}$ Für High-Tech-Unternehmen ist es deshalb besonders schwierig, in einer Nische dauerhaft eine sichere Wettbewerbsposition aufzubauen.

Einen weiteren Schwerpunkt bildet die Frage, welche Wettbewerbsvorteile mit Geschäftsfeldstrategien in High-Tech-Märkten angestrebt werden sollen. ${ }^{37}$ Wettbewerbsvorteile lassen sich nur durch Unternehmensleistungen erzielen, die für den Kunden wichtige, wahmehmbare Leistungsmerkmale aufweisen und im Vergleich zur Konkurrenz dauerhaft überlegen sind. ${ }^{38}$ Ansatzpunkte hierzu werden grundsätzlich im Aufbau von Kosten- oder Differenzierungsvorteilen gesehen. ${ }^{39}$ Während die günstige Kostenposition i.d.R. über eine Niedrigpreispolitik an die

Vgl. z.B. Haedrich, G., Tomczak, Th., Strategische Markenfuihrung, Berlin, Stuttgart 1990, S. 110 ff.; Meffert, H., Marketing, Grundlagen der Absatzpolitik, a.a.O., S. 101 ff.; Freter, H., Marktsegmentierung, Stuttgart u.a. 1983, S. 109 ff.

So ist der Markt für CD-ROM-Laufwerke (Compact Disc Read Only Memory) im Zeitraum von 1991 bis 1993 von 36 auf 300 Tsd. Stck. angestiegen. Für das Jahr 1994 wird mit einem Marktvolumen von 800 Tsd. Stck. gerechnet. Vgl. o.V., Der rasche Siegeszug der glitzernden Scheibe, in: Werben \& Verkaufen, 1993, Nr. 39, S. 56 f.

Vgl. hierzu die Ausführungen im Teil A Kap. 1 und Teil B Kap. 1.2 der Arbeit.

In diesem Zusammenhang werden in der Literatur auch die Begriffe Wettbewerbsstrategie, wettbewerbsorientierte Marketingstrategie oder abnehmergerichtete Wettbewerbsstrategie verwendet. Siehe hierzu z.B. Meffert, H., Marketing-Management, a.a.O., S. 126 ff.; Porter, M., Wettbewerbsstrategie, a.a.O., S. 62 ff.

Vgl. Backhaus, K., Investitionsgütermarketing, a.a.O., S. 29 ff.; Simon, H., Management strategischer Wettbewerbsvorteile, in: Wettbewerbsvorteile und Wettbewerbsfähigkeit, Hrsg.: Simon, H., Stuttgart 1988, S. 2 ff. Im folgenden werden die Begriffe Wettbewerb und Konkurrenz synonym verwendet. Siehe hierzu Link, U., Strategische Konkurrenzanalyse im Konsumgütermarketing, Idstein 1988, S. 42.

Porter unterscheidet zudem, ob die Wettbewerbsvorteile auf einem Gesamt- oder Teilmarkt erzielt werden sollen. Porter, M., Wettbewerbsvorteile, a.a.O., S. $31 \mathrm{ff}$. In der vorliegenden Untersuchung wird die Frage der Marktabdeckung gesondert betrachtet, da sie allein keinen Wettbewerbsvorteil darstellt. Siehe hierzu auch Meffert, H., MarketingManagement, a.a.O., S. 123 f. 
Nachfrager weitergegeben wird, basieren Differenzierungsvorteile häufig auf einer besseren Produktqualität. Obwohl der auf Porter zurückgehende Ansatz in der Literatur und Praxis breiten Anklang gefunden hat, ${ }^{40}$ sind für eine Analyse von Marketingstrategien in High-Tech-Märkten vor allem zwei Aspekte zu berücksichtigen.

Einerseits ist davon auszugehen, daß Unternehmen in High-Tech-Märkten nicht nur eine Preis- oder Qualitätsorientierung verfolgen, sondern z.T. beide Strategiedimensionen miteinander kombinieren. ${ }^{41}$ Beispielhaft sind japanische Unternehmen der Mikroelektronik- bzw. Werkzeugmaschinenindustrie zu nennen, die in den letzten Jahren durch eine aggressive Preispolitik und Sicherstellung einer guten Produktqualität hohe Markterfolge erzielen konnten. Andererseits wird die Zahl wettbewerbsrelevanter Strategiedimensionen in High-TechMärkten größer sein als die von Porter vorgeschlagene Qualitäts-PreisDichotomie. ${ }^{42}$ Aufgrund der hohen Technologiedynamik und -komplexität in High-Tech-Märkten ist zu erwarten, daß Wettbewerbsvorteile auch durch eine starke Technologieorientierung realisiert werden können.

Innerhalb der Geschäftsfeldstrategien wird außerdem das Timing von Technologie- oder Produktinnovationen festgelegt. Ein High-Tech-Unternehmen hat dabei zu entscheiden, ob der Markteintritt über eine Pionier-, früher oder später Folgerposition stattfinden soll. ${ }^{43}$ Aufgrund steigender F\&E-Aufwendungen und

Vgl. stellvertretend Böcker, F., Marketing, 5. Aufl., Stuttgart 1994, S. 465 f.; Hax, A.C., Majluf, N.S., Strategisches Management, Ein integratives Konzept aus dem MIT, Frankfurt a.M., New York 1991, S. 50; Haedrich, G., Tomczak, Th., Strategische Markenfuhrung, a.a.O., S. 108 ff.; Meffert, H., Marketing, Grundlagen der Absatzpolitik, a.a.O., S. 105 f.

41 Vgl. Faulkner, D., Bowman, C., Generic strategies and congruent organizational structures: Some suggestions, in: European Management Journal, Vol. 10 (1992), S. 494 ff.; Meffert, H., Strategische Unternehmensfuhrung und Marketing, a.a.O., S. 42; Wright, P., Research notes and communications - A refinement of Porter's strategies, in: Strategic Management Joumal, Vol. 8 (1987), S. $93 \mathrm{ff}$.

Vgl. Miller, D., The generic strategy trap, in: The Journal of Business Strategy, 1992, No. 1, S. 37 ff.; Bolz, J., Wettbewerbsorientierte Standardisierung der internationalen Marktbearbeitung, a.a.O., S. $28 \mathrm{ff}$.

Vgl. Vidal, M., Wettbewerbsstrategien für Pionierunternehmen, Wiesbaden 1993, S. 2

ff.; Perillieux, R., Strategisches Timing von F\&E und Markteintritt bei innovativen 
gleichzeitig sinkender Technologie- bzw. Produktlebenszyklen besitzt die Wahl des Markteintrittszeitpunktes einen zentralen Stellenwert bei der Formulierung von Marketingstrategien in High-Tech-Märkten.

Bei einer weiterführenden Konkretisierung von Marketingstrategien können auf einer stärker detaillierten Planungsebene Marktteilnehmerstrategien und Marketinginstrumentestrategien voneinander abgegrenzt werden. ${ }^{44}$ Durch Marktteilnehmerstrategien ${ }^{45}$ werden insbesondere die Verhaltensweisen gegenüber wichtigen Abnehmern, Konkurrenten und Absatzmittlern fixiert. ${ }^{46}$ Marktteilnehmerbezogene Strategien können als Präzisierung von Geschäftsfeldstrategien verstanden werden. Die Preisfuihrerschaftsstrategie, verbunden mit einem späten Markteinstieg, läßt sich beispielsweise dahingehend konkretisieren, da $\beta$ eine abnehmergerichtete Preis-Mengen-, konkurrenzbezogene Konfrontations-, absatzmittlerorientierte Umgehungs- und anspruchsgruppenbezogene Anpassungsstrategie verfolgt wird. ${ }^{47}$ Im Rahmen der Gestaltung von Marktteilnehmerstrategien ist jedoch eine Vielzahl weiterer Strategiekombinationen möglich. ${ }^{48}$ Wenn darüber hinaus im Hinblick auf die Marktabdeckung mehrere Teilmärkte bearbeitet werden, führt dies häufig zur Entwicklung unterschiedlicher Marktteilnehmerstrategien für die einzelnen Marktsegmente.

Produkten, in: Integriertes Technologie- und Innovationsmanagement, Konzepte zur Stärkung der Wettbewerbskraft von High-Tech-Unternehmen, Hrsg.: Booz, Allen \& Hamilton, Berlin 1991, S. 23 ff.; Remmerbach, K.-U., Markteintrittsentscheidungen, a.a.O., S. $51 \mathrm{ff}$.

Vgl. Meffert, H., Marketing-Management, a.a.O., S. 123 f.; HeB, G., Marktsignale und Wettbewerbsstrategie, Stuttgart 1991, S. 110 ff.; Müller, W., Planung von MarketingStrategien, Die Entwicklung von Marketing-Strategien im strategischen MarketingPlanungsprozeß, Frankfurt a.M., Bern, New York 1986, S. 43 ff.

In der neueren Literatur wird diesbezüglich auch von Stakeholder-Strategien gesprochen. Siehe hierzu z.B. Savage, G.T., et al., Strategies for assessing and managing organizational stakeholders, in: Academy of Management, Vol. 5 (1991), S. 61 ff.; Freeman, E.R., Stratecic management, A stakeholder approach, Boston u.a. 1984, S. $126 \mathrm{ff}$.

Vgl. z.B. Meffert, H., Marketing-Management, a.a.O., S. 141 ff.; Heß, G., Marktsignale und Wettbewerbsstrategie, a.a.O., S. $235 \mathrm{ff}$.

Vgl. Benkenstein, M., Strategisches Marketing-Management in High-Tech-Branchen, a.a.O., S. 12 f.; Remmerbach, K.-U., Markteintrittsentscheidungen, a.a.O., S. 299 ff.

Vgl. z.B. Becker, J., Marketing-Konzeption, a.a.O., S. 311 ff.; Meffert, H., MarketingManagement, a.a.O., S. $123 \mathrm{ff}$. 
Die Entwicklung von Marketinginstrumentestrategien beinhaltet die Festlegung leistungs-, preis-, kontrahierungs- und kommunikationspolitischer Entscheidungstatbestände für unterschiedliche Produkte oder Produktgruppen eines Unternehmens. $^{49}$ Aufgrund der Erklärungsbedürftigkeit vieler High-TechProdukte bekommen vor allem Servicemaßnahmen in der Produktpolitik und persönliche Verkaufsaktivitäten in der Kommunikationspolitik eine strategische Bedeutung. Instrumentebezogene Strategien können hierbei als Präzisierung von Geschäftsfeldstrategien auf einer operativen Planungsebene verstanden werden.

Die von einigen Autoren $^{50}$ vorgenommene Gleichsetzung einer Marketingstrategie mit der Kombination mehrerer Marketinginstrumenteaktivitäten erscheint mit Blick auf High-Tech-Märkte problematisch, da die komplexe Entscheidungssituation in diesen Märkten eine langfristige Orientierung erfordert, die über den eher kurzfristigen Charakter einzelner Instrumentemaßnahmen hinausgeht. ${ }^{51}$ Es ist deshalb davon auszugehen, daß in High-Tech-Unternehmen die Entscheidungen zur Ausgestaltung von Marketinginstrumenten auf einer der Marketingstrategie untergeordneten Ebene getroffen werden. ${ }^{52}$

Bei der Auseinandersetzung mit den Marktteilnehmer- und Marketinginstrumentestrategien in High-Tech-Märkten bleibt zusammenfassend festzu-

49 Vgl. z.B. Meffert, H., Marketing, Grundlagen der Absatzpolitik, a.a.O., S. 114 ff.; Merkle, E., Technologiemarketing: Ein Ansatz zur Bewältigung des technologischen Wandels, in: Marketing ZFP, 1984, Nr. 1, S. 9 ff.

Vgl. Aaby, N.E., McGann, A.F., Corporate strategy and the role of navigational marketing, in: European Journal of Marketing, Vol. 23 (1989), No. 10, S. 18 ff.; Haedrich, G., Entwicklung von Marketingstrategien, in: Marketing ZFP, 1983, Nr. 3, S. 175 ff.; Hoffmann, K., Wolff, V., Zur Systematik von Absatzstrategien als Grundlage langfristig wirkender Entscheidungen im Absatzbereich, in: Jahrbuch der Absatz- und Verbrauchsforschung, 1977, S. $164 \mathrm{ff}$.

Vgl. hierzu z.B. Marone, J.G., Winning in high-tech markets, the role of general management, Boston, Mass. 1993, S. 12 ff.; Backhaus, K., Investitionsguitermarketing, a.a.O., S. 149 ff.; Remmerbach, K.-U., Markteintrittsentscheidungen, a.a.O., S. 75 ff.

Vgl. zu dieser Einschätzung auch Köhler, R., Beiträge zum Marketing-Management, 2. Aufl., Stuttgart 1991, S. 84 ff.; Meffert, H., Marketing-Management, Grundlagen der Absatzpolitik, a.a.O., S. 25; Fronhoff, B., Die Gestaltung von Marketingstrategien, Köln 1986, S. 89 ff.; Wieselhuber, N., Strategische Marketingplanung, in: Strategisches Marketing, Hrsg.: Wieselhuber, N., Töpfer, A., Landsberg a.L. 1984, S. 67 ff., Steffenhagen, H., Der Strategiebegriff in der Marketingplanung, a.a.O., S. $55 \mathrm{f}$. 
halten, daß in der betriebswirtschaftlichen Forschung gefordert wird, den Begriff der Marketingstrategie inhaltlich nicht $\mathrm{zu}$ weit $\mathrm{zu}$ fassen. ${ }^{53}$ Eine Zusammenfassung aller abnehmer-, konkurrenz-, absatzmittler- und anspruchsgruppenbezogenen sowie sämtlicher leistungs-, preis-, kontrahierungs- und kommunikationspolitischen Strategieoptionen bzw. strategischen Kombinationsmöglichkeiten unter einer Marketingstrategie würde diesem Anliegen jedoch entgegenstehen. Der vorliegenden Untersuchung in High-Tech-Märkten liegt deshalb ein engeres Strategieverständnis zugrunde. ${ }^{54} \mathrm{Im}$ folgenden wird daher unter einer Marketingstrategie in Anlehung an Meffert ${ }^{55}$ ein auf globaler Planungsebene festgelegter bedingter, langfristiger Verhaltensplan verstanden, der beinhaltet, welche Produkt-/Marktkombinationen, Wettbewerbsvorteile und Markteintrittszeitpunkte zur Erreichung der Unternehmens- und Marketingziele in High-Tech-Märkten angestrebt werden.

Bei Betrachtung von Marketingstrategien in High-Tech-Märkten wird es aus managementorientierter Sicht von besonderem Interesse sein, zu erfahren, welche Marketingstrategien in High-Tech-Märkten verfolgt werden und welche hiervon am ehesten zur Erzielung von Wettbewerbsvorteilen geeignet sind. In diesem Zusammenhang wurde in den letzten Jahren verstärkt die Eignung des Konzeptes der strategischen Gruppen zur Identifikation und Bewertung von Marketingstrategien hervorgehoben. In den weiteren Ausführungen wird deshalb das Konzept der strategischen Gruppen skizziert und seine besondere Relevanz für High-Tech-Märkte hinsichtlich der Bereitstellung wettbewerbsstrategischer Informationen und der Identifikation erfolgreicher Marketingstrategien im Wettbewerb näher beleuchtet. Im Anschluß daran werden die zentralen Erklärungs-

Vgl. z.B. Fronhoff, B., Die Gestaltung von Marketingstrategien, a.a.O., S. 92; Steffenhagen, H., Der Strategiebegriff in der Marketingplanung, a.a.O., S. 40 f.

Vgl. zu dieser Einschätzung auch Hannig, U., Die Entwicklung wettbewerbsorientierter Marketingstrategien auf Basis des Konzeptes der strategischen Gruppen, Frankfurt a.M. u.a. 1993 , S. 23 ff.; Wüthrich, H.A., Neuland des strategischen Denkens, a.a.O., S. 48 ff.; Fronhoff, B., Die Gestaltung von Marketingstrategien, a.a.O., S. 92 ff.; Schreyögg, G., Unternehmensstrategie, Berlin, New York 1984, S. 124 ff.; Steffenhagen, H., Der Strategiebegriff in der Marketingplanung, a.a.O., S. $37 \mathrm{ff}$.

Vgl. Meffert, H., Marketing und strategische Unternehmensfuihrung - ein wettbewerbsorientierter Kontingenzansatz, a.a.O., S. 665 f.; Meffert, H., Marketing, Grundlagen der Absatzpolitik, a.a.O., S. 55. 
ansätze sowie die herangezogenen Einflußfaktorengruppen ${ }^{56} \mathrm{zu}$ strategischen Gruppen erörtert.

\section{Erklärungsansätze zu strategischen Gruppen in High-Tech-Märkten}

Als strategische Gruppe in High-Tech-Märkten können grundsätzlich diejenigen Unternehmen bezeichnet werden, die eine ähnliche Marketingstrategie verfolgen. Es sind hierbei zwei Extremformen denkbar, wie sich strategische Gruppen zusammensetzen. Wenn sämtliche Unternehmen in High-Tech-Märkten ein gleiches strategisches Verhalten aufweisen, existiert nur eine einzige strategische Gruppe. Unterscheiden sich die strategischen Verhaltensweisen aller Unternehmen voneinander, dann bildet jedes High-Tech-Unternehmen eine separate strategische Gruppe. Empirische Forschungen der Vergangenheit haben gezeigt, $\mathrm{da} ß$ sich in einem Wirtschaftszweig ${ }^{57}$ i.d.R eine kleine Zahl strategischer Gruppen bildet. Hergert und Miles/Snow/Sharfman konnten in umfassenden Untersuchungen in verschiedenen Branchen nachweisen, daß in den einzelnen Branchen maximal vier und im Durchschnitt drei strategische Gruppen existieren. ${ }^{58}$

Mit dem Konzept der strategischen Gruppen lassen sich in High-Tech-Märkten aus wissenschaftlicher und praxisbezogener Perspektive vor allem folgende Zielsetzungen verfolgen: ${ }^{59}$

Im folgenden werden die Begriffe Einflußfaktor, Bestimmungsfaktor und Einflußgröße synonym verwendet.

57 Im folgenden werden die Begriffe Branche, Wirtschaftszweig, Industrie und Markt synonym verwendet. Vgl. hierzu z.B. Fronhoff, B., Die Gestaltung von Marketingstrategien, a.a.O., S. 169 f.; Mauthe, K.D., Strategische Analyse, München 1984, S. 198.

Vgl. Miles, R.E., Snow, Ch.C., Sharfman, M.P., Industry variety and performance, in: Strategic Management Journal, Vol. 14 (1993), S. 168 ff.; Hergert, M., Causes and consequences of strategic grouping in U.S. Manufacturing Industries, in: International Studies of Management and Organization, Vol. 18 (1987), No. 1, S. $27 \mathrm{ff}$.

Vgl. Meffert, H., Marketing-Management, a.a.O., S. 53 f.; Homburg, Ch., Sütterlin, St., Strategische Gruppen: Ein Survey, in: ZfB, 1992, Nr. 6, S. 652 ff.; Bauer, H.H., Unternehmensstrategie und Strategische Gruppen, in: Unternehmensdynamik, Hrsg.: 
- Verringerung der Komplexität der Wettbewerbsanalyse und Verbesserung der Prognose der Wettbewerbsentwicklung in High-Tech-Märkten durch Identifikation weniger strategischer Gruppen mit ähnlichen wettbewerbsstrategischen Verhaltens- und Reaktionsmustern.

- Identifikation von Erfolgspotentialen und Risiken unterschiedlicher Marketingstrategien im brancheninternen Wettbewerb zur Ableitung von Gestaltungsempfehlungen für die Strategieplanung in High-Tech-Märkten.

- Erklärung langfristiger Profitabilitätsunterschiede zwischen Unternehmen in High-Tech-Märkten durch die Zugehörigkeit zu verschiedenen strategischen Gruppen.

Vor dem Hintergrund der besonderen wettbewerbsstrategischen Herausforderungen in High-Tech-Märkten liefert die Analyse strategischer Gruppen einen wichtigen Erkenntnisgewinn für High-Tech-Unternehmen sowie für die betriebswirtschaftliche Forschung. Die Identifikation strategischer Gruppen in High-Tech-Märkten kann die durch hohe Entwicklungs- und Wettbewerbsdynamik geprägte Komplexität der Wettbewerbsanalyse reduxieren und ist zur Informationsbeschaffung fuir High-Tech-Unternehmen in bestehenden und neuen Märkten geeignet. Aufgnund der aufgezeigten Technologieintegration sehen sich High-Tech-Unternehmen vielfach mit der Situation konfrontiert, daß neue Konkurrenten in etablierte Wettbewerbsstrukturen eindringen. Umgekehrt treten High-Tech-Unternehmen als Anbieter von innovativen Technologien in neue Märkte ein, deren Spielregeln ihnen nicht hinreichend bekannt sind.$^{60}$ Durch die Kenntnis von Anzahl und Art der strategischen Gruppen lassen sich Implika-

Kistner, K.-P., Schmidt, R., Wiesbaden 1991, S. 394; Porter, M., Wettbewerbsstrategie, a.a.O., S. $186 \mathrm{ff}$.

Vgl. Kodama, F., Technologiefusion - der Weg zum Erfolg auf Zukunftsmärkten, in: Harvard Business Manager, 1993, Nr. 1, S. 45; Meffert, H., Strategische Unternehmensfuihrung und Marketing, a.a.O., S. 67 ff.; Remmerbach, K.-U., Markteintrittsentscheidungen, a.a.O., S. $111 \mathrm{ff}$. 
tionen im Hinblick auf die Ausprägung der Wettbewerbsintensität und die Strategiegestaltung für einen neu eindringenden High-Tech-Anbieter ableiten.

Die Wettbewerbsintensität in High-Tech-Märkten wird vor allem durch die Anzahl und relative Größe der strategischen Gruppen, das Ausmaß der Zielgruppenüberschneidung und den Grad der Produkt- und Technologiedifferenzierung bestimmt. Eine intensive Konkurrenzbeziehung besteht i.d.R., wenn eine hohe Zahl großer strategischer Gruppen ähnliche Produkte an vergleichbare Zielgruppen verkauft. ${ }^{61}$ Aufgrund der zunehmenden Technologieintegration und der Ausweitung von Produktfunktionen in High-Tech-Märkten können immer breitere Zielgruppen angesprochen werden, so daß zwischen den strategischen Gruppen grundsätzlich ein intensiver Wettbewerb zu erwarten ist. Für High-Tech-Unternehmen stellt sich somit die Frage, welche Erkenntnisse die Analyse strategischer Gruppen bieten kann, um trotz der hohen Zielgruppenüberschneidung eine Erfolgsposition zu sichern und gegebenenfalls Mobilitätsbarrieren aufzubauen.

Die Wettbewerbsintensität in High-Tech-Märkten wird zudem durch die strategische Distanz zwischen den strategischen Gruppen beeinflußt. ${ }^{62}$ Sie beschreibt den Grad der Verschiedenheit der von den Gruppen gewählten Marketingstrategien, die in bestimmten Strategiedimensionen wie der Qualitäts- oder Kostenorientierung zum Ausdruck kommt. Eine geringe strategische Distanz zwischen den strategischen Gruppen kann zu einer hohen Wettbewerbsverbundenheit führen. ${ }^{63}$ Dies liegt darin begründet, daß der Wechsel zwischen

61 Vgl. Bauer, H.H., Unternehmensstrategie und Strategische Gruppen, a.a.O., S. 394; Fronhoff, B., Die Gestaltung von Marketingstrategien, a.a.O., S. 214; McGee, J., Strategic groups: A bridge between industry structure and strategic management?, in: Strategic marketing and management, Hrsg.: Thomas, H., Gardner, D., Chicester u.a. 1985, S. 295 f.

Vgl. Porter, M., Wettbewerbsstrategie, a.a.O., S. 188 ff.; Harrigan, K.R., An application of clustering for strategic group analysis, in: Strategic Management Journal, Vol. 6 (1985), S. 70; Thomas, H., Gardner, D., Introduction to conference papers on competitive strategy and strategic competition, in: Strategic marketing and management, Hrsg.: Thomas, H., Gardner, D., Chicester u.a. 1985, S. 270.

Porter vertritt in diesem Zusammenhang die Meinung, daB eine hohe strategische Distanz zu einem intensiven Wettbewerb führt. Siehe hierzu Porter, M., Wettbewerbsstrategie, a.a.O., S. 189. Dieser Auffassung kann jedoch nicht zugestimmt werden, denn je 
strategischen Gruppen mit ähnlichen Strategieschwerpunkten, z.B. einer Innovations- und Differenzierungsausrichtung, i.d.R. leichter ist als zwischen sehr verschiedenen wettbewerbsstrategischen Grundhaltungen wie der Innovationsund Kostenorientierung. ${ }^{64}$

Neben der Analyse der aktuellen Wettbewerbssituation in High-Tech-Märkten bietet die Erfassung von strategischen Gruppen auch die Möglichkeit, die zukünftige Entwicklung der auf die High-Tech-Anbieter einwirkenden Wettbewerbskräfte besser $\mathrm{zu}$ prognostizieren, da Unternehmen der gleichen strategischen Gruppe ähnlich auf Veränderungen im Wettbewerbsumfeld reagieren. ${ }^{65}$ Dies erlaubt High-Tech-Unternehmen, relevante Wettbewerbsveränderungen und deren Auswirkungen auf die gesamte strategische Gruppe rechtzeitig zu erkennen. Beispielsweise hängt die Bedrohung einer qualitätsorientierten strategischen High-Tech-Gruppe durch Substitutionsprodukte maßgeblich von technologischen Neuentwicklungen ab. Durch eine frühzeitige Identifikation der sich anbahnenden technologischen Entwicklungen stehen High-Tech-Unternehmen dieser strategischen Gruppe größere Gestaltungsfreiheiten in der Anpassung zur Verfügung.

Die Analyse strategischer Gruppen in High-Tech-Märkten kann darüber hinaus einen Beitrag zur Bewertung von Strategieoptionen eines High-Tech-Anbieters leisten. Durch Betrachtung der zentralen konkurrenzbezogenen Strategiedimensionen und des dahinterstehenden Unternehmensverhaltens läßt sich die grundsätzliche Entscheidung der Wahl einer strategischen Gruppe oder die Frage

unterschiedlicher die strategischen Gruppen hinsichtlich der strategischen Entscheidungsvariablen aufgrund divergierender Zielsetzungen, Ressourcen- bzw. Know-how-Gegebenheiten agieren, desto eher wird der Wettbewerbsdruck abnehmen.

Vgl. Hannig, U., Die Entwicklung wettbewerbsorientierter Marketingstrategien auf Basis des Konzeptes der strategischen Gruppen, a.a.O., S. 164; Fronhoff, B., Die Gestaltung von Marketingstrategien, a.a.O., S. 210 f.; Harrigan, K.R., An application of clustering for strategic group analysis, a.a.O. S. $63 \mathrm{ff}$.

Vgl. Joas, A., Konkurrenzforschung als Erfolgspotential im strategischen Marketing, Augsburg 1990, S. 155 f.; Homburg, Ch., Sütterlin, St., Strategische Gruppen: Ein Survey, a.a.O., S. 653; Porter, M., Wettbewerbsstrategie, a.a.O., S. 202 f.; Aaker, D.A. Strategic Market Management, New York u.a. 1984, S. $60 \mathrm{ff}$. 
des Gruppenwechsels konkretisieren. ${ }^{66}$ Ein Unternehmen mit begrenzten finanziellen bzw. personellen Ressourcen und geringen Know-how-Fähigkeiten wird in einer innovationsorientierten High-Tech-Gruppe langfristig kaum erfolgreich agieren können. Sofern zwischen den strategischen Gruppen Erfolgsunterschiede existieren, können die High-Tech-Unternehmen unter Abwägung der zu überwindenden Mobilitätsbarrieren auch einen Wechsel der strategischen Gruppe in Erwägung ziehen.

In der wissenschaftlichen Diskussion zum Konzept der strategischen Gruppen wurde die Erklärung von Erfolgsunterschieden als ein zentrales Erkenntnisziel herausgestellt. ${ }^{67}$ Es ist deshalb nicht überraschend, daß in der Mehrzahl der empirischen Untersuchungen versucht worden ist, die Unterschiede im Markterfolg von Unternehmen einer Branche anhand der Zugehörigkeit zu verschiedenen strategischen Gruppen zu analysieren. ${ }^{68}$ Bezug nehmend auf das Konzept der strategischen Gruppen lassen sich Profitabilitätsunterschiede damit begründen, daß die langfristige Entwicklung des Gewinnpotentials in einem Wirtschaftszweig insbesondere von der Wettbewerbsintensität zwischen strategischen Gruppen beeinflußt wird. ${ }^{69}$ Die Analyse strategischer High-Tech-Gruppen kann einen wichtigen Beitrag leisten, die in High-Tech-Märkten bestehenden Erfolgsunterschiede zu erklären.

Vgl. Homburg, Ch., Sütterlin, St., Strategische Gruppen: Ein Survey, a.a.O., S. 652; Wolfrum, B., Strategische Gruppen, in: DBW, 1992, Nr. 3, S. 417; Porter, M., Wettbewerbsstrategie, a.a.O., S. $199 \mathrm{ff}$.

Vgl. stellvertretend Oster, Sh., Modern competitive analysis, New York, Oxford 1990, S. 71 ff.; Homburg, Ch., Sütterlin, St., Strategische Gruppen: Ein Survey, a.a.O., S. 638; McGee, J., Thomas, H., Strategic groups: Theory, research and taxonomy, in: Strategic Management Journal, Vol. 7 (1986), S. $142 \mathrm{ff}$.

Vgl. stellvertretend Cool, K.O., Schendel, D., Performance differences among strategic group members, in: Strategic Management Journal, Vol. 9 (1988), S. 220 f.; Harrigan, K.R., An application of clustering for strategic group analysis, a.a.O., S. $62 \mathrm{ff}$.; Dess, G.G., Davis, P.S., Porter's (1980) generic strategies as Determinants of strategic group membership and organizational performance, in: Academy of Management Journal, Vol. 27 (1984), No. 3, S. 477 ff.

Vgl. z.B. Habib, M.M., Victor, B., Strategy, structure, and performance of U.S. Manufacturing and Service MNCs: A comparative analysis, in: Strategic Management Journal, Vol. 12 (1991), S. 595 ff.; Porter, M., Wettbewerbsstrategie, a.a.O., S. 191 ff. 
Zur Erklärung der Ursachen für ein gleichartiges bzw. unterschiedliches strategisches Unternehmensverhalten in High-Tech-Märkten und daraus resultierend für die Existenz von strategischen Gruppen lassen sich zahlreiche Ansätze der betriebswirtschaftlichen Forschung heranziehen.

Aus wettbewerbstheoretischer Perspektive ${ }^{70}$ werden die wesentlichen Grundlagen $\mathrm{zu}$ strategischen Gruppen vor allem in der Industrial Organization Forschung und strategischen Konkurrenzforschung gesehen. ${ }^{71}$ Den Ansätzen der Industrial Organization ${ }^{72}$ liegt die Annahme zugrunde, daß die Struktur einer Branche das Verhalten und letztlich den Erfolg von Unternehmen bestimmt ("Structure-Conduct-Performance"-Paradigma). In dem traditionellen Paradigma von Bain und Mason wird eine einseitig kausale Beziehung zwischen Marktstruktur, Marktverhalten und Markterfolg unterstellt. ${ }^{73}$ Mit Blick auf das strategische Verhalten in High-Tech-Märkten ist jedoch von wechselseitigen Wirkungsbeziehungen auszugehen. So wird z.B. auch die Marktstruktur beeinflußt, wenn die Niedrigpreisstrategie einiger High-Tech-Unternehmen zum Ausscheiden von Grenzanbietern und damit zur Verringerung der Anbieterzahl fuihrt. ${ }^{74}$ Weitere Defizite der Industrial Organization Forschung sind darin zu

Zur Übersicht der wettbewerbstheoretischen Forschung und ihrer Bedeutung für die Marketingwissenschaft siehe insbesondere Fritz, W., Ansätze der Wettbewerbstheorie aus Sicht der Marketingwissenschaft, in: DBW, 1990, Nr. 4, S. 491 ff. Vgl. auch Görgen, W., Strategische Wettbewerbsforschung, Bergisch Gladbach, Köln 1992, S. 4 ff.

Vgl. z.B. Porter, M., The contribution of industrial organization to strategic management, in: Academy of Management Review, Vol. 6 (1981), No. 4, S. 609 ff.; Porter, M., The structure within industries and companies Performance, in: Review of Economics and Statistics, Vol. 61 (1979), S. 215 ff.

Vgl. stellvertretend Herdzina, K. Wirtschaftliches Wachstum, Strukturwandel und Wettbewerb, Berlin 1981, S. 220 f.; Kaufer, E., Industrieökonomik, München 1980, S. 7 ff. In der Literatur werden die Begriffe Theorie der Industrial Organization, Industrieökonomik und Wettbewerbstheorie häufig gleichgesetzt. Vgl. z.B. Cox, H., Hübner, H., Entwicklung und Stand der wettbewerbstheoretischen Diskussion, in: Handbuch des Wettbewerbs, Hrsg.: Cox, H., Jens, U., Markert, K., München 1981 S. 18; Sheperd, W.G., The economics of industrial organization, Englewood Cliffs, N.J. 1979, S. 3.

Vgl. Bain, J.S., Industrial organization, 2. Aufl., New York 1968, S. 6 ff., Mason, E.S., Economic concentration and the monopoly problem, Cambridge 1957, S. 5 ff.

Aufgrund dieser Kritik am einseitig deterministischen "Structure-Conduct-Performance"Paradigma wurde von einigen Autoren die Integration entsprechender Interdependenzen und Aufnahme von Marktstruktur beeinflussenden "basic conditions" (technologische 
sehen, daß das individuelle wettbewerbsstrategische Unternehmensverhalten und dessen Einfluß auf den Unternehmenserfolg unzureichend analysiert werden. ${ }^{75}$

Im Rahmen der strategischen Konkurrenzforschung wird die Betrachtung brancheninterner Wettbewerbsbedingungen stärker in den Vordergrund gerückt. ${ }^{76}$ Aus Unternehmenssicht besitzt die Analyse verhaltensbezogener Einflußgrößen des Wettbewerbs in High-Tech-Märkten eine besondere Bedeutung. Die Intensität des Wettbewerbs hängt einerseits von Art und Umfang der Rivalität zwischen den etablierten High-Tech-Unternehmen ab. Sie wird andererseits durch das Verhalten der Nachfrager und Lieferanten sowie durch die Substitution von Produkten bzw. Technologien und die Bedrohung neuer Konkurrenten in HighTech-Märkten bestimmt. ${ }^{77}$ In diesem Zusammenhang ist vor allem der Einfluß des Technologie- bzw. Preiswettbewerbs auf das strategische Verhalten von High-Tech-Unternehmen und die Bildung strategischer Gruppen.zu hinterfragen.

Für eine detaillierte Analyse des Bedrohungspotentials neuer High-Tech-Anbieter kann auf das Konzept der Mobilitätsbarrieren zurückgegriffen werden. ${ }^{78}$

Veränderungen, Preiselastizität etc.) vorgeschlagen. Vgl. z.B. Scherer, F.M., Industrial market structure and economic performance, 2. Aufl., Chicago 1980, S. 4 ff.

Vgl. z.B. Fronhoff, B., Die Gestaltung von Marketingstrategien, a.a.O., S. 173 ff.; Porter, M., The contribution of industrial organization to strategic management, a.a.O., S. $614 \mathrm{ff}$.

Porter unterscheidet im Rahmen der strategischen Wettbewerbsanalyse zwischen der Strukturanalyse von Branchen, der Analyse strategischer Gruppen (brancheninterne Strukturanalyse) und der individuellen Konkurrenzanalyse. Vgl. Porter, M., Wettbewerbsstrategie, a.a.O., S. 25 ff. Zur Kennzeichnung der Branchen- und Konkurrenzanalyse siehe z.B. Rohleder, P.J., Unternehmens- und Geschäftsfeldstrategien, in: Marketing, Hrsg.: Fischer, G., Landsberg a.L. 1992, S. $11 \mathrm{ff.;}$ Kreikebaum, H., Strategische Unternehmensplanung, 3. Aufl., Stuttgart u.a. 1989, S. 45 ff. Zur Unterscheidung von strategischer und operativer Konkurrenzanalyse siehe z.B. Link, U., Strategische Konkurrenzanalyse im Konsumgütermarketing, a.a.O., S. 34 ff. Zur Abgrenzung von Benchmarking ggü. Konkurrenzanalyse siehe z.B. Watson, G.H., The Benchmarking Workbook, Cambridge, Norwalk 1992, S. 5 ff.; Leibfried, K., McNair, C.J., Benchmarking, New York 1992, S. 17 ff.

Vgl. Joas, A., Konkurrenzforschung als Erfolgspotential im strategischen Marketing, a.a.O., S. 96 ff.; Porter, M., Wettbewerbsstrategie, a.a.O., S. 178. Von einigen Autoren werden als weitere Determinanten des brancheninternen Wettbewerbs das Verhalten staatlicher und politischer Organisationen angeführt. Siehe z.B. Meffert, H., Strategische Unternehmensführung und Marketing, a.a.O., S. $38 \mathrm{f}$.

Vgl. z.B. Minderlein, M., Markteintrittsbarrieren und strategische Verhaltensweisen, in: ZfB, 1990, Nr. 2, S. 156 ff.; Caves, R.E., Porter, M., From entry barriers to mobility 
Mobilitätsbarrieren $^{79}$, wie z.B. Betriebsgrößenersparnisse, hohe technologiespezifische Umstellkosten und unternehmenskulturelle Gegebenheiten, erschweren den Wechsel ${ }^{80}$ zwischen strategischen Gruppen in High-Tech-Märkten. Der Eintritt in eine andere strategische Gruppe ist dabei häufig nur unter großem finanziellen und zeitlichen Aufwand zu realisieren und beinhaltet eine hohe Unsicherheit hinsichtlich der Zielerreichung. Die Existenz von Mobilitätsbarrieren liefert damit auch eine Begründung dafür, daß High-Tech-Unternehmen unterschiedliche Marketingstrategien verfolgen, obwohl nicht alle strategischen Verhaltensweisen gleich erfolgreich sind. ${ }^{81}$

Zur Fundierung von Erkenntnissen über Erfolgsunterschiede zwischen strategischen Gruppen in High-Tech-Märkten können darüber hinaus Ansätze der Erfolgsfaktorenforschung berücksichtigt werden. ${ }^{82}$ Die Erfolgsfaktoren-

barriers: Conjectural decisions and contrived deterrence to new competition, in: Quarterly Journal of Economics, Vol. 91 (1977), S. 250 ff. Zur Unterscheidung des Konzeptes der strategischen Gruppen und des Konzeptes der Mobilitätsbarrieren siehe u.a. Hannig, U., Die Entwicklung wettbewerbsorientierter Marketingstrategien auf Basis des Konzeptes der strategischen Gruppen, a.a.O., S. 105 ff.; Fritz, W., Ansätze der Wettbewerbstheorie aus Sicht der Marketingwissenschaft, a.a.O., S. 498.

Markteintrittsbarrieren schützen demgegenüber strategische Gruppen vor dem Eindringen neuer Konkurrenten in High-Tech-Märkte. Zur Unterscheidung von Mobilitäts- und Markteintrittsbarrieren vgl. z.B. Kolbe, C., Eintrittsbarrieren und die Eintrittsfähigkeit potentieller Konkurrenten, Göttingen 1991, S. 51 ff.; Meffert, H., Marktein- und austrittsbarrieren, in: DBW, 1987, Nr. 5, S. 629 f.; Yip, G.S., Barriers to entry, Lexington, Mass., Toronto 1982, S. 17 ff.

Bei asymmetrischen Mobilitätsbarrieren ist ein strategischer Gruppenwechsel lediglich in eine Richtung schwerer durchzufuihren. Vgl. hierzu z.B. Hatten, K.J., Hatten, M.L., Strategic groups, asymmetrical mobility barriers and contestability, in: Strategic Management Journal, Vol. 8 (1987), S. 334 f.

81 Vgl. Caves, R.E., Ghemawat, P., Identifying mobility barriers, in: Strategic Management Journal, Vol. 13 (1992), S. 2 ff.; McGee, J., Thomas, H., Strategic groups: A useful linkage between industry structure and strategic management?, BEBR, Faculty Working Paper No. 1100, College of Commerce and Business Administration, University of Illinois at Urbana-Champaign, December 1984, S. 3; Hatten, K.J., Schendel, D.E., Heterogenity within industry, in: Journal of Industrial Economics, Vol. 26 (1977), No. 2, S. $97 \mathrm{ff}$.

Vgl. hierzu z.B. Hannig, U., Die Entwicklung wettbewerbsorientierter Marketingstrategien auf Basis des Konzeptes der strategischen Gruppen, a.a.O., S. 131 ff.; Homburg, Ch., Sütterlin, St., Strategische Gruppen: Ein Survey, a.a.O., S. 654 ff.; Preiß, F.J., Strategische Erfolgsfaktoren im Software-Marketing, Frankfurt a.M. u.a. 1991, S. 37 ff.; Trommsdorff, V., Erfolgsfaktorenforschung, Produktinnovationen und Schnittstelle Marketing - F\&E, Arbeitspapier des Instituts fuir Betriebswirtschaftslehre, Berlin 1990, S. 2 ff. 
forschung beruht auf der Annahme, daß der Erfolg oder Mißerfolg eines Unternehmens letztlich nur durch einige wenige Einflußgrößen determiniert wird. ${ }^{83} \mathrm{Im}$ Hinblick auf das strategische Verhalten von High-Tech-Unternehmen erscheint es bedeutsam zu untersuchen, ob z.B. in High-Tech-Märkten eher eine technologiedominante oder eine nachfragebezogene Innovationsausrichtung erfolgversprechend ist.

Unterschiede im wettbewerbsstrategischen Verhalten von High-TechUnternehmen können auch in den Zielsetzungen begründet liegen. ${ }^{84}$ Aus entscheidungsorientierter Sicht bietet es sich an, die Zusammenhänge zwischen den Unternehmenszielen und den Strategieschwerpunkten unterschiedlicher strategischer Verhaltensgruppen in High-Tech-Märkten zu untersuchen. ${ }^{85}$ Es ist hierbei von Interesse $\mathrm{zu}$ erfahren, ob beispielsweise eine technologiebezogene Strategieausrichtung von High-Tech-Unternehmen eher unter Gesichtspunkten der Marktstellung (z.B. Sicherung der Wettbewerbsposition in bestehenden Märkten, Erschließung neuer Märkte) oder der Unternehmensrentabilität (z.B. Gewinnsteigerung, Kostensenkung) erfolgt.

Aufgrund der besonderen Bedeutung einer wettbewerbsorientierten Ausrichtung von Marketingstrategien in High-Tech-Märkten kann auch auf Erkenntnisse der strategischen Unternehmensführung zurückgegriffen werden. In diesem

83 Für einen Überblick ausgewählter Studien zur Erfolgsfaktorenforschung siehe vor allem Fritz, W., Marketing - ein Schlüsselfaktor des Unternehmenserfolges?, in: Marketing ZFP, 1990, Nr. 2, S. 92 ff. Zur Unterscheidung zwischen strategischen Erfolgsfaktoren und strategischen Erfolgspositionen siehe z.B. Pümpin, C., Management strategischer Erfolgspositionen, Bern, Stuttgart 1982, S. 29 ff. Zur Unterscheidung von qualitativen und quantitativen Erfolgsfaktoren siehe z.B. Patt, P.J., Strategische Erfolgsfaktoren im Einzelhandel, Frankfurt a.M. u.a. 1988, S. 6 ff.

Zur Beziehung zwischen Unternehmenszielen und Unternehmensstrategie siehe z.B. Raffée, H., Fritz, W., Dimensionen und Konsistenz der Führungskonzeption von Industrieunternehmen - Ergebnisse einer empirischen Untersuchung, in: ZfbF, 1992, Nr. 4, S. 314 ff.; Heinen, E., Einfuihrung in die Betriebswirtschaftslehre, 9. Aufl., Wiesbaden 1986, S. 49 ff.; Meffert, H., Marketing, Grundlagen der Absatzpolitik, a.a.O., S. 49 ff.

Vgl. hierzu z.B. Conant, J.S., Mokwa, M.P., Varadarajan, R.P., Strategic types, distinctive marketing competencies and organizational performance: a multiple measuresbased study, in: Strategic Management Journal, Vol. 11 (1990), S. 370 ff.; Hawes, J.M., Crittenden, W.F., A taxonomy of competitive retailing strategies, in: Strategic Management Journal, Vol. 5 (1984), S. 277 ff. 
Zusammenhang besitzt die Analyse der Beziehungen zwischen dem wettbewerbsstrategischen Verhalten von High-Tech-Unternehmen (z.B. Qualitäts- vs. Kostenorientierung) und der verfolgten Marktfeld- (z.B. Marktpenetration vs. Produktentwicklung) bzw. Timingstrategie (z.B. Pionier vs. später Folger) einen hohen Stellenwert. ${ }^{86}$

Die Einbeziehung von Erfahrungen aus der verhaltenswissenschaftlichen Organisationsforschung kann dazu dienen, den Einfluß unternehmenskultureller Merkmale auf das wettbewerbsstrategische Verhalten von High-Tech-Unternehmen zu erklären. ${ }^{87}$ Hierbei ist insbesondere der Frage nachzugehen, inwieweit sich strategische Gruppen in High-Tech-Märkten in Abhängigkeit von der Unternehmenskultur herausbilden. ${ }^{88}$ Die Analyse der strategie- und kulturbezogenen Gemeinsamkeiten innerhalb sowie Unterschiede zwischen strategischen Gruppen können hierzu erste Hinweise geben.

Eine stärker ganzheitliche Perspektive liegt den Ansätzen der situativen Organisationsforschung $^{89}$ zugrunde, die das strategische Unternehmensverhalten in Abhängigkeit von unternehmensinternen (z.B. strukturelle und kulturelle

Vgl. z.B. Meffert, H., Marketing-Management, a.a.O., S. 127 f.; Becker, J., MarketingKonzeption, a.a.O., S. $311 \mathrm{ff}$; Bolz, J., Wettbewerbsorientierte Standardisierung der internationalen Marktbearbeitung, a.a.O., S. 27 ff.; Remmerbach, K.-U., Markteintrittsentscheidungen, a.a.O., S. 84 ff.; Meffert, H., Marketing und strategische Unternehmensführung - ein wettbewerbsorientierter Kontingenzansatz, a.a.O., S. 665 ff.

Zur Beziehung zwischen Unternehmenskultur und Unternehmensstrategie siehe z.B. Pümpin, C., Koller, H.P., Die Bedeutung der Unternehmenskultur für die Unternehmensstrategie, in: Die Unternehmenskultur: ihre Grundlagen und ihre Bedeutung für die Führung der Unternehmung, Hrsg.: Lattmann, Ch., Heidelberg 1990, S. 304 ff.; Scholz, Ch., Organisationskultur: die 4 Erfolgsprinzipien, Wiesbaden 1990, S. 108 ff.; Meffert, H., Hafner, K. Unternehmenskultur und marktorientierte Unternehmensfuihrung, Bestandsaufnahme und Wirkungsanalyse, Arbeitspapier Nr. 35 der Wissenschaftlichen Gesellschaft für Marketing und Unternehmensfuihrung e.V., Hrsg.: Meffert H., Wagner, H., Münster 1987, S. 31 ff.

Vgl. z.B. Dvir, D., Segev, E., Shenhar, A., Research notes and communications Technology's varying impact on the success of strategic business units within the miles and snow typology, in: Strategic Management Journal, Vol. 14 (1993), S. 155 ff.; Herbert, Th., Deresky, H., Generic strategie's: An empirical investigation of typology validity and strategy content, in: Strategic Management Journal, Vol. 8 (1987), S. $136 \mathrm{ff}$.

$\mathrm{Zu}$ den Ansätzen der situativen Organisationsforschung vgl. z.B. Kieser, A., Kubicek, H., Organisation, 3. Aufl., Berlin, New York 1993, S. 45 ff.; Meffert, H., MarketingTheorie, in: Vahlens Großes Marketinglexikon, Hrsg.: Diller, H., München 1992, S. 701. 
Merkmale) und -externen (z.B. Merkmale der Technologie-, Markt- und Abnehmersituation) Kontextfaktoren betrachten..$^{90}$ In diesem Zusammenhang weisen neuere Erklärungsansätze der situativen Organisationsforschung auf die Bedeutung des "strategischen Fit" ${ }^{91}$ hin, der eine Abstimmung des strategischen Unternehmensverhaltens auf die Umweltsituation und eine Übereinstimmung von Unternehmensstrategie und -kultur beinhaltet. ${ }^{92}$ Mit Blick auf das wettbewerbsstrategische Verhalten von High-Tech-Unternehmen ist es von besonderem Interesse $\mathrm{zu}$ analysieren, inwieweit ein Fit zwischen der Marketingstrategie und der Umweltsituation besteht und sich hierdurch Erfolgsunterschiede in High-TechMärkten erklären lassen. ${ }^{93}$

In der Literatur wird an situativen Forschungsansätzen u.a. die Kritik geäußert, daß wichtige Kontextmerkmale vernachlässigt werden. Siehe z.B. Kieser, A., Der situative Ansatz, in: Organisationstheorien, Hrsg.: Kieser, A., Stuttgart, Berlin, Köln 1993, S. 175 ff.; Türk, K., Neuere Entwicklungen in der Organisationsforschung, Stuttgart 1989, S. 1 ff.; Lehnert, St., Die Bedeutung von Kontingenzansätzen für das Strategische Management, Frankfurt, Bern, New York 1983, S. 119 ff.

Zum Konzept des "strategischen Fit" siehe insbesondere Remmerbach, K.-U., Markteintrittsentscheidungen, a.a.O., S. 33; Scholz, Ch., Strategisches Management, Ein integrativer Ansatz, Berlin, New York 1987, S. 61 ff.; Venkatraman, N., Camillus, J.C., Exploring the concept of "Fit" in strategic management, in: Academy of Management Review, Vol. 9 (1984), S. 513 ff.; Miles, R.E., Snow, Ch.C., Fit, failure and the hall of fame, in: California Management Review, Vol. 26 (1984), No. 3, S. 10 ff. Im folgenden werden die Begriffe Fit, Stimmigkeit und Kongruenz gleichgesetzt. Siehe hierzu z.B. Drazin, R., Van de Ven, A., Alternative forms of fit in contingency theory, in: Administrative Science Quarterly, Vol. 30 (1985), No. 4, S. 514 ff.

Vgl. z.B. Staehle, W.H., Management, 5. Aufl., München 1990, S. 58 ff.; Miller, D., Relating Porter's business strategies to environment and structure, in: Academy of Management Journal, Vol. 31 (1988), No. 2, S. 283 ff.; Murray, J.A., A contingency view of Porter's generic strategies, in: The Academy of Management Review, Vol. 13 (1988), No. 2, S. 390 ff.; Miller, D., The genesis of configuration, in: Academy of Management Review, Vol. 12 (1987), No. 4, S. 686 ff.; Fry, L.W., Smith, D.A., Congruence, contingency and theory building, in: Academy of Management Review, Vol. 12 (1987), No. 1, S. $117 \mathrm{ff}$.

Vgl. z.B. McArthur, A.W., Nystrom, P.C., Environmental dynamism, complexity and munificence as moderators of strategy-performance relationships, in: Journal of Business Research, Vol. 23 (1991), S. 349 ff.; Meffert, H., Marketingstrategien in unterschiedlichen Marktsituationen, in: Handbuch des Marketing, Hrsg.: Bruhn, M., München 1989, S. 278 ff.; Lawless, M.W., Finch, L.K., Choice and determinism: A test of Hrebiniak and Joyce's framework on strategy-environment fit, in: Strategic Management Journal, Vol. 10 (1989), S. 352 ff.; Kim, L., Lim, Y., Environment, generic strategies and performance in rapidly developing country: a taxonomic approach, in: Academy of Management Journal, Vol. 31 (1988), S. $803 \mathrm{ff}$. 
Mit dem Konzept der strategischen Gruppen wird in der vorliegenden Untersuchung in Anlehnung an die aufgezeigten Einflußfaktorengruppen eine Analyse des wettbewerbsstrategischen Verhaltens und eine Typologisierung von Marketingstrategien in High-Tech-Märkten vorgenommen.

\section{Zielsetzung und Gang der Untersuchung}

Die generelle Zielsetzung der Untersuchung besteht in der Identifikation und Analyse strategischer Gruppen in High-Tech-Märkten. Mit der Untersuchung sind anknüpfend an das Grundkonzept der strategischen Gruppen deskriptive und explikative Teilziele verbunden, die sich an dem in der Abbildung 2 dargestellten allgemeinen Bezugsrahmen orientieren. Im Überblick sind vor allem folgende Teilziele der Untersuchung zu nennen:

1. Theoriegeleitete Ableitung von Variablen zur Operationalisierung, Beschreibung und Erklärung des Konstruktes der strategischen Gruppen in High-TechMärkten

2. Bildung strategischer Gruppen in High-Tech-Märkten und Analyse der Ausgestaltungsformen

3. Erklärung der Zugehörigkeit zu strategischen Gruppen in High-Tech-Märkten anhand ausgewählter Unternehmensvariablen

4. Erklärung von Erfolgsunterschieden in High-Tech-Märkten durch die Zugehörigkeit zu bestimmten strategischen Gruppen

Auf Grundlage einer theoriegestützten Ableitung zentraler Strategiemerkmale ist zu überprüfen, ob strategische Gruppen in einer homogenen Umfeldsituation ${ }^{94}$ 


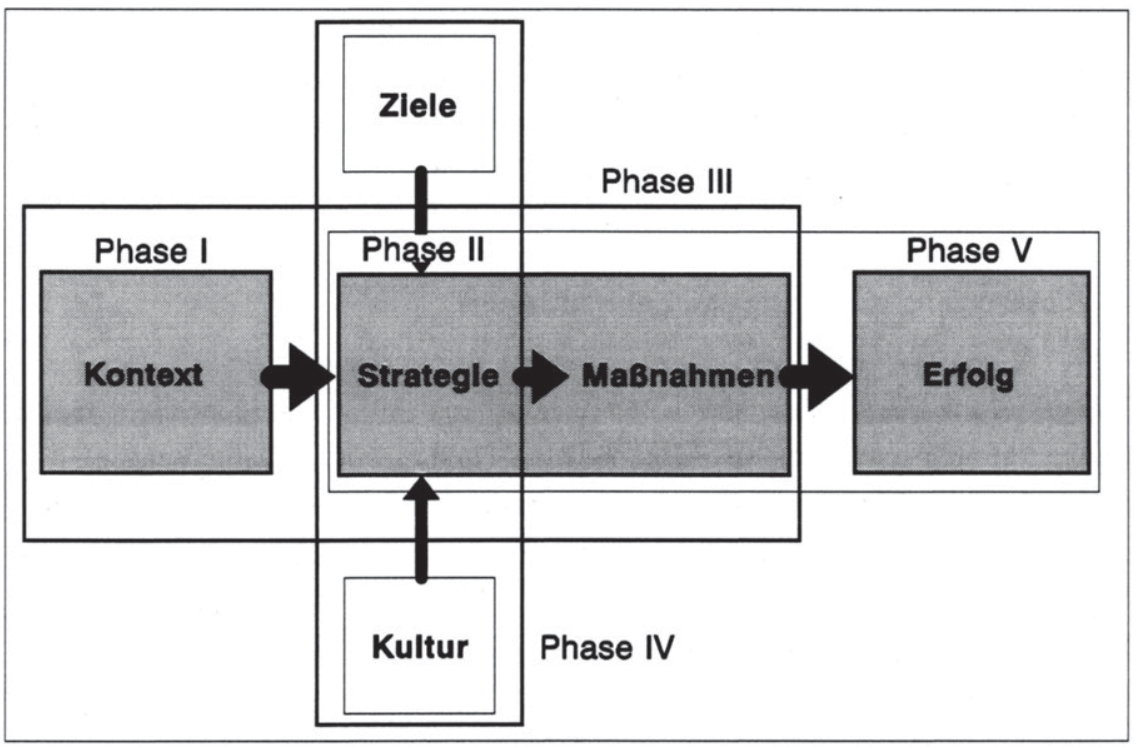

Abb. 2: Allgemeiner Bezugsrahmen zur Systematisierung der Analyseschritte des Forschungsvorhabens

des High-Tech-Marktes ermittelt werden können. Das Problem der Identifizierung eines relevanten Untersuchungsbereiches mit homogenen Umfeldbedingungen wird zuvor durch eine multidimensionale Abgrenzung anhand ausgewählter Umfeldvariablen (Phase I) zu lösen versucht. Weiterhin ist zu untersuchen, ob strategische Gruppen in High-Tech-Märkten auf Basis der abgeleiteten Strategiemerkmale oder verdichteter Basisstrategien gebildet werden. Die anschließende deskriptive Analyse (Phase II) beschränkt sich nicht nur auf die Darstellung der wettbewerbsstrategischen Verhaltensdimensionen, sondern versucht auch die maßnahmenbezogenen Umsetzungsschwerpunkte der strategischen Gruppen in High-Tech-Märkten zu analysieren. Hierdurch soll Aufschluß darüber gewonnen werden, inwieweit die Marketingstrategien tatsächlich im operativen Unternehmensverhalten umgesetzt werden.

Der deskriptiven Untersuchung schließt sich eine explikative Analyse an. Einerseits wird versucht, anhand der zur Verfügung stehenden empirischen Daten ziel- 
und kulturbezogene Unterschiede zwischen den strategischen Gruppen zu untersuchen (Phase III). Hieraus können Aussagen darüber abgeleitet werden, ob bestimmte Zielsysteme und Unternehmenskulturen das Entstehen strategischer Gruppen in High-Tech-Märkten begründen und ggf. Mobilitätsbarrieren zwischen verschiedenen strategischen Verhaltensgruppen bilden. Andererseits werden die strategischen Gruppen im Hinblick auf ihre Erfolgswirkung (Phase IV) analysiert. Hieraus sollen wichtige Implikationen darüber gewonnen werden, welche Marketingstrategien in High-Tech-Märkten eine Erfolgsposition sichern helfen.

Mit den oben beschriebenen Teilzielen und Analyseschritten des Forschungsvorhabens ist bereits der Gang der Untersuchung vorgezeichnet. In Kapitel B bildet die Kennzeichnung des Konzeptes der strategischen Gruppen und die Übertragung auf High-Tech-Märkte den Ausgangspunkt. Hieran anknüpfend werden die zur Identifikation homogener Umfeldsituationen notwendigen Kontextvariablen ${ }^{95}$ spezifiziert. Hinsichtlich der Bildung strategischer Gruppen in HighTech-Märkten werden nach einer Bestandsaufnahme wichtiger Strategieansätze die für die vorliegende Untersuchung relevanten Strategiedimensionen theoriegestützt abgeleitet. Auf Grundlage des empirischen Bezugsrahmens der Untersuchung erfolgt anschließend eine empirische Validierung des Ansatzes zur Bildung strategischer Gruppen in High-Tech-Märkten (vgl. Abbildung 3). ${ }^{96} \mathrm{Im}$ Mittelpunkt der Diskussion steht die Kennzeichnung der durch eine Verdichtung

Die vorliegende Untersuchung beschränkt sich aus Gründen der Komplexitätsreduktion auf die Einbeziehung zentraler Kontextvariablen zur Kennzeichnung der Unternehmensumwelt in High-Tech-Märkten. Vgl. hierzu Laux, H., Liermann, F., Grundlagen der Organisation, Die Steuerung von Entscheidungen als Grundproblem der Betriebswirtschaftslehre, 3. Aufl., Berlin u.a. 1993, S. 57 ff. Zur Abgrenzungsproblematik von Kontextvariablen siehe z.B. Muchna, C., Strategische Marketing-Früherkennung auf Investitionsgütermärkten, Wiesbaden 1988, S. $102 \mathrm{f}$.

Aufgabe des Bezugsrahmens ist es, theoretische Grundlagen fuir die Ermittlung strategischer Gruppen in High-Tech-Märkten zu strukturieren. Aus wissenschaftstheoretischer Sicht erfuillt ein Bezugsrahmen im Hinblick auf die logische Konsistenz und Operationalisierbarkeit nicht die strengen Anforderungen, die an eine Theorie gestellt werden. Siehe hierzu insbesondere RöBl, D., Die Entwicklung eines Bezugsrahmens und seine Stellung im ForschungsprozeB, in: Journal für Betriebswirtschaft, 1990, Nr. 2, S. 99 ff.; Kubicek, H., Heuristischer Bezugsrahmen und heuristisch angelegte Forschungsdesigns als Elemente einer Konstruktionsstrategie empirischer Forschung, in: Empirische und handlungstheoretische Forschungskonzeption in der Betriebswirtschaftslehre, Hrsg.: Köhler, R., Stuttgart 1977, S. 3 ff. 


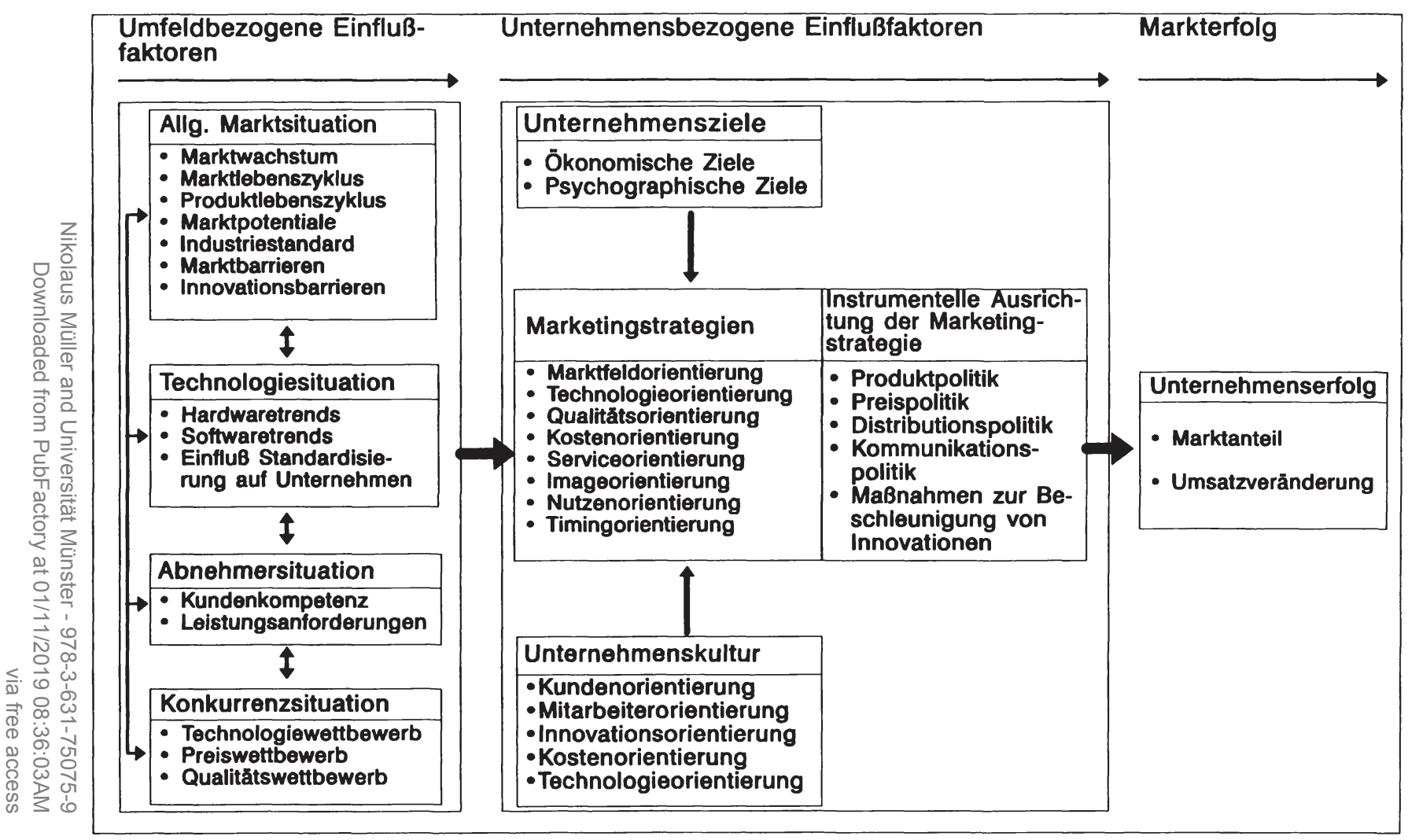

Abb. 3: Bezugsrahmen der empirischen Untersuchung 
wettbewerbsstrategischer Verhaltensdimensionen gewonnenen Basisstrategien sowie die Analyse der Strategieschwerpunkte der identifizierten strategischen Gruppen.

In Kapitel $\mathrm{C}$ werden die strategischen Gruppen hinsichtlich der operativen Ausgestaltungsformen einer konzeptionellen und empirischen Betrachtung unterzogen. Es wird überprüft, in welcher Form die spezifischen Strategieschwerpunkte der strategischen Gruppen in den Marketingaktivitäten umgesetzt werden und welcher Stellenwert den verschiedenen Maßnahmen zur Beschleunigung von Innovationen beigemessen wird. Anschließend werden die zwischen den strategischen Gruppen in High-Tech-Märkten bestehenden Unterschiede im Hinblick auf die verfolgten Unternehmensziele und die Unternehmenskultur theoretisch und empirisch analysiert. Es wird hierbei auch erörtert, inwieweit ein Fit zwischen dem wettbewerbsstrategischen Verhalten und der kulturellen Grundorientierung der strategischen Gruppen in High-Tech-Märkten besteht. Im Anschluß daran wird basierend auf konzeptionellen Überlegungen empirisch untersucht, welchen Markterfolg die High-Tech-Unternehmen in den verschiedenen strategischen Gruppen unter dem Gesichtspunkt eines Strategie-UmweltFit realisieren.

In Kapitel D werden auf der Grundlage der ermittelten Ergebnisse Implikationen für das Management in High-Tech-Märkten abgeleitet. Darüber hinaus wird aufgezeigt, welche Schlußfolgerungen sich für die weiterführende wissenschaftliche Forschung ergeben. 


\section{B. Typologisierung von Marketingstrategien in High-Tech- Märkten auf der Grundlage strategischer Gruppen}

\section{Theoriegeleitete Analyse strategischer Gruppen in High-Tech-Märkten}

Im folgenden werden zunächst die theoretischen Grundlagen des Konzeptes der strategischen Gruppen näher betrachtet, indem eine Begriffsklärung und Inhaltsbeschreibung erfolgt. Anschließend findet eine Bestandsaufnahme der aktuellen Forschung zu strategischen Gruppen im Hinblick auf die Übertragung bestehender Operationalisierungsansätze auf High-Tech-Märkte statt.

\subsection{Grundlagen zum Konzept der strategischen Gruppen}

Der Begriff strategische Gruppe ${ }^{1}$ wurde ursprünglich durch Hunt geprägt. Hunt untersuchte den brancheninternen Wettbewerb in der amerikanischen Haushaltsgeräteindustrie und stellte fest, daß sich das wettbewerbsstrategische Unternehmensverhalten bezüglich der Merkmale $^{2}$ Ausma $B$ der vertikalen Integration, Grad der Produktdiversifikation und Grad der Produktdifferenzierung deutlich unterschied. Anhand dieser Dimensionen konnten die Unternehmen der Haus-

1 Hierbei sind die Begriffe strategische Gruppe, strategische Allianz, strategische Familie und strategisches Netzwerk voneinander abzugrenzen. Die strategische Allianz ist eine Kooperation von mindestens zwei Unternehmen auf einer gleichen Wertschöpfungsstufe mit dem Ziel, die Wettbewerbsposition auszubauen bzw. abzusichern. Vgl. Voigt, S., Strategische Allianzen, Modisches Schlagwort oder Antwort auf globale Herausforderungen?, in: WiSt, 1993, Nr. 5, S. 246 f.; Backhaus, K., Piltz, K., Strategische Allianzen, in: ZfbF, Sonderheft 27, 1989, S. 2. Eine strategische Familie wird durch mehrere Unternehmen gebildet, die in einer Abnehmer-Lieferanten-Beziehung stehen und deren Strategien komplementär sind. Vgl. Albach, H., Strategische Allianzen, strategische Gruppen und strategische Familien, in: ZfB, 1992, Nr. 6, S. 665. Unter einem strategischen Netzwerk wird eine Zusammenarbeit von mehr als zwei Unternehmen auf einer gleichen und unterschiedlichen Wertschöpfungsstufe verstanden. Vgl. demgegenüber Backhaus, K., Meyer, M., Strategische Allianzen und strategische Netzwerke, in: WiSt, 1993, Nr. 7, S. 332.

2

In den weiteren Ausfuihrungen werden die Begriffe Merkmal, Dimension und Variable gleich verwendet. Vgl. hierzu auch Meffert, H., Marketingforschung und Käuferverhalten, 2. Aufl., Wiesbaden 1992, S. $183 \mathrm{ff}$. 
haltsgeräteindustrie in vier Verhaltenscluster eingeteilt werden, die Hunt als strategische Gruppen bezeichnete. ${ }^{3}$

Demnach bilden diejenigen Unternehmen einer Branche eine strategische Gruppe, die hinsichtlich bestimmter strategischer Dimensionen ein vergleichbares Verhalten zeigen. Ein Vergleich zahlreicher nachfolgender Untersuchungen zeigt, daß der Begriff der strategischen Gruppen weitgehend einheitlich aufgefaßt wird:

- Porter versteht unter einer strategischen Gruppe die "Unternehmen in einer Branche, die dieselbe oder eine ähnliche Strategie verfolgen".4

McGee und Thomas sind der Meinung, daß "the natural way to assign firms to strategic groups is by reference to the characteristics of their strategies with group members displaying similar strategies".5

Von Homburg und Sütterlin wird die Ansicht vertreten, daß eine strategische Gruppe eine "Gruppe von Unternehmen einer Branche [darstellt], die eine ähnliche Wettbewerbsstrategie verfolgen".6

- Oster beschreibt strategische Gruppen als "Clusters of firms within an industry that share certain critical asset configurations and follow common strategies".7

In der Gesamtschau der Definitionsbemühungen sind zwei zentrale konstitutive Merkmale von strategischen Gruppen zu erkennen. Zum einen wird eine vergleichbare Umfeldsituation im Sinne eines spezifischen Branchenkontextes hervorgehoben. Zum anderen wird ein homogenes wettbewerbsstrategisches Verhal-

Vgl. Hunt, M.S., Competition in the major home appliance industry, Diss., Harvard University 1972, S. 57 ff. Im Rahmen der empirischen Untersuchung wurde jedoch keine Analyse der brancheninternen Rentabilitätsunterschiede durchgefuihrt. Siehe hierzu auch McGee, J., Thomas, H., Strategic groups: Theory, research and taxonomy, a.a.O., S. 142.

4 Porter, M., Wettbewerbsstrategie, a.a.O., S. 177.

5 McGee, J., Thomas, H., Strategic groups: Theory, research and taxonomy, a.a.O., S. 142.

6 Homburg, Ch., Sütterlin, St., Strategische Gruppen: Ein Survey, a.a.O., S. 137.

7 Oster, Sh., Modern competitive analysis, a.a.O., S. 368. 
ten als kennzeichnend für Unternehmen einer strategischen Gruppe angesehen. ${ }^{8}$ Auf Basis der konstitutiven Merkmale von strategischen Gruppen wird der weiteren Untersuchung folgende Arbeitsdefinition zugrunde gelegt:

Eine strategische Gruppe umfaßt diejenigen Unternehmen, die in einer gleichartigen Umfeldsituation ein homogenes Verhalten bezüglich der wettbewerbsrelevanten strategischen Entscheidungsvariablen ${ }^{9}$ zeigen.

Als Indikator für die "vergleichbare Umfeldsituation" der Unternehmen dient i.d.R. die Zugehörigkeit zu einer gleichen Branche oder die Bearbeitung eines gleichen Marktes. Es wird davon ausgegangen, daß ein gleichartiger Wettbewerb bei denjenigen Unternehmen vorliegt, deren Produkte dieselben oder ähnliche Nachfragerdürfnisse befriedigen. ${ }^{10}$ Zur Bildung von wettbewerbsstrategischen Verhaltensgruppen sind deshalb ausschließlich Unternehmen zu berücksichtigen, die einen gleichen Branchen- oder Marktbezug aufweisen.

Eine Literaturdurchsicht zeigte, daß den empirischen Studien hinsichtlich der Identifikation strategischer Gruppen überwiegend eine angebotsbezogene Beurteilung des wettbewerbsstrategischen Verhaltens zugrunde liegt. Hierbei ist die Untersuchung von Hannig, bei der strategische Gruppen aus Abnehmersicht $(n=85)$ gebildet werden, als Ausnahme anzusehen. Vgl. Hannig, U., Die Entwicklung wettbewerbsorientierter Marketingstrategien auf Basis des Konzeptes der strategischen Gruppen, a.a.O., S. $195 \mathrm{ff}$. An diesem Vorgehen ist zu kritisieren, daß die Qualität der Beurteilung der strategischen Leistungsdimensionen von der nachfragerspezifischen Kenntnis der betrachteten Unternehmen abhängt. Weiterhin stellen die ermittelten strategischen Dimensionen Durchschnittswerte dar, die zudem durch die Wahl des Stichprobenumfangs beeinflußt werden können. Es besteht außerdem bezüglich des Konzeptes der strategischen Gruppen die Gefahr einer Begriffs- und Inhaltsverwirrung. In der vorliegenden Untersuchung wird deshalb eine angebotsbezogene Sicht vertreten.

Strategische Entscheidungen im Wettbewerb umfassen alle langfristig wichtigen, unternehmensexternen und -internen Verhaltensweisen und richten sich auch auf die Gestaltung der Unternehmensstruktur und -kultur. Es wird daher unterstellt, daß wettbewerbsstrategische Entscheidungsvariablen strategie-, struktur- und kulturbezogene Aspekte beinhalten. Zur Bedeutung strategischer, struktureller bzw. kultureller Unternehmensmerkmale im Wettbewerb siehe z.B. Frese, E., Grundlagen der Organisation, Konzepte Prinzipien - Strukturen, 5. Aufl., Wiesbaden 1993, S. 295 ff.; Fritz, W., Marketing - ein Schlüsselfaktor des Unternehmenserfolges?, a.a.O., S. 92 ff.; Meffert, H., Zur Bedeutung von Konkurrenzstrategien im Marketing, in: Marketing ZFP, 1985, Nr. 1, S. 14 ff.

Vgl. Bauer, H.H., Unternehmensstrategie und Strategische Gruppen, a.a.O., S. 402; Link, U., Strategische Konkurrenzanalyse im Konsumgütermarketing, a.a.O., S. 44 ff.; Bain, J.S., Industrial organization, a.a.O., S. 124 f.; Mason, E.S., Economic concentration and the monopoly problem, a.a.O., S. $5 \mathrm{ff}$. 
Hinsichtlich der Konkretisierung des "homogenen wettbewerbsstrategischen Verhaltens" finden sich in der Literatur unterschiedliche Operationalisierungsansätze. Aufbauend auf einer kritischen Reflexion der bestehenden Ansätze ist im Rahmen der weiteren Arbeit zu prüfen, welche spezifischen strategischen Verhaltensdimensionen für eine Typologisierung von Marketingstrategien in High-TechMärkten einzubeziehen sind.

\section{2 Übertragung des Konzeptes der strategischen Gruppen auf High-Tech- Märkte}

Bei der Übertragung des Konzeptes der strategischen Gruppen auf High-TechMärkte treten grundlegende konzeptionelle Probleme auf, die bis heute in der Forschung zu strategischen Gruppen nicht oder nur unzureichend thematisiert worden sind.

In bezug auf das konstitutive Merkmal einer homogenen Umweltsituation zeigt eine Bestandsaufnahme der Untersuchungen zu strategischen Gruppen, daß eine kritische Auseinandersetzung mit dem Problem der Branchen- bzw. Marktabgrenzung bisher meist vernachlässigt worden ist. ${ }^{11}$ Dies fuihrte in der empirischen Forschung ${ }^{12}$ dazu, da $B$ in einigen Untersuchungen strategische Gruppen nicht nur in einer Branche, sondern branchenübergreifend i.S. allgemeiner Strategietypologien bzw. strategischer Grundhaltungen gebildet wurden. ${ }^{13}$

Vgl. hierzu auch Hannig, U., Die Entwicklung wettbewerbsorientierter Marketingstrategien auf Basis des Konzeptes der strategischen Gruppen, a.a.O., S. 83 ff.; Bauer, H.H., Unternehmensstrategie und Strategische Gruppen, a.a.O., S. 400.

Vgl. stellvertretend die Untersuchungen von Baubin, Miller und Harrigan. Baubin, Th., Effiziente Wachstumsstrategien der informationstechnischen Industrie, Diss., Vallendar 1990, S. 35 ff.; Miller, A., A taxonomy of technological settings, with related strategies and performance levels, in: Strategic Management Journal, Vol. 9 (1988), S. 240 ff.; Harrigan, K.R., An application of clustering for strategic group analysis, a.a.O., S. $55 \mathrm{ff}$. Weitere empirische Studien, denen eine branchenübergreifende Erfassung strategischer Gruppen zugrunde liegt, sind der Übersicht im Anhang I der Arbeit zu entnehmen.

Aus diesem Grund unterscheiden Bauer und Hannig im Rahmen der Auseinandersetzung mit dem Begriff der strategische Gruppe zwischen einer "competing unit" und einer "analytical unit". Im ersten Fall stellen strategische Gruppen Teilstrukturen einer Branche dar, die miteinander in Wettbewerbsbeziehungen stehen. Bei einem Verständnis der strategischen Gruppe als analytisches Konstrukt werden Unternehmen mit einem ähn- 
Aufgrund der Entwicklungsdynamik, Technologieintegration und Ausweitung der kundenspezifischen Nutzenanforderungen in High-Tech-Märkten ergibt sich das Problem, daß eine produktbezogene, nachfrageorientierte oder auf der Branchenstatistik beruhende Marktabgrenzung ${ }^{14}$ keine geeignete Grundlage für das Untersuchungsfeld von strategischen Gruppen liefert. Der Markt wird i.d.R. zu eng definiert, und es besteht die Gefahr, daß das Verhalten relevanter Wettbewerber nicht berücksichtigt wird. ${ }^{15}$ In diesem Zusammenhang stellt das Konzept der dreidimensionalen Marktabgrenzung nach Abell, in dem die Technologie explizit als Abgrenzungsmerkmal berücksichtigt wird, einen umfassenderen Ansatz dar. Die Abgrenzung des relevanten Marktes erfolgt hierbei anhand der drei Dimensionen potentielle Abnehmergruppen (customer groups), Abnehmer-

lichen strategischen Verhalten branchenunabhängig zusammengefaßt. Nach Bauer führt eine branchenübergreifende Analyse zur Identifikation unterschiedlicher "strategischer Stile", die mit den von Porter ermittelten generischen Strategietypen (Preis-Kostenfuihrerschaft, Differenzierungsstrategie, Konzentrationsstrategie) oder den von Miles/Snow abgeleiteten strategischen Grundhaltungen ("Prospector", "Analyzer", "Defender", "Reactor") vergleichbar sind. Siehe hierzu Bauer, H.H., Unternehmensstrategie und Strategische Gruppen, a.a.O., S. 400 f.; Hannig, U., Die Entwicklung wettbewerbsorientierter Marketingstrategien auf Basis des Konzeptes der strategischen Gruppen, a.a.O., S. 83 ff.; Porter, M., Wettbewerbsstrategie, a.a.O., S. 62 ff.; Miles, R.E., Snow, Ch.C., Organizational strategy, structure and process, New York 1978, S. 29.

14 High-Tech-Märkte lassen sich grundsätzlich nach räumlichen, zeitlichen und sachlichen Gesichtspunkten abgrenzen. In räumlicher Hinsicht wird betrachtet, welches geographische Absatzgebiet High-Tech-Unternehmen bearbeiten. Die zeitbezogene Marktabgrenzung orientiert sich an der Dauer und Stabilität der Nachfrage. Bei der sachlichen Marktabgrenzung werden High-Tech-Unternehmen berücksichtigt, zwischen denen Wettbewerbsbeziehungen aufgrund ähnlicher Produktleistungen, Technologien oder Kundenbedürfnisse bestehen. Im Vordergrund der betriebswirtschaftlichen Forschung stehen insbesondere Fragestellungen bezüglich der sachlichen Abgrenzung von Märkten auf der Grundlage unternehmens-, produkt- bzw. nachfragebezogener Ansätze. Siehe hierzu vor allem Bauer, H.H., Marktabgrenzung: Konzeption und Problematik von Ansätzen und Methoden zur Abgrenzung und Strukturierung von Märkten unter besonderer Berücksichtigung von marketingtheoretischen Verfahren, Berlin 1989, S. 65 ff. u. 108 ff.; Backhaus, K., Die Abgrenzung des relevanten Marktes vor dem Hintergrund kartellrechtlicher Mißbrauchsaufsicht, Arbeitspapier Nr. 18 des Instituts für Unternehmensführung und Unternehmensforschung, Bochum 1979, S. 3 ff.

15 Vgl. Görgen, W., Strategische Wettbewerbsforschung, a.a.O., S. 83 f.; Backhaus, K., Investitionsgütermarketing, a.a.O., S. 151 ff.; Joas, A., Konkurrenzforschung als Erfolgspotential im strategischen Marketing, a.a.O., S. 82 ff.; Link, U., Strategische Konkurrenzanalyse im Konsumgütermarketing, a.a.O., S. $52 \mathrm{ff}$. In der Literatur wird die enge Beziehung zwischen absatz- und wettbewerbsbezogener Marktabgrenzung hervorgehoben. Siehe hierzu z.B. Baum, H., Der relevante Markt als Problem der Wettbewerbspolitik, in: Wirtschaft und Wettbewerb, 1980, Nr. 6, S. $401 \mathrm{ff}$. 
funktionen (customer functions) und verwendbare Technologien (alternative technologies). ${ }^{16}$

Durch Analyse der Abnehmerdimension wird bestimmt, welche Nachfragergruppen in Abhängigkeit von der Ausprägung des Bedarfs und des voraussichtlichen Kaufverhaltens in High-Tech-Märkten zu bearbeiten sind. Die zweite Abgrenzungsdimension konkretisiert die Abnehmerfunktionen, indem festgelegt wird, welche Kundenbedürfnisse mit den High-Tech-Produktleistungen befriedigt werden sollen. Die Technologiedimension beschreibt alternative Möglichkeiten, wie die Abnehmerfunktionen erfüllt werden können. Es ist hierbei zu entscheiden, welche spezifischen Technologien zur Lösung der Kundenprobleme in HighTech-Märkten eingesetzt werden. ${ }^{17}$

In der folgenden Abbildung 4 ist eine Abgrenzung ausgewählter High-TechMärkte nach dem Konzept von Abell dargestellt. Die dreidimensionale Abgrenzung der High-Tech-Märkte bietet den Vorteil, daß anhand der Technologiedimension, Kundenbedürfnisse und Abnehmergruppen die aktuelle Wettbewerbssituation differenzierter untersucht werden kann. Allerdings zeigt sich hierin auch die besondere Schwierigkeit, eine dauerhaft gültige Marktabgrenzung vorzunehmen.

16 Vgl. Abell, D.F., Defining the business, Englewood Cliffs, N.J. 1980, S. $18 \mathrm{ff}$.

17 Im Rahmen der dreidimensionalen Marktabgrenzung tritt sowohl ein Komplexitäts- als auch ein Reihenfolgeproblem auf. Das Komplexitätsproblem besteht darin, daB mit zunehmender Differenzierung der Abgrenzungsdimensionen die zu analysierenden abnehmer-, funktions- und technologiespezifischen Marktsegmente exponentiell zunehmen. Bei einer sukzessiven Marktabgrenzung ist zu entscheiden, in welcher Reihenfolge die einzelnen Dimensionen einfließen. Es bleibt hierbei festzuhalten, da $B$ eine abnehmerorientierte Vorgehensweise primär auf das Erkennen und die Ausnutzung von Marktchancen ausgerichtet ist. Demgegenüber berücksichtigt ein funktions- oder technologiebezogener Planungsansatz stärker die unternehmensspezifischen Ressourcen. Vgl. hierzu Birkelbach, R., Strategische Geschäftsfeldplanung im Versicherungssektor, in: Marketing ZFP, 1988, Nr. 3, S. 237 f.; Meffert, H., Marketing, Grundlagen der Absatzpolitik, a.a.O., S. 99 f.; Krups, M., Marketing innovativer Dienstleistungen am Beispiel elektronischer Wirtschaftsinformationsdienste, Frankfurt a.M. u.a. 1985, S. 49 ff. u. 75 $\mathrm{ff}$. In der vorliegenden Untersuchung werden beide Problembereiche nicht weiter vertieft. 


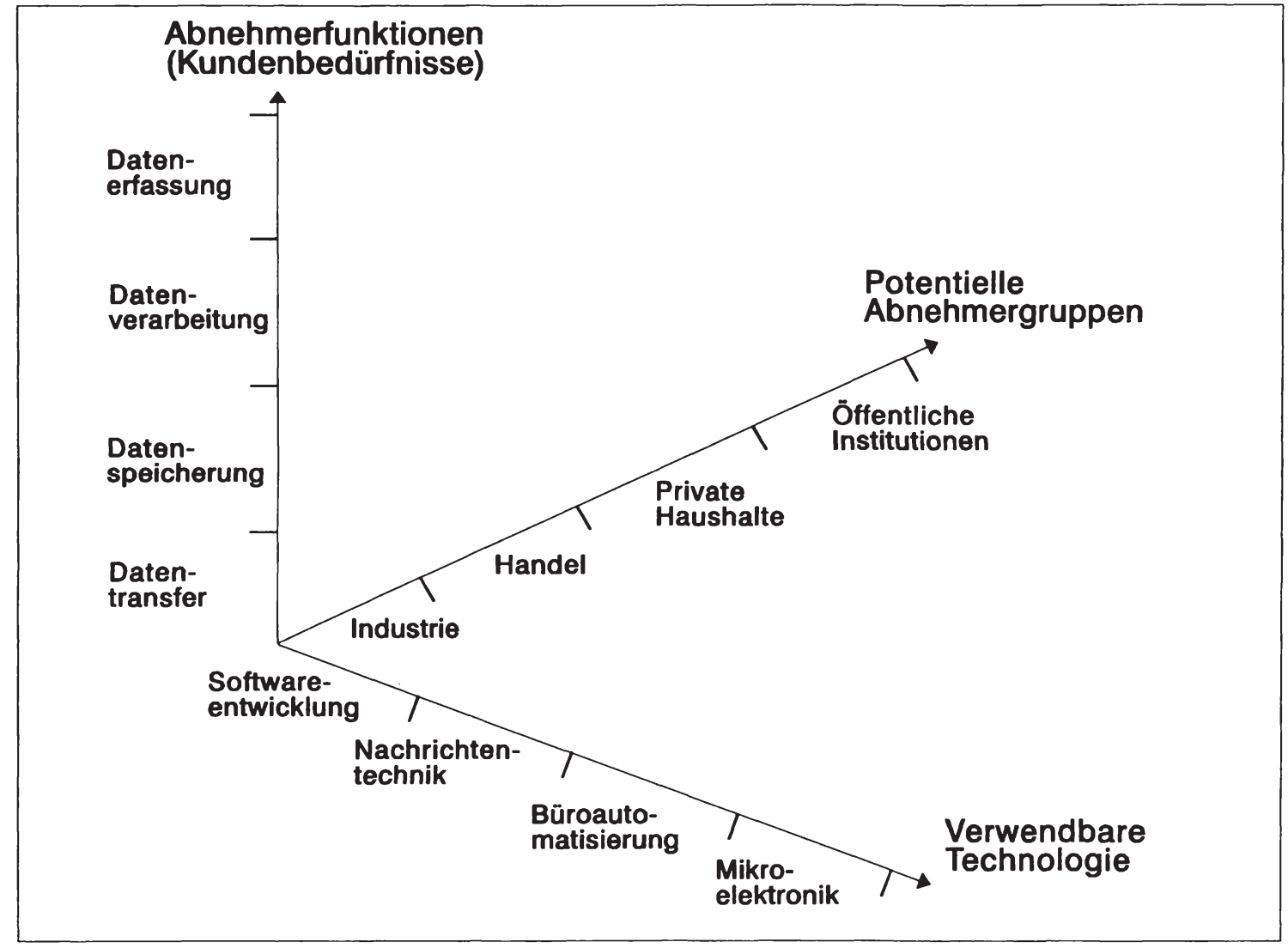

Abb. 4: Dreidimensionale Abgrenzung ausgewählter High-Tech-Märkte 
Die High-Tech-Märkte sind von einer schnellen Integration ursprünglich getrennter Technologien geprägt. So sind die Mikroelektronik und die klassische Nachrichtentechnik in der Telekommunikation aufgegangen. Die Telekommunikation wächst wiederum in Verbindung mit der Datenverarbeitung und der Büroautomatisierung zu dem gemeinsamen Bereich der Informations- und Kommunikationstechnologie zusammen. ${ }^{18}$ Der Trend zur Digitalisierung und Multimedialisierung führt derzeit $\mathrm{zu}$ einer verstärkten Technologieintegration in der Softwareentwicklung, der Unterhaltungsindustrie und der Verlagsbranche. ${ }^{19}$

Die Kundenbedürfnisse (Abnehmerfunktionen) weiten sich in High-TechMärkten kontinuierlich aus. Durch speicherfähige Schreibmaschinen und Computer werden die Funktionen der Datenerfassung, -verarbeitung und -speicherung erfullt. Das zusätzliche Kundenbedürfnis des Datentransfers führte zum Angebot von Schreibmaschinen, die auch als Telefax-Gerät genutzt werden. Die Schaffung der Voraussetzungen zur Vernetzung von Computern und das Angebot von Computern in Verbindung mit einem Modem dienen dem gleichen Zweck. ${ }^{20} \mathrm{Daß}$ dieser Trend im Bereich der Informations- und Kommunikationstechnologie gegenwärtig an Dynamik gewinnt, zeigt sich u.a. an der Entwicklung des sog. "Personal Digital Assistant", der die Produkteigenschaften eines MobilfunkTelefons, Telefax-Gerätes und portablen Computers miteinander verbindet. ${ }^{21}$

Vgl. Dussauge, P., Hart, St., Ramanantsoa, B., Strategic technology management, a.a.O., S. 28 ff.; Meffert, H., Strategische Unternehmensfuhrung und Marketing, a.a.O., S. 59; Backhaus, K. Weiber, R., Technologieintegration und Marketing, a.a.O., S. 35; Meffert, H., Klassische Funktionenlehre und marktorientierte Führung, Integrationsperspektiven aus Sicht des Marketing, in: Integration und Flexibilität, Eine Herausforderung an die allgemeine Betriebswirtschaftslehre, Hrsg.: Adam, D., Backhaus, K., Meffert, H., Wagner, H., Münster 1989, S. 378; Mayndel, E., Technologie-Akzeptanz im Unternehmen: Mitarbeiter für neue Informationstechnologien gewinnen, Wiesbaden 1987 , S. $18 \mathrm{ff}$.

Vgl. Schrape, K., Medienmärkte im Umbruch?, in: Planung und Analyse, 1994, Nr. 1, S. 19 ff.; Meffert, J., Standards als Integrationsinstrument in der Computer- und Kommunikationsindustrie, a.a.O., S. 106; Kodama, F., Technologiefusion - der Weg zum Erfolg auf Zukunftsmärkten, a.a.O., S. $41 \mathrm{ff}$.

Vgl. Meffert, J., Standards als Integrationsinstrument in der Computer- und Kommunikationsindustrie, a.a.O., S. 115 f. Green, K. Creating demand for biotechnology, a.a.O., S. 172 ff.; Meffert, H., Strategische Unternehmensfuihrung und Marketing, a.a.O., S. 59.

Vgl. o.V., Zeitungen in Amerika experimentieren mit elektronischen Medien, in: FAZ vom 03.08.1993, S. 14. 
Darüber hinaus ist eine Abgrenzung der High-Tech-Märkte auf Grundlage von Abnehmergruppen als problematisch anzusehen. High-Tech-Unternehmen, die sich mit ihren Produktleistungen bisher auf eine bestimmte Zielgruppe, z.B. Großunternehmen oder mittelständische Unternehmen, konzentriert haben, können ihr Produktangebot oftmals ohne größere Schwierigkeiten auf weitere Abnehmergruppen, z.B. Privatkunden, ausdehnen. Dies läßt sich am Beispiel des strategischen Verhaltens von Unternehmen der Computerindustrie verdeutlichen. Während IBM seine Computer ab Mitte der 70er Jahre nicht mehr nur an gewerbliche, sondern auch an private Abnehmer verkauft, führten Apple und Siemens/Nixdorf eine Zielgruppenausweitung in umgekehrter Weise durch. ${ }^{22}$

Indem die High-Tech-Märkte in bezug auf die verwendete Technologie und die Abnehmerfunktionen rasch zusammenwachsen sowie bezüglich der Abnehmergruppen unzureichend dauerhaft zu trennen sind, ergibt sich für die High-TechUnternehmen häufig das Problem einer zu engen Abgrenzung des relevanten Marktes. Es besteht hierdurch die Gefahr, daß wichtige Konkurrenten übersehen werden und die aktuelle Wettbewerbssituation damit falsch eingeschätzt wird. Dies kann wiederum zu einer unvollständigen Beurteilung von strategischen Gestaltungsoptionen und zu einer Fehleinschätzung von Erfolgsunterschieden in High-Tech-Märkten führen.

Angesichts des Problems der Abgrenzung relevanter High-Tech-Märkte anhand traditioneller Branchenstatistiken, einzelner Produktkategorien, Technologien bzw. Nachfragergruppen wird in der vorliegenden Untersuchung ein multidimensionaler Ansatz zur Bildung gleichartiger Umfeldsituationen gewählt. ${ }^{23}$

Vgl. Marone, J.G., Winning in high-tech markets, a.a.O., S. 65 ff.; Dussauge, P., Hart, St., Ramanantsoa, B., Strategic technology management, a.a.O., S. 28 ff.; Samli, A.C., Wills, J.R., Strategies for marketing computers and related products, in: Industrial Marketing Management, Vol. 15 (1986), S. 27 ff.

Vgl. zu diesem Vorgehen auch Kieser, A., Kubicek, H., Organisation, a.a.O., S. 204 ff.; Bauer, H.H., Marktabgrenzung: Konzeption und Problematik von Ansätzen und Methoden zur Abgrenzung und Strukturierung von Märkten unter besonderer Berücksichtigung von marketingtheoretischen Verfahren, a.a.O., S. 258 ff.; RöBl, D., Ein Stufenplan zur Marktabgrenzung, Wien 1986, S. 75 ff. Zu Problemen einer mehrdimensionalen Marktabgrenzung siehe z.B. Horst, B., Ein mehrdimensionaler Ansatz zur Segmentierung von Investitionsgütermärkten, Diss., Köln 1988, S. 343. 
Hierbei werden zur Kennzeichnung von High-Tech-Märkten relevante Kontextvariablen, z.B. technologische Trends, Nutzenanforderungen der Nachfrager, Preis- und Technologiewettbewerb, simultan berücksichtigt. ${ }^{24}$

Neben der Schaffung einer homogenen Umweltsituation liegt ein weiteres konzeptionelles Problem in der Ableitung von Operationalisierungsvariablen zur Identifikation strategischer Gruppen. Im Hinblick auf das vorliegende Forschungsvorhaben erscheint es zweckmäßig, aus der Vielzahl möglicher Operationalisierungsvariablen nur solche Variablen zur Bildung strategischer Gruppen in High-Tech-Märkten einzubeziehen, die den Unternehmen in einem spezifischen Branchenkontext ein größtmögliches Differenzierungspotential im Wettbewerb bieten und sich dadurch zum Aufbau von Wettbewerbsvorteilen eignen. Bei einer Bewertung der überwiegend empirisch gestützten Forschung zum Untersuchungsbereich der strategischen Gruppen lassen sich grundsätzlich zwei Klassen von Operationalisierungsansätzen unterscheiden. ${ }^{25}$ Der ersten Klasse sind empirische Untersuchungen zuzurechnen, bei denen strategische Gruppen auf der Grundlage von unternehmensbezogenen Variablen, die Strategie- und Strukturmerkmale umfassen, gebildet werden.

Beispielhaft fuir die Erfassung strategischer Gruppen anhand von Strategiemerkmalen ist die empirische Studie von Hawes und Crittenden anzufuihren. Im Rahmen der Untersuchung wurden amerikanische Handelsunternehmen bezüglich ihrer Strategien für markenlose Lebensmittel analysiert und auf Basis von 18 Marketingvariablen (u.a. Produkt, Promotion, Preis und Display) drei strategischen Gruppen zugeordnet. ${ }^{26}$ Neben "aggressiven Initiatoren" konnten "konservative Reagierer" und "passive Verteidiger" ermittelt werden. Insgesamt

Vgl. hierzu im Detail die Ausführungen im Teil B Kap. 2.1 der Arbeit.

In diesem Zusammenhang wurden die zur Verfügung stehenden empirischen Studien zu strategischen Gruppen einer Analyse unterzogen. Da bisher kaum empirische Arbeiten aus High-Tech-Märkten vorliegen, gab eine Durchsicht der vorhandenen Studien wertvolle Hinweise für die vorliegende Untersuchung. Eine Übersicht wichtiger empirischer Studien ist im Anhang I der Arbeit enthalten.

Vgl. Hawes, J.M., Crittenden, W.F., A taxonomy of competitive retailing strategies, a.a.O., S. $278 \mathrm{ff}$. 
konnte nachgewiesen werden, daß "aggressive Initiatoren" deutlich erfolgreicher sind als die beiden anderen strategischen Verhaltensgruppen.

Die vorliegenden Forschungsansätze zur Bildung strategischer Gruppen anhand von Strategiemerkmalen sind auf High-Tech-Märkte nur eingeschränkt übertragbar. Dies liegt darin begründet, daß die in den Untersuchungen berücksichtigten Strategievariablen meist für ein individuelles Untersuchungsdesign abgeleitet wurden. ${ }^{27}$ Die Forschungsansätze enthalten deshalb neben allgemeingültigen, branchenübergreifenden Strategievariablen, die die Qualitäts-, Innovations- und Kostenorientierung der Unternehmen charakterisieren, auch spezielle branchenbezogene strategische Verhaltensvariablen, z.B. Promotion bzw. Zweitplazierung. ${ }^{28}$

Als Beispiel für die Identifikation strategischer Gruppen anhand von Strukturmerkmalen ist die Untersuchung von Heinemann zu nennen. Auf der Grundlage von 26 strukturbezogenen Variablen der Bereiche Warengruppe, Kundengruppe und Handelsfunktion konnten sechs strategische Gruppen im textilen Facheinzelhandel klassifiziert werden. ${ }^{29}$ Es zeigte sich, daß zwischen den strategischen Gruppen deutliche Unterschiede bezüglich der Marktbearbeitungsmaßnahmen Sortiments-, Service-, Kommunikations- und Preispolitik bestehen.

Siehe hierzu die Übersicht im Anhang I der Arbeit, in der die in den empirischen Untersuchungen berücksichtigten Strategievariablen zur Bildung strategischer Gruppen dargestellt sind.

Vgl. Hawes, J.M., Crittenden, W.F., A taxonomy of competitive retailing strategies, a.a.O., S. 277 ff. Siehe hierzu u.a. Dess, G.G., Davis, P.S., Porter's (1980) generic strategies as determinants of strategic group membership and organizational performance, a.a.O., S. $470 \mathrm{ff}$.; Hatten, K.J., Hatten, M.L., Strategic groups, asymmetrical mobility barriers and contestability, a.a.O., S. $330 \mathrm{ff}$.

Die von Heinemann erfaßten strategischen Gruppen waren: große Herrenausstatter mit jung-hochwertiger Betonung, hochwertig-qualitätsorientierte Bekleidungsfachgeschäfte mit sportlicher Note, gehobene DOB-Spezialisten, preis- und qualitätsorientierte Bekleidungsfachgeschäfte, konsumorientierte Vollsortimenter mit qualitäts- und preisorientierter Note und modisch-legere Trendsetter. Vgl. Heinemann, G., Betriebstypenprofilierung und Erlebnishandel, Wiesbaden 1989, S. 64 ff.; Meffert, H., Heinemann, G., Strategische Gruppen im Handel, Eine empirische Analyse am Beispiel des textilen Einzelhandels, in: Handelsforschung 1989, Hrsg.: Trommsdorff, V., Wiesbaden 1989, S. 124 ff. 
Gegen die Berücksichtigung von Strukturvariablen im Rahmen der strategischen Gruppenbildung ist grundsätzlich einzuwenden, daß mit strategischen Entscheidungen auch Veränderungen struktureller Art einhergehen können, um einen Fit zwischen Strategie und Struktur ${ }^{30}$ sicherzustellen. ${ }^{31}$ Die strategische Entscheidung, als Kostenführer im Markt zu agieren, kann jedoch auf der Grundlage unterschiedlicher Strukturen erfolgen, z.B. durch den Aufbau zusätzlicher Produktionskapazitäten mit höheren Degressionseffekten bzw. durch die Auslagerung von Produktions- und Logistikfunktionen. Gleiche Strategieschwerpunkte können somit auf Basis unterschiedlicher Unternehmensstrukturen verfolgt werden. ${ }^{32}$ Hieraus folgt, daß die Bildung strategischer Gruppen anhand von Strukturvariablen ggf. nicht hinreichend die strategische Grundorientierung i.S. einer Wettbewerbsdifferenzierung erkennen läßt. Auf die Einbeziehung von Strukturvariablen zur Identifikation strategischer Gruppen in High-Tech-Märkten wird deshalb verzichtet.

Der zweiten Klasse von Operationalisierungsansätzen sind empirische Forschungen zuzuordnen, die neben unternehmensbezogenen auch umfeld- und markterfolgsspezifische Merkmale bei der Bildung strategischer Gruppen berücksichtigen. Umfeldbezogene Merkmale wurden z.B. in der Studie von Dess und Davis einbezogen. Es wurden dabei 21 unternehmens- (u.a. Produktqualität, Preisniveau, Image, Service) und umfeldbezogene (Marktwachstum) Variablen zu

Der Organisationsstruktur lassen sich in einer weiteren Begriffsfassung Aspekte der Aufbau- und Ablauforganisation sowie Fragestellungen im Zusammenhang mit der Gestaltung organisationaler Systeme zuordnen. Meffert, H., Marketing-Management, a.a.O., S. 325 ff.; Kieser, A., Kubicek, H., Organisation, a.a.O., S. 16 ff.; Bühner, R., Betriebswirtschaftliche Organisationslehre, 4. Aufl., München, Wien 1991, S. 5 ff.

Beispielsweise stellt Chandler heraus, daß die Unternehmensstruktur durch das strategische Verhalten beeinflußt wird ("structure-follows-strategy"). Vgl. Kreikebaum, H., Strategische Unternehmensplanung, a.a.O., S. 113 ff.; Chandler, A.D. Jr., Strategy and structure, Chapters in the history of industrial enterprise, Cambridge, Mass., London 1962, S. $20 \mathrm{ff}$.

Vgl. Habib, M.M., Victor, B., Strategy, structure, and performance of U.S. Manufacturing and Service MNCs: A comparative analysis, a.a.O., S. 595 ff.; Conant, J.S., Mokwa, M.P., Varadarajan, R.P., Strategic types, distinctive marketing competencies and organizational performance: a multiple measures-based study, a.a.O., S. $370 \mathrm{ff}$.; Nayyar, P., Research notes and communications strategic groups: A comment, in: Strategic Management Journal, Vol. 10 (1989), S. 103; Fronhoff, B., Die Gestaltung von Marketingstrategien, a.a.O., S. 209.; Kamani, A., Generic competitive strategies - an analytical approach, in: Strategic Management Joumal, Vol. 5 (1984), No. 4, S. 367 ff. 
drei wettbewerbsstrategischen Dimensionen ("differenziertes Marketing", "niedrige Kosten", "Spezialisierung") verdichtet und hiermit vier strategische Verhaltensgruppen in der farbenherstellenden Industrie erfaßt. ${ }^{33}$ Die identifizierten kostenorientierten Farbenhersteller sind primär an einer Effizienzsteigerung in der Beschaffung und der Produktion interessiert. Die "Differenzierungsorientierten" messen vor allem der Neuproduktentwicklung, der Werbung und der Markenbekanntheit einen besonderen Stellenwert bei. Bei den Unternehmen, die eine Fokussierungsstrategie verfolgen, sind die Entwicklung von Spezialprodukten und die Servicepolitik von großer Bedeutung. Das Verhalten in der letzten strategischen Gruppe besitzt keinen eindeutigen Strategieschwerpunkt. Dess und Davis konnten nachweisen, daß zwischen den strategischen Gruppen der farbenproduzierenden Industrie deutliche Erfolgsunterschiede bestehen. ${ }^{34}$

Bei der Auswahl von Umfeldvariablen im Rahmen der Identifikation strategischer Gruppen lassen sich allgemeine (globale Umwelt) und spezielle (aufgabenspezifische Umwelt) Kontextmerkmale unterscheiden. ${ }^{35}$ Während allgemeine Kontextvariablen, z.B. Marktwachstum, rechtliche und politische Rahmenbedingungen, die Unternehmen einer Branche i.d.R. in gleicher Weise determinieren, wird das Unternehmensverhalten durch spezielle Situationsvariablen, wie Bedarfsveränderungen, Preis- und Qualitätswettbewerb unter-

Vgl. Dess, G.G., Davis, P.S., Porter's (1980) generic strategies as Determinants of strategic group membership and organizational performance, a.a.O., S. $470 \mathrm{ff}$.

Von den kostenorientierten Farbenherstellern werden die höchste Gesamtkapitalrentabilität und zweithöchste Umsatzsteigerung erzielt. Differenzierungsorientierte Unternehmen stehen bei der Rentabilität an zweiter und beim Umsatzwachstum an dritter Stelle. Unternehmen, die eine Fokussierungsstrategie verfolgen, erreichen zwar die höchste Umsatzsteigerung, realisieren jedoch gleichzeitig die niedrigste Gesamtkapitalrentabilität. Die farbenproduzierenden Unternehmen der vierten strategischen Gruppe liegen hinsichtlich der Rentabilität an vorletzter und der Umsatzsteigerung an letzter Position. Vgl. Dess, G.G., Davis, P.S., Porter's (1980) generic strategies as Determinants of strategic group membership and organizational performance, a.a.O., S. 482.

$\mathrm{Zu}$ einer vergleichbaren Systematisierung siehe auch Staehle, W.H., Management, a.a.O., S. 97; Meffert, H., Marktanalyse, in: Handwörterbuch der Planung, Hrsg.: Szyperski, N., Stuttgart 1989, Sp. 1021 ff.; Meffert, H., Marketing, Einfuihrung in die Absatzpolitik, 6. Aufl., Wiesbaden 1982, S. 45 ff. 
schiedlich stark beeinflußt. ${ }^{36} \mathrm{Da}$ das Konzept der strategischen Gruppen eine homogene Umfeldsituation für die beobachteten Unternehmen unterstellt, dürfen Kontextvariablen der globalen und aufgabenbezogenen Umwelt nicht zur Bildung strategischer Gruppen in High-Tech-Märkten herangezogen werden.

Beispielhaft für die Bildung strategischer Gruppen auf Basis von Erfolgsmerkmalen (z.B. Umsatz, Marktanteil) ist die empirische Analyse von Porter zu sehen, bei der Unternehmen der Konsumgüterindustrie anhand des relativen Branchenumsatzes klassifiziert wurden. ${ }^{37}$ Unternehmen mit einem relativen Branchenumsatz von mindestens $30 \%$ wurden in der strategischen Gruppe der "Leader" und die übrigen Konsumgüterhersteller in der Gruppe der "Follower" zusammengefaßt. Im Rahmen der Untersuchung versuchte Porter nachzuweisen, inwieweit der Unternehmenserfolg durch 15 strategische unternehmensinterne und -externe Dimensionen erklärt wird. Es konnten jedoch keine signifikanten Ergebnisse festgestellt werden. ${ }^{38}$

Der Ermittlung von strategischen Gruppen anhand von Erfolgsvariablen wie dem Umsatz oder dem Marktanteil ist entgegenzuhalten, daß bei einer anschließenden Analyse brancheninterner Erfolgsunterschiede Unternehmen einer überdurchschnittlich erfolgreichen strategischen Gruppe i.d.R. auch hinsichtlich weiterer Erfolgsgrößen (z.B. Gewinn, Umsatzrentabilität) besonders erfolgreich sein

36 Im Hinblick auf High-Tech-Märkte ist davon auszugehen, daß innerhalb der globalen Umwelt technologische Rahmenbedingungen einen unterschiedlich starken EinfluB auf das Verhalten von High-Tech-Unternehmen ausüben. Siehe hierzu die Ausführungen im Teil B Kap. 2 der Arbeit.

Vgl. Porter, M., The structure within industries and companies performance, a.a.O., S. $215 \mathrm{ff}$.

38 Porter stellte dennoch fest, daß "Leader" vor allem in den Produktbereichen erfolgreicher sind, die durch hohe Werbeausgaben (Softdrinks, Getreideprodukte) oder F\&E-Ausgaben (Medikamente) gekennzeichnet sind. "Follower" agieren insbesondere in den Konsumgüterbereichen profitabler, die stark fragmentiert (Optik, Zeitschriften, Teppiche) sind bzw. in denen Degressionseffekte eine geringe Bedeutung (Bekleidung, Schuhe, Fleischprodukte) haben. Vgl. Porter, M., The structure within industries and companies performance, a.a.O., S. $222 \mathrm{ff}$. 
werden und umgekehrt. ${ }^{39} \mathrm{Da}$ in dem vorliegenden Forschungsvorhaben u.a. analysiert werden soll, welche Erfolgswirkung unterschiedliche Marketingstrategien in High-Tech-Märkten haben, erscheint es nicht sinnvoll, Erfolgsvariablen in einen Ansatz zur Bildung strategischer Gruppen in High-TechMärkten zu integrieren.

Im Zusammenhang mit der methodischen Vorgehensweise zur Bildung strategischer Gruppen in High-Tech-Märkten zeigt eine Bestandsaufnahme der empirischen Forschung, daß grundsätzlich sog. "typologische" bzw. multivariate Analysemethoden zur Abgrenzung strategischer Gruppen herangezogen werden. ${ }^{40}$

Bei den sog. "typologischen" Ansätzen wird ein "strategischer Raum" anhand von i.d.R. zwei a priori festgelegten strategischen Schlüsseldimensionen (z.B. Qualitäts- und Preisorientierung) aufgespannt, in dem die Unternehmen positioniert und anschließend zu strategischen Gruppen zusammengefaßt werden. ${ }^{41} \mathrm{Als}$ besondere Schwäche der sog. "typologischen" Analysemethoden ist die Subjektivität in der Auswahl der Gruppierungsvariablen und in der Bildung strategischer Gruppen zu nennen. ${ }^{42}$ Dabei wird oftmals die Relevanz der verwendeten Gruppierungsdimensionen nicht genügend hinterfragt und nicht überprüft, ob eine andere Art der Klassifikation zu einer höheren Homogenität der strategischen Gruppen führt. In der Literatur zu strategischen Gruppen wird als eine weitere

Vgl. Barney, J.B., Hoskisson, R.E., Strategic groups: Untested assertions and research proposals, in: Managerial and Decision Economics, Vol. 11 (1990), S. 187 ff.; Nayyar, P., Research notes and communications strategic groups: A comment, a.a.O., S. 103.

Eine vollständige Übersicht über die in den empirischen Studien verwendeten Analysemethoden ist im Anhang I der Arbeit zu finden.

41 Diese graphische Form der Gruppenbildung wird auch als "strategische Karte" bzw. "strategische Matrix" bezeichnet. Vgl. hierzu u.a. Bauer, H.H., Unternehmensstrategie und Strategische Gruppen, a.a.O., S. 406; McNannee, P., McHugh, M., Mapping competitive groups in the clothing industry, in: Long Range Planning, Vol. 22 (1989), S. 89 ff.; Porter, M., Wettbewerbsstrategie, a.a.O., S. 178 f. u. 204 ff.

Vgl. stellvertretend Kumar, N., Mobility barriers and profitability of multinational and local enterprises in Indian manufacturing, in: The Journal of Industrial Economics, Vol. 38 (1990), No. 4, S. 450 ff.; Hatten, K.J., Hatten, M.L., Strategic groups, asymmetrical mobility barriers and contestability, a.a.O., S. 331; Harrigan, K.R., An application of clustering for strategic group analysis, a.a.O., S. 58; Newman, H.M., Strategic groups and the structure-performance relationships, in: The Review of Economics and Statistics, Vol. 60 (1978), S. 418 ff. 
Schwäche der sog. "typologischen" Analysemethoden die Beschränkung auf jeweils zwei Gruppierungsvariablen hervorgehoben. ${ }^{43}$

Für eine dem Konzept der strategischen Gruppen entsprechenden Gruppierung von High-Tech-Unternehmen ist deshalb der Einsatz multivariater Analysemethoden erforderlich. Diese bieten den Vorteil einer objektiven Selektion relevanter wettbewerbsstrategischer Schlüsseldimensionen (Regressions-, Diskriminanzanalyse) und der Verdichtung von Variablen (Faktoranalyse), die miteinander korrelieren. Darüber hinaus wird eine größtmögliche Homogenität bei der Bildung strategischer Gruppen (Cluster-, Varianzanalyse) sichergestellt. ${ }^{44}$ Es ist hervorzuheben, da $\beta$ in neueren empirischen Forschungen die Identifikation strategischer Gruppen überwiegend auf der Grundlage einer Clusteranalyse erfolgt, die auch in der vorliegenden Untersuchung zur Bildung von strategischen Unternehmensgruppen in High-Tech-Märkten herangezogen wird. ${ }^{45}$

Eine zusammenfassende Übersicht der dargestellten Ansätze und ausgewählter weiterer Untersuchungen zur Bildung strategischer Gruppen im Hinblick auf den

43 Vgl. stellvertretend Oster, Sh., Intraindustry structure and the ease of strategic change, a.a.O., S. 378 ff.; Newman, H.M., Strategic groups and the structure-performance relationships, a.a.O, 418 ff.; Porter, M., Consumer behavior, retailer power and manufacturing strategy in consumer goods industries, Diss., Harvard University 1973, S. 20 ff.

Vgl. stellvertretend Hannig, U., Die Entwicklung wettbewerbsorientierter Marketingstrategien auf Basis des Konzeptes der strategischen Gruppen, a.a.O., S. 201 ff.; Bauer, H.H., Unternehmensstrategie und Strategische Gruppen, a.a.O, S. 405 f.; Lawless, M.W., Finch, L.K. Choice and determinism: A test of Hrebiniak and Joyce's framework on strategy-environment fit, a.a.O., 353 ff.; Harrigan, K.R., An application of clustering for strategic group analysis, a.a.O., S. 58 ff.; Hinterhuber, H., Kirchebner, M., Die Analyse strategischer Gruppen von Unternehmungen, in: ZfB, 1983, Nr. 9, S. 857 ff.

Vgl. hierzu u.a. Carroll, Ch., Lewis, P., Thomas, H., Developing competitive strategies in retailing, in: Long Range Planning, Vol. 25 (1992), No. 2, S. 82 ff.; Fiegenbaum, A., Thomas, H., Strategic Groups and Performance: The U.S. Insurance Industry, 1970-84, in: Strategic Management Journal, Vol. 11 (1990), S. 197 ff.; Lawless, M.W., Finch, L.K. Choice and determinism: A test of Hrebiniak and Joyce's framework on strategyenvironment fit, a.a.O., 353 ff.; Heinemann, G., Betriebstypenprofilierung und Erlebnishandel, a.a.O., S. 64 ff.; Dess, G.G., Davis, P.S., Porter's (1980) generic strategies as Determinants of strategic group membership and organizational performance, a.a.O., S. $470 \mathrm{ff}$.; Hawes, J.M., Crittenden, W.F., A taxonomy of competitive retailing strategies, a.a.O., S. $278 \mathrm{ff}$. 
Branchenbezug, die Gruppierungsvariablen und die methodische Vorgehensweise ist den folgenden Abbildungen 5-7 zu entnehmen. ${ }^{46}$

46 Eine ausführliche Kennzeichnung der in den einzelnen empirischen Studien untersuchten Branchen, verwendeten Gruppierungsvariablen und Methodik ist in der Übersicht im Anhang I der Arbeit zu finden. Die Aufstellung wurde auf der Grundlage der in der Literatur bereits erstellten Übersichten erstellt und aktualisiert. Siehe in diesem Zusammenhang vor allem Reger, R.K., Huff, A.S., Strategic groups: A cognitive perspective, in: Strategic Management Journal, Vol. 14 (1993), S. 103 ff.; Homburg, Ch., Sütterlin, St., Strategische Gruppen: Ein Survey, a.a.O., S. 641 ff.; Thomas, H., Venkatraman, N., Research on strategic groups: Progress and prognosis, in: Journal of Management Studies, Vol. 25 (1988), No. 6, S. 541 ff. 


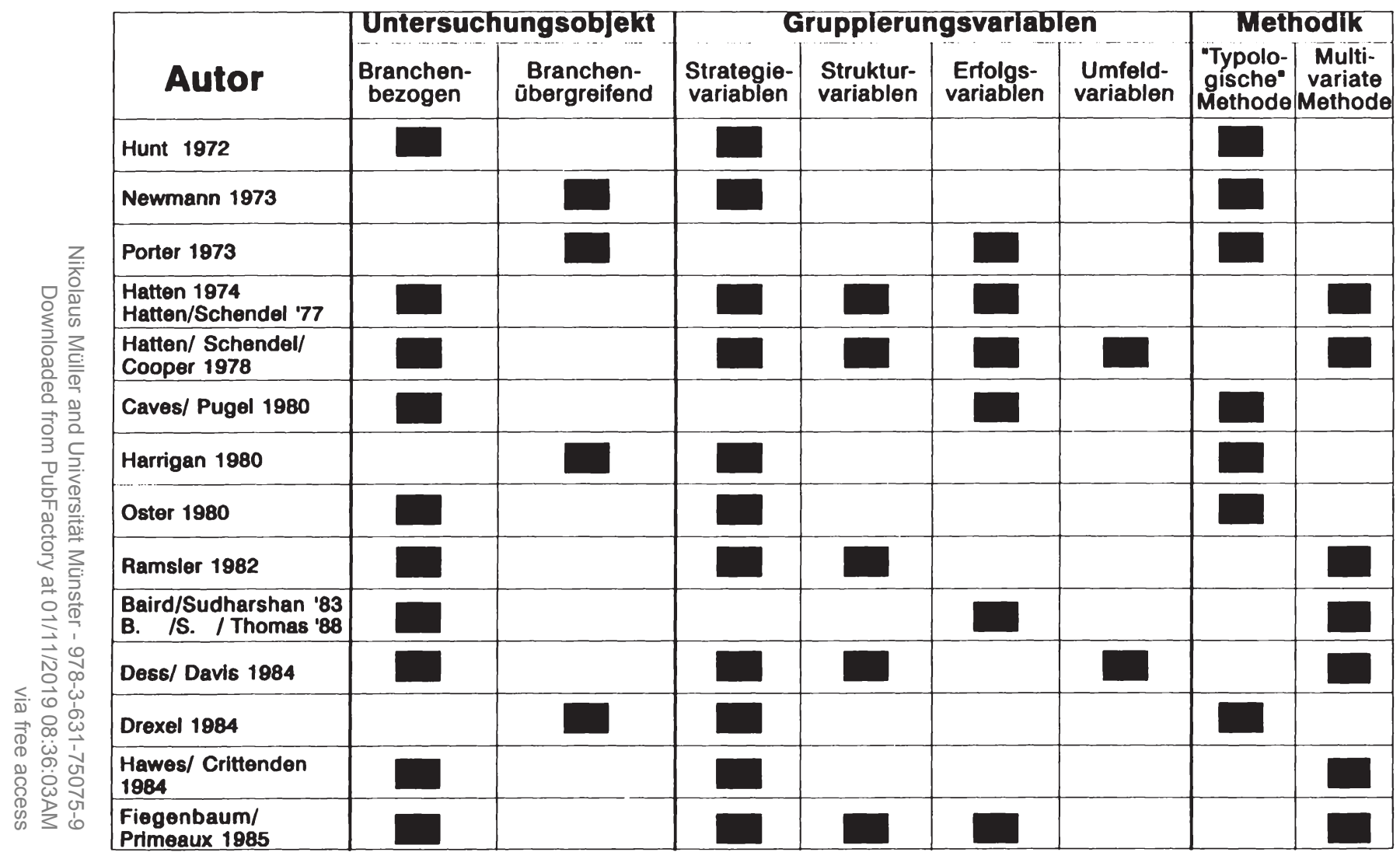

Abb. 5: Obersicht ausgewăhlter empirischer Studien zur Bildung strategischer Gruppen 


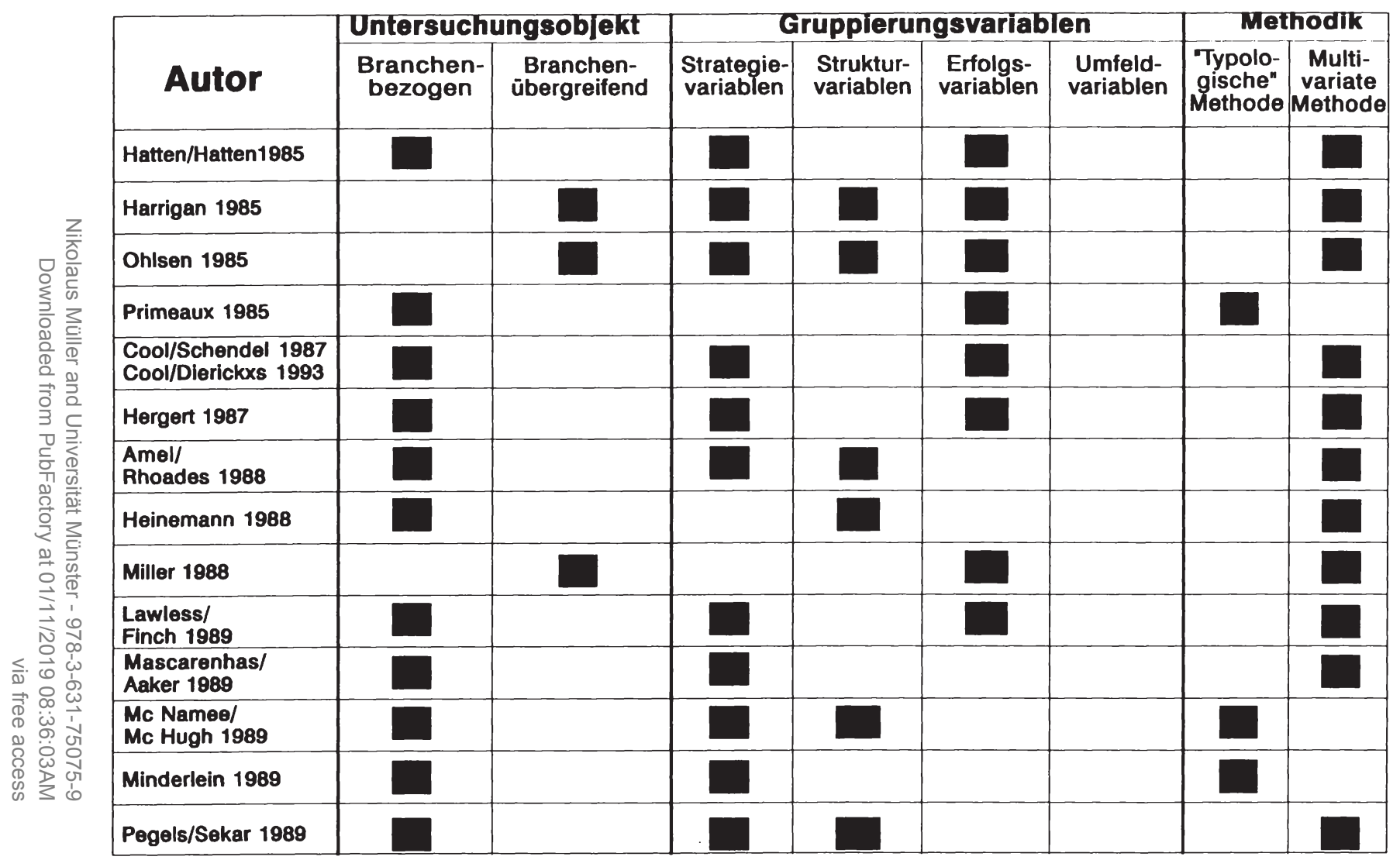

Abb. 6: Ubersicht ausgewăhlter empirischer Studien zur Bildung strategischer Gruppen (Fortsetzung) 


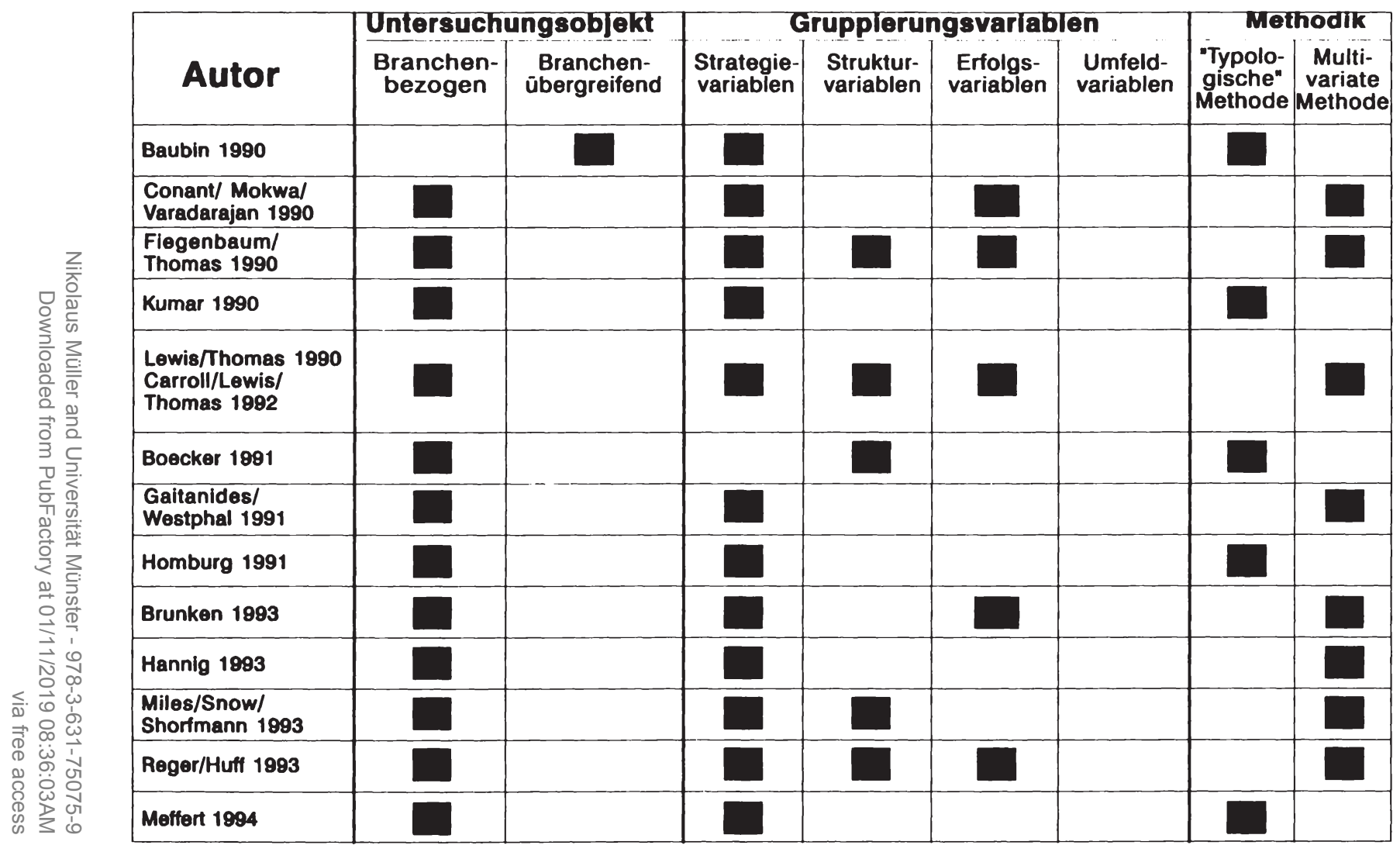

Abb. 7: Obersicht ausgewåhlter empirischer Studien zur Bildung strategischer Gruppen (Fortsetzung) 


\section{Theoriegeleitete Analyse von Determinanten zur Bildung strategischer Gruppen in High-Tech-Märkten}

Ziel der folgenden Ausführungen ist zunächst die Ermittlung geeigneter Kontextdimensionen zur Identifikation homogener Umfeldsituationen in High-TechMärkten. Es werden hierzu Dimensionen der Unternehmensumwelt herausgearbeitet, die für das wettbewerbsstrategische Verhalten von High-Tech-Unternehmen von Bedeutung sind. Diese Vorgehensweise erscheint deshalb notwendig, da die High-Tech-Märkte aus den oben angeführten Gründen keinen homogenen Untersuchungsbereich darstellen. Im Anschluß daran erfolgt nach einer Bestandsaufnahme von Strategieansätzen eine Ableitung zentraler Strategiedimensionen zur Typologisierung ${ }^{47}$ von Marketingstrategien in High-Tech-Märkten auf der Grundlage strategischer Gruppen.

\subsection{Kennzeichnung der Unternehmensumwelt und Ableitung zentraler Kontextdimensionen zur Bildung strategischer Gruppen in High-Tech- Märkten}

Zur Strukturierung der Unternehmensumwelt werden in der Literatur unterschiedliche Ansätze vorgestellt. ${ }^{48}$ Für die vorliegende Untersuchung in High-Tech-

47 In der folgenden Typologisierung werden High-Tech-Unternehmen auf Basis mehrerer wettbewerbsstrategischer Verhaltensdimensionen $\mathrm{zu}$ strategischen Gruppen zusammengefaßt. Zu einer ähnlichen methodischen Vorgehensweise vgl. Kirchgeorg, M., Ökologieorientiertes Unternehmensverhalten, Wiesbaden 1990, S. 142 ff. Siehe in diesem Zusammenhang auch Braunschweig, E., Typen als Zielgruppen-Kriterien, in: "Typologien", Hrsg.: Heinrich Bauer Stiftung, Velbert 1975, S. 12 f.; Knoblich, H., Die typologische Methode in der Betriebswirtschaftslehre, in: WiSt, 1972, Nr. 4, S. 142 f. Zudem werden die Begriffe Typologisierung, numerische Klassifikation und numerische Taxonomie synonym verwendet. Vgl. hierzu z.B. Staehle, W.H., Management, a.a.O., S. 60; McKelvey, B., Organizational systematics: Taxonomic lessons from biology, in: Management Science, Vol. 24 (1978), No. 13, S. 1430; Vogel, F., Probleme und Verfahren der numerischen Klassifikation, Göttingen 1975, S. 5 f. $\mathrm{Zu}$ einem Vergleich dieser Begriffe siehe z.B. Degens, P.O., Hermes, H.J., Opitz, O. (Hrsg.), Die Klassifikation und ihr Umfeld, Frankfurt a.M. 1986; Niemeier, J., Wettbewerbsumwelt und interne Konfiguration, Frankfurt a.M., Bern, New York 1986, S. $30 \mathrm{ff}$.

Vgl. z.B. Jehle, E., Unternehmung und gesellschaftliche Umwelt, Stuttgart 1980, S. 82 ff. Zu Problemen bei der Abgrenzung und Bestimmung der Unternehmensumwelt siehe 
Märkten wird eine Untergliederung der unternehmensexternen Kontextsituation in eine globale und aufgabenbezogene Umwelt vorgenommen. ${ }^{49}$ Der globalen Umwelt (Makroumwelt) werden technologische, gesamtwirtschaftliche, politischrechtliche, sozio-kulturelle und ökologische Kontextvariablen zugerechnet. Innerhalb der Aufgabenumwelt werden u.a. Abnehmer, Konkurrenten, Zulieferer, Geldgeber Handels- und Verbraucherorganisationen als Interaktionspartner angesehen. ${ }^{50}$ Mit Blick auf die globale Umwelt ist für High-Tech-Unternehmen vor allem die Analyse der technologischen Rahmenbedingungen von Interesse, während im Vordergrund der Aufgabenumwelt die Betrachtung der allgemeinen marktbezogenen sowie der speziellen abnehmer- und konkurrenzbezogenen Umfeldsituation steht. ${ }^{51}$

\subsection{Technologiebezogene Umweltsituation}

Die Technologiesituation in High-Tech-Märkten ist durch eine hohe technologische Dynamik und Komplexität gekennzeichnet, die die Unternehmen vor besondere Herausforderungen stellt. Im Hinblick auf die Technologiedynamik ${ }^{52}$

z.B. Schreyögg, G., Umwelt, Technologie und Organisationsstruktur, Bern, Stuttgart 1978 , S. 80 ff.

$\mathrm{Zu}$ einer vergleichbaren Systematisierung siehe auch HeB, G., Marktsignale und Wettbewerbsstrategie, a.a.O., S. 65; Staehle, W.H., Management, a.a.O., S. 97; Raffee, H., Wiedmann, K.-P., Marketingumwelt 2000, in: Marketing 2000, Perspektiven zwischen Theorie und Praxis, Hrsg.: Schwarz, Chr., Wiesbaden 1987, S. 186 ff.; Meffert, H., Marketing, Einfuihrung in die Absatzpolitik, a.a.O., S. $46 \mathrm{ff}$.

Vgl. z.B. Meffert, H., Kirchgeorg, M., Marktorientiertes Umweltmanagement, Stuttgart 1992; S. 60 f.; Steinmann, H., Schreyögg, G., Management, Stuttgart 1990, S. 137 ff.; Meffert, H., Marktanalyse, in: Handwörterbuch der Planung, a.a.O., Sp. 1022.

In der vorliegenden Untersuchung wird auf die Einbeziehung anderer Kontextvariablen der globalen Umwelt und Aufgabenumwelt verzichtet, da vor allem den technologie-, markt-, abnehmer- und konkurrenzbezogenen Kontextdimensionen in High-Tech-Märkten eine besondere Bedeutung zukommt. Zudem wird dieses Vorgehen aus Gründen der Komplexitätsreduktion gewählt. Vgl. zu dieser Einschätzung auch HeB, G., Marktsignale und Wettbewerbsstrategie, a.a.O., S. 102 f.; Laux, H., Liermann, F., Grundlagen der Organisation, Die Steuerung von Entscheidungen als Grundproblem der Betriebswirtschaftslehre, a.a.O., S. $57 \mathrm{ff}$.

Die Dynamik umfaßt die Häufigkeit, Stärke und Unregelmäßigkeit von Änderungen der technologiebezogenen Umweltfaktoren in High-Tech-Märkten. Zum Begriff der Dynamik siehe Kieser, A., Kubicek, H., Organisation, a.a.O., S. 371 ff.; Jacobs, F., Produktinnovation bei komplexer Technologie, Organisationstheoretische Analyse der Bedin- 
ist festzustellen, daß hiervon sowohl der Entwicklungproze $B$ als auch die Vermarktungsphase von Technologien und technologischen Anwendungen betroffen sind (vgl. Abbildung 8). ${ }^{53}$

Die Unternehmen müssen einerseits immer höhere F\&E-Aufwendungen über einen länger werdenden Entwicklungszeitraum erbringen, um technologische Neuerungen in High-Tech-Märkten hervorzubringen. So sind beispielsweise im Bereich der Elektrotechnik die Innovationsaufwendungen in den Jahren 1980 bis 1988 von 10 Mrd. DM auf knapp 20 Mrd. DM angestiegen, während sich die Entwicklungszeiten im gleichen Zeitraum kontinuierlich verlängert haben. ${ }^{54}$ Andererseits werden die technologischen Neuheiten ab dem Markteinführungszeitpunkt laufend durch alternative Technologien bedroht. Während zu Beginn der Markteinfuihrung i.d.R. zahlreiche Technologien im Wettbewerb um den Industriestandard ${ }^{55}$ stehen, werden etablierte Technologien anschließend häufig durch verbesserte oder neue Technologien verdrängt. ${ }^{56}$

gungen und Prozesse von Produktinnovationen auf der Grundlage einer empirischen Untersuchung der Branche "Bürokommunikation", Frankfurt a.M. u.a. 1988, S. 90.

Vgl. Popper, E.T., Buskirk, B.D., Technology life cycles in industrial markets, in: Industrial Marketing Management, Vol. 21 (1992), S. 23 ff.; Perillieux, R., Strategisches Timing von F\&E und Markteintritt bei innovativen Produkten, a.a.O., S. 26; Remmerbach, K.-U., Markteintrittsentscheidungen, a.a.O., S. 136 ff.; Ansoff, H.I., Implanting strategic management, Englewood Cliffs, N.J. 1984, S. $102 \mathrm{ff}$.

Vgl. Benkenstein, M., Strategisches Marketing-Management in High-Tech-Branchen, a.a.O., S. 8 f.; Perillieux, R., Wittkemper, G., Ziele und Module eines integrierten Technologie- und Innovationsmanagements, a.a.O., S. 15.

Unter einem Standard wird eine von der Mehrzahl der Marktteilnehmer akzeptierten Spezifikation bei einem bestimmten technologischen Produkt, System oder einer Komponente in High-Tech-Märkten verstanden. Vgl. Kleinaltenkamp, M., Der Einfluß der Normung und Standardisierung auf die Diffusion technischer Innovationen, in: "Neue Informationstechnologien von CIM-Systemen auf der Basis teilautonomer flexibler Fertigungsstrukturen", Arbeitspapier Nr. 187 des SFB, Bochum 1990, S. 2 ff. Zur Abgrenzung von Standard, Norm und Typ siehe Meffert, J., Standards als Integrationsinstrument in der Computer- und Kommunikationsindustrie, a.a.O., S. $14 \mathrm{ff}$.

Vgl. Große-Oetringhaus, W., Mega- und Supratechnologien: Kundenorientierte Wege zum Verständnis und zur Gestaltung des Technologiewandels, in: Technologie-Marketing, Hrsg.: Töpfer, A., Sommerlatte, T., Landsberg a.L. 1991, S. 127 ff. 


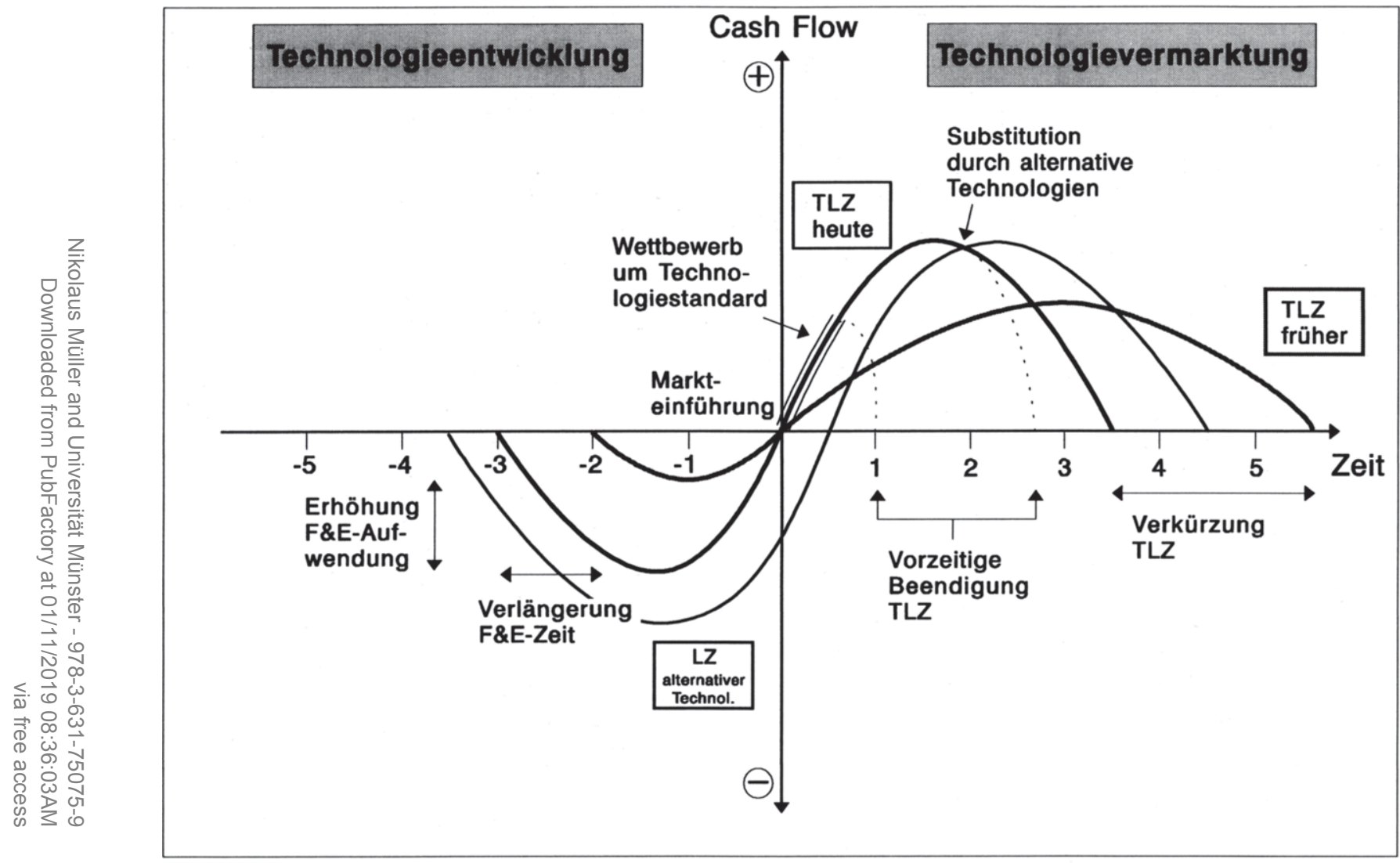

Abb. 8: Technologiedynamik in High-Tech-Mărkten 
Beides hat eine vorzeitige Beendigung von Technologie- bzw. Produktlebenszyklen in High-Tech-Märkten zur Folge und beinhaltet für die High-Tech-Unternehmen die Gefahr einer unzureichenden Amortisation der getätigten F\&E-Aufwendungen. In High-Tech-Märkten lassen sich zahlreiche Beispiele für eine Verkürzung von Lebenszyklen technologieintensiver Produkte finden. So haben sich die Produktlebenszyklen in der Elektrotechnik- und Computerindustrie von 1980 bis 1990 im Durchschnitt um $46 \%$ verkürzt. ${ }^{57}$ Für die High-Tech-Unternehmen ergibt sich hieraus eine hohe strategische Unsicherheit bezüglich der Erfolgsträchtigkeit neuer Technologien und technologischer Anwendungen. ${ }^{58}$

Die in High-Tech-Märkten vorhandene hohe Komplexität ${ }^{59}$ resultiert insbesondere aus dem raschen Zusammenwachsen ursprünglich getrennter Absatzmärkte und Technologien. ${ }^{60}$ Beispielhaft für die fortschreitende Technologieintegration sind das Zusammengehen der Glas-, Kabel-, und Elektrotechnik zur Herstellung faseroptischer Produkte sowie der Elektronik, Kristalltechnik und Optik zur Produktion optoelektronischer Güter zu nennen. ${ }^{61}$ Vor diesem Hintergrund wird insgesamt deutlich, daß die ausgeprägte Technologiedynamik und -komplexität technologische Diskontinuitäten ${ }^{62}$ in High-Tech-Märkten auslöst und eine Inten-

57 Vgl. Bullinger, H.-J., Wasserloos, G., Der Wettbewerbsfaktor Zeit muß konsequent genutzt werden, Die Stellschraube anziehen, in: Computerwoche vom 12.04.91, S. 10; Gahl, A., Die Konzeption strategischer Allianzen, Berlin 1991, S. 25.

Vgl. Benkenstein, M., Technikfolgenabschätzung, in: DBW, 1993, Nr. 3, S. 423 f.; MacInnis, M., Heslop, L.A., Market planning in a high-tech environment, a.a.O., S. 107 ff.; Benkenstein, M., Modelle technologischer Entwicklungen als Grundlage für das Technologiemanagement, a.a.O., S. 498 ff.; Porter, M., Wettbewerbsstrategie, a.a.O., S. $274 \mathrm{ff}$.

59 Die Technologiekomplexität wird vor allem durch die hohe Zahl und Verschiedenheit von Einflußfaktoren verursacht, die bei der Vermarktung von High-Tech-Produkten zu berücksichtigen sind. Vgl. Jacobs, F., Produktinnovation bei komplexer Technologie, a.a.O., S. 90 .

Siehe hierzu die ausfihhrlichen Erläuterungen im Teil A Kap. 1 und Teil B Kap. 1.2 der Arbeit.

61 Vgl. Kodama, F., Technologiefusion - der Weg zum Erfolg auf Zukunftsmärkten, a.a.O., S. 42 f.; Benkenstein, M., Strategisches Marketing-Management in High-Tech-Branchen, a.a.O., S. 8.

Technologische Diskontinuitäten kennzeichnen abrupte technologiebezogene Veränderungen im Unternehmensumfeld. Zum Begriff der Diskontinuität siehe Ansoff, H.I., Die Bewältigung von Überraschungen und Diskontinuitäten durch die Unternehmensfuihrung - Strategische Reaktionen auf schwache Signale, in: Planung und Kontrolle, Hrsg.: 
sivierung des preis- bzw. technologiebezogenen Wettbewerbs zwischen den High-Tech-Unternehmen bewirkt.

Darüber hinaus ist von den Unternehmen zu prüfen, inwieweit Änderungen in den technologiebezogenen Rahmenbedingungen, die z.B. durch Technologieintegration, -verbesserungen, -innovationen oder Durchsetzung eines Industriestandards verursacht werden, eine zunehmende Standardisierung bzw. Individualisierung von Technologien, Bestandteilen des Produktkerns, Benutzeranwendungen und Services zur Folge haben. Es ist davon auszugehen, daß der gegenwärtige Trend $\mathrm{zu}$ "offenen Systemen" die Standardisierung von technologischen Anwendungen und Hardwarekomponenten fördert. ${ }^{63}$ Eine stärkere Standardisierung zeichnet sich beispielsweise im Computerbereich bei der Hard- und Software ab. "Geschlossene Systeme" haben sich als nicht mehr wettbewerbsfähig erwiesen, seitdem es weitgehend einheitliche Standards ermöglichen, Hardwarekomponenten nahezu beliebig zu kombinieren. Im Softwarebereich wird zudem erwartet, daß der Anteil der Standardprogramme am Gesamtmarkt in den Jahren 1991 bis 1997 weltweit von ca. $15 \%$ auf über $50 \%$ ansteigen wird. ${ }^{64}$ Der Einfluß technologischer Standardisierungs- bzw. Individualisierungsentwicklungen ist darüber hinaus bezüglich einzelner prozeß- (z.B. Beschaffung, Produktion) und absatzmarktgerichteter (z.B. Marketing, technischer Kundendienst) Unternehmenstätigkeiten zu untersuchen, da die Wettbewerbsfähigkeit von High-TechUnternehmen durch die Anpassungsfähigkeit der einzelnen Unternehmensbereiche an die technologischen Veränderungen determiniert wird. ${ }^{65}$

Steinmann, H., München 1981, S. 233 ff.; Drucker, P., The age of discontinuity, New York 1968, S. 3 ff.

Vgl. Rominski, D., Wie kommen Computerhersteller über den Berg?, Dynamik aus neuer Offenheit, in: Absatzwirtschaft, 1992, Nr. 3, S. 30 ff. Zur Kennzeichnung von "offenen Systemen" siehe z.B. Meffert, J., Standards als Integrationsinstrument in der Computerund Kommunikationsindustrie, a.a.O., S. $25 \mathrm{ff}$.

Vgl. Vollmer, M., Anwendungsarchitekturen bestimmen die Lösungswelt: Trend zur Standardisierung beeinfluBt Softwareangebot, in: Computer Zeitung, 1993, Nr. 45, S. 17.

Vgl. Meffert, J., Standards als Integrationsinstrument in der Computer- und Kommunikationsindustrie, a.a.O., S. 65 ff., Saad, K.N., Roussel, Ph.A., Tiby, C., Management der F\&E-Strategie, Hrsg.: Little, A.D., Wiesbaden 1991, S. 122 f.; Servatius, H.-G., Schwerpunkte und Methoden des Management von Technologien, in: Technologie-Marketing, Hrsg.: Töpfer, A., Sommerlatte T., Landsberg a.L. 1991, S. 55 ff. 


\subsection{Marktbezogene Umweltsituation}

Im Rahmen der Analyse der allgemeinen Marktsituation ist für High-TechUnternehmen von Bedeutung, Informationen über die Marktentwicklung und den Ausschöpfungsgrad des Marktpotentials zu erhalten. Eine Betrachtung der HighTech-Märkte auf der Grundlage des Marktlebenszykluskonzeptes ${ }^{66}$ kann eine erste Orientierungshilfe hinsichtlich der Beurteilung des Marktpotentials und der damit zusammenhängenden strategischen Verhaltensalternativen bieten. ${ }^{67}$ Dies läßt sich beispielhaft anhand der in Abbildung 9 dargestellten High-Tech-Märkte aufzeigen. ${ }^{68}$

Es wird deutlich, daß sich die Nachrichtentechnik, Büroautomatisierungstechnik und Softwareentwicklung im Vergleich zur Biotechnologie und Umwelttechnik in einer fortgeschrittenen Entwicklungsphase des Marktlebenszyklus befinden. Aufgrund einer deutlich gestiegenen Nachfrage sind diese Märkte auch durch höhere Wachstumsraten gekennzeichnet. Beispielsweise ist der Umsatz im PC-SoftwareMarkt in Deutschland von 1992 auf 1993 um ca. 30 \% gestiegen. ${ }^{69}$ Gleichzeitig ist jedoch festzustellen, daß die Anbieterzahl in den stark wachsenden HighTech-Märkten rasch zunimmt. So sind beispielsweise im Zeitraum 1983 bis 1985

Zur Kennzeichnung des Lebenszykluskonzeptes siehe vor allem Höft, U., Lebenszykluskonzepte, Grundlage für das strategische Marketing- und Technologiemanagement, Berlin 1992, S. 15 ff.; Meffert, H., Interpretation und Aussagewert des Lebenszyklus-Konzeptes, in: Neuere Ansätze der Marketingtheorie, Hrsg.: Hammann, P., Kroeber-Riel, W., Meyer, C.W., Berlin 1974, S. 85 ff. Zur Abgrenzung von Markt-, Technologie- und Produktlebenszyklus siehe z.B. Benkenstein, M., Modelle technologischer Entwicklungen als Grundlage für das Technologiemanagement, a.a.O., S. 503 f.; Remmerbach, K.-U., Markteintrittsentscheidungen, a.a.O., S. $136 \mathrm{f}$.

Vgl. Kotler, Ph., Bliemel, F., Marketing-Management, 7. Aufl., Stuttgart 1992, S. 543 ff.; Meffert, H., Marketingstrategien in unterschiedlichen Marktsituationen, a.a.O., München 1989, S. 280. Zur Kritik am Lebenszykluskonzept vgl. z.B. Kreilkamp, E., Strategisches Management und Marketing, Berlin, New York 1987, S. $137 \mathrm{ff}$.

Bei der Verwendung des Lebenszykluskonzeptes wird in der jüngeren Literatur häufig ein Vierphasenmodell verwendet. Vgl. hierzu z.B. Drazin, R., Kazanjian, R.K., A reanalysis of Miller and Friesen's life cycle data, in: Strategic Management Journal, 1990, No. 4, S. 319 ff.; Meffert, H., Strategische Unternehmensfiihrung und Marketing, a.a.O., S. $53 \mathrm{ff}$.

Vgl. o.V., PC-Software-Umsatz in Europa, in: Computerwoche, 1994, Nr. 1, S. 48. 


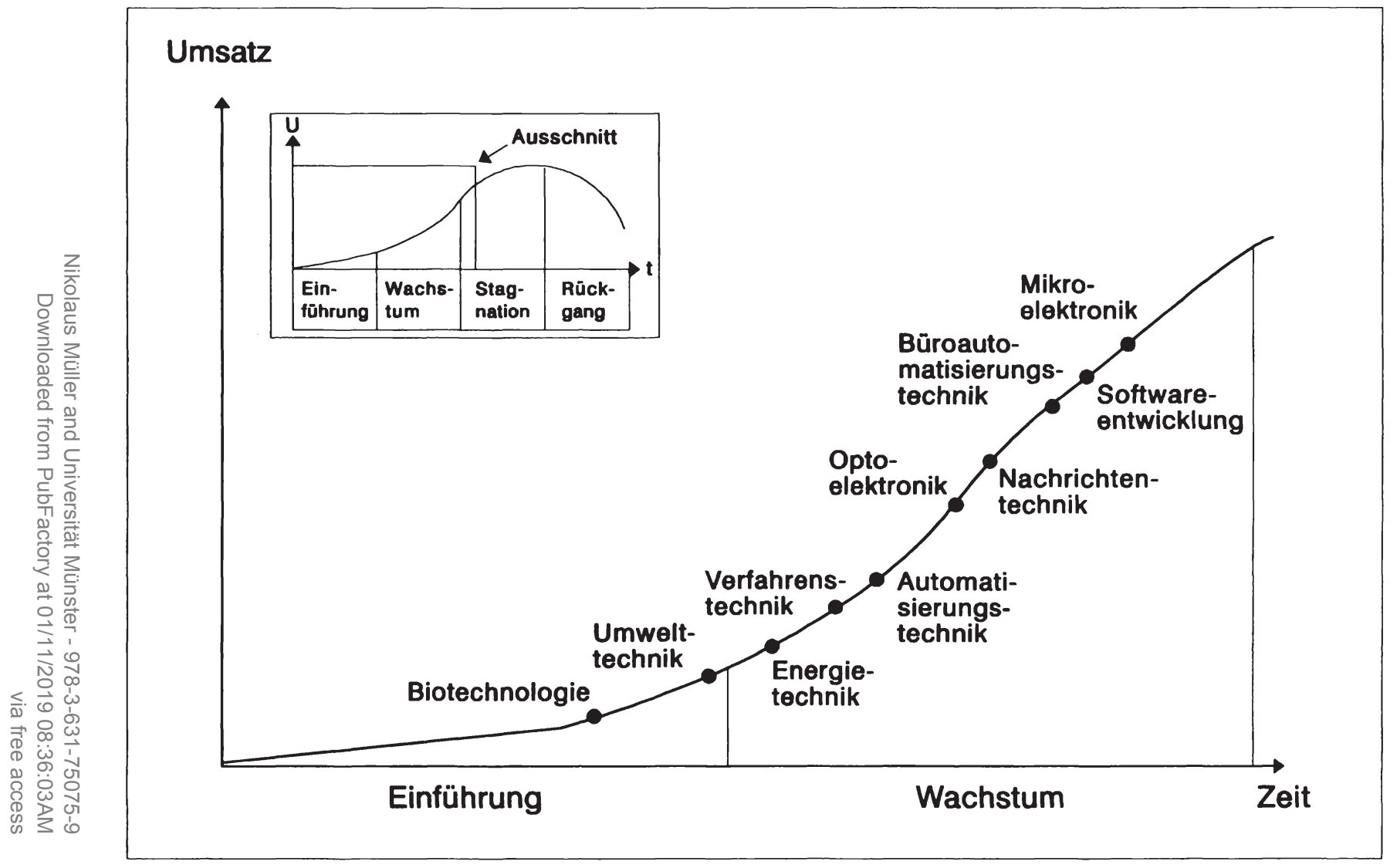

Abb. 9: Ausgewăhlte High-Tech-Mărkte im Marktlebenszyklus 
pro Jahr durchschnittlich 57 neue Unternehmen in den Bereich der Nachrichtentechnik eingetreten. ${ }^{70}$ Für die bereits im Markt tätigen High-Tech-Unternehmen ergibt sich hieraus eine spürbare Erhöhung des Wettbewerbs.

Markt- und innovationsspezifische Barrieren prägen die Entwicklung der allgemeinen Marktsituation in High-Tech-Märkten. ${ }^{71}$ Durch marktbezogene Barrieren, z.B. in Form eines hohen technologischen Leistungsniveaus etablierter HighTech-Anbieter, einer schnellen Abfolge technologischer Innovationen oder der Durchsetzung eines produkt- bzw. technologiebezogenen Industriestandards, werden die Eintrittsfähigkeit potentieller Konkurrenten und damit die Wettbewerbsintensität zwischen den High-Tech-Unternehmen beeinflußt. ${ }^{72}$ Innovationsbarrieren, die auf der Unternehmensseite u.a. in einer Verlängerung der Technologie- bzw. Produktentwicklungszeiten und auf der Nachfragerseite insbesondere in Kaufwiderständen begründet liegen, beeinträchtigen den schnellen Aufbau einer erfolgreichen Marktposition. ${ }^{73}$

\subsection{Abnehmerbezogene Umweltsituation}

Bei der Betrachtung der Abnehmersituation in High-Tech-Märkten wird deutlich, daß eine starke technologie- und leistungsbezogene Unsicherheit das Verhal-

70 Vgl. Posselt, Th., Mobilitätsverhalten von Unternehmen, Wiesbaden 1993, S. 154 f. u. $160 \mathrm{f}$.

71 Vgl. Kolbe, C., Eintrittsbarrieren und die Eintrittsfähigkeit potentieller Konkurrenten, S. 51 ff.; Merkle, E., Technologiemarketing, a.a.O., S. 7; Yip, G.S., Barriers to entry, S. 17 ff.; Walters, M., Marktwiderstände und Marketingplanung, a.a.O., S. 34 ff.

Vgl. Meffert, J., Standards als Integrationsinstrument in der Computer- und Kommunikationsindustrie, a.a.O., S. 107 ff.; o.V., Innovation: Wettlauf der Erfinder, in: Focus, 1993, Nr. 13, S. 186 ff.; Milling, P., Schwierigkeiten bei der Umsetzung des neuen Wissens, a.a.O., S. 7. Hinsichtlich der Durchsetzung eines Industriestandards siehe die Ausführungen im Teil B Kap. 2.31 und 2.32 der Arbeit.

Vgl. Baaken, Th., Technologie-Marketing, a.a.O., S. 295 f.; Remmerbach, K.-U., Markteintrittsentscheidungen, a.a.O., S. 118 ff.; Walters, M., Marktwiderstände und Marketingplanung, a.a.O., S. 37 ff. Zu weiteren Ursachen unternehmensbezogener Innovationsbarrieren siehe die Ausführungen zur Technologiedynamik im Teil B Kap. 2.11 der Arbeit. 
ten der $\mathrm{Abnehmer}^{74}$ bestimmten, die häufig zu einem hohen wahrgenommenen Kaufrisiko und dadurch zu Kaufwiderständen bzw. zu einer Kaufzurückhaltung führt. ${ }^{75}$ Eine in diesem Zusammenhang durchgeführte Studie zur Akzeptanz von Technologien und technologischen Produkten zeigt, daß High-Tech-Unternehmen dem Abbau der Kaufwiderstände und -zurückhaltung zukünftig einen größeren Stellenwert beizumessen haben. ${ }^{76}$

Zur Reduzierung der Kaufwiderstände und -zurückhaltung ist es hierbei aus Anbietersicht von Interesse, nähere Informationen sowohl über die an High-TechProdukte gerichteten Leistungsanforderungen als auch über die technologischen Kenntnisse der High-Tech-Anwender zu erhalten. Die Anforderungen der Nachfrager konzentrieren sich vor allem auf die Nutzungsmöglichkeiten, den Komplexitätsgrad und die Benutzerfreundlichkeit von High-Tech-Produkten. ${ }^{77}$ Es ist hierbei festzustellen, daß die High-Tech-Anwender eine kontinuierliche Ausweitung der Nutzungsmöglichkeiten erwarten, ${ }^{78}$ während gleichzeitig die damit einhergehende Komplexitätssteigerung zu deutlichen Akzeptanzproblemen fuihren

Im folgenden werden unter High-Tech-Abnehmern private und institutionelle Nachfrager technologischer Produkte zusammengefaßt. Zur Kennzeichung des Verhaltens privater und institutioneller Nachfrager vgl. z.B. Meffert, H., Marketingforschung und Käuferverhalten, a.a.O., S. 47 ff. u. S. 141 ff.; Backhaus, K., Investitionsgütermarketing, a.a.O., S. $49 \mathrm{ff}$.

Vgl. Wieandt, A., Zum Marketing von Innovationen, a.a.O., S. 22 ff.; Backhaus, K., Weiber, R., Systemtechnologien - Herausforderung des Investitionsgütermarketing, a.a.O., S. 78 f. Zu einer weitergehenden Betrachtung hinsichtlich der Ursachen von Kaufwiderständen siehe auch die Ausführungen im Teil A Kap. 1 der Arbeit.

Nach dieser Untersuchung können 50 \% der befragten Personen in Deutschland als "Technologie-Skeptiker" bezeichnet werden, und es empfinden sogar $75 \%$ ein Unbehagen gegenüber dem schnellen technologischen Wandel. Bei der Einschätzung einzelner Produkte ist beispielsweise zu erkennen, daß ein Drittel der Befragten noch nie einen Computer benutzt hat. Vgl. o.V., Jeder Zweite hat Angst vor neuer Technik, in: PCWelt, 1993, Nr. 12, S. 15; o.V., Studie: Technologie-Akzeptanz, in: asw, 1993, Nr. 11, S. 26.

Vgl. Schaible, J., Hönig, A., High-Tech-Marketing in der Praxis, a.a.O., S. 26 ff.; Sommerlatte, T., Töpfer, A., Die Integration von Technologie und Marketing als strategischer Erfolgsfaktor: Übersicht und Einordnung der Beiträge, in: Technologie-Marketing, Hrsg.: Töpfer, A., Sommerlatte T., Landsberg a.L. 1991, S. 19; Zimmermann, A., High-Tech-Marketing, in: Thexis, 1987, Nr. 1, S. 18 f.; Merkle, E., Technologiemarketing, a.a.O., S. 10; Walters, M., Marktwiderstände und Marketingplanung, a.a.O., S. 156 ff.

Zur Ausweitung der Nutzungsmöglichkeiten in High-Tech-Märkten siehe die Ausführungen im Teil A Kap. 1 und Teil B Kap. 1.2 der Arbeit. 
kann. Dies trifft in High-Tech-Märkten insbesondere auf Systemtechnologien wie z.B. Bürokommunikationssysteme, Verkehrsleitsysteme und CIM-Systeme zu. ${ }^{79}$

Von den High-Tech-Unternehmen wird deshalb häufig eine stärkere problemlösungsbezogene Kompeten $z^{80}$ gefordert, die auch in einer leichten Handhabung technologischer Anwendungen zum Ausdruck kommen soll. Die besondere Bedeutung der Bedienungsfreundlichkeit von High-Tech-Produkten wird durch eine in den USA bei aktuellen und potentiellen PC-Benutzern durchgefuihrte Untersuchung unterstrichen. Laut dieser Umfrage wünschen sich $80 \%$ bzw. $92 \%$ der befragten Personen, daß die am meisten genutzten Dateien bei Arbeitsbeginn automatisch geöffnet und die bearbeiteten Daten bei Arbeitsende automatisch gespeichert werden. Darüber hinaus legen ca. $90 \%$ großen Wert auf eine leicht verständliche Gebrauchsanleitung bei der Konfiguration von Computer- und Peripheriegeräten sowie auf gut nachvollziehbare Diagnose- und Hilfsprogramme bei auftretenden Anwendungsproblemen. ${ }^{81}$

Bei der Entwicklung und Bereitstellung von High-Tech-Produkten sind neben den spezifischen Leistungsanforderungen auch die technologiebezogenen Kenntnisse der Nachfrager zu berücksichtigen. Anhaltspunkte über das vorhandene technologische Know-how kann dabei die Betrachtung der bestehenden Erfahrungen und der Schulungsbereitschaft der High-Tech-Verwender sowie der Anlernzeit bei neuen High-Tech-Anwendungen liefern. ${ }^{82}$

Vgl. z.B. Backhaus, K., Späth, G.-M., Applikationen prägen die zum Einsatz kommende Technik: Hochgeschwindigkeits-Netze sind aus Anwendersicht unumgänglich, in: Computerwoche, 1993, Nr. 6, S. 23 f.; ISI (Hrsg.), Technologie am Beginn des 21. Jahrhunderts, Karlsruhe 1993, S. 70 ff. u. $133 \mathrm{ff}$.

Zum Begriff der Kompetenz sowie zur Abgrenzung von Fach- und Problemlösungskompetenz siehe z.B. Rasche, Chr., Kernkompetenzen, in: DBW, 1993, Nr. 3, S. 425 ff.; Weiss, P., Die Kompetenz von Systemanbietern: Ein neuer Ansatz im Marketing für Systemtechnologien, a.a.O., S. 59 ff.; Backhaus, K., Weiss, P.A., Kompetenz - Die entscheidende Dimension im Marketing, in: Harvard Manager, 1989, Nr. 3, S. $111 \mathrm{f}$.

Vgl. Riggs, B., Der Personalcomputer der Zukunft soll Strom sparen und "mitdenken": Amerikaner fordern von Herstellern bedienungsfreundlichere PC's, in: Computer Zeitung, 1993, Nr. 48, S. 21.

Vgl. Baaken, Th., Launen, M., Software-Marketing, München 1993, S. 18 ff.; Bittner, L., Ursachen und Folgen der steigenden Bedeutung des DV-Service, in: Software-Marketing, Hrsg.: Wimmer, F., Bittner, L., Wiesbaden 1992, S. 168 ff.; Benkenstein, M., 


\subsection{Konkurrenzbezogene Umweltsituation}

Die aufgezeigten technologie- und abnehmerbezogenen Kontextbedingungen haben einen maßgeblichen Einfluß auf die Konkurrenzsituation in High-TechMärkten. Sie wird zudem durch Art und Umfang des strategischen Verhaltens von High-Tech-Unternehmen bestimmt. Hierbei ist der technologie- und preisbezogene Wettbewerb näher zu untersuchen. Die Entwicklung des Technologiewettbewerbs kommt insbesondere in der technologischen Dynamik (u.a. Technologieverbesserungen bzw. -innovationen in kurzen Zeitabständen) und der Komplexität (u.a. Technologieintegration) in High-Tech-Märkten zum Ausdruck. ${ }^{83}$

Die Intensität des Preiswettbewerbs wird einerseits durch das preispolitische Verhalten der High-Tech-Anbieter determiniert. Beispielsweise kann ein Unternehmen, das mit einer Produkt- bzw. Technologieneuheit in einen High-TechMarkt eintritt, zur Abschöpfung von Pioniergewinnen einen hohen Einfuihrungspreis (Skimming-Strategie) wählen oder zur Erreichung einer schnellen Marktdurchdringung und zur Abschreckung anderer eintrittsfähiger High-Tech-Unternehmen eine niedrige Preissetzung (Penetrations-Strategie) favorisieren. Die Stärke des Preiswettbewerbs wird andererseits durch die Preisbereitschaft der Nachfrager beeinflußt, die in einigen High-Tech-Märkten wie der Mikroelektronik und der Automatisierungstechnik besonders stark ausgeprägt ist. ${ }^{84}$

High Tech-Marketing, a.a.O., S. 398; Backhaus, K., Weiss, P.A., Kompetenz - Die entscheidende Dimension im Marketing, a.a.O., S. 111.

Vgl. Wolfrum, B., Technologiestrategien im strategischen Management, a.a.O., S. 25 ff.; Porter, M., Wettbewerbsvorteile, a.a.O., S. 219 ff.; Perillieux, R., Der Zeitfaktor im strategischen Technologie-Management, a.a.O., S. 120 ff.; Sommerlatte, T., Dechamps, J.-Ph., Der strategische Einsatz von Technologien, a.a.O., S. 56 ff. In der nachfolgenden empirischen Untersuchung werden die Technologiedynamik und -komplexität aufgrund des starken Einflusses auf die Wettbewerbsintensität innerhalb der Wettbewerbssituation berücksichtigt. Siehe hierzu auch die Ausführungen im Teil B Kap. 1.1 der Arbeit.

Vgl. Meffert, J., Standards als Integrationsinstrument in der Computer- und Kommunikationsindustrie, a.a.O., S. 103 ff.; Meffert, H., Preispolitik, in: Handwörterbuch des Rechnungswesens, 3. Aufl., Hrsg.: Chmielewicz, K., Schweitzer, M., Stuttgart 1993, Sp. 1589 f.; Backhaus, K. Investitionsgütermarketing, a.a.O., S. 183 ff.; Maringer, A., Preisverfall mikroelektronischer Bauelemente am Beispiel DRAM-Speicherbauelemente, a.a.O., S. 423 ff. Zur Kennzeichung von Skimming- und Penetrations-Strategie siehe z.B. Meffert, H., Marketing Management, a.a.O., S. 214 f. 
Bei Betrachtung des Unternehmensverhaltens in High-Tech-Märkten lassen sich anhand der Kriterien Leistungsangebot der Unternehmen und Preisbereitschaft der Nachfrager vier typische Wettbewerbssituationen charakterisieren (vgl. Abbildung 10). ${ }^{85}$

Es herrscht ein geringer Wettbewerb in High-Tech-Märkten, wenn sich die angebotenen technologischen Leistungen deutlich voneinander unterscheiden und ein geringes Preisbewußtsein auf der Nachfragerseite vorliegt. Diese Situation ist zunächst in der Einführungsphase von Technologien und technologischen Anwendungen anzutreffen, in der die High-Tech-Anbieter noch einen großen Handlungsspielraum im Hinblick auf die Markterschließung und den Aufbau von Mobilitätsbarrieren haben.

Ausgelöst durch ein deutliches Nachfragewachstum treten zahlreiche Unternehmen in High-Tech-Märkte ein und sind bestrebt, die bestehenden Technologieangebote $\mathrm{zu}$ imitieren oder durch Eigenentwicklungen $\mathrm{zu}$ verdrängen (Technologiewettbewerb). In dieser Situation besteht noch eine hohe Preisbereitschaft, da die High-Tech-Verwender hohe Leistungsanforderungen haben und über eine unzureichende Markttransparenz verfügen. Der Wettbewerb zwischen den High-Tech-Anbietern wird deshalb überwiegend auf der Technologieebene geführt.

Ein eingeschränkter Wettbewerb kann sich in High-Tech-Märkten entwickeln, wenn es den vorhandenen Unternehmen gelingt, bestimmte Differenzierungsvorteile aufzubauen, z.B. durch einen zuverlässigen Service oder durch eine kompetente Beratung, die schwer nachzuahmen sind. Diese Wettbewerbsvorteile stellen Markteintrittsbarrieren für potentielle Konkurrenten und Mobilitätsbarrieren für High-Tech-Unternehmen aus anderen strategischen Gruppen dar.

85 Vgl. Specht, G., Zörgiebel, W., Technologieorientierte Wettbewerbsstrategien, in: Strategisches Marketing, Hrsg.: Raffée, H., Wiedmann, K.-P., Stuttgart 1985, S. 504 f.; Servatius, H.-G., Methodik des strategischen Technologie-Managements, Grundlage für erfolgreiche Innovationen, Berlin 1985, S. 273 ff. 


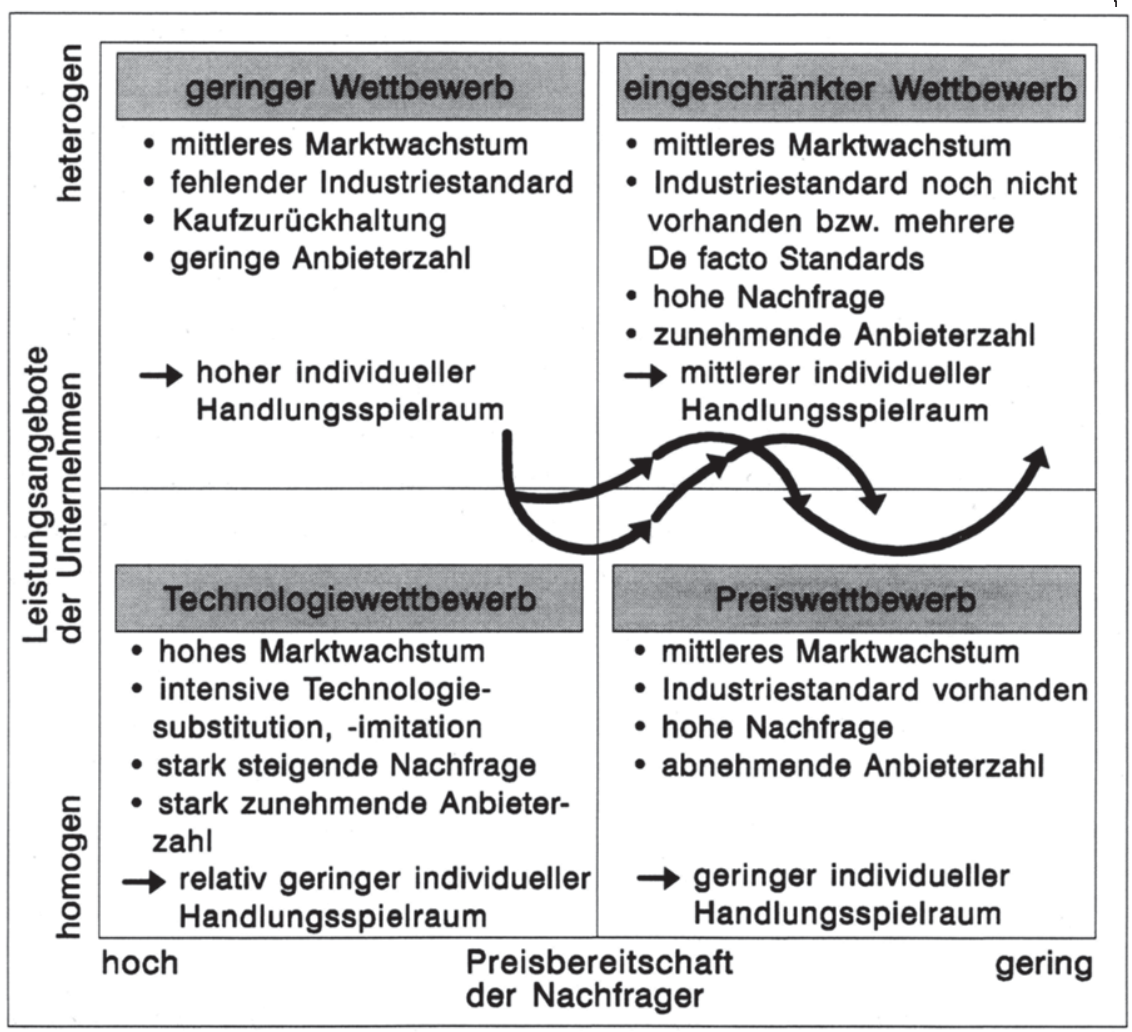

Abb 10: Typische Wettbewerbssituationen in High-Tech-Märkten

Die High-Tech-Nachfrager sind in dieser Wettbewerbssituation, die durch individuelle Leistungsangebote geprägt ist, trotz einer grundsätzlich geringen Preisbereitschaft noch zu Preiszugeständnissen bereit. ${ }^{86}$

Wenn die Technologien und technologischen Anwendungen zunehmend homogener werden und die Preisbereitschaft der High-Tech-Anwender kontinuierlich abnimmt, sehen sich Unternehmen in High-Tech-Märkten einem massiven Preiswettbewerb ausgesetzt. Der Handlungsspielraum für die Unternehmen ist hierbei oftmals auf die Ausnutzung von Degressions- und Erfahrungskurveneffekten be- 
grenzt. Zum Zeitpunkt eines technologischen Durchbruchs oder einer Technologieintegration besteht für die High-Tech-Unternehmen jedoch auch die Möglichkeit, sich durch neue Technologien und technologische Leistungsangebote von den Wettbewerbern zu differenzieren. Es entsteht hierdurch wiederum eine Situation mit einem eingeschränkten Wettbewerb. ${ }^{87}$

Es bleibt weiterhin festzuhalten, daß die aufgezeigten Wettbewerbssituationen meist in einer zeitlichen Reihenfolge stehen. ${ }^{88}$ Während High-Tech-Märkte zu Beginn durch eine geringe Wettbewerbsintensität gekennzeichnet sind, rückt der technologieorientierte Wettbewerb anschließend stärker in den Vordergrund. Im weiteren Zeitablauf werden sich in Abhängigkeit von Art und Umfang der Differenzierungsvorteile ein eingeschränkter bzw. durch den Absatzpreis dominierter Wettbewerb herausbilden.

Abschließend sind in der Abbildung 11 die relevanten technologie-, markt-, abnehmer- und konkurrenzbezogenen Kontextdimensionen dargestellt, die zur Kennzeichung von High-Tech-Märkten geeignet erscheinen und im Rahmen der empirischen Analyse zur Identifikation homogener Umfeldsituationen berücksichtigt werden. ${ }^{89}$

In diesem Zusammenhang soll auf der Grundlage der empirischen Daten folgende Basishypothese ${ }^{90}$ überprüft werden:

Vgl. Backhaus, K.,Investitionsgütermarketing, a.a.O., S. 171 f.; Meffert, H., Strategische Unternehmensführung und Marketing, a.a.O., S. 77.

Vgl. Meffert, H., Strategische Unternehmensfuihrung und Marketing, a.a.O., S. 53 ff.; Porter, M., Wettbewerbsstrategie, a.a.O., S. 559 ff.; Perillieux, R., Der Zeitfaktor im strategischen Technologie-Management, a.a.O., S. $47 \mathrm{ff}$.

In der vorliegenden Untersuchung erfolgte eine Komplexitätsreduzierung dahingehend, da $B$ aus der Vielzahl möglicher Kontextmerkmale wenige zentrale Dimensionen auf Grundlage der aufgezeigten Ausführungen sowie aufgrund von Plausibilitätsüberlegungen ausgewählt wurden. Vgl. zu dieser Einschätzung auch HeB, G., Marktsignale und Wettbewerbsstrategie, a.a.O., S. 102 f.; Laux, H., Liermann, F., Grundlagen der Organisation, a.a.O., S. 57 ff.

Für die nachfolgende empirische Analyse wird eine Unterscheidung zwischen Tendenzund Basishypothesen vorgenommen. Tendenzhypothesen werden formuliert, wenn sich konkrete Wirkungsrichtungen zwischen abhängigen und unabhängigen Variablen ableiten lassen. Die Festlegung von Basishypothesen erfolgt, wenn keine eindeutigen Aussagen 
H UM In High-Tech-Märkten lassen sich verschiedene homogene Umfeldsituationen anhand von markt-, technologie-, abnehmer- und konkurrenzbezogenen Kontextmerkmalen identifizieren, die sich signifikant voneinander unterscheiden.

\subsection{Ansätze zur Systematisierung von Marketingstrategien in High-Tech- Märkten}

In der folgenden Diskussion werden zentrale Strategieansätze hinsichtlich ihrer Eignung zur Typologisierung von Marketingstrategien in High-Tech-Märkten untersucht. Eine Bestandsaufnahme der Literatur zeigt, daß eine Strategietypologisierung einerseits anhand von Zeit- und Technologiedimensionen vorgenommen wird. ${ }^{91}$ In den Systematisierungsansätze werden andererseits neben Zeitund Technologie- auch stärker wettbewerbsbezogene Strategieaspekte berücksichtigt. ${ }^{92}$

getroffen werden können und eine erste Orientierung im Untersuchungsverlauf angestrebt wird. Siehe hierzu auch Kirchgeorg, M., Ökologieorientiertes Unternehmensverhalten, a.a.O., S. 50; Bruhn, M., Das soziale BewuBtsein von Konsumenten, Wiesbaden 1978, S. 104; Kopp, M., Hypothesenformulierung in der Absatzforschung, Berlin 1972, S. 537 ff.

91 In der Literatur werden häufig technologie- und zeitbezogene Strategiedimensionen unzureichend voneinander getrennt. Es fuihrt dazu, da $B$ einige Autoren eine Technologieführerschaft mit einer Pionierposition und eine Technologiefolgerschaft mit einer Folgerposition gleichsetzen. Vgl. zu dieser Kritik z.B. Wolfrum, B., Strategisches Technologiemanagement, Wiesbaden 1991, S. 225 ff. und die dort angegebene Literatur. In der vorliegenden Untersuchung wird deshalb explizit zwischen technologie- und zeitbezogenen Strategiedimensionen unterschieden. Die zeitbezogenen Strategieaspekte werden hierbei im Rahmen der Analyse der zeit- und technologieorientierten sowie der wettbewerbsorientierten Systematisierungsansätze näher betrachtet.

Vgl. zu dieser Einschätzung und zu älteren Untersuchungen, die in der vorliegenden Arbeit nicht berücksichtigt werden, insbesondere Schewe, G., Imitationsmanagement, Nachahmung als Option des Technologiemanagements, Stuttgart 1992, S. 41 ff.; Remmerbach, K.-U., Markteintrittsentscheidungen, a.a.O., S. 40 ff.; Perillieux, R., Der Zeitfaktor im strategischen Technologie-Management, a.a.O., S. $135 \mathrm{ff}$. 


\begin{tabular}{|c|c|}
\hline Al:Omelne Markistuation & Abnehmersituation \\
\hline $\begin{array}{l}\text { - Unausgeschöpftes Marktpotential } \\
\text { - Marktwachstum } \\
\text { - Marktlebenszyklus } \\
\text { - Produktlebenszyklus } \\
\text { - Fehlender Industriestandard } \\
\text { - Marktbarrieren } \\
\text { - Innovationsbarrieren }\end{array}$ & $\begin{array}{l}\text { - Technologie Know-how } \\
\text { - Anlernzeit } \\
\text { - Schulungsbereitschaft } \\
\text { - Nutzenerwartungen } \\
\text { - Komplexität der Anwendungen } \\
\text { - Leichte Handhabung }\end{array}$ \\
\hline Technologlesturtion & Konkurronzaltuation \\
\hline $\begin{array}{l}\text { - Standardisierung der Technologie } \\
\text { - Standardisierung des Produktkerns } \\
\text { - Standardisierung der Benutzeranwendungen } \\
\text { - Standardisierung des Service } \\
\text { - EinfluB der Tech.-stand. auf das Marketing } \\
\text { - EinfluB der Tech.-stand. auf F\&E } \\
\text { - EinfluB der Tech.-stand. auf das Produktdesign } \\
\text { - EinfluB der Tech.-stand. auf den Tech. Kundendienst } \\
\text { - EinfluB der Tech.-stand. auf die Beschaffung } \\
\text { - EinfluB der Tech.-stand. auf die Produktion } \\
\text { - EinfluB der Tech.-stand. auf die Logistik }\end{array}$ & $\begin{array}{l}\text { - Preiswettbewerb } \\
\text { - Preisorientierung } \\
\text { - Preissenkung (Beschaffungseinsp.) } \\
\text { - Preiserosion } \\
\text { - Verkürzte PLZ } \\
\text { - Technologiewettbewerb } \\
\text { - Qualitătswettbewerb (Tech.- dynamik) }\end{array}$ \\
\hline
\end{tabular}

Abb. 11: Determinanten der spezifischen Unternehmenssituation in High-Tech-Märkten 


\subsection{Zeit- und Technologieorientierte Strategieansätze}

\section{Einen Überblick über wichtige zeit- und technologieorientierte Systematisie-} rungsansätze gibt Abbildung 12. Hierbei ist zu erkennen, daß einige Autoren Marketingstrategien auf Grundlage des technologiebezogenen Inventions- bzw. Innovationstiming systematisieren. ${ }^{93}$ Diese stärker unter zeitlichen Gesichtspunkten gewählte Vorgehensweise liegt den Arbeiten von Spital, Crawford, Beuttel, Schnaars und Perillieux bzw. Specht/Perillieux zugrunde. ${ }^{94}$

Der Strategieansatz von Perillieux wird an dieser Stelle beispielhaft skizziert. Bei der Betrachtung des strategischen Innovationsmanagement ${ }^{95}$ von Unternehmen der Maschinenbauindustrie setzte sich Perillieux eingehend mit zeitbezogenen Strategieaspekten auseinander. Als Systematisierungskriterien zur Typologisie-

93 Durch das Inventionstiming wird der Zeitpunkt festgelegt, an dem ein Unternehmen mit der Entwicklung neuer Technologien bzw. technologischer Anwendungen beginnt. Im Rahmen des Innovationstiming wird der Markteintrittszeitpunkt einer technologischen Neuerung bestimmt. Siehe hierzu z.B. Wolfrum, B., Technologiestrategien im strategischen Management, a.a.O., S. 30 f.; Leder, M., Innovationsmanagement - Ein Überblick, in: Innovationsmanagement, Theorie und Praxis im Kulturvergleich, Hrsg.: Albach, H., Wiesbaden 1990, S. 6 ff.; Perillieux, R., Der Zeitfaktor im strategischen Technologie-Management, a.a.O., S. $120 \mathrm{ff}$.

Vgl. Specht, G., Perillieux, R., Erfolgsfaktoren technischer Führer- und Folgerpositionen auf Investitionsgütermärkten, in: ZfbF, 1988, S. 209 ff.; Perillieux, R., Der Zeitfaktor im strategischen Technologie-Management, a.a.O., S. 166 ff.; Schnaars, St. P., When entering growth markets, are pioneers better than poachers?, in: Business Horizons, Vol. 29 (1986), No. 3-4, S. 27 ff.; Beuttel, W., Marketingstrategien in schnell wachsenden Märkten, in: Handbuch Strategisches Marketing, Hrsg.: Wieselhuber, N., Töpfer, A., Landsberg a.L. 1984, S. 308 ff.; Crawford, J.D., New products management, Homewood, Ill. 1983, S. 100 ff.; Spital, F., Gaining market share advantage in the semiconductor industry by lead time in innovation, in: Research on technological innovation, management and policy, Hrsg.: Rosenbloom, R.S., Greenwich, Conn. 1983, S. 55 ff.

In diesem Zusammenhang sind das Technologie, F\&E- und Innovationsmanagement voneinander abzugrenzen. Das Technologiemanagement umfaßt alle Aktivitäten zum Aufbau von technologischen produkt- oder prozeßbezogenen Leistungspotentialen eines Unternehmens. Vgl. z.B. Specht, G., Technologiemanagement: Grundgedanken zum Gegenstand und zugleich Sammelrezension, in: DBW, 1992, Nr. 4, S. 548 f. Das F\&EManagement beinhaltet sämtliche Unternehmenstätigkeiten, die zu einem materiellen bzw. immateriellen Gegenstand fuihren. Brockhoff, K., Forschung und Entwicklung, 2. Aufl., München, Wien 1989, S. 23 f. Das Innovationsmanagement schlieBt alle Unternehmensaktivitäten ein, die von der Ideengewinnung bis zur Markteinführung eines neuen Produktes reichen. Vgl. z.B. Gerpott, T., Wittkemper, G., Verkürzung der Produktentwicklungszeiten, in: Integriertes Technologie und Innovationsmanagement, Konzepte zur Stärkung der Wettbewerbskraft von High-Tech-Unternehmen, Hrsg.: Booz, Allen \& Hamilton, Berlin 1991, S. 119 ff. 


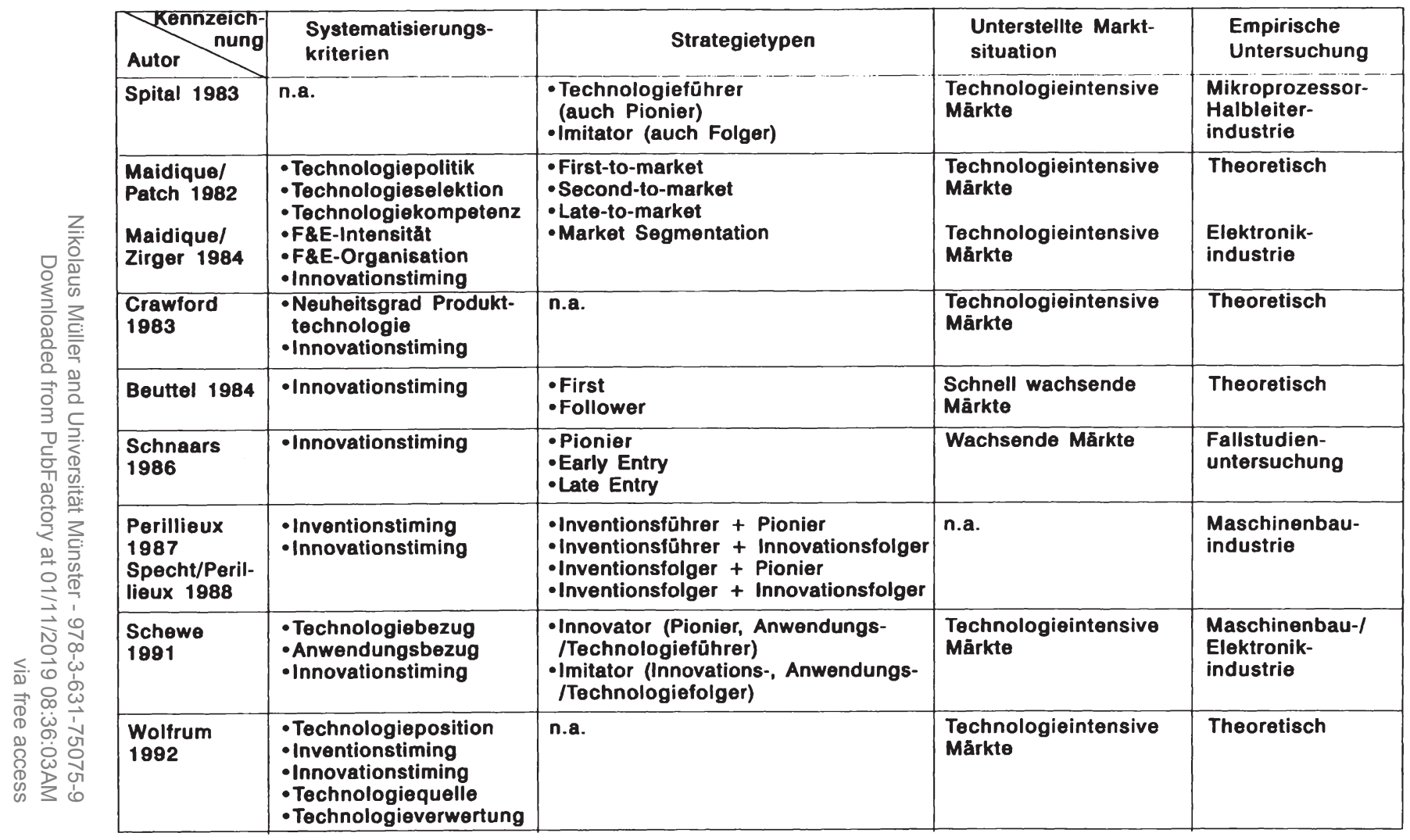

Abb. 12: Zentrale Zeit- und technologieorientierte Ansätze zur Systematisierung von Marketingstrategien in High-Tech-Märkten 
rung technologiebezogener Marketingstrategien wurden das Inventions- sowie das Innovationstiming herangezogen und dabei jeweils zwischen einer Führerund Folgerposition unterschieden. ${ }^{96}$ Im Rahmen der Untersuchung konnte Perillieux nachweisen, daß Inventionsführer überwiegend als Pionier in den Markt eintreten, während Inventionsfolger meist einen späteren Markteintrittszeitpunkt wählen. Keine der identifizierten Strategietypen stellte sich jedoch als signifikant erfolgreicher heraus. ${ }^{97}$

Von manchen Autoren wird eine stärkere Differenzierung der technologischen Verhaltensdimensionen vorgenommen. Hierzu zählen die Untersuchungen von Maidique/Patch bzw. Maidique/Zirger, Schewe und Wolfrum. ${ }^{98}$ Der technologieorientierte Strategieansatz von Wolfrum wird im folgenden beispielhaft dargelegt. Wolfrum befaßte sich intensiv mit dem strategischen Unternehmensverhalten in technologieintensiven Märkten. Als wesentliche Elemente der Strategieplanung wurden das angestrebte Leistungsniveau, das Inventionstiming, der Markteinfuihrungszeitpunkt, die Bezugsquelle und die Art der Verwertung von Technologien bzw. technologischen Anwendungen angesehen. ${ }^{99}$ Wolfrum verwendete diese

Hinsichtlich der Folgerposition beim Innovationstiming wurde eine Differenzierung zwischen einer frühen und späten Folgerschaft vorgenommen. $\mathrm{Da}$ in der empirischen Analyse keine grundlegenden Verhaltensunterschiede zwischen frühen und späten Innovationsfolgern festgestellt werden konnten, wird hierauf in der vorliegenden Untersuchung nicht weiter eingegangen. Vgl. hierzu Perillieux, R., Der Zeitfaktor im strategischen Technologie-Management, a.a.O., S. $202 \mathrm{ff}$.

Vgl. Specht, G., Perillieux, R., Erfolgsfaktoren technischer Führer- und Folgerpositionen auf Investitionsguitermärkten, a.a.O., S. 216 ff.; Perillieux, R., Der Zeitfaktor im strategischen Technologie-Management, a.a.O., S. $202 \mathrm{ff}$.

Schewe, G., Imitationsmanagement, Nachahmung als Option des Technologiemanagements, a.a.O., S. 14 f. u. 18 ff.; Wolfrum, B., Technologiestrategien im strategischen Management, a.a.O., S. 26 ff.; Maidique, M.A., Zirger, B.J., A study of success and failure in product innovation: The case of U.S. electronics industry, in: IEEE, Stanford University, 1984, No. 4, S. 192 ff.; Maidique, M.A., Hayes, R.H., The art of hightechnology management, in: Sloan Management Review, Vol. 26 (1984), S. 17 ff.; Maidique, M.A., Patch, P., Corporate strategy and technological policy, in: Readings in the management of innovation, Hrsg.: Tushman, M.L., Moore, W.L., Cambridge 1982, S. $275 \mathrm{ff}$.

Als Bezugsquelle für das technologische Know-how bieten sich z.B. die unternehmensinterne F\&E, der Erwerb von Lizenzen, Unternehmenskooperationen oder die Vergabe von Forschungsaufträgen an. Die Verwertung des technologischen Wissens kann beispielsweise durch eine Lizenzvergabe sowie durch eine eigenständige bzw. gemeinschaftliche Technologievermarktung erfolgen. Siehe hierzu Wolfrum, B., Strategisches Technologiemanagement, a.a.O., S. 293 ff.; Remmerbach, K.U., Markteintritts- 
technologiebezogenen Strategiedimensionen jedoch nicht zur Identifikation alternativer Strategietypen. ${ }^{100}$

Es wird insgesamt deutlich, daß in den zeitbezogenen Strategieansätzen unzureichend auf den Aspekt der technologischen Leistungsführerschaft oder -folgerschaft eingegangen wird. Dies ist als problematisch anzusehen, da das technologische Leistungsniveau die Wettbewerbsposition von Unternehmen maßgeblich beeinflußt. Eine grundsätzliche Schwäche der zeit- und technologieorientierten Strategieansätze stellt die mangelnde Berücksichtigung wettbewerbsstrategischer Verhaltensdimensionen dar. Es wird beispielsweise nicht betrachtet, ob ein technologie- bzw. innovationsführendes Unternehmen eine Qualitäts- oder eine Kostenführerschaft anstrebt.

\subsection{Wettbewerbsorientierte Strategieansätze}

Eine Übersicht wichtiger Strategieansätze, die wettbewerbsbezogenen Verhaltensaspekten einen größeren Stellenwert beimessen, zeigt Abbildung 13. Im Gegensatz zu den zeit- und technologieorientierten Ansätzen werden hierbei insbesondere das technologische Leistungsniveau bzw. die angestrebte Wettbewerbsposition von Unternehmen zur Strategietypologisierung herangezogen. ${ }^{101}$ Von den wettbewerbsorientierten Systematisierungen wird im folgenden beispielhaft der Ansatz von Specht und Zörgiebel gekennzeichnet. ${ }^{102}$

entscheidungen, a.a.O., S. 22 f.; Perillieux, R., Der Zeitfaktor im strategischen Technologie-Management, a.a.O., S. $129 \mathrm{ff}$.

100

Vgl. Hofmaier, R., Das "Magische Viereck" strategischen I+T-Marketings als Basis eines ganzheitlichen I+T-Marketingansatzes, in: Investitionsgüter- und High-Tech-Marketing, Hrsg.: Hofmaier, R., Landsberg a.L. 1992, S. 76 ff.; Nyström, H., Technological and market innovation, strategies for product and company development, Chichester u.a. 1990, S. 256 ff.; Porter, M., Wettbewerbsvorteile, a.a.O., S. 239 ff.; Remmerbach, K.U., Markteintrittsentscheidungen, a.a.O., S. 75 ff.; Servatius, H.-G., Methodik des strategischen Technologie-Managements, a.a.O., S. 266 ff.; Porter, M., The technological dimensions of competitive strategy, in: Research on technological innovation, management and policy, Hrsg.: Rosenbloom, R.S., Greenwich, Conn. 1983, S. 1 ff.

Vgl. Specht, G., Zörgiebel, W., Technologieorientierte Wettbewerbsstrategien, a.a.O., S. 494 ff.; Zörgiebel, W.W., Technologie in der Wettbewerbsstrategie, a.a.O., S. $109 \mathrm{ff}$. 


\begin{tabular}{|c|c|c|c|c|c|}
\hline \multirow{6}{*}{ 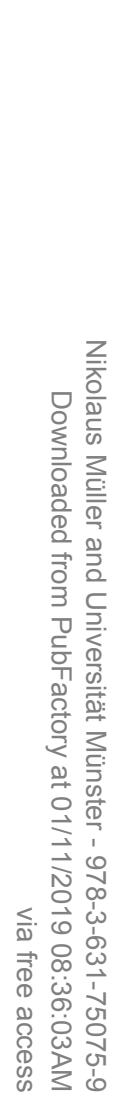 } & $\begin{array}{l}\text { Kennzeich- } \\
\text { Autor nung } \\
\text { Porter } \\
1983\end{array}$ & $\begin{array}{l}\text { Systematisierungs- } \\
\text { kriterien } \\
\text { - Technologieposition } \\
\text { - Wettbowerbsposition }\end{array}$ & $\begin{array}{l}\text { Strategietypen } \\
\text { - Technologiefūhrer (auch Pionier) + } \\
\text { Leistungsführer } \\
\text { - Technologieführer (auch Plonler) + } \\
\text { Kostenführer } \\
\text { - Technologiefolger (auch Innovations- } \\
\text { folger) + Leistungsführer } \\
\text { - Technologiefolger (auch Innovations- } \\
\text { folger) + Kostenführer }\end{array}$ & $\begin{array}{l}\text { Unterstellte Markt- } \\
\text { situation } \\
\text { Technologieintensive } \\
\text { Mărkte }\end{array}$ & $\begin{array}{l}\text { Empirische } \\
\text { Untersuchung } \\
\text { Theoretisch }\end{array}$ \\
\hline & \begin{tabular}{|l|} 
Servatius \\
1985 \\
\end{tabular} & $\begin{array}{l}\text { - Innovationstiming } \\
\text { - Wettbewerbsposition }\end{array}$ & n.a. & Chemische Industrie & Theoretisch \\
\hline & $\begin{array}{l}\text { Zörgiebel } \\
1983 \\
\text { Specht/ } \\
\text { Zörgiebel } \\
1985\end{array}$ & $\begin{array}{l}\text { - Wettbewerbsposition } \\
\text { - Innovationstiming } \\
\text { - Markterfassung }\end{array}$ & $\begin{array}{l}8 \text { Strategietypen durch Kombination } \\
\text { von Wettbewerbsvorteil (Leistungs-, } \\
\text { Kostenführer), Innovationstiming (Pio- } \\
\text { nier=Technologiefūhrer vs. Innova- } \\
\text { tlons- = Technologlefolger) u. Markt- } \\
\text { erfassung (Gesamt- vs. Teilmarkt) }\end{array}$ & n.a. & Theoretisch \\
\hline & $\begin{array}{l}\text { Remmerbach } \\
1988\end{array}$ & $\begin{array}{l}\text { primår } \\
\text { - Innovationstiming } \\
\text { sekundār } \\
\text { - Technologieposition } \\
\text { - Wettbewerbsposition } \\
\text { - Markterfassung } \\
\text { - Marktbearbeitung }\end{array}$ & $\begin{array}{l}\text { Auf Basis des Innovationstiming (Pio- } \\
\text { nier, Früher Folger, Später Folger) } \\
\text { erfolgt Konkretisierung der übrigen } \\
\text { Strategieoptionen }\end{array}$ & $\begin{array}{l}\text { Einfūhrungs-, } \\
\text { Wachstumsphase } \\
\text { von Mārkten }\end{array}$ & Theoretisch \\
\hline & \begin{tabular}{|l|} 
Nystrōm \\
1980
\end{tabular} & $\begin{array}{l}\text { - Technologieposition } \\
\text { - Marketingposition }\end{array}$ & $\begin{array}{l}\text { - Innovator } \\
\text { - Technologieführer } \\
\text { - Marktführer } \\
\text { - Industrieller Folger }\end{array}$ & n.a. & Theoretisch \\
\hline & $\begin{array}{l}\text { Hofmaier } \\
1992\end{array}$ & $\begin{array}{l}\text { - Technologieposition } \\
\text { - Wettbewerbsposition } \\
\text { - Markterfassung }\end{array}$ & n.a. & $\begin{array}{l}\text { Investitionsgūtermārkte/ } \\
\text { High-Tech-Mărkte }\end{array}$ & Theoretisch \\
\hline
\end{tabular}

Abb. 13: Zentrale wettbewerbsorientierte Ansätze zur Systematisierung von Marketingstrategien in High-Tech-Murkten 
Im Rahmen der konzeptionellen Überlegungen von Specht und Zörgiebel erfolgte eine Konkretisierung des wettbewerbsstrategischen Unternehmensverhaltens anhand von drei Strategiedimensionen. Bei der angestrebten Wettbewerbsposition wurde zwischen dem Aufbau von Differenzierungs- bzw. Kostenvorteilen und bei der Markterfassung zwischen der Gesamtmarktabdeckung bzw. der Konzentration auf eine Nische unterschieden. Im Hinblick auf das Innovationstiming wurden die Pionier- und die Technologieführerposition sowie die Innovationsund die Technologiefolgerschaft gleichgesetzt. ${ }^{103}$ Aus der Kombination der wettbewerbsstrategischen Dimensionen leiteten Specht und Zörgiebel insgesamt acht verschiedene Strategietypen ab. ${ }^{104}$

Kritisch bleibt anzumerken, daß in den wettbewerbsorientierten Strategieansätzen häufig nicht zwischen einer technologischen Führer- und Folgerschaft und einer timingbezogenen Führer- und Folgerposition unterschieden wird. Demzufolge würden Unternehmen bei einer Technologiefuihrerschaft immer als Pionier und bei einer technologischen Folgerschaft immer als Innovationsfolger in einen Markt eintreten. Es folgt hieraus, daß sich das Spektrum der möglichen strategischen Verhaltensoptionen nicht hinreichend abbilden läßt. Als weitere Schwäche dieser Systematisierungsansätze ist die Begrenzung der wettbewerbsstrategischen Verhaltensoptionen i.d.R. auf die Qualitäts- oder Kostenorientierung von Unternehmen anzusehen.

103 Vgl. Specht, G., Zörgiebel, W., Technologieorientierte Wettbewerbsstrategien, a.a.O., S. 495 u. 497.

104 Die Strategietypen lauten: Segmentspezifische Technologiefürungs- u. Individualisierungsstrategie, Segmentspezifische Technologiefolger- u. Individualisierungsstrategie, Generelle Technologiefuihrungs- u. Individualisierungsstrategie, Generelle Technologiefolger- u. Individualisierungsstrategie, Segmentspezifische Technologiefuihrungs- u. Standardisierungsstrategie, Segmentspezifische Technologiefolger- u. Standardisierungsstrategie, Generelle Technologiefuhrungs- u. Standardisierungsstrategie sowie Generelle Technologiefolger- u. Standardisierungsstrategie. Vgl. Specht, G., Zörgiebel, W., Technologieorientierte Wettbewerbsstrategien, a.a.O., S. $496 \mathrm{ff}$. 


\subsection{Kritische Würdigung}

In einer zusammenfassenden Beurteilung der vorliegenden Systematisierungsansätze wird deutlich, daß bislang integrative Strategieansätze fehlen. Weder die zeit- und technologiebezogenen Systematisierungen noch die wettbewerbsorientierten Ansätze können für sich allein eine problemadäquate Grundlage zur Typologisierung von Marketingstrategien in High-Tech-Märkten bilden. In beiden Fällen werden jeweils wichtige Strategiedimensionen aus der Analyse ausgeschlossen und somit die Anforderung nach einer vollständigen Erfassung der zentralen Systematisienungsmerkmale nicht hinreichend erfüllt. ${ }^{105}$

Ein weiterer Kritikpunkt richtet sich auf die unzureichende Trennung wichtiger Strategiedimensionen. In den Ansätzen von Spital, Porter und Specht/Zörgiebel werden die technologie und timingbezogene Führerposition sowie die technologie- und timingbezogene Folgerschaft gleichgesetzt. ${ }^{106}$ Es ergibt sich hieraus einerseits das Problem, daß die Strategietypen nicht eindeutig zu charakterisieren sind. Andererseits werden bestimmte Strategiekombinationen vernachlässigt, die für Unternehmen in High-Tech-Märkten durchaus von Interesse sind. Beispielsweise kann es für ein technologieführendes Unternehmen durchaus sinnvoll sein, erst als Folger in einen Markt einzutreten, um aus den Erfahrungen eines Pionierunternehmens zu lernen oder einen besseren Überblick über die Marktentwicklung zu erhalten.

Insgesamt bleibt festzuhalten, daß sich die aufgezeigten Systematisierungsansätze nur bedingt für eine Typologisierung von Marketingstrategien in High-TechMärkten auf der Grundlage strategischer Gruppen übertragen lassen. In den weiteren Ausführungen werden deshalb unter Berücksichtigung der vorliegenden

$105 \mathrm{Vgl}$. zu diesem Problem auch Michel, K., Technologie im strategischen Management, 2. Aufl., Berlin 1991, S. 110 ff.; Wolfrum, B., Strategisches Technologiemanagement, a.a.O., S. $225 \mathrm{ff}$.

Vgl. Wolfrum, B., Strategisches Technologiemanagement, a.a.O., S. 225 ff.; Specht, G., Zörgiebel, W., Technologieorientierte Wettbewerbsstrategien, a.a.O., S. 495 u. 497.; Porter, M., The technological dimensions of competitive strategy, a.a.O., S. 1 ff.; Spital, F., Gaining market share advantage in the semiconductor industry by lead time in innovation, a.a.O., S. $55 \mathrm{ff}$. 
Forschungsansätze in einem eigenständigen Ansatz geeignete Strategiedimensionen zur Bildung strategischer Gruppen in High-Tech-Märkten abgeleitet. ${ }^{107}$ Es werden hierbei marktfeld-, wettbewerbs- und timinggerichtete Strategiedimensionen generiert, die das spezifische strategische Verhalten von High-TechUnternehmen abbilden und denen eine hohe Relevanz im Hinblick auf die Schaffung von Wettbewerbsvorteilen zukommt. ${ }^{108}$

\subsection{Ableitung zentraler Strategiedimensionen zur Bildung strategischer Gruppen in High-Tech-Märkten}

\subsection{Marktfeldbezogene Strategiedimensionen}

Eine wichtige strategische Grundsatzentscheidung in High-Tech-Märkten stellt die Form der Marktfeldbearbeitung i.S. der Produkt-Markt-Kombination von Ansoff dar. ${ }^{109}$ Von den High-Tech-Unternehmen wird dabei festgelegt, inwiefern

107 Damit wird einem wesentlichen Kritikpunkt an empirischen Untersuchungen zu strategischen Gruppen Rechnung getragen, der in einer ungenügenden Berücksichtigung von spezifischen Branchengegebenheiten liegt. Vgl. hierzu z.B. Hannig, U., Die Entwicklung wettbewerbsorientierter Marketingstrategien auf Basis des Konzeptes der strategischen Gruppen, a.a.O., S. 144 f.; McGee, J., Thomas, H., Strategic groups: Theory, research and taxonomy, a.a.O., S. 149.

Vgl. Meffert, H., Marketing-Management, a.a.O., S. 123 f.; Manns, J.R., Produktinnovationen als Ergebnis der Koordination von F\&E und Marketing, Ursachen, Auswirkungen und Lösungsmöglichkeiten, dargestellt am Beispiel eines Unternehmens der mobilen Kommunikation, Ludwigsburg, Berlin 1992, S. 78 f.; Backhaus, K., Investitionsgütermarketing, a.a.O., S. 177 ff.; Wolfrum, B., Technologiestrategien im strategischen Management, a.a.O., S. 25 ff.; Dellmann, K., Einflußfaktoren der Erfolgsdynamik, in: Unternehmensdynamik, Hrsg.: Kistner, K.-P., Schmidt, R., Wiesbaden 1991, S. 427; Porter, M., Wettbewerbsstrategie, a.a.O., S. 273 ff. Zur Notwendigkeit der Komplexitätsreduzierung bei der Ableitung von Strategiedimensionen siehe auch HeB, G., Marktsignale und Wettbewerbsstrategie, a.a.O., S. 102 f.; Remmerbach, K.-U., Markteintrittsentscheidungen, a.a.O., S. 50; Knoblich, H., Die typologische Methode in der Betriebswirtschaftslehre, a.a.O., S. 143.

109 Aufgrund der Kritik, daß die Marktfeldstrategie von Ansoff ausschließlich für Wachstumsmärkte Gültigkeit hat, wurde von einigen Autoren die Strategiealternative des Rückzugs bzw. der Elimination hinzugefuigt. Siehe hierzu u.a. Meffert, H., MarketingManagement, a.a.O., S. 123 f.; Müller, W., Planung von Marketing-Strategien, a.a.O., S. $90 \mathrm{ff}$. Diese Strategieoption ist in der vorliegenden Untersuchung aufgrund des hohen 
vorhandene oder neue Produkt- und Technologiekonzepte in bestehenden oder neuen High-Tech-Märkten angeboten werden. ${ }^{110}$

Im Rahmen der Marktpenetrationsstrategie wird versucht, das Marktpotential gegenwärtiger High-Tech-Märkte durch die Vermarktung vorhandener Produktund Technologieleistungen auszuschöpfen. Bei dieser Strategie soll im wesentlichen durch eine Intensivierung der Marketingaktivitäten die Nutzungsintensität bestehender Kunden gesteigert bzw. die Anzahl der Verwender (z.B. bisherige Nichtverwender, Verwender von Konkurrenzprodukten) erhöht werden. ${ }^{11}$

Angesichts der hohen F\&E-Aufwendungen der High-Tech-Unternehmen und der Notwendigkeit einer schnellen Amortisation besitzt die Marktpenetrationsstrategie eine besondere Bedeutung. Grundlage für eine erfolgreiche Marktdurchdringung bildet häufig die Durchsetzung eines technologischen De facto-Standards ${ }^{112}$, wie z.B. das Betriebssystem DOS, die Benutzeroberfläche Windows im PC-Bereich, TOP (Technical Office Protocol) in der Bürokommunikation oder CAN (Car Area Network) in der Automobilelektronik. ${ }^{113}$ Ansatzpunkte für die Etablierung eines De facto-Standards können anbietergerichtet in der proaktiven

Marktwachstums der betrachteten High-Tech-Märkte jedoch zu vernachlässigen. Vgl. o.V., PC-Markt wächst weltweit um 16 Prozent, a.a.O., S. 9; Meffert, H., Lamnek, S., Maisberger, P., High-Tech-Marketing, a.a.O., S. 23.

110 Vgl. Meffert, H., Marketing-Management, a.a.O., S. 109 ff.; Becker, J., Marketing-Konzeption, a.a.O., S. 123 ff.; Kotler, Ph., Bliemel, F., Marketing-Management, a.a.O., S. 64 ff.; Schildhauer, Th., Strategisches Software-Marketing, Übersicht und Bewertung, Wiesbaden 1992, S. 34 ff.; Hofmaier, R., Ein systematischer I+T-Marketing-Gesamtansatz - Vom klassischen Verkaufs- zum Produktmanagement hin zum "integrierten" Segment- und Erfolgspotentialmanagement, in: Investitionsgüter- und High-Tech-Marketing, Hrsg.: Hofmaier, R., Landsberg a.L. 1992, S. 154 f.; Roberts, E.B., Berry, Ch., Entering new business: Selecting strategies for success, in: The Best of MIT's Sloan Management Review, Cambridge, Mass. 1986, S. 57 ff.; Ansoff, H.I., ManagementStrategie, München 1966, S. 13 ff.

111 Vgl. Dussauge, P., Hart, St., Ramanantsoa, B., Strategic technology management, a.a.O., S. 90 f. ; Meffert, H., Marketing, Grundlagen der Absatzpolitik, a.a.O., S. 91 .

112 Ein De facto-Standard besteht, wenn ein technologisches Produkt, System oder eine Komponente so stark in High-Tech-Märkten penetriert ist, daß sich die Marktteilnehmer bei strategischen Entscheidungen daran orientieren. Vgl. Meffert, J., Standards als Integrationsinstrument in der Computer- und Kommunikationsindustrie, a.a.O., S. 17.

113 Bühner, R., Technologieorientierung als Wettbewerbsstrategie, a.a.O., S. 397; Backhaus, K., Investitionsgütermarketing, a.a.O., S. $368 \mathrm{ff}$. 
Vergabe von Lizenzen bzw. in der Schaffung technologischer Kompatibilität und nachfragergerichtet in der frühzeitigen Ansprache innovationsfreudiger Anwender bzw. professioneller Anwender liegen. Durch eine gezielte nachfragerbezogene Informationspolitik gelang es beispielweise IBM sein Netzsystem "Token Rings" als De facto-Standard erfolgreich im Markt durchzusetzen. Der Marktanteil für lokale Netze ist hierbei im Zeitraum 1889 bis 1993 von $13 \%$ auf ca. $40 \%$ angestiegen. ${ }^{114}$

Weiterhin können technologiespezifische Faktoren die schnelle Durchdringung eines High-Tech-Marktes erfordern. Beispielhaft sei das Angebot sog. Kritische Masse-Systeme (z.B. Btx, Videotext) erwähnt, die eine gewisse Anzahl von Nachfragern erreichen müssen, damit ein stabiler Diffusionsprozeß einsetzt. Dies bildet wiederum die Grundlage dafür, da $B$ ein Anwendernutzen durch Interaktion mit anderen Systemnutzern gewährleistet werden kann. ${ }^{115}$

Die Ausgestaltung der Marktpenetrationsstrategie ist zudem in Abhängigkeit vom Lebenszyklus der jeweiligen Technologie zu sehen. Es wird hierbei zwischen einer Schrittmacher-, Schlüssel- und Basistechnologie unterschieden. ${ }^{116}$ Schrittmachertechnologien befinden sich in einem frühen Entwicklungsstadium und bieten für die Zukunft erhebliche Wettbewerbschancen. Schlüsseltechnologien sind Technologien, die sich in der Wachstumsphase befinden und als Produktund Verfahrenstechnologie zur Ausnutzung von Leistungs- und Kostenvorteilen

114 Vgl. Garud, R., Kumaraswamy, A., Changing competitive dynamics in network industries: An exploration of sun microsystems' open systems strategy, in: Strategic Management Journal, Vol. 14 (1993), S. 353 ff.; HeB, G., Marktsignale und Wettbewerbsstrategie, a.a.O., S. 6 ff.; Easingwood, Ch., Beard, Ch., High technology launch strategies in the U.K., in: Industrial Marketing Management, Vol. 18 (1989), S. $128 \mathrm{ff}$.

115 Vgl. Weiber, R., Die Diffusion von Kritische Masse-Systemen, Wiesbaden 1992, S. 64 ff.; Backhaus, K., Investitionsgütermarketing, a.a.O., S. 386 ff.; Meffert, H., Marketing und neue Medien, Stuttgart 1985, S. 37 ff.; Middelhoff, Th., Walters, M., Akzeptanz neuer Medien - Eine empirische Analyse aus Unternehmenssicht, Arbeitspapier Nr. 27 des Instituts für Marketing der Universität Münster, Hrsg.: Meffert, H., Münster 1981, S. $11 \mathrm{ff}$.

116 Vgl. Höft, U., Lebenszykluskonzepte, a.a.O., S. 74 ff.; Saad, K.N., Roussel, Ph.A., Tiby, C., Mangement der F\&E-Strategie, a.a.O., S. 69 f.; Servatius, H.-G., Methodik des strategischen Technologie-Managements, a.a.O., S. 116 ff.; Little, A.D. (Hrsg.), The strategic management of technology, European Management Forum, Davos 1981, S. 12 ff. 
bereits einen Beitrag leisten. Eine breite Marktdurchdringung haben Basistechnologien vollzogen, die eine wesentliche Grundlage für die industrielle Anwendung von Produkt-, Verfahrens- und Anwendungstechnologien bilden. ${ }^{117}$ Die Wettbewerbspotentiale von Unternehmensleistungen, die auf Basistechnologien beruhen, werden als vergleichsweise gering eingestuft, da alle Wettbewerber diese Technologien weitgehend beherrschen. Für High-Tech-Unternehmen bildet demzufolge insbesondere die Marktpenetration erfolgversprechender Leistungsangebote auf Basis von Schrittmacher- und Schlüsseltechnologien eine attraktive strategische Stoßrichtung. ${ }^{118}$

Die Marktentwicklungsstrategie basiert auf der Überlegung, für vorhandene High-Tech-Produkte einen oder mehrere neue Absatzmärkte zu finden bzw. zu entwickeln. Dies kann insbesondere durch eine geographische Marktausdehnung, den Aufbau zusätzlicher Distributionskanäle und die Erschließung neuer Marktsegmente erfolgen. ${ }^{119}$

Vielfach bietet auch die voranschreitende Technologieintegration Möglichkeiten, neue Märkte zu erschließen, auf denen die High-Tech-Unternehmen bisher noch nicht tätig waren. Für High-Tech-Unternehmen stellt die Bearbeitung zusätzlicher Marktsegmente eine wichtige strategische Wachstumsquelle dar. Beispielsweise werden Mikroprozessoren heute nicht nur für Produkte der Mikroelektronik und der Bürokommunikation hergestellt, sondern auch im Bereich der Verfahrenstechnik und der Automatisierungstechnik eingesetzt. ${ }^{120}$

117 Zur Unterscheidung von Produkt-, Verfahrens- und Anwendungstechnologien siehe z.B. Meyer, N., Hebelpunkte des strategischen Entwicklungsmanagement, in: TechnologieMarketing, Hrsg.: Töpfer, A., Sommerlatte, T., Landsberg a.L. 1991, S. 93. Im Vordergrund der vorliegenden Arbeit steht vor allem die Betrachtung von Produkt- und Anwendungstechnologien.

Vgl. Green, K., Creating demand for biotechnology, a.a.O., S. 172 ff.; Meyer, N., Hebelpunkte des strategischen Entwicklungsmanagement, a.a.O., S. 94 ff.; Rüdiger, B., Schlüsseltechnologien: Die Herausforderungen für die 90er Jahre, a.a.O., S. 42 ff.; Remmerbach, K.-U., Markteintrittsentscheidungen, a.a.O., S. $137 \mathrm{ff}$.

119 Vgl. Schildhauer, Th., Strategisches Software-Marketing, a.a.O., S. 37 f.; Kotler, Ph., Bliemel, F., Marketing-Management, a.a.O., S. 66 f. technologien: Die Herausforderungen für die 90er Jahre, a.a.O., S. 52.; Saad, K.N., 
Mit der Produktentwicklungsstrategie wird angestrebt, die in den angestammten High-Tech-Märkten vorhandenen Wachstumspotentiale durch Entwicklung neuer Produkt- und Technologiekonzepte zu nutzen. Als grundlegende Alternativen bieten sich die Modifikation bestehender High-Tech-Produkte und die Schaffung von Produkt- bzw. Technologieinnovationen i.S. echter Marktneuheiten an. ${ }^{121}$

Als Beispiel für eine Produktentwicklungsstrategie läßt sich das von ITT im Jahr 1984 eingeführte ABS (Anti-BlockierSystem) nennen. Aufgrund der hohen Nachfrage nach ABS-Produkten sind bis zum Jahr 1994 bereits 10 Mio. Stück verkauft worden. In diesem Zeitraum konnte ITT seine Position als Marktführer erfolgreich behaupten und verfuigt über einen Anteil am Weltmarkt von $26 \%$. Da 1994 lediglich $34 \%$ der Neuwagen mit einem ABS ausgerüstet sind, ist auch in der Zukunft mit einem starken Marktwachstum zu rechnen. ${ }^{122}$

Insbesondere wenn Technologien im Lebenszyklus zu Basistechnologien geworden sind, lassen sich langfristige Wachstumschancen i.d.R. nur noch durch die Einfuihrung neuer Produkt- und Anwendungstechnologien erzielen. Aufgrund der in High-Tech-Branchen vorherrschenden hohen Technologiedynamik besitzt darüber hinaus die kontinuierliche Entwicklung von Produktinnovationen eine besondere Bedeutung. So ist beispielsweise im Bereich der Mikroelektronik einerseits bei Speicherchips eine schnelle Abfolge von Neuproduktentwicklungen festzustellen. Bestehende Speicherchip-Generationen werden hierbei in Abstän-

Roussel, Ph.A., Tiby, C., Management der F\&E-Strategie, a.a.O., S. 69 f.; Servatius, H.G., Methodik des strategischen Technologie-Managements, a.a.O., S. $106 \mathrm{ff}$.

Zum Begriff der Innovation und zur Abgrenzung von Produkt- und Prozeßinnovationen vgl. Manns, J.R., Produktinnovationen als Ergebnis der Koordination von F\&E und Marketing, Ursachen, Auswirkungen und Lösungsmöglichkeiten, a.a.O., S. 8 f.; Pfeiffer, St., Technologie-Frühaufklärung, a.a.O., S. 40 ff. Im Vordergrund der weiteren Diskussion stehen primär produktbezogene Innovationen, da ihnen eine besondere Bedeutung für den Aufbau komparativer Wettbewerbsvorteile zukommt. Siehe hierzu Weber, M., Unternehmerische Marktstrategien bei dynamischem Wettbewerb, Diss., Münster 1992, S. 100 ff.; Ostmeier, H., Ökologieorientierte Produktinnovationen, Frankfurt a.M. u. a. 1990, S. 10.

Vgl. o.V., Innovation: Wettlauf der Erfinder, a.a.O., S. 187. 
den von ca. 3 Jahren durch leistungsfähigere Produktgenerationen substituiert. ${ }^{123}$ Andererseits wird in der Abbildung 14 deutlich, daß auch die Entwicklung von Mikroprozessoren rasch voranschreitet. Es ist hierbei zu erkennen, daß sich die Zahl der Transistoren auf einem Mikroprozessor im Durchschnitt alle zwei Jahre verdoppelt. Während ein 8088 Prozessor Anfang der 80er Jahre noch mit 29 Tsd. Transistoren bestückt war, beinhaltet der Pentium (586 Prozessor) 1993 schon 3,1 Mio. Transistoren und erbringt eine 200 fach höhere Leistung als sein Vorgänger. ${ }^{124}$

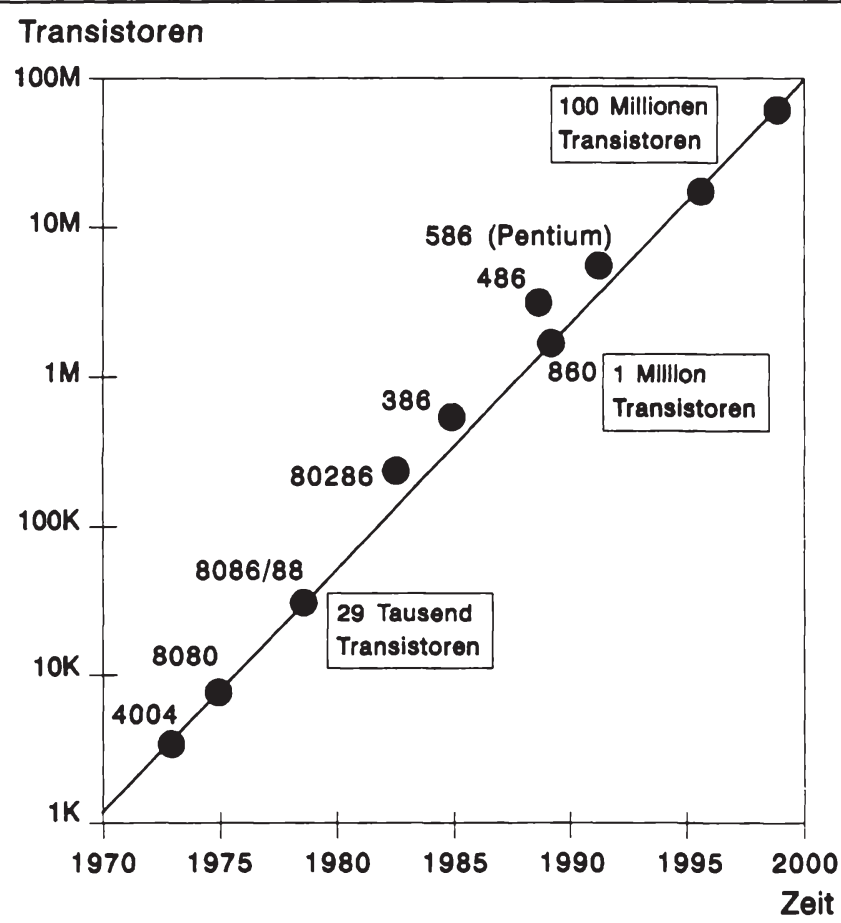

Abb. 14: Entwicklungsdynamik bei Mikroprozessoren (Quelle: Intel 1993)

123 Vgl. Milling, P., Schwierigkeiten bei der Umsetzung des neuen Wissens, a.a.O., S. 7; Benkenstein, M., Strategisches Marketing-Management in High-Tech-Branchen, a.a.O., S. 9; Backhaus, K., Investitionsgütermarketing, a.a.O., S. 11.

124 Vgl. o.V., Der unendlich schrumpfende Transistor, in: Computer Zeitung vom 12.08.1993, S. 19. 
Bei der Diversifikationsstrategie liegt der Schwerpunkt der Unternehmenstätigkeiten auf der Entwicklung neuer Produkt- bzw. Technologiekonzepte für neue High-Tech-Märkte. In Abhängigkeit von der mit dieser Strategie beabsichtigten Risikostreuung und unter Synergiegesichtspunkten ist zwischen einer horizontalen, vertikalen und lateralen Vorgehensweise zu unterscheiden. ${ }^{125}$

Im Rahmen der häufig anzutreffenden horizontalen Diversifikation wird das bestehende Produktprogramm durch die Vermarktung verwandter High-TechProdukte ausgeweitet. Ein High-Tech-Unternehmen, das bisher Lasersysteme zur Materialbearbeitung (u.a. Trennen, Schweißen und Bohren) im Stahl- und Fahrzeugbau produziert, kann beispielsweise durch das Angebot von Laseranwendungen in der Medizin (Augenheilkunde, Tumorbehandlung) neue High-TechMärkte erschließen. ${ }^{126}$

Bei der vertikalen Diversifikation wird die Tiefe des Produktprogramms entweder durch Rückwärtsintegration in Richtung des Beschaffungsmarktes oder durch Vorwärtsintegration in Richtung des Absatzmarktes vergrößert. ${ }^{127}$ In der Computerindustrie ist eine vertikale Diversifikation i.S. einer Vorwärtsintegration z.B. von Intel betrieben worden, der ursprünglich als Komponentenhersteller lediglich Prozessoren herstellte und mittlerweile als Endgeräteanbieter auch "Portable" Computer vertreibt.

Die laterale Diversifikation stellt einen Vorstoß in völlig neue Produkt- bzw. Technologiebereiche dar, $\mathrm{zu}$ denen die bisherigen Unternehmensaktivitäten in keinem sachlichen Zusammenhang stehen. Sie wird insbesondere von Unternehmen verfolgt, die die fortschreitende Technologieintegration nutzen, um an

Vgl. Schildhauer, Th., Strategisches Software-Marketing, a.a.O., S. 45 ff.; Meffert, H., Marketing-Management, a.a.O., S. 111.

126 Vgl. Marone, J.G., Winning in high-tech markets, a.a.O., S. 67 ff.; Rüdiger, B., Schlüsseltechnologien: Die Herausforderungen für die 90er Jahre, a.a.O., S. 50 f.; Roberts, E.B., Entrepreneurs in high technology: Lessons from MIT and beyond, New York u.a. 1991, S. $291 \mathrm{ff}$.

Vgl. Benkenstein, M., Henke, N., Vertikale Integration - Eine transaktionskostentheoretische Interpretation, Arbeitspapier Nr. 39 des Instituts für Marketing, Hrsg.: Meffert, H., Münster 1990, S. 2 ff. 
den in High-Tech-Märkten bestehenden Wachstumspotentialen zu partizipieren und eine gegenüber den Wettbewerbern verteidigbare Marktposition aufzubauen. Als Beispiel für eine laterale Diversifikationsstrategie in High-Tech-Märkten kann die Expansionspolitik von Daimler-Benz angeführt werden. Durch den Zukauf von MTU (Triebwerkhersteller), Dornier (Luft- und Raumfahrt), AEG (Elektrounternehmen) und MBB (Luft- und Raumfahrt) erreichte Daimler-Benz den Zugang zu High-Tech-Märkten und stärkte gleichzeitig die technologische Wettbewerbsposition im bestehenden Automobilgeschäft. In der Unterhaltungsindustrie sind zur Sicherung von technologieinduzierten Wachstumspotentialen (u.a. digitale TV- und Tonbandtechnik, "virtuelle" Hard- und Softwaretechnik) die Columbia Pictures Entertainment von Sony, die MCA Inc. von Matsushita und die Warner Brothers von Microsoft übernommen worden. ${ }^{128}$

Für die Marktbearbeitungsstrategie läßt sich zusammenfassend festhalten, daß die gegenwärtige und zukünftige Wettbewerbssituation in High-Tech-Märkten sowie die Erfolgsposition eines Anbieters maßgeblich durch die von den High-TechUnternehmen festgelegte strategische Stoßrichtung beeinflußt werden. Eine von High-Tech-Anbietern verfolgte Strategie der Marktpenetration führt i.d.R. zu einer Verschärfung der aktuellen Wettbewerbssituation. Demgegenüber wird die Intensität des zukünftigen Wettbewerbs in High-Tech-Märkten bei der Produktentwicklungsstrategie meist durch die kontinuierliche Schaffung von Leistungsverbesserungen und bei der Marktentwicklungs- bzw. Diversifikationsstrategie vor allem durch den Anstieg der Anzahl der Konkurrenten zunehmen. ${ }^{129}$ Darüber hinaus ist zu erwarten, daß die High-Tech-Unternehmen die einzelnen Strategieoptionen auch miteinander kombinieren, wodurch sich die Wettbewerbs-

Vgl. Kodama, F., Technologiefusion - der Weg zum Erfolg auf Zukunftsmärkten, a.a.O., S. 45; Becker, J., Marketing-Konzeption, a.a.O., S. 143; Schildhauer, Th., Strategisches Software-Marketing, a.a.O., S. 46 f.; Samli, A.C., Wills, J.R., Strategies for marketing computers and related products, a.a.O., S. $27 \mathrm{ff}$.

129 Vgl. Brezski, E., Konkurrenzforschung im Marketing, Wiesbaden 1993, S. 58; Backhaus, K., Investitionsgütermarketing, a.a.O., S. 10 f.; Homburg, Ch., Sütterlin, St., Strategische Gruppen: Ein Survey, a.a.O., S. 652 ff.; Römer, E., Konkurrenzforschung, in: ZfB, 1988, H. 4, S. 490; Ewald, A., Organisation des strategischen TechnologieManagements, Stufenkonzept zur Implementierung einer integrierten Technologie- und Marktplanung, Berlin 1989, S. 182f.; Porter, M., Wettbewerbsstrategie, a.a.O., S. 186 ff. 
intensität und -dynamik in den High-Tech-Märkten erneut verstärken wird. ${ }^{130} \mathrm{Da}$ mit dem Konzept der strategischen Gruppen das aktuelle und potentielle strategische Verhalten von High-Tech-Unternehmen abgebildet werden soll, sind die Strategiedimensionen der Marktbearbeitung bei der Bildung strategischer Gruppen in High-Tech-Märkten zu berücksichtigen.

\subsection{Wettbewerbsbezogene Strategiedimensionen}

Im Rahmen der Gestaltung einer abnehmergerichteten Wettbewerbsstrategie treffen die High-Tech-Anbieter die Entscheidung, in welcher Form komparative Wettbewerbsvorteile aufgebaut werden sollen. ${ }^{131}$ Wie bereits angefuihrt, stehen Unternehmen in High-Tech-Märkten vor besonderen wettbewerbsstrategischen Herausforderungen, so daß die Wahl einer geeigneten abnehmergerichteten Wettbewerbsstrategie zur Erzielung und Erhaltung einer Erfolgsposition von zentraler Bedeutung ist. ${ }^{132}$ Zur Profilierung der High-Tech-Unternehmen im Wettbewerbsumfeld bieten sich anknüpfend an die traditionelle Diskussion zum Aufbau von Wettbewerbsvorteilen grundsätzlich die Strategie der Differenzierung und der Kostenführerschaft an. ${ }^{133}$

130 Vgl. Becker, J., Marketing-Konzeption, a.a.O., S. 149 ff.; Marone, J.G., Winning in high-tech markets, a.a.O., S. 65 ff.; Samli, A.C., Wills, J.R., Strategies for marketing computers and related products, a.a.O., S. $27 \mathrm{ff}$.

Vgl. Meffert, H., Marketing-Management, a.a.O, S. 126 f.; Backhaus, K., Investitionsguitermarketing, a.a.O., S. 17 ff.; Simon, H., Management strategischer Wettbewerbsvorteile, in: ZfB, 1988, S. $463 \mathrm{ff}$.

Siehe hierzu die Ausführungen in Teil A Kap. 1 und Teil B Kap. 2 der Arbeit.

Vgl. Dussauge, P., Hart, St., Ramanantsoa, B., Strategic technology management, a.a.O., S. 43 ff.; Benkenstein, M., Strategisches Marketing-Management in High-TechBranchen, a.a.O., S. 13. In der Literatur werden die Differenzierungs- und Präferenzstrategie sowie die Kostenfuhrerschafts- und Preis-Mengen-Strategie i.d.R. gleichgesetzt. Vgl. hierzu z.B. Backhaus, K., Investitionsgütermarketing, a.a.O., S. 177 ff.; Haedrich, G., Tomczak, Th., Strategische Markenfuihrung, a.a.O., S. 118. Diese Sicht liegt auch den folgenden Ausfuihrungen zugrunde, obwohl eine genaue Betrachtung zeigt, da $B$ die Unternehmensleistungen bei der Differenzierungs- und Kostenführerschaftsstrategie immer in Relation zur Konkurrenz gesehen werden und die Analyse von differenzierungs- bzw. kostenbezogenen Wettbewerbsvorteilen über alle Wertaktivitäten hinweg erfolgt. Siehe zu dieser Einschätzung z.B. Meffert, H., Marketing-Management, a.a.O., S. 126 f. u. 137; Meffert, H., Benkenstein, M., Wertkette, in: DBW, 1989, Nr. 6, 
Mit einer ausgeprägten Kostenorientierung wird versucht, die Stückkosten durch eine konsequente Ausnutzung von Skalen- und Erfahrungseffekten sowie durch produktivitätssteigernde Verfahrensverbesserungen unter das Niveau der wichtigsten Konkurrenten zu senken. Während Skaleneffekte vor allem durch eine Nutzung von Kostendegressionen bei steigender Ausbringungsmenge (Degressionseffekte) und eine effizientere Ausschöpfung der Unternehmenskapazitäten (Mengeneffekte) erzielt werden, lassen sich Erfahrungseffekte insbesondere durch eine verbesserte Arbeitsteilung (Spezialisierungseffekte) und eine effizientere Gestaltung der Arbeitsabläufe (Lerneffekte) realisieren. ${ }^{134}$ Indem der hieraus resultierende Kostenvorsprung über eine Politik der niedrigen Absatzpreise an die High-Tech-Nachfrager weitergegeben wird, lassen sich komparative Konkurrenzvorteile aufbauen. ${ }^{135}$ Kostenorientierte Strategien bieten darüber hinaus die Möglichkeit, in preissensiblen Segmenten eine schnelle Diffusion neuer Technologien zu forcieren, um frühzeitig einen faktischen Standard durchzusetzen und die Amortisationsperiode zu verkürzen.

Im Zusammenhang mit der Erzielung von Kostenvorteilen wird in der jüngeren betriebswirtschaftlichen Literatur die Möglichkeit der Auslagerung von Unternehmensaktivitäten (Outsourcing) verstärkt diskutiert. ${ }^{136}$ Als Vorzüge des Outsourcing werden unter Kostengesichtspunkten insbesondere eine stärkere Variabilisierung von Fixkosten und eine Flexibilitätserhöhung durch kurzfristig

S. 786. Für Porter stellt die Konzentration auf eine Marktnische eine weitere strategische StoBrichtung dar. Siehe hierzu Porter, M., Wettbewerbsvorteile, a.a.O., S. 35 ff. Aufgrund der oftmals dynamischen Entwicklung kleinerer High-Tech-Märkte und des häufigen Zusammengehens ursprünglich getrennter High-Tech-Märkte erscheint es jedoch nicht sinnvoll, diese strategische StoBrichtung weiter zu betrachten. Siehe hierzu im Detail die Ausführungen in Teil A Kap. 2 und Teil B Kap. 1.2 der Arbeit.

Für eine differenzierte Betrachtung der skalen- und erfahrungsspezifischen Teileffekte siehe z.B. Kloock, J., Sabel, H., Schuhmann, W., Die Erfahrungskurve in der Unternehmenspolitik, in: ZfB, 1987, Ergänzungsheft Nr. 2, S. 8 ff.; Ghemawat, P., Dauerhafte Wettbewerbsvorteile aufbauen, in: Harvard Manager, 1987, Nr. 2, S. 105 f.

Vgl. Meffert, H., Marketing-Management, a.a.O., S. 135 ff.; Fröhling, O., Dynamisches Kostenmanagement, Konzeptionelle Grundlagen und praktische Umsetzung im Rahmen eines strategischen Kosten- und Erfolgs-Controlling, München 1994, S. 109 ff.; Porter, M., Wettbewerbsvorteile, a.a.O., S. $93 \mathrm{ff}$.

Siehe hierzu stellvertretend Meffert, H., Erfolgreiches Marketing in der Rezession, Strategien und Maßnahmen in engeren Märkten, Wien 1994, S. 48 ff.; Herzig, H., Outsourcing - Chancen und Grenzen, in: Lean Marketing, Hrsg.: Geyer, D., Bauer, A., Landsberg a.L. 1993, S. $167 \mathrm{ff}$. 
auf- und abbaubare Kapazitäten genannt. Für High-Tech-Unternehmen, die einerseits hohe F\&E-, Produktions- und Marktinvestitionen tätigen müssen und anderseits mit einer hohen Unsicherheit der Nachfrageentwicklung konfrontiert werden, schafft die Auslagerung von Wertschöpfungsaktivitäten größere Gestaltungsfreiheiten. Es ist jedoch darauf zu achten, daß ein Outsourcing nicht zum Abfluß von wichtigem technologischem Know-how und zu einer hohen Abhängigkeit von Zulieferern führt. Auf der Grundlage einer systematischen Überprüfung der Wertschöpfungstätigkeiten sind deshalb diejenigen Unternehmensleistungen selbst zu erbringen, die eine große wettbewerbsstrategische Relevanz in High-TechMärkten haben und relativ geringe Kosteneinsparungspotentiale bieten. ${ }^{137}$

Die Strategie der Kostenführerschaft wurde in High-Tech-Märkten vor allem von japanischen Unternehmen in den 80er Jahren verfolgt. In der Mikroelektronik ist es den japanischen High-Tech-Unternehmen gelungen, durch eine Standardisierung der Produktion bei gleichzeitiger Sicherung des technologischen Knowhows und eine aggressive Preispolitik hohe Marktanteile zu gewinnen. So haben die zehn größten japanischen Hersteller von Speicher-Chips in den Jahren 1980 bis 1986 bei einer durchschnittlichen Preissenkung (\$/bit) von ca. $30 \%$ pro Jahr den kumulierten Weltmarktanteil von $25 \%$ auf über $60 \%$ mehr als verdoppeln können. ${ }^{138}$

Als Beispiel für eine erfolgreiche Kostenführerschaftstrategie im Bereich der Glasfasertechnik ist die Corning Incorporated zu nennen. Durch eine Reduzierung der Glasfaserpreise im Zeitraum von 1980 bis 1990 von ca. 1,6 auf 0,1 \$/Meter war das Unternehmen in der Lage, seine Marktposition kontinuierlich auszubauen und wichtige Wettbewerber (z.B. Philips) aus dem Markt zu verdrängen. ${ }^{139}$

137 Vgl. Meffert, H., Erfolgreiches Marketing in der Rezession, a.a.O., S. 49 ff.; Venkatesan, R., Make or Buy: Die Stärken des Endproduktes schützen, in: Harvard Business Manager, 1993, Nr. 2, S. 99 ff.; Hanser, P. Marketing-Outsourcing, Schlankheitskur mit Risiko, in: asw, 1993, Nr. 8, S. $34 \mathrm{ff}$.

Vgl. Backhaus, K., Investitionsgütermarketing, a.a.O., S. 183 ff.; Maringer, A., Preisverfall mikroelektronischer Bauelemente am Beispiel DRAM-Speicherbauelemente, a.a.O., S 424 ff.; Meffert, H., Strategische Unternehmensfuhrung und Marketing, a.a.O., S. 42.

Marone, J.G., Winning in high-tech markets, a.a.O., S. $163 \mathrm{ff}$. 
Eine zentrale Voraussetzung für die Durchführung der Kostenführerschaftsstrategie besteht darin, daß die Höhe des Produktpreises von den Kunden als dominantes Kaufkriterium eingestuft wird. In den High-Tech-Märkten wird die Kaufentscheidung jedoch neben dem Absatzpreis auch durch weitere produktund unternehmensspezifische Leistungen bestimmt. Empirische Untersuchungen in High-Tech-Märkten haben gezeigt, daß vor allem in einem hohen technologischen Know-how, einer hohen Produktqualität, einem guten Serviceangebot und einem positiven Unternehmensimage Differenzierungsmöglichkeiten und damit Wettbewerbsvorteile liegen können. ${ }^{140}$

Angesichts der hohen Technologiedynamik in High-Tech-Märkten nimmt die Technologieorientierung innerhalb der abnehmergerichteten Wettbewerbsstrategie einen besonderen Stellenwert ein. Die High-Tech-Unternehmen legen hierbei das technologische Leistungsniveau durch die Entscheidung fest, ob hinsichtlich der unternehmensrelevanten Produkt- und Anwendungstechnologien eine Führer- oder Folgerposition angestrebt wird. ${ }^{141}$

Der Aufbau einer technologischen Führerschaft ist insbesondere bei Schrittmacher- (z.B. Biotechnologie, Umwelttechnik) und Schlüsseltechnologien (z.B. Bürokommunikation, Nachrichtentechnik) sinnvoll, da Schrittmachertechnologien eine grundlegende Voraussetzung für die zukünftige Wettbewerbsfähigkeit von High-Tech-Unternehmen bilden und Schlüsseltechnologien die aktuelle Erfolgsposition im High-Tech-Wettbewerb maßgeblich bestimmen.

140 Vgl. hierzu z.B. Reinecke, S., Rezession und Strukturwandel in der Informationstechnologie-Branche: Problemanalyse und Erfolgsansätze, Forschungskolloquium IfMFAH, Münster 1994, S. 17 ff.; Hannig, U., Die Entwicklung wettbewerbsorientierter Marketingstrategien auf Basis des Konzeptes der strategischen Gruppen, a.a.O., S. 197 ff.; Baaken, Th., Launen, M., Software-Marketing, a.a.O., S. 113 ff.; Preiß, F.J., Strategische Erfolgsfaktoren im Software-Marketing, in: Software-Marketing, Hrsg.: Wimmer, F., Bittner, L., Wiesbaden 1992, S. 45 ff. u. 62 ff.; Minderlein, M., Markteintrittsbarrieren und Unternehmensstrategie, Wiesbaden 1989, S. 294 ff.; Aaker, D.A., Kriterien zur Identifikation dauerhafter Wettbewerbsvorteile, in: Wettbewerbsvorteile und Wettbewerbsfähigkeit, Hrsg.: Simon, H., Stuttgart 1988, S. 39 ff.

Vgl. Meffert, H., Marketing-Management, a.a.O., S. 211; Wolfrum, B., Technologiestrategien im strategischen Management, a.a.O., S. 25 ff.; Porter, M., Wettbewerbsvorteile, a.a.O., S. 225 ff.; Servatius, G., Methodik des strategischen TechnologieManagement, a.a.O., S. $266 \mathrm{ff}$. 
Welche wirtschaftliche Bedeutung hierbei Schrittmachertechnologien in der Zukunft haben werden, stellt eine Studie der Economic Planning Agency heraus, in der die Entwicklung wichtiger Zukunftstechnologien prognostiziert wird. ${ }^{142}$ Einige Ergebnisse dieser Untersuchung lassen sich anhand ausgewählter Produkte und Anwendungen aus dem Bereich der Informations- und Kommunikationstechnologie verdeutlichen, die in Abbildung 15 dargestellt sind.

Im Bereich der Mikroelektronik wird erwartet, daß sog. Terabyte-Speicher, die gegenüber heutigen Chips ein vielfach größeres Speichervolumen besitzen, im Jahr 2030 marktfähig sein werden. Als Einsatzfelder werden Hochleistungsrechner, "intelligente" Haushaltsgeräte, Informations- und Nachrichtensysteme und die Automobilindustrie gesehen. Bei Terabyte-Speichern wird mit einem Marktpotential von ca. 48 Mrd. DM gerechnet. In der Bioelektronik werden Biosensoren, die organische Materialien (z.B. Antigene, Mikroben) mit physikalischchemischen Bauelementen kombinieren, voraussichtlich zu Beginn des Jahres 2000 für die klinische Analyse, künstliche Organe und Produktionsprozesse bzw. Messungen im Umweltbereich eingesetzt. Eine interessante Anwendungstechnologie stellen sog. "sich selbst erweiternde" Datenbanksysteme dar, die selbständig über die Berechung und Modifikation von Daten entscheiden. Für das Jahr 2020 wird mit der praktischen Realisierung dieser Technologie gerechnet, die für Verwender von Informations- und Adressdatenbeständen von Interesse sein wird. Das Marktpotential für Biosensoren und "sich selbst erweiternde" Datenbanksysteme wird jeweils auf ca. $16 \mathrm{Mrd}$. DM geschätzt. ${ }^{143}$

142 Vgl. EPA (Hrsg.), Technologieprognose für das Jahr 2010, Tokio 1992, S. 1 ff. Vergleichbare Studien sind vom ISI sowie vom BMFT durchgeführt worden. Zu den Ergebnissen siehe Little, A.D. (Hrsg.), Management erfolgreicher Produkte, Wiesbaden 1994, S. 65 ff.; ISI (Hrsg.), Technologie am Beginn des 21. Jahrhunderts, a.a.O., S. 45 ff.; BMFT (Hrsg.), Deutscher Delphi-Bericht zur Entwicklung von Wissenschaft und Technik, Bonn 1993, S. 135 ff.

Vgl. EPA (Hrsg.), Technologieprognose für das Jahr 2010, Tokio 1992, S. 1 ff. 


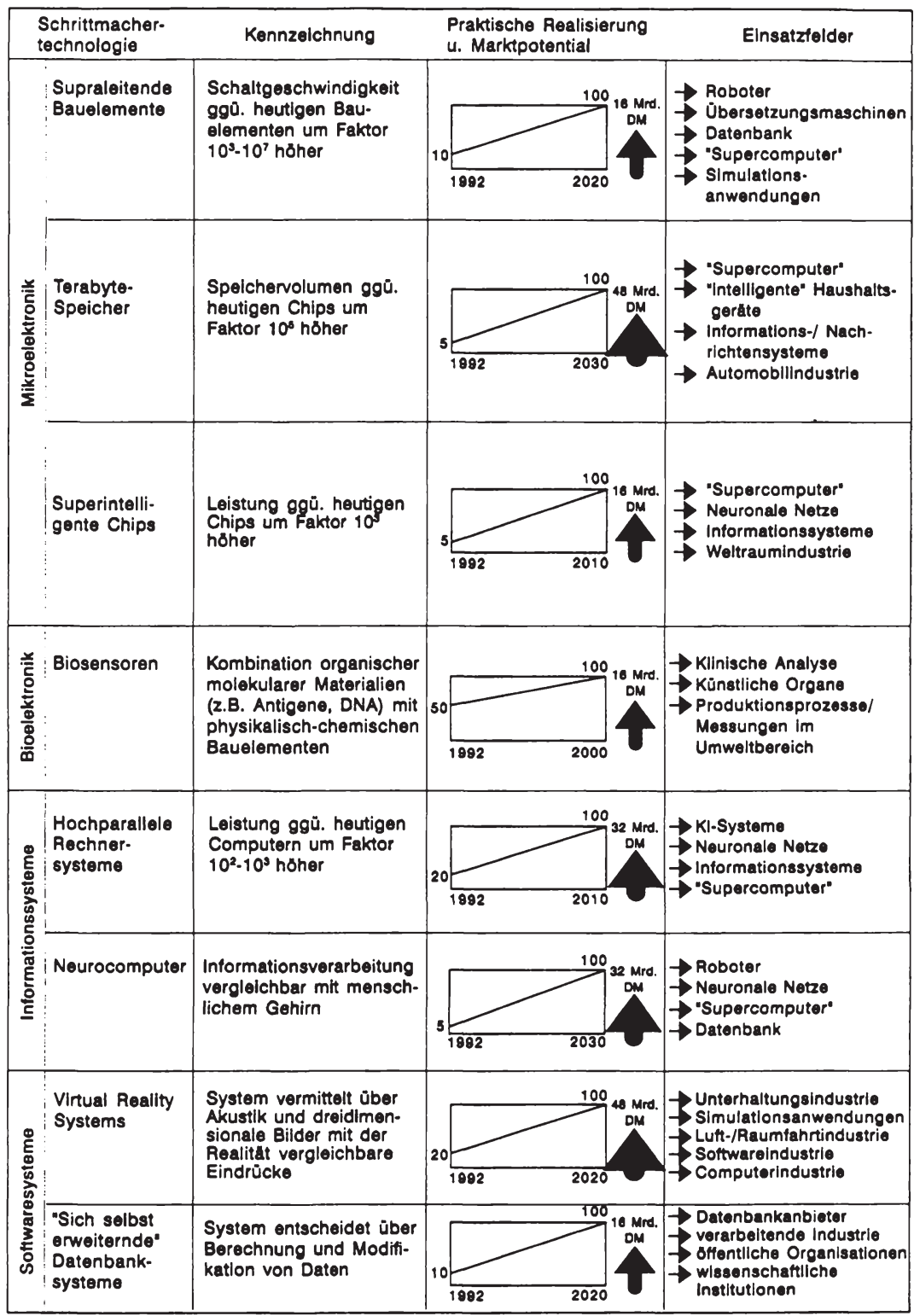

Abb. 15: Prognose der Entwicklung ausgewählter Produkte und Anwendungen aus dem Bereich der Informations- und Kommunikationstechnologie (Quelle: EPA 1992) 
Es bleibt festzuhalten, daß sich für High-Tech-Unternehmen der frühzeitige Aufbau von technologischem Know-how bei gegenwärtigen Schrittmachertechnologien, die ab dem Jahr 2000 den Status von Schlüsseltechnologien erlangen werden, nicht nur aus wettbewerbspolitischen Gesichtspunkten, sondern auch zur Ausnutzung von Wachstumschancen anbietet.

Im Vergleich zu Schrittmacher- und Schlüsseltechnologien werden Basistechnologien in High-Tech-Märkten von der Mehrzahl der Unternehmen beherrscht. Sie werden zwar noch zur Herstellung technologischer Produkte und Anwendungen (z.B. Speicher-Chips in der Mikroelektronik) benötigt, bieten jedoch relativ geringe Möglichkeiten für eine dauerhafte Wettbewerbsdifferenzierung. Für HighTech-Unternehmen ist es deshalb bei Basistechnologien i.d.R. ausreichend, eine technologische Folgerposition i.S. einer technologischen Präsenz einzunehmen. ${ }^{144}$

Zum Aufbau von Wettbewerbsvorteilen in High-Tech-Märkten ist eine alleinige Ausrichtung am technologisch Machbaren i.S. eines "technology-push" häufig nicht ausreichend. Es besteht hierbei die Gefahr, daß Produkt- und Anwendungskonzepte ohne genügende Berücksichtigung nachfragebezogener Bedürfnisse entwickelt werden und sich deshalb nicht im Markt durchsetzen. Als Beispiele hierfür lassen sich das von Philips/Grundig entwickelte Videosystem 2000 und die Bildplatte von RCA nennen. ${ }^{145}$

Aufgrund der hohen Kundenanforderungen an die Anwendungsmöglichkeiten und die Benutzerfreundlichkeit von High-Tech-Produkten ergibt sich für die HighTech-Anbieter die Notwendigkeit, der Nutzenorientierung ${ }^{146}$ i.S. eines "market-

144 Vgl. Little, A.D. (Hrsg.), Management erfolgreicher Produkte, a.a.O., S. 75 ff.; Rüdiger, B., Schlüsseltechnologien: Die Herausforderungen für die 90er Jahre, a.a.O., S. 52; Saad, K.N., Roussel, Ph.A., Tiby, C., Management der F\&E-Strategie, a.a.O., S. 69 f.; Porter, M., Wettbewerbsvorteile, a.a.O., S. 239 ff.; Sommerlatte, T., Deschamps, J.-Ph., Der strategische Einsatz von Technologien, a.a.O., S. $46 \mathrm{ff}$.

145 Vgl. Heß, G., Kampf um den Standard!, Erfolgreiche und gescheiterte Standardisierungsprozesse - Fallstudien aus der Praxis, Stuttgart 1993, S. 69 ff.; Link, J., Überlegen mit starken Produkten, in: asw, Sondernummer Oktober 1993, S. 193 f.; Milling, P., Schwierigkeiten bei der Umsetzung des neuen Wissens, a.a.O., S. 7.

146 In der Literatur wird im Zusammenhang mit der Nutzenorientierung von Unternehmen auch die Bedeutung der "Kundennähe" hervorgehoben. Siehe hierzu z.B. Albers, S., 
pull" eine größere strategische Bedeutung beizumessen. ${ }^{147}$ Die Unternehmen sind hierbei einerseits gefordert, sich möglichst frühzeitig mit den Nutzenerwartungen der aktuellen und potentiellen Nachfrager auseinanderzusetzen und dies innerhalb des Innovationsprozesses zu berücksichtigen. Als Beispiel für eine bedarfsinduzierte technologische Produktinnovation kann die von Philips entwickelte Glühlampe angeführt werden, die im Vergleich zu herkömmlichen Lampen einen deutlich geringeren Energieverbrauch und eine achtmal längere Lebensdauer aufweist und damit dem Wunsch nach größerer Wirtschaftlichkeit und Langlebigkeit nachkommt. ${ }^{148}$ Andererseits sind die bestehenden Produkt- und Technologieangebote laufend hinsichtlich des aus Kundensicht wahrgenommenen KostenNutzen-Verhältnisses zu überprüfen, um rechtzeitig Anpassungen im Leistungsangebot vornehmen zu können. ${ }^{149}$

In diesem Zusammenhang stellte Cooper in einer empirischen Analyse der Innovationspolitik von Investitionsgüterunternehmen fest, daß die Kombination von "technology-push"- und "market-pull"-Orientierung i.S. einer "balanced-strategy"

Eggert, K., Kundennähe - Strategie oder Schlagwort, in: Marketing ZFP, 1988, Nr. 1, S. $5 \mathrm{ff}$.

Vgl. Backhaus, K., Investitionsgütermarketing, a.a.O., S. 8 f.; Meffert, H., Erfolgreiches Marketing in den neunziger Jahren, in: Marketing im Umbruch - Chancen und Gefahren für Unternehmer, Zürich 1991, S. 27; Kühn, R., Methodische Überlegungen zum Umgang mit der Kundenorientierung im Marketing-Management, in: Harvard Manager, 1987, H. 3, S. 28 ff.; Sommerlatte, T., Töpfer, A., Die Integration von Technologie und Marketing als strategischer Erfolgsfaktor: Übersicht und Einordnung der Beiträge, a.a.O., S. 16 ff.; Brogsitter, B., Kundennutzen - Herzstück der ganzheitlichen Firmenstrategie, in: Harvard Manager, 1988, H. 4, S. 113 ff.; Wittek, B., Strategien auf Kundennutzen aufbauen, in: Wettbewerbsvorteile und Wettbewerbsfähigkeit, Hrsg. Simon, H., Stuttgart 1988, S. 66 ff.; Merkle, E., Technologiemarketing, a.a.O., S. 10.

Vgl. Deutsch, Chr., Reiner Selbstmord, in: Wirtschaftswoche, 1993, Nr. 21, S. 58 f.

Vgl. Little, A.D. (Hrsg.), Management erfolgreicher Produkte, a.a.O., S. 21 ff. u. S. 95 ff.; Backhaus, K., Was heiBt Investitionsgütermarketing?, Arbeitspapier Nr. 15 des Betriebswirtschaftlichen Instituts für Anlagen und Systemtechnologien, Münster 1992, S. 16 ff.; Zabel, S., High-Tech-Produkte brauchen ein spezielles Marketing, in: BddW, 1992, Nr. 161, S. 1; Eisele, R., Industriemärkte im Wandel - Auswirkungen auf die Unternehmensfuihrung, in: Marketing-Schnittstellen: Herausforderungen für das Management, Hrsg.: Specht, G., Silberer, G., Engelhardt, W.H., Stuttgart 1989, S. 329 f.; Bender, H.O., Das Marketing von Technologieprodukten, in: asw, 1988, Nr. 8, S. 117 ff.; Cooper, R.G., Predevelopment activities determine new product success, in: Industrial Marketing Management, Vol. 17 (1988), S. 237 ff.; Yeager, R.L., Strategy development: Engineering the customer-oriented marketing-strategy, in: Business Marketing, 1985, S. $61 \mathrm{ff}$. 
zur Erlangung einer Erfolgsposition besonders geeignet ist. ${ }^{150} \mathrm{Zu}$ einem vergleichbaren Ergebnis sind auch Abele und Christiaens bei einer Untersuchung von High-Tech-Unternehmen u.a. aus den Bereichen der Mikroelektronik, Büroautomatisierungstechnik und Biotechnologie gekommen. ${ }^{151}$

Eine weitere grundlegende Strategiedimension zur Schaffung von Wettbewerbsvorteilen in High-Tech-Märkten ist in der Qualitätsorientierung ${ }^{152}$ von HighTech-Unternehmen zu sehen. Hierbei kann zwischen einer anbieterbezogenen technisch-objektiven und einer nachfragebezogenen subjektiv-wahrgenommenen Qualität (relative Qualität) von technologischen Produktleistungen unterschieden werden. ${ }^{153}$ Zum Aufbau einer dominanten Wettbewerbsposition ist jedoch entscheidend, daß die Produktleistungen eines High-Tech-Unternehmens aus Nachfragersicht im Vergleich zur Konkurrenz eine überlegene Qualität aufweisen. In diesem Zusammenhang konnte in empirischen Untersuchungen auf Basis der PIMS-Datenbank nachgewiesen werden, daß die relative Produktqualität

150 Vgl. hierzu Cooper, R.G., New product strategies: What distinguishes the top performers, in: Journal of Product Innovation Management, 1984, Nr. 2, S. 160 ff.; Cooper, R.G., Overall corporate strategies for new product programms, in: Industrial Marketing Management, Vol. 14 (1985), S. 184 ff. Siehe in diesem Zusammenhang auch Fritz, W., Marktorientierte Unternehmensführung und Unternehmenserfolg, Stuttgart 1992, S. 167; Gierl, H., Kotzbauer, N., Der Einfluß des F\&E-Aufwandes auf den wirtschaftlichen Erfolg von Industrieunternehmen, in: ZfbF, 1992, Nr. 11, S. 980 ff.; Brockhoff, K., Forschung und Entwicklung, a.a.O., S. $113 \mathrm{ff}$.

151 Vgl. Van den Abele, P., Christiaens, I., Strategies of belgian high-tech firms, in: Industrial Marketing Management, Vol. 15 (1986), S. $305 \mathrm{ff}$.

152 In der Literatur wird häufig zwischen einer Produkt- und Servicequalität unterschieden. Vgl. hierzu z.B. Meffert, H., Marketing-Management, a.a.O., S. 129; Birkelbach, R., Qualitätsmanagement in Dienstleistungscentern, Frankfurt a.M. u.a. 1993, S. 13 ff.; Büker, B., Qualitätsbeurteilung investiver Dienstleistungen, Operationalisierungsansätze an einem Beispiel zentraler EDVDienste, Frankfurt a.M. u.a. 1991, S. $11 \mathrm{ff}$. In der vorliegenden Untersuchung wird diese Einschätzung auch vertreten, indem sich die Qualitätsorientierung auf die Produktqualität und die Serviceorientierung auf die Servicequalität richtet. Für eine ausfuihrliche Kennzeichung des Qualitätsbegriffs siehe insbesondere Witte, A., Integrierte Qualitätssteuerung im Total Quality Management, Münster, Hamburg 1993, S. 9 ff.

153 Vgl. Meffert, H., Marketing-Management, a.a.O., S. 129 f.; Backhaus, K., Investitionsgütermarketing, a.a.O., S. 180 f.; Benkenstein, M., Strategisches Marketing-Management in High-Tech-Branchen, a.a.O., S. 13; Kotler, Ph., Bliemel, F., MarketingManagement, a.a.O., S. 459 ff.; Simon, H., Management strategischer Wettbewerbsvorteile, a.a.O., S. $3 \mathrm{ff}$. 
einen hohen positiven Einfluß auf den Unternehmenserfolg (Marktanteil, ROI) hat. ${ }^{154}$

Von den High-Tech-Unternehmen ist bei der Festlegung von Umfang und Stärke der Qualitätsorientierung zu prüfen, welche Teilkomponenten die relative wahrgenommene Qualität beinhaltet. Mit Blick auf High-Tech-Märkte lassen sich vor allem die Qualitätsdimensionen Normgerechtigkeit, Gebrauchsnutzen, Ausstattung, Haltbarkeit und Zuverlässigkeit unterscheiden. ${ }^{155}$

Normgerechtigkeit bedeutet, inwieweit bei der Entwicklung neuer High-TechProdukte bestehende technische Normen und Standards berücksichtigt werden. Eine weitgehende Kompatibilität zwischen vergleichbaren technologischen Produktkonzepten wird oftmals von High-Tech-Nachfragern im Hinblick auf eine spätere Leistungserweiterung oder einen möglichen Systemwechsel gefordert. ${ }^{156}$ Der Gebrauchsnutzen kommt in wichtigen produktspezifischen Funktionsmerkmalen zum Ausdruck, die in der Computerindustrie Eigenschaften wie Leistungsfähigkeit (z.B. Verarbeitungsgeschwindigkeit, Speichervolumen) und Wirtschaftlichkeit (z.B. Preis-/Leistungsverhältnis, Stromverbrauch) umfassen. In einem engen Zusammenhang zum Gebrauchsnutzen steht die Ausstattung von High-Tech-Produkten, die den Grundnutzen eines Produktes um bestimmte

154 Vgl. Meffert, H., Marketing-Management, a.a.O., S. 62 f.; Hildebrandt, L., Wettbewerbssituation und Unternehmenserfolg, Empirische Analysen, in: ZfB, 1992, Nr. 10, S. 1072 ff.; Engelhardt, W.H., Herausforderungen des Total Quality Management aus wettbewerbsorientierter Sicht, in: Kosten, Qualität und Zeit als Wettbewerbsvorteile, Was bringt Total Quality Management?, Dokumentationspapier Nr. 75 der Wissenschaftlichen Gesellschaft für Marketing und Unternehmensfuihrung e.V., Hrsg.: Meffert H., Wagner, H., Backhaus, K., Münster 1992, S. 22; Kim, L., Lim, Y., Environment, generic strategies and performance in rapidly developing country: a taxonomic approach, a.a.O., S. 802 ff.; Meyer, J., Qualität als strategische Wettbewerbswaffe, in: Wettbewerbsvorteile und Wettbewerbsfähigkeit, Hrsg. Simon, H., Stuttgart 1988, S. $77 \mathrm{ff}$.

Vgl. Meffert, H., Marketing-Management, a.a.O., S. 129 ff.; Kotler, Ph., Bliemel, F., Marketing-Management, a.a.O., S. 463 ff. Für eine Betrachtung möglicher Qualitätsdimensionen siehe insbesondere Garvin, D.A., Die acht Dimensionen der Produktqualität, in: Harvard Manager, 1988, Nr. 3, S. 66 ff.

Vgl. Meffert, J., Standards als Integrationsinstrument in der Computer- und Kommunikationsindustrie, a.a.O., S. 142 ff.; Backhaus, K., Investitionsgütermarketing, a.a.O., S. 396 ff.; Kleinaltenkamp, M., Der Einflub der Normung und Standardisierung auf die Diffusion technischer Innovationen, a.a.O., S. 2 ff. 
Zusatznutzen (bei Computern z.B. ergonomische Tastatur, strahlungsarmer Monitor) ergänzt. ${ }^{157}$

Die Haltbarkeit stellt ein Maß für die Lebensdauer technologischer Produkte dar, die durch die Häufigkeit der Produktverwendung bis zum Zeitpunkt des Funktionsausfalls bestimmt wird. Die Zuverlässigkeit eines High-Tech-Produktes beschreibt die Wahrscheinlichkeit, mit der in einem bestimmten Zeitintervall keine Funktionsstörung auftritt. Aufgrund von teuren Ausfall- und Wartezeiten, die bei High-Tech-Produkten entstehen können, besitzt die Zuverlässigkeit technologischer Produktleistungen gerade in diesen Märkten eine besondere Bedeutung. Das Unternehmen Tandy Computer entwickelte deshalb ein Produktkonzept, bei dem mehrere Rechner parallel arbeiten, wodurch der Ausfall eines Gerätes nicht $\mathrm{zu}$ einem Gesamtausfall, sondern nur zu einer langsameren Bearbeitung von weniger wichtigen Funktionen fuihrt. Dieses Sicherheitskonzept wird von Tandy insbesondere an Unternehmen verkauft, die auf einen On-Line-Betrieb angewiesen sind (z.B. Banken, Börsen und Handelsunternehmen) und bei denen ein Systemstillstand nicht auftreten darf. ${ }^{158}$ Im Rahmen der Konkretisierung der Qualitätsorientienung über die aufgezeigten Qualitätsdimensionen wird von den High-Tech-Unternehmen insgesamt festgelegt, welches Qualitätsniveau für die technologischen Produktleistungen angestrebt wird.

Neben der Qualitätsorientierung bildet die Serviceorientierung ${ }^{159}$ eine wichtige Differenzierungsdimension im Wettbewerb. Aufgrund fortschreitender Techno-

157 Vgl. Meffert, H., Marketing-Management, a.a.O., S. 130; Meffert, H., Strategien zur Profilierung von Marken, in: Marke und Markenartikel als Instrumente des Wettbewerbs, Hrsg.: Dichtl, E., Eggers, W., München 1992, S. 130 f.; Porter, M., Wettbewerbsvorteile, a.a.O., S. $192 \mathrm{f}$.

Vgl. Meffert, H., Marketing-Management, a.a.O., S. 130.; Witte, A., Integrierte Qualitätssteuerung im Total Quality Management, a.a.O., S. $65 \mathrm{f}$.

159 In der vorliegenden Untersuchung werden die Begriffe Service-, Dienst- und Kundendienstleistungen synonym verwendet. Vgl. hierzu z.B. Meffert, H., Marketing-Management, a.a.O., S. 131; Bauche, K., Segmentierung von Kundendienstleistungen auf investiven Märkten, Frankfurt a.M. u.a. 1994; S. 8. Für eine ausfuihrliche Betrachtung dieser Begriffe siehe Meffert, H., Dienstleistungsmarketing, in: Handwörterbuch des Marketing, Hrsg.: Köhler, R., Tietz, B., Zentes, J., 2. Aufl., Stuttgart 1994 (im Druck); Zerr, K., Jugel, St., Dienstleistungen als strategisches Element eines Technologie-Marketing, Arbeitspapier Nr. 68 des Instituts für Marketing, Hrsg.: Dichtl, E., Geist, M., 
logieintegration und hoher Komplexität der High-Tech-Anwendungen bieten z.B. die Entwicklung individueller Beratungs-, Schulungs- und Wartungsleistungen, das Angebot von sog. "value-added"-Services sowie die Vermarktung von FullService-Konzepten den High-Tech-Unternehmen die Möglichkeit, sich stärker gegenüber den Wettbewerbern zu differenzieren und hierdurch Wettbewerbsvorteile aufzubauen. ${ }^{160}$

$\mathrm{Da}$ im Servicebereich noch weitreichende Profilierungschancen bestehen, bestätigt eine von Arthur D. Little durchgefuihrte Untersuchung, in der sich $62 \%$ der befragten Unternehmen über Mängel in der Lieferzuverlässigkeit, in der Beratung und im Kundendienst ihrer Zulieferer beschwerten. ${ }^{161}$ Im Hinblick auf die Serviceorientierung sind von den High-Tech-Unternehmen Umfang und Intensität der Serviceleistungen näher zu bestimmen.

Bezüglich der Wahl des Serviceumfangs bietet sich eine Differenzierung zwischen sog. Muß-, Soll- und Kann-Leistungen an. ${ }^{162} \mathrm{Zu}$ Muß-Leistungen gehören alle Dienste, die eine erstmalige Nutzung eines High-Tech-Produktes ermöglichen (z.B. Anlieferung und Installation einer Telefonanlage) oder aus markt-

Raffée, H., Mannheim 1989, S. 6 ff. Zur Kennzeichung der konstitutiven Merkmale von Serviceleistungen siehe z.B. Meffert, H., Marktorientierte Führung von Dienstleistungsunternehmen - Neuere Entwicklungen in Theorie und Praxis, Arbeitspapier Nr. 78 der Wissenschaftlichen Gesellschaft für Marketing und Unternehmensführung e.V., Hrsg.: Meffert H., Wagner, H., Backhaus, K., Münster 1993, S. 5 ff.

Vgl. o.V., Kaum Gewinne im Einkaufstempel: Vobis-Kunden wollen mehr Beratung, in: Werben \& Verkaufen, 1994, Nr. 6, S. 12; o.V., Komlexe Systeme erfordern mehr Beratung, in: Computer Zeitung, 1993, Nr. 48, S. 21; Barnes, J.G., Glynn, W.J., The customer wants service: Why technology is no longer enough, in: Journal of Marketing Management, Vol. 9 (1993), S. 43 ff.; Hannig, U., Die Entwicklung wettbewerbsorientierter Marketingstrategien auf Basis des Konzeptes der strategischen Gruppen, a.a.O., S. 185 f.; Benkenstein, M., High Tech-Marketing, a.a.O., S. 398; Bittner, L., Ursachen und Folgen der steigenden Bedeutung des DV-Service, a.a.O., S. 168 ff.; Porter, M., Wettbewerbsstrategie, a.a.O., S. 175.

161 Vgl. Little, A.D. (Hrsg.), Management erfolgreicher Produkte, a.a.O., S. 49 f.

162 Vgl. Meffert, H., Erfolgreiches Marketing in der Rezession, a.a.O., S. 100; Bauche, K. Segmentierung von Kundendienstleistungen auf investiven Märkten, a.a.O., S. 10 f.; Müller, W., Service-"Tuning" als Wettbewerbsvorteils-Konzept im Marketing von KfzZulieferunternehmen und Herstellern, in: Investitionsgüter- und High-Tech-Marketing, Hrsg.: Hofmaier, R., Landsberg a.L. 1992, S. 540 ff.; Meffert, H., Kundendienstpolitik, Eine Bestandsaufnahme zu einem komplexen Marketinginstrument, in: Marketing ZFP, 1987, Nr. 2, S. 97 f. 
bedingten Gründen vom High-Tech-Unternehmen zu erbringen sind (z.B. Garantiezusagen). Den Soll-Leistungen werden Maßnahmen zugerechnet, die auf Grundlage von Erfahrungen bzw. Prognosen bezüglich der Materialermüdung und Lebensdauer bestimmter High-Tech-Komponenten abzuschätzen sind und den High-Tech-Verwendern als Wartungs-, Pflege- oder Reparaturdienste bereitgestellt werden. Von manchen Computerherstellern werden z.B. ein 24-StundenService oder ein spezieller Versicherungsschutz angeboten. ${ }^{163}$

Im Vergleich zu Muß- bzw. Soll-Diensten, die von den Wettbewerbern meist in ähnlicher Form erfüllt werden, bieten Kann-Leistungen umfangreiche Differenzierungsmöglichkeiten. Kann-Leistungen werden in Abhängigkeit von den spezifischen Kundenbedürfnissen und den Kosten-/Nutzenerwartungen der Hauptleistungsnehmer entwickelt. Einer Zielgruppe professioneller Computeranwender (Mehr-Platz-Computer) können beispielsweise Veranstaltungen zum Netzwerkmanagement und zur Optimierung von Systemanwendungen angeboten werden. ${ }^{164}$

Hinsichtlich der Serviceintensität wird von den High-Tech-Unternehmen festgelegt, mit welchem Qualitätsniveau die Servicedienste vermarktet werden. Es ist hierbei darauf $\mathrm{zu}$ achten, daß die Serviceleistungen auf das vorhandene technologische Produktkonzept abgestimmt sind und ein vergleichbares Preis/Qualitätsniveau aufweisen. So können z.B. Computeranbieter bei preisgünstigen Standardprodukten eher auf Dienstleistungen wie Produktanlieferung und Garan-

163 Vgl. Little, A.D. (Hrsg.), Management erfolgreicher Produkte, a.a.O., S. 51; Bauche, K., Segmentierung von Kundendienstleistungen auf investiven Märkten, a.a.O., S. 11; Läbe, M., Stolpmann, F., Dienst am Kunden total?, Wie die Investitionsgüterindustrie mit dem Service umgeht, in: asw, Sondernummer Oktober 1993, S. 27 f.; Wegwart, J., Preis- und Kontrahierungspolitik im Kundendienst unter besonderer Berücksichtigung von Wartungs- oder Call-Service, in: Kundendienst-Management, Hrsg.: Meffert, H., Frankfurt a.M., Bern 1982, S. 121 f.

164 Vgl. Meffert, J., Standards als Integrationsinstrument in der Computer- und Kommunikationsindustrie, a.a.O., S. 143 ff.; Bauche, K., Segmentierung von Kundendienstleistungen auf investiven Märkten, a.a.O., S. 12 ff.; Baaken, Th., Qualifizierung des Kunden als integrierte Aufgabe im Technologie-Marketing, in: Technologie-Marketing, Hrsg.: Töpfer, A., Sommerlatte, T., Landsberg a.L. 1991, S. 216 ff.; Remmerbach, K.U., Value Added Marketing, in: Marktforschung \& Management, 1990, Nr. 2, S. 13 ff. 
tiezusagen verzichten als bei erklärungsbedürftigen und wartungsintensiven Hochleistungsrechnern. ${ }^{165}$

Neben einer Wettbewerbsdifferenzierung bietet das Angebot von Servicemaßnahmen den Unternehmen auch die Möglichkeit, an Wachstumspotentialen in High-Tech-Märkten zu partizipieren. ${ }^{166}$ Bei zahlreichen High-Tech-Unternehmen ist deshalb ein Trend zu einer stärkeren Serviceorientierung festzustellen. IBM steigerte beispielsweise den Anteil der Software- und Serviceleistungen am Gesamtumsatz in den Jahren 1985 bis 1992 von $26 \%$ auf $41 \%$. Bis zum Jahr 1996 wird geplant, den Software- und Serviceumsatz auf $50 \%$ am Gesamtgeschäft zu erhöhen. ${ }^{167}$ Eine aktuelle Studie aus dem High-Tech-Bereich zeigt, daß Serviceleistungen mittlerweile auch selbständig vermarktet werden. Als ein zentrales Ergebnis dieser Untersuchung wurde festgestellt, daß $33 \%$ der befragten High-Tech-Unternehmen ihren Kunden über die Hälfte der Serviceleistungen und $50 \%$ der Befragten sogar alle Servicedienste mit Ausnahme von Kulanz und Gewährleistungen separat in Rechnung stellen. ${ }^{168}$

Im Rahmen der Strategieplanung wird eine stärkere Berücksichtigung der Imageorientierung $^{169}$ aufgrund der in High-Tech-Märkten bestehenden hohen

165 Vgl. Meffert, H., Erfolgreiches Marketing in der Rezession,a.a.O., S. 102 f.; Little, A.D. (Hrsg.), Management erfolgreicher Produkte, a.a.O., S. 60; Bauche, K., Segmentierung von Kundendienstleistungen auf investiven Märkten, a.a.O., S. $150 \mathrm{ff}$.

Vgl. o.V., Komplexe Systeme erfordern mehr Beratung, in: Computer Zeitung, 1993, Nr. 48, S. 21; Baaken, Th., Launen, M., Software-Marketing, a.a.O., S. 41 ff.; Mager, H.J., Siegberg, U., Erfolgsfaktor "Technologie-Innovation" bei der Entwicklung medizintechnischer Großgeräte, in: Technologie-Marketing, Hrsg.: Töpfer, A., Sommerlatte, T., Landsberg a.L. 1991, S. 483 ff.; Schamann, H.-D., Qualifizierung der Mitarbeiter als Bestandteil eines erfolgreichen Technologie-Management, in: Technologie-Marketing, Hrsg.: Töpfer, A., Sommerlatte, T., Landsberg a.L. 1991, S. $226 \mathrm{ff}$.

167 Vgl. Henkel, H.O., Serviceleistungen sind eigenständige Produkte, in: FAZ vom 07.07.1993, S. B6.

168 In der Studie wurden 135 Unternehmen in Deutschland aus den Bereichen Maschinenbau, Elektrotechnik/Elektronik, Datenverarbeitung/Büromaschinen und Feinmechanik/Optik untersucht. Vgl. hierzu Läbe, M., Stolpmann, F., Dienst am Kunden total?, a.a.O., S. $22 \mathrm{ff}$.

169 Zur Kennzeichung der Begriffe Image und Einstellung siehe insbesondere Meffert, $\mathrm{H}$., Marketingforschung und Käuferverhalten, a.a.O., S. 55 ff.; Kroeber-Riel, W., Konsumentenverhalten, 5. Aufl., München 1992, S. 160 ff. Für eine Beschreibung von Produkt- und Unternehmensimage vgl. u.a. Meffert, H., Marketing-Management, a.a.O., S. 
Unsicherheit der Nachfrager und der daraus resultierenden Kaufzurückhaltung notwendig. Neue High-Tech-Produkte werden oftmals auf Basis von Schrittmacher- bzw. Schlüsseltechnologien entwickelt, die vielfach noch nicht genügend ausgereift sind (z.B. CIM-Systeme, Computer Integrated Office-Systeme) und deren Leistungsfähigkeit deshalb weitgehend unbekannt ist. ${ }^{170}$ Die High-TechUnternehmen haben zudem häufig noch keinen Produkt- und Technologiestandard in den High-Tech-Märkten durchsetzen können. Das Risiko einer Fehlentscheidung wird daher von den Nachfragern als besonders hoch eingeschätzt. Diesem Problem können High-Tech-Anbieter durch den Aufbau eines Image entgegenwirken, das z.B. ihre Erfahrung und Zuverlässigkeit im Umgang mit Produkt- und Anwendungstechnologien besonders herausstellt. ${ }^{171}$

In diesem Zusammenhang ist sicherzustellen, daß das produkt- und unternehmensbezogene Image möglichst gut mit dem Qualitätsniveau der Produkt- und Serviceleistungen übereinstimmt, da andernfalls ein Glaubwürdigkeits- und Vertrauensverlust bei den High-Tech-Verwendern auftritt. Welche negativen Konsequenzen dies für Unternehmen haben kann, läßt sich anhand der S-Klasse von Mercedes verdeutlichen. Aufgrund gravierender Qualitätsmängel bei einzelnen Baugruppen, die mit den Qualitätsansprüchen des Unternehmens nicht in Einklang standen, wurden die hohen Preisforderungen für die Fahrzeuge nicht mehr akzeptiert. Aus diesem Grund wechselten zahlreiche Interessenten vor allem aus dem Ausland zu vergleichbaren japanischen Konkurrenzmodellen. ${ }^{172}$

134; Meffert, H., Strategien zur Profilierung von Marken, a.a.O., S. $147 \mathrm{ff}$. In der vorliegenden Untersuchung wird zwar auch zwischen diesen Imageformen unterschieden, aber auf eine umfassende Betrachtung verzichtet.

170 Siehe hierzu im Detail die Ausfuhrungen in Teil A Kap. 1. und Teil B Kap. 2.13 der Arbeit.

171 Vgl. Wieandt, A., Zum Marketing von Innovationen, a.a.O., S. 23; Backhaus, K., Investitionsgütermarketing, a.a.O., S. 350 ff. u. S. 401 ff.; Weiss, P., Die Kompetenz von Systemanbietern: Ein neuer Ansatz im Marketing für Systemtechnologien, a.a.O., S. $40 \mathrm{f}$; Nyström, H., Technological and market innovation, strategies for product and company development, a.a.O., S. 117 ff.; Trommsdorff, V., Schuster, H., Strategie- und Imageplanung für junge Technologiefirmen, in: asw, 1985, Nr. 9, S. $116 \mathrm{ff}$.

172 Vgl. Little, A.D. (Hrsg.), Management erfolgreicher Produkte, a.a.O., S. 54. Siehe in diesem Zusammenhang auch Meffert, H., Heinemann, G., Operationalisierung des Imagetransfers, in: Marketing ZFP, 1990, Nr. 1, S. 5 ff.; Simon, H., Goodwill und Marketingstrategie, Wiesbaden 1985, S. $78 \mathrm{ff}$. 
Darüber hinaus kommt der Imagebildung in High-Tech-Märkten eine besondere Bedeutung zu, wenn die Produkt- und Anwendungstechnologien aus Abnehmersicht zunehmend homogener werden. Die High-Tech-Unternehmen werden hierbei vor die Aufgabe gestellt, sich über einen einprägsamen und unverwechselbaren kommunikativen Auftritt von den Wettbewerbern zu differenzieren und eine dauerhafte Position im "Evoked-Set" der Nachfrager zu sichern. ${ }^{173}$ In der Computerindustrie ist ein solches Vorgehen beispielsweise bei Rechnern von Apple (Macintosh) und Prozessoren von Intel ("Intel-Inside") anzutreffen. ${ }^{174}$

Es bleibt insgesamt festzuhalten, daß sich die Unternehmen in High-Tech-Märkten verstärkt mit der Frage auseinandersetzen, ob sie im Wettbewerbsumfeld eine Imagefuihrerschaft anstreben. Dies wird auch durch eine aktuelle Untersuchung im Bereich der Informations- und Kommunikationstechnologie bestätigt, in der 73 \% der befragten Unternehmen die Bedeutung einer stärkeren Imageorientierung innerhalb der Marketingstrategie hervorhoben. ${ }^{175}$

\subsection{Timingbezogene Strategiedimensionen}

Neben der Festlegung der Marktbearbeitungsstrategie und der abnehmergerichteten Wettbewerbsstrategie bildet die Formulierung der Timingstrategie einen wichtigen Entscheidungstatbestand in High-Tech-Märkten. Im Rahmen der Timingstrategie bestimmt ein High-Tech-Unternehmen, ob es mit einer neuen Technologie bzw. einem neuen Produkt als Pionier, früher Folger oder später

173 Vgl. Meffert, H., Marketingforschung und Käuferverhalten, a.a.O., S. 40 f.; Backhaus, K., Investitionsguitermarketing, a.a.O., S. 102 ff. u. 428; Hall, R., The strategic analysis of intangible resources, in: Strategic Management Journal, Vol. 13 (1992), S. $140 \mathrm{ff}$.

174 Vgl. Jörg, G., Die Abstimmung zwischen Zielgruppenkampagnen und Marktkommunikation am Beispiel eines ausgewählten Informationstechnologie-Anbieters, in: Investitionsgüter- und High-Tech-Marketing, Hrsg.: Hofmaier, R., Landsberg a.L. 1992, S. 410 ff.

Vgl. Reinecke, S., Rezession und Strukturwandel in der Informationstechnologie-Branche: Problemanalyse und Erfolgsansätze, a.a.O., S. $15 \mathrm{f}$. 
Folger in einen Markt eintritt. ${ }^{176}$ Die strategischen Eintrittsoptionen können anhand der Einfuihrungs- und Wachstumsphase eines High-Tech-Marktes und der Zeiträume, in denen sich das Wettbewerbsumfeld neu strukturiert (Shake-OutPhasen), charakterisiert werden (vgl. Abbildung 16).

Als Pionier geht ein High-Tech-Unternehmen mit Produkt- bzw. Technologieinnovationen zuerst in einen entstehenden Markt, wobei der Strategieschwerpunkt $\mathrm{zu}$ diesem Zeitpunkt im Marktaufbau und in der Markterschließung liegt. Ein zentraler Vorteil der Pionierstrategie besteht darin, daß die in dieser Marktphase bestehende Monopolsituation zum Aufbau von Eintrittsbarrieren genutzt werden kann, die den Marktzugang für Folgerunternehmen erschweren. ${ }^{177}$

Angebotsseitig können Markteintrittsbarrieren in der frühzeitigen Etablierung eines produkt- bzw. technologiebezogenen Industrie- oder De facto-Standards, in der Absicherung von technologischem Know-how durch Patente und in der Bindung von Vorlieferanten bzw. Absatzmittlern liegen. ${ }^{178}$ Im Zusammenhang mit der Bildung von Marktbarrieren über Patente ist festzustellen, daß von deutschen High-Tech-Unternehmen beispielsweise in der Informations- und Kommunikationstechnologie ca. 1.500, in der Umwelttechnik ca. 800 sowie in der Bio- und

176 Vgl. Meffert, H., Marketing-Management, a.a.O., S. 207 ff.; Wolfrum, B., Strategisches Technologiemanagement, a.a.O., S. 263 ff.; Benkenstein, M., Innovationsmanagement in High-Tech-Branchen, Freiräume für Pioniere, in: asw, 1993, Nr. 6, S. 104 ff.; Perillieux, R., Strategisches Timing von F\&E und Markteintritt bei innovativen Produkten, a.a.O., S. 23 ff.; Zahn, E., Innovations- und Technologiemanagement, in: Technologie- und Innovationsmanagement, Hrsg.: Zahn, E., Berlin, München 1986, S. 10 ff.; Remmerbach, K.-U., Markteintrittsentscheidungen, a.a.O., S. $51 \mathrm{ff}$.

Vgl. Vidal, M., Wettbewerbsstrategien für Pionierunternehmen, a.a.O., S. 13 ff. u. 140 ff.; Weber, M., Unternehmerische Marktstrategien bei dynamischem Wettbewerb, a.a.O., S. 82 ff.; Minderlein, M., Markteintrittsbarrieren und Unternehmensstrategie, a.a.O., S. 303 ff.; Meffert, H., Strategische Unternehmensführung und Marketing, a.a.O., S. 72 f.

178 Vgl. Meffert, H., Marketing-Management, a.a.O., S. 212 f.; Backhaus, K., Investitionsgütermarketing, a.a.O., S. 199 f. u. 368 ff.; Alpert, F.H., Kamins, M.A., Graham, J.L., An examination of reseller buyer attitudes toward order of brand entry, in: Journal of Marketing, Vol. 56 (1992), S. 26 ff.; Bühner, R., Technologieorientierung als Wettbewerbsstrategie, a.a.O., S. 397. 


\section{Branchenumsatz}

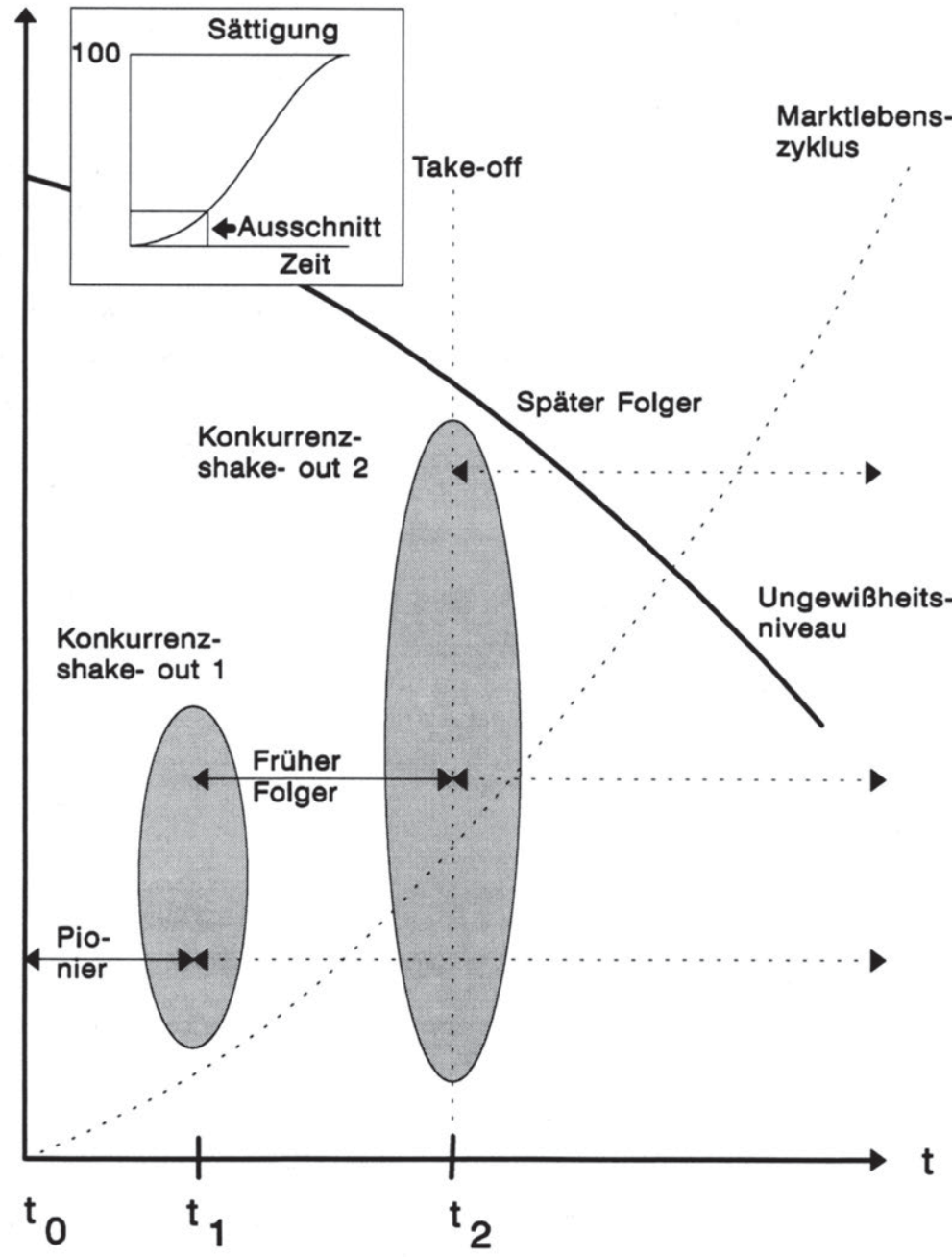

Eintrittskontinuum

$\longleftrightarrow$ Phase der Marktbearbeitung und -behauptung

Abb. 16: Abgrenzung strategischer Timigoptionen (Quelle: Remmerbach, K.-U., Markteintrittsstrategien, Wiesbaden 1988, S. 55) 
Gentechnik ca. 375 Patentanmeldungen im Jahr 1989 abgegeben worden sind. ${ }^{179}$ Markteintrittsbarrieren lassen sich nachfrageseitig aufbauen, indem eine kontinuierliche Verbesserung der Produktkonzepte und die Schaffung eines progressiven Technologieimages zu einer engen Kundenbindung führen.

Als ein wesentlicher Nachteil dieser Strategie sind die hohen Markterschließungskosten zu nennen, die der Pionier i.d.R. allein zu tragen hat, während der Nutzen einer Markterschließung auch den nachfolgenden High-Tech-Unternehmen zufallen kann. Ein weiteres Problem ist darin zu sehen, daß die Entwicklung eines High-Tech-Marktes zum Zeitpunkt des Eintritts noch weitgehend unsicher ist. Hieraus ergibt sich die Gefahr, daß die vom Pionier aufgebauten Wettbewerbsvorteile durch Nachfrageänderungen und technologische Sprünge obsolet werden. ${ }^{180}$

Von einem High-Tech-Unternehmen, das eine frühe Folgerstrategie realisiert, wird der Markteintritt nach dem Pionier und vor der take-off Phase des Marktes vollzogen. Der frühe Folger hat dabei in Abhängigkeit von der Wettbewerbsstärke zu entscheiden, ob das Verhalten des Pioniers toleriert und ggf. imitiert oder eine Konfrontationsstrategie angestrebt wird. Entsprechend der gewählten Strategieausrichtung erfolgt der Eintritt in die strategische Gruppe des Pioniers oder die Bildung einer neuen strategischen Verhaltensgruppe, wodurch sich die Konkurrenzsituation im High-Tech-Markt neu strukturiert (shake-out 1). ${ }^{181}$

Vgl. Gerstenberger, W., Zur Wettbewerbsposition der deutschen Industrie im HighTech-Bereich, in: Ifo-Schnelldienst, 1992, Nr. 13, S. 19 ff. Für eine detaillierte Betrachtung siehe o.V., Technologie, Gebremste Patentaktivität, in: Außen-Wirtschaft, 1994, Nr. 3, S. 22 ff.; Schmoch, U., Schnöring, Th., Wie steht es um die Wettbewerbsposition der Telekommunikationsgeräteindustrie in Europa?, Diskussionsbeitrag Nr. 104 des Wissenschaftlichen Instituts für Kommunikationsdienste, Bad Honnef 1993, S. 23 ff.; Faust, K., Technologische Wettbewerbsposition im Licht der Ifo-Patentstatistik, in: IfoSchnelldienst, 1992, Nr. 32, S. 11 ff.; Fischer, G., Schwarzer, U., Außer Atem, in: Manager Magazin, 1992, Nr. 11, S. 243 ff.

180 Vgl. Czepiel, J.A., Competitive marketing strategy, a.a.O., S. 261 ff.; Perillieux, R., Strategisches Timing von F\&E und Markteintritt bei innovativen Produkten, a.a.O., S. 25 ff.; Meffert, H., Strategische Unternehmensfuihrung und Marketing, a.a.O., S. 68; Remmerbach, K.-U., Markteintrittsentscheidungen, a.a.O., S. 59 f.

181 Vgl. Meffert, H., Marketing-Management, a.a.O., S. 209 f.; Schewe, G., Imitationsmanagement, Nachahmung als Option des Technologiemanagements, a.a.O., S. 58 ff.; Remmerbach, K.-U., Markteintrittsentscheidungen, a.a.O., S. 56. 
Sofern der frühe Folger die Markteintrittsbarrieren überwindet, treffen für ihn in abgeschwächter Form auch die mit der Pionierstrategie verbundenen Vorteile zu, da die Umfeldsituation der des Pioniers ähnlich ist. Mit dieser Eintrittsoption ist zudem die Chance verbunden, aus den Fehlern des Pioniers zu lernen und dadurch die Kosten der Technologie- bzw. Produktentwicklung sowie der Markterschließung zu senken. Ein zentrales Risiko der frühen Folgerstrategie besteht darin, daß die vom Pionier aufgebauten Eintrittsbarrieren im High-Tech-Markt nicht oder nur unzureichend überwunden werden können. ${ }^{182}$

Für späte Folger ist die Eintrittsphase nach dem take-off aufgrund der größtenteils abgebauten Kaufwiderstände durch ein starkes Marktwachstum gekennzeichnet. Da der High-Tech-Markt zu diesem Zeitpunkt bereits durch den Pionier und frühen Folger besetzt ist, liegt der Strategieschwerpunkt später Folgerstrategien auf der Bildung von Wettbewerbsvorteilen und der Sicherung einer erfolgreichen Marktposition. Durch die Art des Markteintrittsverhaltens der späten Folger, z.B. in Form einer aggressiven Imitationsstrategie, wird die Wettbewerbsdynamik im High-Tech-Markt bestimmt und eine Neustrukturierung der High-Tech-Anbieter eingeleitet (shake-out 2). ${ }^{183}$

Ein zentraler Vorteil dieser Strategie liegt darin, daß die wesentlichen Abnehmerbedürfnisse und Marktgegebenheiten bekannt sind und von den späten Folgern im Rahmen der Gestaltung einer wettbewerbsgerichteten Marketingstrategie frühzeitig berücksichtigt werden können. Als Nachteile sind vor allem die gestiegenen Markteintrittsbarrieren und der Verzicht auf Pioniergewinne im High-Tech-Markt zu nennen. ${ }^{184}$

182 Vgl. Hannig, U., Die Entwicklung wettbewerbsorientierter Marketingstrategien auf Basis des Konzeptes der strategischen Gruppen, a.a.O., S. 120 ff.; Schewe, G., Imitationsmanagement, Nachahmung als Option des Technologiemanagements, a.a.O., S. 100 ff.; Meffert, H., Strategische Unternehmensfuihrung und Marketing, a.a.O., S. 72 f.; Remmerbach, K.-U., Markteintrittsentscheidungen, a.a.O., S. $60 \mathrm{ff}$.

Vgl. Meffert, H., Marketing-Management, a.a.O., S. 219 ff.; Carpenter, G.S., Nakamoto, K., Competitive strategies for late entry into a market with a dominant brand, in: Management Science, Vol. 36 (1990), No. 10, S. 1269 ff.; Porter, M., Wettbewerbsstrategie, a.a.O., S. $422 \mathrm{ff}$.

Vgl. Schewe, G., Die Innovation im Wettbewerb, in: ZfB, 1992, Nr. 9, S. 980 ff.; Minderlein, M., Markteintrittsbarrieren und Unternehmensstrategie, a.a.O., S. 238 f.; 
In der empirischen Forschung, die sich mit dem Markterfolg von Timingstrategien auseinandersetzt, konnte bisher nicht die Vorteilhaftigkeit einer Timingoption nachgewiesen werden. ${ }^{185}$ Beispielsweise zeigten Robinson und Fornell in einer Untersuchung auf Basis der PIMS-Datenbank, da $\beta$ die Pionierstrategie bei Investitionsgüterherstellern besonders erfolgreich ist. ${ }^{186}$ Demgegenüber stellte eine empirische Studie von Booz, Allen \& Hamilton heraus, daß für Unternehmen der Maschinenbauindustrie eine frühe bzw. späte Folgerschaft im Vergleich zur Pionierposition zu einem größeren Markterfolg führt. ${ }^{187}$

Es bleibt insgesamt festzuhalten, daß aussagefähige Schlußfolgerungen über die Vorteilhaftigkeit von Timingstrategien nur unter Beachtung weiterer unternehmensbezogener Strategiedimensionen wie beispielsweise der wettbewerbsstrategischen Grundausrichtung und situativer Umfeldbedingungen sowie der Preis- bzw. Technologiedynamik gezogen werden können. ${ }^{188}$ Dies soll auch bei der Typologisierung von Marketingstrategien in High-Tech-Märkten auf der Grundlage strategischer Gruppen berücksichtigt werden.

Remmerbach, K.-U., Markteintrittsentscheidungen, a.a.O., S. 63 ff.; Perillieux, R., Der Zeitfaktor im strategischen Technologie-Management, a.a.O., S. $126 \mathrm{ff}$.

185 Für eine Übersicht ausgewählter Studien zum Erfolg von Timingstrategien vgl. insbesondere Kerin, R.A., Varadarajan, P.R., Peterson, R.A., First-mover advantage: A synthesis, conceptual framework and research propositions, in: Journal of Marketing, Vol. 56 (1992), S. $35 \mathrm{ff}$. Siehe hierzu auch Perillieux, R., Strategisches Timing von F\&E und Markteintritt bei innovativen Produkten, a.a.O., S. 27 ff. sowie die Ausfuihrungen in Teil B Kap. 2.21 der Arbeit.

186 Vgl. Robinson, W.T., Fornell, C. Market pioneering and sustainable market share advantages, The PIMS-Letter on Business Strategy, No. 39, Strategic Planning Institute, Cambridge, Mass. 1986, S. 2 ff.

187 Vgl. Perillieux, R., Strategisches Timing von F\&E und Markteintritt bei innovativen Produkten, a.a.O., S. $36 \mathrm{ff}$.

188 Vgl. Vidal, M., Wettbewerbsstrategien für Pionierunternehmen, a.a.O., S. 134 ff.; Lambkin, M., Pioneering new markets: A comparison of market share winners and losers, in: International Journal of Research in Marketing, Vol. 9 (1992), S. 9 ff.; Robinson, W.T., Fornell, C., Sullivan, M., Are market pioneers intrinsically stronger than later entrants?, in: Strategic Management Journal, Vol. 13 (1992), S. 613 ff.; Backhaus, K., Investitionsgütermarketing, a.a.O., S. 204 ff.; Schewe, G., Imitationsmanagement, Nachahmung als Option des Technologiemanagements, a.a.O., S. 82 ff.; Perillieux, R., Strategisches Timing von F\&E und Markteintritt bei innovativen Produkten, a.a.O., S. 32 ff.; Remmerbach, K.-U., Markteintrittsentscheidungen, a.a.O., S. 70 ff. 
Zusammenfassend sind in der Abbildung 17 die zur Profilierung im Wettbewerb und zum Aufbau von Wettbewerbsvorteilen für High-Tech-Unternehmen relevanten Strategiedimensionen dargestellt, die im Rahmen der Bildung strategischer Gruppen in High-Tech-Märkten einbezogen werden.

\begin{tabular}{|l|l|}
\hline Bezugsebene & Strategiedimensionen \\
\hline Marktfeldstrategie & - Marktpenetration \\
& - Marktentwicklung \\
& - Produktentwicklung \\
& - Diversifikation \\
\hline Wettbewerbsstrategie & - Kostenorientierung \\
& - Technologieorientierung \\
& - Nutzenorientierung \\
& - Qualitätsorientierung \\
& - Serviceorientierung \\
& - Imageorientierung \\
\hline Timingstrategie & - Pionierstrategie \\
& - Frühe Folgerstrategie \\
& - Späte Folgerstrategie \\
\hline
\end{tabular}

Abb. 17: Übersicht wettbewerbsrelevanter Strategiedimensionen in High-TechMärkten

Vor dem Hintergrund der abgeleiteten Strategievariablen wird in der vorliegenden Untersuchung zum einen folgende Basishypothese aufgestellt:

H ST1 In High-Tech-Märkten lassen sich marktfeld-, wettbewerbs- und timingbezogene Strategiedimensionen zu Basisstrategien verdichten. 
Zum anderen werden im Hinblick auf das Konzept der strategischen Gruppen unter Bezugnahme auf die vorherigen Ausfuihrungen folgende Basishypothesen aufgestellt:

H ST2 Strategische Gruppen in High-Tech-Märkten unterscheiden sich hinsichtlich der von ihnen verfolgten Strategieschwerpunkte innerhalb einer homogenen Umfeldsituation.

\section{Empirische Analyse strategischer Gruppen in High-Tech-Märkten}

Vorbereitend zur Analyse strategischer Gruppen wird zunächst untersucht, ob sich in High-Tech-Märkten allgemeine Basisstrategien identifizieren lassen. Neben einer Beschreibung und Überprüfung der abgeleiteten strategischen Grundhaltungen wird betrachtet, inwieweit die Basisstrategien mit sog. generischen Marketingstrategien aus der Literatur übereinstimmen und ob für HighTech-Märkte weitere strategische Grundhaltungen von besonderer Relevanz sind. Im Hinblick auf das Konzept der strategischen Gruppen wird untersucht, ob strategische Gruppen in High-Tech-Märkten anhand der ermittelten Basisstrategien oder der einzelnen strategischen Verhaltensdimensionen gebildet werden sollen. ${ }^{189}$

Zur Typologisierung von Marketingstrategien in High-Tech-Märkten auf der Grundlage strategischer Gruppen wird gemäß der konzeptionellen Vorüberlegungen ein zweistufiges Vorgehen gewählt. In einem ersten Schritt werden homogene Umfeldsituationen identifiziert, die anhand der bereits dargestellten Situationsvariablen charakterisiert und überprüft werden. ${ }^{190}$ Innerhalb des zweiten Analyseschritts werden in den homogenen Umfeldsituationen strategische Grup-

$189 \mathrm{Zu}$ einem vergleichbaren Vorgehen siehe z.B. Kirchgeorg, M., Ökologieorientiertes Unternehmensverhalten, a.a.O, S. $137 \mathrm{ff}$.

Vgl. hierzu auch die Ausführungen in Teil B Kap. 1.2 und Kap. 2.1 der Arbeit. 
pen ermittelt und ihre Strategieschwerpunkte herausgearbeitet. ${ }^{191}$ In diesem Zusammenhang wird auch betrachtet, ob sich in den verschiedenen Kontextsituationen ähnliche strategische Gruppen identifizieren lassen. Hierdurch lassen sich möglicherweise generalisierende Aussagen über die Ausrichtung von strategischen Gruppen treffen. Der empirischen Untersuchung wird eine Beschreibung der Stichprobenauswahl und -struktur einer 1990 durchgeführten Unternehmensbefragung zum Themenbereich "High-Tech-Marketing" sowie eine Kennzeichung der im folgenden verwendeten statistischen Analysemethoden vorangestellt.

\subsection{Design der empirischen Untersuchung}

Der empirischen Überprüfung des Bezugsrahmens liegt eine schriftliche Befragung von 1.800 High-Tech-Unternehmen zum Themenbereich "High-Tech-Marketing" zugrunde. ${ }^{192}$

Dem vorliegenden Forschungsvorhaben liegen hieraus Daten von 385 Unternehmen vor, die den in der Abbildung 18 aufgezeigten High-Tech-Branchen zugeordnet werden können. Eine weitere Beschreibung der Unternehmen anhand der Unternehmensgröße ist der Abbildung 19 zu entnehmen.

Für die High-Tech-Befragung wurde ein sechsseitiger Fragebogen konzipiert, der insgesamt 45 Fragen umfaßt. ${ }^{193}$ Anhand der in der Untersuchung enthaltenen standardisierten Fragen war die Ermittlung zahlreicher Kontext-, Ziel-, Strategie-, Instrumente-, Kultur- und Erfolgsvariablen möglich.

191 Vgl. hierzu auch die Ausführungen Teil B Kap. 1.2, Kap. 2.2 und Kap. 2.3 der Arbeit.

192 Die Befragung wurde vom Institut für Marketing in Zusammenarbeit mit dem Wirtschaftsmagazin "high Tech" und der Unternehmensberatung Maisberger \& Partner im Herbst 1990 durchgefuihrt. Vgl. hierzu Meffert, H., Lamnek, S., Maisberger, P., HighTech-Marketing, a.a.O., S. 9 ff.

193 Ein Exemplar des Fragebogens ist im Anhang III der Arbeit enthalten. 


\begin{tabular}{|l|c|c|}
\hline Bereiche & Anzahl & Anteil \\
\hline 01. Automatisierungstechnik & 53 & $13,8 \%$ \\
\hline 02. Mikroelektronik & 38 & $9,9 \%$ \\
\hline 03. Softwareentwicklung & 27 & $7,0 \%$ \\
\hline 04. Büroautomatisierungstechnik & 26 & $6,8 \%$ \\
\hline 05. Nachrichtentechnik & 24 & $6,2 \%$ \\
\hline 06. Verfahrenstechnik & 19 & $4,9 \%$ \\
\hline 07. Optoelektronik/Laser & 12 & $3,1 \%$ \\
\hline 08. Energietechnik & 11 & $2,9 \%$ \\
\hline 09. Umwelttechnik & 11 & $2,9 \%$ \\
\hline 10. Biotechnologie & 8 & $2,1 \%$ \\
\hline 11. Sonstige & 156 & $40,4 \%$ \\
\hline Summe & $\mathbf{3 8 5}$ & $\mathbf{1 0 0 , 0 \%}$ \\
\hline
\end{tabular}

Abb. 18: Verteilung der Unternehmen auf die High-Tech-Branchen

\begin{tabular}{|c|c|c|}
\hline $\begin{array}{c}\text { Unternehmensgrößenklassen } \\
\text { (Beschäftigtenanzahl) }\end{array}$ & Anzahl & Anteil \\
\hline $1-99$ & 65 & $16,9 \%$ \\
\hline $100-499$ & 120 & $31,2 \%$ \\
\hline $500-999$ & 47 & $12,2 \%$ \\
\hline $1.000-2.999$ & 66 & $17,1 \%$ \\
\hline $3.000-4.999$ & 20 & $5,2 \%$ \\
\hline $5.000-9.999$ & 22 & $5,7 \%$ \\
\hline 10.000 und mehr & 45 & $11,7 \%$ \\
\hline Summe & 385 & $\mathbf{1 0 0 , 0 \%}$ \\
\hline
\end{tabular}

Abb. 19: Verteilung der High-Tech-Unternehmen nach der Unternehmensgröße 
Die statistische Datenauswertung erfolgte mit Hilfe des PC-gestützten Softwarepaketes SPSS für Windows Version 5.01. Als multivariate Analysemethoden wurden Korrelations-, Faktoren-, Cluster-, Diskriminanz- und Varianzanalysen durchgefuihrt. ${ }^{194}$ Zur Untersuchung von Merkmalsunterschieden zwischen den ermittelten Umfeldsituationen und zwischen den identifizierten strategischen Gruppen in High-Tech-Märkten wurde bei metrisch skalierten Variablen der FTest, der Scheffé-Test und der T-Test sowie bei nominalskalierten Variablen der Chi-Quadrat-Test verwendet. ${ }^{195}$

\subsection{Identifikation von Basisstrategien in High-Tech-Märkten}

Zur Ermittlung von Basisstrategien in High-Tech-Märkten wurde eine explorative Faktorenanalyse verwendet. ${ }^{196}$ In diesem Zusammenhang wurde zunächst die

Vgl. zu den einzelnen statistischen Verfahren Backhaus, K., et al., Multivariate Analysemethoden, 7. Aufl., Berlin u.a. 1993, S. 56 ff.; Meffert, H., Marketingforschung und Käuferverhalten, a.a.O., S. 252 ff.; Hammann, P., Erichson, B., Marktforschung, 2. Aufl., Stuttgart, New York 1990, S. 198 ff.; Berekoven, L., Eckert, W., Ellenrieder, P., Marktforschung, Methodische Grundlagen und praktische Anwendung, 4. Aufl., Wiesbaden 1989, S. 210 ff. Im Rahmen der schriftlichen Befragung wurden die meisten Daten mit Hilfe von Ratingskalen erhoben. Da Ratingskalen die mathematischen Voraussetzungen von Intervallskalen erfullen, dürfen die o.g. statistischen Operationen eingesetzt werden. Vgl. hierzu Kroeber-Riel, W., Konsumentenverhalten, a.a.O., S. 186; Meffert, H., Marketingforschung und Käuferverhalten, a.a.O., S. 185. Für eine kritische Betrachtung siehe auch Nieschlag, R., Dichtl, E., Hörschgen, H., Marketing, 16. Aufl., Berlin 1991, S. 649.

Mit dem F-Test wird überprüft, ob die verwendeten Kontext- und Strategievariablen einen signifikanten EinfluB auf die Gruppenbildung haben. Vgl. Hartung, J., Statistik, München, Wien 1989, S. 616. Der Scheffé-Test untersucht anhand der Mittelwertunterschiede der Gruppierungsvariablen die Signifikanz der ermittelten strategischen Gruppen. Die Anwendung des Scheffe-Tests gegenüber anderen Mittelwertvergleichstests ist vor allem bei ungleichen Gruppengrößen zu empfehlen. Bauer, F., Datenanalyse mit SPSS, Berlin u.a. 1984, S. 84 f. Anhand des T-Tests wird geprüft, ob die Erfolgsunterschiede zwischen den strategischen High-Tech-Gruppen signifikant sind. Vgl. Bleymüller, J., Gehlert, G., Gülicher, H., Statistik für Wirtschaftswissenschaftler, 5. Aufl., München 1988, S. 111 ff. Mit dem Chi-Quadrat-Test wird untersucht, ob eine Beziehung zwischen zwei nominalskalierten Variablen besteht. Vgl. Brosius, G., SPSS/PC+ Advanced Statistics und Tables, Hamburg, New York 1989, S. 217 ff.; Bleymüller, J., Gehlert, G., Gülicher, H., Statistik für Wirtschaftswissenschaftler, a.a.O, S. $134 \mathrm{ff}$.

Vgl. Backhaus, K., et al., Multivariate Analysemethoden, a.a.O., S. 189 ff.; Meffert, H., Marketingforschung und Käuferverhalten, a.a.O., S. 257 ff.; Weiber, R., Faktorenanalyse, Eine anwendungsorientierte computergestützte Einführung mit Übungen, St. Gallen 1984, S. 4 ff. Zur Unterscheidung zwischen der explorativen und konfirma- 
Eignung der Strategiedimensionen im Hinblick auf die Faktorenbildung mit Hilfe des Kaiser-Meyer-Olkin-Kriteriums überprüft. ${ }^{197}$

Mit dem Kaiser-Meyer-Olkin-Kriterium läßt sich die Stärke der Zusammengehörigkeit der Strategievariablen untersuchen. Es werden dabei Prüfgrößen auf Basis einer Anti-Image-Korrelationsmatrix berechnet, die zur Beurteilung aller bzw. einzelner Strategiedimensionen dienen und Aussagen darüber zulassen, welche Strategievariablen in eine Faktorenanalyse einzubeziehen sind. Eine Berechnung der Prüfwerte ${ }^{198}$ zeigte, daß ein relativ gutes Gesamtergebnis (MSA $>0,75$ ) vorliegt. ${ }^{199}$ Für die Strategievariable früher Folger wurde jedoch lediglich ein MSA-Wert von 0,32 ermittelt. Das Ergebnis liegt in der geringen Korrelation $(<0,1)$ dieser Variable mit den übrigen Strategiedimensionen begründet. Die Unabhängigkeit der Variable früher Folger ist möglicherweise ein Hinweis dafür, $\mathrm{da} ß$ sie eine eigenständige Basisstrategie in High-Tech-Märkten darstellt. Für die weitere empirische Analyse erschien es daher sinnvoll, die Strategievariable früher Folger aufgrund des schlechten MSA-Wertes von 0,32 zwar aus der weiteren faktorenanalytischen Betrachtung auszuschließen, ${ }^{200}$ sie aber im Rahmen der inhaltlichen Interpretation der Basisstrategien zu berücksichtigen.

torischen Faktorenanalyse siehe z.B. Backhaus, K., et al., Multivariate Analysemethoden, a.a.O., S. $251 \mathrm{f}$. $\mathrm{Zu}$ den in der empirischen Analyse einbezogenen Strategievariablen siehe Teil B Kap. 2.3 der Arbeit.

197 In der Literatur wird die Notwendigkeit einer Überprüfung der Eignung der Ausgangsdaten für faktoranalytische Zwecke betont. In diesem Zusammenhang hat sich das Kaiser-Meyer-Olkin-Kriterium als ein sehr gutes Prüfverfahren erwiesen. Vgl. hierzu und zur Kennzeichung des Kaiser-Meyer-Olkin-Kriteriums z.B. Backhaus, K., et al., Multivariate Analysemethoden, a.a.O., S. 201 ff.; Stewart D.W., The application and misapplication of factor analysis in marketing research, in: Journal of Marketing Research, Vol. 18 (1981), S. 57 f.; Dziuban, C.D., Shikey, E.C., When is a correlation matrix appropriate for factor analysis?, in: Psychological Bulletin, Vol. 81 (1974), S. 359.

Die ermittelten Prüfgrößen sind im Anhang II der Arbeit zu finden. Die MSA-Werte der einzelnen Strategiedimensionen stehen hierbei auf der Diagonalen der Anti-Image-Korrelationsmatrix.

199 Im Hinblick auf die Interpretation der MSA-Werte siehe auch Backhaus, K, et al., Multivariate Analysemethoden, a.a.O., S. 205; Siefke, A., Die Eignung der Faktorenanalyse zur Beurteilung des Evoked Set, unver. Diplomarbeit, Münster 1992, S. 27 ff.; Kaiser, H.F., Rice, J., Mark IV, in: Educational and Psychological Measurement, Vol. 34 (1974), S. 111 ff.; Kaiser, H.F., A second generation little jiffy, in: Psychometrica, Vol. 35 (1970), S. 405.

200 Dieses Vorgehen wurde durch eine erneute Ermittlung der Prüfgrößen für die reduzierte Anzahl der Strategiedimensionen bestätigt, bei der sich die Ergebnisse sowohl insgesamt 
Im Anschluß an diese Vorüberlegungen wurden die ausgewählten Strategiedimensionen über eine explorative Faktorenanalyse zu Basisstrategien verdichtet. Anhand des Kaiser-Kriteriums ${ }^{201}$ konnten vier Faktoren extrahiert werden, die in der Abbildung 20 in aufbereiteter Form dargestellt sind. ${ }^{202}$

Beim ersten Faktor sind hohe positive Faktorladungen bezüglich der Strategievariablen Technologie-Führerschaft, Pionier und Produktentwicklung zu erkennen. ${ }^{203}$ Eine hohe negative Faktorladung besteht bei der Strategiedimension später Folger. Da diese strategischen Verhaltensdimensionen Ausdruck einer starken Innovationsorientierung bei der Entwicklung und Vermarktung von High-TechProdukten sind, läßt sich der erste Faktor als produktbezogene Innovationsstrategie interpretieren. Durch diesen Faktor werden insgesamt 25,7 \% der Varianz aller Strategievariablen erklärt.

Demgegenüber trägt der zweite Faktor mit 11,6 \% zur Erklärung der Gesamtvarianz bei. Im Vordergrund stehen hierbei das Streben nach einer Service-, Image-, Nutzen- und Qualitäts-Führerschaft. Durch diese Strategiedimensionen wird eine Basisstrategie in High-Tech-Märkten gekennzeichnet, die mehrere Ansatzpunkte zur Profilierung im Wettbewerbsumfeld vereint und deshalb als differenzierte Profilierungsstrategie bezeichnet werden kann.

als auch für die einzelnen Strategievariablen verbesserten. Siehe hierzu die MSA-Werte im Anhang II der Arbeit, die ohne die Strategiedimension früher Folger berechnet wurden.

201 Bei Verwendung des Kaiser-Kriteriums werden nur Faktoren mit einem Eigenwert gröBer als eins generiert. Der Eigenwert gibt hierbei die durch den jeweiligen Faktor erklärte Varianz aller untersuchten Strategiedimensionen wieder. Siehe hierzu z.B. Meffert, H., Marketingforschung und Käuferverhalten, a.a.O., S. 262 ff.; Bleymüller, J., Multivariate Analyse für Wirtschaftswissenschaftler, unver. Manuskript, Münster 1989, S. $101 \mathrm{ff}$.

Als Schätzverfahren wurde in der vorliegenden Untersuchung die Hauptkomponentenmethode angewendet. Vgl. hierzu z.B. Bleymüller, J., Multivariate Analyse für Wirtschaftswissenschaftler, a.a.O., S. 96 ff. Für eine Unterscheidung von Hauptkomponenten- und Hauptachsenmethode siehe z.B. Backhaus, K. et al., Multivariate Analysemethoden, a.a.O., S. 221 f. Zur besseren Interpretation der Faktorenstruktur wurde zudem eine Varimax-Rotation durchgefuihrt. Vgl. hierzu z.B. Bleymüller, J., Multivariate Analyse für Wirtschaftswissenschaftler, a.a.O., S. 106 ff.; Weiber, R., Faktorenanalyse, a.a.O., S. $63 \mathrm{ff}$.

Für die Interpretation der Faktoren werden nur Strategievariablen mit einer Faktorladung $>0,5$ herangezogen. Vgl. zu dieser Einschätzung Backhaus, $\mathrm{K}$., et al., Multivariate Analysemethoden, a.a.O., S. 254. Eine vollständige Dokumentation der Faktorenstruktur ist im Anhang II der Arbeit zu finden. 


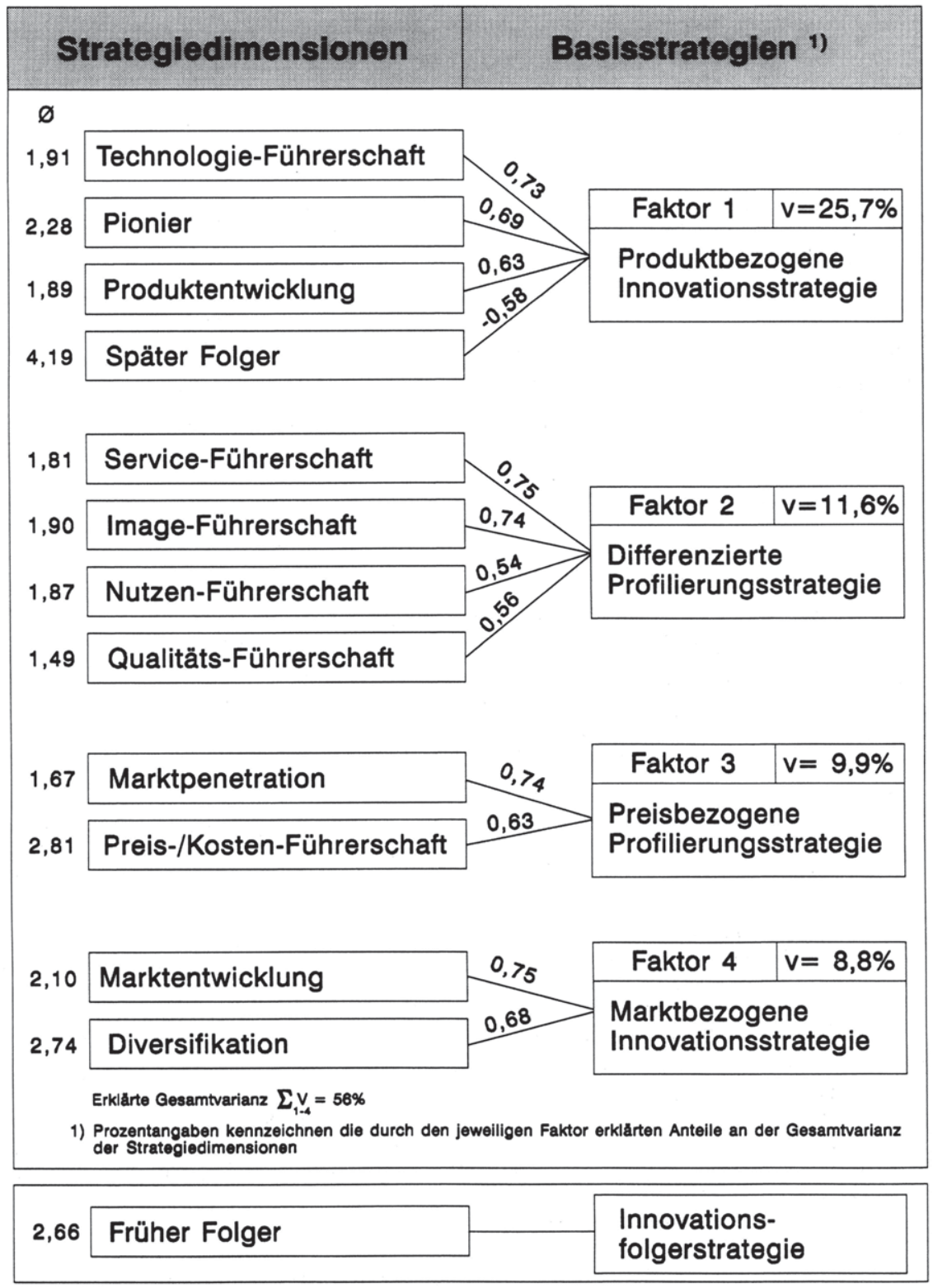

Abb. 20: Faktoranalytisch verdichtete Basisstrategien in High-Tech-Märkten 
Der dritte Faktor zeichnet sich durch hohe positive Faktorladungen bei den Strategievariablen Marktpenetration und Preis-/Kosten-Führerschaft aus. Beide strategischen Verhaltensdimensionen sind zum Aufbau von kosten- bzw. preisbezogenen Wettbewerbsvorteilen in High-Tech-Märkten besonders gut geeignet. In diesem Zusammenhang läßt sich der dritte Faktor als preisbezogene Profilierungstrategie beschreiben. Durch diesen Faktor werden 9,9 \% der Gesamtvarianz erklärt.

Der vierte Faktor stellt eine als marktbezogene Innovationsstrategie zu bezeichnende Basisstrategie in High-Tech-Märkten dar. Sowohl die Marktentwicklung als auch die Diversifikation, die durch eine deutliche Marktwachstumsorientierung geprägt sind, stellen die zentralen Strategievariablen des vierten Faktors dar. Insgesamt werden durch diesen Strategiefaktor $8,8 \%$ der Varianz der untersuchten strategischen Verhaltensdimensionen erklärt.

Vor dem Hintergrund der aufgezeigten Basisstrategien ist auch auf die Strategieoption der frühen Folgerschaft einzugehen. Das Verhalten des frühen Folgers ist dadurch gekennzeichnet, daß das Unternehmen mit technologischen Produktneuheiten erst nach dem Pionier in einen Markt eintritt. ${ }^{204} \mathrm{Da}$ eine Folgerposition bezüglich des Innovationstiming vorliegt, läßt sich die Strategiedimension auch als innovationsbezogene Folgerstrategie charakterisieren. ${ }^{205}$ Ein Grund für diese Strategieausrichtung ist darin zu sehen, daß der frühe Folger von den Erfahrungen des Pioniers profitieren möchte, um die Kosten der Markterschließung zu reduzieren. Neben einer ausgeprägten Kostenorientierung kann ein weiterer Grund für diese Strategieausrichtung darin liegen, daß der frühe Folger erst die Markteinfuihrung einer technologischen Produktinnovation durch den Pionier abwartet, um wichtige Informationen hinsichtlich der anwendungsbezogenen Schwächen des High-Tech-Produktes und der Ursachen von Kaufwiderständen zu sammeln. Die Informationen können dahingehend berücksichtigt werden, daß die Qualität 
des eigenen Produktes verbessert bzw. weitere Serviceleistungen angeboten werden. $^{206}$

Eine synoptische Betrachtung der Mittelwertausprägungen der Strategiedimensionen in der Abbildung 20 zeigt, daß für die High-Tech-Unternehmen als Basisstrategien insbesondere die produktbezogene Innovationsstrategie sowie die differenzierte Profilierungsstrategie von Bedeutung sind. Dies läßt darauf schliessen, daß diesen Basisstrategien in High-Tech-Märkten eine hohe Relevanz im Hinblick auf den Aufbau und die Sicherung strategischer Wettbewerbsvorteile zukommt. Im Vergleich dazu messen die High-Tech-Unternehmen der preisbezogenen Profilierungsstrategie und der marktbezogenen Innovationsstrategie einen geringeren Stellenwert bei. Es kann für die preisbezogene Basisstrategie dahingehend interpretiert werden, daß eine lediglich auf einem Preisvorteil basierende Marketingstrategie zur Differenzierung im Wettbewerb allein nicht ausreicht. Die vergleichsweise geringe Bedeutung der marktbezogenen Innovationsstrategie läßt sich damit erklären, daß diese Basisstrategie von High-Tech-Unternehmen hohe Marktinvestitionen erfordert und zudem eine hohe strategische Unsicherheit durch geringe Markterfahrungen beinhaltet.

Um eine zuverlässigere Aussage über die Validität der ermittelten Basisstrategien machen zu können, wurde ein Stabilitätstest durchgefuihrt. ${ }^{207}$ Bei einem Stabilitätstest erfolgt ausgehend von der über das Kaiser-Kriterium automatisch generierten Faktorenlösung eine sukzessive Reduzierung der Anzahl der Faktoren über mehrere Teststufen hinweg. In diesem Zusammenhang werden diejenigen

Vgl. Meffert, H., Marketing-Management, a.a.O., S. 218; Hannig, U., Die Entwicklung wettbewerbsorientierter Marketingstrategien auf Basis des Konzeptes der strategischen Gruppen, a.a.O., S. 121.; Backhaus, K., Investitionsgütermarketing, a.a.O., S. 201 f.; Remmerbach, K.-U., Markteintrittsentscheidungen, a.a.O., S. 61 f. u. $189 \mathrm{ff}$.

$\mathrm{Zu}$ einem ähnlichen Vorgehen siehe z.B. Niemeier, J., Wettbewerbsumwelt und interne Konfiguration, a.a.O., S. $160 \mathrm{ff}$; Fritz, W., Warentest und Konsumgüter-Marketing, Wiesbaden 1984, S. 336 ff.; Gabele, E., Einführung in die Geschäftsfeldorganisation, Tübingen 1981, S. $120 \mathrm{ff}$. 
Variablen als stabil angesehen, die in mehr als der Hälfte der Analyseschritte Faktorladungen $>0,5$ aufweisen. ${ }^{208}$

In der Abbildung 21 ist das Ergebnis des Stabilitätstests für die strategischen Grundhaltungen in High-Tech-Märkten zu sehen. ${ }^{209}$ Es zeigt sich, daß bei der Vier-Faktorenlösung nur Doppelladungen bei den Strategiedimensionen Qualitäts-Führerschaft und später Folger auftreten. Bei dem dreifaktoriellen Ergebnis sind lediglich die strategischen Verhaltensvariablen Technologie-Führerschaft und bei der zweifaktoriellen Lösung die Variablen Marktentwicklung und Preis/Kosten-Führerschaft durch eine Faktorladung $<0,5$ gekennzeichnet. Aufgrund der guten Ergebnisse sind die identifizierten Basisstrategien in High-Tech-Märkten als sehr stabil zu bezeichnen. Die Hypothese $\mathrm{H}$ Stl ist deshalb anzunehmen.

In einer abschließenden Würdigung läßt sich die Frage beantworten, inwieweit die in High-Tech-Märkten ermittelten Basisstrategien mit generischen Marketingstrategien aus der Literatur übereinstimmen. Es wird hierbei deutlich, daß die differenzierte und die preisbezogene Profilierungsstrategie mit den auf Porter zurückgehenden Wettbewerbsstrategien (Preis-/Kosten- bzw. Differenzierungsführerschaft) korrespondieren. ${ }^{210}$ Darüber hinaus zeigt sich, daß die produkt- und die marktbezogene Innovationsstrategie in High-Tech-Märkten eigenständige strategische Grundhaltungen darstellen. Dieses Ergebnis wird auch durch aktuelle empirische Untersuchungen bestätigt, die in technologieintensiven Märkten durchgeführt wurden. ${ }^{211}$

208 Im Gegensatz zu den Arbeiten von Niemeier und Fritz, die im Hinblick auf die Stabilität von Variablen lediglich Faktorladungen $>0,4$ fordern, werden in der vorliegenden Untersuchung Faktorladungen $>0,5$ als notwendig vorausgesetzt. Siehe hierzu auch Backhaus, K., et al., Multivariate Analysemethoden, a.a.O., S. 254.

Siehe dazu auch die Faktorenstruktur furr die vier-, drei- und zweifaktorielle Lösung mit Faktorladungen > 0,4 im Anhang II der Arbeit.

210 Vgl. in diesem Zusammenhang auch Meffert, H., Marketing-Management, a.a.O., S. 113 ff.; Backhaus, K., Investitionsgütermarketing, a.a.O., S. 177 ff.; Becker, J., MarketingKonzeption, a.a.O., S. 154 ff.; Kotler, Ph., Bliemel, F., Marketing-Management, a.a.O., S. 459 ff.; Porter, M., Wettbewerbsstrategie, a.a.O., S. $63 \mathrm{ff}$.

211 Vgl. Miles, R.E., Snow, Ch.C., Sharfman, M.P., Industry variety and performance, a.a.O., S. 168 ff.; Kim, L., Lim, Y., Environment, generic strategies and performance in rapidly developing country: a taxonomic approach, a.a.O., S. 804 ff.; Gaitanides, M., Westphal, J., Strategische Gruppen und Unternehmenserfolg - Ergebnisse einer empiri- 


\begin{tabular}{|l|c|c|c|c|}
\hline $\begin{array}{c}\text { Faktoren- } \\
\text { Strategie- } \\
\text { dimensionen }\end{array}$ & $\mathbf{F = 4}$ & $\mathbf{F = 3}$ & $\mathbf{F = 2}$ & $\begin{array}{c}\text { Stabiles } \\
\text { Ergebnis }\end{array}$ \\
\hline Marktpenetration & A & A & A & ja \\
\hline Marktentwicklung & A & A & B & ja \\
\hline Produktentwicklung & A & A & A & ja \\
\hline Diversifikation & A & A & A & ja \\
\hline Technologie-Führerschaft & A & B & A & ja \\
\hline Preis-/Kosten-Führersch. & A & A & B & ja \\
\hline Qualitäts-Führerschaft & C & A & A & ja \\
\hline Service-Führerschaft & A & A & A & ja \\
\hline Image-Führerschaft & A & A & A & ja \\
\hline Nutzen-Führerschaft & A & A & A & ja \\
\hline Pionier & A & A & A & ja \\
\hline Später Folger & C & A & A & ja \\
\hline
\end{tabular}

$A=$ Strategiedimensionen mit Faktorladungen von $0,50<L_{1}<1,00$

$B=$ Strategiedimensionen mit Faktorladungen von $L_{1} \leq 0,50$

$C=$ Strategiedimensionen mit Doppel- oder Mehrfachladungen von $L_{i}>0,40$

Abb. 21: Stabilitätstests für die Basisstrategien in High-Tech-Märkten

Im Hinblick auf die Verwendung der verdichteten Basisstrategien zur Identifikation strategischer Gruppen in High-Tech-Märkten bleibt festzuhalten, daß eine Clusteranalyse auf der Grundlage der Basisstrategien zu einem hohen Informationsverlust von $44 \%$ führen würde, da durch die vorliegende Faktorenlösung lediglich $56 \%$ der Gesamtvarianz der Strategievariablen erklärt werden. In der

schen Studie, in: Zeitschrift für Planung, 1991, Nr. 3, S. 254 ff.; Robinson, R.B., Pearce, J.A., Planned patterns of strategic behavior and their relationship to business-unit performance, in: Strategic Management Journal, Vol. 9 (1988), S. 44 ff. Siehe in diesem Zusammenhang auch Benkenstein, M., Die Reduktion der Fertigungstiefe als betriebswirtschaftliches Entscheidungsproblem, unver. Habil., Münster 1992, S. 71 ff.; Bolz, J., Wettbewerbsorientierte Standardisierung der internationalen Marktbearbeitung, a.a.O., S. $28 \mathrm{ff}$. 
weiteren Untersuchung erfolgt deshalb die Bildung strategischer Gruppen in High-Tech-Märkten anhand der einzelnen Strategiedimensionen. ${ }^{212}$

\subsection{Identifikation homogener Situationscluster in High-Tech-Märkten}

Zur Identifikation homogener Umfeldsituationen in High-Tech-Märkten wurden die technologie-, markt-, abnehmer- und konkurrenzbezogenen Kontextvariablen $^{213}$ in eine Clusteranalyse einbezogen. ${ }^{214}$

In diesem Zusammenhang war vorab zu klären, ob alle Kontextmerkmale in die Clusteranalyse einfließen dürfen. Es wurden hierzu die statistischen Voraussetzungen bezüglich der Korrelation, Standardisierung und Konstanz überprüft. ${ }^{215}$ Eine vorgeschaltete Korrelationsanalyse ergab, daß zwischen den Umfeldvariablen keine oder nur geringe Beziehungen (jeweils $<0,5$ ) bestehen. Eine Standardisierung der Kontextvariablen, bei der die absolute Streuung bei einzel-

212 Die Ergebnisse der Clusteranalyse werden unter Berücksichtigung der ermittelten strategischen Grundhaltungen dargestellt. Vgl. hierzu auch Pues, C., MarkterschlieBungsstrategien bundesdeutscher Unternehmen in Osteuropa, Eine Analyse der Ausgestaltungsformen und Einflußfaktoren auf empirischer Grundlage, Diss., Münster 1994, S. 139; Meffert, H., Marketingforschung und Käuferverhalten, a.a.O., S. 268; Kirchgeorg, M., Ökologieorientiertes Unternehmensverhalten, a.a.O, S. 143.

213 Die Kontextvariablen sind im einzelnen dem Teil B Kap. 2.1 der Arbeit sowie dem Fragebogen im Anhang II der Arbeit zu entnehmen.

214 Zur Ermittlung der Umfeldsituationen stehen eine Reihe unterschiedlicher Algorithmen zur Verfügung. Für die vorliegende Untersuchung wird aus den hierarchisch-agglomerativen Cluster-Algorithmen das Ward-Verfahren ausgewählt. Es bildet besonders homogene Cluster, da die Unternehmen vereinigt werden, die die Streuung (Varianz) in einer Gruppe möglichst wenig erhöhen. Vgl. hierzu Backhaus, K., et al., Multivariate Analysemethoden, a.a.O., S. 285 ff.; Kern, W., Hagemeister, St., Konzeption und Problematik der Clusteranalyse bei betriebswirtschaftlichen Anwendungen, in: WISU, 1986, Nr. 2, S. 29 ff. Das Ward-Verfahren findet in der empirischen Forschung überwiegend Anwendung, da es die Unternehmen zumeist den richtigen Gruppen zuordnet und zu sehr stabilen Partitionen führt. Siehe insbesondere Bergs, S., Optimalität bei ClusterAnalysen, Diss., Münster 1981, S. 96 f. Die Anzahl der Cluster wurde anhand des Elbow-Kriteriums ermittelt. Es wird hierbei davon ausgegangen, da $B$ die optimale Clusteranzahl dann erreicht ist, wenn eine weitere Reduzierung der Clusterzahl zu einem relativ starken Heterogenitätszuwachs fuihrt. Vgl. hierzu z.B. Backhaus, K., et al., Multivariate Analysemethoden, a.a.O., S. 307 f.; Meffert, H., Marketingforschung und Käuferverhalten, a.a.O., S. 277 f.

Vgl. Backhaus, K., et al., Multivariate Analysemethoden, a.a.O., S. 312 ff. 
nen Variablen verloren geht, war nicht notwendig, da alle Merkmale das gleiche Skalenniveau und die gleiche Spannweite besitzen. ${ }^{216}$ Weiterhin liegt keine Konstanz $\mathrm{z}^{217}$ der Merkmalsausprägungen der Kontextdimensionen vor. Es war somit zulässig, sämtliche umfeldbezogenen Variablen in die Clusteranalyse aufzunehmen.

Im folgenden werden die Ergebnisse der Clusteranalyse zunächst anhand der gruppenbildenden Situationsvariablen analysiert. Zur Beschreibung der Umfeldsituationen werden anschließend zusätzliche Marktvariablen herangezogen. ${ }^{218}$ Auf Basis der Gruppierungsvariablen ließen sich drei unterschiedliche Umfeldsituationen identifizieren. Zur Beschreibung der Situationen werden die in der Abbildung 22 dargestellten Mittelwertabweichungen der Kontextmerkmale herangezogen. ${ }^{219}$

\section{Situation A: Geringer Wettbewerb $(n=104)$}

Die Umfeldsituation dieser Unternehmen zeichnet sich durch einen geringen Preiswettbewerb und eine geringe Preiserosion aus. Die Wirkung von Kosteneinsparungen im Beschaffungsbereich auf die Absatzpreise und die Preisorientierung der Abnehmer ist ebenfalls unterdurchschnittlich ausgeprägt. Die Merkmale bezüglich des technologiebezogenen Wettbewerbs (Technologiewettbewerb, Qualitätswettbewerb aufgrund Technologiedynamik, verkürzte PLZ) sind ebenfalls von geringer Wichtigkeit. Für die allgemeine Marktsituation läßt sich fest-

216 Brosius stellt heraus, daß die Standardisierung der Gruppierungsvariablen zu absoluten Streuverlusten bei den einzelnen Variablen fuihrt. Hierdurch kann sich das Ergebnis der Clusteranalyse verschlechtern. Bei gleichem Skalenniveau (Ratingskala) und gleicher Spannweite (1 bis 5) der gruppenbildenden Variablen ist es deshalb sinnvoll, auf eine Standardisierung zu verzichten. Vgl. Brosius, G., SPSS/PC+ Advanced Statistics und Tables, a.a.O., S. $178 \mathrm{f}$.

217 Konstante Merkmale sind Variablen, die keinerlei Streuung besitzen und deshalb für alle Objekte die gleiche Ausprägung aufweisen. Vgl. Backhaus, K., et al., Multivariate Analysemethoden, a.a.O., S. 314.

Vgl. in diesem Zusammenhang auch die Abbildung 11 der Arbeit.

219 Die absoluten Mittelwertabweichungen der einzelnen Kontextmerkmale sind im Anhang II der Arbeit zu finden. 


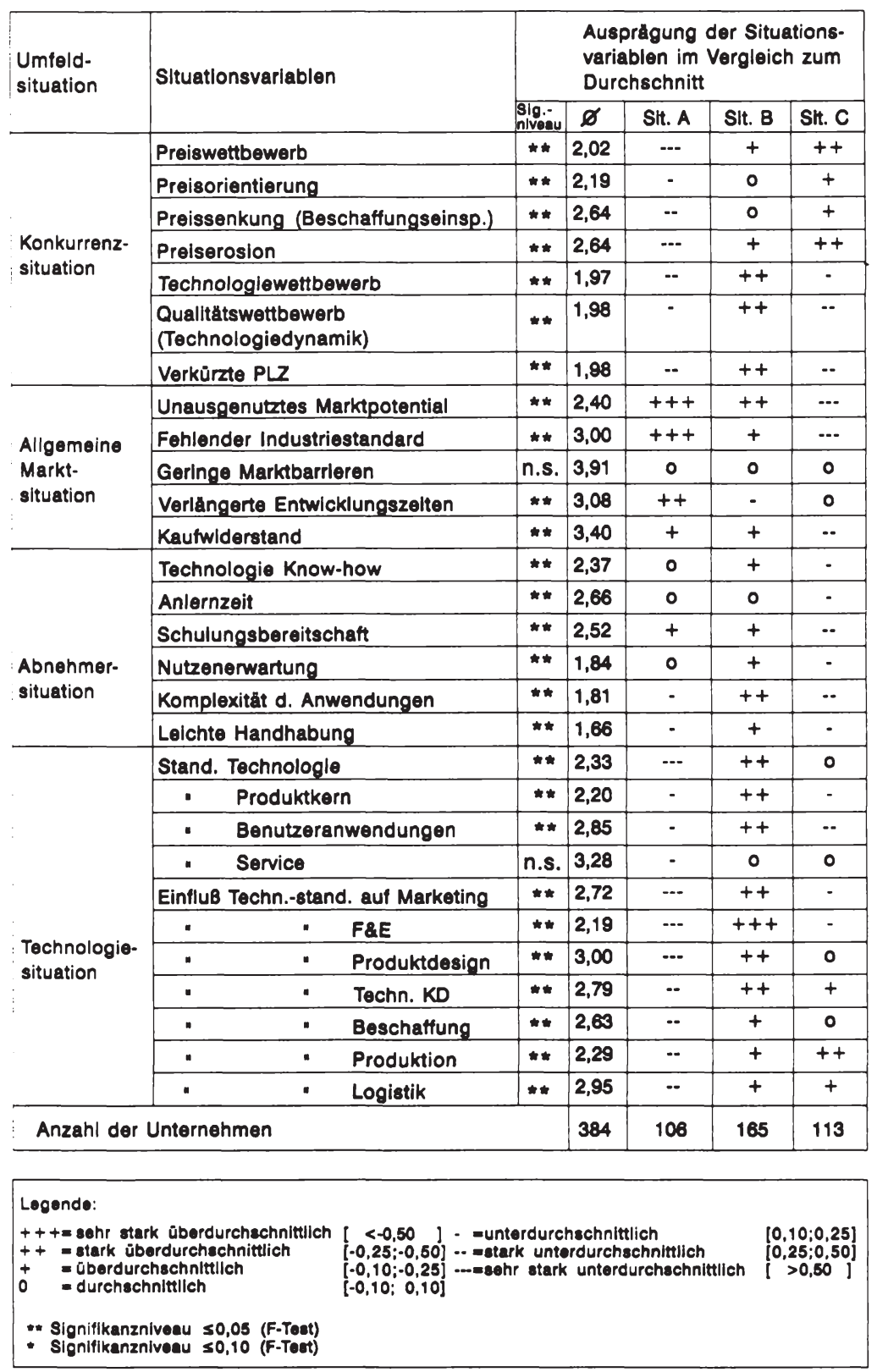

Abb. 22: Beschreibung der Situationscluster in High-Tech-Märkten 
halten, daß das Marktpotential relativ wenig ausgeschöpft ist und ein Industriestandard noch weitgehend fehlt. Darüber hinaus besitzen die meisten Merkmale innerhalb der Abnehmer- und Technologiesituation eine unterdurchschnittliche Ausprägung. Zusammenfassend bleibt festzuhalten, daß Situation A durch eine sehr geringe Preis- und Technologiedynamik, eine sehr hohe Marktdynamik und einen stark unterdurchschnittlichen technologiebezogenen Standardisierungsdruck gekennzeichnet ist. Es kann hieraus geschlossen werden, daß sich die Technologien und technologischen Anwendungen noch in einer relativ frühen Entwicklungsphase befinden und die High-Tech-Unternehmen demzufolge lediglich mit einem geringen Wettbewerb konfrontiert werden.

\section{Situation B: Technologiewettbewerb $(n=165)$}

Innerhalb der Umfeldsituation B kommt den preisbezogenen Merkmalen insgesamt nur eine durchschnittliche Bedeutung zu. Es ist jedoch im Gegensatz zu den Umfeldsituationen $\mathrm{A}$ und $\mathrm{C}$ festzustellen, daß die High-Tech-Unternehmen in dieser Situation von einer starken Technologiedynamik betroffen sind. Dies kommt in der überdurchschnittlichen Ausprägung der Technologievariablen zum Ausdruck. In diesem Zusammenhang wird auch deutlich, daß die High-TechUnternehmen einem starken technologiebedingten Standardisierungsdruck ausgesetzt sind. Hierbei weisen sämtliche Merkmale mit Ausnahme des Service auf eine überdurchschnittlich hohe Standardisierung hin. Die allgemeine Marktsituation wird primär durch ein unzureichend ausgeschöpftes Marktpotential geprägt. Für die Abnehmersituation gilt, daß die Komplexität der Anwendungen, die Nutzenerwartungen und die Anforderungen an eine leichte Handhabung von besonderer Wichtigkeit sind. Es zeigt sich insgesamt, daß in Situation B eine besonders hohe Technologiedynamik, eine hohe Marktdynamik, hohe Leistungsanforderungen der Abnehmer und ein stark überdurchschnittlicher technologischer Standardisierungsdruck vorherrscht. Aufgrund der hohen Relevanz der technologiebezogenen Kontextmerkmale sind die High-Tech-Unternehmen in dieser Situation gefordert, sich in einem intensiven Technologiewettbewerb $\mathrm{zu}$ behaupten. 


\section{Situation C: Preiswettbewerb $(n=113)$}

Im Vergleich zu den ersten beiden Umfeldsituationen unterliegen die High-TechUnternehmen in der Situation C einer besonders hohen Preisdynamik. Daher wird den Variablen des preisbezogenen Wettbewerbs eine stark überdurchschnittliche Bedeutung beigemessen, während die technologiebezogenen Merkmale von einer unterdurchschnittlichen Wichtigkeit sind. Die Marktsituation ist vor allem durch ein weitgehend ausgeschöpftes Marktpotential und einen etablierten Industriestandard geprägt. Innerhalb der Abnehmersituation besitzen sowohl Merkmale, die den Kundenkenntnissen zugeordnet werden (Technologie Know-how, Verkürzung der Anlernzeit, Schulungsbereitschaft) als auch Variablen, die als Leistungsanforderungen zu interpretieren sind (Nutzenerwartungen, Komplexität der Anwendungen, leichte Handhabung) einen geringen Stellenwert. Dies kann ein Indiz dafür sein, daß die Technologien und technologischen Anwendungen in dieser Umfeldsituation schon weitgehend ausgereift sind. Sie werden deshalb zunehmend von High-Tech-Verwendern gekauft, die vergleichsweise geringe Anforderungen an die Produkt- und Serviceleistung stellen und über relativ wenig technologiespezifische Kenntnisse verfügen. Als Beispiele hierfür sind private Nachfrager im PC-Markt, im Markt für Standardsoftware oder im Mobilfunkmarkt zu nennen. Im Hinblick auf die Technologiesituation ist zu erkennen, daß die Produktion, die Logistik und der technische Kundendienst von der Technologiestandardisierung besonders beeinflußt werden. Demgegenüber sind die übrigen Standardisierungsmerkmale nur durchschnittlich bzw. leicht unterdurchschnittlich ausgeprägt. Zusammenfassend läßt sich konstatieren, daß die Situation $C$ durch eine sehr hohe Preisdynamik, eine sehr geringe Technologiedynamik und eine stark unterdurchschnittliche Marktdynamik bestimmt wird. Die High-Tech-Unternehmen werden hierbei vor die Aufgabe gestellt, sich in einem preisintensiven Wettbewerbsumfeld zu profilieren.

Es läßt sich insgesamt festhalten, daß die ermittelten Situationscluster sehr unterschiedliche Umfeldsituationen in den untersuchten High-Tech-Märkten abbil- 
den. ${ }^{220}$ Aus diesem Grunde ist dieser vorbereitende Analyseschritt zur Bildung strategischer Gruppen als besonders wichtig anzusehen.

Die identifizierten Situationscluster werden im folgenden anhand der Merkmale Marktwachstum (bezogen auf Deutschland), Produktlebenszyklus, Marktlebenszyklus und Branchenzugehörigkeit näher charakterisiert.

Das Marktwachstum in den verschiedenen Umfeldsituationen ist in der Abbildung 23 dargestellt. Es ist hierbei zu erkennen, daß ein signifikanter Zusammenhang zwischen dem Marktwachstum in Deutschland und den Umfeldsituationen besteht $(\alpha<0,01)$. Situation A (geringer Wettbewerb) besitzt mit $20 \%$ das höchste Marktwachstum. Es bestätigt die in dieser Umfeldsituation bestehende besonders hohe Markt- und geringe Preisdynamik. Im Vergleich dazu ist das Marktwachstum in Situation B, die durch eine hohe Marktdynamik und einen ausgeprägten Technologiewettbewerb geprägt ist, mit $15 \%$ schon deutlich niedriger. Das unterdurchschnittliche Marktwachstum von $8 \%$ in Situation C bekräftigt die schwache Ausprägung der Marktdynamik und den intensiven Preiswettbewerb dieser Umfeldsituation.

\begin{tabular}{|l|c|c|c|c|}
\hline & Durchschnitt & Sit. A & Sit. B & Sit. C \\
\hline $\begin{array}{l}\text { Marktwachstum } \\
\text { Deutschland }\end{array}$ & $14 \%$ & $20 \%$ & $15 \%$ & $8 \%$ \\
\hline Signifikanzniveau $\alpha<0,01$ (T-Test) & & & \\
\hline
\end{tabular}

Abb. 23: Marktwachstum in den Situationsclustern

Die zeitliche Veränderung des Produktlebenszyklus in den verschiedenen Situationsclustern wird in Abbildung 24 beschrieben. Es zeigt sich, daß eine signifikante Beziehung zwischen den Produktlebenszyklen und den Kontextsituationen vorliegt $(\alpha<0,05)$. Situation B ist durch eine rasche Verkürzung der Produktlebenszyklen gekennzeichnet. Als Grund hierfür kann der intensive Technologie- 
wettbewerb gesehen werden, der in dieser Kontextsituation existiert. ${ }^{221}$ Demgegenüber sind die Produktlebenszyklen innerhalb der Situationen A und C, die eine geringe Technologiedynamik aufweisen, deutlich länger.

\begin{tabular}{|l|c|l|l|l|}
\hline PLZ & Durchschnitt & Sit. A & Sit. B & Sit. C \\
\hline $1987-1990$ & 5,22 J. & 5,94 J. & 4,57 J. & 5,61 J. \\
\hline 1990 & 4,42 J. & 5,06 J. & 3,81 J. & 4,84 J. \\
\hline $1990-1993$ & 3,83 J. & 4,90 J. & 2,95 J. & 4,28 J. \\
\hline \multicolumn{5}{|l}{ Signifikanzniveau $\alpha<0,05$ (T-Test) } \\
\hline
\end{tabular}

Abb. 24: Produktlebenszyklen in den Situationsclustern

\begin{tabular}{|l|c|c|c|c|}
\hline Marktwachstum & $\begin{array}{l}\text { Anzahl der } \\
\text { Unternehmen }\end{array}$ & Sit. A & Sit. B & Sit. C \\
\hline schnelles Wachstum & 75 & $22 \%$ & $24 \%$ & $12 \%$ \\
\hline Wachstum & 228 & $65 \%$ & $60 \%$ & $59 \%$ \\
\hline Sättigung & 72 & $13 \%$ & $16 \%$ & $29 \%$ \\
\hline Signifikanzniveau $\alpha<0,05$ (Chi-Quadrat-Test) \\
\hline
\end{tabular}

Abb. 25: Verteilung der Marktwachstumsphasen in den Situationsclustern

Abbildung 25 zeigt die Verteilung der Marktwachstumsphasen in den alternativen Umfeldsituationen. Es wird deutlich, daß eine signifikante Beziehung zwischen den Marktwachstumsphasen und den Umfeldsituationen existiert $(\alpha<0,05)$. In den Situationen A (geringer Wettbewerb) und B (Technologiewettbewerb) befinden sich doppelt so viele Unternehmen in einer schnellen Wachstumsphase wie im Vergleich zur Situation C (Preiswettbewerb). Umgekehrt sehen sich $29 \%$ der Unternehmen in Situation C mit einem stagnierenden Markt konfrontiert, gegenüber nur $13 \%$ und $16 \%$ in Situation A und B.

Die Verteilung der High-Tech-Branchen auf die unterschiedlichen Kontextsituationen ist in der Abbildung 26 dargestellt. Es wird deutlich, daß ein signifikanter

221 Siehe dazu auch die Darstellungen im Teil B Kap. 2.11 der Arbeit. 
Zusammenhang zwischen den Branchen und den Umfeldsituationen besteht $(\alpha<$ 0,1 ). In diesem Zusammenhang ist jedoch festzustellen, daß einzelne Branchen nicht genau einer Umfeldsituation zugeordnet werden können. Ein Grund hierfür kann in einer zu weit gefaßten Abgrenzung der High-Tech-Märkte liegen, sofern man auf die in der Befragung zugrunde gelegte Branchenzuordnung Bezug nimmt. So umfaßt beispielsweise die Mikroelektronik einerseits Unternehmen, die hochleistungsfähige Prozessoren herstellen und deshalb mit einer hohen Technologiedynamik (Situation B) konfrontiert werden. Andererseits zählen zu dieser Branche auch Unternehmen, die relativ einfache Speicherchips produzieren und sich mit einem hohen Preiswettbewerb (Situation C) auseinanderzusetzen haben.

\begin{tabular}{|l|c|c|c|c|}
\cline { 2 - 5 } \multicolumn{1}{c|}{} & \begin{tabular}{c} 
Anzahl \\
\multicolumn{1}{c|}{ Unternehmen }
\end{tabular} & Situation A & Situation B & Situation C \\
\hline Mikroelektronik & 38 & $18 \%$ & $58 \%$ & $24 \%$ \\
\hline Optoelektronik & 12 & $25 \%$ & $42 \%$ & $33 \%$ \\
\hline Softwareentwicklung & 27 & $48 \%$ & $48 \%$ & $4 \%$ \\
\hline Biotechnologie & 8 & $38 \%$ & $38 \%$ & $24 \%$ \\
\hline $\begin{array}{l}\text { Automatisierungs- } \\
\text { technik }\end{array}$ & 53 & $22 \%$ & $38 \%$ & $40 \%$ \\
\hline Nachrichtentechnik & 24 & $29 \%$ & $38 \%$ & $33 \%$ \\
\hline Büroautomatisierung & 26 & $7 \%$ & $73 \%$ & $20 \%$ \\
\hline Verfahrenstechnik & 19 & $42 \%$ & $32 \%$ & $26 \%$ \\
\hline Energietechnik & 11 & $36 \%$ & $36 \%$ & $28 \%$ \\
\hline Umwelttechnik & 11 & $45 \%$ & $36 \%$ & $19 \%$ \\
\hline & $\sum 229$ & \multicolumn{2}{|c|}{ Signifikanzniveau $\alpha<0,1$ (Chi-Quadrat-Test) } \\
\hline
\end{tabular}

Abb. 26: Verteilung der High-Tech-Branchen in den Situationsclustern

Ähnliche Beispiele lassen sich für die Automatisierungstechnik, die Nachrichtentechnik und die Büroautomatisierung finden. In der Branche der Softwareentwicklung befinden sich Unternehmen, die Standardsoftware bei einem hohen Technologiewettbewerb (Situation B) vermarkten. Demgegenüber 
gilt für Unternehmen, die individuelle Softwarelösungen anbieten, noch eine hohe Marktdynamik (Situation A). ${ }^{222}$

Insgesamt wird deutlich, daß unterschiedliche Kontextsituationen in den produktbezogen abgegrenzten High-Tech-Branchen anzutreffen sind. Für das Konzept der strategischen Gruppen läßt sich die Schlußfolgerung ziehen, daß die Identifikation von strategischen Gruppen in einer High-Tech-Branche aufgrund der mangelnden Homogenität der Umfeldsituationen nicht möglich ist. Das konstitutive Merkmal der vergleichbaren Umfeldsituation für die High-Tech-Unternehmen wird hingegen durch die in der vorliegenden Untersuchung durchgeführte Bildung homogener Situationscluster erfüllt. ${ }^{223}$

Die Überprüfung der Güte der Trennung der Situationscluster erfolgte auf Grundlage einer Diskriminanzanalyse ${ }^{224}$ und von Mittelwertvergleichstests ${ }^{225}$ anhand der clusterbildenden Kontextvariablen.

Innerhalb der Diskriminanzanalyse stellt die zu $85 \%$ richtige Zuordnung der Unternehmen zu den Umfeldsituationen ein gutes Ergebnis dar. ${ }^{226}$ Die relativ

Siehe zu dieser Einschätzung auch Meffert, J., Standards als Integrationsinstrument in der Computer- und Kommunikationsindustrie, a.a.O., S. 103 ff.; Hannig, U., Die Entwicklung wettbewerbsorientierter Marketingstrategien auf Basis des Konzeptes der strategischen Gruppen, a.a.O., S. 212 ff.; Marone, J.G., Winning in high-tech markets, the role of general management, a.a.O., S. $163 \mathrm{ff}$.; Maringer, A., Preisverfall mikroelektronischer Bauelemente am Beispiel DRAM-Speicherbauelemente, S. $424 \mathrm{ff}$.

Vgl. hierzu auch die Diskussion im Teil B Kap. 1.1 und 1.2 der Arbeit.

$\mathrm{Zu}$ einem vergleichbaren Vorgehen siehe z.B. Heinemann, G., Betriebstypenprofilierung und Erlebnishandel, a.a.O., S. 82 ff.; Zur Methodik der Diskriminanzanalyse vgl. u.a. Meffert, H., Marketingforschung und Käuferverhalten, a.a.O., S. 279 ff.; Bleymüller, J., Multivariate Analyse für Wirtschaftswissenschaftler, a.a.O., S. 228 ff.; Aaker, D.A., Day, G.S., Marketing Research, 3. Aufl., New York u.a. 1986, S. 466 ff.

Vgl. Backhaus, K., et al., Multivariate Analysemethoden, a.a.O., S. 56 ff. u. 309 ff.; Hartung, J., Statistik, a.a.O., S. 609 ff.; Bleymüller, J., Gehlert, G., Gülicher, H., Statistik für Wirtschaftswissenschaftler, a.a.O., S. $115 \mathrm{ff}$.

Das Ergebnis der diskriminatorischen Klassifikation der Situationscluster ist dem Anhang II der Arbeit zu entnehmen. Die Zufallswahrscheinlichkeit ohne Zurücklegen beträgt 33 $\%$. Bei Berücksichtigung der Größenunterschiede zwischen den Situationsclustern ergeben sich für die Situationen A, B und C Zufallswahrscheinlichkeiten ohne Zurücklegen von $28 \%, 43 \%$ und $29 \%$. Das vorliegende Klassifikationsergebnis ist in beiden Fällen als sehr zufriedenstellend zu bezeichnen. Vgl. hierzu z.B. Backhaus, K, et al., Multivariate Analysemethoden, a.a.O., S. 116. 
geringen Werte für Wilks' Lambda von 0,2535 und 0,5097 weisen auf eine gute Trennqualität der ermittelten Diskriminanzfunktionen hin. Der durchgeführte ChiQuadrat-Test zeigt, daß die Umfeldsituationen mit einem $\alpha<0,0001$ signifikant trennen. Weiterhin verdeutlichen die hohen kanonischen Korrelationskoeffizienten von 0,7090 und 0,7002 die starke Beziehung zwischen der Diskriminanzfunktion und den Gruppierungsvariablen. ${ }^{227}$

Im Rahmen der Mittelwertvergleichstests ergab der durchgeführte F-Test, daß die in der Clusteranalyse berücksichtigten Situationsmerkmale, bis auf die Variablen geringe Marktbarrieren und Service, mit einem $\alpha<0,05$ sehr gut trennen. ${ }^{228}$ Der Scheffé-Test zeigte darüber hinaus, daß die Mehrzahl der konkurrenz-, markt-, abnehmer- und technologiebezogenen Merkmale in den drei identifizierten Situationsclustern auch untereinander signifikant verschieden sind. ${ }^{229}$

Es bleibt zusammenfassend festzuhalten, daß sich in High-Tech-Märkten homogene Umfeldsituationen auf Basis der spezifizierten markt-, technologie-, abnehmer- und konkurrenzbezogenen Kontextdimensionen bilden lassen. Aufgrund der signifikanten Unterschiede zwischen den Situationen "geringer Wettbewerb", "Technologiewettbewerb" und "Preiswettbewerb" ist die Hypothese H UM anzunehmen.

\subsection{Bildung strategischer Gruppen in den Situationsclustern}

Die Ermittlung strategischer High-Tech-Gruppen innerhalb der identifizierten homogenen Kontextsituationen erfolgte mit Hilfe einer Clusteranalyse ${ }^{230}$ auf

227 Die Diskriminanzfunktionen, die Werte für Wilks' Lambda bzw. die kanonischen Korrelationskoeffizienten und die Ergebnisse des Chi-Quadrat-Tests sind dem Anhang II der Arbeit zu entnehmen.

228 Zu den Ergebnissen des F-Tests siehe auch die Abbildung 22 der Arbeit.

Die Ergebnisse des F-Tests und Scheffe-Tests sind im Anhang II der Arbeit zu finden.

Zur Bildung von strategischen Gruppen in den homogenen Umfeldsituationen wird in der vorliegenden Untersuchung das Ward-Verfahren verwendet und zur Bestimmung der Clusteranzahl das Elbow-Kriterium herangezogen. Vgl. Backhaus, K., et al., Multivariate 
Basis der abgeleiteten marktfeld-, wettbewerbs- und timingbezogenen Strategievariablen. ${ }^{231}$ Bei Berücksichtigung der statistischen Voraussetzungen hinsichtlich der Korrelation, Standardisierung und Konstanz konnten alle strategischen Verhaltensdimensionen in die Clusteranalyse einbezogen werden. ${ }^{232}$ In den Umfeldsituationen geringer Wettbewerb, Technologiewettbewerb und Preiswettbewerb wurden jeweils drei strategische Gruppen ermittelt, die in einem Überblick in Abbildung 27 dargestellt sind.

Im Vordergrund der folgenden Ausführungen steht zunächst die Beschreibung der strategischen High-Tech-Gruppen in Situation A. Anschließend werden in einer situationsübergreifenden Betrachtung die Gemeinsamkeiten und Unterschiede in den Strategieschwerpunkten der strategischen Gruppen in High-Tech-Märkten herausgestellt. Zur Interpretation der strategischen Gruppen in den unterschiedlichen Situationsclustern werden die in der Abbildung 28 gezeigten Mittelwertabweichungen der Strategievariablen verwendet und dabei vor allem die strategischen Verhaltensdimensionen hervorgehoben, die den jeweiligen Strategietyp charakterisieren. ${ }^{233}$

In der Situation A, die aufgrund der hohen Markt- sowie der geringen Preisbzw. Technologiedynamik durch einen geringen Wettbewerb gekennzeichnet ist, lassen sich die High-Tech-Unternehmen der differenzierungs-, kosten- oder innovationsorientierten strategischen Verhaltensgruppe zuordnen.

Analysemethoden, a.a.O., S. 292 ff.; Meffert, H., Marketingforschung und Käuferverhalten, a.a.O., S. 277 f.; Bergs, S., Opimalität bei Cluster-Analysen, a.a.O., S. 96 f. Fragebogen im Anhang II der Arbeit zu entnehmen.

Vgl. Backhaus, K., et al., Multivariate Analysemethoden, a.a.O., S. 312 ff.; Brosius, G., SPSS/PC+ Advanced Statistics and Tables, a.a.O., S. 178 f. Siehe zu den statistischen Voraussetzungen auch die Ausführungen im Teil B Kap. 3.2 der Arbeit.

Die absoluten Mittelwertabweichungen der einzelnen Strategiedimensionen der strategischen High-Tech-Gruppen in den Situationen A, B und C sind im Anhang II der Arbeit zu finden. 


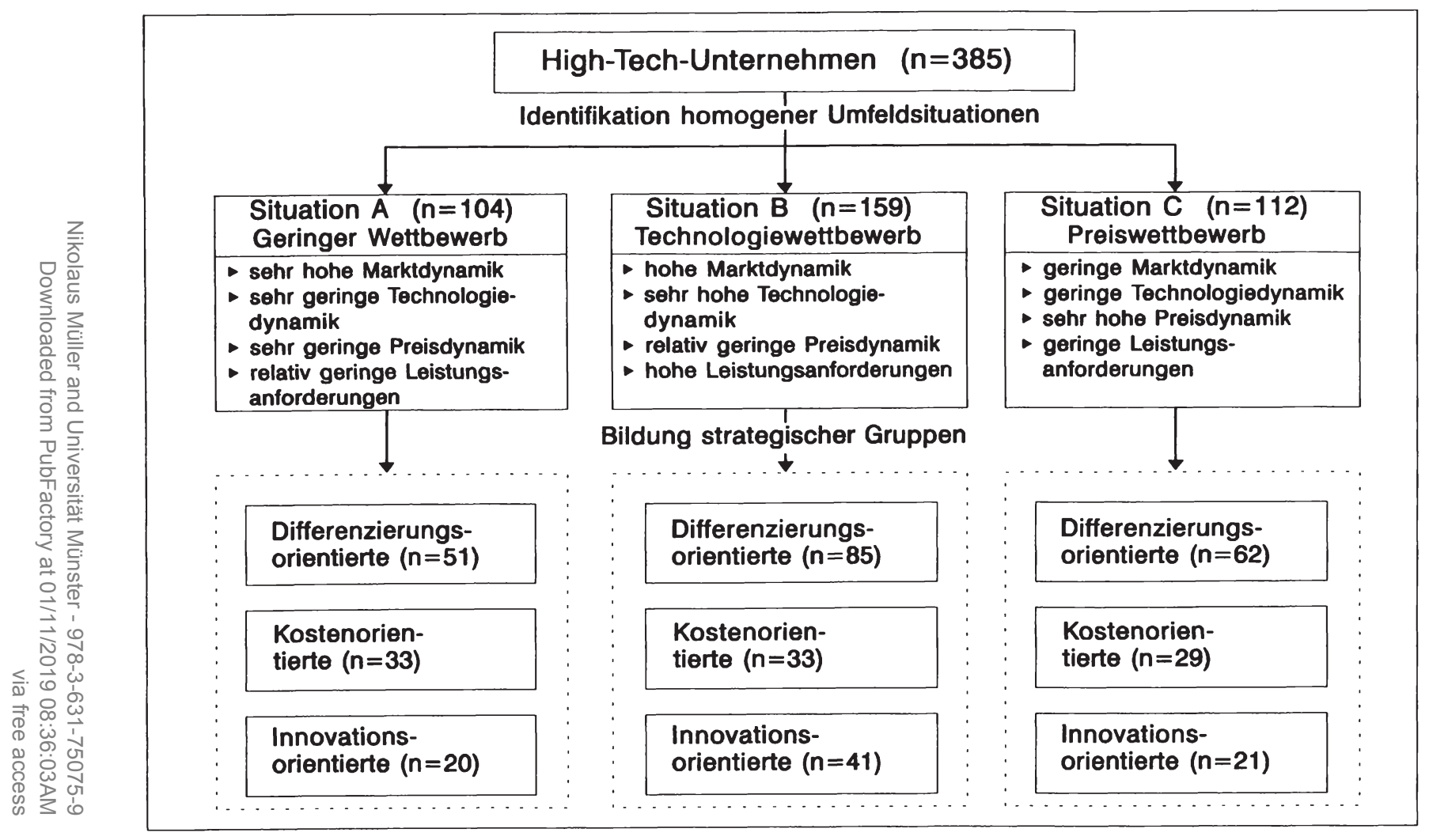

Abb. 27: Übersicht der einzelnen High-Tech-Gruppen in den einzelnen Umfeldsituationen 


\section{Strategische Gruppe 1: Differenzierungsorientierte $(\mathrm{n}=\mathbf{5 1})$}

Die Unternehmen dieser strategischen Gruppe messen der Marktdurchdringung sowie der Markt- und Produktentwicklung als Dimensionen der Marktfeldbearbeitung einen überdurchschnittlichen Stellenwert bei. Es fällt auf, daß primär die Erreichung einer Qualitäts-, Service-, Image- und Nutzen-Führerschaft im Vordergrund der Strategieausrichtung steht. Weiterhin ist die Technologieorientierung hoch ausgeprägt. Demgegenüber besitzt die Preis/Kostenorientierung für diese High-Tech-Unternehmen eine stark unterdurchschnittliche Bedeutung. Bei Produktinnovationen nehmen "Differenzierungsorientierte" eine klare Pionierposition ein. Es wird insgesamt deutlich, daß die High-Tech-Unternehmen der ersten strategischen Gruppe insbesondere die beiden Basisstrategien produktbezogene Innovationsstrategie und differenzierte Profilierungsstrategie miteinander kombinieren.

\section{Strategische Gruppe 2: Kostenorientierte $(n=33)$}

In der zweiten strategischen Gruppe ist bei der Marktfeldstrategie die Strategievariable Marktpenetration überdurchschnittlich ausgeprägt, während die übrigen strategischen Verhaltensdimensionen vernachlässigt werden. Es zeigt sich, daß der Strategieschwerpunkt für die Unternehmen dieses Strategieclusters in der Preis-/Kostenorientierung liegt. Im Vergleich dazu sind die übrigen Differenzierungsdimensionen von sehr geringer Relevanz. "Kostenorientierte" treten mit Produktneuerungen vor allem als früher und später Folger in den Markt ein. Zusammenfassend bleibt festzuhalten, daß für Unternehmen der zweiten strategischen Gruppe allein die Basisstrategie der preisbezogenen Profilierung von besonderer Bedeutung ist. Darüber hinaus wird deutlich, da $\beta$ in High-Tech-Märkten die Strategieoption der frühen Folgerschaft mit der Preis-/Kosten-Führerschaft kombiniert wird. 


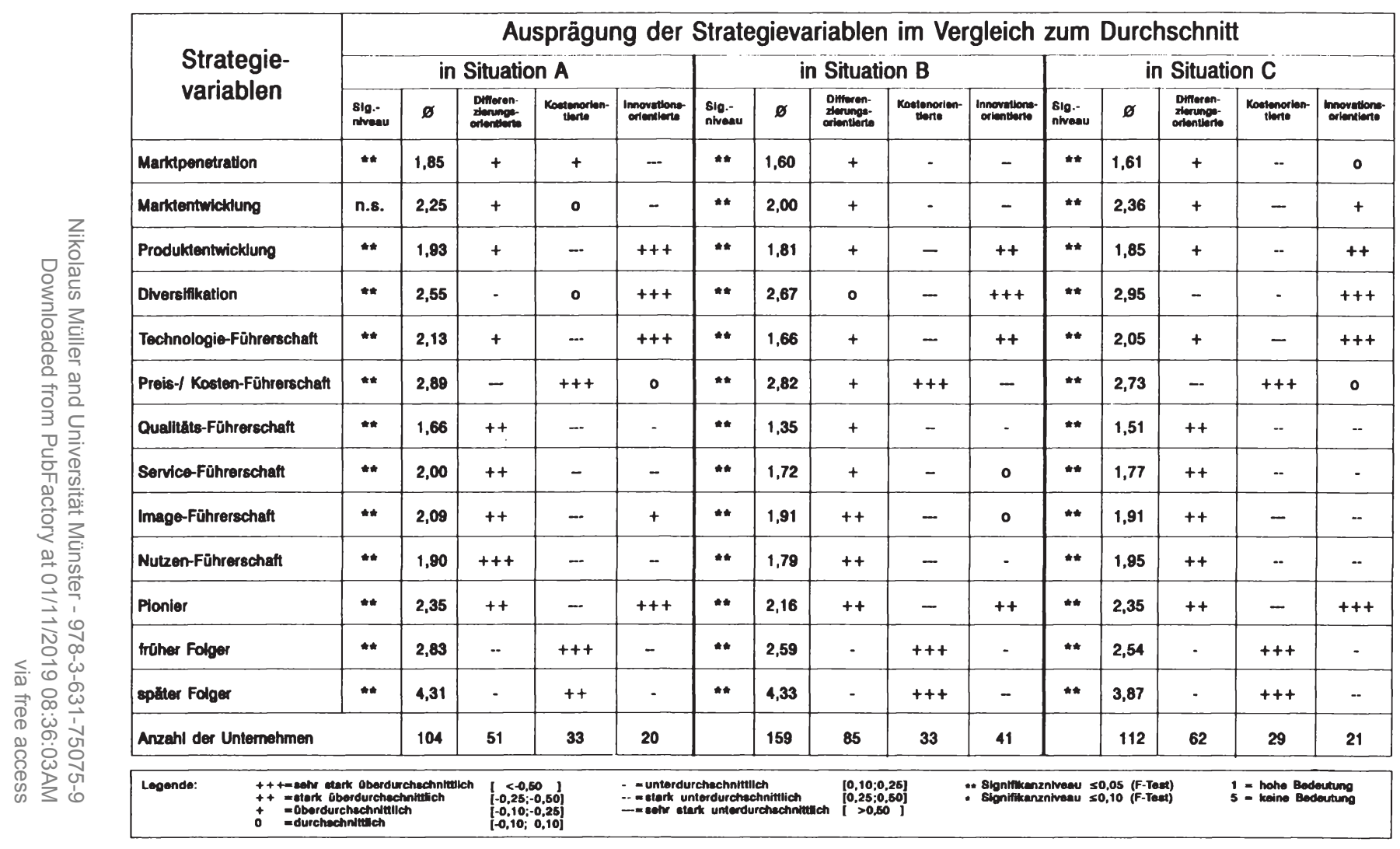

Abb. 28: Beschreibung der strategischen High-Tech-Gruppen in den Umfeldsituationen 


\section{Strategische Gruppe 3: Innovationsorientierte $(n=20)$}

Innerhalb der Marktfeldbearbeitung sehen "Innovationsorientierte" die Produktentwicklung und die Diversifikation als besonders wichtig an. Demgegenüber sind die Marktdurchdringung und die Marktentwicklung von stark untergeordneter Bedeutung. Gegenüber den "Differenzierungsorientierten" streben die High-Tech-Unternehmen des dritten Strategieclusters in deutlich stärkerem Maße eine Technologie-Führerschaft an. Die Innovationsausrichtung kommt zudem in der überdurchschnittlichen Ausprägung der Produktentwicklung und der Pionierorientierung zum Ausdruck. Den restlichen wettbewerbs- und timingbezogenen Strategiedimensionen wird meist ein geringer Stellenwert zugesprochen. Es ist insgesamt zu erkennen, daß bei Unternehmen dieser strategischen Gruppe die produktbezogene Innovationsstrategie eine zentrale strategische Grundhaltung darstellt. Im Hinblick auf die Basisstrategie der marktbezogenen Innovation läßt sich feststellen, daß die innovationsorientierten High-Tech-Unternehmen lediglich der strategischen Verhaltensdimension Diversifikation eine besondere Bedeutung beimessen, während eine Marktentwicklung eher von differenzierungsorientierten Unternehmen verfolgt wird.

Eine Betrachtung der Kontextsituationen A, B und C zeigt, daß sich in jeder Situation vergleichbare strategische High-Tech-Gruppen identifizieren lassen, die entweder stärker differenzierungs-, kosten- oder innovationsorientiert ausgerichtet sind. Eine Überprüfung der Hypothese $\mathrm{H} \mathrm{St2}$, die besagt, daß sich die strategischen Gruppen bezüglich der von ihnen verfolgten Strategieschwerpunkte in den einzelnen homogenen Kontextsituationen unterscheiden, erfolgte unter Zuhilfenahme der Diskriminanzanalyse ${ }^{234}$ und der Mittelwertvergleichstests ${ }^{235}$. Die im Rahmen der Diskriminanzanalyse berechneten Prüfkriterien zur Beurteilung der

Vgl. Meffert, H., Marketingforschung und Käuferverhalten, a.a.O., S. 279 ff.; Lewis, P., Thomas, H., The linkage between strategy, strategic groups and performance in the U.K. Retail Grocery Industry, in: Strategic Management Journal, Vol. 11 (1990), S. 390 ff.; Bleymüller, J., Multivariate Analyse für Wirtschaftswissenschaftler, a.a.O., S. 228 ff.; Aaker, D.A., Day, G.S., Marketing Research, a.a.O., S. $466 \mathrm{ff}$.

Vgl. Backhaus, K. et al., Multivariate Analysemethoden, a.a.O., S. 56 ff. u. 309 ff.; Hartung, J., Statistik, a.a.O., S. 609 ff.; Bleymüller, J., Gehlert, G., Gülicher, H., Statistik für Wirtschaftswissenschaftler, a.a.O., S. $115 \mathrm{ff}$. 
Trennschärfe der strategischen High-Tech-Gruppen sind in Abbildung 29 dargestellt.

Die Klassifikationsergebnisse der Diskriminanzanalyse sind sehr zufriedenstellend, da die High-Tech-Unternehmen den strategischen Gruppen in allen drei Situationen mit über $96 \%$ richtig zugeordnet werden. ${ }^{236}$ Die gute Trennqualität der ermittelten Diskriminanzfunktionen wird durch die relativ niedrigen Wilks' Lambda Werte unterstrichen. Die hohen kanonischen Korrelationskoeffizienten zeigen, daß zwischen der Diskriminanzfunktion und den Gruppierungsvariablen eine deutliche Beziehung besteht. Der Chi-Quadrat-Test verdeutlicht, daß sich die strategischen Gruppen in den einzelnen Umfeldsituationen mit einem $\alpha<0,0001$ signifikant unterscheiden. ${ }^{237}$

\begin{tabular}{|l|l|l|l|}
\hline Prüfkriterien & Sit. A & Sit. B & Sit. C \\
\hline richtige & $96 \%$ & $96 \%$ & $98 \%$ \\
Klassifikation & & & \\
\hline \multirow{2}{*}{ Wilks' Lambda } & 0,1855 & 0,1593 & 0,1335 \\
\cline { 2 - 4 } & 0,5397 & 0,5191 & 0,5283 \\
\hline \multirow{2}{*}{$\begin{array}{l}\text { kanonische } \\
\text { Korrelationskoeff. }\end{array}$} & 0,8102 & 0,8326 & 0,8645 \\
\cline { 2 - 4 } Chi-Quadrat-Test & $\alpha<0,01$ & 0,6934 & 0,6868 \\
\hline
\end{tabular}

Abb. 29: Beurteilung der Trennschärfe der strategischen

High-Tech-Gruppen anhand ausgewählter Prüfkriterien

236 Die Ergebnisse der diskriminatorischen Klassifikation der strategischen Gruppen in den Situationen A, B und C sind dem Anhang II der Arbeit zu entnehmen. Die Zufallswahrscheinlichkeiten ohne Zurücklegen betragen jeweils $33 \%$. Bei Berücksichtigung der Größenunterschiede zwischen den strategischen High-Tech-Gruppen in den Situationen A, B und C ergeben sich Wahrscheinlichkeiten ohne Zurücklegen von $49 \%$ für Differenzierungsorientierte, von $32 \%$ für Kostenorientierte und von $19 \%$ fur Innovationsorientierte in Situation A und entsprechend $53 \%, 21 \%$ bzw. $26 \%$ in Situation B und 55 $\%, 26 \%$ bzw. $19 \%$ in Situation C. In beiden Fällen sind die vorliegenden Klassifikationsergebnisse als sehr zufriedenstellend zu bezeichnen. Siehe hierzu z.B. Backhaus, K., et al., Multivariate Analysemethoden, a.a.O., S. 116.

237 Die Diskriminanzfunktionen, die Werte für Wilks' Lambda bzw. die kanonischen Korrelationskoeffizienten und die Ergebnisse der Chi-Quadrat-Tests sind dem Anhang II der Arbeit zu entnehmen. 
Innerhalb der Mittelwertvergleichstests bestätigt der F-Test, daß die in der Clusteranalyse einbezogenen strategischen Verhaltensdimensionen in den Situationen A, B und C bis auf die Strategievariable Marktentwicklung in Situation A mit einem $\alpha<0,05$ signifikant trennen. ${ }^{238}$ Der durchgefuihrte Scheffé-Test verdeutlicht weiterhin, daß zwischen den identifizierten strategischen Gruppen auch bezüglich der marktfeld-, wettbewerbs- und timingbezogenen Strategiedimensionen signifikante Unterschiede bestehen. ${ }^{239}$ Aufgrund der aufgezeigten Ergebnisse ist die Hypothese $\mathrm{H} \mathrm{St} 2$ anzunehmen.

Die situationsübergreifende Betrachtung hatte gezeigt, daß die strategischen High-Tech-Gruppen in den Umfeldsituationen A (geringer Wettbewerb), B (Technologiewettbewerb) und C (Preiswettbewerb) ähnliche Strategieschwerpunkte aufweisen. In diesem Zusammenhang ist es von Interesse, zu untersuchen, inwieweit das strategische Verhalten der High-Tech-Unternehmen durch die jeweilige Umfeldsituation beeinflußt wird.

Es wird hierbei deutlich, daß die jeweiligen strategischen Gruppen in den Umfeldsituationen A, B und C grundsätzlich gleichartige Strategieschwerpunkte verfolgen (vgl. Abbildung 28). "Differenzierungsorientierte" streben insbesondere in den Strategiedimensionen Qualität, Service, Image und Nutzen nach einer Führerschaft. Die Technologie- und Pionierorientierung sowie die Produktentwicklung sind ebenfalls von Bedeutung, jedoch im Vergleich zu den "Innovationsorientierten" deutlich geringer ausgeprägt. Darüber hinaus werden die strategischen Verhaltensvariablen Marktpenetration und Marktentwicklung als wichtig eingeschätzt. Es läßt sich hieraus schließen, daß die Unternehmen dieser strategischen Gruppe in allen drei Situationen eine Wettbewerbsprofilierung in bestehenden und neuen High-Tech-Märkten durch den Aufbau von Differenzierungsvorteilen über eine starke Nutzen-, Qualitäts-, Service- und Imageorientierung sowie durch eine produktbezogene Innovationsstrategie anstreben.

$\mathrm{Zu}$ den Ergebnissen des F-Tests für die strategischen High-Tech-Gruppen in den Situationen A, B und C vergleiche die Abbildung 28 der Arbeit. in den Situationen A, B und C sind im Anhang I der Arbeit zu finden. 
Bei der strategischen Gruppe der kostenorientierten Unternehmen konzentriert sich das wettbewerbsstrategische Verhalten auf die Erreichung einer Preis/Kosten-Führerschaft, die mit einer innovationsbezogenen Folgerposition einhergeht. Im Vordergrund der Strategieausrichtung der strategischen Gruppe der "Innovationsorientierten" steht eine deutliche Technologie- und Pionierorientierung, die mit einer ausgeprägten Produktentwicklung und Diversifikation verbunden ist. Der Zusammenhang der strategischen Verhaltensdimensionen deutet darauf hin, daß Unternehmen, die in High-Tech-Märkten eine technologische Führerschaft anstreben und an der Entwicklung neuer Produktleistungen arbeiten, beabsichtigen, diese Differenzierungspotentiale über eine Pionierposition in vorhandenen und neuen Absatzmärkten frühzeitig auszunutzen.

Im Rahmen der Untersuchung der differenzierungs-, kosten- und innovationsorientierten High-Tech-Unternehmen in den Kontextsituationen A, B und C ist auch $\mathrm{zu}$ erkennen, daß die vergleichbaren strategischen Gruppen bezüglich einzelner strategischer Verhaltensdimensionen starke Unterschiede aufweisen (vgl. Abbildung 28). ${ }^{240}$ So wird die Bedeutung der Technologie-Führerschaft und die Pionierorientierung von den "Differenzierungsorientierten" in Situation B aufgrund des sehr starken Technologiewettbewerbs deutlich wichtiger eingeschätzt als in den beiden anderen Situationen. Die Erreichung einer Nutzen-Führerschaft besitzt in Situation C, die durch eine sehr hohe Preisdynamik geprägt ist, einen geringeren Stellenwert als in den Situationen A und B. Bei der strategischen Gruppe der kostenorientierten Unternehmen sind die Preis-/Kostenorientierung und der Markteintritt als später Folger in Situation C von größerer Bedeutung als in den beiden anderen Umfeldsituationen. Bei den "Innovationsorientierten" ist festzustellen, daß sie der Produktentwicklung in der Situation A eine höhere Relevanz beimessen als in den Situationen B und C.

Um die Stärke der Unterschiede zwischen den vergleichbaren strategischen Gruppen in High-Tech-Märkten insgesamt besser beurteilen zu können, wurden

240 Siehe hierzu auch die Tabelle $\mathrm{zu}$ den absoluten Mittelwertabweichungen der einzelnen Strategiedimensionen der strategischen High-Tech-Gruppen in den Situation A, B und C im Anhang II der Arbeit. 
die Strategiedimensionen zu den drei Profilierungsfaktoren Differenzierungs-, Technologie- und Preisvorteil verdichtet. ${ }^{241}$ Die Abbildung 30 zeigt die strategischen Verhaltensgruppen in den Situationen A, B und C, die in einem durch die Profilierungsfaktoren aufgespannten dreidimensionalen Raum eingeordnet worden sind. Es wird hierbei deutlich, daß der Preisvorteil der "Differenzierungsorientierten" in den Situationen B und C deutlich größer ist als in der Situation A. Bei den kostenorientierten High-Tech-Unternehmen ist der Technologievorteil in Situation B im Vergleich zu den übrigen Umfeldsituationen deutlich geringer. Von "Innovationsorientierten" wird ein Differenzierungsvorteil in einem stärkeren Maße in der Situation B als in den Situationen A und C angestrebt.

Es läßt sich zusammenfassend festhalten, daß von den jeweiligen strategischen Gruppen in den verschiedenen Kontextsituationen grundsätzlich gleichartige Strategieschwerpunkte verfolgt werden. Darüber hinaus wurde deutlich, daß bei den vergleichbaren strategischen High-Tech-Gruppen nur zum Teil situationsbedingte Unterschiede im strategischen Verhalten festgestellt werden können. In diesem Zusammenhang ist es für die weitere Untersuchung von Interesse, zu analysieren, ob sich die differenzierungs-, kosten- und innovationsorientierten strategischen High-Tech-Gruppen trotz der geringen Strategieunterschiede dennoch im operativen Verhalten, in den Zielsetzungen und in der Unternehmenskultur in den Situationen A, B und C unterscheiden.

241 Zur Bestimmung der Profilierungsfaktoren wurden die Ausprägungen der jeweiligen Strategiedimensionen aufsummiert und durch die Anzahl der Variablen dividiert. Hierbei wurden der Profilierungsfaktor Innovationsvorteil anhand der Strategievariablen Produktentwicklung, Diversifikation, Technologie-Führerschaft, Pionier und der Faktor Differenzierungsvorteil anhand der Variablen Qualitäts-, Service-, Image-, NutzenFührerschaft, Marktentwicklung sowie der Faktor Preisvorteil anhand der Variablen Marktpenetration, Preis-/Kosten-Führerschaft, früher Folger, später Folger gebildet. 


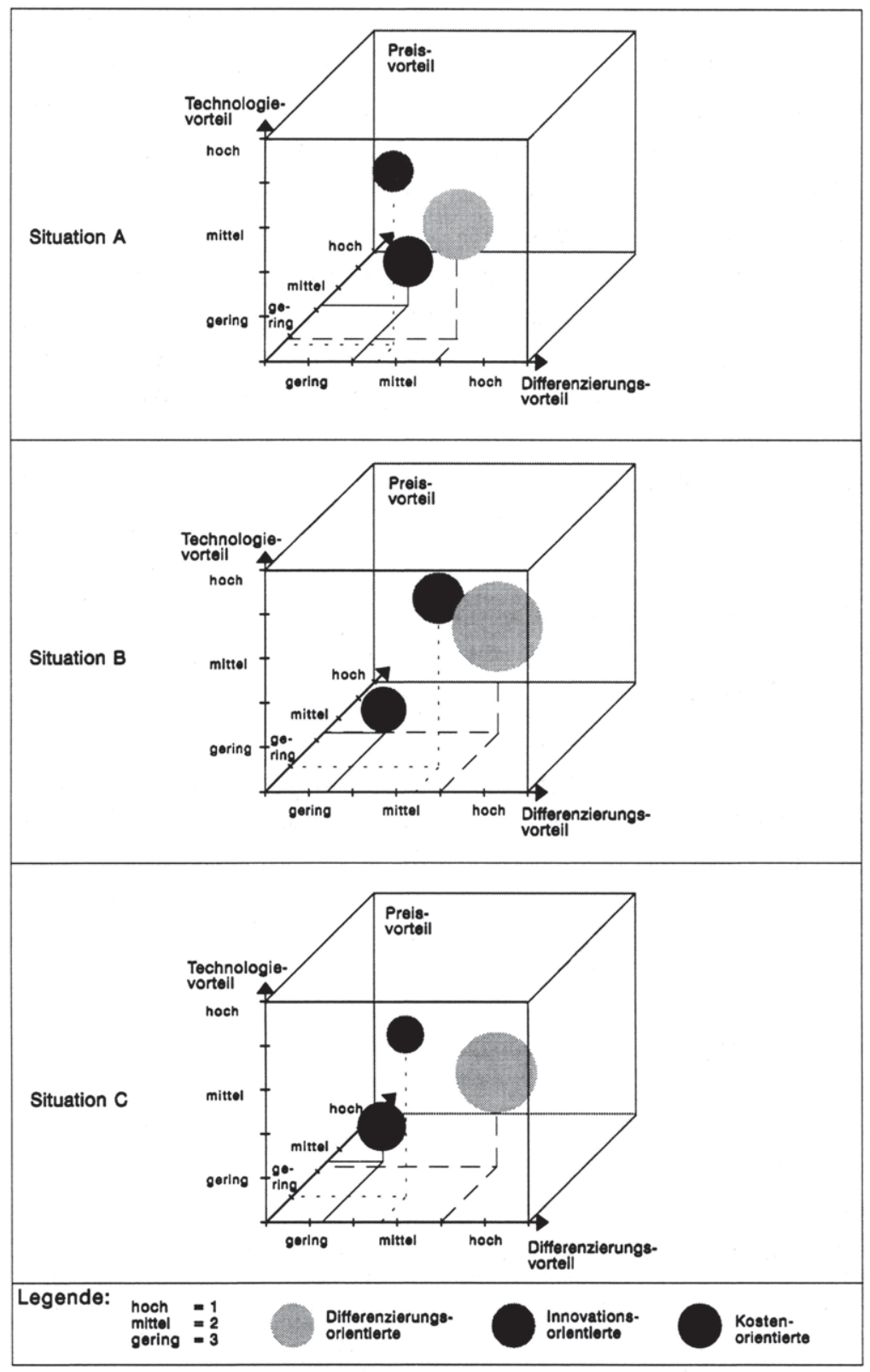

Abb. 30: Darstellung der High-Tech-Gruppen im dreidimensionalen Raum 


\section{Ausgestaltungsformen und Einflußfaktoren der Marketing- strategien von strategischen Gruppen in High-Tech-Märkten}

Im Mittelpunkt der weiteren Betrachtung steht die hypothesengestützte Untersuchung der Ausgestaltungsformen und Einflußfaktoren der Marketingstrategien von strategischen High-Tech-Gruppen in den homogenen Umfeldsituationen. Auf Basis der im empirischen Bezugsrahmen ${ }^{1}$ systematisierten Unternehmensvariablen werden in einem deskriptiven Analyseschritt zunächst die Instrumenteschwerpunkte der strategischen Gruppen hinsichtlich der Marketingmaßnahmen sowie der Maßnahmen zur Verkürzung der Entwicklungszeiten und zur Beschleunigung der Vermarktung untersucht. Hierdurch sollen Erkenntnisse über die operative Umsetzung von Marketingstrategien in High-Tech-Märkten vermittelt werden. Im Anschluß daran erfolgt eine Untersuchung der ziel- und kulturbezogenen Unterschiede der strategischen Verhaltensgruppen, um Erkenntnisse darüber zu erlangen, ob die Unternehmensziele und die Unternehmenskultur Einfluß auf die Bildung strategischer High-Tech-Gruppen haben. In einem abschlieBenden Analyseschritt wird überprüft, welchen Beitrag das Konzept der strategischen Gruppen zur Erklärung der Erfolgsunterschiede in High-Tech-Märkten leistet. $^{2}$

Vgl. hierzu die Abbildung 3 im Teil A Kap. 4 der Arbeit.

2 Die im Rahmen dieses Analyseschrittes einbezogenen Markterfolgsvariablen sind in dem empirischen Bezugsrahmen aufgefuhrt. Siehe hierzu die Abbildung 3 im Teil A Kap. 4 der Arbeit. 


\section{Instrumentelle Ausrichtung der Marketingstrategien von strategischen Gruppen in High-Tech-Märkten}

\subsection{Unternehmensaktivitäten im Marketing-Mix-Bereich}

\subsection{Ansatzpunkte zur Ausgestaltung von Marketingstrategien im Marketing-Mix-Bereich}

Ansatzpunkte zur Ausgestaltung von Marketingstrategien in High-Tech-Märkten lassen sich im Marketing-Mix-Bereich in der Produkt-, Kontrahierungs-, Kommunikations- und Distributionspolitik finden. ${ }^{3}$

Die Produktpolitik umfaßt alle marktgerichteten Aktivitäten, die sich auf die Gestaltung des Leistungsprogramms von Unternehmen beziehen. Von den HighTech-Unternehmen sind innerhalb der Produktpolitik vor allem Entscheidungen hinsichtlich Produktinnovation, Produktpflege, Produktqualität, Serviceleistungen, Benutzerfreundlichkeit und Design technologischer Anwendungen sowie Sortimentstiefe und -breite zu fällen. ${ }^{4}$

In High-Tech-Märkten besitzt die Produktinnovation aufgrund der ausgeprägten Technologiedynamik generell einen hohen Stellenwert. Eine kontinuierliche Produktpflege ist oftmals notwendig, um die bei der Markteinführung von Innovationen bestehenden anwendungstechnischen Probleme zu beheben. Sie bietet

$\mathrm{Zu}$ einer vergleichbaren Sytematisierung der Marketing-Mix-Bereiche siehe auch Kaas, K.P., Marketing-Mix, in: Vahlens Großes Marketinglexikon, Hrsg.: Diller, H., München 1992, S. 682 ff.; Schaible, J., Hönig, A., High-Tech-Marketing in der Praxis, a.a.O., S. 60 ff.; Meffert, H., Konsumgütermarketing, in: Handwörterbuch der Betriebswirtschaftlehre, 5. Aufl., Band II, Hrsg.: Wittmann, et al., Stuttgart 1992, Sp. 2249 ; Meffert, H., Marketing, Grundlagen der Absatzpolitik, a.a.O., S. 116 ff.; Merkle, E., Technologiemarketing: Ein Ansatz zur Bewältigung des technologischen Wandels, a.a.O., S. 9 ff.; Meffert, H., Marketing als Führungsaufgabe, Aspekte des Investitionsgütermarketing, in: Datascope, 1972, Nr. 7, S. 8 ff.

$4 \quad \mathrm{Zu}$ den Bestandteilen der Produktpolitik vgl. Meffert, H., Erfolgreiches Marketing in der Rezession, a.a.O., S. 92 ff.; Kotler, Ph., Bliemel, F., Marketing-Management, a.a.O., S. 621 ff.; Meffert, H., Marketing, Grundlagen der Absatzpolitik, a.a.O., S. 361 ff. 
zudem die Möglichkeit, frühzeitig Veränderungen in den Nachfragebedürfnissen zu berücksichtigen. ${ }^{5}$

Ansatzpunkte zum Abbau von Kaufwiderständen auf der Nachfragerseite liegen in der Sicherung einer gleichbleibend hohen Produktqualität und in der Bereitstellung geeigneter Serviceleistungen. Die technologische und leistungsbezogene Unsicherheit von High-Tech-Anwendern läßt sich hierbei vor allem durch das Angebot spezieller Pre-Sale-Services wie z.B. Problemanalysen, technische, organisatorische oder finanzielle Beratungen sowie After-Sale-Services wie beispielsweise Implementierung der Produktanwendungen im Gesamtsystem oder Einführungsschulungen verringern. Darüber hinaus kommt den Servicemaßnahmen aufgrund der Beratungs- und Erklärungsbedürftigkeit vieler High-TechProdukte eine besondere Bedeutung zu. ${ }^{6}$

Von den High-Tech-Nachfragern werden oftmals hohe Anforderungen an die Benutzerfreundlichkeit von High-Tech-Produkten gestellt. Als Ansatzpunkte zur Verbesserung der Benutzerfreundlichkeit sind u.a. eine mögliche Leistungsanpassung an individuelle Kundenbedürfnisse, eine ergonomische Handhabung, eine hohe Robustheit bei Bedienungsfehlern sowie klar strukturierte und leicht verständliche Dokumentationen zu nennen. ${ }^{7}$ Ein unverwechselbares und gut einprägsames produktbezogenes Design dient insbesondere der leichteren Wiedererkennung und der besseren optischen Differenzierung gegenüber Konkurrenzprodukten. $^{8}$

Vgl. hierzu und zu den folgenden Darstellungen auch die Ausfuihrungen in Teil B Kap. 2.1 und 2.3 der Arbeit.

6

Vgl. Backhaus, K., Investitionsgütermarketing, a.a.O., S. 257 f.; Meffert, H., Programmierte Flops im Technologiemarketing, in: HighTech, 1990, Nr. 9, S. 56; Meffert, H., Kundendienstpolitik, a.a.O., S. 94. Zur Kennzeichung von Pre-Sale- und After-SaleServices siehe z.B. Bauche, K., Segmentierung von Kundendienstleistungen auf investiven Märkten, a.a.O., S. 7 f.

Vgl. Baaken, Th., Launen, M., Software-Marketing, a.a.O., S. $129 \mathrm{ff}$.

8 Vgl. Willumeit, K., Heike, G., Strategische CI-Entwicklung für Unternehmen mit Technologieprodukten am Beispiel der SIHI-Gruppe, in: Technologie-Marketing, Hrsg.: Töpfer, A., Sommerlatte, T., Landsberg a.L. 1991, S. 256 f.; Englert, W.H., Marketing von Standard-Anwendungssoftware, München 1977, S. $146 \mathrm{ff}$. 
Im Hinblick auf die Sortimentsbreite ist die Anzahl der Produktlinien von HighTech-Unternehmen festzulegen. Das Produktprogramm eines Computerherstellers kann sich beispielsweise aus den Produktlinien Großrechner, Personal Computer und Laptop zusammensetzen. Durch die Sortimentstiefe wird die Anzahl der Produkttypen innerhalb einer Produktlinie bestimmt, z.B. bei Personal Computern durch Rechner mit einem 386er-, 486er- oder 586er-Prozessor. Vor dem Hintergrund der hohen F\&E-Aufwendungen und der dynamischen Entwicklungen in High-Tech-Märkten ist die sortimentsbezogene Ausrichtung eines Unternehmens ständig kritisch zu hinterfragen. Das Angebot einer überschaubaren Anzahl von Produkttypen trägt auch dazu bei, daß die High-Tech-Nachfrager nicht durch eine Vielzahl ähnlicher Leistungsangebote verunsichert werden und damit zusätzliche Kaufwiderstände entstehen. ${ }^{9}$

Wenn die Produkt- und Serviceleistungen von High-Tech-Unternehmen keine Differenzierungspotentiale bieten, rücken häufig kontrahierungspolitische Maßnahmen in den Vordergrund des operativen Marketingverhaltens. Die Marketingstrategien werden hierbei vor allem durch Entscheidungen über die Preisund Konditionengestaltung der Leistungsangebote konkretisiert. ${ }^{10}$

Bei der Preisbestimmung ist einerseits darauf zu achten, daß ein ausgewogenes Preis-/Leistungsverhältnis besteht. Andererseits sind die Absatzpreise in Abhängigkeit von der Preisbereitschaft der High-Tech-Nachfrager festzulegen. So lassen sich beispielsweise bei individuellen Softwarelösungen im Vergleich zu Standardsoftware-Produkten i.d.R. höhere Preisforderungen durchsetzen. ${ }^{11}$

9 Vgl. Meffert, H., Marketing-Management, a.a.O., S. 214; Backhaus, K., Investitionsgütermarketing, a.a.O., S. 256. Zur Kennzeichnung der Begriffe Sortimentsbreite und tiefe siehe z.B. Meffert, H., Marketing, Grundlagen der Absatzpolitik, a.a.O., S. $361 \mathrm{ff}$.

Vgl. Meffert, H., Programmierte Flops im Technologiemarketing, a.a.O., S. 56. Zu den Bestandteilen der Kontrahierungspolitik siehe Meffert, H., Erfolgreiches Marketing in der Rezession, a.a.O., S. 116 ff.; Backhaus, K., Investitionsgütermarketing, a.a.O., S. 296 ff.; Meffert, H., Marketing, Grundlagen der Absatzpolitik, a.a.O., S. 118 f., Simon, H., Preismanagement, 2. Aufl., Wiesbaden 1992, S. 12 ff.

11 Vgl. Baaken, Th., Launen, M., Software-Marketing, a.a.O., S. 150 ff.; Backhaus, K., Investitionsgütermarketing, a.a.O., S. 296 ff.; Shanklin, W.L., Ryans, J.K., Essentials of marketing high technology, a.a.O., S. $258 \mathrm{ff}$. 
Von den High-Tech-Unternehmen ist bei der Preisfestsetzung außerdem zu entscheiden, ob ein "Preisbündel" oder ein "Preisbaukasten" vermarktet wird. Im ersten Fall werden mehrere technologiebezogene Produkt- und Serviceleistungen zusammengefaßt und zu einem Preis angeboten, der unterhalb der Summe der Einzelpreise liegt. Ein Vorteil der "Preisbündelung" besteht darin, da $ß$ es den High-Tech-Kunden schwer fällt, Referenzpreise über die Einzelleistungen zu bilden und dadurch der preispolitische Spielraum für die High-Tech-Anbieter größer wird. ${ }^{12}$ Bei der Bildung von "Preisbaukästen" setzt sich das Gesamtangebot (z.B. Personal Computer) aus komplementären Teilleistungen zusammen (z.B. Rechnerkomponenten, Monitor, Tastatur), die preislich getrennt ausgewiesen werden. Dies bietet den High-Tech-Unternehmen die Gelegenheit, den Nachfragern den gesamten Leistungsumfang $\mathrm{zu}$ verdeutlichen und gleichzeitig diejenigen Teilleistungen besonders herauszustellen, bei denen ein Wettbewerbsvorteil besteht. ${ }^{13}$

Im Rahmen der Konditionengestaltung werden von den High-Tech-Unternehmen Rabatte, Absatzkredite und Zahlungsbedingungen spezifiziert. Bei Rabatten handelt es sich um Preisnachlässe fuir bestimmte Leistungen der High-Tech-Abnehmer. Funktionsrabatte werden u.a. für die Übernahme logistischer Aufgaben und Zeitrabatte z.B. zu einer bestimmten Saison gewährt. Mengenrabatte orientieren sich häufig am Umsatzvolumen und Treuerabatte an der Dauer der Zusammenarbeit mit einem Kunden. Die Preisreduzierung über Rabatte dient vor allem dazu, Umfang und Intensität des Kaufverhaltens von High-Tech-Verwendern zu stimulieren. Demgegenüber bildet die Bereitstellung günstiger Absatzkredite und Zahlungsbedingungen eine wichtige Voraussetzung dafür, daß der Kauf von High-Tech-Produkten mit einem größeren Investitionsvolumen überhaupt zu realisieren ist. ${ }^{14}$

12 Vgl. Meffert, H., Erfolgreiches Marketing in der Rezession, a.a.O., S. 122 f.; Simon, H., Preisbündelung, in: ZfB, 1992, Nr. 11, S. 1228 ff.

Vgl. Meffert, H., Erfolgreiches Marketing in der Rezession, a.a.O., S. 123 f.; Diller, H., Preisbaukästen als preispolitische Option, in: WiSt, 1993, Nr. 6, S. 272 ff.; Meffert, H., Marketing, Grundlagen der Absatzpolitik, a.a.O., S. $338 \mathrm{ff}$.

Vgl. Baaken, Th., Launen, M., Software-Marketing, a.a.O., S. 153 f.; Backhaus, K., Investitionsgütermarketing, a.a.O., S. 298 ff.; Meffert, H., Marketing, Grundlagen der Absatzpolitik, a.a.O., S. $346 \mathrm{ff}$. 
Die High-Tech-Unternehmen beschäftigen sich im Rahmen der Kommunikationspolitik mit der bewußten Gestaltung der absatzmarktgerichteten Informationen zum Zweck der Verhaltensbeeinflussung aktueller und potentieller HighTech-Nachfrager. Persönlicher Verkauf, Werbung, Public Relations, Sponsoring und Verkaufsförderung bilden hierbei wichtige Entscheidungstatbestände. ${ }^{15}$

In High-Tech-Märkten wird dem persönlichen Verkauf aufgrund des hohen wahrgenommenen Investitionsrisikos auf der Nachfragerseite ein besonderer Stellenwert beigemessen. Es ist häufig erst durch einen persönlichen Kontakt zwischen Hersteller und Nachfrager möglich, die individuellen Probleme und Bedürfnisse der High-Tech-Anwender zu erfahren und ihnen die produkt- und servicebezogenen Leistungsvorteile zu demonstrieren. Aus diesen Beratungs- und Verkaufsgesprächen werden auch Informationen bezüglich der Verbesserung der technologischen Leistungsangebote gewonnen. ${ }^{16}$

Public Relations- und Sponsoring-Aktionen bieten den High-Tech-Unternehmen die Gelegenheit, die Unternehmensbekanntheit zu erhöhen und das produkt- bzw. unternehmensbezogene Image zu verbessern. Public Relations kommt weiterhin die Aufgabe zu, die Beziehung zu wichtigen Interessengruppen (z.B. staatlichen Institutionen) zu pflegen. Die High-Tech-Anbieter haben hierdurch die Möglichkeit, frühzeitig Informationen über Veränderungen der unternehmensrelevanten

15 Zu den Bestandteilen der Kommunikationspolitik vgl. Meffert, H., Integrierte Marktkommunikation, unver. Skript, Münster 1991, S. 81 ff.; Backhaus, K., Investitionsgütermarketing, a.a.O., S. 280 ff.; Meffert, H., Industriewerbung, in: Technische Mitteilungen, 1972, Nr. 4, S. 155 f. Das Kommunikationsinstrument Messen/Ausstellungen wurde im Rahmen der schriftlichen Befragung nicht erhoben. Es wird daher trotz seiner Bedeutung für die Vermarktung von High-Tech-Produkten nicht näher erläutert.

Vgl. Baaken, Th., Launen, M., Software-Marketing, a.a.O., S. 156 f.; Meffert, H., Programmierte Flops im Technologiemarketing, a.a.O., S. 56.; Meffert, H., Remmerbach, K.-U., Marketingstrategien in jungen Märkten, a.a.O., S. 339; Shanklin, W.L., Ryans, J.K., Essentials of marketing high technology, a.a.O., S. $213 \mathrm{f}$. Zur Unterscheidung der Begriffe Direct Marketing, Direktkommunikation und persönlicher Verkauf siehe z.B. Meffert, H., Integrierte Marktkommunikation, a.a.O., S. 99 ff.; Meffert, H., Marktorientierte Unternehmensfuihrung und Direct Marketing, in: Handbuch Direct Marketing, Hrsg.: Dallmer, H., 6. Aufl., Wiesbaden 1990, S. 38 ff. 
Rahmenbedingungen (z.B. neue gesetzliche Regelungen zur Genforschung) zu erhalten. ${ }^{17}$

Durch die Werbung sollen die potentiellen High-Tech-Nachfrager über den Nutzen der technologischen Leistungsangebote informiert werden. In diesem Zusammenhang werden i.d.R. hohe Anforderungen an die Gestaltung der Werbebotschaft gestellt, da der Anwendungsnutzen von High-Tech-Produkten oftmals schwer zu erklären ist. Die High-Tech-Unternehmen werden deshalb vor die Herausforderung gestellt, die wichtigsten produktspezifischen Leistungsmerkmale $\mathrm{zu}$ identifizieren und in einer prägnanten und leicht verständlichen Form zu übermitteln. Eine wesentliche Zielsetzung ist hierbei, über eine einprägsame Werbung in das "Evoked Set" der High-Tech-Verwender zu gelangen und dadurch bei Kaufentscheidungen vorrangig berücksichtigt zu werden. ${ }^{18}$

Mit dem Einsatz von Verkaufsförderungsmaßnahmen in High-Tech-Märkten wird primär versucht, eine vorübergehende Absatz- bzw. Umsatzsteigerung durch Schaffung zusätzlicher Anreize für die Außendienstmitarbeiter, den Handel und die Nachfrager $\mathrm{zu}$ realisieren. Im Vordergrund der verkaufspersonal- und handelsbezogenen Verkaufsförderung stehen Verkaufshilfen (z.B. Computer Aided-Selling-Systeme) und leistungsbezogene Anreize (z.B. Geld- und Sachprämien). Nachfragebezogene Verkaufsförderungsaktionen lassen sich i.d.R. für weniger erklärungsbedürftige High-Tech-Produkte (z.B. Personal Computer, Kopierer, Drucker) durchführen. ${ }^{19}$

17 Vgl. Backhaus, K., Investitionsgütermarketing, a.a.O., S. 281; Bruhn, M., Sponsoring als Instrument der Unternehmenskommunikation, Erscheinungsformen, Planungskonzepte und Integrationsaspekte, in: Sponsoring, Dokumentationspapier Nr. 55 der Wissenschaftlichen Gesellschaft für Marketing und Unternehmensfuihrung e.V., Hrsg.: Meffert H., Wagner, H., Münster 1989, S. 3 ff.; Meffert, H., Marketing, Grundlagen der Absatzpolitik, a.a.O., S. $493 \mathrm{ff}$.

Vgl. Rieger, G., Die kommunikative Gestaltung des "Markenauftritts" von I+T-Produkten, in: Investitionsgüter- und High-Tech-Marketing, Hrsg.: Hofmaier, R., Landsberg a.L. 1992, S. 422 ff.; Backhaus, K., Investitionsgütermarketing, a.a.O., S. 280 ff. u. 425 ff.; Meffert, H., Integrierte Marktkommunikation, a.a.O., S. 81 ff.; Shanklin, W.L., Ryans, J.K., Essentials of marketing high technology, a.a.O., S. 209 ff.; Rexroad, R.A., High Technology Marketing Management, New York 1983, S. 199 ff. 
Die Distributionspolitik beinhaltet sämtliche Aktivitäten, die sich auf den Weg eines Produktes vom Hersteller zum Kunden beziehen. Von den High-TechUnternehmen werden dabei im Marketingbereich Entscheidungen über die Gestaltung der Absatzwege (Absatzkanäle) und der logistischen Systeme getroffen. $^{20}$

Bei der Wahl der Absatzwege in High-Tech-Märkten kommt dem direkten Vertrieb eine besondere Bedeutung zu. Aufgrund der Komplexität und der Erklärungsbedürftigkeit vieler technologischer Leistungsangebote ist eine regelmäßige Interaktion mit den High-Tech-Kunden notwendig. Außerdem bildet der direkte Vertrieb eine wichtige Voraussetzung dafür, daß eine enge Kundenbindung und damit eine hohe Firmentreue aufgebaut werden kann. ${ }^{21}$ Ein indirekter Vertrieb bietet sich beispielsweise für Unternehmen an, die weniger anspruchsvolle bzw. relativ preiswerte High-Tech-Produkte (z.B. Mobil-Telefone, Fax-Geräte) verkaufen. ${ }^{22} \mathrm{Zu}$ diesem Zweck werden selbständige Absatzmittler eingeschaltet. ${ }^{23}$

Die High-Tech-Anbieter haben durch entsprechende Maßnahmen in den logistischen Systemen dafür zu sorgen, daß die technologischen Leistungen in der gewünschten Spezifikation und Menge zum richtigen Zeitpunkt den Kunden

S. 492. Für eine ausführliche Kennzeichnung der verkaufspersonal-, handels-, und nachfragerorientierten Verkaufsförderung siehe z.B. Meffert, H., Integrierte Marktkommunikation, a.a.O., S. $95 \mathrm{ff}$.

$\mathrm{Zu}$ den Bestandteilen der Distibutionspolitik vgl. Meffert, H., Erfolgreiches Marketing in der Rezession, a.a.O., S. 160 ff.; Nieschlag, R., Dichtl, E., Hörschgen, H., Marketing, a.a.O., S. 370 ff.; Shanklin, W.L., Ryans, J.K., Essentials of marketing high technology, a.a.O., S. 246 ff.; Meffert, H., Marketing, Grundlagen der Absatzpolitik, a.a.O., S. 421 ff.

Vgl. Baaken, Th., Launen, M., Software-Marketing, a.a.O., S. 141 ff.; Backhaus, K., Investitionsgütermarketing, a.a.O., S. 263 ff.; Remmerbach, K.-U., Markteintrittsentscheidungen, a.a.O., S. 97 f.; Meffert, H., Marketing, Grundlagen der Absatzpolitik, a.a.O., S. $425 \mathrm{ff}$.

Vgl. Baaken, Th., Launen, M., Software-Marketing, a.a.O., S. 144 ff.; Backhaus, K., Investitionsgütermarketing, a.a.O., S. 265 ff.; Stratz, R., Erfolgsfaktoren im PC-Handel und Möglichkeiten der Zusammenarbeit mit Herstellern, in: Investitionsgüter- und HighTech-Marketing, Hrsg.: Hofmaier, R., Landsberg a.L. 1992, S. 557 ff.; Merkle, E., Technologiemarketing: Ein Ansatz zur Bewältigung des technologischen Wandels, a.a.O., S. $12 \mathrm{f}$.

$\mathrm{Zu}$ unterschiedlichen Formen des indirekten Vertriebs siehe z.B. Meffert, H., MarketingManagement, a.a.O., S. $176 \mathrm{ff}$. 
erreichen. Von den Unternehmen sind hierzu vor allem die Transportmittel und dienstleister sowie Art und Standort der Lagerhaltung festzulegen. ${ }^{24}$ Die spezifische Ausgestaltung der logistischen Systeme wird unter Berücksichtigung der von den High-Tech-Nachfragern bezüglich der Lieferzuverlässigkeit und -genauigkeit gestellten Anforderungen und unter Kosten-/Nutzen-Gesichtspunkten vorgenommen.

Vor dem Hintergrund der im Überblick dargestellten Möglichkeiten der instrumentellen Ausrichtung von High-Tech-Anbietern stellt sich die Frage, in welcher Form die Marketingstrategien der strategischen Gruppen im operativen Unternehmensverhalten umgesetzt werden und welche Instrumentekombinationen hierbei gewählt werden. In diesem Zusammenhang wird folgende Basishypothese formuliert:

H MM1 Die Marketingstrategien von strategischen Gruppen in High-TechMärkten werden in den Marketingmaßnahmen umgesetzt. Dabei unterscheiden sich die strategischen Gruppen hinsichtlich der von ihnen durchgeführten Marketingmaßnahmen innerhalb einer Umfeldsituation.

Auf der Grundlage der empirischen Ergebnisse zu den Marketingstrategien der strategischen High-Tech-Gruppen in den homogenen Umfeldsituationen sollen im weiteren folgende Basishypothesen überprüft werden:

H MM2 In den verschiedenen homogenen Umfeldsituationen in High-TechMärkten lassen sich bei den vergleichbaren strategischen Gruppen ähnliche Instrumentekombinationen identifizieren.

H MM3 Bei den vergleichbaren strategischen Gruppen, die in den verschiedenen homogenen Umfeldsituationen in High-Tech-Märkten ähnliche Instrumentekombinationen realisieren, lassen sich situationsbedingte Unterschiede im operativen Marketingverhalten feststellen. 


\subsection{Empirische Analyse der Marketingmaßnahmen von strategischen Gruppen}

In den folgenden Ausführungen werden die Marketingmaßnahmen der strategischen High-Tech-Gruppen zunächst in der Umfeldsituation A gekennzeichnet. Im Anschluß daran werden in einer situationsübergreifenden Analyse die Gemeinsamkeiten und Unterschiede der Instrumenteschwerpunkte der vergleichbaren strategischen Gruppen in High-Tech-Märkten erläutert. Zur Beschreibung der strategischen Gruppen in den verschiedenen Umfeldsituationen werden die in Abbildung 31 gezeigten Mittelwertabweichungen der Instrumentevariablen herangezogen und dabei die Besonderheiten in den Ausgestaltungsformen der Marketinginstrumente dargelegt. ${ }^{25}$

In der Situation A messen die differenzierungsorientierten High-Tech-Anbieter der Produktpolitik eine hohe Bedeutung bei. So sind die Benutzerfreundlichkeit und der Service stark überdurchschnittlich und die Produktinnovation, die Produktpflege, die Produktqualität, das Design sowie die Sortimentstiefe/-breite überdurchschnittlich ausgeprägt. Im Vergleich dazu besitzen die kontrahierungspolitischen Maßnahmen Preis und Konditionen einen untergeordneten Stellenwert. Für die Unternehmen dieser strategischen Gruppe sind weiterhin alle kommunikationspolitischen Maßnahmen (Werbung, Verkaufsförderung, Public Relations, Sponsoring) mit Ausnahme des persönlichen Verkaufs relevant. Innerhalb der Distributionspolitik werden darüber hinaus die Absatzwegegestaltung und logistische Marketingaktivitäten als überdurchschnittlich wichtig eingeschätzt.

Trotz des geringen Preiswettbewerbs in Situation A steht für die kostenorientierten Unternehmen primär die Kontrahierungspolitik im Vordergrund des Marketing-Mix. Die Preis- und Konditionengestaltung werden hierbei als überdurchschnittlich wichtig beurteilt. Von den kostenorientierten High-Tech-Anbie- 


\begin{tabular}{|c|c|c|c|c|c|c|c|c|c|c|c|c|c|c|c|}
\hline \multirow{2}{*}{$\begin{array}{l}\text { Marketing- } \\
\text { instrument- } \\
\text { variablen }\end{array}$} & \multicolumn{15}{|c|}{$\begin{array}{l}\text { Ausprägung der Marketi } \\
\text { in Situation A }\end{array}$} \\
\hline & $\begin{array}{l}\text { 819.- } \\
\text { ntrean }\end{array}$ & $\varnothing$ & amponos & Kootenorion & Innovetions- & 819.- & $\varnothing$ & $\begin{array}{l}\text { Oillecen- } \\
\text { cherumea- } \\
\text { orteriterto }\end{array}$ & Koemenorten- & Innoverons- & sig. & $\varnothing$ & $\begin{array}{l}\text { Dimeren } \\
\text { clerveap. } \\
\text { orlentionto }\end{array}$ & $\begin{array}{l}\text { Koolenorten- } \\
\text { thorto }\end{array}$ & $\begin{array}{c}\text { Impovedions. } \\
\text { orteviterts }\end{array}$ \\
\hline Produktpflege & $\bullet$ & 1,86 & + & 0 & -- & n.8. & 1,80 & + & $\mathbf{0}$ & 0 & \# & 1,81 & + & - & + \\
\hline Produktinnovation & - & 1,67 & + & - & ++ & ** & 1,51 & + & - & ++ & $\star$ & 1,84 & + & - & ++ \\
\hline Benutzerfreundlichkeit & 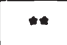 & 2,00 & ++ & - & - & \#* & 1,61 & ++ & $\cdots$ & 0 & $\bullet$ & 1,85 & + & - & + \\
\hline Design & - & 3,02 & + & - & - & $\star$ & 2,57 & + & - & - & n.s. & 2,44 & + & - & 0 \\
\hline Produktqualitât & 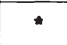 & 1,49 & + & - & - & * & 1,26 & + & - & 0 & * & 1,29 & + & - & 0 \\
\hline Sortimentstiefe/-breite & n.8. & 2,62 & 0 & $\mathbf{0}$ & - & n.8. & 2,62 & + & - & - & * & 2,17 & + & - & - \\
\hline Service & \# & 2,03 & ++ & - & - & $\bullet$ & 1,63 & + & $\cdots$ & - & $\star *$ & 1,72 & ++ & $\cdots$ & 0 \\
\hline Preis & n.s. & 2,33 & - & + & 0 & 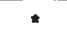 & 1,96 & 0 & + & - & * & 1,87 & 0 & ++ & - \\
\hline Konditionen & n.s. & 2,70 & 0 & + & - & n.s. & 2,36 & 0 & + & - & * & 2,44 & 0 & + & - \\
\hline Werbung & - & 2,67 & + & - & $\cdots$ & - & 2,52 & + & -- & 0 & - & 2,45 & + & - & + \\
\hline Persónllcher Verkauf & n.s. & 1,80 & 0 & + & - & n.s. & 1,85 & - & + & 0 & n.s. & 2,02 & 0 & + & 0 \\
\hline Verkaufeförderung & 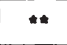 & 2,58 & + & - & -- & ** & 2,24 & + & -- & - & n.s. & 2,29 & + & 0 & - \\
\hline Public Relations & - & 2,57 & + & - & 0 & n.s. & 2,45 & + & - & - & 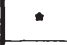 & 2,66 & + & - & 0 \\
\hline Sponsoring & n.s. & 4.45 & + & - & - & n.s. & 3,84 & 0 & 0 & 0 & n.s. & 4.13 & + & - & - \\
\hline Absatrwegegestaltung & $*$ & 2,85 & ++ & - & - & - & 2,64 & + & -- & 0 & $\bullet$ & 2,62 & + & $\cdots$ & 0 \\
\hline Logistik & \#* & 3,06 & ++ & 0 & - & $\star$ & 2,54 & + & 0 & - & $\star$ & 2,45 & + & $\cdots$ & 0 \\
\hline \multicolumn{2}{|l|}{ Anzahl der Unternehmen } & 104 & 51 & 33 & 20 & & 159 & 51 & 33 & 41 & & 112 & 62 & 29 & 21 \\
\hline 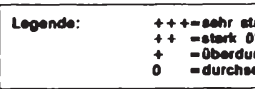 & \multicolumn{4}{|c|}{ 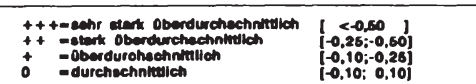 } & \multicolumn{5}{|c|}{$\begin{array}{l}{[0.10 ; 0,26]} \\
10.25 ; 0,50] \\
i>0,50]\end{array}$} & 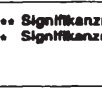 & $\begin{array}{l}\text { entraeus } \\
\text { entreacu }\end{array}$ & 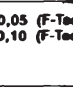 & & \multicolumn{2}{|c|}{ 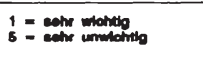 } \\
\hline
\end{tabular}

Abb. 31: Bedeutung der Marketinginstrumente fur die strategischen High-Tech-Gruppen in den Umfeldsituationen 
tern werden hingegen produkt- und distributionspolitische Maßnahmen vernachlässigt. Innerhalb der Kommunikationspolitik ist der persönliche Verkauf überdurchschnittlich ausgeprägt, während den restlichen kommunikationsbezogenen Maßnahmen eine geringe Relevanz zukommt.

Bei den innovationsorientierten High-Tech-Unternehmen nimmt im Rahmen der Produktpolitik die Produktinnovation eine dominante Position ein. Hierbei wird der Produktinnovation von den "Innovationsorientierten" ein deutlich größerer Stellenwert beigemessen als von den "Differenzierungsorientierten". Es fällt weiterhin auf, daß die übrigen produktbezogenen Maßnahmen vernachlässigt werden. So sind die Produktqualität und das Design unterdurchschnittlich und die Produktpflege, die Benutzerfreundlichkeit, die Sortimentstiefe/-breite sowie der Service stark unterdurchschnittlich ausgeprägt. Von den Unternehmen dieser strategischen High-Tech-Gruppe werden außerdem sämtliche kontrahierungs-, kommunikations- und distributionspolitischen Marketinginstrumente als weniger wichtig beurteilt.

Als Ergebnis bleibt festzuhalten, daß in der Umfeldsituation A die Strategieschwerpunkte der High-Tech-Anbieter konsistent in den operativen Marketingaktivitäten umgesetzt werden (vgl. Abbildungen 28 und 31). ${ }^{26}$ Die differenzierungsorientierten Unternehmen legen entsprechend ihrem Streben nach einer Qualitäts-, Service-, Image- und Nutzenführerschaft eine besondere Betonung auf die Produkt- und Kommunikationspolitik. Bei den kostenorientierten High-TechUnternehmen kommt die starke Preis/-Kostenorientierung in der hohen Bedeutung der kontrahierungspolitischen Maßnahmen zum Ausdruck. Für die "Innovationsorientierten", die primär eine Technologiefuihrerschaft anstreben, steht die Produktinnovation im Vordergrund des Marketing-Mix.

Eine Analyse des operativen Marketingverhaltens der strategischen High-TechGruppen in den verschiedenen Umfeldsituationen A, B und C zeigt weiterhin, daß

Siehe in diesem Zusammenhang auch die absoluten Mittelwertabweichungen der einzelnen Strategievariablen sowie der einzelnen Marketinginstrumentevariablen der strategischen High-Tech-Gruppen in Situationen A, B und C im Anhang II der Arbeit. 
die Marketingstrategien in gleicher Weise in den Marketingmaßnahmen umgesetzt werden und daß dabei vergleichbare Instrumentekombinationen realisiert werden (vgl. Abbildungen 28 und 31). ${ }^{27}$ Auf der Grundlage dieser Erkenntnisse und unter Berücksichtigung der Ergebnisse der Mittelwertvergleichstests sind die Hypothesen $\mathrm{H}$ MM1 und $\mathrm{H}$ MM2 insgesamt zu bestätigen. ${ }^{28}$

Im Hinblick auf die Hypothese $\mathrm{H}$ MM3 wird untersucht, ob sich strategische Gruppen, die in den verschiedenen homogenen Umfeldsituationen ähnliche Instrumenteschwerpunkte verfolgen, in der Ausgestaltung einzelner Maßnahmen im Marketingbereich unterscheiden. Eine Betrachtung der in Abbildung 31 dargestellten Mittelwertabweichungen der Instrumentevariablen verdeutlicht, daß die vergleichbaren strategischen High-Tech-Gruppen einzelnen Marketingmaßnahmen in den Umfeldsituationen A, B und C eine unterschiedlich hohe Bedeutung beimessen. ${ }^{29}$ Für die differenzierungsorientierten Unternehmen sind die Produktinnovation, die Benutzerfreundlichkeit und die Produktqualität vor allem in der Situation B besonders wichtig, in der eine sehr hohe Technologiedynamik herrscht. Die Bedeutung von Servicemaßnahmen und Aktivitäten innerhalb der Marketinglogistik ist in den Situationen B und C ungleich höher als in der Umfeldsituation A.

Die "Kostenorientierten" legen auf die Preis- und die Konditionengestaltung in den Situationen B und C im Vergleich zur Situation A einen größeren Wert. Von den innovationsorientierten High-Tech-Herstellern wird die Produktinnovation in der Umfeldsituation B als besonders wichtig eingeschätzt. Demgegenüber besitzt die Produktpflege die größte Relevanz in der Situation C, die durch einen sehr

Siehe hierzu auch die absoluten Mittelwertabweichungen der einzelnen Strategievariablen sowie der einzelnen Marketinginstrumentevariablen der strategischen HighTech-Gruppen in Situationen A, B und C im Anhang II der Arbeit.

$\mathrm{Zu}$ den Ergebnissen der Mittelwertvergleichstests siehe Abbildung 31 der Arbeit. Es ist in diesem Zusammenhang darauf hinzuweisen, da $B$ für die Marketinginstrumente Sortimentstiefe/ -breite, Konditionen, persönlicher Verkauf und Sponsoring in der Mehrzahl der Umfeldsituationen keine signifikanten Ergebnisse festgestellt werden konnten.

Siehe hierbei auch die absoluten Mittelwertabweichungen der einzelnen Marketinginstrumentevariablen der strategischen High-Tech-Gruppen in Situationen A, B und C im Anhang II der Arbeit. 
hohen Preiswettbewerb gekennzeichnet ist. Darüber hinaus halten die "Innovationsorientierten" die Benutzerfreundlichkeit technologischer Anwendungen in den Situationen B und $\mathrm{C}$ für besonders wichtig. Zusammenfassend läßt sich feststellen, daß sich die strategischen High-Tech-Gruppen in den Umfeldsituationen $\mathrm{A}, \mathrm{B}$ und $\mathrm{C}$ vergleichsweise gering im operativen Marketingverhalten unterscheiden. Die Hypothese $\mathrm{H}$ MM3 wird somit nur eingeschränkt bestätigt.

\subsection{Unternehmensaktivitäten zur Verkürzung der Entwicklungszeiten und Beschleunigung der Vermarktung von High-Tech-Produkten}

\subsection{Ansatzpunkte zur Verkürzung der Entwicklungszeiten und Beschleu- nigung der Vermarktung von High-Tech-Produkten}

In High-Tech-Märkten werden die Unternehmen bei der Entwicklung und Vermarktung technologischer Produkte mit einer hohen Technologiedynamik konfrontiert. ${ }^{30}$ Es ergibt sich hieraus die Notwendigkeit, durch den Einsatz geeigneter Maßnahmen die Entwicklungszeiten von High-Tech-Produkten zu reduzieren und die Vermarktung zu beschleunigen. In diesem Zusammenhang lassen sich Ansatzpunkte in der instrumentellen Ausrichtung des Unternehmensverhaltens im F\&E-, Produktions- und Marketingbereich sowie in der Organisation und Führung aufzeigen. ${ }^{31}$

Vgl. hierzu und zu den folgenden Beiträgen auch die Ausführungen im Teil B Kap. 2 der vorliegenden Untersuchung.

31 Der folgenden Diskussion liegt als konzeptioneller Analyserahmen die Wertkette zugrunde. Zu einer ähnlichen Vorgehensweise vgl. auch Ostmeier, H., Ökologieorientierte Produktinnovationen, a.a.O., S. 178 ff.; Kirchgeorg, M., Ökologieorientiertes Unternehmensverhalten, a.a.O., S. 51 ff. Für eine Kennzeichung der Wertkette als Gestaltungsinstrument operativer Unternehmensaktivitäten siehe z.B. Meffert, H., Die Wertkette als Instrument einer integrierten Unternehmensplanung, in: Der Integrationsgedanke in der Betriebswirtschaftslehre, Hrsg.: Delfmann, W., Wiesbaden 1989, S. 258 ff. 
Die High-Tech-Unternehmen legen im F\&E-Bereich fest, durch welche Maßnahmen das technologische Wissen zur Hervorbringung von Innovationen generiert werden soll. Als Alternativen zur Verringerung der Entwicklungszeiten bieten sich grundsätzlich ein erhöhter Ressourceneinsatz in der unternehmensinternen F\&E, eine Kooperation ${ }^{32}$ mit anderen Unternehmen und ein Zukauf externen Know-hows an. ${ }^{33}$

Durch einen erhöhten Ressourceneinsatz in der eigenen F\&E haben die HighTech-Anbieter die Möglichkeit, frühzeitig exklusives technologisches Know-how aufzubauen, das eine Grundlage zur Sicherung von Wettbewerbsvorteilen bildet. Die Unternehmen können den Entwicklungsprozeß uneingeschränkt beeinflussen und damit besser auf die Marketingstrategie abstimmen. Es besteht auch nicht die Gefahr einer technologischen Abhängigkeit von anderen High-Tech-Herstellern. ${ }^{34}$

Steigende F\&E-Aufwendungen und die Komplexität von High-Tech-Produkten machen jedoch häufig Kooperationen mit anderen High-Tech-Anbietern unumgänglich. Die Unternehmen werden hierbei vor die Aufgabe gestellt, durch entsprechende vertragliche Vereinbarungen und vertrauensbildende Maßnahmen wie z.B. regelmäßiger Informationsaustausch oder die Bildung einer gemeinsamen Projektgruppe für ein Anreiz-/Beitrags-Gleichgewicht zwischen den Kooperationspartnern zu sorgen. ${ }^{35}$

32 Zum Vergleich der Begriffe Unternehmenskooperation und strategische Allianz siehe z.B. Backhaus, K, Meyer, M., Strategische Allianzen und strategische Netzwerke, a.a.O., S. 330 f.; Gahl, A., Die Konzeption strategischer Allianzen, a.a.O., S. 32 ff.

Siehe zu einer vergleichbaren Einteilung auch Meffert, H., Remmerbach, K.-U., Marketingstrategien in jungen Märkten, a.a.O., $333 \mathrm{f}$.

Vgl. Dussauge, P., Hart, St., Ramanantsoa, B., Strategic technology management, a.a.O., S. 92; Wolfrum, B., Strategisches Technologiemanagement, a.a.O., S. 294 ff.; Ewald, A., Organisation des strategischen Technologie-Managements, a.a.O., S. 202 ff.; Perillieux, R., Der Zeitfaktor im strategischen Technologie-Management, a.a.O., S. 131.

Vgl. Meffert, H., Marketing-Management, a.a.O., S. 157 ff.; Bronder, Ch., Kooperationsmanagement, Unternehmensdynamik durch strategische Allianzen, Frankfurt a.M., New York 1993, S. 19 ff.; Gahl, A., Die Konzeption strategischer Allianzen, a.a.O., S. 18 ff.; Backhaus, K., Die Macht der Allianz, in: asw, 1987, Nr. 11, S. 127 ff. Für eine ausfuihrliche Darstellung zu Unternehmenskooperationen siehe z.B. Bronder, Ch., Kooperationsmanagement, a.a.O., S. $64 \mathrm{ff}$. 
Durch Unternehmenskooperationen und Know-how Zukäufe sind die High-TechHersteller in der Lage, sich fehlendes technologisches Wissen in einem relativ kurzen Zeitraum anzueignen. Ein Know-how Zukauf erfolgt durch Akquisition technologischer Kenntnisse sowie den Erwerb zeitlich befristeter Nutzungsrechte, wie z.B. Einfach-, Exklusiv- Lizenzen. Von den High-Tech-Unternehmen wird dabei bereits existierendes technologisches Know-how übernommen. Diesem Verhalten liegt eine Strategie der Inventions- und i.d.R. auch Innovationsfolgerschaft zugrunde. ${ }^{36}$

In der Produktion stellen die Reduzierung der Fertigungstiefe, die Optimierung der Durchlaufzeiten und die Just-in-time-Beschaffung Ansatzpunkte zur Verringerung der Entwicklungszeiten und Beschleunigung der Vermarktung von HighTech-Produkten dar. ${ }^{37}$

Eine geringe Fertigungstiefe gibt den High-Tech-Herstellern die Gelegenheit, ihre finanziellen und personellen Ressourcen stärker auf die Entwicklung einer kleinen Anzahl im Unternehmen produzierbarer Produktkomponenten und technologischer Teilsysteme $\mathrm{zu}$ konzentrieren. Darüber hinaus ist eine Fertigungstiefenreduktion sinnvoll, wenn potentielle Zulieferer bestimmte Produktbestandteile kostengünstiger produzieren oder bei einzelnen Komponenten über ein spezielles Know-how verfügen. Empirische Untersuchungen haben in diesem Zusammenhang gezeigt, daß die Dauer von F\&E-Projekten nachhaltig durch die Einbeziehung von Zulieferern verkürzt wurde, die in Abstimmung mit dem Her-

Vgl. Dussauge, P., Hart, St., Ramanantsoa, B., Strategic technology management, a.a.O., S. 126 ff.; Wolfrum, B., Strategisches Technologiemanagement, a.a.O., S. 294 ff.; Hauschildt, J., Innovationsstrategien und ihre organisatorischen Konsequenzen, in: Strategieentwicklung, Hrsg.: Riekhof, H.-Ch., Stuttgart 1989, S. 259 ff.; Perillieux, R., Der Zeitfaktor im strategischen Technologie-Management, a.a.O., S. $133 \mathrm{f}$.

Siehe in diesem Zusammenhang auch Meffert, H., Erfolgreiches Marketing in der Rezession, a.a.O., S. 41 ff.; Benkenstein, M., Die Reduktion der Fertigungstiefe als betriebswirtschaftliches Entscheidungsproblem, a.a.O., S. 50 ff.; Dichtl, E., Produktauslagerung und Fertigungstiefe als Determinanten der Wertschöpfung, in: MarketingSchnittstellen: Herausforderungen für das Management, Hrsg.: Specht, G., Silberer, G., Engelhardt, W.H., Stuttgart 1989, S. 94 ff. 
steller ausgewählte Produktkomponenten selber entwickelten. ${ }^{38}$ Entscheidungen über die Fertigungstiefe sind stets unter Berücksichtigung der von den High-TechAnbietern verfolgten Marketingstrategie zu treffen. Beruht der Wettbewerbsvorteil eines Unternehmens z.B. auf der hohen Produktqualität, ist von einem Fremdbezug abzusehen, wenn bei den extern zu beziehenden Komponenten ein gleichbleibendes Qualitätsniveau nicht sichergestellt werden kann. ${ }^{39}$

Die Durchlaufzeiten werden in High-Tech-Märkten häufig durch eine Standardisierung der Produktionsprozesse gesenkt. Eine Optimierung der Durchlaufzeiten in der Produktion führt insbesondere bei großen Ausbringungsmengen zu deutlichen Kosteneinsparungen, die über eine Politik der niedrigen Absatzpreise eine schnelle Vermarktung technologischer Produktleistungen fördert. ${ }^{40}$

Die Just-in-time-Beschaffung eröffnet den Unternehmen in der Einführungsphase von High-Tech-Produkten eine größere Handlungsfreiheit bei der Festlegung der Liefermengen in Art und Umfang. Die High-Tech-Hersteller sind hierdurch in der Lage, sich rascher an absatzmarkt- (z.B. Nachfrageboom) und technologiebezogene (z.B. Durchbruch einer Schrittmachertechnologie, Substitution einer Basistechnologie) Veränderungen anzupassen. ${ }^{41}$

Vgl. Gerpott, T., Wittkemper, G., Verkürzung der Produktentwicklungszeiten, a.a.O., S. 132 f. Siehe hierzu auch Hofmaier, R., Das "Magische Viereck" strategischen I+T-Marketings als Basis eines ganzheitlichen I+T-Marketingansatzes, a.a.O., S. $88 \mathrm{ff}$.

Vgl. Benkenstein, M., Integriertes Innovationsmanagement - Ansatzpunkte zum "lean innovation", in: Marktforschung und Management, 1993, Nr. 1, S. 23 f.; Benkenstein, M., Henke, N., Vertikale Integration - Eine transaktionskostentheoretische Interpretation, a.a.O., S. 19 ff.; Dichtl, E., Produktauslagerung und Fertigungstiefe als Determinanten der Wertschöpfung, a.a.O., S. $95 \mathrm{ff}$.

Vgl. Bühner, R., Betriebswirtschaftliche Organisationslehre, a.a.O., S. 178 ff. Zur Gestaltung von Durchlaufzeiten in Unternehmen siehe z.B. Kaluza, B., Klenter, G., Zeit als strategischer Erfolgsfaktor von Industrieunternehmen, Teil II: Erfolgskritische Komponenten des strategischen Erfolgsfaktors Zeit, Diskussionsbeitrag Nr. 176 des Fachbereichs Wirtschaftswissenschaft, Duisburg 1993, S. $68 \mathrm{ff}$.

Vgl. Meffert, H., Marketing-Management, a.a.O., S. 135 ff.; Bühner, R., Betriebswirtschaftliche Organisationslehre, a.a.O., S. 229 ff.; Dichtl, E., Produktauslagerung und Fertigungstiefe als Determinanten der Wertschöpfung, a.a.O., S. 95 ff. Zur Kennzeichnung unterschiedlicher Just-in-time-Konzepte vgl. z.B. Backhaus, K., Investitionsgütermarketing, a.a.O., S. $330 \mathrm{ff}$. 
Innerhalb des Marketing läßt sich der Verkauf technologischer Neuheiten durch eine globale Vermarktung, Vertriebskooperationen, eine Zusammenarbeit mit Pilotkunden und eine Erhöhung des Werbedrucks intensivieren. ${ }^{42}$

Aufgrund der kurzen Amortisationszeiten in High-Tech-Märkten ergibt sich häufig die Notwendigkeit einer globalen Vermarktung von High-Tech-Produkten (z.B. medizinische Geräte wie Herzschrittmacher, Nieren- und Gallensteinzertrümmerer, Kernspin-Tomographie-Apparate) bzw. technischer Komponenten (z.B. Speicher-Chips, Prozessoren). Eine globale Marktbearbeitung trägt zu einer Risikostreuung bei, da Nachfrageschwankungen in den einzelnen Ländermärkten insgesamt kompensiert werden. Wenn High-Tech-Anbieter mit technologischen Innovationen gleichzeitig in den zentralen Weltmärkten vertreten sind, lassen sich gegenüber den Wettbewerbern Markteintrittsbarrieren aufbauen, die wiederum den Diffussionsprozeß der eigenen High-Tech-Produkte absichern. ${ }^{43}$

Eine Zusammenarbeit mit Pilotkunden bietet die Möglichkeit, wichtige Nachfragerbedürfnisse in der Entwicklungsphase und notwendige Leistungsanpassungen in der Vermarktungsphase von High-Tech-Produkten frühzeitig zu berücksichtigen. Die High-Tech-Hersteller sparen hierdurch Kosten und erhöhen die Akzeptanz für die technologischen Produktangebote bei den Nachfragern. ${ }^{44}$

Siehe in diesem Zusammenhang auch Bronder, Ch., Kooperationsmanagement, Unternehmensdynamik durch strategische Allianzen, a.a.O., S. 19 ff.; Schürmann, U., Erfolgsfaktoren der Werbung im Produktlebenszyklus, Frankfurt a.M. u.a. 1992, S. 78 ff. u. 155 ff.; Große-Oetringhaus, W., Internationale Marketingstrategien von Groß-Unternehmen in den Investitionsgüter- und High-Tech-Märkten, a.a.O., S. 31 ff.; Davidow, W.H., Marketing High Technology, An Insider's View, New York, London 1986, S. 118 ff.

Vgl. Meffert, H., Marketing-Management, a.a.O., S. 453 ff.; Mager, H.J., Siegberg, U., Erfolgsfaktor "Technologie-Innovation" bei der Entwicklung medizintechnischer Großgeräte, a.a.O., S. 483 ff. Backhaus, K., Strategien auf sich verändernden Weltmärkten Chancen und Risiken, in: DBW, 1989, Nr. 4, S. 470 ff. Zu weiteren Aspekten der Globalisierung der Unternehmenstätigkeit siehe z.B. Meffert, H., Bolz, J., Globale Wettbewerbsstrategien und länderübergreifende Integration - Status und Entwicklungsperspektiven, Arbeitspapier Nr. 70 der Wissenschaftlichen Gesellschaft für Marketing und Unternehmensfuihrung e.V., Hrsg.: Meffert H., Wagner, H., Backhaus, K., Münster 1992, S. $6 \mathrm{ff}$.

Vgl. Meffert, H., Marketing-Management, a.a.O., S. 214; Walters, M., Marktwiderstände und Marketingplanung, a.a.O., S. 155 ff.; Meffert, H., Die Durchsetzung von Innovationen in der Unternehmung und im Markt, in: ZfB, 1976, Nr. 2, S. 98. 
Vertriebskooperationen werden oftmals von Unternehmen angestrebt, die sich kurzfristig einen Zugang zu High-Tech-Märkten verschaffen wollen. Die Firma AT\&T ging beispielsweise mit Philips und Olivetti eine Kooperation ein, um deren Vertriebswege in Europa für die Vermarktung der eigenen Produkte zu nutzen. ${ }^{45}$ Vertriebskooperationen sind darüber hinaus erforderlich, wenn die High-Tech-Hersteller nur über begrenzte personelle Kapazitäten und Finanzmittel verfügen. ${ }^{46}$

Durch einen hohen Werbedruck zu Beginn der Produkteinführung können die Unternehmen den relevanten High-Tech-Anwendern die neuen oder verbesserten technologischen Produkte schneller bekannt machen und dadurch frühzeitig Interesse an den Leistungsangeboten wecken. Es ist weiterhin möglich, daß die HighTech-Anbieter über eine intensive Werbung ein unternehmensspezifisches Image aufbauen wollen, wie z.B. ein progressives technologisches Unternehmensimage, welches zur Profilierung im Wettbewerb geeignet ist. ${ }^{47}$

Im Hinblick auf die Beschleunigung von Innovationsvorhaben ist von den HighTech-Unternehmen im Bereich der Organisation und Führung zu entscheiden, welche Bedeutung interdisziplinäre Teams und die Mitarbeitermotivation haben. In diesem Zusammenhang ist auch festzulegen, ob Interaktionen zwischen den Mitarbeitern eher unter Gesichtspunkten der Flexibilität oder der Zeiteffizienz erfolgen sollen. ${ }^{48}$

45 Vgl. Backhaus, K. Strategien auf sich verändernden Weltmärkten - Chancen und Risiken, a.a.O., S. 474.

Vgl. Bronder, Ch., Kooperationsmanagement, Unternehmensdynamik durch strategische Allianzen, a.a.O., S. 28 ff.; Gahl, A., Die Konzeption strategischer Allianzen, a.a.O., 24 ff.

Vgl. Meffert, H., Remmerbach, K.-U., Marketingstrategien in jungen Märkten, a.a.O., S. 338. Siehe für eine Übersicht empirischer Untersuchungen zum Einfluß des Werbebudgets auf den Absatz von Produkten z.B. Assmus, G., Farley, J.U., Lehmann, D.R., How advertising affects sales: Meta analysis of econometric results, in: Journal of Marketing Research, 1984, S. 65 ff.

Siehe in diesem Zusammenhang auch Gerpott, T., Wittkemper, G., Verkürzung der Produktentwicklungszeiten, a.a.O., S. 137 ff.; Schmelzer, H.J., Buttermilch, K.-H., Reduzierung der Entwicklungszeiten in der Produktentwicklung als ganzheitliches Problem, in: ZfbF, 1988, Sonderheft Nr. 23, S. 47 ff.; Meffert, H., Die Durchsetzung von Innova- 
Bei der Bildung interdisziplinärer Teams werden Mitarbeiter aus verschiedenen Unternehmensfunktionen in einer Projektgruppe zusammengefaßt. Dies führt i.d.R. zu einer effizienteren und schnelleren Koordination, da der Abstimmungsbedarf zwischen den an der Produktentwicklung beteiligten Abteilungen, z.B. F\&E, Produktion und Marketing, deutlich reduziert wird. Durch einen intensiven Informationsaustausch der Spezialisten innerhalb des Projektteams lassen sich zudem Entwicklungsmängel frühzeitiger erkennen und beheben. ${ }^{49}$

Beim Aufbau von Anreizsystemen stoßen die High-Tech-Unternehmen auf erhebliche Probleme, da der Innovationserfolg technologischer Neuerungen aufgrund der langen Entwicklungszeiten einzelnen Mitarbeitern kaum zuzurechnen ist. In diesem Zusammenhang wurde in einer empirischen Studie erfolgreicher innovationsorientierter Unternehmen aus den Bereichen Chemie, Elektronik, Maschinen- und Fahrzeugbau untersucht, welche Maßnahmen zur Mitarbeitermotivation eingesetzt werden. Es zeigt sich, daß die Projektmitarbeiter i.d.R. ein relativ hohes Grundgehalt erhalten und darüber hinaus primär durch nichtmaterielle Anreize wie z.B. Lob oder eine stärkere Einbindung in die Informationsprozesse motiviert werden. ${ }^{50}$

tionen in der Unternehmung und im Markt, a.a.O., S. 78 ff.; Meffert, H., Die Flexibilität in betriebswirtschaftlichen Entscheidungen, unver. Habil., München 1968, S. 138 ff.

Vgl. Schaible, J., Hönig, A., High-Tech-Marketing in der Praxis, a.a.O., S. 99 ff.; Benkenstein, M., Integriertes Innovationsmanagement - Ansatzpunkte zum "lean innovation", a.a.O.; S. 23 f.; Gerpott, T., Wittkemper, G., Verkürzung der Produktentwicklungszeiten, a.a.O., S. 139 ff. Zu einer ausfuihrlichen Darstellung der Teamorganisation siehe z.B. Bühner, R., Betriebswirtschaftliche Organisationslehre, a.a.O., S. $169 \mathrm{ff}$. Zu Koordinationsproblemen zwischen F\&E und Marketing siehe z.B. Manns, J.R., Produktinnovationen als Ergebnis der Koordination von F\&E und Marketing, Ursachen, Auswirkungen und Lösungsmöglichkeiten, a.a.O., S. $17 \mathrm{ff}$.

Vgl. De Pay, D., Kulturspezifische Determinanten der Organisation von Innovationsprozessen, in: Innovationsmanagement: Theorie und Praxis im Kulturvergleich, Hrsg.: Albach, H., Wiesbaden 1990, S. 144 ff. Siehe hierzu auch Schneider, D.J., Müller, M.E., Durch Koordination zu marktgerechten Innovationen - Schnittstellengestaltung zwischen F\&E und Marketing, in: Marktforschung und Management, 1993, Nr. 1, S. 8 ff.; Tebbe, K., Die Organisation von Produktinnovationsprozessen, Stuttgart 1990, S. 49 ff.; Schmelzer, H.J., Buttermilch, K.-H., Reduzierung der Entwicklungszeiten in der Produktentwicklung als ganzheitliches Problem, a.a.O., S. 62 ff.; Schuster, J., Management compensation in high technology companies, Lexington, Mass. 1984, S. $73 \mathrm{ff}$. 
Die Informationsprozesse zwischen den Funktionsbereichen und damit die Koordination von Innovationsprojekten werden maßgeblich davon beeinflußt, ob das Mitarbeiterverhalten durch eine starke Flexibilität oder Zeiteffizienz geprägt ist. Eine hohe Flexibilität der Mitarbeiter ist erforderlich, wenn völlig neue und schlecht zu strukturierende Entwicklungsvorhaben vorliegen und die Mitarbeiter nicht auf Erfahrungen zurückgreifen können. Bei technologischen Neuheiten, die eher den Charakter von Verbesserungsinnovationen haben, lassen sich hingegen konkrete Zeitvorgaben zur Beschleunigung der Entwicklungszeiten bestimmen. ${ }^{51}$

Ausgehend von den dargelegten Ansatzpunkten der instrumentellen Ausrichtung von Unternehmen in High-Tech-Märkten ist es von Interesse zu erfahren, in welcher Form die Marketingstrategien der strategischen Gruppen in den Maßnahmen zur Verkürzung der Entwicklungszeiten und Beschleunigung der Vermarktung von High-Tech-Produkten umgesetzt werden und welche Maßnahmenschwerpunkte dabei verfolgt werden. Es wird hierzu auf der Grundlage der empirischen Daten folgende Basishypothese überprüft:

H II1 Die Marketingstrategien von strategischen Gruppen in High-TechMärkten werden in den Maßnahmen zur Verkürzung der Entwicklungszeiten und Beschleunigung der Vermarktung von High-Tech-Produkten umgesetzt. Die strategischen Gruppen unterscheiden sich hinsichtlich der von ihnen durchgefuihrten Unternehmensaktivitäten innerhalb einer Umfeldsituation.

Auf Basis der empirischen Ergebnisse zu den Marketingstrategien der strategischen High-Tech-Gruppen in den homogenen Umfeldsituationen werden für die weitere Analyse folgende Basishypothesen formuliert:

51 Vgl. Gerpott, T., Wittkemper, G., Verkürzung der Produktentwicklungszeiten, a.a.O., S. 142 f.; Domsch, M., Gerpott, T., Akzeptanz von Zeitkontrollen in der industriellen Forschung und Entwicklung, in: ZfbF, 1988, Sonderheft Nr. 23, S. 93 ff.; Benkenstein, M., F\&E und Marketing, Wiesbaden 1987, S. 84 ff.; Meffert, H., Marketing-Flexibilität als Erfolgsfaktor der Unternehmung, in: Thexis, 1985, Nr. 4, S. $10 \mathrm{f}$. 
H II2 In den verschiedenen homogenen Umfeldsituationen in High-TechMärkten lassen sich bei den vergleichbaren strategischen Gruppen ähnliche Maßnahmenschwerpunkte finden.

H II3 Bei den vergleichbaren strategischen Gruppen, die in den verschiedenen homogenen Umfeldsituationen in High-Tech-Märkten ähnliche Maßnahmenschwerpunkte verfolgen, lassen sich situationsbedingte Unterschiede im operativen Unternehmensverhalten identifizieren.

1.22 Empirische Analyse der Maßnahmen zur Verkürzung der Entwicklungszeiten und Beschleunigung der Vermarktung von HighTech-Produkten von strategischen Gruppen

In der weiteren Analyse wird zunächst das operative Verhalten der strategischen High-Tech-Gruppen in den Bereichen F\&E, Produktion und Marketing sowie Organisation und Führung in der Situation A dargestellt. Anschließend wird untersucht, welche Gemeinsamkeiten und Unterschiede in der instrumentellen Ausrichtung der vergleichbaren strategischen Gruppen in den Umfeldsituationen A, B, und C bestehen. In Abbildung 32 sind die Mittelwertabweichungen der Maßnahmenvariablen zu sehen. ${ }^{52}$ Hierdurch wird aufgezeigt, welche Bedeutung die strategischen Gruppen den Maßnahmen zur Verkürzung der Entwicklungszeiten und Beschleunigung der Vermarktung technologischer Produkte beimessen.

Für die differenzierungsorientierten High-Tech-Hersteller haben die Maßnahmen aus dem F\&E-Bereich in der Situation A einen untergeordneten Stellenwert. In der Produktion wird die Just-in-time-Beschaffung und im Marketing die Erhöhung des Werbedrucks als besonders wichtig eingeschätzt. Die restlichen produktions- und marketingbezogenen Aktivitäten werden von den Unternehmen schen High-Tech-Gruppen in Situationen A, B und C sind dem Anhang II der Arbeit zu entnehmen. 


\begin{tabular}{|c|c|c|c|c|c|c|c|c|c|c|c|c|c|c|c|}
\hline \multirow{2}{*}{$\begin{array}{l}\text { Variablen zur } \\
\text { Beschleunigung } \\
\text { von Innovationen }\end{array}$} & \multicolumn{15}{|c|}{ 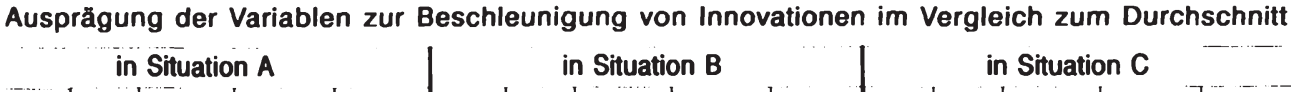 } \\
\hline & $\begin{array}{l}819 . \\
\text { ntroen }\end{array}$ & $\boldsymbol{\varnothing}$ & 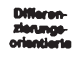 & Konenortent & morvetionse & $\begin{array}{l}\text { Slg. } \\
\text { ntveau }\end{array}$ & $\varnothing$ & 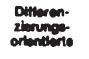 & Koedenoriter- & morventome & $\begin{array}{l}\text { sig. } \\
\text { niveaus }\end{array}$ & $\varnothing$ & $\begin{array}{l}\text { Demoran. } \\
\text { zienuge- } \\
\text { ortontionto }\end{array}$ & $\begin{array}{l}\text { Kontonorien. } \\
\text { titerto }\end{array}$ & Imovetions:- \\
\hline Know-how Zukeur & $\because$ & 2,67 & o & $+t$ & - & * & 2,18 & + & + & $-\cdot$ & - & 2,48 & + & o & 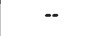 \\
\hline Erholmer Reseourconolneatz & $\cdots$ & 2,41 & o & - & ++ & * & 2,30 & + & - & + & * & 2,30 & + & -- & + \\
\hline Unternehmenckooperation & 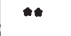 & 2,61 & - & ○ & +++ & * & 2,12 & - & + & -- & * & 2,57 & o & ++ & $-\cdot$ \\
\hline Fertigungetiofenreduktion & n.s. & 2,97 & o & + & + & n.s. & 2,58 & o & + & $\mathbf{0}$ & - & 2,68 & o & ++ & o \\
\hline Just-in-time-Boechaffung & * & 2,75 & + & - & -- & n.6. & 2,32 & + & o & - & * & 2,32 & + & o & -- \\
\hline Optimierung Durchieutzethen & n.s. & 1,82 & o & + & - & n.s. & 1,67 & o & + & o & n.s. & 1,62 & ○ & + & - \\
\hline Globale Vermarktung & n.8. & 2,88 & - & ० & 0 & n.8. & 2,50 & o & ० & o & * & 2,55 & o & $\cdot$ & ++ \\
\hline Kooperation mi Pllokkunden & - & 2,13 & o & - & ++ & n.s. & 1,86 & $\mathbf{0}$ & - & + & - & 2,17 & o & - & + \\
\hline Erhohung Werbedruck & - & 2,81 & + & ○ & - & • & 2,72 & + & - & o & n.s. & 2,83 & + & - & o \\
\hline Vertriebekooperationen & n.s. & 2,64 & - & ० & + & n.s. & 2,26 & - & o & 0 & - & 3,06 & 0 & - & ++ \\
\hline Mitarbehtermotwation & n.8. & 1,50 & + & ० & + & \#* & 1,45 & + & 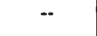 & + & $*$ & 1,67 & + & - & + \\
\hline Inlerdlexiplinire Toame & n.s. & 2,12 & ० & - & o & n.s. & 1,88 & 0 & o & + & 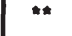 & 2,28 & o & - & + \\
\hline Floxibuinin U-Grundeatz & n.8. & 1,79 & + & - & o & - & 1,67 & + & - & - & n.s. & 1,76 & + & - & o \\
\hline Zetheffizienz U-Grundeatz & n.8. & 2,10 & $\cdot$ & + & o & n.s. & 1,87 & - & + & o & - & 2,15 & - & + & + \\
\hline \multicolumn{2}{|l|}{ Anzahl der Unternehmen } & 104 & 51 & 33 & 20 & & 159 & 85 & 33 & 41 & & 112 & 62 & 29 & 21 \\
\hline 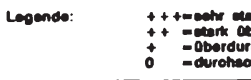 & 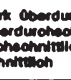 & chnver & $\begin{array}{r}1<-0 \\
-0.25 \\
1-0.10 \\
1-0.10:\end{array}$ & & 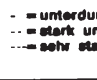 & at woner & Ithon & ch $\begin{array}{l}10.10 ; \\
10,25 ; \\
1>0\end{array}$ & & $\begin{array}{l}\text { ontminanz } \\
\text { onthikenx }\end{array}$ & 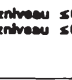 & Te & & 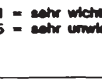 & tiots \\
\hline
\end{tabular}

Abb. 32: Bedeutung der Maßnahmen zur Verkurzung der Entwicklungszeiten und Beschleunigung der Vermarktung für die strategischen High-Tech-Gruppen in den Umfeldsituationen 
dieser strategischen Gruppe vernachlässigt. Die "Differenzierungsorientierten" legen in der Organisation und Führung einen großen Wert auf die Mitarbeitermotivation und eine flexible Unternehmenspolitik.

Von den F\&E-Aktivitäten sehen die High-Tech-Anbieter der zweiten strategischen Gruppe den Zukauf von Know-how als außerordentlich wichtig an. Im Produktionsbereich zeigt sich die Kostenorientierung in der hohen Bedeutung der Fertigungstiefenreduktion und der Optimierung der Produktionszeiten. Die kostenorientierten Unternehmen messen den marketingbezogenen Instrumenten einen geringen Stellenwert bei. Im Bereich der Organisation und Führung richtet sich das Interesse insbesondere auf eine hohe Zeiteffizienz im Unternehmensverhalten.

Für die innovationsorientierten High-Tech-Anbieter sind die Erhöhung des Ressourceneinsatzes und die Intensivierung von Unternehmenskooperationen im F\&E-Bereich von besonderer Relevanz. Mit Ausnahme der Reduktion der Fertigungstiefen werden die produktionsspezifischen Maßnahmen als wenig wichtig eingestuft. Im Marketing werden der Zusammenarbeit mit Pilotkunden und Vertriebskooperationen eine überdurchschnittliche Bedeutung zugesprochen. In der Organisation und Führung favorisieren die Unternehmen dieser strategischen Gruppe Maßnahmen zur Motivation der Mitarbeiter.

Es zeigt sich insgesamt, daß die strategischen Gruppen in Situation A entsprechend ihrer Strategieausrichtung unterschiedliche Schwerpunkte bei den Maßnahmen zur Verkürzung der Entwicklungszeiten und Beschleunigung der Vermarktung von High-Tech-Produkten setzen (vgl. Abbildungen 28 und 32). ${ }^{53}$ Die differenzierungsorientierten High-Tech-Anbieter sind bestrebt, den Innovationsproze $\beta$ durch ein flexibles, die Mitarbeiter motivierendes Verhalten sowie durch eine starke Werbeunterstützung zu beschleunigen. Durch eine Just-in-timeBeschaffung lassen sich kurzfristige Anpassungen in den technologischen 
Leistungsangeboten realisieren. Von den kostenorientierten Unternehmen, deren Strategieschwerpunkt auf der Erreichung einer Preis-/Kostenfuhrerschaft liegt, werden vor allem Maßnahmen zum Aufbau von Kosten- und Zeitvorteilen in der Produktion und in der Organisation und Führung durchgeführt. Die innovationsorientierten High-Tech-Anbieter versuchen primär, die Entwicklungszeiten von Innovationen durch eine Intensivierung der F\&E-Aktivitäten, eine Kooperation mit Pilotkunden und eine Mitarbeitermotivation zu verkürzen.

Eine situationsübergreifende Untersuchung der differenzierungs-, kosten- und innovationsorientierten strategischen Gruppen in High-Tech-Märkten verdeutlicht, daß die Marketingstrategien in den Umfeldsituationen A, B und C in einem gleichen operativen Unternehmensverhalten mit ähnlichen Maßnahmenschwerpunkten konkretisiert werden (vgl. Abbildungen 28 und 32). ${ }^{54}$ Aufgrund der wenig zufriedenstellenden Ergebnisse der Mittelwertvergleichstests sind die Hypothesen $\mathrm{H}_{\mathrm{II} 1}$ und $\mathrm{H}_{\mathrm{II} 2}$ insgesamt abzulehnen. ${ }^{55}$

Im Rahmen einer situationsübergreifenden Betrachtung der vergleichbaren strategischen High-Tech-Gruppen lassen sich jedoch auch Unterschiede in der Ausprägung der Maßnahmen zur Verkürzung der Entwicklungszeiten und Beschleunigung der Vermarktung von High-Tech-Produkten identifizieren (vgl. Abbildung 32). ${ }^{56}$ Für die differenzierungsorientierten High-Tech-Anbieter besitzt der Zukauf von technologischem Know-how vor allem in der Situation B eine hohe Bedeutung, in der ein hoher Technologiewettbewerb besteht. Die Just-in-time-Beschaffung ist in den Umfeldsituationen B und C wichtiger als in der Situation A.

Siehe hierzu auch die absoluten Mittelwertabweichungen der einzelnen instrumentebezogenen Ausgestaltungsformen der strategischen High-Tech-Gruppen in Situationen A, B und C im Anhang II der Arbeit.

$\mathrm{Zu}$ den Ergebnissen der Mittelwertvergleichstests siehe Abbildung 32 der Arbeit. Es ist hierbei zu erkennen, daß für die Variablen Fertigungstiefenreduktion, Optimierung der Durchlaufzeiten, globale Vermarktung, Vertriebskooperationen, Mitarbeitermotivation, Interdisziplinäre Teams, Flexibilität und Zeiteffizienz in der Mehrzahl der Umfeldsituationen keine signifikanten Ergebnisse vorliegen.

Vgl. hierzu die absoluten Mittelwertabweichungen der instrumentebezogenen Ausgestaltungsformen der strategischen High-Tech-Gruppen in den Situationen A, B und C im Anhang II der Arbeit. 
Die "Kostenorientierten" legen in der Umfeldsituation A im Vergleich zu den Situationen B und C einen geringeren Wert auf die Optimierung der Durchlaufzeiten. Weiterhin werden dem Know-how Zukauf in Situation B sowie Kooperationen mit fremden Unternehmen und der Fertigungstiefenreduktion in den Situationen B und C ein hoher Stellenwert eingeräumt. Von den innovationsorientierten High-Tech-Unternehmen werden insbesondere F\&E-Kooperationen in der Situation A und die Zusammenarbeit mit Pilotkunden in den Situationen A und $\mathrm{B}$ bevorzugt. Die Notwendigkeit einer globalen Vermarktung von High-TechProdukten wird primär in der Umfeldsituation $C$ gesehen, die durch eine hohe Preisdynamik geprägt ist. Insgesamt läßt sich festhalten, daß bei den strategischen Gruppen in den Situationen A, B und C vergleichsweise geringe situationsbedingte Unterschiede in der instrumentellen Ausrichtung bestehen. Die Hypothese $\mathrm{H}_{\text {II } 3}$ wird deshalb lediglich eingeschränkt bestätigt.

\section{Unternehmensbezogene Einflußfaktoren der Marketingstrategien von strategischen Gruppen in High-Tech-Märkten}

\subsection{Unternehmensziele als Einflußfaktoren der strategischen Gruppen}

In den weiteren Ausführungen erfolgt eine theoriegestützte und empirische Analyse des Zusammenhangs zwischen den Unternehmenszielen und den Marketingstrategien von strategischen Gruppen in High-Tech-Märkten. Hierdurch sollen Erkenntnisse darüber gewonnen werden, ob Unterschiede in den Zielsetzungen der High-Tech-Anbieter das Entstehen verschiedener strategischer Verhaltensgruppen begründen.

\subsection{Theoretische Grundlagen zum Zusammenhang zwischen Unter- nehmenszielen und Marketingstrategien von strategischen Gruppen}

Unternehmensziele stellen Orientierungs- bzw. Richtgrößen dar, an denen sich das strategische und operative Unternehmensverhalten $\mathrm{zu}$ ihrer Realisierung 
orientiert. ${ }^{57}$ Eine zieladäquate Ausrichtung der Unternehmenstätigkeit ist in den durch eine hohe Unsicherheit und Komplexität gekennzeichneten High-TechMärkten besonders wichtig, da die aus den dynamischen Umweltveränderungen resultierenden Herausforderungen mit einem reaktiven Anpassungsverhalten kaum zu bewältigen sind. Es ist deshalb überraschend, daß bisher in der Literatur zur strategischen Unternehmensführung in High-Tech-Märkten und in der wissenschaftlichen Forschung zum Konzept der strategischen Gruppen eine Betrachtung von Unternehmenszielen und ihrem Einfluß auf die Gestaltung von Marketingstrategien weitgehend vernachlässigt worden ist. Mit den folgenden Ausfuihrungen wird versucht, diesem Defizit Rechnung zu tragen.

Im Rahmen der Zielplanung ${ }^{58}$ werden unter Berücksichtigung der Unternehmensgrundsätze $\mathrm{e}^{59}$ und der Unternehmensidentität ${ }^{60}$ konkrete Handlungsziele formuliert, die vor allem im Hinblick auf die Zielinhalte zu präzisieren sind. ${ }^{61}$ Als eine

Vgl. Meffert, H., Marketing, Grundlagen der Absatzpolitik, a.a.O., S. 74; Schreyögg, G., Unternehmensstrategie, a.a.O.; S. 87; Heinen, E., Grundlagen betriebswirtschaftlicher Entscheidungen, Das Zielsystem der Unternehmung, 3. Aufl., Wiesbaden 1976, S. 23 ff. Zur Unterscheidung von Unternehmens- und Marketingzielen siehe z.B. Meffert, H., Marketing-Management, a.a.O., S. 93 ff.; Becker, J., Marketing-Konzeption, a.a.O., S. $11 \mathrm{ff}$.

Zur Kennzeichnung der Zielplanung siehe z.B. Meffert, H., Marketing-Management, a.a.O., S. 78 ff.; Becker, J., Marketing-Konzeption, a.a.O., S. $25 \mathrm{ff}$.

In den Unternehmensgrundsätzen sind Unternehmenszweck und -philosophie dokumentiert und bekommen dadurch einen verbindlichen Charakter. Während der Unternehmenszweck Art und Bedeutung der Unternehmensleistungen verdeutlicht, kennzeichnet die Unternehmensphilosophie die Grundeinstellung eines Unternehmens zu seiner Umwelt und das Verhältnis zu den zentralen Ziel- und Anspruchsgruppen. Vgl. hierzu z.B. Meffert, H., Marketing-Management, a.a.O., S. 79 ff.; Kreikebaum, H., Strategische Unternehmensplanung, a.a.O., S. 46 ff.; Gabele, E., Kretschmer, H., Unternehmensgrundsätze, Zürich 1986, S. 27 ff.

Hierbei sind die Begriffe Unternehmensidentität und -kultur voneinander abzugrenzen. An der Unternehmensidentität sollen unternehmensintern und -extern das Verhalten, die Kommunikation und der visuelle Auftritt ausgerichtet werden. In der Unternehmenskultur kommen die historisch gewachsenden Normen, Wertvorstellungen und Denkhaltungen zum Ausdruck, die das aktuelle Verhalten der Mitarbeiter und das gegenwärtige Erscheinungsbild des Unternehmens prägen. Vgl. hierzu auch Meffert, H., MarketingManagement, a.a.O., S. 79 ff. u. 425 ff.; Meffert, H., Strategische Unternehmensführung und Marketing, a.a.O., S. $337 \mathrm{f}$.

Unternehmensziele werden darüber hinaus nach Ausmaß, Raum-, Zeit- und Segmentbezug spezifiziert. Vgl. Heinen, E., Einfihrung in die Betriebswirtschaftslehre, a.a.O., S. 52. Für eine ausfuihrliche Betrachtung dieser Zieldimensionen siehe z.B. Becker, J., Marketing-Konzeption, a.a.O., S. 20 ff.; Meffert, H., Marketing, Grundlagen der Absatz- 
zentrale Zielsetzung wird das Erreichen eines Unternehmensgewinns angesehen. Dies liegt darin begründet, daß i.d.R. ein angemessener Gewinn die Grundlage für die Existenz und den dauerhaften Fortbestand eines Unternehmens bildet. In diesem Zusammenhang wurde in aktuellen empirischen Untersuchungen zur Zielforschung festgestellt, daß Unternehmen trotz der grundsätzlich hohen Relevanz des Gewinnziels auch weitere Zielsetzungen, wie z.B. Umsatz-, Marktanteils-, Produktivitäts- und Imageziele verfolgen. ${ }^{62}$

Zur Kennzeichnung ausgewählter Unternehmensziele in High-Tech-Märkten lassen sich die Zielinhalte den Marktstellungs-, Rentabilitäts-, Finanzierungs-, Sozial- und Prestigeziele zuordnen. ${ }^{63}$

Für die High-Tech-Unternehmen sind die Marktstellungsziele wie z.B. Umsatz, Marktanteil, Sicherung der Wettbewerbsposition und Markterschließung von besonderer Bedeutung. Ein Grund hierfür ist darin zu sehen, daß in High-TechMärkten oftmals mehrere Produkt- und Anwendungstechnologien im Wettbewerb um den Industriestandard stehen. ${ }^{64}$ Die Marktdurchsetzung einer Technologie ist dabei stark abhängig von dem erreichten Marktanteil. Beispielhaft ist das von JVC entwickelte VHS-System anzuführen, welches sich gegenüber dem Beta-

politik, a.a.O., S. 81 ff. Zur Unterscheidung ökonomischer und psychographischer Zielinhalte siehe z.B. Meffert, H., Marketing-Management, a.a.O., S. 96 ff.; Schürmann, U., Erfolgsfaktoren der Werbung im Produktlebenszyklus, a.a.O., S. $51 \mathrm{ff}$.

Vgl. hierzu insbesondere Fritz, W., Marktorientierte Unternehmensführung und Unternehmenserfolg, a.a.O., S. 248 ff.; Meffert, H., Kirchgeorg, M., Umweltschutz als Unternehmensziel, in: Marketing-Schnittstellen: Herausforderungen für das Management, Hrsg.: Specht, G., Silberer, G., Engelhardt, W.H., Stuttgart 1989, S. 186 ff.; Meffert, H., Strategische Unternehmensführung und Marketing, a.a.O., S. 40 f.; Fritz, W., et al., Unternehmensziele in Industrie und Handel, in: DBW, 1988, Nr. 4., S. 378 ff.; Niebauer, F.F., Das PIMS-Programm und Portfolio-Management, in: Strategische Unternehmensplanung, Hrsg.: Hahn, D., Taylor, B., Würzburg, Wien 1983, S. 135 ff.; Heinen, E., Grundlagen betriebswirtschaftlicher Entscheidungen, a.a.O., S. 59 ff.

Siehe zu einer vergleichbaren Systematisierung auch Meffert, H., Marketing-Management, a.a.O., S. 90 f.; Becker, J., Marketing-Konzeption, a.a.O., S. 12 f.; Ulrich, P., Fluri, E., Management, 3. Aufl., Bern, Stuttgart 1984, S. 81 f. Einer weiteren Kategorie sind umweltschutzbezogene Unternehmensziele zuzurechnen. Vgl. dazu Ostmeier, H., Ökologieorientierte Produktinnovationen, a.a.O., S. 109 ff.; Kirchgeorg, M., Ökologieorientiertes Unternehmensverhalten, a.a.O., S. $232 \mathrm{ff}$. $\mathrm{Da}$ in der vorliegenden Untersuchung keine Umweltschutzziele erhoben worden sind, wird hierauf nicht weiter eingegangen. 
System von Sony und dem Videosystem 2000 von Philips/Grundig im Videorecordermarkt erfolgreich durchgesetzt hat. ${ }^{65}$ Mit Blick auf die Timingstrategie ist die Sicherung einer Wettbewerbsposition in den Shake-Out-Phasen wichtig, da sich die Wettbewerbssituation zu diesen Zeitpunkten neu strukturiert. ${ }^{66}$ Die Markterschließung besitzt vor allem bezüglich der Nutzung von Wachstumspotentialen im Rahmen einer Marktentwicklungs- und Diversifikationsstrategie einen besonderen Stellenwert. ${ }^{67}$

Zwischen den Marktstellungszielen und den rentabilitätsbezogenen Zielsetzungen wie z.B. kurzfristiger bzw. langfristiger Gewinn, Produktivitätssteigerung in den Unternehmensfunktionen und Kostenreduktion besteht eine Mittel-ZweckBeziehung. ${ }^{68}$ Eine Marktanteilssteigerung führt beispielsweise aufgrund der Skalen- und Erfahrungseffekte i.d.R. zu einer Reduktion der Stückkosten im Unternehmen. ${ }^{69}$ In Bezug auf den Unternehmensgewinn bleibt festzuhalten, daß die Gewinnerzielung Vorraussetzung für den langfristigen Bestand des Unternehmens ist. Es ist hierbei jedoch insbesondere von Pionierunternehmen zu berücksichtigen, da $B$ in der Markteinführungsphase von High-Tech-Produkten aufgrund der

Vgl. Heß, G., Kampf um den Standard!, a.a.O., S. 53 ff.

66 Vgl. Raffée, H., Fritz, W., Dimensionen und Konsistenz der Führungskonzeption von Industrieunternehmen - Ergebnisse einer empirischen Untersuchung, a.a.O., S. 312 f.; Meffert, H., Marketing und strategische Unternehmensfuhrung - ein wettbewerbsorientierter Kontingenzansatz, a.a.O., S. 662 f. Siehe in diesem Zusammenhang auch die Erläuterungen im Teil B Kap. 2.33 der Arbeit.

Vgl. Wieselhuber, N., Erschließung von neuen Wachstumsquellen durch Diversifikation, in: Handbuch Strategisches Marketing, Hrsg.: Wieselhuber, N., Töpfer, A., Landsberg a.L. 1984, S. 426 ff. Siehe hierzu auch die Darstellungen im Teil B Kap. 2.31 der Arbeit.

Vgl. Meffert, H., Strategische Unternehmensfuihrung und Marketing, a.a.O., S. 40 f.; Meffert, H., Marketing, Grundlagen der Absatzpolitik, a.a.O., S. 78 f. Die Mittel-ZweckBeziehungen von Zielen lassen sich in einem Zielsystem zusammenfassen. Zwischen einzelnen Zielen besteht hierbei ein komplementärer, neutraler oder konfliktärer Zusammenhang. Vgl. hierzu z.B. Kupsch, P., Unternehmensziele, Stuttgart, New York 1979, S. 26 ff.; Heinen, E., Grundlagen betriebswirtschaftlicher Entscheidungen, a.a.O., S. 104 f.; Meffert, H., Unternehmensziele, in: Jahrbuch des Marketing, Hrsg.: Schöttle, K.M., Essen 1971, S. $29 \mathrm{ff}$.

Vgl. Meffert, H., Marketing-Management, a.a.O., S. 60 ff.; Remmerbach, K.-U., Markteintrittsentscheidungen, a.a.O., S. 15 f.; Kloock, J., Sabel, H., Schuhmann, W., Die Erfahrungskurve in der Unternehmenspolitik, a.a.O., S. 8 ff. Siehe hierzu auch die Ausführungen im Teil B Kap. 2.2 der Arbeit. 
hohen Aufwendungen für die Produktentwicklung und den Marktaufbau kaum Gewinne erwirtschaftet werden. ${ }^{70}$

In diesem Zusammenhang gewinnen auch die finanziellen Ziele wie z.B. Kreditwürdigkeit in High-Tech-Märkten zunehmend an Bedeutung. ${ }^{71}$ Für die Unternehmen stellt die Kreditwürdigkeit immer wieder eine kritische Zielgröße dar, die oftmals nur durch die Bereitstellung von Risikokapital zu erfüllen ist. ${ }^{72}$ Darüber hinaus sind von den High-Tech-Anbietern Prestigeziele wie z.B. Imageverbesserung und Sozialziele wie z.B. Mitarbeiterzufriedenheit festzulegen. Mitarbeiterbezogenen Sozialzielen ist hierbei eine besondere Aufmerksamkeit beizumessen, da das Know-how der Mitarbeiter in High-Tech-Märkten häufig einen Engpaßfaktor darstellt. ${ }^{73}$

Vor dem Hintergrund der aufgezeigten Ziele in High-Tech-Märkten ist aus entscheidungsorientierter Perspektive der Frage nachzugehen, inwieweit ein Zusammenhang zwischen den Unternehmenszielen und den Marketingstrategien von strategischen Gruppen besteht. ${ }^{74}$ Es ist hierbei u.a. von Interesse, zu erfahren,

Vgl. Remmerbach, K.-U., Markteintrittsentscheidungen, a.a.O., S. 16; Shanklin, W.L., Ryans, J.K., Essentials of marketing high technology, a.a.O., S. 145 f.; McKenna, R., Borrus, M., Cohen, S., Industrial policy and international competition in high technology, Part I: Blocking capital formation, in: California Management Review, 1984, No. 2, S. $15 \mathrm{ff}$.

71 Vgl. Roberts, E.B., Entrepreneurs in high technology: Lessons from MIT and beyond, a.a.O., S. $124 \mathrm{ff}$.

Vgl. Roberts, E.B., Entrepreneurs in high technology: Lessons from MIT and beyond, a.a.O., S. $188 \mathrm{ff}$. Zu den Finanzierungsformen des Venture Capital siehe auch Hurry, D., Miller, A.T., Bowmann, E.H., Calls on high-technology: Japanese exploration of venture capital investments in the united states, in: Strategic Management Journal, Vol. 13 (1992), S. 86 ff.; Ewald, A., Organisation des strategischen Technologie-Managements, a.a.O., S. 217 ff.; Faselow, K.-H., Stedler, H., Venture Capital in Deutschland, in: Die Bank, 1988, S. $555 \mathrm{ff}$.

Vgl. Meffert, H., Marketing-Management, a.a.O., S. 91 f.; Schmelzer, H.J., Buttermilch, K.-H., Reduzierung der Entwicklungszeiten in der Produktentwicklung als ganzheitliches Problem, a.a.O., 63 f.; Maidique, M.A., Hayes, R.H., The art of high-technology management, in: Sloan Management Review, Vol. 26 (1984), S. 17 ff.

Im Hinblick auf das strategische Unternehmensverhalten übernehmen Unternehmensziele eine Steuerungs-, Koordinations- und Kontrollfunktion. Zu den Funktionen von Unternehmenszielen siehe vor allem Kupsch, P., Unternehmensziele, a.a.O., S. 15 ff.; Meffert, H., Unternehmensziele, a.a.O., S. 23 f.; Heinen, E., Das Zielsystem der Unternehmung, Wiesbaden 1966, S. $18 \mathrm{ff}$. 
ob eine langfristige Gewinnorientierung auch $\mathrm{zu}$ einer wachstumsorientierten Strategieausrichtung der High-Tech-Unternehmen führt, die z.B. in der Produktbzw. Marktentwicklung zum Ausdruck kommt. Darüber hinaus erscheint es bedeutsam, zu untersuchen, ob ein Zusammenhang zwischen bestimmten Marktstellungszielen wie z.B. der Erschließung neuer High-Tech-Märkte und der Strategieausprägung der strategischen Gruppen im Hinblick auf die Marktentwicklung bzw. Diversifikation festzustellen ist. Für die weitere Analyse läßt sich folgende Basishypothese formulieren, die einer empirischen Überprüfung unterzogen wird:

$\mathrm{H}_{\mathrm{Zl}}$ In High-Tech-Märkten besteht ein Zusammenhang zwischen den Unternehmenszielen und den Marketingstrategien von strategischen Gruppen. Die strategischen Gruppen unterscheiden sich daher hinsichtlich der von ihnen verfolgten Unternehmensziele innerhalb einer homogenen Umfeldsituation.

Auf der Grundlage der empirischen Ergebnisse zu den Marketingstrategien der strategischen High-Tech-Gruppen in den homogenen Umfeldsituationen werden für die weitere Analyse folgende Basishypothesen formuliert:

H Z2 In den verschiedenen homogenen Umfeldsituationen in High-TechMärkten lassen sich bei den vergleichbaren strategischen Gruppen ähnliche Zielprioritäten identifizieren.

H Z3 Bei den vergleichbaren strategischen Gruppen, die in den verschiedenen homogenen Umfeldsituationen in High-Tech-Märkten ähnliche Zielprioritäten haben, lassen sich situationsbedingte Unterschiede in der Bedeutung der Unternehmensziele feststellen. 


\subsection{Empirische Analyse der Unternehmensziele von strategischen Gruppen}

In den folgenden Ausführungen werden zunächst die Unternehmensziele der strategischen Gruppen in der Umfeldsituation A aufgezeigt. Danach wird in einer situationsübergreifenden Betrachtung analysiert, welche Gemeinsamkeiten und Unterschiede in den Zielprioritäten der vergleichbaren strategischen High-TechGruppen bestehen. Die Beschreibung der empirischen Ergebnisse zu den Unternehmenszielen der strategischen Gruppen erfolgt anhand der in Abbildung 33 dargestellten Mittelwertabweichungen der Zielausprägungen. ${ }^{75}$

In der Umfeldsituation A sind bei den differenzierungsorientierten High-TechAnbietern die Marktstellungsziele Sicherung der Wettbewerbsfähigkeit, langfristige Gewinnerzielung, Marktanteilssicherung und Markterschließung überdurchschnittlich ausgeprägt. Darüber hinaus sehen die Unternehmen dieser strategischen Gruppe die Imageverbesserung und die Mitarbeitermotivation als besonders wichtig an. Den übrigen Unternehmenszielen wird eine untergeordnete Bedeutung zugesprochen.

Bei den kostenorientierten High-Tech-Unternehmen steht die Erreichung kurzfristiger Gewinne im Vordergrund der Zielsetzungen. Die Kostenorientierung kommt außerdem in der überdurchschnittlichen Bewertung der Unternehmensziele Kostenreduktion, Produktivitätssteigerung in der Produktion und Kreditwürdigkeit zum Ausdruck. Die restlichen Zieldimensionen werden von den Unternehmen der zweiten strategischen Gruppe vernachlässigt.

In der Situation A, die durch eine hohe Marktdynamik gekennzeichnet ist, wird die Markterschließung von den innovationsorientierten Unternehmen für überdurchschnittlich wichtig gehalten. Den übrigen Marktstellungs-, Rentabilitäts-, Finanz-, Sozial- und Prestigezielen messen die "Innovationsorientierten" eine geringe Bedeutung bei.

Die absoluten Mittelwertabweichungen der einzelnen Zielvariablen der strategischen High-Tech-Gruppen in den Situationen A, B und C sind dem Anhang II der Arbeit zu entnehmen. 


\begin{tabular}{|c|c|c|c|c|c|c|c|c|c|c|c|c|c|c|c|}
\hline \multirow{3}{*}{ Zielvariablen } & \multicolumn{15}{|c|}{ Ausprägung der Zielvariablen im Vergleich zum Durchschnitt } \\
\hline & \multicolumn{5}{|c|}{ in Situation A } & \multicolumn{5}{|c|}{ in Situation B } & \multicolumn{5}{|c|}{ in Situation C } \\
\hline & $\begin{array}{l}\text { 810.- } \\
\text { niveav }\end{array}$ & Ø & 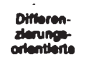 & Koetemortion. & Imoverations: & $\begin{array}{l}\text { Sto.- } \\
\text { ntveeru }\end{array}$ & $\varnothing$ & 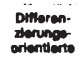 & Roetenorien- & $\begin{array}{l}\text { Innovetione- } \\
\text { orientierto }\end{array}$ & \begin{tabular}{|l|l} 
Sig.- \\
niveas
\end{tabular} & $\varnothing$ & $\begin{array}{l}\text { Dimeron- } \\
\text { ziennges:- } \\
\text { orientionto }\end{array}$ & $\begin{array}{l}\text { Kowlenorien- } \\
\text { tiertio }\end{array}$ & morvatione \\
\hline $\begin{array}{l}\text { Sicherung Wettbewerbs- } \\
\text { poshtion }\end{array}$ & - & 1,84 & + & - & - & - & 1,67 & 0 & - & + & * & 1,94 & 0 & - & + \\
\hline Umsatz & n.s. & 2,19 & 0 & 0 & 0 & n.s. & 2,01 & 0 & 0 & - & n.s. & 2,05 & 0 & 0 & - \\
\hline Marktanteil & n.s. & 2,26 & + & - & 0 & * & 2,10 & + & -- & 0 & n.s. & 2,05 & + & - & 0 \\
\hline Markterschliebung & * & 2,13 & + & - & + & n.s. & 1,81 & 0 & - & + & $*$ & 2,21 & + & - & $+t$ \\
\hline kurztr. Gowinn & \# & 2.78 & - & ++ & 0 & ** & 2,38 & 0 & ++ & -. & $\bullet$ & 2,24 & 0 & + & - \\
\hline langtr. Gowinn & n.s. & 1,63 & + & - & 0 & ** & 1,72 & + & -- & 0 & n.s. & 1,86 & 0 & - & + \\
\hline Kostenreduktion & 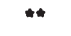 & 2,08 & 0 & + & - & $\#$ & 1,86 & + & + & -- & * & 1,85 & 0 & + & - \\
\hline Kreditwôrdigkeit & n.s. & 2,52 & 0 & + & - & - & 2,44 & + & -- & - & ** & 2,47 & 0 & - & ++ \\
\hline $\begin{array}{l}\text { Produktivitătsetelgerung } \\
\text { F\&E }\end{array}$ & n.8. & 2,39 & 0 & 0 & 0 & - & 1,81 & + & - & - & ** & 2,28 & - & $\cdots$ & + \\
\hline $\begin{array}{l}\text { Produktivitâtssteigerung } \\
\text { Produktion }\end{array}$ & n.s. & 2,09 & 0 & + & - & $\bullet$ & 1,72 & 0 & + & - & • & 1,77 & + & + & - \\
\hline $\begin{array}{l}\begin{array}{l}\text { Produktivitatssteigerung } \\
\text { Vertrieb }\end{array} \\
\end{array}$ & . & 2,02 & 0 & 0 & $\cdots$ & n.8. & 1,77 & 0 & 0 & $\cdot$ & $\star$ & 2,02 & + & - & + \\
\hline Mitarbelterzulriedenheit & - & 2,28 & + & - & - & • & 2,12 & + & - & 0 & • & 2,56 & + & - & + \\
\hline Imageverbesserung & $\bullet$ & 2,17 & + & - & $\mathbf{0}$ & ** & 2,03 & + & -- & + & n.s. & 2,15 & + & - & 0 \\
\hline \multicolumn{2}{|l|}{ Anzahl der Unternehmen } & 104 & 51 & 33 & 20 & & 159 & 85 & 33 & 41 & & 112 & 62 & 29 & 21 \\
\hline 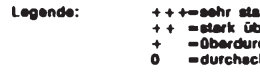 & \multicolumn{4}{|c|}{ 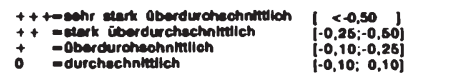 } & 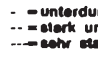 & $\begin{array}{l}\text { oheohnt } \\
\text { chordunch } \\
\text { cit unter }\end{array}$ & 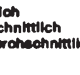 & \multicolumn{2}{|c|}{ on $\begin{array}{l}{[0.10: 0.26]} \\
{[0.25} \\
{[>0.50,1}\end{array}$} & 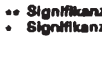 & $\begin{array}{l}\text { zntwoeau } \\
\text { enlveau }\end{array}$ & $0.05 \mathrm{Fi}$ & & \multicolumn{2}{|c|}{ 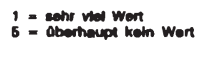 } \\
\hline
\end{tabular}

Abb. 33: Bedeutung der Unternehmensziele fur die strategischen High-Tech-Gruppen in den Umfeldsituationen 
Insgesamt wird deutlich, daß in der Umfeldsituation A ein Zusammenhang zwischen den Zielsetzungen und den Marketingstrategien der differenzierungs-, kosten- und innovationsorientierten High-Tech-Unternehmen besteht und sich die strategischen Gruppen in der Ausprägung der Unternehmensziele unterscheiden (vgl. Abbildungen 28 und 33). ${ }^{76}$ Für die "Differenzierungsorientierten" sind Zielsetzungen relevant, die die langfristige Stellung in High-Tech-Märkten betreffen. Entsprechend werden von den Unternehmen innerhalb der Marketingstrategie die Marktpenetration und die Marktentwicklung als wichtig beurteilt. Es ist weiterhin festzustellen, daß die überdurchschnittliche Bedeutung des Imageziels in dieser strategischen Gruppe mit dem Streben nach einer Image-Führerschaft korrespondiert. Der hohe Stellenwert des mitarbeiterbezogenen Sozialziels weist darauf hin, daß der Aufbau einer dauerhaften Service-Führerschaft eine hohe Mitarbeiterzufriedenheit voraussetzt.

Das Unternehmensverhalten der kostenorientierten High-Tech-Anbieter ist durch eine kurzfristige Gewinnorientierung geprägt. Aus diesem Grund werden den Strategieoptionen Produktentwicklung, Marktentwicklung und Diversifikation, die hohe Vorlaufinvestitionen erfordern, eine geringe Bedeutung beigemessen. Demgegenüber ist bei den Unternehmen dieser strategischen Gruppe zu erkennen, daß ein Zusammenhang zwischen den rentabilitätsorientierten Unternehmenszielen Kostenreduktion bzw. Produktivitätssteigerung in der Produktion und der Strategieausrichtung Erreichung einer Preis-/Kosten-Führerschaft besteht. Bei den innovationsorientierten High-Tech-Herstellern steht die Markterschließung im Vordergrund der Unternehmensziele. Sie räumen daher auch der Diversifikation und der Pionierorientierung im Rahmen der Marketingstrategie einen überdurchschnittlich hohen Stellenwert ein.

Die Analyse der Unternehmensziele der differenzierungs-, kosten- und innovationsorientierten High-Tech-Unternehmen in den verschiedenen homogenen Umfeldsituationen zeigt, daß den vergleichbaren strategischen High-Tech-Gruppen dimensionen sowie der einzelnen Zielvariablen der strategischen High-Tech-Gruppen in den Situationen A, B und C im Anhang II der Arbeit. 
ähnliche Zielprioritäten zugrunde liegen (vgl. Abbildungen 28 und 33). ${ }^{77}$ Aufgrund der aufgezeigten Zusammenhänge zwischen den Zielen und den Marketingstrategien der strategischen High-Tech-Gruppen und aufgrund der Ergebnisse der Mittelwertvergleichstests sind die Hypothesen $\mathrm{H} \mathrm{Z1}$ und $\mathrm{H}$ Z2 insgesamt anzunehmen. $^{78}$

Bezüglich der Hypothese $\mathrm{H} \mathrm{Z3}$ ist zu prüfen, ob bei den vergleichbaren strategischen Gruppen, die in den Umfeldsituationen A, B und C ähnliche Zielschwerpunkte besitzen, situationsbedingte Unterschiede in der Bedeutung einzelner Unternehmensziele bestehen. Auf Basis der in Abbildung 33 dargestellten Mittelwertabweichungen der Zieldimensionen ist festzustellen, daß die "Differenzierungsorientierten" auf die Realisierung langfristiger Gewinne und die Mitarbeitermotivation in den Umfeldsituationen A und B im Vergleich zur Situation $\mathrm{C}$ einen größeren Wert legen. ${ }^{79}$ Der Produktivitätssteigerung in der F\&E wird vor allem in Situation B und der Produktivitätssteigerung in der Produktion in Situation C eine besondere Relevanz zugesprochen. Die unterschiedliche Bedeutung der Unternehmensziele läßt sich damit erklären, daß bei der Zielplanung in der Situation B technologische und in der Situation C kostenbezogene Aspekte eine stärkere Berücksichtigung finden.

Die kostenorientierten Unternehmen messen der Erzielung kurzfristiger Gewinne, der Kostenreduktion und der Produktivitätssteigerung in der Produktion in Situation A, in der noch kein Technologie- bzw. Preiswettbewerb herrscht, einen besonders geringen Stellenwert bei. Von der strategischen Gruppe der "Innovationsorientierten" werden die Markterschließung in Situation B und die Kreditwürdigkeit in Situation $C$ als besonders wichtig angesehen. Darüber hinaus

Siehe in diesem Zusammenhang auch die absoluten Mittelwertabweichungen der einzelnen Strategiedimensionen sowie der einzelnen Zielvariablen der strategischen High-TechGruppen in den Situationen A, B und C im Anhang II der Arbeit.

$\mathrm{Zu}$ den Ergebnissen der Mittelwertvergleichstests siehe die Abbildung 33 der Arbeit. Es zeigt sich hierbei, daß fur die Zielvariablen langfristiger Gewinn, Umsatz und Marktanteil in der Mehrzahl der Umfeldsituationen keine signifikanten Ergebnisse vorliegen.

Vgl. hierzu auch die absoluten Mittelwertabweichungen der einzelnen Strategiedimensionen sowie der einzelnen Zielvariablen der strategischen High-Tech-Gruppen in den Situationen A, B und C im Anhang II der Arbeit. 
legen die innovationsorientierten High-Tech-Unternehmen vor allem in den Situationen B und C einen großen Wert auf die Sicherung der Wettbewerbsfähigkeit. Ein Grund für die wachsende Bedeutung dieses Marktstellungsziels liegt darin, daß die Wettbewerbsdynamik in den beiden Umfeldsituationen B und C im Vergleich zur Situation A deutlich höher ist. Es bleibt zusammenfassend festzuhalten, daß sich die strategischen High-Tech-Gruppen in ihren Zielprioritäten in den verschiedenen Umfeldsituationen vergleichsweise gering unterscheiden. Somit ist die Hypothese $\mathrm{H}$ Z3 nur eingeschränkt zu bestätigen.

\subsection{Unternehmenskultur als Einflußfaktor der strategischen Gruppen}

Im Mittelpunkt der folgenden Betrachtung steht die Analyse des Zusammenhangs zwischen der Unternehmenskultur und den Marketingstrategien von strategischen Gruppen. Es sollen hierdurch Erfahrungen gesammelt werden, ob Unterschiede in der Unternehmenskultur die Bildung bestimmter strategischer Gruppen begünstigen.

\subsection{Theoretische Grundlagen zum Zusammenhang zwischen Unternehmenskultur und Marketingstrategien von strategischen Gruppen}

Die Unternehmenskultur ${ }^{80}$ bildet ein System von Wertvorstellungen, Verhaltensnormen sowie Denk- und Handlungsweisen, durch die das Verhalten der Mitarbeiter im gesamten Unternehmen geprägt wird. ${ }^{81}$ Eine nähere Untersuchung der

80 In der Literatur werden die Begriffe Unternehmenskultur, Organisationskultur, Corporate Culture und Organizational Culture weitgehend synonym verwendet. Vgl. hierzu u.a. Scholz, Ch., Organisationskultur, Zwischen Schein und Wirklichkeit, in: ZfbF, 1988, Nr. 3, S. 244; Staerkle, R., Wechselwirkungen zwischen Unternehmenskultur und Organisationsstruktur, in: Integriertes Management, Hrsg.: Probst, G.J., Siegwart, H., Bern, Stuttgart 1985, S. 552. Zur Abgrenzung der Unternehmenskultur von funktionsbereichsbezogenen Subkulturen wie z.B. der Marketing- oder der F\&E-Kultur siehe z.B. Meffert, H., Strategische Unternehmensführung und Marketing, a.a.O., S. $352 \mathrm{ff}$.

Zum Begriff und den Merkmalen der Unternehmenskultur siehe z.B. Meffert, H., Marketing-Management, a.a.O., S. 426 ff.; Meffert, H., Hafner, K., Unternehmenskultur und 
Kultur von High-Tech-Anbietern ist von besonderem Interesse, da die Entwicklungsdynamik in High-Tech-Märkten hohe Anforderungen an die Innovationsund Anpassungsfähigkeit der Unternehmen und deren Mitarbeiter stellt. ${ }^{82}$ Die Unternehmenskultur übt einen besonderen Einfluß auf die Präferenzstruktur des Management bei der Planung von Marketingstrategien aus. ${ }^{83}$ So sind die Strategieentscheidungen bei einem High-Tech-Anbieter, der eine starke innovationsorientierte Kultur aufweist, u.a. durch eine hohe Risikofreudigkeit und Flexibilität des Management geprägt. ${ }^{84}$

Fragestellungen zur verhaltensprägenden Wirkung der Unternehmenskultur stehen seit Mitte der 80er Jahre verstärkt im Interesse der betriebswirtschaftlichen Forschung. Hierbei ist jedoch zu erkennen, daß sich die Forschungen zum Kultur-Strategie-Zusammenhang zu meist auf eine theoretische Analyse beschränken. ${ }^{85}$ Empirisch gesicherte Erkenntnisse zur Beziehung zwischen der

marktorientierte Unternehmensführung, a.a.O., S. 4 ff.; Steinbacher, F., Kultur, Begriff, Theorie, Funktion, Stuttgart 1976, S. 33.

Vgl. Dussauge, P., Hart, St., Ramanantsoa, B., Strategic technology management, a.a.O., S. 197 ff.; Ewald, A., Organisation des strategischen Technologie-Managements, a.a.O., S. 294 ff.; Kieser, A., Unternehmenskultur und Innovation, in: Unternehmenskultur und Unternehmensfiihrung, Dokumentationspapier Nr. 39 der Wissenschaftlichen Gesellschaft für Marketing und Unternehmensführung e.V., Hrsg.: Meffert H., Wagner, H., Münster 1987, S. 20 ff.; Heinen, E., Unternehmenskultur als Gegenstand der Betriebswirtschaftslehre, in: Unternehmenskultur, Perspektiven für Wissenschaft und Praxis, Hrsg.: Heinen, E., München, Wien 1987, S. 2 ff.

Die Unternehmenskultur hat zudem einen maßgeblichen Einfluß auf die Strategieimplementierung. Vgl. hierzu z.B. Hilker, J., Marketingimplementierung, a.a.O., S. 73 ff. u. 291 ff.; Pümpin, C., Bedeutung der Unternehmenskultur für die Unternehmensfuihrung, in: Unternehmenskultur und Unternehmensfuihrung, Dokumentationspapier Nr. 39 der Wissenschaftlichen Gesellschaft für Marketing und Unternehmensfuihrung e.V., Hrsg.: Meffert H., Wagner, H., Münster 1987, S. 8.

Vgl. Bleicher, K., Organisation: Strategien, Strukturen, Kulturen, 2. Aufl., Wiesbaden 1990, S. 785 ff.; Kieser, A., Unternehmenskultur und Innovation, a.a.O., S. 23 ff.; Schuster, L., Widmer, A.W., Theorie und Praxis der Unternehmenskultur, in: ZfO, 1984, Nr. 8, S. 492 f.

$\mathrm{Zu}$ konzeptionellen Überlegungen im Hinblick auf den Kultur-Strategie-Fit siehe z.B. Meffert, H., Marketing-Management, a.a.O., S. 440 ff.; Pümpin, C., Koller, H.P., Die Bedeutung der Unternehmenskultur für die Unternehmensstrategie, a.a.O., S. 304 ff.; Hinterhuber, H., Wettbewerbsstrategie, a.a.O., S. 224 ff.; Bleicher, K., Zum Verhältnis von Kulturen und Strategien der Unternehmung, in: Organisationskultur: Phänomen Philosophie - Technologie, Hrsg.: Dülfer, E., Stuttgart 1988, S. 96 ff.; Scholz, Ch., Corporate culture and strategy, The problem of strategic fit, in: Long Range Planning, Vol. 20 (1987), S. 78 ff.; Deshpandé, R., Parasuraman, A., Linking corporate culture to strategic planning, in: Business Horizons, 1986, No. 3, S. $28 \mathrm{ff}$. 
Unternehmenskultur und dem strategischen Unternehmensverhalten liegen in High-Tech-Märkten bislang kaum vor. ${ }^{86}$

Vor diesem Hintergrund wird für die folgende Diskussion auf die allgemeine Literatur zur Kulturforschung zurückgegriffen. Um zu einer differenzierten Betrachtung der Kultur von High-Tech-Unternehmen zu gelangen, wird die Analyse anhand der kulturellen Grundorientierungen vorgenommen, die in einem hohen Kunden-, Mitarbeiter-, Innovations-, Kosten- bzw. Technologiebezug zum Ausdruck kommen. ${ }^{87}$ Im Hinblick auf die Wirkungsbeziehung zwischen Kultur und Strategie wird unterstellt, daß die Unternehmenskultur als unabhängige Bestimmungsgröße das strategische Verhalten von High-Tech-Anbietern beeinflußt. ${ }^{88}$

In High-Tech-Märkten besitzt die Kundenorientierung der Unternehmen eine besondere Relevanz, da ein enger Kundenkontakt und eine gute Kenntnis der Anwenderprobleme die wesentlichen Voraussetzungen für die Entwicklung marktfähiger technologischer Produkte darstellen. Ein starker Kundenbezug ist

86 Erste Hinweise über den Kultur-Strategie-Zusammenhang von High-Tech-Unternehmen gibt die empirische Untersuchung von Meffert/Hafner. Vgl. hierzu Meffert, H., Hafner, K., Unternehmenskultur praxistauglich?, in: asw, Sondernummer Oktober 1988, S. 28 ff.; Meffert, H., Hafner, K., Poggenpohl, M., Unternehmenskultur und Unternehmensfühung, Ergebnisse einer empirischen Untersuchung, Arbeitspapier Nr. 43 der Wissenschaftlichen Gesellschaft furr Marketing und Unternehmensfiihrung e.V., Hrsg.: Meffert H., Wagner, H., Münster 1988, S. 12 ff. Siehe in diesem Zusammenhang auch Meffert, H., Kirchgeorg, M., Marktorientiertes Umweltmanagement, a.a.O., S. 317 ff.

$87 \mathrm{Zu}$ einer vergleichbaren Systematisierung kultureller Grundorientierungen siehe auch Meffert, H., Marketing-Management, a.a.O., S. 440; Meffert, H., Hafner, K., Unternehmenskultur praxistauglich?, a.a.O., S. 26 ff.; Pümpin, C., Bedeutung der Unternehmenskultur für die Unternehmensführung, a.a.O., S. 6. Die aufgefuhrten kulturellen Grundorientierungen wurden auch im Rahmen einer Diplomarbeit am Institut für Marketing am Beispiel von High-Tech-Unternehmen empirisch nachgewiesen. Siehe hierzu Wübben, J., Die Unternehmenskultur als Bestimmungsfaktor marktorientierter Unternehmensstrategien, unver. Diplomarbeit, Münster 1991, S. 26 ff. Eine Darstellung der auf Basis einer konfirmatorischen Faktorenanalyse ermittelten kulturellen Grundorientierungen ist auch im Anhang II der Arbeit enthalten.

Vgl. zu dieser Einschätzung auch Heinen, E., Unternehmenskultur als Gegenstand der Betriebswirtschaftslehre, a.a.O., S. 43. In der vorliegenden Untersuchung wird auf eine Betrachtung der wechselseitigen Beziehungen zwischen Unternehmenskultur und -strategie sowie zwischen Unternehmenskultur und -zielen verzichtet. Siehe hierzu u.a. Meffert, H., Marketing-Management, a.a.O., S. 88 f. u. 438 f.; Meffert, H., Hafner, K., Unternehmenskultur und marktorientierte Unternehmensführung, a.a.O., S. $27 \mathrm{ff}$. 
daher insbesondere bei High-Tech-Anbietern wichtig, die eine Nutzen-Führerschaft anstreben. ${ }^{89}$

Die Mitarbeiterorientierung bildet eine zentrale Grundlage der qualitätsorientierten Marketingstrategien. Eine hohe Identifikation der Mitarbeiter mit dem Unternehmen und das Bewußtsein, für die Qualität der High-Tech-Produkte verantwortlich zu sein, fördert die Einsatzbereitschaft und motiviert zu einem eigenständigen Handeln. Dies bietet die Möglichkeit, einen hohen Qualitätsstandard im Unternehmen zu verankern, der auf einem von allen Mitarbeitern gleichermaßen getragenen Qualitätsverständnis beruht. Ein ausgeprägtes qualitätsbezogenes Anspruchsniveau der Mitarbeiter trägt außerdem dazu bei, den Umfang der Qualitätskontrollen während der Leistungserstellung zu reduzieren. ${ }^{90}$

Ein dominanter Einfluß der Innovationsorientierung ist im Hinblick auf die Produktentwicklung und Diversifikation zu vermuten. Unternehmerische Flexibilität, Kreativität und Risikofreudigkeit eröffnen den High-Tech-Anbietern in einem dynamischen Wettbewerbsumfeld die Chance, mit technologischen Produktneuheiten in bestehenden und neuen Märkten dauerhaft Wettbewerbsvorteile aufzubauen und zu sichern. Darüber hinaus ist eine deutliche Innovationsorientierung bei Unternehmen zu erwarten, die überwiegend als Pioniere in High-Tech-Märkte eintreten. ${ }^{91}$

Vgl. Frese, E., Grundlagen der Organisation, a.a.O., S. 307 ff.; Meffert, H., Hafner, K., Poggenpohl, M., Unternehmenskultur und Unternehmensführung, a.a.O., S. 41.; Pümpin, C., Wüthrich, H.A., Die Marketing-Kultur als betrieblicher Wert von strategischer Bedeutung, in: Thexis, 1985, Nr. 4, S. 23 f.; Peters, Th., Waterman, R., Auf der Suche nach Spitzenleistungen, Landsberg a.L. 1984, S. 39. Siehe hierzu auch die Ausfuihrungen im Teil B Kap. 2.32 der Arbeit.

Vgl. Meffert, H., Marketing-Management, a.a.O., S. 446 f.; Witte, A., Integrierte Qualitätssteuerung im Total Quality Management, a.a.O., S. 103 ff.; Puimpin, C., Bedeutung der Unternehmenskultur für die Unternehmensführung, a.a.O., S. 13.; Heinen, E., Unternehmenskultur als Gegenstand der Betriebswirtschaftslehre, a.a.O., S. 2 ff.

Vgl. Tylecote, A., Demirag, I., Short-Termism: Culture and structures as factors in technological innovation, in: Technological change and company strategies, Hrsg.: Coombs, R., Saviotti, P., Walsh, V., London u.a. 1992, S. 211 ff.; Meffert, H., Hafner, K., Poggenpohl, M., Unternehmenskultur und Unternehmensfuhrung, a.a.O., S. 41; Kieser, A., Unternehmenskultur und Innovation, a.a.O., S. 23 ff. Siehe hierzu auch die Darstellungen im Teil B Kap. 2.31 und Kap. 2.33 der Arbeit. 
Bei einer intensiven Kostenorientierung der Kultur werden die Unternehmen i.d.R. den Strategieoptionen einen untergeordneten Stellenwert beimessen, die hohe Vorlaufinvestitionen und ein erhebliches Entwicklungs- und Vermarktungsrisiko beinhalten wie z.B. die Technologie- oder die Innovations-Führerschaft. Es ist vielmehr damit zu rechnen, daß die High-Tech-Hersteller preis-/ kostenbezogene Marketingstrategien verfolgen, denen eine produktionsorientierte, eher innengerichtete Sicht des Management zugrunde liegt. ${ }^{92}$

Aufgrund der Technologiedynamik in High-Tech-Märkten kommt der Technologieorientierung als kulturelle Grundausrichtung grundsätzlich eine hohe Bedeutung zu. Dies liegt darin begründet, daß die konsequente Weiterentwicklung von produkt- und anwendungsbezogenen Schrittmachertechnologien eine wesentliche Voraussetzung zur Sicherung der gegenwärtigen Wettbewerbsposition und der frühzeitige Aufbau von Know-How bei Schlüsseltechnologien die Grundlage zur Schaffung zukünftiger Differenzierungspotentiale darstellen. ${ }^{93}$

Eine einseitige Technologieorientierung birgt jedoch auch die Gefahr der Etablierung einer stark innengerichteten Unternehmenskultur, die allein durch die Wertvorstellungen und Denkhaltungen der F\&E-Mitarbeiter geprägt wird. Es besteht hierbei das Risiko, daß sich die High-Tech-Unternehmen bei der Produktentwicklung primär am technologisch Machbaren orientieren und dadurch wesentliche Nachfragerbedürnisse übersehen. Als Beispiel hierfür ist der Computerhersteller DEC zu nennen. Das Unternehmen brachte Mitte der 80er Jahre einen neuen Computer auf den Markt, der den Konkurrenzprodukten bezüglich der Rechnerleistung deutlich überlegen war, aber auf wenig Akzeptanz bei den Nachfragern traf. Der Mißerfolg lag u.a. darin begründet, daß die High-TechAnwender vermehrt benutzerfreundliche Personal-Computer für Textverarbeitung

Vgl. Frese, E., Grundlagen der Organisation, a.a.O., S. 30 ff.; Meffert, H., Strategische Unternehmensfuihrung und Marketing, a.a.O., S. 350; Meffert, H., Hafner, K., Unternehmenskultur und marktorientierte Unternehmensführung, a.a.O., S. $38 \mathrm{f}$. Siehe hierzu auch die Ausführungen im Teil B Kap. 2.32 der vorliegenden Untersuchung.

Vgl. Meffert, H., Marketing-Management, a.a.O., S. 445; Dussauge, P., Hart, St., Ramanantsoa, B., Strategic technology management, a.a.O., S. $197 \mathrm{ff}$. Siehe in diesem Zusammenhang auch die Erörterungen in Teil B Kap. 2.11, Kap. 2.31 und Kap. 2.32 der Arbeit. 
und Desktop-Anwendungen forderten und dies von DEC nicht rechtzeitig erkannt wurde. ${ }^{94}$ Um Nachfrageveränderungen frühzeitig identifizieren zu können, ist daher in der Kultur technologieorientierter High-Tech-Unternehmen i.d.R. auch eine Kundenorientierung stärker zu verankem.

Ausgehend von den skizzierten kulturellen Grundorientierungen in High-TechMärkten ist es von Interesse, zu erfahren, inwieweit ein Zusammenhang zwischen der Kultur und dem strategischen Unternehmensverhalten vorliegt. Unterschiede in der Kulturausprägung der verschiedenen strategischen Verhaltensgruppen können dabei Aufschluß darüber geben, ob die Unternehmenskultur Einfluß auf die Bildung strategischer High-Tech-Gruppen hat. In diesem Zusammenhang wird für die empirische Analyse folgende Basishypothese formuliert:

H K1 In High-Tech-Märkten besteht ein Zusammenhang zwischen der Unternehmenskultur und den Marketingstrategien von strategischen Gruppen. Die strategischen Gruppen unterscheiden sich daher in ihrer Unternehmenskultur innerhalb einer homogenen Umfeldsituation.

Auf der Grundlage der empirischen Ergebnisse zu den Marketingstrategien der strategischen High-Tech-Gruppen in den homogenen Umfeldsituationen werden in der weiteren Analyse folgende Basishypothesen überprüft:

$\mathrm{H}_{\mathrm{K} 2}$ In den verschiedenen homogenen Umfeldsituationen in High-TechMärkten weisen die vergleichbaren strategischen Gruppen ähnliche kulturelle Grundorientierungen auf.

$\mathrm{H}_{\mathrm{K} 3}$ Bei den vergleichbaren strategischen Gruppen, die in den verschiedenen homogenen Umfeldsituationen in High-Tech-Märkten ähnliche kulturelle Grundorientierungen besitzen, sind situationsbedingte Unterschiede in der Kulturausprägung festzustellen. 


\subsection{Empirische Analyse der Unternehmenskultur von strategischen Gruppen}

In den weiteren Ausführungen wird die Unternehmenskultur der strategischen High-Tech-Gruppen in der Situation A gekennzeichnet. Im Anschluß daran werden in einer situationsübergreifenden Analyse die Gemeinsamkeiten und Unterschiede der kulturellen Grundorientierungen der strategischen Gruppen in HighTech-Märkten untersucht. Zur Beschreibung der strategischen Verhaltensgruppen in den verschiedenen homogenen Umfeldsituationen werden die in der Abbildung 34 dargestellten Mittelwertabweichungen der Kulturvariablen herangezogen. ${ }^{95}$

In der Umfeldsituation A wird der mitarbeiterbezogenen Grundorientierung von den differenzierungsorientierten High-Tech-Anbietern eine zentrale Bedeutung beigemessen. Hierbei werden das Vertrauen in die Mitarbeiter und die Eigenständigkeit der Mitarbeiter als besonders wichtig angesehen. Es ist außerdem zu erkennen, daß in dieser strategischen High-Tech-Gruppe die Kulturdimensionen Kenntnis der Kundenprobleme und Technologieorientierung überdurchschnittlich ausgeprägt sind. Im Vergleich dazu sind die innovations- und kostenbezogenen Grundorientierungen in der Kultur der "Differenzierungsorientierten" kaum verankert.

Die High-Tech-Unternehmen der zweiten strategischen Gruppe legen großen Wert auf die kostenbezogenen Kulturmerkmale. Das Kostenbewußtsein ist überdurchschnittlich und die Kostenorientierung stark überdurchschnittlich ausgeprägt. In der Situation A, in der eine starke Marktdynamik herrscht, sehen die "Kostenorientierten" zudem die enge Kundenbeziehung als besonders relevant an. Andere kulturelle Grundorientierungen sind für die kostenorientierten HighTech-Hersteller von nachrangiger Bedeutung. nen Kulturvariablen der strategischen High-Tech-Gruppen in den Situationen A, B und C im Anhang II der Arbeit. 


\begin{tabular}{|c|c|c|c|c|c|c|c|c|c|c|c|c|c|c|c|}
\hline \multirow{3}{*}{ Kulturvariablen } & \multicolumn{15}{|c|}{ Ausprāgung der Kulturvariablen im Vergleich zum Durchschnitt } \\
\hline & \multicolumn{5}{|c|}{ in Situation A } & \multicolumn{5}{|c|}{ in Situation B } & \multicolumn{5}{|c|}{ in Situation C } \\
\hline & $\begin{array}{l}\text { sig. } \\
\text { nwoosu }\end{array}$ & $\varnothing$ & Ompon- & Konenoriter & Imoventions & \begin{tabular}{|l} 
8se.-. \\
nhroesu
\end{tabular} & $\varnothing$ & 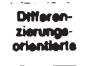 & rostenoriter & Imoventerse- & $\begin{array}{l}\text { sia. } \\
\text { nveasu }\end{array}$ & $\varnothing$ & 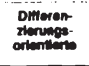 & rostanorten- & mnovertions. \\
\hline $\begin{array}{l}\text { Enge } \\
\text { Kundenbeziehungen }\end{array}$ & n.s. & 1,49 & 0 & + & - & n.s. & 1,39 & 0 & 0 & - & $*$ & 1,62 & + & - & 0 \\
\hline $\begin{array}{l}\text { Kenntnis der } \\
\text { Kundenprobleme }\end{array}$ & - & 1,74 & + & 0 & - & * & 1,66 & 0 & - & + & $*$ & 1,82 & + & - & 0 \\
\hline Vertrauen in Mitarbelter & - & 2,04 & + & - & - & $*$ & 1,86 & + & - & 0 & ** & 2,12 & + & - & - \\
\hline $\begin{array}{l}\text { Elgenstândigkeit der } \\
\text { Mitarbeher }\end{array}$ & * & 2,25 & + & - & - & n.s. & 1,93 & 0 & 0 & 0 & $\cdot$ & 2,30 & + & - & $\mathbf{0}$ \\
\hline $\begin{array}{l}\text { Einsatzbereitschatt der } \\
\text { Mitarbelter }\end{array}$ & n.s. & 1,89 & 0 & 0 & - & n.s. & 1,74 & 0 & 0 & 0 & $\cdot$ & 1,88 & + & - & - \\
\hline $\begin{array}{l}\text { Konsequente Zlel- } \\
\text { veriolgung }\end{array}$ & n.s. & 2,46 & 0 & 0 & 0 & $*$ & 2,28 & + & - & 0 & n.s. & 2,44 & 0 & - & - \\
\hline Risikofreudigkelt & n.8. & 3,14 & 0 & 0 & 0 & - & 2,92 & + & - & 0 & n.s. & 3,31 & 0 & - & $\mathbf{0}$ \\
\hline Flexibilitât & n.s. & 2.41 & 0 & 0 & 0 & n.s. & 2,20 & 0 & - & 0 & n.s. & 2,51 & 0 & 0 & 0 \\
\hline Kreativităt & $*$ & 2,75 & 0 & - & ++ & $*$ & 2,52 & + & - & + & • & 2,89 & 0 & - & ++ \\
\hline KostenbewuBtsein & * & 2,95 & 0 & + & - & n.s. & 2,60 & 0 & 0 & 0 & n.s. & 2,74 & 0 & 0 & 0 \\
\hline Kostenorientierung & $*$ & 2,82 & - & ++ & - & $*$ & 2,58 & 0 & ++ & - & n.s. & 2,57 & 0 & 0 & -- \\
\hline Technologleorlentiorung & • & 2,28 & + & - & + & * & 1,65 & 0 & - & + & ** & 2,09 & + & - & ++ \\
\hline Technologiebewubtsein & 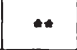 & 2,61 & 0 & $-\cdot$ & ++ & * & 2,07 & 0 & - & + & •* & 2,59 & + & - & ++ \\
\hline Anzahl der Unternehmen & & 104 & 51 & 33 & 20 & & 159 & 85 & 33 & 41 & & 112 & 62 & 29 & 21 \\
\hline 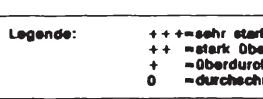 & 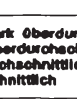 & echnn & ten $\begin{array}{c}1<-2 \\
1-0.28 \\
1-0.10 \\
1-0,10 ;\end{array}$ & & $=4 \mathrm{~mm}$ & & . & & & Yonmenternz & an & $\begin{array}{l}05 \\
10 \\
10 \\
F-1\end{array}$ & & the & \\
\hline
\end{tabular}

Abb. 34: Bedeutung der Kulturdimensionen fur die strategischen High-Tech-Gruppen 
Bei den innovationsorientierten High-Tech-Herstellern stehen die Technologieorientierung, das Technologiebewußtsein und die Kreativität im Vordergrund der Unternehmenskultur. Es wird deutlich, daß die Unternehmen dieser strategischen Gruppe den übrigen Kulturmerkmalen der innovationsorientierten Grundhaltung einen geringen Stellenwert beimessen. Darüber hinaus werden die übrigen kulturellen Grundorientierungen als unterdurchschnittlich wichtig eingestuft.

Als Ergebnis bleibt festzuhalten, daß in der Situation A tendenziell ein Zusammenhang zwischen der Unternehmenskultur und den Strategieschwerpunkten der High-Tech-Anbieter besteht und sich die strategischen Gruppen in der Ausprägung der Kulturmerkmale unterscheiden (vgl. Abbildungen 28 und 34) ${ }^{96} \mathrm{Bei}$ den "Differenzierungsorientierten", die u.a. eine Qualitäts-, Service- und NutzenFührerschaft anstreben, stehen insbesondere die Kunden- und Mitarbeiterorientierung im Vordergrund der Unternehmenskultur. Dies ist ein Hinweis dafür, daß die kulturelle Ausrichtung das strategische Verhalten der differenzierungsorientierten High-Tech-Anbieter beeinflußt. Diese Aussage ist allerdings aufgrund der geringen Übereinstimmung der innovationsorientierten Kulturmerkmale mit den Strategieoptionen der Produktentwicklung und der Pionierorientierung zu relativieren.

Ein starker Kultur-Strategie-Zusammenhang ist bei den kostenorientierten HighTech-Herstellern zu erkennen. Bei den Unternehmen steht daher die Realisierung einer Preis-/Kosten-Führerschaft im Mittelpunkt der Marketingstrategie. Das strategische Verhalten der innovationsorientierten High-Tech-Anbieter wird vor allem durch eine technologiebezogene kulturelle Grundhaltung bestimmt. Aus diesem Grund legen die Unternehmen einen besonderen Wert auf die Erreichung einer Technologie-Führerschaft. Bei der Betrachtung der Kultur der "Innovationsorientierten" zeigt sich jedoch auch, daß die innovationsorientierten Kulturmerkmale mit Ausnahme der Kreativität nur schwach ausgeprägt sind. Es läßt sich daraus schließen, daß im Hinblick auf die Strategieoptionen Produkttionen A, B und C im Anhang II der Arbeit. 
entwicklung, Diversifikation und Pionierorientierung lediglich ein geringer Kultur-Strategie-Fit besteht.

In einer situationsübergreifenden Untersuchung der Unternehmenskultur der differenzierungs-, kosten- und innovationsorientierten High-Tech-Unternehmen wird deutlich, daß die vergleichbaren strategischen Gruppen in den Umfeldsituationen A, B und C ähnliche kulturelle Grundorientierungen besitzen (vgl. Abbildung 28 und 34). ${ }^{97}$ Aufgrund der z.T. geringen Kultur-Strategie-Zusammenhänge bei den strategischen High-Tech-Gruppen und aufgrund der teilweise unbefriedigenden Ergebnisse der Mittelwertvergleichstests sind die Hypothesen $\mathrm{H}_{\mathrm{K}} 1$ und $\mathrm{H}_{\mathrm{K} 2}$ insgesamt nur eingeschränkt zu bestätigen. ${ }^{98}$

Hinsichtlich der Hypothese $\mathrm{H} \mathrm{K} 3_{\mathrm{K}}$ ist $\mathrm{zu}$ analysieren, ob bei den vergleichbaren strategischen High-Tech-Gruppen, die in den verschiedenen homogenen Umfeldsituationen eine ähnliche kulturelle Grundausrichtung aufweisen, situationsbedingte Unterschiede in der Kulturausprägung bestehen. Ein Vergleich der in der Abbildung 34 gezeigten Mittelwertabweichungen macht deutlich, da $B$ in der Unternehmenskultur der differenzierungs- und innovationsorientierten HighTech-Anbieter die technologiebezogenen Kulturmerkmale eine Bedeutungsveränderung erfahren. ${ }^{99}$ Die strategische Gruppe der "Differenzierungsorientierten" räumt den Kulturmerkmalen Technologieorientierung und Technologiebewußtsein primär in der Umfeldsituation B einen besonderen Stellenwert ein. Dies läßt sich damit erklären, daß die in der Situation B vorherrschende Technologiedynamik zu Anpassungen in den Wertvorstellungen

97 Siehe hierzu auch die absoluten Mittelwertabweichungen der einzelnen Kulturvariablen der strategischen High-Tech-Gruppen in den Situationen A, B und C im Anhang II der Arbeit.

$\mathrm{Zu}$ den Ergebnissen der Mittelwertvergleichstests siehe die Abbildung 34 der Arbeit. Es ist hierbei anzumerken, da $B$ für die Kulturvariablen enge Kundenbeziehungen, Einsatzbereitschaft der Mitarbeiter, konsequente Zielverfolgung, Risikofreudigkeit, Flexibilität, Kreativität und KostenbewuBtsein in der Mehrzahl der Umfeldsituationen keine signifikanten Ergebnisse vorliegen.

Vgl. hierzu auch die absoluten Mittelwertabweichungen der einzelnen Kulturvariablen der strategischen High-Tech-Gruppen in den Situationen A, B und C im Anhang II der Arbeit. 
und Denkhaltungen der Mitarbeiter in den differenzierungsorientierten HighTech-Unternehmen führt.

Aus dem gleichen Grund legen die "Innovationsorientierten" in der Situation B einen größeren Wert auf die technologieorientierte Kulturausrichtung als in den beiden anderen Umfeldsituationen. Für die innovationsorientierten High-TechAnbieter ist darüber hinaus die Kenntnis der Kundenprobleme in Situation B von überdurchschnittlicher Relevanz. Ein Grund hierfür ist darin zu sehen, daß die Unternehmen die Notwendigkeit erkennen, eine intensive Technologieorientierung mit einem hohen Kundenbezug zu verbinden, um der Gefahr einer stark innengerichteten Unternehmenskultur vorzubeugen. Trotz der aufgezeigten Kulturunterschiede bleibt insgesamt anzumerken, da $\$$ sich die vergleichbaren strategischen Gruppen in der Ausprägung der kulturellen Grundorientierungen in den Umfeldsituationen A, B und C nur wenig voneinander unterscheiden. Die Hypothese $\mathrm{H}_{\mathrm{K}} 3$ ist somit abzulehnen.

\section{Analyse des Markterfolges der Marketingstrategien von strategischen Gruppen in High-Tech-Märkten}

Ziel der folgenden Betrachtung ist es, die Erfolgswirksamkeit von Marketingstrategien in High-Tech-Märkten zu analysieren. Nach einer Erörterung ausgewählter Studien zum Markterfolg des strategischen Unternehmensverhaltens von High-Tech-Anbietern wird in der vorliegenden Untersuchung überprüft, ob zwischen den strategischen Gruppen signifikante Erfolgsunterschiede bestehen.

\subsection{Theoretische Grundlagen zum Markterfolg der Marketingstrategien von strategischen Gruppen}

Eine Bestandsaufnahme der Literatur zum Markterfolg von Marketingstrategien in High-Tech-Märkten läßt zwei Forschungsschwerpunkte erkennen. In den Untersuchungen wird einerseits die Erfolgswirkung unterschiedlicher Wettbe- 
werbsstrategien von High-Tech-Unternehmen analysiert. ${ }^{100}$ Andererseits wird in den empirischen Forschungen die Vorteilhaftigkeit alternativer Timingstrategien in High-Tech-Märkten untersucht. ${ }^{101}$

In einer Studie von Kim und Lim wurde der Markterfolg abnehmergerichteter Wettbewerbsstrategien von Unternehmen der Elektronikindustrie untersucht. ${ }^{102}$ Es wurden hierbei vier verschiedene Typen von Unternehmensstrategien identifiziert. Die Unternehmen des ersten Strategietyps sind durch eine deutliche Preis/Kostenorientierung gekennzeichnet. Andere Elektronikhersteller verfolgten eine produktbezogene Innovationsstrategie oder eine Differenzierungsstrategie auf der Grundlage einer hohen Produkt- und Servicequalität sowie einer starken Imageorientierung. Der letzte Strategietyp wird durch Anbieter repräsentiert, die kein eindeutiges Strategieprofil aufweisen. Im Rahmen der empirischen Analysen kommen Kim und Lim zu dem Ergebnis, daß die kosten- und innovationsorientierten Unternehmen eine höhere Gesamtkapitalrentabilität erzielen als die differenzierungsorientierten High-Tech-Anbieter. Im Vergleich dazu realisieren

100 Siehe in diesem Zusammenhang u.a. Diller, H., Lücking, M.A., Kaffenberger, A., Das "Schicksal" von Marktführern, Eine empirische Analyse in 29 Märkten für technische Gebrauchsgüter, Arbeitspapier Nr. 26 des Lehrstuhls für Marketing, Erlangen-Nürnberg 1993, S. 6 ff.; Fritz, W., Marktorientierte Unternehmensführung und Unternehmenserfolg, in: Marketing ZFP, 1993, Nr. 4, S. 239 ff.; Roberts, E.B., Entrepreneurs in high technology: Lessons from MIT and beyond, a.a.O., S. 298 ff.; Töpfer, A., Marketing für Start-up-Geschäfte mit Technologieprodukten, a.a.O., S. 176 ff.; Lilien, G.L., Yoon, E., Determinants of new industrial product performance: A strategy reexamination of the empirical literature, in: IEEE Transactions on Engineering Management, Vol. 36 (1989), No. 1, S. 3 ff.; Cooper, R.G., Project new products: Factors in new product success, in: European Journal of Marketing, 1980, No. 5-6, S. 277 ff. Vgl. hierzu auch die Erläuterungen in Teil B Kap. 2.2 und Kap. 2.3 der Arbeit.

Vgl. hierzu u.a. Robinson, W.T., Fornell, C., Sullivan, M., Are market pioneers intrinsically stronger than later entrants?, a.a.O., S. 613 ff.; Perillieux, R., Strategisches Timing von F\&E und Markteintritt bei innovativen Produkten, a.a.O., S. 25 ff. Zu einer Übersicht ausgewählter Studien zum Erfolg von Timingstrategien siehe Kerin, R.A., Varadarajan, P.R., Peterson, R.A., First-mover advantage: A synthesis, conceptual framework and research propositions, a.a.O., S. 35 ff.; Specht, G., Perillieux, R., Erfolgsfaktoren technischer Führer- und Folgerpositionen auf Investitionsgütermärkten, a.a.O., S. 209 ff.; Remmerbach, K.-U., Markteintrittsentscheidungen, a.a.O., S. 40 ff. u. $66 \mathrm{ff}$.

Vgl. Kim, L., Lim, Y., Environment, generic strategies and performance in rapidly developing country: a taxonomic approach, a.a.O., S. $807 \mathrm{ff}$. 
die differenzierungsorientierten Elektronikhersteller die größte Umsatzsteigerung. ${ }^{103}$

$\mathrm{Zu}$ entgegengesetzten Erkenntnissen sind Doyle und Hooley in einer Untersuchung englischer Unternehmen der verarbeitenden Industrie gekommen. ${ }^{104} \mathrm{Sie}$ konnten nachweisen, daß Unternehmen mit einer starken Differenzierungsausrichtung gegenüber kostenorientierten Herstellern deutlich erfolgreicher sind. So ist der Markterfolg bei den differenzierungsorientierten Unternehmen in bezug auf die Erfolgsgrößen Umsatz, Marktanteil und ROI signifikant höher. ${ }^{105}$ Als Zwischenfazit bleibt festzuhalten, daß die Forschungen zur Erfolgswirkung abnehmergerichteter Wettbewerbsstrategien in High-Tech-Märkten bislang zu keinem eindeutigen Ergebnis geführt haben.

Speziellere Hinweise über den Markterfolg des strategischen Verhaltens von High-Tech-Unternehmen liefern empirische Studien, die im Bereich der Erfolgsfaktorenforschung anzusiedeln sind. ${ }^{106}$ Von Neugebauer wurden beispielsweise das strategische Unternehmensverhalten und die Faktoren des Unternehmens-

Vgl. in diesem Zusammenhang auch Robinson, R.B., Pearce, J.A., Planned patterns of strategic behavior and their relationship to business-unit performance, a.a.O., S. $43 \mathrm{ff}$;; Dess, G.G., Davis, P.S., Porter's (1980) generic strategies as Determinants of strategic group membership and organizational performance, a.a.O., S. $470 \mathrm{ff}$. Zur Untersuchung von Dess und Davis siehe auch die Ausführungen im Teil B Kap. 1.2 der Arbeit.

Vgl. Doyle, P., Holley, G.J., Strategic orientation and corporate performance, in: International Journal of Research in Marketing, Vol. 9 (1992), S. 62 ff.

Vgl. in diesem Zusammenhang auch Slater, St.F., Narver, J.C., Does competitive environment moderate the market orientation-performance relationship?, in: Journal of Marketing, Vol. 58 (1994), S. 47 ff.; Day, G.S., Wensley, R., Assessing advantage: A framework for diagnosing competitive superiority, in: Journal of Marketing, Vol. 52 (1988), No. 2, S. $17 \mathrm{ff}$.

Vgl. hierzu z.B. Baaken, Th., Launen, M., Software-Marketing, a.a.O., S. 114 f.; Preiß, F.J., Strategische Erfolgsfaktoren im Software-Marketing, a.a.O., S. $54 \mathrm{ff}$.; Fritz, W., Marketing - ein Schlüsselfaktor des Unternehmenserfolges?, a.a.O., S. 92 ff.; Buschmann, E., et al., Der Software-Markt in der Bundesrepublik Deutschland, Hrsg.: Gesellschaft fuir Mathematik und Datenverarbeitung, GMD-Bericht Nr. 167, St. Augustin 1989, S. 94 ff.; Poth, L.G., Erfolgsfaktoren von Marktfuihrern, Ergebnisse einer Studie im Fachbereich Wirtschaft der Fachhochschule Düsseldorf, in: Apitz, K., et al., Erfolgsfaktoren von Marktfiihrem, Landsberg a.L. 1989, S. 195 ff.; Maidique, M.A., Hayes, R.H., The art of high-technology management, a.a.O., S. 17 ff.; Pümpin, C., Management strategischer Erfolgspositionen, a.a.O., S. $135 \mathrm{ff}$. Siehe in diesem Zusammenhang auch Homburg, Ch., Sütterlin, St., Strategische Gruppen: Ein Survey, a.a.O., S. 654 ff. 
erfolges bei Anbietern von Standardsoftware analysiert. ${ }^{107}$ Es zeigte sich hierbei, daß der Markterfolg im wesentlichen durch die Qualität der Produktleistungen, den Umfang der Serviceangebote und das Image der Software-Anbieter bestimmt werden.

In einer aktuellen Untersuchung im Bereich der Informations- und Kommunikationstechnologie identifizierte Reinecke vergleichbare Erfolgsfaktoren. ${ }^{108}$ Darüber hinaus wurde festgestellt, daß erfolgreiche High-Tech-Unternehmen häufiger eine Technologieführerschaft anstreben als weniger erfolgreiche Unternehmen. ${ }^{109} \mathrm{Zu}$ einem ähnlichen Ergebnis kam Roberts in einer empirischen Analyse des strategischen Unternehmensverhaltens in der Computer- und Softwareindustrie. ${ }^{110}$ Es wurde hierbei deutlich, daß eine ausgeprägte Technologieorientierung der HighTech-Hersteller eine wesentliche Voraussetzung für den Markterfolg bildet. ${ }^{111}$

Hinsichtlich der Erfolgswirksamkeit von Timingstrategien in High-TechMärkten lassen sich in der Literatur mehrere Untersuchungen finden. ${ }^{1{ }^{12}}$ In einer

Vgl. Neugebauer, U., Das Software-Unternehmen, Empirische Untersuchungen des Unternehmensverhaltens und der Faktoren des Unternehmenserfolges, GMD-Bericht Nr. 157, München, Wien 1986, S. $88 \mathrm{ff}$.

Vgl. Reinecke, S., Rezession und Strukturwandel in der Informationstechnologie-Branche: Problemanalyse und Erfolgsansätze, a.a.O., S. 17 f. $\mathrm{Zu}$ Studien mit ähnlichen Ergebnissen siehe auch Hannig, U., Die Entwicklung wettbewerbsorientierter Marketingstrategien auf Basis des Konzeptes der strategischen Gruppen, a.a.O., S. 197 ff.; Preiß, F.J., Strategische Erfolgsfaktoren im Software-Marketing, a.a.O., S. 154 ff. Zur Bedeutung der Qualität als Erfolgsfaktor siehe auch Hildebrandt, L., Wettbewerbssituation und Unternehmenserfolg, a.a.O., S. $1072 \mathrm{ff}$.

109 Vgl. Reinecke, S., Rezession und Strukturwandel in der Informationstechnologie-Branche: Problemanalyse und Erfolgsansätze, a.a.O., S. 20.

110 Vgl. Roberts, E.B., Entrepreneurs in high technology: Lessons from MIT and beyond, a.a.O., S. $298 \mathrm{ff}$.

111 Diese Aussage ist zu relativieren mit Blick auf aktuelle Forschungen zum Zusammenhang zwischen F\&E-Intensität und Markterfolg. Vgl. dazu Pinkwart, A., Chaos und Unternehmenskrise, Wiesbaden 1992, S. 83 ff.; Gierl, H., Kotzbauer, N., Der EinfluB des F\&E-Aufwandes auf den wirtschaftlichen Erfolg von Industrieunternehmen, a.a.O., S. $983 \mathrm{ff}$. In beiden Untersuchungen wurde eine konvexe Beziehung zwischen dem F\&EAufwand und den Erfolgsgrößen Umsatz und Gewinn ermittelt. Zu einer Übersicht ausgewählter Studien, in denen auch eine positive Beziehung zwischen F\&E-Intensität und Markterfolg festgestellt wurde, siehe Gierl, H., Kotzbauer, N., Der EinfluB des F\&EAufwandes auf den wirtschaftlichen Erfolg von Industrieunternehmen, a.a.O., S. $980 \mathrm{ff}$.

Siehe hierzu auch die Ausführungen in Teil B Kap. 2.2 und Kap. 2.3 der Arbeit. 
empirischen Analyse des Markteintrittsverhaltens von Herstellern medizinischtechnischer Geräte wurde von Mitchell ermittelt, daß Unternehmen, die eine Pionierstrategie verfolgen, einen höheren Marktanteil realisieren als Gerätehersteller mit einer Folgerstrategie. ${ }^{113}$ Einen positiven Zusammenhang zwischen Timingverhalten und Marktanteil konnten auch Parry und Bass bei Unternehmen der verarbeitenden Industrie nachweisen. ${ }^{114}$

Andere empirische Studien zeigten hingegen, daß der Markteintritt als früher oder später Folger im Vergleich zur Pionierposition zu einem gleichen bzw. größeren Markterfolg in bezug auf den Marktanteil führt. Flaherty stellte beispielsweise in einer Untersuchung amerikanischer Unternehmen aus dem Bereich der Mikroelektronik einen geringen negativen Zusammenhang zwischen einem frühen Markteintrittsverhalten und dem Marktanteil fest. ${ }^{115}$ In einer empirischen Analyse der Timingstrategien französischer High-Tech-Unternehmen des verarbeitenden Gewerbes kamen Lilian und Yoon zu dem Ergebnis, daß die Strategie der späten Folgerschaft besonders erfolgreich ist. ${ }^{116}$ Die aufgezeigten Forschungen zur Erfolgswirkung von Markteintrittsstrategien in High-Tech-Märkten verdeutlichen,

113 Vgl. Mitchell, W., Dual clocks: Entry order influence on incumbent and newcomer market share and survival when specialized assets retain their value, in: Strategic Management Journal, 1991, No. 1, S. 87 ff.

114 Vgl. Perry, M., Bass, F.M., When to lead or follow?, It depends, in: Marketing Letters, 1990, No. 1, S. 189 ff. Zu Studien mit vergleichbaren Ergebnissen siehe u.a. Lambkin, M., Pioneering new markets: A comparison of market share winners and losers, a.a.O., S. 9 ff.; Robinson, W.T., Sources of market pioneer advantages: The case of industrial goods industries, in: Journal of Marketing Research, Vol. 25 (1988), S. 89 ff.; Robinson, W.T., Fornell, C., Market pioneering and sustainable market share advantages, a.a.O., S. 2 ff.; Spital, F., Gaining market share advantage in the semiconductor industry by lead time in innovation, a.a.O., S. $55 \mathrm{ff}$.

115 Vgl. Flaherty, M.T., Market-Share, technology leadership and competition in international semiconductor markets, in: Research on technological innovation, management and policy, Hrsg.: Rosenbloom, R.S., Greenwich, Conn. 1983, S. 75 ff. Zu Untersuchungen, in denen vergleichbare Ergebnisse ermittelt wurden, siehe z.B. Srinivasan, K., Pioneering versus early following in new product markets, Diss., University of California, Los Angeles 1990, S. 45 ff.; Miller, A., Gartner, W.B., Wilson, R., Entry order, Market share and competitive advantage: A study of their relationships in new corporate ventures, in: Journal of Business Venturing, Vol. 4 (1989), S. $199 \mathrm{ff}$.

116 Vgl. Lilien, G.L., Yoon, E., The timing of competitive market entry: An exploratory study of new industrial products, in: Management Journal, Vol. 36 (1990), S. $570 \mathrm{ff}$. 
daß es bislang nicht gelungen ist, die Vorteilhaftigkeit einer Timingoption eindeutig nachzuweisen.

Vor dem Hintergrund der z.T. widersprüchlichen empirischen Ergebnisse in bezug auf den Markterfolg von abnehmergerichteten Wettbewerbsstrategien sowie von Timingstrategien erscheint es von Interesse, zu untersuchen, ob zwischen den Marketingstrategien der strategischen High-Tech-Gruppen Erfolgsunterschiede bestehen. Hierzu wird folgende Basishypothese überprüft:

H E1 In High-Tech-Märkten unterscheiden sich die Marketingstrategien der strategischen Gruppen bezüglich des Markterfolges innerhalb der einzelnen Umfeldsituationen.

Mit Blick auf die identifizierten Situationen in High-Tech-Märkten bietet sich außerdem eine situationsübergreifende Analyse der Erfolgswirkung der Marketingstrategien von vergleichbaren strategischen Gruppen an. Hierdurch sollen Erkenntnisse darüber gewonnen werden, in welcher Umfeldsituation eine bestimmte strategische High-Tech-Gruppe besonders erfolgreich ist. Beispielsweise ist es von Interesse, zu erfahren, ob bei einem hohen Technologiewettbewerb diejenige strategische Gruppe den höchsten Markterfolg realisiert, die primär eine technologieorientierte Marketingstrategie verfolgt. Entsprechend kann der Frage nachgegangen werden, ob High-Tech-Unternehmen mit einer ausgeprägten Preis-/Kostenorientierung bei einem hohen Preiswettbewerb überdurchschnittlich erfolgreich sind. In diesem Zusammenhang wird im Rahmen der weiteren Analyse folgende Tendenzhypothese untersucht:

H E2 In High-Tech-Märkten sind in Abhängigkeit von der Umfeldsituation Unterschiede in der Erfolgswirkung der Marketingstrategien von vergleichbaren strategischen Gruppen festzustellen. 


\subsection{Empirische Analyse des Markterfolges der Marketingstrategien von strategischen Gruppen}

In den weiteren Ausführungen wird die Erfolgswirkung der strategischen Gruppen in den Situationen A, B und C untersucht. Als Bestimmungsgrößen für den Markterfolg der High-Tech-Unternehmen werden die prozentuale Umsatzsteigerung und der Marktanteil in Deutschland herangezogen. ${ }^{117}$ Der Betrachtung der prozentualen Umsatzveränderung liegt der Gedanke zugrunde, daß prozentuale Größen einen aussagefähigeren Indikator für den Markterfolg des strategischen Unternehmensverhaltens darstellen als absolute Werte. Die Analyse der Marktanteile erscheint sinnvoll, da sie als zentrale marktbezogene Erfolgsgröße Aufschluß darüber gibt, welche Marketingstrategien in High-Tech-Märkten zum Aufbau einer Wettbewerbsvorteilsposition besonders geeignet sind. Zudem wird in der empirischen Forschung nachgewiesen, daß ein positiver Zusammenhang zwischen dem Markanteil und dem ökonomischen Erfolg (z.B. ROI) von HighTech-Unternehmen besteht. ${ }^{118}$

In Abbildung 35 sind die durchschnittlichen Umsatzsteigerungen der strategischen High-Tech-Gruppen innerhalb der Umfeldsituationen in den Jahren 1988 bis 1990 dargestellt. ${ }^{119}$ In Situation A, in der ein geringer Technologie- und

117 Zu einer vergleichbaren Vorgehensweise bezüglich der Untersuchung des Markterfolges von Unternehmen siehe z.B. Cool, K.O., Dierickx, I., Rivalery, Strategic Groups and firm profitability, in: Strategic Management Journal, Vol. 14 (1993), S. 50; Doyle, P., Holley, G.J., Strategic orientation and corporate performance, a.a.O., S. 71; Bolz, J., Wettbewerbsorientierte Standardisierung der internationalen Marktbearbeitung, a.a.O., S. 177.; Lewis, P., Thomas, H., The linkage between strategy, strategic groups and performance in the U.K. Retail Grocery Industry, a.a.O., S. 388. Auf andere Erfolgsgrößen konnte im Rahmen der Befragung nicht zurückgegriffen werden.

Vgl. zum Zusammenhang von Marktanteil und ROI in der Investitionsgüterindustrie insbesondere Schwalbach, J., Marktanteil und Unternehmensgewinn, in: ZfB, 1988, Nr. 4, S. 540. Zur Beziehung zwischen Marktanteil und ROI siehe auch Meffert, H., Marketing-Management, a.a.O., S. 60 f.; Buzzell, R.D., Gale, B.T., Das PIMS-Programm, Strategien und Unternehmenserfolg, Wiesbaden 1989, S. $80 \mathrm{ff}$.

119 Die absoluten Umsätze der strategischen Gruppen innerhalb der Umfeldsituationen A, B und $C$ in den Jahren 1988-1990 sind im Anhang II der Arbeit zu finden. Bei der Untersuchung der umsatzbezogenen Erfolgswirkung der strategischen Gruppen wird davon ausgegangen, daß die High-Tech-Unternehmen ihre Marketingstrategie im Beobachtungszeitraum 1988 bis 1990 kaum verändert haben. 


\begin{tabular}{|c|c|c|c|c|c|}
\hline & & $\varnothing$ & 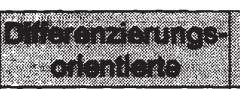 & 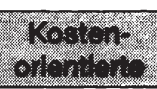 & (. \\
\hline \multirow{2}{*}{ 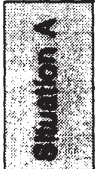 } & $\triangle \begin{array}{r}\text { Umsatz } \\
1988 / 89\end{array}$ & $+8,5 \%$ & $+8,6 \%$ & $+6,4 \%$ & $+10,6 \%$ \\
\hline & $\triangle \underset{1989 / 90}{\text { Umsatz }}$ & $+9,1 \%$ & $+11,7 \%$ & $+4,2 \%$ & $+7,8 \%$ \\
\hline \multirow{2}{*}{$\frac{1}{\frac{6}{6}}$} & $\triangle \underset{1988 / 89}{\text { Umsatz }}$ & $+10,3 \%$ & $+10,8 \%$ & $+7,8 \%$ & $+10,4 \%$ \\
\hline & $\triangle \underset{1989 / 90}{\text { Umsatz }}$ & $+9,3 \%$ & $+8,6 \%$ & $+7,6 \%$ & $+10,6 \%$ \\
\hline \multirow{2}{*}{$\frac{x}{2}+2$} & $\triangle \underset{1988 / 89}{\text { Umsatz }}$ & $+10,4 \%$ & $+11,3 \%$ & $+10,1 \%$ & $+8,2 \%$ \\
\hline & $\triangle \underset{1989 / 90}{\text { Umsatz }}$ & $+9,3 \%$ & $+10,3 \%$ & $+10,3 \%$ & $+5,4 \%$ \\
\hline
\end{tabular}

Abb. 35: Durchschnittliche Umsatzveränderungen der strategischen High-TechGruppen innerhalb der Umfeldsituationen in den Jahren 1988-1990

Preiswettbewerb herrscht, ist zu erkennen, da $\beta$ die differenzierungsorientierten High-Tech-Unternehmen im Jahr 1989 mit einer Umsatzsteigerung von 8,6 \% lediglich leicht über dem Durchschnitt liegen. Demgegenüber kommt der Erfolg der "Differenzierungsorientierten" im Jahr 1990 in der hohen Umsatzveränderung von 11,7 \% zum Ausdruck. Die "Kostenorientierten" realisieren in den Jahren 1989 und 1990 ein stark unterdurchschnittliches Umsatzwachstum. Es zeigt sich, daß die innovationsorientierten High-Tech-Unternehmen mit einer Umsatzsteigerung von 10,6\% im Jahr 1989 deutlich überdurchschnittlich erfolgreich sind. Im Vergleich dazu liegt die Umsatzzunahme von 7,8 \% im Jahr 1990 unterhalb des Durchschnitts. Zusammenfassend läßt sich feststellen, daß die "Differenzierungsorientierten" und die "Innovationsorientierten" in der Umfeldsituation A im Vergleich zu den kostenorientierten High-Tech-Anbietern erfolgreicher sind.

Die strategische Gruppe der "Differenzierungsorientierten" setzt in der Situation B, die durch einen deutlichen Technologiewettbewerb gekennzeichnet ist, im Jahr 1989 einen überdurchschnittlichen Umsatzzuwachs von 10,8\% durch. Es wird jedoch deutlich, daß die Unternehmen im Jahr 1990 mit einer Umsatzsteigerung 
von 8,6 \% nicht den Durchschnitt aller High-Tech-Anbieter erreichen. Die Umsatzveränderung der kostenorientierten High-Tech-Hersteller liegt in beiden Jahren unterhalb des Durchschnitts. Der Erfolg der "Innovationsorientierten" in den Jahren 1989 und 1990 kommt in der überdurchschnittlichen Umsatzsteigerung von $10,4 \%$ und 10,6\% zum Ausdruck. Als Ergebnis ist festzuhalten, $\mathrm{da} ß$ tendenziell die strategische Gruppe der innovationsorientierten Unternehmen in der Situation B am erfolgreichsten ist.

In der Situation C, die durch einen hohen Preiswettbewerb geprägt ist, sind die differenzierungsorientierten High-Tech-Unternehmen sowohl im Jahr 1989 mit 11,3\% als auch im Jahr 1990 mit 10,3 \% überdurchschnittlich erfolgreich. Weiterhin ist zu erkennen, daß die strategische Gruppe der "Kostenorientierten" mit 10,1 \% im Jahr 1989 und mit 10,3 \% im Jahr 1990 hohe Umsatzsteigerungen verwirklicht. Die innovationsorientierten Unternehmen realisieren in den Jahren 1989 und 1990 lediglich unterdurchschnittliche Umsatzzuwächse. Es wird insgesamt deutlich, daß in Situation $C$ die differenzierungs- und die kostenorientierten High-Tech-Anbieter erfolgreicher sind als die innovationsorientierten Unternehmen.

Abbildung 36 zeigt, welche Marktanteile durchschnittlich von den strategischen High-Tech-Gruppen im Jahr 1990 erreicht werden. ${ }^{120}$ Hierbei ist zu erkennen, da $B$ die differenzierungsorientierten High-Tech-Unternehmen in Situation A mit einem Marktanteil von 46,44 \% sehr erfolgreich sind. Die strategische Gruppe der "Kostenorientierten" liegt demgegenüber mit einem Marktanteil von 35,52\% deutlich unter dem Durchschnitt. Von den innovationsorientierten Unternehmen wird mit 45,18\% ein überdurchschnittlicher Marktanteil erreicht. Es bleibt festzuhalten, da $\beta$ die differenzierungs- und innovationsorientierten High-Tech-Anbieter in Situation A gegenüber den Unternehmen der strategischen Gruppe der "Kostenorientierten" einen größeren Markterfolg erzielen.

Bei der Analyse der marktanteilsbezogenen Erfolgswirkung der strategischen Gruppen wird unterstellt, daß die High-Tech-Unternehmen ihre Marketingstrategie im Jahr 1990 kaum verändert haben. 


\begin{tabular}{|c|c|c|c|c|c|}
\hline & & $\phi$ & \begin{tabular}{|c|}
$\begin{array}{c}\text { Differenzierungs- } \\
\text { orientiente }\end{array}$ \\
\end{tabular} & $\begin{array}{l}\text { Kosten- } \\
\text { orlentierte }\end{array}$ & $\begin{array}{c}\text { Innovations- } \\
\text { orlentierte }\end{array}$ \\
\hline 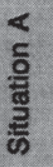 & $\begin{array}{c}\text { MA } \\
\text { Deutschland }\end{array}$ & 42,54 & 46,44 & 35,52 & 45,18 \\
\hline $\begin{array}{c}\infty \\
\frac{5}{0} \\
\frac{\mathrm{c}}{3} \\
\frac{0}{3}\end{array}$ & $\begin{array}{c}\text { MA } \\
\text { Deutschland }\end{array}$ & 40,79 & 40,22 & 36,12 & 45,07 \\
\hline$\frac{0}{\frac{5}{0}}$ & $\begin{array}{c}\text { MA } \\
\text { Deutschland }\end{array}$ & 38,22 & 40,41 & 37,37 & 31,87 \\
\hline
\end{tabular}

Abb. 36: Durchschnittliche Marktanteile der strategischen High-Tech-Gruppen innerhalb der Umfeldsituationen im Jahr 1990

In Situation B erreichen die "Differenzienungsorientierten" mit 40,22 \% beinahe einen durchschnittlichen Marktanteil. Der Marktanteil der kostenorientierten High-Tech-Unternehmen liegt mit 36,12 \% klar unterhalb des Durchschnitts. Die "Innovationsorientierten" erreichen mit 45,07\% im Vergleich zu den beiden anderen strategischen Gruppen den höchsten Marktanteil. Insgesamt wird deutlich, daß in Situation B die innovationsorientierten Anbieter erfolgreicher sind als die "Differenzierungsorientierten" und die "Kostenorientierten".

Die differenzierungsorientierten High-Tech-Hersteller liegen in der Situation C mit einem Marktanteil von 40,41 \% klar über dem Durchschnitt von 38,22 \%. Von den kostenorientierten Unternehmen werden mit 37,37 \% unterdurchschnittliche Marktanteile realisiert. Die "Innovationsorientierten" befinden sich bei einem Marktanteil von lediglich 31,87\% deutlich unterhalb des Durchschnitts. Insgesamt zeigt sich, daß insbesondere die strategische Gruppe der "Differenzierungsorientierten" in Situation C erfolgreich ist. 
Es läßt sich zusammenfassend festhalten, daß die Analyse des Markterfolges der strategischen High-Tech-Gruppen anhand der Erfolgsgrößen Umsatzwachstum und Marktanteil überwiegend zu gleichen Ergebnissen führt. In der Umfeldsituation A sind die innovations- und differenzierungsorientierten High-TechAnbieter überdurchschnittlich erfolgreich. Der Erfolg der "Innovationsorientierten" liegt u.a. darin begründet, daß ein hohes technologisches Know-how und eine kontinuierliche Produktverbesserung den Unternehmen ermöglicht, $\mathrm{zu}$ den High-Tech-Nachfragern frühzeitig eine enge Bindung aufzubauen. Der marktbezogene Erfolg der "Differenzierungsorientierten" läßt sich damit erklären, $\mathrm{da} ß$ es den High-Tech-Unternehmen gelingt, durch die Sicherstellung eines produktbezogenen Qualitätsstandards, das Angebot benutzerfreundlicher technologischer Anwendungen und spezieller Serviceleistungen die in der Situation A vorhandenen Kaufwiderstände und die Kaufzurückhaltung der potentiellen Anwender zu reduzieren und Präferenzen für die eigenen Leistungsangebote zu bilden.

In der Umfeldsituation B ist die strategische Gruppe der "Innovationsorientierten" am erfolgreichsten. Ein Grund hierfür liegt darin, daß eine starke Technologieund Pionierorientierung den High-Tech-Anbietern die Möglichkeit bietet, in einer Situation mit einer hohen Technologiedynamik Wettbewerbsvorteile gegenüber den Konkurrenten aufzubauen. Die innovationsorientierten Unternehmen scheinen darüber hinaus die hohen Leistungsanforderungen der High-Tech-Nachfrager durch eine kontinuierliche Schaffung technologischer Produktneuheiten besonders gut erfüllen zu können.

Die differenzierungsorientierten Unternehmen erzielen in der Umfeldsituation C einen überdurchschnittlichen Markterfolg. Dies ist ein Hinweis dafür, daß zahlreiche High-Tech-Anwender trotz einer ausgeprägten Preisorientierung die Qualität der technologischen Produktleistungen und das Angebot individueller Servicedienste bei der Kaufentscheidung berücksichtigen. Bei der strategischen Gruppe der "Kostenorientierten" ergeben sich unterschiedliche Ergebnisse für Umsatzwachstum und Marktanteil. Die kostenorientierten High-Tech-Hersteller verzeichnen einerseits eine überdurchschnittliche Umsatzsteigerung, sind jedoch 
in bezug auf den Markanteil unterdurchschnittlich erfolgreich. Der vergleichsweise schlechte Markterfolg der kostenorientierten Unternehmen im Vergleich zu den differenzierungsorientierten High-Tech-Anbietern in der Situation C läßt sich u.a. damit erklären, daß der alleinige Aufbau von Preis-/Kostenvorteilen zur Profilierung im Wettbewerb nicht ausreicht.

Zur Überprüfung der beschriebenen Ergebnisse wurde im Rahmen der Varianzanalyse ein T-Test durchgeführt. ${ }^{121}$ Die bei den strategischen High-Tech-Gruppen innerhalb der einzelnen Umfeldsituationen festgestellten Unterschiede in den Erfolgsmerkmalen Umsatzveränderung und Marktanteil lassen sich hierbei nur z.T. bestätigen. ${ }^{122}$ Als wesentliche Ursache hierfür ist die Heterogenität der Branchenstruktur der untersuchten High-Tech-Unternehmen anzuführen. So ist beispielsweise der Erfolg eines Unternehmens mit einem Marktanteil von $15 \%$ in einer High-Tech-Branche, in der der Marktfuihrer einen Anteil von $25 \%$ besitzt, besser zu bewerten als ein Marktanteil von $15 \%$ in einer Branche mit einem Marktanteil von $60 \%$ des größten Wettbewerbers. Es ist deshalb zweckmäßig, die Erfolgsmerkmale Umsatzwachstum und Marktanteil relativ in bezug auf die jeweilige Branche auszudrücken. Als Normierungsgröße wird hierbei die Umsatzveränderung bzw. der Marktanteil des jeweils größten Unternehmens der einzelnen Branchen herangezogen. ${ }^{123}$

\section{Umsatzveränderung des Unternehmens i}

Relative in der Branche $\mathrm{j}$ in der Periode $\mathrm{k}$ in \%

Umsatzver- = änderung

Umsatzveränderung des größten Unternehmens der Branche $\mathrm{j}$ in der Periode $\mathrm{k}$ in \%

121 Vgl. Bleymüller, J., Gehlert, G., Gülicher, H., Statistik für Wirtschaftswissenschaftler, a.a.O., S. $111 \mathrm{ff}$.

Siehe hierzu die Ergebnisse des T-Tests im Anhang II der Arbeit.

123 Vgl. zu einer ähnlichen Vorgehensweise z.B. Kirchgeorg, M., Ökologieorientiertes Unternehmensverhalten, a.a.O., S. 210; Böbel, I., Wettbewerb und Industriestruktur, Berlin u.a. 1984, S. 6 f. 


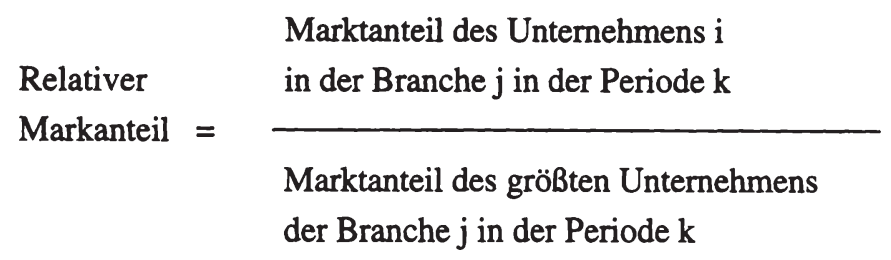

In den Abbildungen 37 und 38 sind die Ausprägungen der gebildeten relativen Markterfolgsgrößen für die strategischen Gruppen in in den Umfeldsituationen A, B und C dargestellt. Bei der Betrachtung des Markterfolges anhand dieser relativen Erfolgsgrößen lassen sich vergleichbare Ergebnisse für die strategischen High-Tech-Gruppen in den einzelnen Situationen feststellen ebenso wie bei der Analyse der Erfolgswirkung der strategischen Gruppen anhand der absoluten Erfolgsgrößen.

Die ermittelten Ergebnisse wurden anschließend innerhalb der Varianzanalyse mit Hilfe des T-Tests überprüft. Abbildung 39 kennzeichnet die Ergebnisse der Mittelwertvergleichstests für die Erfolgsgrößen relative Umsatzveränderung und relativer Marktanteil der strategischen High-Tech-Gruppen. Es wird deutlich, daß vor allem die identifizierten Erfolgsunterschiede bei den differenzierungs- und kostenorientierten sowie bei den kosten- und innovationsgerichteten High-TechUnternehmen in den Umfeldsituationen A und B signifikant sind. Die in Situation $C$ u.a. festgestellten Unterschiede im Markterfolg zwischen den strategischen Gruppen der "Differenzierungsorientierten" und "Innovationsorientierten" wurden ebenfalls bestätigt. Die Ergebnisse können deshalb insgesamt als zufriedenstellend bezeichnet werden.

Als Fazit bleibt festzuhalten, daß die in den High-Tech-Märkten bestehenden Erfolgsunterschiede durch die Bildung von strategischen Gruppen erklärt werden können. Es hat sich hierbei gezeigt, daß die differenzierungs- und innovationsorientierten Untemehmen in Situation A, die "Innovationsorientierten" in Situation B und die "Differenzierungsorientierten" in Situation C besonders erfolgreich agieren. Auf der Grundlage dieser Erkenntnisse und der Ergebnisse der Mittelwertvergleichstests sind die Hypothesen $\mathrm{H}_{\mathrm{E} 1}$ und $\mathrm{H}_{\mathrm{E} 2}$ insgesamt zu bestätigen. 


\begin{tabular}{|c|c|c|c|c|c|}
\hline & & $\varnothing$ & $\begin{array}{c}\text { Differenzienungs } \\
\text { orlondierto }\end{array}$ & $\begin{array}{l}\text { Kosten } \\
\text { oriendiente }\end{array}$ & $\begin{array}{l}\text { Innovalione- } \\
\text { orientlente }\end{array}$ \\
\hline \multirow{2}{*}{ 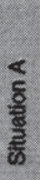 } & $\begin{array}{c}\mathrm{re} \triangle \mathrm{Umsatz} \\
1988 / 89\end{array}$ & $+28,67 \%$ & $+31,26 \%$ & $+20,20 \%$ & $+41,38 \%$ \\
\hline & $\begin{array}{c}\mathrm{rel} \triangle \text { Umsatz } \\
1989 / 90\end{array}$ & $+28,67 \%$ & $+32,67 \%$ & $+23,97 \%$ & $+30,39 \%$ \\
\hline \multirow{2}{*}{$\frac{\stackrel{\infty}{\delta}}{\frac{8}{3}}$} & $\begin{array}{c}\text { reß } \triangle \text { Umsatz } \\
1988 / 89\end{array}$ & $+23,64 \%$ & $+23,90 \%$ & $+15,14 \%$ & $+28,84 \%$ \\
\hline & $\begin{array}{c}\text { rel } \triangle \text { Umsatz } \\
1989 / 90\end{array}$ & $+26,23 \%$ & $+26,04 \%$ & $+18,66 \%$ & $+31,51 \%$ \\
\hline \multirow{2}{*}{$\begin{array}{l}0 \\
\text { ह } \\
\text { 莹 } \\
\frac{3}{5}\end{array}$} & $\begin{array}{c}\text { reß } \triangle \text { Umsatz } \\
1988 / 89\end{array}$ & $+16,28 \%$ & $+17,49 \%$ & $+14,17 \%$ & $+10,79 \%$ \\
\hline & 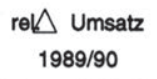 & $+20,56 \%$ & $+24,08 \%$ & $+16,61 \%$ & $+11,01 \%$ \\
\hline
\end{tabular}

Abb. 37: Relative durchschnittliche Umsatzveränderung der strategischen HighTech-Gruppen innerhalb der Umfeldsituation in den Jahren 1988-1990

\begin{tabular}{|c|c|c|c|c|c|}
\hline & & $\varnothing$ & $\begin{array}{l}\text { Outararvierunge- } \\
\text { arlenterte }\end{array}$ & $\begin{array}{l}\text { Kocton- } \\
\text { onlonterise }\end{array}$ & $\begin{array}{l}\text { Innovationse } \\
\text { oflentlerte }\end{array}$ \\
\hline है & $\begin{array}{l}\text { relattver MA } \\
\text { Deutschland }\end{array}$ & 0,5049 & 0,5495 & 0,4202 & 0,5441 \\
\hline$\frac{\infty}{8}$ & $\begin{array}{l}\text { relativer MA } \\
\text { Deutschland }\end{array}$ & 0,4842 & 0,4765 & 0,4200 & 0,5445 \\
\hline$\frac{0}{8}$ & $\begin{array}{l}\text { relativer MA } \\
\text { Deutschland }\end{array}$ & 0,4452 & 0,4676 & 0,4416 & 0,3750 \\
\hline
\end{tabular}

Abb. 38: Relative durchschnittliche Marktanteile der strategischen High-TechGruppen innerhalb der Umfeldsituationen im Jahr 1990 


\begin{tabular}{|c|c|c|c|c|c|c|c|c|c|}
\hline \multirow{3}{*}{$\begin{array}{l}\text { Erfolgs- } \\
\text { variablen }\end{array}$} & \multicolumn{3}{|c|}{ Situation A } & \multicolumn{3}{|c|}{ Situation B } & \multicolumn{3}{|c|}{ Situation C } \\
\hline & \multicolumn{3}{|c|}{ T-Test } & \multicolumn{3}{|c|}{ T-Test } & \multicolumn{3}{|c|}{ T-Test } \\
\hline & $1 / 2$ & $1 / 3$ & $2 / 3$ & $1 / 2$ & $1 / 3$ & $2 / 3$ & $1 / 2$ & $1 / 3$ & $2 / 3$ \\
\hline $\begin{array}{l}\text { rel. } \Delta \\
\text { Umsatz } \\
1988 / 89\end{array}$ & $\#$ & & & 1 & & 11 & & 11 & \\
\hline $\begin{array}{l}\text { rel. } \Delta \\
\text { Umsatz } \\
1989 / 90\end{array}$ & 8 & & & 11 & & $y$ & & 1 & \\
\hline $\begin{array}{c}\text { rel. MA } \\
\text { Deutschland }\end{array}$ & & & 11 & & 1 & & & 11 & 1 \\
\hline Legende & $\begin{array}{l}=\boldsymbol{\alpha} \\
=\boldsymbol{\alpha} \\
=\mathrm{ni}\end{array}$ & $\begin{array}{l}05 \\
1 \\
\text { signi }\end{array}$ & & $\begin{array}{l}1= \\
2= \\
3=\end{array}$ & $\begin{array}{l}\text { feren: } \\
\text { steno } \\
\text { hovati }\end{array}$ & $\begin{array}{l}\text { ungso } \\
\text { tierte } \\
\text { orient }\end{array}$ & ierte & & \\
\hline
\end{tabular}

Abb. 39: Mittelwertvergleichstest der relativen Erfolgsgrößen 


\section{Zusammenfassung und Implikationen}

\section{Zusammenfassende Würdigung der Untersuchungsergebnisse}

In High-Tech-Märkten werden die Unternehmen aufgrund der hohen Entwicklungsdynamik und der zunehmenden Technologieintegration vor besondere Herausforderungen gestellt. Aus Sicht der Praxis und betriebswirtschaftlichen Forschung ist es von besonderem Interesse, Informationen über das Wettbewerbsverhalten in High-Tech-Märkten zu erhalten und $\mathrm{zu}$ erfahren, welche Marketingstrategien zur Erzielung von Wettbewerbsvorteilen und zur Sicherung einer dauerhaften Erfolgsposition in High-Tech-Märkten besonders geeignet sind. Angesichts der in diesem Zusammenhang bestehenden Erkenntnisdefizite wurde in der vorliegenden Untersuchung versucht, eine theoretische Aufarbeitung und empirische Fundierung des Untersuchungskomplexes zu leisten. Es wurden hierzu auf der Grundlage des Konzeptes der strategischen Gruppen das strategische und operative Verhalten von High-Tech-Unternehmen, die Bedeutung ziel- und kulturbezogener Einflußfaktoren auf das strategische Unternehmensverhalten sowie die Erfolgswirkung von Marketingstrategien in High-Tech-Märkten analysiert. Im Hinblick auf die eingangs formulierten Zielsetzungen und die zu prüfenden Hypothesen des Untersuchungsvorhabens lassen sich auf Basis der empirischen Analysen folgende zentrale Schlußfolgerungen ableiten:

(1) In High-Tech-Märkten konnten im Rahmen einer Faktorenanalyse vier Basisstrategien identifiziert werden. Die erste Basisstrategie, die als produktbezogene Innovationsstrategie zu bezeichnen ist, wird durch die Produktentwicklung sowie eine Technologie- und Pionierorientierung geprägt. Bei der differenzierten Profilierungsstrategie steht die Erreichung einer Qualitäts-, Service-, Image- und Nutzen-Führerschaft im Vordergrund. Die preisbezogene Profilierungsstrategie vereint die Strategievariablen Marktpenetration und Preis-/Kosten-Führerschaft, während die marktbezogene Innovationsstrategie die Dimensionen Marktentwicklung und Diversifikation zusammenfuihrt. Bei der Analyse der Basisstrategien wurde festgestellt, daß die differenzierte und die preisbezogene Profi- 
lierungsstrategie mit den Wettbewerbsstrategien von Porter korrespondieren, während die produkt- und die marktbezogene Innovationsstrategie in High-Tech-Märkten eigenständige strategische Grundhaltungen darstellen.

(2) Der gewählte zweistufige Operationalisierungsansatz hat sich als tragfähige Grundlage zur Typologisierung von Marketingstrategien in HighTech-Märkten erwiesen. Angesichts der Problematik einer traditionell branchenbezogenen Marktabgrenzung in dynamischen und von technologischen Integrationstendenzen geprägten High-Tech-Märkten wurden zunächst über einen multidimensionalen Abgrenzungsansatz homogene Umfeldsituationen ermittelt, in denen anschließend strategische Gruppen identifiziert werden konnten.

Hierbei wurde deutlich, daß sich die gebildeten Umfeldsituationen signifikant voneinander unterscheiden. In Situation A werden die High-TechUnternehmen mit einer sehr hohen Marktdynamik sowie einem sehr geringen Preis- und Technologiewettbewerb konfrontiert. Situation B ist vor allem durch eine deutliche Technologiedynamik und hohe Leistungsanforderungen der High-Tech-Nachfrager gekennzeichnet. Im Vergleich dazu wird Situation $C$ durch einen ausgeprägten Preiswettbewerb bestimmt.

Die Typologisierung von Marketingstrategien auf der Basis strategischer Gruppen in den homogenen Umfeldsituationen erfolgte anhand von konzeptionell abgeleiteten marktfeld-, wettbewerbs- und timingbezogenen Strategiedimensionen. Dabei konnte eine Bestandsaufnahme der bisherigen Forschungsansätze zum Konzept der strategischen Gruppen nur in begrenztem Umfang Hinweise zur Ableitung relevanter Strategievariablen für die strategische Gruppenbildung in High-Tech-Märkten liefern. Dies liegt im wesentlichen darin begründet, daß Wettbewerbsvorteile und Erfolgspositionen in den bislang untersuchten Märkten durch spezifische strategische Verhaltensweisen erzielt wurden, die nur eingeschränkt auf andere 
Branchen zu übertragen sind. Die Operationalisierung des Konstruktes der strategischen Gruppen wurde daher auf den spezifischen Kontext von High-Tech-Märkten zugeschnitten. Eine Aufarbeitung zentraler Strategieansätze zeigte darüber hinaus, daß weder zeit- und technologiebezogene Systematisierungen noch wettbewerbsorientierte Ansätze für sich alleine eine problemadäquate Grundlage zur Typologisierung von Marketingstrategien in High-Tech-Märkten bilden.

Im Rahmen der strategischen Gruppenbildung wurden in Situation A drei Verhaltensgruppen ermittelt, die signifikante Unterschiede in den Strategieschwerpunkten aufweisen. Die identifizierten Gruppen sind jeweils durch eine deutliche Differenzierungs-, Kosten- oder Innovationsorientierung gekennzeichnet. "Differenzierungsorientierte" streben primär eine Qualitäts-, Service-, Image- und Nutzen-Führerschaft an und nehmen bei der Einführung von Produktneuheiten eine Pionierposition ein. Unternehmen, die der zweiten strategischen Gruppe angehören, legen den Strategieschwerpunkt auf die Preis-/Kostenorientierung und treten entweder als früher oder später Folger in den Markt ein. Für die innovationsorientierten High-Tech-Anbieter sind die Erreichung einer Technologie-Führerschaft, die Produktentwicklung und der Markteintritt als Pionier von besonderer Bedeutung.

Eine situationsübergreifende Analyse des strategischen Unternehmensverhaltens zeigte, daß sich in den Situationen A, B und C strategische Verhaltensgruppen mit vergleichbaren Strategieschwerpunkten identifizieren lassen. Durch dieses Ergebnis wird die hohe Validität der ermittelten strategischen High-Tech-Gruppen unterstrichen.

Darüber hinaus wurde festgestellt, daß die Strategieschwerpunkte der vergleichbaren strategischen Gruppen in den jeweiligen Situationen unterschiedlich stark ausgeprägt sind. Die differenzierungsorientierten HighTech-Anbieter legen in der Situation B aufgrund des starken Technologiewettbewerbs einen deutlich höheren Wert auf die Technologie- und 
Pionierorientierung als in den beiden anderen Umfeldsituationen. Für die "Kostenorientierten" kommt der Preis-/Kostenorientierung und dem Markteintritt als später Folger in Situation C, in der ein intensiver Preiswettbewerb herrscht, die höchste Bedeutung zu. Die strategische Gruppe der "Innovationsorientierten" mißt der Produktentwicklung in der Situation A den größten Stellenwert bei. Da bei den vergleichbaren High-TechGruppen insgesamt jedoch lediglich geringe Strategieunterschiede bestehen, läßt sich mit der vorliegenden Untersuchung nur bedingt bestätigen, daß die Umfeldsituation einen Einfluß auf das strategische Unternehmensverhalten ausübt.

Eine weitere Zielsetzung des Forschungsvorhabens bestand in der Analyse der Ausgestaltungsformen der strategischen Gruppen in High-Tech-Märkten.

(3) Hierbei konnte nachgewiesen werden, daß die Strategieschwerpunkte der strategischen High-Tech-Gruppen auch in den Marketingmaßnahmen umgesetzt werden. Bei der Betrachtung der strategischen High-Tech-Gruppen in den Umfeldsituationen A, B und C wurden ähnliche Instrumentekombinationen deutlich. Für die "Differenzierungsorientierten" stehen primär die Produkt- und Kommunikationspolitik, für die "Kostenorientierten" die kontrahierungspolitischen Maßnahmen und für die "Innovationsorientierten" die Produktinnovation im Vordergnund des Marketing-Mix.

Die situationsübergreifende Untersuchung der Instrumentekombinationen der vergleichbaren strategischen Gruppen zeigte Unterschiede in der Ausgestaltung einzelner Marketingmaßnahmen auf. Die differenzierungsorientierten High-Tech-Anbieter legen vor allem in der Situation B einen besonderen Wert auf die Produktinnovation, die Benutzerfreundlichkeit und die Produktqualität. Serviceleistungen und Aktivitäten in der Marketinglogistik sind insbesondere in der Situation C relevant. Die "Kostenorientierten" beurteilen die kontrahierungspolitischen Maßnahmen in den Umfeldsituationen $\mathrm{B}$ und $\mathrm{C}$ als besonders wichtig. Von den "Innovationsorientierten" werden der Produktpflege in der Situation C und der 
Benutzerfreundlichkeit in den Umfeldsituationen $B$ und $C$ die größte Bedeutung beigemessen. Trotz dieser Unterschiede bleibt festzuhalten, da $B$ die marktgerichteten Maßnahmen der vergleichbaren strategischen Gruppen in den unterschiedlichen Situationen sehr ähnlich sind. Dies ist ein Hinweis dafür, daß die Umfeldsituation das operative Verhalten im Marketing-Mix-Bereich nur bedingt beeinflußt.

(4) Im Rahmen der Analyse der Unternehmensaktivitäten zur Verkürzung der Entwicklungszeiten und Beschleunigung der Vermarktung von HighTech-Produkten wurde festgestellt, daß von den strategischen High-TechGruppen unterschiedliche Maßnahmenschwerpunkte gesetzt werden. Von den "Differenzierungsorientierten" werden die Just-in-time-Beschaffung, die Erhöhung des Werbedrucks sowie Maßnahmen zur Motivation der Mitarbeiter und Erhöhung der Unternehmensflexibilität präferiert. Für die kostenorientierten High-Tech-Unternehmen stehen die Fertigungstiefenreduktion, die Optimierung der Durchlaufzeiten und die Verbesserung der Zeiteffizienz im Mittelpunkt der Betrachtung. Von der strategischen Gruppe der "Innovationsorientierten" werden ein erhöhter Ressourceneinsatz in der F\&E, Kooperationen mit Pilotkunden sowie die Mitarbeitermotivation als besonders wichtig eingeschätzt. Die identifizierten Maßnahmenunterschiede bezüglich der Aktivitäten zur Verkürzung der F\&E und der Beschleunigung der Vermarktung von High-Tech-Produkten sind jedoch lediglich als Tendenzaussagen zu verstehen, da sie im Rahmen der Mittelwertvergleichstests nicht hinreichend validiert werden konnten.

Die Betrachtung der Maßnahmenschwerpunkte der vergleichbaren strategischen Gruppen in den Umfeldsituationen A, B und C verdeutlicht Unterschiede in der Ausprägung einzelner Maßnahmen. Für die "Differenzierungsorientierten" besitzt der Know-how Zukauf vor allem in der Situation B und die Just-in-time-Beschaffung in den Umfeldsituationen $\mathrm{B}$ und $\mathrm{C}$ eine hohe Bedeutung. Die "Kostenorientierten" legen in den Situationen B und C großen Wert auf die Optimienung der Durchlaufzeiten. Weiterhin werden dem Zukauf von technologischem Know-how in Situa- 
tion B sowie Unternehmenskooperationen und der Reduktion der Fertigungstiefe in den Situationen B und C ein hoher Stellenwert eingeräumt. Die "Innovationsorientierten" bevorzugen insbesondere in der Situation A F\&E-Kooperationen, in den Situationen A und B die Zusammenarbeit mit Pilotkunden und in der Situation $C$ eine globale Vermarktung von HighTech-Produkten. Insgesamt zeigen sich auch hier relativ geringe situationsübergreifende Unterschiede bei den vergleichbaren strategischen Gruppen. Es ist somit davon auszugehen, daß die jeweiligen Umfeldsituation lediglich einen geringen Einfluß auf das operative Unternehmensverhalten ausübt.

Ein weiteres Forschungsziel lag in der Analyse ziel- und kulturbezogener Einflußfaktoren der Marketingstrategien von strategischen High-Tech-Gruppen, um Erkenntnisse darüber zu gewinnen, ob bestimmte Zielsetzungen und kulturelle Grundhaltungen die Bildung strategischer Gruppen in High-Tech-Märkten begünstigen.

(5) Die Analyse der Unternehmensziele ergab, daß sich die Marketingstrategien auch in den Zielen der differenzierungs-, kosten- und innovationsorientierten High-Tech-Hersteller widerspiegeln. Bei den "Differenzierungsorientierten" korrespondieren in den Situationen A, B und C die Zielsetzungen, die die langfristige Marktstellung betreffen, mit den Strategiedimensionen der Marktpenetration und -entwicklung sowie das Imageziel mit dem Streben nach einer Image-Führerschaft. Die überdurchschnittliche Bedeutung des Sozialziels Mitarbeitermotivation ist ein Hinweis dafür, daß eine hohe Mitarbeiterzufriedenheit eine wichtige Voraussetzung zur Realisierung einer Qualitäts-, Service- und Nutzen-Führerschaft darstellt. Die "Kostenorientierten" messen der Realisierung kurzfristiger Gewinne, der Kostenreduktion und der Produktivitätssteigerung im Produktionsbereich einen hohen Stellenwert bei. Diese Unternehmensziele stehen in einer engen Beziehung zur Strategieausrichtung der Preis-/ Kosten-Führerschaft. Bei den "Innovationsorientierten" besitzt die Zielsetzung der Markterschließung eine besondere Relevanz. Entsprechend 
werden die Diversifikation und Pionierorientierung für überdurchschnittlich wichtig gehalten.

In einer Untersuchung der vergleichbaren strategischen Gruppen in den Situationen A, B und C waren einige situationsbedingte Unterschiede in der Ausprägung einzelner Unternehmensziele zu erkennen. Für die "Differenzierungsorientierten" sind die Realisierung langfristiger Gewinne und die Mitarbeitermotivation in den Situationen A und B, die Produktivitätssteigerung in der F\&E in Situation B und die Produktivitätssteigerung in der Produktion in Situation C von besonderer Bedeutung. Dies läßt sich damit begründen, da $B$ die differenzierungsorientierten High-Tech-Anbieter bei der Zielplanung in der Situation B technologische und in der Situation C kostenbezogene Aspekte stärker berücksichtigen. Die kostenorientierten Unternehmen messen der Erzielung kurzfristiger Gewinne, der Kostenreduktion und der Produktivitätssteigerung in der Produktion in Situation A, in der noch kein Technologie- bzw. Preiswettbewerb herrscht, einen relativ geringen Stellenwert bei. Von den "Innovationsorientierten" werden die Markterschließung in Situation B, die Kreditwürdigkeit in Situation C und die Sicherung der Wettbewerbsfähigkeit in den Situationen B und C als besonders wichtig angesehen. Die wachsende Bedeutung des Marktstellungsziels läßt sich darauf zurückfuihren, daß die Wettbewerbsdynamik in den Umfeldsituationen B und C im Vergleich zur Situation A deutlich höher ist. Die vergleichsweise geringen situationsübergreifenden Zielunterschiede bei den vergleichbaren strategischen Gruppen deuten jedoch darauf hin, daß die Umfeldsituation die Zielplanung der High-TechHersteller insgesamt nur relativ wenig beeinflußt.

(6) Die Analyse der Unternehmenskultur zeigte, daß die vergleichbaren strategischen Gruppen in den Umfeldsituationen A, B und C eine ähnliche kulturelle Grundorientierung aufweisen. Von den "Differenzierungsorientierten", die insbesondere eine Qualitäts-, Service- und NutzenFührerschaft verfolgen, werden der Kunden- und Mitarbeiterorientierung eine besondere Bedeutung beigemessen. Dies weist darauf hin, da $B$ die 
kulturelle Ausrichtung das strategische Verhalten der differenzierungsorientierten High-Tech-Anbieter beeinflußt. Bei den "Kostenorientierten" zeigt sich der enge Kultur-Strategie-Zusammenhang in der kostenbezogenen kulturellen Grundhaltung und dem Streben nach einer Preis-/Kosten-Führerschaft. Durch die technologiebezogene Kulturausrichtung wird das strategische Verhalten der "Innovationsorientierten" geprägt. Dies kommt in dem Streben nach einer Technologie-Führerschaft zum Ausdruck. Es wird jedoch auch deutlich, daß trotz der hohen Bedeutung der Kreativität lediglich eine schwache Innovationsorientierung in der Unternehmenskultur verankert ist. Hieraus folgt, daß bezüglich der Strategiedimensionen Produktentwicklung, Diversifikation und Pionierorientierung nur ein geringer Kultur-Strategie-Fit besteht. Insgesamt bleibt festzuhalten, daß die vorliegenden Erkenntnisse aufgrund der z.T. schwachen Kultur-Strategie-Zusammenhänge und der z.T. schlechten Ergebnisse der Mittelwertvergleichstests nur Tendenzaussagen darstellen.

Die situationsübergreifende Betrachtung der Unterschiede in den kulturellen Grundhaltungen der vergleichbaren strategischen Gruppen konnte keinen Nachweis für die These erbringen, daß die Unternehmenskultur der High-Tech-Anbieter durch die Umfeldsituation beeinflußt wird.

Die Abbildungen 40 und 41 zeigen eine Übersicht der Gemeinsamkeiten und Unterschiede der vergleichbaren strategischen High-Tech-Gruppen in den Umfeldsituationen A, B und C in bezug auf das strategische Unternehmensverhalten sowie die Ausgestaltungsformen und Einflußfaktoren der Marketingstrategien von strategischen Gruppen.

Ein zusätzliches Untersuchungsziel bestand darin, mit dem Konzept der strategischen Gruppen die in den High-Tech-Märkten bestehenden Erfolgsunterschiede zu erklären. 


\begin{tabular}{|c|c|c|c|}
\hline \multirow{3}{*}{ 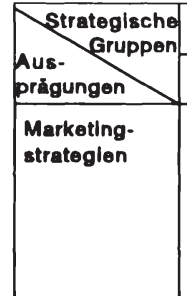 } & \multirow{2}{*}{$\begin{array}{c}\text { Differenzierungsorientierte } \\
\text { Situationen } A, B, C \\
\end{array}$} & \multirow{3}{*}{$\begin{array}{l}\text { Kostenorientierte } \\
\text { Situationen A,B,C } \\
\text {-Preis-/Kosten-F. } \\
\text { - Früher Folger } \\
\text { - Spāter Folger }\end{array}$} & \multirow{3}{*}{$\begin{array}{l}\text { Innovationsorientierte } \\
\text { Situationen A,B,C } \\
\text { - Produktentwicklung } \\
\text { - Technologie-F. } \\
\text { - Pionier }\end{array}$} \\
\hline & & & \\
\hline & $\begin{array}{ll}\text { - Marktpenetration } & \text {-Service-F. } \\
\text { - Marktentwicklung } & \text {-Image-F. } \\
\text { - Produktentwicklung } & \text { - Nutzen-F. } \\
\text { - Technologie-F. } & \text {-Pionier } \\
\text { - Qualităts-F. } & \end{array}$ & & \\
\hline $\begin{array}{l}\text { Marketing- } \\
\text { Instrumente }\end{array}$ & \begin{tabular}{|ll}
-Produktpflege & -Werbung \\
- Produktinnovation & - Verkaufsförderung \\
- Benutzerfreund- & -Public Relations \\
lichkeit & -Absatzwege- \\
- Design & gestaltung \\
- Produktqualität & - Logistik \\
- Service &
\end{tabular} & $\begin{array}{l}\text {-Preis } \\
\text { - Konditionen } \\
\text {-Persōnlicher Verkauf }\end{array}$ & - Produktinnovationen \\
\hline $\begin{array}{l}\text { MaBnahmen } \\
\text { zur Vorkar- } \\
\text { zung d. F\&E u } \\
\text { Beschlount- } \\
\text { gung der } \\
\text { Vormarktung } \\
\text { v. Hlgh-Tech- } \\
\text { Produkton }\end{array}$ & $\begin{array}{l}\text { - Just-in-time Beschaffung } \\
\text { - Erhöhung Werbedruck } \\
\text { - Mitarbeitermotivation } \\
\text { - Flexibilitāt U.-grundsatz }\end{array}$ & $\begin{array}{l}\text { - Fertigungstlefenreduktion } \\
\text {-Optimierung Durchlautzeiten } \\
\text {-Zeiteffizienz U.-grundsatz }\end{array}$ & $\begin{array}{l}\text { - Erhōhter Ressourceneinsatz } \\
\text { - Kooperation mit Pilotkunden } \\
\text { - Mitarbeitermotivation }\end{array}$ \\
\hline $\begin{array}{l}\text { Unter- } \\
\text { nehmens- } \\
\text { ziele }\end{array}$ & $\begin{array}{l}\text { - Marktanteil } \\
\text { - MarkterschlieBung } \\
\text { - Langfr. Gewinn } \\
\text { - Imageverbesserung } \\
\text { - Mitarbeitermotivation }\end{array}$ & $\begin{array}{l}\text {-Kurzfr. Gewinn } \\
\text { - Kostenreduktion } \\
\text { - Produktivitätssteigerung F\&E }\end{array}$ & $\begin{array}{l}\text { - MarkterschlieBung } \\
\text { - Langfr. Gewinn }\end{array}$ \\
\hline $\begin{array}{l}\text { Unter- } \\
\text { nehmens- } \\
\text { kultur }\end{array}$ & $\begin{array}{l}\text { - Kenntnis d. Kundenprobleme } \\
\text { - Vertrauen in Mitarbeiter } \\
\text { - Eigenståndigkeit d. Mitarbeiter } \\
\text { - Technologieorientierung }\end{array}$ & - Kostenorientierung & $\begin{array}{l}\text { - Kreativităt } \\
\text { - Technologieorientierung } \\
\text { - Technologiebewußtsein }\end{array}$ \\
\hline
\end{tabular}

Abb. 40: Situationsubergreifende Gemeinsamkeiten der strategischen High-Tech-Gruppen 


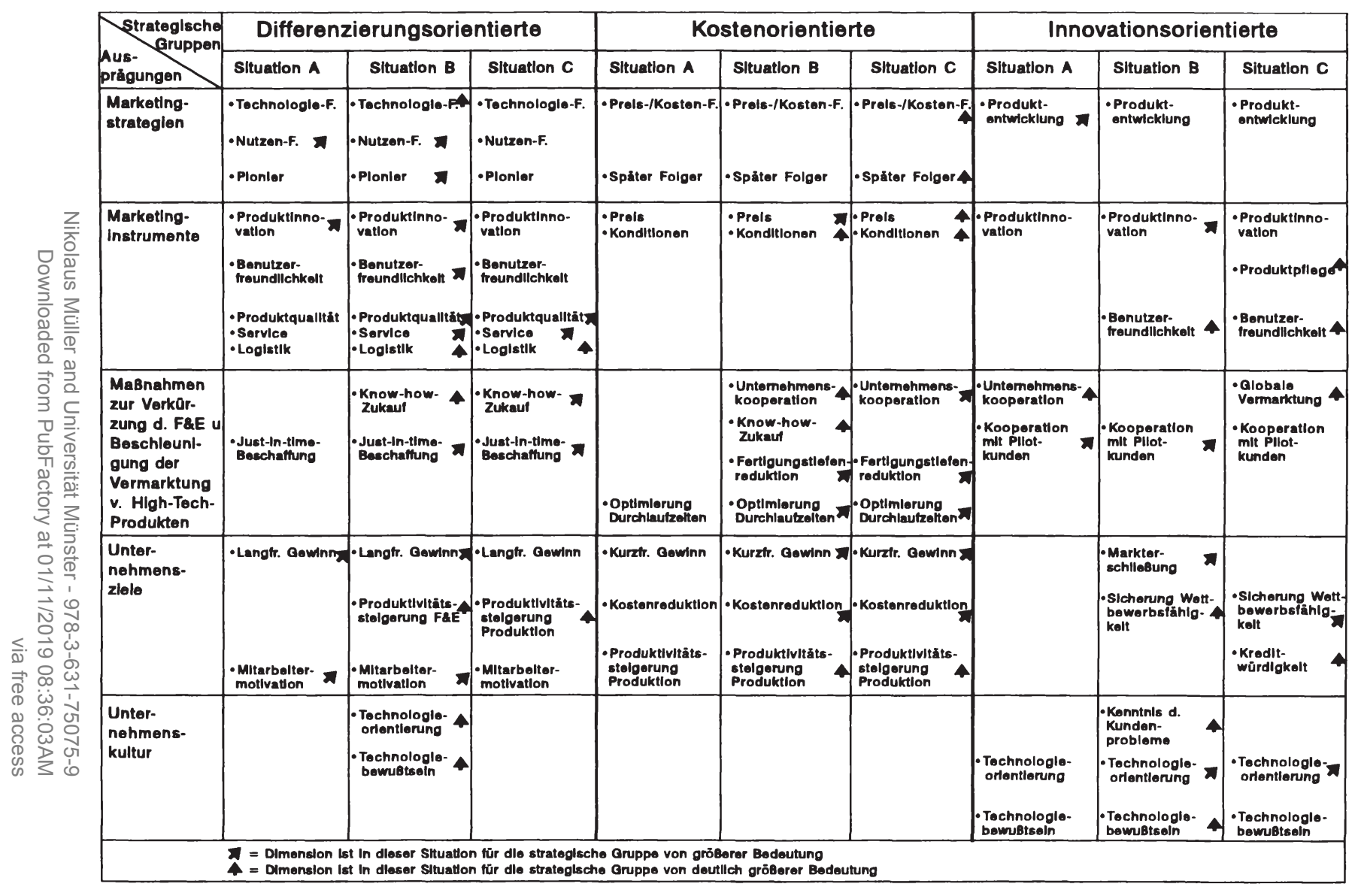

Abb. 41: Situationsübergreifende Unterschiede der strategischen High-Tech-Gruppen 
(7) Hierbei wurde nachgewiesen, daß sich die strategischen Gruppen im Hinblick auf die Erfolgsgrößen relative Umsatzsteigerung und relativer Marktanteil klar unterscheiden. In Situation A sind die differenzierungs- und innovationsorientierten High-Tech-Unternehmen besonders erfolgreich. Bei den "Differenzierungsorientierten" läßt sich der Markterfolg darauf zurückführen, daß die Unternehmen durch einen gleichbleibenden Qualitätsstandard, benutzerfreundliche High-Tech-Produkte und individuelle Serviceangebote in der Lage sind, die Kaufwiderstände der potentiellen Nachfrager zu senken und Präferenzen für die eigenen Produktangebote zu schaffen. Der Erfolg der "Innovationsorientierten" ist vor allem damit zu erklären, daß ein hohes technologisches Know-how und eine kontinuierliche Produktverbesserung zu einer frühzeitigen Kundenbindung führen.

Die strategische Gruppe der "Innovationsorientierten" ist in Situation B am erfolgreichsten, in der eine hohe Technologiedynamik besteht. Ein Grund hierfür ist darin zu sehen, daß sich in dieser Umfeldsituation insbesondere durch eine starke Technologie- und Pionierorientierung Wettbewerbsvorteile aufbauen lassen. Die innovationsorientierten Unternehmen können zudem die hohen Leistungsanforderungen der High-Tech-Anwender durch eine kontinuierliche Hervorbringung technologischer Produktneuheiten besonders gut erfüllen.

In Situation C sind die "Differenzierungsorientierten" überdurchschnittlich erfolgreich. Dies ist ein Indiz dafür, daß die High-Tech-Anwender trotz einer deutlichen Preisorientierung im Rahmen der Kaufentscheidung einen besonderen Wert auf die Qualität der technologischen Produktanwendungen und die Bereitstellung spezieller Serviceleistungen legen. Der relativ schlechte Markterfolg der kostenorientierten Unternehmen läßt sich damit begründen, daß der alleinige Aufbau von Preis-/Kostenvorteilen auch in einer Umfeldsituation, die durch eine hohe Preisdynamik gekennzeichnet ist, zur Differenzierung im Wettbewerbsumfeld und Schaffung einer Erfolgsposition nicht ausreicht. 
In einer zusammenfassenden Würdigung bleibt festzuhalten, daß die vorliegende Untersuchung eine erste umfassendere Bestandsaufnahme des strategischen und operativen Verhaltens deutscher High-Tech-Anbieter darstellt. Darüber hinaus konnten grundlegende Erkenntnisse über den Einfluß der Unternehmensziele und der Unternehmenskultur auf das strategische Verhalten sowie die Erfolgswirkung unterschiedlicher Marketingstrategien in High-Tech-Märkten gewonnen werden. Mit dem Forschungsansatz, diese Analyse auf Basis des Konzeptes der strategischen Gruppen vorzunehmen, kommt der vorliegenden Arbeit der Charakter einer Basisuntersuchung zu. Hierbei wurde deutlich, daß die in der Literatur zur strategischen Unternehmensführung in High-Tech-Märkten bislang weitgehend unabhängig diskutierten Fragestellungen bezüglich der Gestaltung der Wettbewerbs- und Timingstrategie nicht losgelöst voneinander betrachtet werden dürfen.

Mit Blick auf die heterogene Stichprobenstruktur und die teilweise geringen Sicherheitswahrscheinlichkeiten der empirischen Analysen ist allerdings darauf hinzuweisen, da $\beta$ aus den ermittelten Forschungsergebnissen oftmals nur Tendenzaussagen abgeleitet werden können. Im Rahmen einer möglichen Replizierung der Untersuchung sind insbesondere folgende Aspekte zu berücksichtigen.

Zur Identifikation homogener Umfeldsituationen wurden technologie-, markt-, abnehmer- und konkurrenzbezogene Kontextdimensionen herangezogen. Angesichts der hohen Entwicklungsdynamik in High-Tech-Märkten gilt es bei Folgeuntersuchungen zu prüfen, ob Veränderungen in der Unternehmensumwelt die Berücksichtigung weiterer Kontextdimensionen der globalen Umwelt wie z.B. ökologischer Rahmenbedingungen sowie der Aufgabenumwelt wie z.B. Zulieferer- und Absatzmittleraspekten erforderlich macht.

In der vorliegenden Arbeit wurde bei der Betrachtung des Abnehmerverhaltens nicht explizit zwischen privaten und institutionellen Nachfragern technologischer Produkte unterschieden. Zukünftige Forschungen in High-Tech-Märkten können sich speziell auf die Analyse privater oder institutioneller Abnehmer konzen- 
trieren, um Besonderheiten im Nachfrageverhalten aufzudecken und den HighTech-Unternehmen dadurch Handlungsempfehlungen zur Bearbeitung der jeweiligen Abnehmergruppe zur Verfügung zu stellen.

Zur Bildung strategischer High-Tech-Gruppen wurden marktfeld-sowie wettbewerbs- und timingbezogene Strategiedimensionen konzeptionell abgeleitet. In zukünftigen Untersuchungen erscheint es sinnvoll, im Rahmen der strategischen Gruppenbildung auch das ökologieorientierte strategische Unternehmensverhalten einzubeziehen, da steigende Anforderungen der Nachfrager hinsichtlich der Umweltverträglichkeit technologischer Produkte u.a. im Computerbereich den High-Tech-Anbietern die Gelegenheit bieten, sich beipielsweise durch eine offensive Umweltschutzstrategie gegenüber den Wettbewerbern zu profilieren. ${ }^{1}$

Die Operationalisierung der Unternehmenskultur erfolgte auf Basis der Experteneinschätzung jeweils eines Mitglieds der untersuchten High-Tech-Unternehmen. Es ergibt sich hierbei das Problem, daß die von den Mitarbeitern tatsächlich gelebte Kultur durch die Befragung einer Person i.d.R. nicht hinreichend erfaßt werden kann. ${ }^{2}$ Darüber hinaus lassen sich kaum Schlußfolgerungen über den Einfluß von Subkulturen (z.B. F\&E-Kultur, Marketingkultur) auf die Unternehmenskultur ableiten. Dem ist in weiterführenden Forschungen zur Unternehmenskultur in High-Tech-Märkten dahingehend Rechnung zu tragen, daß Mitarbeiter aus verschiedenen Funktionsbereichen hinsichtlich ihrer Wertvorstellungen, Verhaltensnormen und Denkhaltungen befragt werden.

In dieser Arbeit wurde jeweils einzeln analysiert, ob Unternehmensziele und Unternehmenskultur die Gestaltung der Marketingstrategien von strategischen Gruppen in High-Tech-Märkten beeinflussen. Es wurde hierbei nicht überprüft,

1 Vgl. Felser, G., Kein Fall für Hacker, Gefährlicher Computermüll: Gifte von Arsen bis Zinn, in: Natur und Umwelt, 1994, Nr. 2, S. 16 ff.; Olschewski, J., Das I+T-Unternehmen in der Verantwortung gegenüber der Öffentlichkeit - Neue Marketingstrategien unter Berücksichtigung von Recycling, Ergonomie und Sicherheit, in: Investitionsgüterund High-Tech-Marketing, Hrsg.: Hofmaier, R., Landsberg a.L. 1992, S. 582 ff.; Kirchgeorg, M., Ökologieorientiertes Unternehmensverhalten, a.a.O., S. $137 \mathrm{ff}$.

2 Vgl. Wever, U., Unternehmeskultur in der Praxis, Frankfurt a.M., New York 1989, S. 123. 
welche Beziehungen zwischen Zielen und Kultur bestehen und welchen Einfluß die Strategie ihrerseits auf die Kultur ausübt. Für nachfolgende Forschungsstudien bietet es sich an, im Rahmen einer integrierten Betrachtung die wechselseitigen Wirkungsbeziehungen zwischen Unternehmenszielen, -kultur und -strategien näher zu untersuchen.

Der Markterfolg von High-Tech-Unternehmen wurde in Abhängigkeit von der verfolgten Marketingstrategie analysiert. Es wurde hierbei nicht untersucht, welchen Einfluß die Strategieimplementierung auf den Unternehmenserfolg in High-Tech-Märkten hat. Bei erfolgreichen Marketingstrategien ist davon auszugehen, daß i.d.R. auch eine gute Strategieumsetzung vorliegt. Demgegenüber können die Ursachen der geringen Erfolgswirkung von Marketingstrategien neben einer "falschen" Strategiewahl auch in der unzureichenden Implementierung einer erfolgversprechenden Marketingstrategie liegen. ${ }^{3}$ Bei zukünftigen Untersuchungen zur Erfolgsfaktorenforschung in High-Tech-Märkten sind daher auch verstärkt Fragestellungen der Strategieimplementierung zu berücksichtigen.

\section{Implikationen für das Management in High-Tech-Unternehmen}

In der vorliegenden Arbeit konnte gezeigt werden, daß das Konzept der strategischen Gruppen in den durch eine hohe Entwicklungs- und Wettbewerbsdynamik geprägten High-Tech-Märkten geeignet ist, die Komplexität der Wettbewerbsanalyse zu verringern. Für das Management bietet es sich hierbei an, in High-Tech-Märkten zuerst strategische Gruppen zu identifizieren und auf dieser Grundlage eine Analyse einzelner Konkurrenten durchzufuihren. Durch die strategische Gruppenbildung werden die High-Tech-Unternehmen zu einer überschaubaren Anzahl von Gruppen mit ähnlichen wettbewerbsstrategischen Verhaltens- und Reaktionsmustern zusammengefaßt. Bei einer strategischen Gruppe, die mit dem eigenen Unternehmen in enger Wettbewerbsbeziehung steht, lassen sich in einer anschließenden Konkurrenzanalyse das strategische Verhalten sowie implementierung, a.a.O., S. 7 ff. 
die Stärken und Schwächen der einzelnen High-Tech-Anbieter differenziert untersuchen. Das Konzept der strategischen Gruppen bietet den High-TechUnternehmen somit eine wichtige Hilfestellung bei der Auswertung wettbewerbsbezogener Informationen.

Die Kenntnis der Erfolgspotentiale von Marketingstrategien in High-Tech-Märkten gibt dem Management wertvolle Hinweise bei Entscheidungen im Zusammenhang mit dem Wechsel einer strategischen Gruppe. ${ }^{4}$ Unter Berücksichtigung der spezifischen Erfolgsposition der innovationsorientierten Unternehmen in der Kontextsituation B ergeben sich beispielsweise für einen differenzierungsorientierten High-Tech-Anbieter bei einem strategischen Gruppenwechsel folgende Konsequenzen. ${ }^{5}$ Im Rahmen des strategischen Unternehmensverhaltens sind der Produktentwicklung und dem Streben nach einer TechnologieFührerschaft eine größere Bedeutung beizumessen. Das Unternehmen ist weiterhin gefordert, die Marketingaktivitäten bezüglich der Produktinnovation zu intensivieren und während der Entwicklung und Vermarktung von High-Tech-Produkten verstärkt Kooperationen mit Pilotkunden einzugehen. Durch den Einsatz entsprechender Maßnahmen ist die Technologieorientierung in der Unternehmenskultur stärker zu verankern.

Auf der Grundlage der vorliegenden Untersuchung lassen sich zudem Aussagen darüber ableiten, welche Verhaltensanpassungen von High-Tech-Unternehmen in dynamischen Umfeldsituationen vorzunehmen sind, um bei einem Situationswechsel in einer erfolgreichen strategischen Gruppe zu verbleiben. Bei einer Änderung der Kontextsituation von einer hohen Marktdynamik zu einem intensiven Preiswettbewerb (Situation A $\rightarrow$ C) lassen sich beispielsweise für einen differenzierungsorientierten High-Tech-Hersteller folgende Implikationen ablei-

$4 \quad$ Vgl. Görgen, W., Van Kerkom, K., Der Wechsel der Wettbewerbsstrategie - Eine kritische Analyse der Bestimmungsfaktoren und Maßnahmen, Arbeitspapier des Instituts für Markt- und Distributionsforschung, Köln 1991, S. 22 ff.; Boeker, W.P., Strategic change - The effects of founding and history, in: Academy of Management Journal, Vol. 32 (1989), No. 3, S. $489 \mathrm{ff}$.

Vgl. in diesem Zusammenhang auch die Abbildungen 28, 31, 32 und 34 der Arbeit. 
ten. ${ }^{6}$ Im Marketingbereich sind Maßnahmen zur Verbesserung der Produktqualität zu ergreifen, zusätzliche Serviceleistungen anzubieten und die logistischen Systeme zu optimieren. Im Hinblick auf die Verkürzung der Entwicklungszeiten und der Beschleunigung der Vermarktung von Produktneuheiten sind ein größeres Gewicht auf den Zukauf von technologischem Know-how und die Justin-time-Beschaffung zu legen.

\section{Implikationen für die Forschung zum Konzept der strategischen Grup- pen}

Vor dem Hintergrund der vorliegenden Untersuchungsergebnisse und der daraus abgeleiteten Implikationen ergeben sich für weiterführende empirische Studien und konzeptionelle Forschungsüberlegungen zum Konzept der strategischen Gruppen weitere interessante Ansatzpunkte:

(1) Bisherige Forschungen zum Problemkreis der strategischen Gruppen haben die Fragestellung der Abgrenzung des relevanten Marktes weitgehend vernachlässigt. In der vorliegenden Untersuchung von sich dynamisch entwickelnden High-Tech-Märkten wird die Schwierigkeit der Marktabgrenzung besonders deutlich. Die Bildung strategischer Gruppen kann jedoch nur einen Erkenntnisgewinn liefern, wenn der Untersuchungsbereich, d.h. der relevante Markt, richtig abgegrenzt wird. Es zeigte sich jedoch, daß die der traditionellen Branchenabgrenzung zugrundeliegende produkt- bzw. technologiebezogene Sichtweise in dynamischen HighTech-Märkten keine geeignete Grundlage zur Erfassung strategischer Gruppen darstellt. In der vorliegenden Untersuchung in High-Tech-Märkten erfolgte deshalb die Abgrenzung homogener Umfeldsituationen anhand eines multidimensionalen Ansatzes. Im Rahmen zukünftiger Forschungen zum Konzept der strategischen Gruppen sind der Abgrenzung des relevanten Marktes und der Ableitung von Abgrenzungskriterien, die z.B. techno-

Vgl. hierzu auch die Abbildungen 28, 31, 32 und 34 der vorliegenden Untersuchung. 
logische Integrationstendenzen sowie abnehmer -und konkurrenzbezogene Besonderheiten berücksichtigen, verstärkt Bedeutung beizumessen.

(2) Anhand des Konzeptes der strategischen Gruppen wurden in dem vorliegenden Forschungsvorhaben die strategischen Verhaltensgruppen der differenzierungs-, kosten- und innovationsorientierten High-Tech-Unternehmen gebildet. Aufgrund der Tatsache, daß die identifizierten strategischen Gruppen in allen drei Umfeldsituationen anzutreffen sind, scheinen diese Marketingstrategien für High-Tech-Märkte eine hohe Validität aufzuweisen, wenngleich die Ausprägungen einzelner Strategiedimensionen situationsspezifisch zu relativieren sind. Da die ermittelten Ergebnisse lediglich für deutsche High-Tech-Anbieter Gültigkeit haben, erscheint es sinnvoll, die vorliegende Untersuchung unter Einbeziehung europäischer, amerikanischer und japanischer High-Tech-Unternehmen zu replizieren. Hierbei kann analysiert werden, ob sich die internationalen HighTech-Unternehmen den gefundenen strategischen High-Tech-Gruppen zuordnen lassen oder ob durch ausländische High-Tech-Anbieter neue strategische Verhaltensgruppen gebildet werden. Angesichts der Bedeutung des globalen Wettbewerbs in High-Tech-Märkten sind weiterführende Forschungen auf diesem Gebiet besonders dringlich.

(3) In der vorliegenden Arbeit wurde gezeigt, daß das Konzept der strategischen Gruppen zur Erklärung von Erfolgsunterschieden in High-TechMärkten geeignet ist. In zukünftigen Forschungen ist das begrenzte Set an marktbezogenen Erfolgsvariablen dieser empirischen Studie durch weitere finanzwirtschaftliche Erfolgsgrößen wie z.B. Gewinn, ROI, Gesamtkapitalrentabilität zu ergänzen.

(4) Im Rahmen der Analyse der Erfolgsunterschiede zwischen strategischen Gruppen in High-Tech-Märkten ist es auch von Interesse, zu untersuchen, welchen Erfolgsbeitrag einzelne strategische Verhaltensdimensionen leisten. Darüber hinaus erscheint es sinnvoll, in weiterfuihrenden Forschungen zu analysieren, welchen Einfluß der Zusammenhang zwischen Kultur 
und Strategie auf den Unternehmenserfolg innerhalb einer strategischen Gruppe hat.

(5) Mit Blick auf den aktuellen Forschungsstand zum Konzept der strategischen Gruppen ist ein weiteres interessantes forscherisches Aufgabenfeld im Zusammenhang mit Fragestellungen zu strategischen Allianzen zu sehen. So ist es beispielsweise denkbar, daß High-Tech-Anbieter aus verschiedenen strategischen Gruppen, zwischen denen geringe Wettbewerbsbeziehungen bestehen, miteinander kooperieren, um an den Wettbewerbsvorteilen des jeweils anderen Unternehmens zu partizipieren. Für Folgeuntersuchungen bietet es sich an, im Rahmen der Analyse strategischer Gruppen in High-Tech-Märkten auch das Kooperationsverhalten der Unternehmen zu berücksichtigen. Es läßt sich hierbei überprüfen, inwieweit der Markterfolg von High-Tech-Unternehmen innerhalb einer strategischen Gruppe durch die Bildung strategischer Allianzen beeinflußt wird.

(6) In der empirischen Analyse wurde deutlich, daß zwischen den differenzierungs- und kostenorientierten sowie den kosten- und innovationsgerichteten High-Tech-Anbietern eine relativ große strategische Distanz besteht. Diese strategische Distanz liegt in den strategischen und operativen Verhaltensweisen sowie in ziel- und kulturbezogenen Charakteristika begründet. Es kann davon ausgegangen werden, daß bestimmte Zielsysteme und Unternehmenskulturen die Zugehörigkeit eines High-TechUnternehmens zu einer bestimmten strategischen Gruppe determinieren und gleichzeitig Mobilitätsbarrieren darstellen. Ein Wechsel zwischen den oben genannten High-Tech-Gruppen ist aufgrund der großen strategischen Distanz kaum zu erwarten. Demgegenüber ist die Wahrscheinlichkeit des strategischen Gruppenwechsels zwischen den "Differenzierungsorientierten" und "Innovationsorientierten" aufgrund einer ähnlichen Strategieausrichtung und aufgrund von Gemeinsamkeiten in den Instrumente- und Kulturausprägungen sehr hoch. In diesem Zusammenhang bietet es sich an, das Wechselverhalten der High-Tech-Unternehmen aus 
beiden strategischen Gruppen im Zeitablauf genauer zu analysieren. Durch eine Längsschnittanalyse lassen sich zudem Veränderungen in der Gruppenstruktur bezüglich der Anzahl, Größe und Stabilität strategischer Gruppen in High-Tech-Märkten beobachten.

(7) Die vorliegende Untersuchung gibt darüber hinaus erste Hinweise über die Anpassungsnotwendigkeiten bei dynamischen Marktentwicklungen. $\mathrm{Da}$ im Rahmen der vorliegenden Studie keine Längsschnittdaten zur Verfügung standen, wurde den Überlegungen ein statischer Vergleich der verschiedenen Umfeldsituationen zugnundegelegt, die nur eine annäherungsweise Überführung in eine dynamische Perspektive erlaubt. Weiterfuihrende Forschungen sollten durch explizite Längsschnittanalysen den Anpassungsnotwendigkeiten von strategischen Gruppen an veränderte Umweltsituationen verstärkt Beachtung schenken.

Insgesamt verdeutlichen die offenen Fragestellungen, daß dem Konzept der strategischen Gruppen aus verschiedenen wissenschaftlichen Forschungsperspektiven zukünftig eine größere Aufmerksamkeit beizumessen ist. 
Nikolaus Müller and Universität Münster - 978-3-631-75075-9 Downloaded from PubFactory at 01/11/2019 08:36:03AM via free access 
ANHANG I

Studien zum Untersuchungsgegenstand

Nikolaus Müller and Universität Münster - 978-3-631-75075-9

Downloaded from PubFactory at 01/11/2019 08:36:03AM

via free access 


\section{Tabellenverzeichnis des Anhangs I}

Tab. 1: Umfassende Übersicht ausgewählter empirischer Studien zur Bildung strategischer Gruppen

Tab. 2: Umfassende Übersicht ausgewählter empirischer Studien zur Bildung strategischer Gruppen (Fortsetzung)

Tab. 3: Umfassende Übersicht ausgewählter empirischer Studien zur Bildung strategischer Gruppen (Fortsetzung)

Tab. 4: Umfassende Übersicht ausgewählter empirischer Studien zur Bildung strategischer Gruppen (Fortsetzung)

Tab. 5: Umfassende Übersicht ausgewählter empirischer Studien zur Bildung strategischer Gruppen (Fortsetzung)

Tab. 6: Umfassende Übersicht ausgewählter empirischer Studien zur Bildung strategischer Gruppen (Fortsetzung)

Tab. 7: Umfassende Übersicht ausgewählter empirischer Studien zur Bildung strategischer Gruppen (Fortsetzung) 


\begin{tabular}{|c|c|c|c|}
\hline Verfasser & $\begin{array}{c}\text { Untersuchungs- } \\
\text { objekt }\end{array}$ & $\begin{array}{l}\text { Gruppierungs- } \\
\text { variablen }\end{array}$ & Methodik \\
\hline $\begin{array}{l}\text { Hunt } \\
1972\end{array}$ & $\begin{array}{l}\text { Haushaltsgerăte- } \\
\text { industrie :ă }\end{array}$ & $\begin{array}{l}\text { - Grad der vertikalen } \\
\text { Integration } \\
\text { - Grad der Produkt- } \\
\text { diversifikation } \\
\text { - Grad der Produkt- } \\
\text { differenzierung }\end{array}$ & $\begin{array}{l}\text { - "Typologische" } \\
\text { Methode }\end{array}$ \\
\hline $\begin{array}{c}\text { Newmann } \\
1973\end{array}$ & $\begin{array}{l}\text { Chemische Indus- } \\
\text { trie (34 Branchen) * }\end{array}$ & $\begin{array}{l}\text { - Grad der vertikalen } \\
\text { Integration }\end{array}$ & $\begin{array}{l}\text { - "Typologische" } \\
\text { Methode }\end{array}$ \\
\hline $\begin{array}{l}\text { Porter } \\
1973\end{array}$ & $\begin{array}{l}\text { Konsumgüterin- } \\
\text { dustrie (38 } \\
\text { Branchen) * }\end{array}$ & $\begin{array}{l}\text { - relativer Branchen- } \\
\text { umsatz }\end{array}$ & $\begin{array}{l}\text { - "Typologische" } \\
\text { Methode }\end{array}$ \\
\hline $\begin{array}{c}\text { Hatten } \\
1974 \\
\text { Hatten/ } \\
\text { Schendel } \\
1977\end{array}$ & Brauereiindustrie $\mathbb{a}$ & $\begin{array}{l}\text { - Konzentrationsmaß } \\
\text { - Unternehmensgröße } \\
\text { - Anzahl der Produk- } \\
\text { tionsstătten } \\
\text { - Alter der Produk- } \\
\text { tionsstătten } \\
\text { - Kapitalintensitāt } \\
\text { der Produktions- } \\
\text { stătten } \\
\text { - Anzahl der Marken } \\
\text { - Preis } \\
\text { - Umsatz }\end{array}$ & - Clusteranalyse \\
\hline $\begin{array}{c}\text { Hatten/ } \\
\text { Schendel/ } \\
\text { Cooper } \\
1978\end{array}$ & Brauereiindustrie $\not{a}$ & $\begin{array}{l}\text { zusătzlich zu den } \\
\text { o.g. Variablen: } \\
\text { - EKJGK-Rendite } \\
\text { - Fusionsquote }\end{array}$ & - Clusteranalyse \\
\hline $\begin{array}{c}\text { Caves/Pugel } \\
1980\end{array}$ & $\begin{array}{l}\text { Herstellendes } \\
\text { Gewerbe } \not\end{array}$ & $\begin{array}{l}\text { - relativer Branchen- } \\
\text { umsatz }\end{array}$ & $\begin{array}{l}\text { - "Typologische" } \\
\text { Methode }\end{array}$ \\
\hline $\begin{array}{l}\text { Harrigan } \\
1980\end{array}$ & $\begin{array}{l}\text { Konsumgūterindus- } \\
\text { trie (7 Branchen)* }\end{array}$ & - Strategische Position & $\begin{array}{l}\text { - "Typologische" } \\
\text { Methode }\end{array}$ \\
\hline $\begin{array}{l}\text { Oster } \\
1980\end{array}$ & $\begin{array}{l}\text { Konsumgüterindus- } \\
\text { trie (19 Branchen) } \not\end{array}$ & $\begin{array}{l}\text { - Werbekosten/ } \\
\text { Umsatz }\end{array}$ & $\begin{array}{l}\text { - "Typologische" } \\
\text { Methode }\end{array}$ \\
\hline $\begin{array}{l}\text { Ramsler } \\
1982\end{array}$ & Banken $\bar{\alpha}$ & $\begin{array}{l}\text { - Produktdifferen- } \\
\text { zierung } \\
\text { - UnternehmensgrōBe } \\
\text { - Geographische } \\
\text { Marktabdeckung }\end{array}$ & - Clusteranalyse \\
\hline
\end{tabular}

Tab. 1: Umfassende Übersicht ausgewählter empirischer Studien zur Bildung strategischer Gruppen 


\begin{tabular}{|c|c|c|c|}
\hline Verfasser & $\begin{array}{c}\text { Untersuchungs- } \\
\text { objekt }\end{array}$ & $\begin{array}{l}\text { Gruppierungs- } \\
\text { variablen }\end{array}$ & Methodik \\
\hline $\begin{array}{c}\text { Baird/ } \\
\text { Sudharshan } \\
1983 \\
\text { Baird/ } \\
\text { Sudharshan/ } \\
\text { Thomas } 1988\end{array}$ & $\begin{array}{l}\text { Bürokommuni- } \\
\text { kationsindustrie } \not{\alpha}\end{array}$ & $\begin{array}{l}\text { - EK/GK-Rendite } \\
\text { - kurzfr. Liquidität } \\
\text { - Gewinn/GK } \\
\text { - Dividendenanteil } \\
\text { - Sollzinsen } \\
\text { - rel. Branchenumsatz }\end{array}$ & $\begin{array}{l}\text { - Faktoranalyse } \\
\text { - Clusteranalyse }\end{array}$ \\
\hline $\begin{array}{c}\text { Dess/Davis } \\
1984\end{array}$ & $\begin{array}{l}\text { Farbenproduzie- } \\
\text { rende Industrie a }\end{array}$ & $\begin{array}{l}21 \text { unternehmensin- } \\
\text { terne und -externe } \\
\text { Variablen u.a.: } \\
\text { - Produkt (Qualităt, } \\
\text { etc.) } \\
\text { - Preis (Niveau, etc.) } \\
\text { - Werbung } \\
\text { - Image (Marke, etc.) } \\
\text { - Innovationen (Pro- } \\
\text { zeBbereich, etc.) } \\
\text { - Service } \\
\text { - Personalqualităt } \\
\text { - Grad der Fremdfin- } \\
\text { anzierung } \\
\text { - Marktwachstum }\end{array}$ & $\begin{array}{l}\text { - Faktoranalyse } \\
\text { - Clusteranalyse }\end{array}$ \\
\hline $\begin{array}{l}\text { Drexel } \\
1984\end{array}$ & $\begin{array}{l}\text { Textil- und Möbel- } \\
\text { handel (mehrere } \\
\text { Branchen) * }\end{array}$ & $\begin{array}{l}\text { - Preisniveau } \\
\text { - Preisimage }\end{array}$ & $\begin{array}{l}\text { - "Typologische" } \\
\text { Methode }\end{array}$ \\
\hline $\begin{array}{c}\text { Hawes/ } \\
\text { Crittenden } \\
1984\end{array}$ & $\begin{array}{l}\text { Lebensmittel- } \\
\text { handel }\end{array}$ & $\begin{array}{l}18 \text { unternehemensin- } \\
\text { terne Variablen u.a.: } \\
\text { - Zielgruppe (Marken- } \\
\text { kăufe, etc. ) } \\
\text { - Produkt (Qualität, } \\
\text { etc.) } \\
\text { - Promotion (Preis- } \\
\text { promotion, etc.) } \\
\text { - Preis (Niveau, etc.) } \\
\text { - Display (Zweitplazie- } \\
\text { rung, etc.) } \\
\text { - Art der Produkt- } \\
\text { beschaffung (regel- } \\
\text { mäßig, etc.) }\end{array}$ & - Varianzanalyse \\
\hline
\end{tabular}

Tab. 2: Umfassende Übersicht ausgewählter empirischer Studien zur Bildung strategischer Gruppen (Fortsetzung) 


\begin{tabular}{|c|c|c|c|}
\hline Verfasser & $\begin{array}{l}\text { Untersuchungs- } \\
\text { objekt }\end{array}$ & $\begin{array}{l}\text { Gruppierungs- } \\
\text { variablen }\end{array}$ & Methodik \\
\hline $\begin{array}{l}\text { Fiegenbaum/ } \\
\text { Primeaux } \\
1985\end{array}$ & $\begin{array}{l}\text { Pharmazeutische } \\
\text { Industrie } \\
\text { Parfumindustrie } \\
\text { Computerin- } \\
\text { dustrie :a }\end{array}$ & $\begin{array}{l}\text { - Investitionen } \\
\text { - Inventar } \\
\text { - F\&E } \\
\text { - Werbung } \\
\text { - Umsatz } \\
\text { - Vermögen } \\
\text { - Grad der Fremdfinan- } \\
\text { zierung } \\
\text { - rel. Wettbewerbs- } \\
\text { stärke }\end{array}$ & - Clusteranalyse \\
\hline $\begin{array}{l}\text { Hatten/ } \\
\text { Hatten } \\
1985\end{array}$ & Brauereiindustrie $\not{\alpha}$ & $\begin{array}{l}\text { - Preis } \\
\text { - Werbung } \\
\text { - Anzahl der Marken } \\
\text { - rel. Marktanteil } \\
\end{array}$ & - Clusteranalyse \\
\hline $\begin{array}{l}\text { Harrigan } \\
1985\end{array}$ & $\begin{array}{l}\text { Einzelhandel (meh- } \\
\text { rere Branchen) }\end{array}$ & $\begin{array}{l}\text { - Umsatz/Angestellter } \\
\text { - Werbekosten/Umsatz } \\
\text { - Umschlag Inventar } \\
\text { - Durchschnittliches } \\
\text { Alter u. Inventar }\end{array}$ & - Clusteranalyse \\
\hline $\begin{array}{l}\text { Ohisen } \\
1985\end{array}$ & $\begin{array}{l}\text { Haushaltsgeräte- } \\
\text { industrie (mehrere } \\
\text { Branchen) }\end{array}$ & $\begin{array}{l}\text { Unternehmensinterne } \\
\text { u.-externe Variablen u.a.: } \\
\text { - Kapazitătsauslastung } \\
\text { - Lagerbestand } \\
\text { - Liquidităt } \\
\text { - Preiswettbewerb } \\
\text { - Markenbekanntheit } \\
\text { - Marktanteil } \\
\end{array}$ & - Clusteranalyse \\
\hline $\begin{array}{l}\text { Primeaux } \\
1985\end{array}$ & $\begin{array}{l}\text { Petrochemische } \\
\text { Industrie } \not \mathbb{~}\end{array}$ & $\begin{array}{l}\text { - rel. Branchenumsatz } \\
\text { - Nettoausgaben }\end{array}$ & $\begin{array}{l}\text { - "Typologische" } \\
\text { Methode }\end{array}$ \\
\hline $\begin{array}{l}\text { Cool/ } \\
\text { Schendel } \\
1987\end{array}$ & $\begin{array}{l}\text { Pharmazeutische } \\
\text { Industrie } \bar{\alpha}\end{array}$ & $\begin{array}{l}15 \text { unternehmensinterne } \\
\text { u.-externe Variablen u.a.: } \\
\text { - Bearbeitete Marktseg- } \\
\text { mente (Umsatz Apothe- } \\
\text { ke/Gesamtumsatz, etc.) } \\
\text { - Umfang Produktpalette } \\
\text { (Marktanteil Apothe- } \\
\text { kengeschât, etc.) } \\
\text { - Geographischer } \\
\text { - Marktanteil } \\
\text { - FeE (Kosten/Umsatz, } \\
\text { - etc) } \\
\text { Produkt (Innovations- } \\
\text { - guote) } \\
\text { - Gesamotion (Umsatz/ } \\
\text { - Gesamtumsatz, etc.) }\end{array}$ & - Clusteranalyse \\
\hline
\end{tabular}

Tab. 3: Umfassende Übersicht ausgewählter empirischer Studien zur Bildung strategischer Gruppen (Fortsetzung) 


\begin{tabular}{|c|c|c|c|}
\hline Verfasser & $\begin{array}{c}\text { Untersuchungs- } \\
\text { objekt }\end{array}$ & $\begin{array}{l}\text { Gruppierungs- } \\
\text { variablen }\end{array}$ & Methodik \\
\hline $\begin{array}{l}\text { Hergert } \\
1987\end{array}$ & $\begin{array}{l}\text { Herstellendes Ge- } \\
\text { werbe (50 Bran- } \\
\text { chen) La }\end{array}$ & $\begin{array}{l}\text { - Werbekosten/Umsatz } \\
\text { - F\&E-Kosten/Umsatz } \\
\text { - Umsatz/GK } \\
\text { - Umsatz der strat. } \\
\text { Geschäftseinheiten/ } \\
\text { Gesamtumsatz } \\
\text { - Marktanteil }\end{array}$ & - Clusteranalyse \\
\hline $\begin{array}{l}\text { Amel/ } \\
\text { Rhoades } \\
1988\end{array}$ & Banken $\mathfrak{a}$ & $\begin{array}{l}15 \text { unternehmensinterne } \\
\text { Variablen u.a.: } \\
\text { - Bankkredite } \\
\text { - Wohnungskredite } \\
\text { - Industriekredite } \\
\text { - Sichteinlagen }\end{array}$ & - Clusteranalyse \\
\hline $\begin{array}{c}\text { Heinemann } \\
1988\end{array}$ & $\begin{array}{l}\text { Textileinzel- } \\
\text { handel } \mathbb{a}\end{array}$ & $\begin{array}{l}26 \text { unternehmensinterne } \\
\text { Variablen u.a.: } \\
\text { - Warengruppe (Herren- } \\
\text { artikel, etc.) } \\
\text { - Kundengruppe(Herren } \\
\text { KGR1, etc.) } \\
\text { - Handelsfunktion (Be- } \\
\text { dienung nach Wunsch, } \\
\text { etc.) }\end{array}$ & - Clusteranalyse \\
\hline $\begin{array}{l}\text { Miller } \\
1988\end{array}$ & $\begin{array}{l}\text { Konsumgüterin- } \\
\text { dustrie } \\
\text { Investitionsgüter- } \\
\text { industrie } \\
\text { Dienstleistungs- } \\
\text { bereich }\end{array}$ & $\begin{array}{l}\text { - Innovationsquote } \\
\text { - Hăufigkeit der Pro- } \\
\text { duktmodifikationen } \\
\text { - Produktentwicklungs- } \\
\text { zeit } \\
\text { - Grad der Eigenent- } \\
\text { wicklung } \\
\text { - rel. Umsatz der Wett- } \\
\text { bewerbsinnovationen }\end{array}$ & - Clusteranalyse \\
\hline $\begin{array}{l}\text { Lawless/ } \\
\text { Finch } \\
1989\end{array}$ & $\begin{array}{l}\text { Herstellendes } \\
\text { Gewerbe (52 } \\
\text { Branchen) * }\end{array}$ & $\begin{array}{l}\text { - Werbekosten/Umsatz } \\
\text { - F\&E-Kosten/Umsatz } \\
\text { - Produktkosten/Umsatz } \\
\text { - Forderungen/Umsatz } \\
\text { - Inventar/Umsatz }\end{array}$ & - Clusteranalyse \\
\hline $\begin{array}{c}\text { Mascarenkas/ } \\
\text { Aaker } \\
1989\end{array}$ & Ölförderindustrie & $\begin{array}{l}\text { - Grad der Internationa- } \\
\text { lităt } \\
\text { - durchschnittliche } \\
\text { Tiefe der Bohrungen } \\
\text { - Anteil Tiefseeförderung }\end{array}$ & - Clusteranalyse \\
\hline
\end{tabular}

Tab. 4: Umfassende Übersicht ausgewählter empirischer Studien zur Bildung strategischer Gruppen (Fortsetzung) 


\begin{tabular}{|c|c|c|c|}
\hline Verfasser & $\begin{array}{l}\text { Untersuchungs- } \\
\text { objekt }\end{array}$ & $\begin{array}{c}\text { Gruppierungs- } \\
\text { variablen }\end{array}$ & Methodik \\
\hline $\begin{array}{l}\text { Mc Name日/ } \\
\text { Mc Hugh } \\
1989\end{array}$ & $\begin{array}{l}\text { Bekleidungs- } \\
\text { industrie } \square\end{array}$ & $\begin{array}{l}\text { - Preis } \\
\text { - Produktdifferenzierung } \\
\text { - Geographische Markt- } \\
\text { abdeckung } \\
\text { - Zielgruppe } \\
\text { - Eigentumsverhältnisse } \\
\text { - Unternehmensgröße }\end{array}$ & $\begin{array}{l}\text { - "Typologische" } \\
\text { Methode }\end{array}$ \\
\hline $\begin{array}{c}\text { Minderlein } \\
1989\end{array}$ & PC-Industrie $\not$ & $\begin{array}{l}\text { - Grad der Marken- } \\
\text { geltung } \\
\text { Wahl des Vertriebs- } \\
\text { weges }\end{array}$ & $\begin{array}{l}\text { - "Typologische" } \\
\text { Methode }\end{array}$ \\
\hline $\begin{array}{c}\text { Pegels/ } \\
\text { Sekar } 1989\end{array}$ & Krankenhäuser $\not{a}$ & $\begin{array}{l}\text { 16 Unternehmensinterne } \\
\text { Variablen u.a.: } \\
\text { - Unterbringungskosten } \\
\text { - Qualităt der Verpfle- } \\
\text { gung } \\
\text { - Qualităt der Betreuung } \\
\text { - Schnelligkeit Behand- } \\
\text { lung } \\
\text { - Standort }\end{array}$ & - MDS \\
\hline $\begin{array}{c}\text { Baubin } \\
1990\end{array}$ & $\begin{array}{l}\text { Informations- } \\
\text { technische In- } \\
\text { dustrie } \\
\text { (mehrere Bran- } \\
\text { chen) }\end{array}$ & $\begin{array}{l}\text { - Anwendungsorientie- } \\
\text { rung der Leistungen } \\
\text { - Technologiespeziali- } \\
\text { sierung der Leistungen }\end{array}$ & $\begin{array}{l}\text { - "Typologische" } \\
\text { Methode }\end{array}$ \\
\hline $\begin{array}{c}\text { Conant/ } \\
\text { Mokwa/ } \\
\text { Varadarajan } \\
1990\end{array}$ & Krankenhăuser $\not a$ & $\begin{array}{l}20 \text { unternehmensinter- } \\
\text { ne und -externe Varia- } \\
\text { blen u.a.: } \\
\text { - Kenntnisse der Markt- } \\
\text { teilnehmer (Wett- } \\
\text { bewerber etc.) } \\
\text { - Service (bifferen- } \\
\text { zierung, etc.) } \\
\text { - Kommunikation } \\
\text { (Effizienz der Public } \\
\text { Relations, etc.) } \\
\text { - Image } \\
\text { - Standort } \\
\text { - ROI }\end{array}$ & - Varianzanalyse \\
\hline
\end{tabular}

Tab. 5: Umfassende Übersicht ausgewählter empirischer Studien zur Bildung strategischer Gruppen (Fortsetzung) 


\begin{tabular}{|c|c|c|c|}
\hline Verfasser & $\begin{array}{l}\text { Untersuchungs- } \\
\text { objekt }\end{array}$ & $\begin{array}{l}\text { Gruppierungs- } \\
\text { variablen }\end{array}$ & Methodik \\
\hline $\begin{array}{l}\text { Fiegenbaum/ } \\
\text { Thomas } 1990\end{array}$ & $\begin{array}{l}\text { Versicherungs- } \\
\text { industrie } \mathbf{a}\end{array}$ & $\begin{array}{l}\text { - Privat- vs. Geschăfts- } \\
\text { versicherungen } \\
\text { - Haus- /Haftpflicht- vs. } \\
\text { Lebensversicherungen } \\
\text { - Diversifikation } \\
\text { - UnternehmensgröBe } \\
\text { - Kosten/Erlőse } \\
\text { - Anteil Rückversicher- } \\
\text { ungen } \\
\text { - Erlöse/Gewinn } \\
\text { - Aktien vs. Obligations- } \\
\text { anlage }\end{array}$ & - Clusteranalyse \\
\hline $\begin{array}{l}\text { Lewis/ } \\
\text { Thomas } 1990 \\
\text { Carroll/Lewis/ } \\
\text { Thomas } 1992\end{array}$ & $\begin{array}{l}\text { Lebensmittel- } \\
\text { handel } \not a\end{array}$ & $\begin{array}{l}\text { - Anzahl der Geschäfte } \\
\text { - Grőße der Geschăfte } \\
\text { - Werbung/Umsatz } \\
\text { - Anzahl Marken } \\
\text { - Anteil Handelsmarken } \\
\text { - rel. Umsatzgroßßen }\end{array}$ & - Clusteranalyse \\
\hline Kumar 1990 & $\begin{array}{l}\text { Herstellendes } \\
\text { Gewerbe } \\
\text { (43 Branchen) }\end{array}$ & $\begin{array}{l}\text { nationale vs. } \\
\text { multinationale } \\
\text { Strategie }\end{array}$ & $\begin{array}{l}\text { - "Typologische" } \\
\text { Methode }\end{array}$ \\
\hline Boeker 1991 & $\begin{array}{l}\text { Brauerei- } \\
\text { industrie } \not{x}\end{array}$ & $\begin{array}{l}\text { - Konzentrationsmaß } \\
\text { - Anzahl der Anbieter } \\
\text { - Absatzmenge } \\
\text { - Preisbestimmungen } \\
\text { - Steuern }\end{array}$ & $\begin{array}{l}\text { - "Typologische" } \\
\text { Methode }\end{array}$ \\
\hline $\begin{array}{c}\text { Gaitanides/ } \\
\text { Westphal } \\
1991\end{array}$ & $\begin{array}{l}\text { Automobilzu- } \\
\text { lieferindustrie } \mathbf{a}\end{array}$ & $\begin{array}{l}\text { - Kostenorientierung } \\
\text { - Differenzierung } \\
\text { (u.a. Qualităt//mage) } \\
\text { - Preis } \\
\text { - Produkt-/ProzeB- } \\
\text { innovation }\end{array}$ & - Clusteranalyse \\
\hline $\begin{array}{c}\text { Homburg } \\
1991\end{array}$ & $\begin{array}{l}\text { Maschinenbau- } \\
\text { industrie } \not a\end{array}$ & $\begin{array}{l}\text { - Breite Produkt- } \\
\text { palette } \\
\text { - Fertigungstiefe }\end{array}$ & $\begin{array}{l}\text { - "Typologische" } \\
\text { Methode }\end{array}$ \\
\hline $\begin{array}{l}\text { Brunken } \\
1993\end{array}$ & Banken $\mathbf{a}$ & $\begin{array}{l}\text { unternehmensinterne und } \\
\text { - externe Variablen u.a.: } \\
\text { - Geschăftsfeldstrategie } \\
\text { - Geographische Markt- } \\
\text { abdeckung } \\
\text { - Produktdifterenzierung } \\
\text { - Standort } \\
\text { - Ertrag }\end{array}$ & - Clusteranalyse \\
\hline
\end{tabular}

Tab. 6: Umfassende Übersicht ausgewählter empirischer Studien zur Bildung strategischer Gruppen (Fortsetzung) 


\begin{tabular}{|c|c|c|c|}
\hline Verfasser & $\begin{array}{c}\text { Untersuchungs- } \\
\text { objekt }\end{array}$ & $\begin{array}{l}\text { Gruppierungs- } \\
\text { variablen }\end{array}$ & Methodik \\
\hline Hannig 1993 & $\begin{array}{l}\text { Computer- } \\
\text { industrie } \not a\end{array}$ & $\begin{array}{l}\text { - Preis-/Leistungs- } \\
\text { verhältnis } \\
\text { - Produktqualităt } \\
\text { - Kompatibilität } \\
\text { - System-/Einsatz- } \\
\text { - beratung } \\
\text { - Nutzenunterstützung } \\
\text { - Zukunftssicherheit }\end{array}$ & $\begin{array}{l}\text { - Clusteranalyse } \\
\text { - MDS }\end{array}$ \\
\hline Hannig 1993 & $\begin{array}{l}\text { Software- } \\
\text { industrie } a\end{array}$ & $\begin{array}{l}\text { - Preis-/Leistungs- } \\
\text { verhältnis } \\
\text { - Produktqualităt } \\
\text { - Informationspolitik } \\
\text { - Hotline } \\
\text { - Bedienungsfreund- } \\
\text { lichkeit } \\
\text { - Zukunftssicherheit }\end{array}$ & $\begin{array}{l}\text { - Clusteranalyse } \\
\text { - MDS }\end{array}$ \\
\hline $\begin{array}{l}\text { Miles/Snow/ } \\
\text { Sharfmann } \\
1993\end{array}$ & $\begin{array}{l}\text { High-Tech- } \\
\text { Industrie } \\
\text { Investitionsgüter- } \\
\text { industrie } \\
\text { Konsumgüter- } \\
\text { industrie } \\
\text { (12 Branchen) } x\end{array}$ & $\begin{array}{l}\text { - Werbekosten/Umsatz } \\
\text { - F\&E-Kosten/Umsatz } \\
\text { - Kapitalintensităt }\end{array}$ & - Clusteranalyse \\
\hline $\begin{array}{l}\text { Reger/ } \\
\text { Huff } 1993\end{array}$ & Banken & $\begin{array}{l}23 \text { unternehmens- } \\
\text { interne Variablen u.a.: } \\
\text { - Geographische } \\
\text { Marktabdeckung } \\
\text { - Geschaftsfeldstrategie } \\
\text { - Standort } \\
\text { - Managementqualität } \\
\text { - Ertrag }\end{array}$ & - Clusteranalyse \\
\hline Meffert 1994 & $\begin{array}{l}\text { Computer- } \\
\text { industrie } \not \alpha\end{array}$ & $\begin{array}{l}\text { - Geschăftstyp } \\
\text { - Geschäftsaktivität } \\
\text { - Produkttyp }\end{array}$ & - "Typologische" \\
\hline
\end{tabular}

* = branchenübergreifende Bildung strategischer Gruppen (i.w.S.)

$\not \alpha$ = branchenbezogene Bildung strategischer Gruppen (i.e.S.)

Tab. 7: Umfassende Übersicht ausgewählter empirischer Studien zur Bildung strategischer Gruppen (Fortsetzung) 
Nikolaus Müller and Universität Münster - 978-3-631-75075-9 Downloaded from PubFactory at 01/11/2019 08:36:03AM via free access 
A NHANG II

Tabellen der empirischen Untersuchungen 


\section{Tabellenverzeichnis des Anhangs II}

Tab. 1: Codierungsplan für die Variablen der empirischen Untersuchung

Tab. 2: Prüfgrößen für alle Strategiedimensionen auf der Grundlage des Kaiser-Meyer-Olkin-Kriteriums

Tab. 3: Prüfgrößen für die Strategiedimensionen mit Ausnahme der Variable früher Folger auf der Grundlage des Kaiser-Meyer-Olkin-Kriteriums

Tab. 4: Ergebnisse der faktoranalytischen Verdichtung der Strategievariablen

Tab. 5: Ergebnisse des Stabilitätstests für die verdichteten Strategievariablen

Tab. 6: Mittelwertabweichungen der Situationscluster in den High-TechMärkten

Tab. 7: Ergebnisse der diskriminatorischen Klassifikation anhand der Situationsvariablen

Tab. 8: Standardisierte Diskriminanzfunktionen für die Situationscluster auf Basis der Situationsvariablen

Tab. 9: Ergebnisse der Mittelwertvergleichstests für die Situationscluster auf Basis der Situationsvariablen

Tab. 10: Mittelwertabweichungen der Strategiedimensionen der strategischen High-Tech-Gruppen in den Situationen A, B und C

Tab. 11: Ergebnisse der diskriminatorischen Klassifikation anhand der Strategievariablen in Situation A

Tab. 12: Standardisierte Diskriminanzfunktionen für die Cluster der strategischen High-Tech-Gruppen in Situation A auf Basis der Strategievariablen

Tab. 13: Ergebnisse der diskriminatorischen Klassifikation anhand der Strategievariablen in Situation B

Tab. 14: Standardisierte Diskriminanzfunktionen für die Cluster der strategischen High-Tech-Gruppen in Situation B auf Basis der Strategievariablen 
Tab. 15: Ergebnisse der diskriminatorischen Klassifikation anhand der Strategievariablen in Situation C

Tab. 16: Standardisierte Diskriminanzfunktionen für die Cluster der strategischen High-Tech-Gruppen in Situation C auf Basis der Strategievariablen

Tab. 17: Ergebnisse der Mittelwertvergleichstests für die Cluster der strategischen High-Tech-Gruppen in Situation A auf Basis der Strategievariablen

Tab. 18: Ergebnisse der Mittelwertvergleichstests für die Cluster der strategischen High-Tech-Gruppen in Situation B auf Basis der Strategievariablen

Tab. 19: Ergebnisse der Mittelwertvergleichstests für die Cluster der strategischen High-Tech-Gruppen in Situation C auf Basis der Strategievariablen

Tab. 20: Mittelwertabweichungen der Marketinginstrumente bei den strategischen High-Tech-Gruppen in den Situationen A, B und C

Tab. 21: Mittelwertabweichungen der Instrumente zur Verkürzung der Entwicklungszeiten und Beschleunigung der Vermarktung bei den strategischen High-Tech-Gruppen in Situationen A, B und C

Tab. 22: Mittelwertabweichungen der Unternehmensziele bei den strategischen High-Tech-Gruppen in den Situationen A, B und C

Tab. 23: Ergebnis der konfirmatorischen Faktorenanalyse zu kulturellen Grundorientierungen in High-Tech-Märkten

Tab. 24: Mittelwertabweichungen der Kulturdimensionen bei den strategischen High-Tech-Gruppen in den Situationen A, B und C

Tab. 25: Durchschnittliche Umsätze der strategischen Gruppen innerhalb der Umfeldsituationen in den Jahren 1988-1990

Tab. 26: Ergebnisse der Mittelwertvergleichstests für die absoluten Erfolgsmerkmale der strategischen High-Tech-Gruppen 
Kontextvariablen

Preiswettbewerb

FM 1 A

Technologiewettbewerb

FM 1 B

Preisorientierung

FM 1 C

Fehlender Industriestandard

FM 1 D

Qualitätswettbewerbs (Tech.-dynamik)

FM $1 \mathrm{E}$

Unausgeschöpftes Marktpotential

FM $1 \mathrm{~F}$

Geringe Marktbarrieren

FM 1 G

Verkürzung PLZ

Verlängerung der Entwicklungszeiten

FM $1 \mathrm{H}$

Kaufwiderstand

FM 1 I

Preiserosion

FM $1 \mathrm{~J}$

FM $1 \mathrm{~K}$

Preissenkung (Beschaffungseinsparung)

FM $1 \mathrm{~L}$

Leichte Handhabung

FM 2 A

Schulungsbereitschaft

FM 2 B

Anlernzeit

FM 2 C

Technologie Know-how

FM 2 D

Nutzenerwartungen

FM 2 E

Komplexität der Anwendungen

FM 2 F

Stand. Technologie

FM 14 A

Stand. Produktkem

FM 14 B

Stand. Service

Stand. Benutzeranwendungen

FM 14 C

FM 14 D

Einfluß Tech.-stand. auf F\&E

FM $15 \mathrm{~A}$

EinfluB Tech.-stand. auf Produktdesign

FM 15 B

Einflu $\beta$ Tech.-stand. auf Beschaffung

FM 15 C

Einfluß Tech.-stand. auf Produktion

FM 15 D

FM $15 \mathrm{E}$

EinfluB Tech.-stand. auf Techn. KD

FM $15 \mathrm{~F}$

Einfluß Tech.-stand. auf Marketing

FM 15 G

Strategievariablen

Marktpenetration

FM 8 A

Marktentwicklung

FM 8 B

Produktentwicklung

FM $8 \mathrm{C}$

Diversifikation

FM 8 D

Technologie-Führerschaft

FM 9 A

Preis-/Kosten-Führerschaft

FM 9 B

Qualitäts-Führerschaft

FM 9 C

FM 9 D

Service-Führerschaft

FM $9 \mathrm{E}$

FM $9 \mathrm{~F}$

Nutzen-Führerschaft

FM $11 \mathrm{~A}$

Pionier

FM 11 B

Früher Folger

FM $11 \mathrm{C}$

Tab. 1: Codierungsplan für die Variablen der empirischen Untersuchung 


\begin{tabular}{|c|c|c|c|c|c|c|c|}
\hline \multicolumn{8}{|c|}{ Anti-image correlation Matrix: } \\
\hline & FM11A & FM11B & FM11C & FM8A & FM8B & FM8C & FM8D \\
\hline \multirow[t]{2}{*}{$\begin{array}{l}\text { FM11A } \\
\text { FM11B } \\
\text { FM11C } \\
\text { FM8A } \\
\text { FM8B } \\
\text { FM8C } \\
\text { FM8D } \\
\text { FM9A } \\
\text { FM9B } \\
\text { FM9C } \\
\text { FM9D } \\
\text { FM9E } \\
\text { FM9F }\end{array}$} & $\begin{array}{r}, 75772 \\
, 21152 \\
, 31593 \\
, 02684 \\
-, 08346 \\
-, 12125 \\
-, 03161 \\
-, 27881 \\
-, 02444 \\
-, 07628 \\
-, 01257 \\
-, 17098 \\
-, 05599\end{array}$ & $\begin{array}{r}.31444 \\
, 08970 \\
.07220 \\
-, 04185 \\
-, 00984 \\
.06925 \\
.00191 \\
-.02378 \\
-.11584 \\
-.01577 \\
-.05140 \\
-.01952\end{array}$ & $\begin{array}{r}, 72908 \\
-, 10413 \\
, 08616 \\
, 00904 \\
, 19776 \\
, 10958 \\
-, 07834 \\
, 11043 \\
-, 01863 \\
-, 08603 \\
, 06816\end{array}$ & $\begin{array}{r}.61477 \\
-, 17105 \\
, 03977 \\
-14260 \\
-, 01759 \\
-, 14842 \\
-, 13541 \\
-, 00525 \\
-, 09051 \\
-, 06514\end{array}$ & $\begin{array}{r}, 68465 \\
, 06810 \\
-, 16097 \\
, 01359 \\
-, 06675 \\
-, 07841 \\
-14003 \\
-, 04447 \\
-, 04791\end{array}$ & $\begin{array}{r}, 80599 \\
-, 14725 \\
-, 12085 \\
-, 04757 \\
-, 14065 \\
.05042 \\
.00777 \\
-, 04743\end{array}$ & $\begin{array}{r}, 61196 \\
, 07694 \\
-, 05743 \\
-, 00074 \\
-, 02723 \\
-, 11696 \\
, 01292\end{array}$ \\
\hline & FM9A & FM9B & FM9C & FM9D & FM9E & FM9F & \\
\hline $\begin{array}{l}\text { FM9A } \\
\text { FM9B } \\
\text { FM9C } \\
\text { FM9D } \\
\text { FM9E } \\
\text { FM9F }\end{array}$ & $\begin{array}{r}, 80980 \\
-, 15149 \\
-, 16449 \\
, 02939 \\
-, 03903 \\
-, 05962\end{array}$ & $\begin{array}{r}, 65851 \\
-, 04201 \\
-, 05056 \\
-, 0276 \\
-, 03135\end{array}$ & $\begin{array}{r}, 82118 \\
-, 19272 \\
-, 19978 \\
-, 07756\end{array}$ & $\begin{array}{r}, 72246 \\
-, 19612 \\
-, 14204\end{array}$ & $\begin{array}{r}, 77494 \\
-, 18240\end{array}$ &, 86447 & \\
\hline \multicolumn{8}{|c|}{$\begin{array}{l}\text { Measures of Sampling Adequacy (MSA) are printed on the diagonal. } \\
\text { Kaiser-Meyer-Olkin Measure of Sampling Adequacy }=, 75457\end{array}$} \\
\hline
\end{tabular}

Tab. 2: Prüfgrößen für alle Strategiedimensionen auf der Grundlage des Kaiser-Meyer-Olkin-Kriteriums

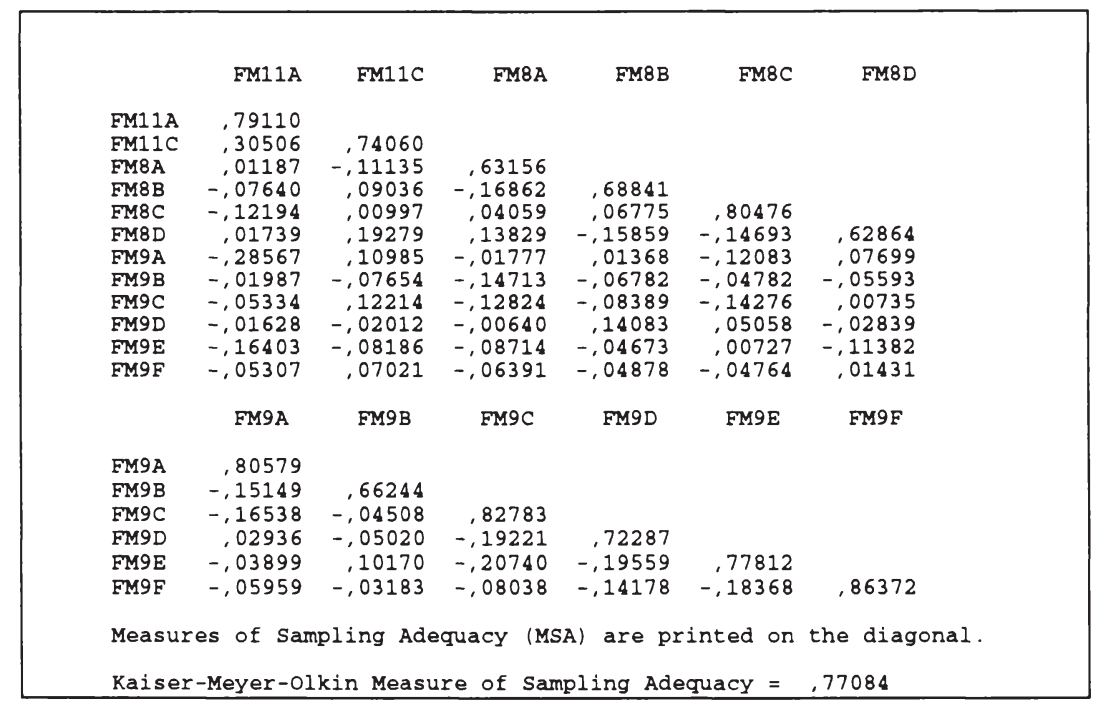

Tab. 3: Prüfgrößen für die Strategiedimensionen mit Ausnahme der Variable früher Folger auf der Grundlage des Kaiser-Meyer-Olkin-Kriteriums 


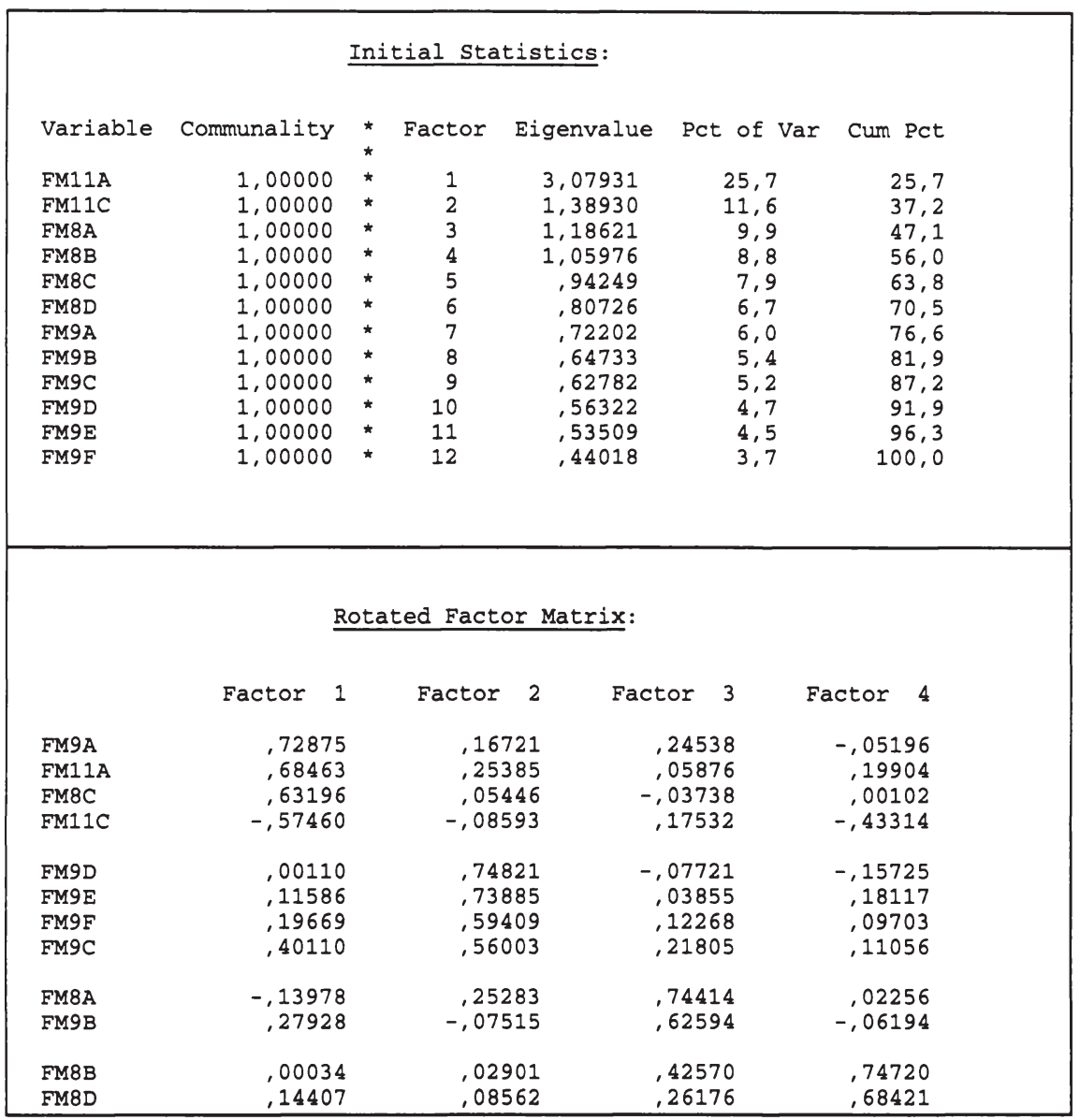

Tab. 4: Ergebnisse der faktoranalytischen Verdichtung der Strategievariablen 


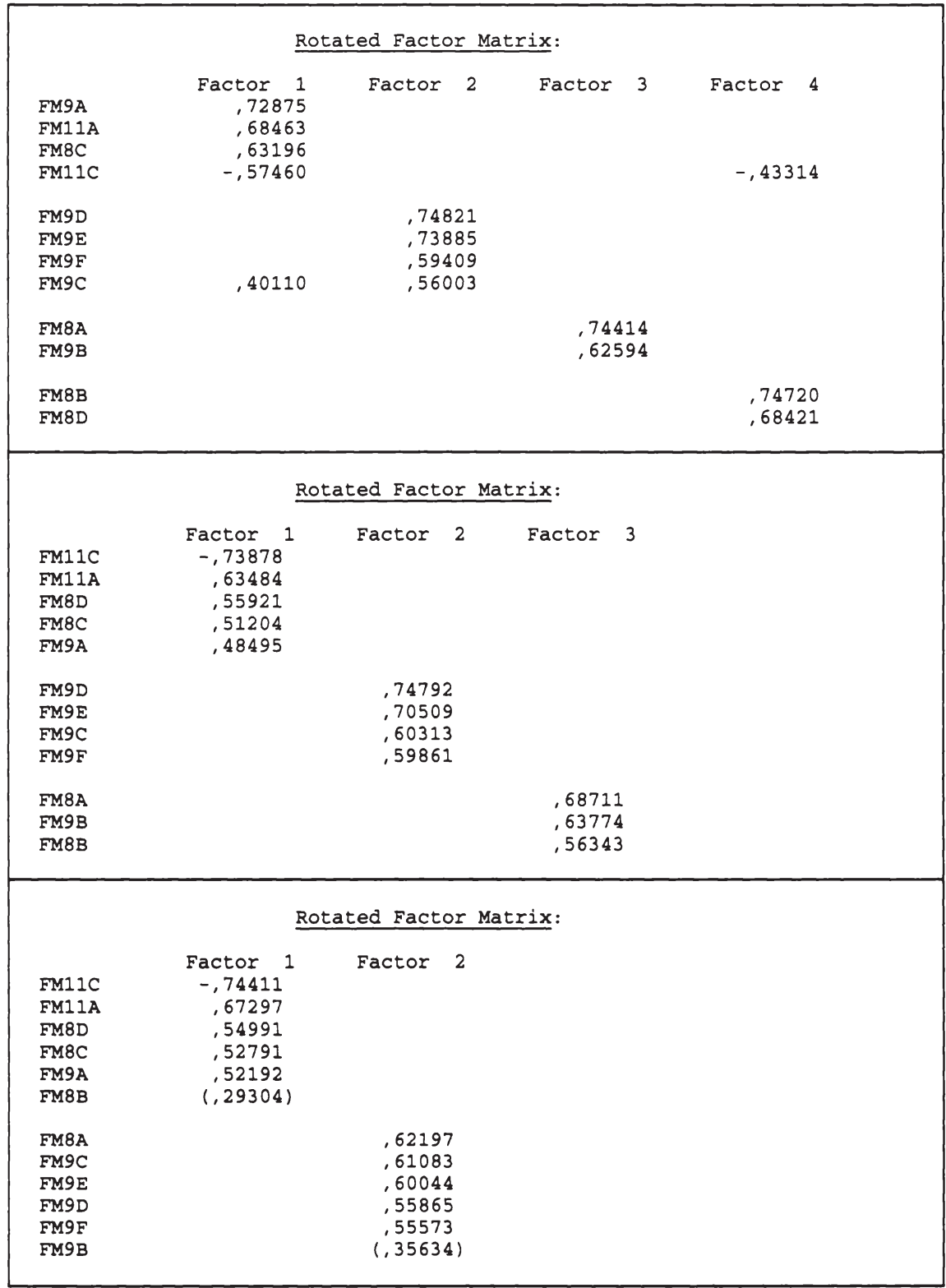

Tab. 5: Ergebnisse des Stabilitätstests für die verdichteten Strategievariablen 


\begin{tabular}{|c|c|c|c|c|c|c|c|}
\hline \multirow[t]{2}{*}{$\begin{array}{l}\text { Umfeld- } \\
\text { situation }\end{array}$} & \multirow{2}{*}{\multicolumn{3}{|c|}{ Situationsvariablen }} & \multicolumn{4}{|c|}{$\begin{array}{l}\text { Ausprāgung der Situations- } \\
\text { variablen im Vergleich zum } \\
\text { Durchschnitt }\end{array}$} \\
\hline & & & & $\varnothing$ & Sit. A & Sit. B & Sit. C \\
\hline \multirow{7}{*}{$\begin{array}{l}\text { Konkurrenz- } \\
\text { situation }\end{array}$} & \multicolumn{3}{|c|}{ Preiswettbewerb } & 2,02 & $+0,58$ & $-0,12$ & $-0,38$ \\
\hline & \multicolumn{3}{|c|}{ Preisorientierung } & 2,19 & $+0,19$ & $-0,04$ & $-0,10$ \\
\hline & \multicolumn{3}{|c|}{ Preissenkung (Beschaffungseinsp.) } & 2,64 & $+0,28$ & $-0,07$ & $-0,12$ \\
\hline & \multicolumn{3}{|c|}{ Preiserosion } & 2,64 & $+0,63$ & $-0,20$ & $-0,26$ \\
\hline & \multicolumn{3}{|c|}{ Technologiewettbewerb } & 1,97 & $+0,25$ & $-0,25$ & $+0,14$ \\
\hline & \multicolumn{3}{|c|}{$\begin{array}{l}\text { Qualitātswettbewerb } \\
\text { (Technologiedynamik) }\end{array}$} & 1,98 & $+0,10$ & $-0,25$ & $+0,26$ \\
\hline & \multicolumn{3}{|c|}{ Verkūrzte PLZ } & 1,98 & $+0,30$ & $-0,37$ & $+0,28$ \\
\hline \multirow{5}{*}{$\begin{array}{l}\text { Allgemeine } \\
\text { Markt- } \\
\text { situation }\end{array}$} & \multicolumn{3}{|c|}{ Unausgenutztes Marktpotential } & 2,40 & $-0,50$ & $-0,25$ & $+0,85$ \\
\hline & \multicolumn{3}{|c|}{ Fehlender Industriestandard } & 3,00 & $-0,50$ & $-0,22$ & $+0,79$ \\
\hline & \multicolumn{3}{|c|}{ Geringe Marktbarrieren } & 3,91 & $+0,08$ & $-0,08$ & $+0,05$ \\
\hline & \multicolumn{3}{|c|}{ Verlångerte Entwicklungszeiten } & 3,08 & $-0,33$ & $+0,18$ & $+0,04$ \\
\hline & \multicolumn{3}{|c|}{ Kaufwiderstand } & 3,40 & $-0,10$ & $-0,19$ & $+0,35$ \\
\hline \multirow{6}{*}{$\begin{array}{l}\text { Abnehmer- } \\
\text { situation }\end{array}$} & \multicolumn{3}{|c|}{ Technologie Know-how } & 2,37 & $+0,02$ & $-0,15$ & $+0,19$ \\
\hline & \multicolumn{3}{|c|}{ Anlernzeit } & 2,66 & $-0,05$ & $-0,08$ & $+0,18$ \\
\hline & \multicolumn{3}{|c|}{ Schulungsbereitschaft } & 2,52 & $-0,18$ & $-0,16$ & $+0,40$ \\
\hline & \multicolumn{3}{|c|}{ Nutzenerwartung } & 1,84 & $+0,07$ & $-0,16$ & $+0,16$ \\
\hline & \multicolumn{3}{|c|}{ Komplexităt d. Anwendungen } & 1,81 & $+0,11$ & $-0,25$ & $+0,25$ \\
\hline & \multicolumn{3}{|c|}{ Leichte Handhabung } & 1,66 & $+0,14$ & $-0,18$ & $+0,15$ \\
\hline & \multicolumn{3}{|c|}{ Stand. Technologie } & 2,33 & $+0,51$ & $-0,36$ & $+0,03$ \\
\hline & \multicolumn{3}{|c|}{ " Produktkern } & 2,20 & $+0,17$ & $-0,27$ & $+0,24$ \\
\hline & \multicolumn{3}{|c|}{ " Benutzeranwendungen } & 2,85 & $+0,20$ & $-0,41$ & $+0,42$ \\
\hline & \multicolumn{3}{|c|}{ " Service } & 3,28 & $+0,14$ & $-0,06$ & $-0,06$ \\
\hline & \multicolumn{3}{|c|}{ EinfluB Techn.-stand. auf Marketing } & 2,72 & $+0,53$ & $-0,47$ & $+0,21$ \\
\hline & $\cdot$ & $"$ & F\&E & 2,19 & $+0,72$ & $-0,57$ & $+0,15$ \\
\hline Techn & $\cdot$ & $"$ & Produktdesign & 3,00 & $+0,62$ & $-0,39$ & $-0,01$ \\
\hline & 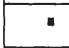 & $"$ & Techn. KD & 2,79 & $-0,26$ & $-0,38$ & $-0,14$ \\
\hline & $\cdot$ & $"$ & Beschaffung & 2,63 & $+0,26$ & $-0,17$ & $+0,02$ \\
\hline & $\cdot$ & $"$ & Produktion & 2,29 & $+0,37$ & $-0,10$ & $-0,25$ \\
\hline & " & $"$ & Logistik & 2,95 & $+0,49$ & $-0,20$ & $-0,17$ \\
\hline Anzahl der Un & erneh & & & 384 & 106 & 165 & 113 \\
\hline
\end{tabular}

Tab. 6: Mittelwertabweichung der Situationscluster in den High-Tech-Märkten 


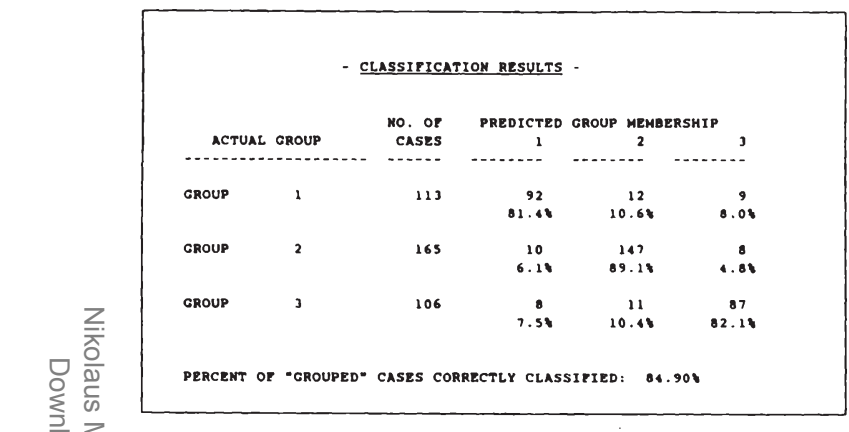

Tab. 7: Ergebnisse der diskriminatorischen Klassifikation anhand der Situationsvariablen

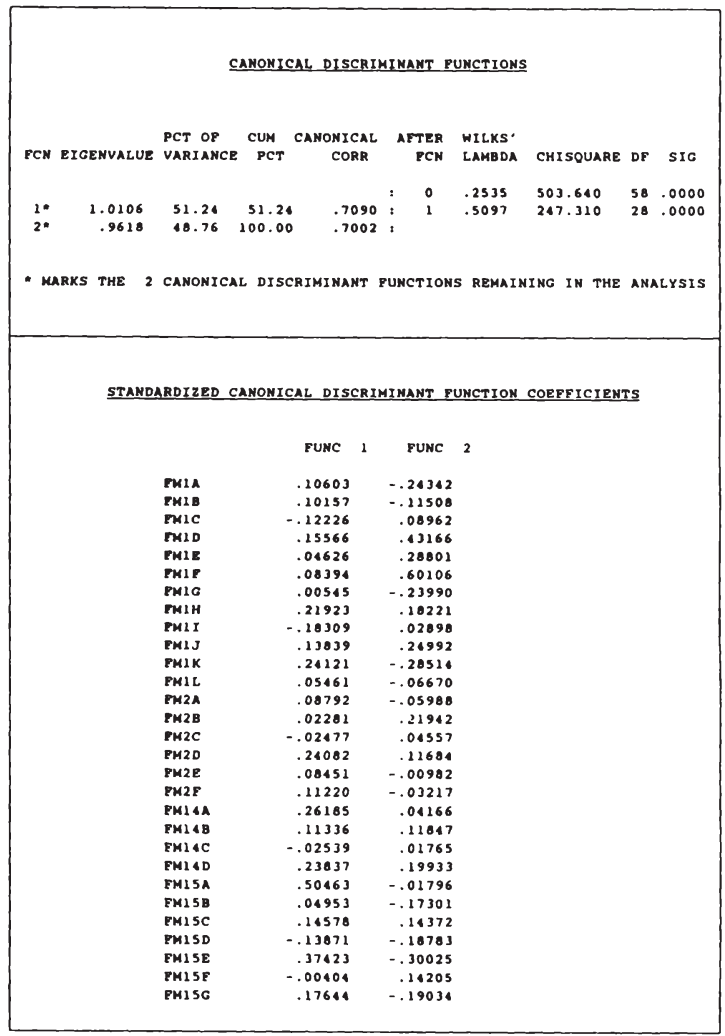

Tab. 8: Standardisierte Diskriminanzunktionen für die Situationscluster auf Basis der Situationsvariablen 

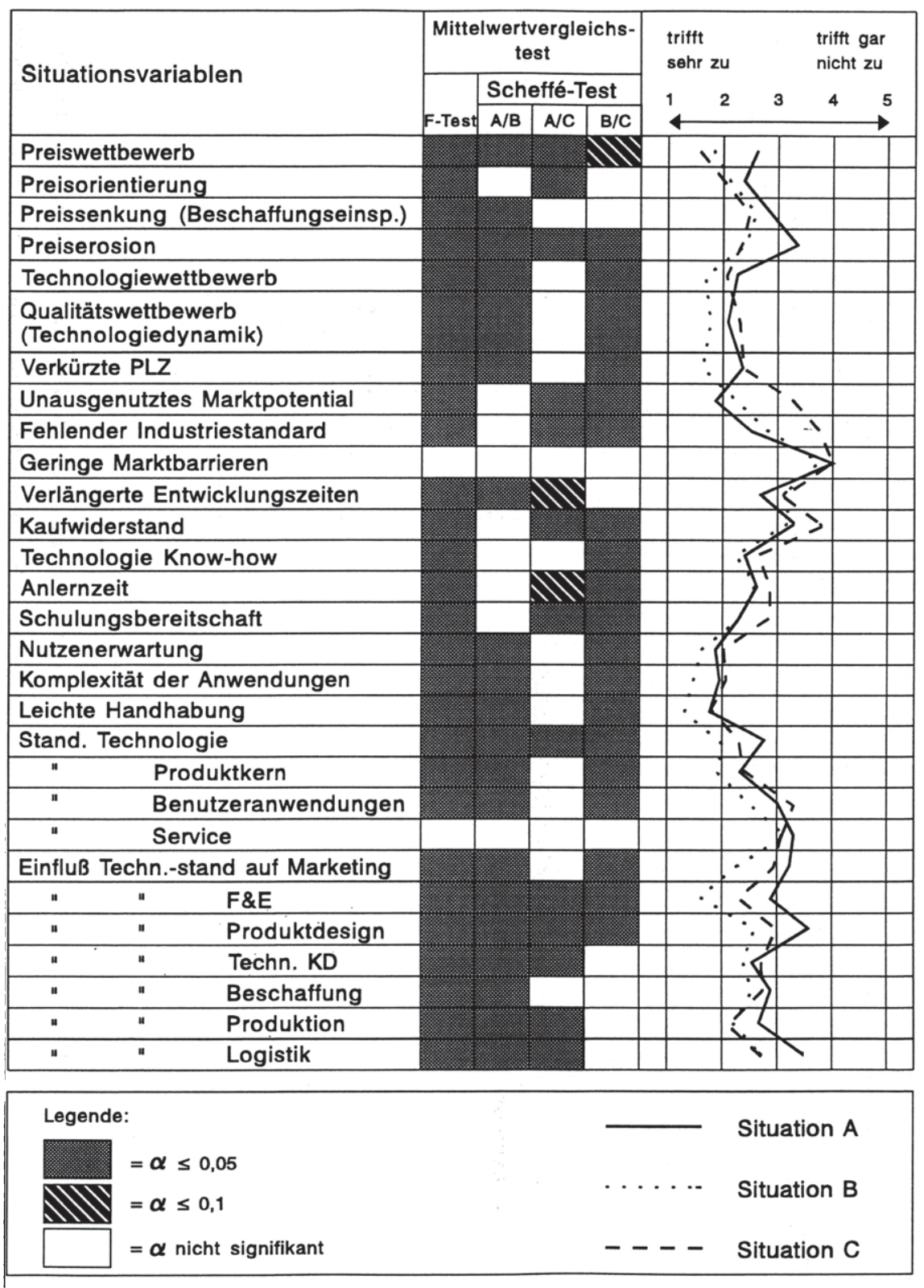

Tab. 9: Ergebnisse der Mittelwertvergleichstests für die Situationscluster auf Basis der Situationsvariablen 


\begin{tabular}{|c|c|c|c|c|c|c|c|c|c|c|c|c|}
\hline \multirow[b]{2}{*}{$\begin{array}{l}\text { Marketinginstrument- } \\
\text { variablen }\end{array}$} & \multicolumn{4}{|c|}{ Situation A } & \multicolumn{4}{|c|}{ Situation B } & \multicolumn{4}{|c|}{ Situation C } \\
\hline & $\boldsymbol{\varnothing}$ & $\begin{array}{l}\text { Diflerenzie- } \\
\text { rungsorient. }\end{array}$ & $\begin{array}{c}\text { Kostenorien- } \\
\text { tierte }\end{array}$ & $\begin{array}{c}\text { Innovations- } \\
\text { orientierte }\end{array}$ & $\varnothing$ & $\begin{array}{l}\text { Diflerenzie- } \\
\text { rungsorient. }\end{array}$ & $\begin{array}{c}\text { Kostenorien- } \\
\text { tierte }\end{array}$ & $\begin{array}{l}\text { Innovations- } \\
\text { orientierte }\end{array}$ & $\boldsymbol{\sigma}$ & $\begin{array}{l}\text { Differenzie- } \\
\text { rungsorient. }\end{array}$ & $\begin{array}{c}\text { Kostenorien- } \\
\text { tierte }\end{array}$ & $\begin{array}{l}\text { Innovations } \\
\text { orientierte }\end{array}$ \\
\hline Produktpflege & 1,86 & $-0,13$ & $+0,08$ & $+0,26$ & 1,80 & $-0,11$ & $+0,08$ & $+0,08$ & 1,81 & $-0,12$ & $+0,44$ & $-0,24$ \\
\hline Produktinnovation & 1,67 & $-0,11$ & $+0,27$ & $-0,27$ & 1,51 & $-0,14$ & $+0,34$ & $\cdot 0,29$ & 1,84 & $-0,11$ & $+0,44$ & $-0,41$ \\
\hline Benutzertreundlichkeit & 2,00 & $-0,27$ & $+0,12$ & $+0,49$ & 1,61 & $-0,26$ & $+0,27$ & $+0,04$ & 1,85 & $-0,15$ & $+0,26$ & $-0,14$ \\
\hline Design & 3,02 & $-0,22$ & $+0,19$ & $+0,23$ & 2,57 & $-0,15$ & $+0,22$ & $+0,13$ & 2,44 & $-0,12$ & $+0,18$ & $-0,09$ \\
\hline Produktqualităt & 1,49 & $-0,12$ & $+0,11$ & $+0,16$ & 1,26 & $-0,15$ & $+0,16$ & $-0,04$ & 1,29 & $-0,14$ & $+0,24$ & $-0,05$ \\
\hline Sortimentstiofe/-breite & 2,62 & $-0,06$ & $+0,08$ & $+0,27$ & 2,62 & $-0,11$ & $+0,12$ & $+0,17$ & 2,17 & $-0,11$ & $+0,14$ & $+0,12$ \\
\hline Service & 2,03 & $-0,34$ & $+0,26$ & $+0,47$ & 1,63 & $-0,12$ & $+0,27$ & $+0,11$ & 1,72 & $-0,27$ & $+0,52$ & $+0,09$ \\
\hline Prois & 2,33 & $+0,12$ & $-0,18$ & $-0,03$ & 1,86 & $-0,03$ & $-0,14$ & $+0,19$ & 1,87 & $-0,03$ & $-0,26$ & $+0,12$ \\
\hline Konditionen & 2,70 & 0,00 & $-0,13$ & $+0,12$ & 2,36 & $-0,06$ & $-0,12$ & $+0,12$ & 2,44 & $+0,05$ & $-0,18$ & $+0,17$ \\
\hline Werbung & 2,67 & $-0,20$ & $+0,15$ & $+0,28$ & 2,52 & $-0,12$ & $+0,27$ & $-0,01$ & 2,45 & $-0,15$ & $+0,14$ & $-0,12$ \\
\hline Persōnlicher Verkaưf & 1,90 & $+0,04$ & $-0,14$ & $+0,15$ & 1,85 & $+0,13$ & $-0,14$ & $-0,05$ & 2,02 & $+0,01$ & $-0,15$ & $+0,03$ \\
\hline Verkautsforderung & 2,59 & $-0,24$ & $+0,11$ & $+0,46$ & 2,24 & $-0,19$ & $+0,43$ & $+0,11$ & 2,29 & $-0,11$ & $+0,10$ & $+0,23$ \\
\hline Public Relations & 2,57 & $-0,16$ & $+0,31$ & $-0,12$ & 2,45 & $-0,12$ & $+0,13$ & $+0,13$ & 2,66 & $-0,14$ & $+0,31$ & $+0,01$ \\
\hline Sponsoring & 4,45 & $-0,15$ & $+0,22$ & $+0,02$ & 3,84 & $+0,05$ & $+0,04$ & $-0,13$ & 4,13 & $-0,11$ & $+0,15$ & $+0,11$ \\
\hline Absatrwegegestaltung & 2,85 & $-0,27$ & $+0,18$ & $+0,31$ & 2,64 & $-0,13$ & $+0,33$ & $+0,02$ & 2,62 &,- 012 & $+0,28$ & 0,00 \\
\hline Logistik & 3,06 & $-0,26$ & $+0,03$ & $+0,62$ & 2,54 & $-0,16$ & $+0,04$ & $+0,31$ & 2,45 & $-0,13$ & $+0,45$ & $-0,05$ \\
\hline Anzahl der Unternehmen & 104 & 51 & 33 & 20 & 159 & 85 & 33 & 41 & 112 & 62 & 29 & 21 \\
\hline
\end{tabular}

Tab. 10: Mittelwertabweichungen der Strategiedimensionen der strategischen High-Tech-Gruppen in den Situationen A, B und C 


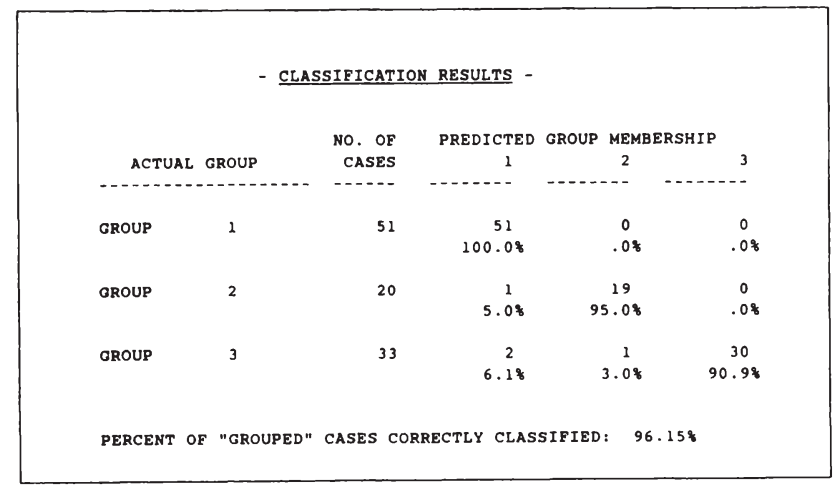

Tab. 11: Ergebnisse der diskriminatorischen Klassifikation anhand der Strategievariablen in Situation A

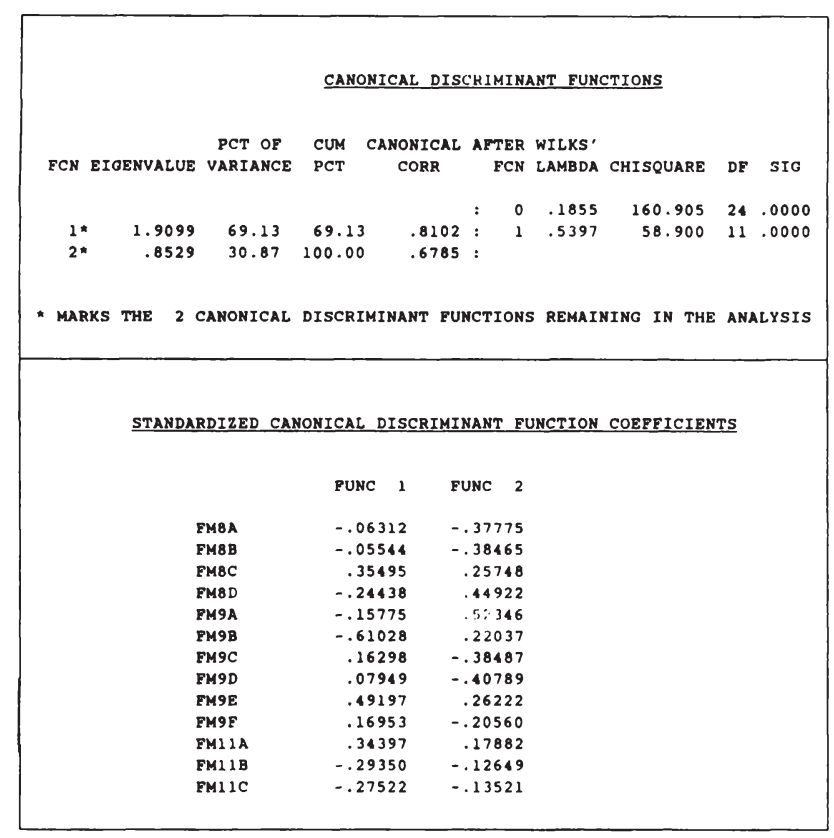

Tab. 12: Standardisierte Diskriminanzfunktionen fuir die Cluster der strategischen High-Tech-Gruppen in Situation A auf Basis der Strategievariablen 


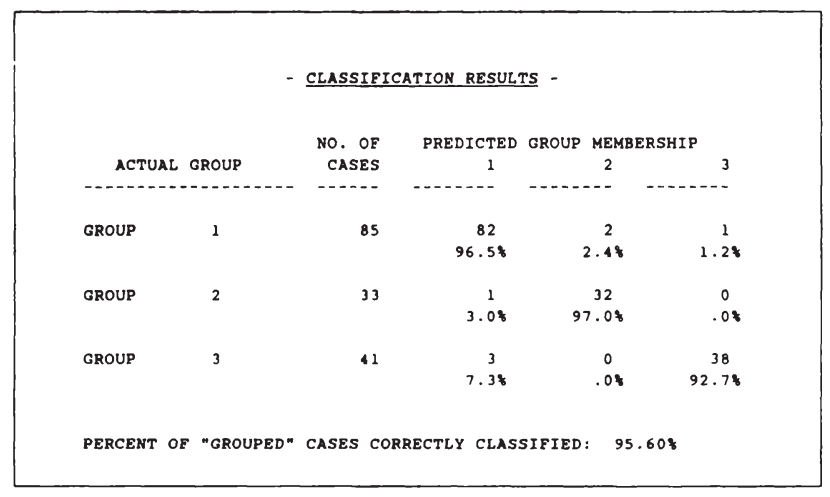

Tab. 13: Ergebnisse der diskriminatorischen Klassifikation anhand der Strategievariablen in Situation B

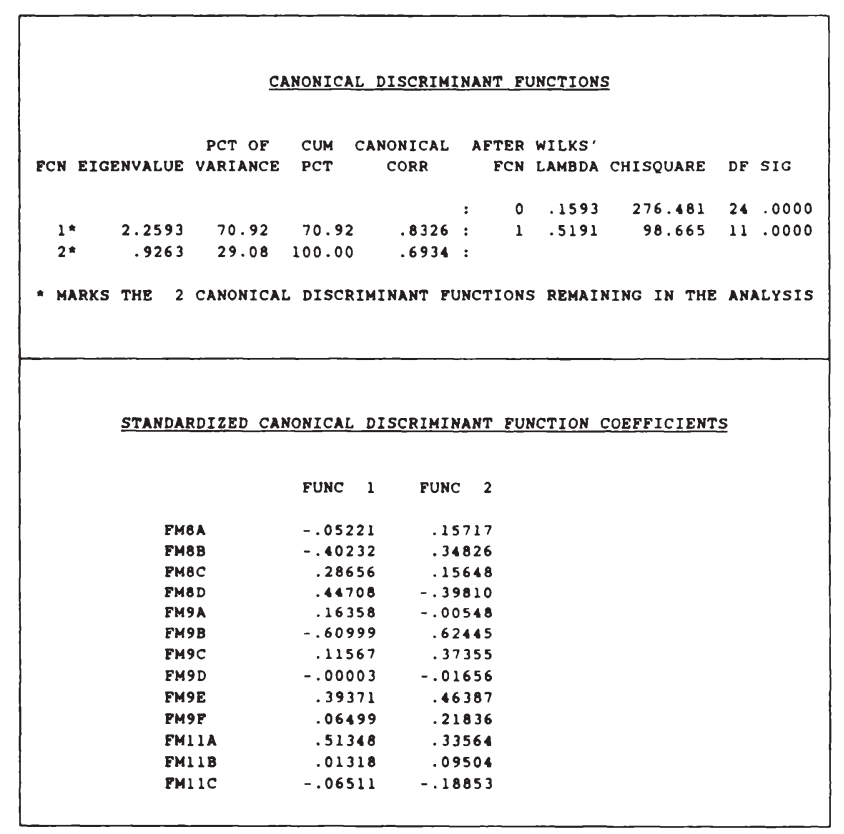

Tab. 14: Standardisierte Diskriminanzfunktionen fuir die Cluster der strategischen High-Tech-Gruppen in Situation B auf Basis der Strategievariablen 
- CLASSIFICATION RESULTS -

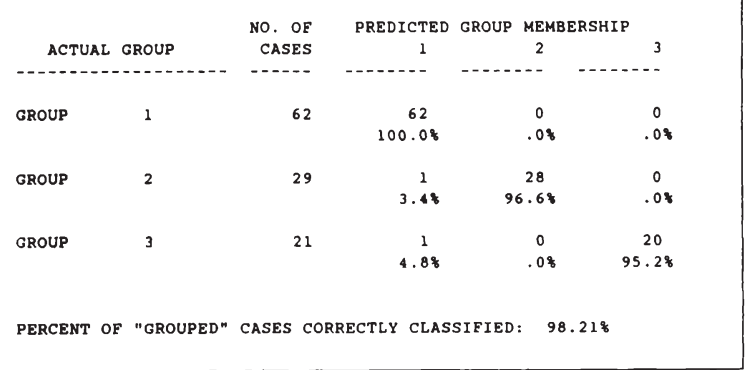

Tab. 15: Ergebnisse der diskriminatorischen Klassifikation anhand der Strategievariablen in Situation C

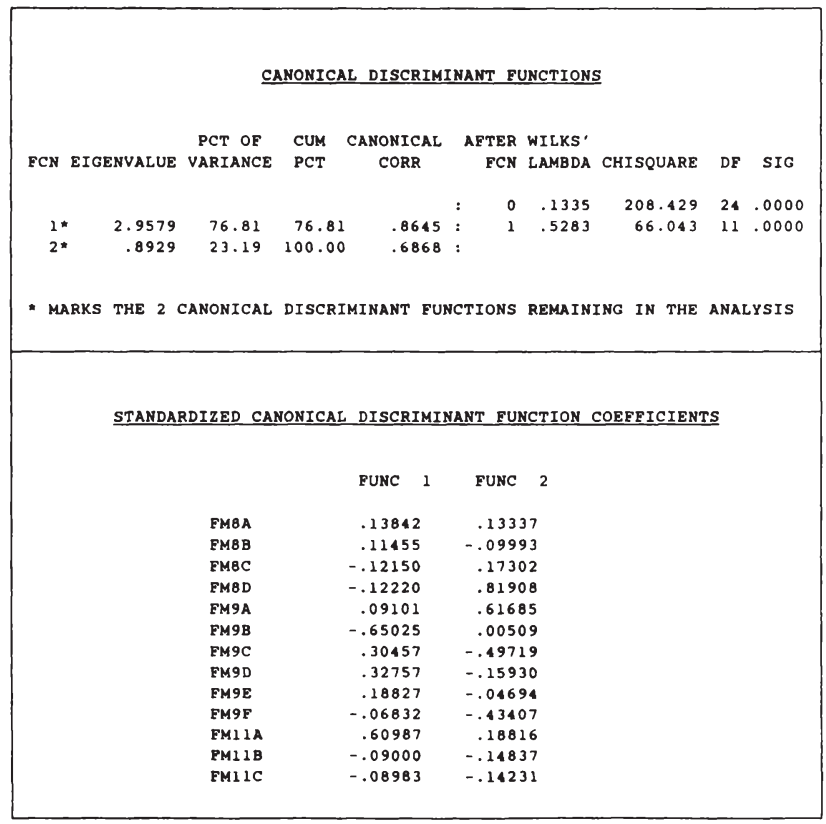

Tab. 16: Standardisierte Diskriminanzfunktionen für die Cluster der strategischen High-Tech-Gruppen in Situation C auf Basis der Strategievariablen 


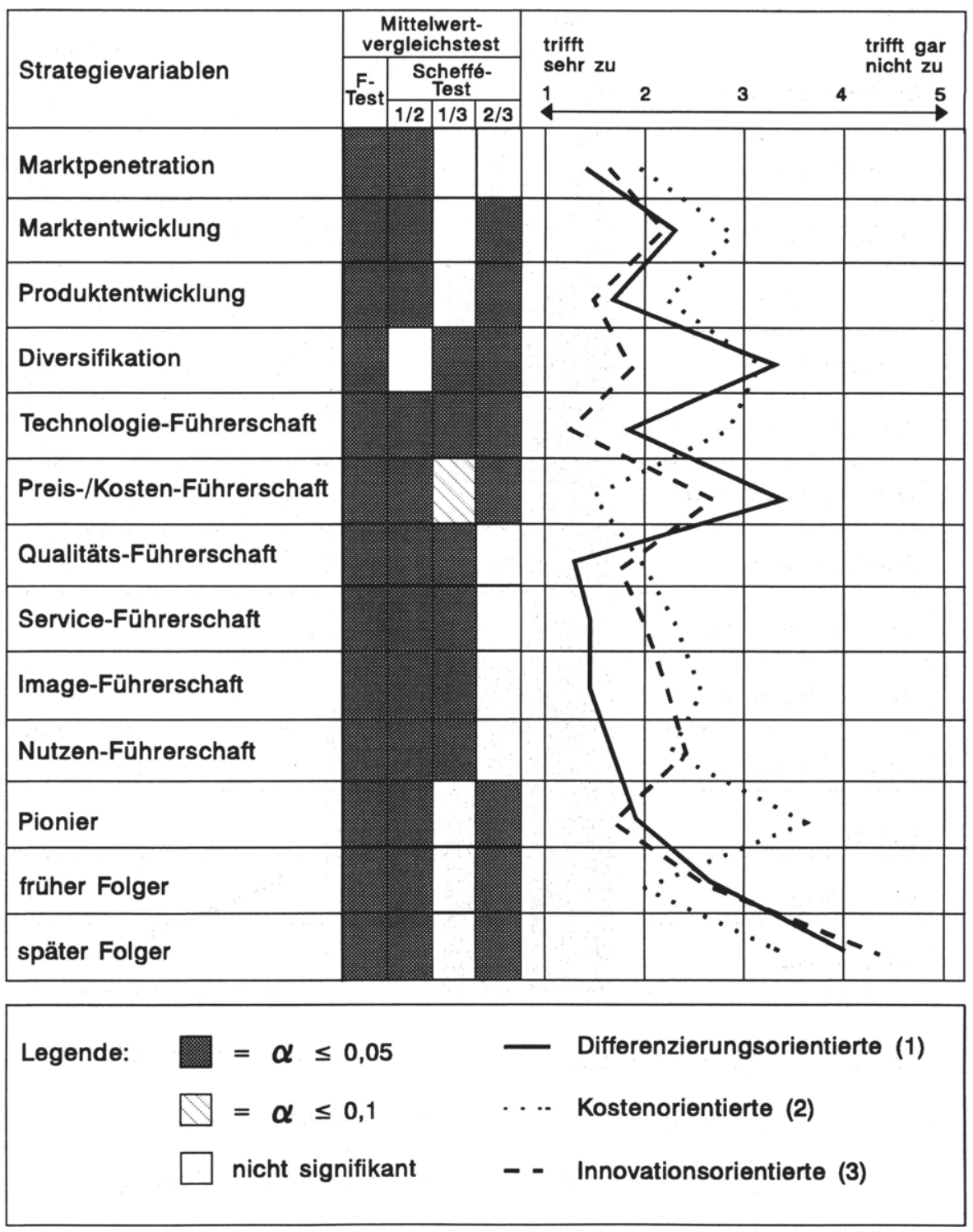

Tab. 17: Ergebnisse der Mittelwertvergleichstests fir die Cluster der strategischen High-Tech-Gruppen in Situationen A auf Basis der Strategievariablen 


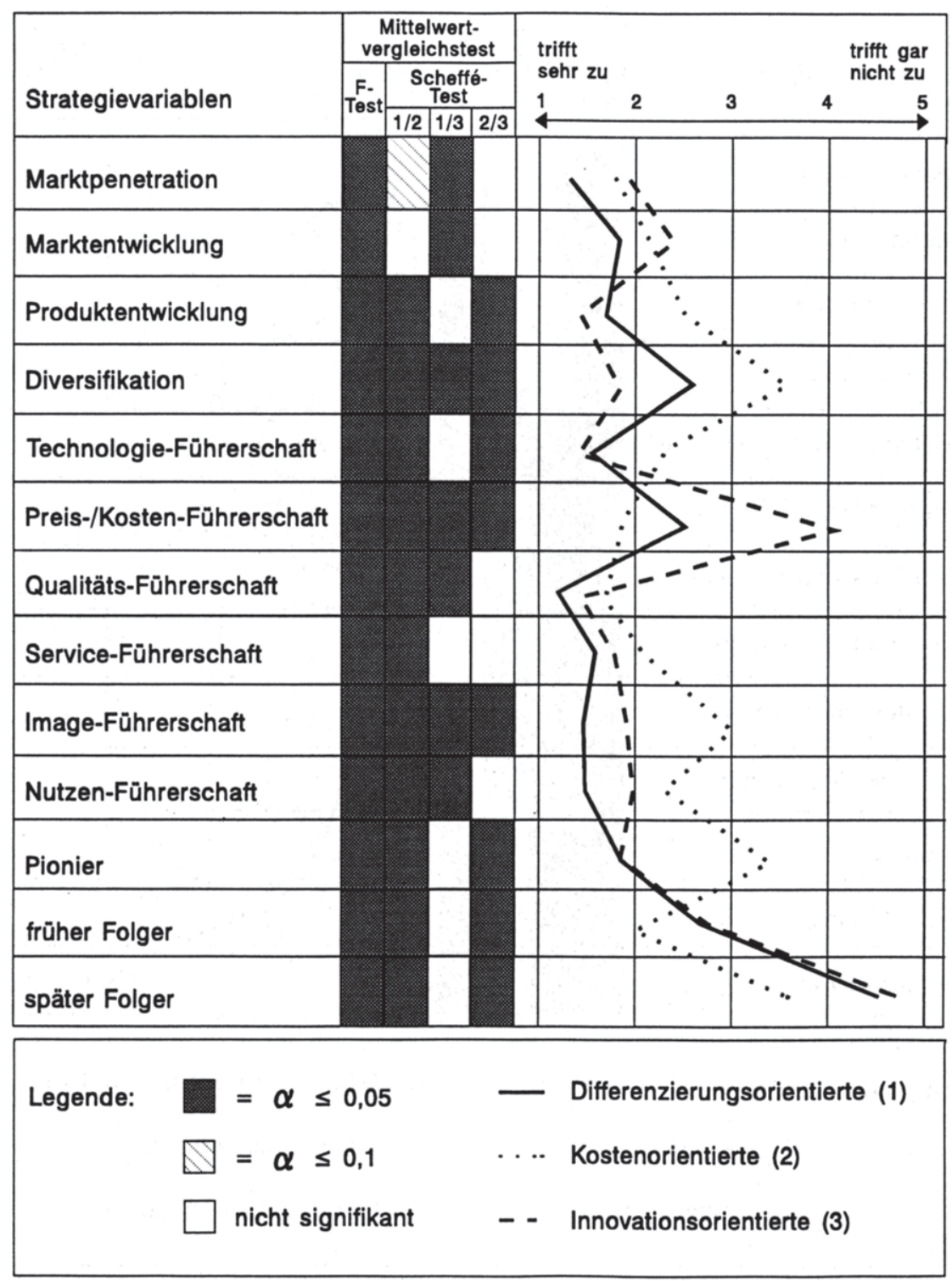

Tab. 18: Ergebnisse der Mittelwertvergleichstests fuir die Cluster der strategischen High-Tech-Gruppen in Situationen B auf Basis der Strategievariablen 


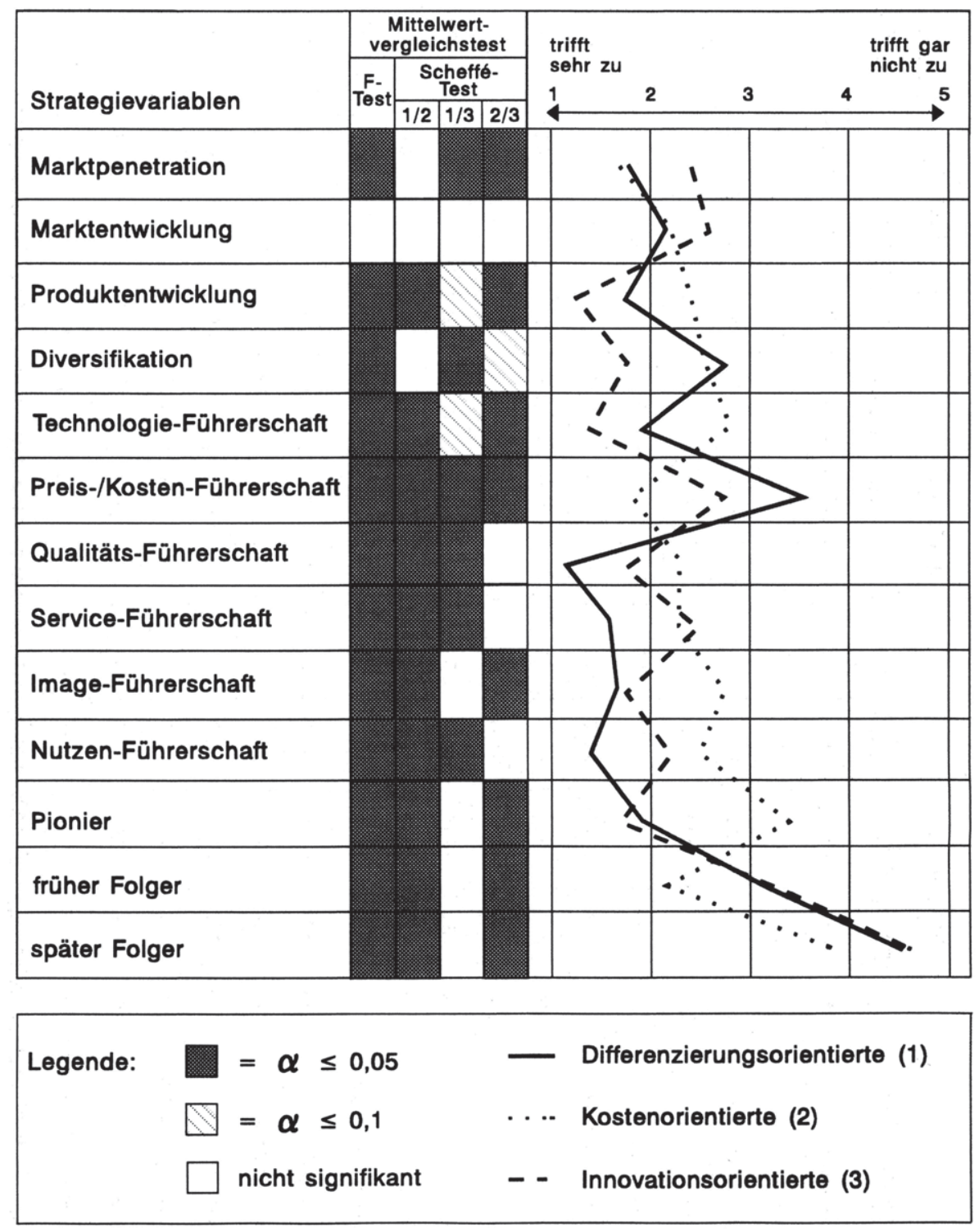

Tab. 19: Ergebnisse der Mittelwertvergleichstests für die Cluster der strategischen High-Tech-Gruppen in Situationen $\mathbf{C}$ auf Basis der Strategievariablen 


\begin{tabular}{|c|c|c|c|c|c|c|c|c|c|c|c|c|}
\hline \multirow[b]{2}{*}{$\begin{array}{l}\text { Marketinginstrument- } \\
\text { variablen }\end{array}$} & \multicolumn{4}{|c|}{ Situation A } & \multicolumn{4}{|c|}{ Situation B } & \multicolumn{4}{|c|}{ Situation C } \\
\hline & $\varnothing$ & $\begin{array}{l}\text { Difterenzie- } \\
\text { rungsorient. }\end{array}$ & $\begin{array}{c}\text { Kostenorien- } \\
\text { tierto }\end{array}$ & $\begin{array}{c}\text { Innovations- } \\
\text { orientierte }\end{array}$ & $\varnothing$ & $\begin{array}{l}\text { Differenzie- } \\
\text { rungsorient. }\end{array}$ & $\begin{array}{c}\text { Kostenorien- } \\
\text { tierte }\end{array}$ & $\begin{array}{l}\text { Innovations- } \\
\text { orientierte }\end{array}$ & $\varnothing$ & $\begin{array}{l}\text { Differenzie- } \\
\text { rungsorient. }\end{array}$ & $\begin{array}{c}\text { Kostenorion- } \\
\text { tierte }\end{array}$ & $\begin{array}{c}\text { Innovations- } \\
\text { orientierte }\end{array}$ \\
\hline Produktpflege & 1,86 & $-0,13$ & $+0,08$ & $+0,26$ & 1,80 & $-0,11$ & $+0,08$ & $+0,08$ & 1,81 & $-0,12$ & $+0,44$ & $-0,24$ \\
\hline Produktinnovation & 1,67 & $-0,11$ & $+0,27$ & $-0,27$ & 1,51 & $-0,14$ & $+0,34$ & $-0,29$ & 1,84 & $-0,11$ & $+0,44$ & $-0,41$ \\
\hline Benutzerfreundlichkeit & 2,00 & $-0,27$ & $+0,12$ & $+0,49$ & 1,61 & $-0,26$ & $+0,27$ & $+0,04$ & 1,85 & $-0,15$ & $+0,26$ & $-0,14$ \\
\hline Design & 3,02 & $-0,22$ & $+0,19$ & $+0,23$ & 2,57 & $-0,15$ & $+0,22$ & $+0,13$ & 2,44 & $-0,12$ & $+0,18$ & $-0,09$ \\
\hline Produktqualitât & 1,49 & $-0,12$ & $+0,11$ & $+0,16$ & 1,26 & $-0,15$ & $+0,16$ & $-0,04$ & 1,29 & $-0,14$ & $+0,24$ & $-0,05$ \\
\hline Sortimentstiefe/-breite & 2,62 & $-0,06$ & $+0,08$ & $+0,27$ & 2,62 & $-0,11$ & $+0,12$ & $+0,17$ & 2,17 & $-0,11$ & $+0,14$ & $+0,12$ \\
\hline Service & 2,03 & $-0,34$ & $+0,26$ & $+0,47$ & 1,63 & $-0,12$ & $+0,27$ & $+0,11$ & 1,72 & $-0,27$ & $+0,52$ & $+0,09$ \\
\hline Preis & 2,33 & $+0,12$ & $-0,18$ & $-0,03$ & 1,96 & $-0,03$ & $-0,14$ & $+0,18$ & 1,87 & $-0,03$ & $-0,26$ & $+0,12$ \\
\hline Konditionen & 2,70 & 0,00 & $-0,13$ & $+0,12$ & 2,36 & $-0,06$ & $-0,12$ & $+0,12$ & 2,44 & $+0,05$ & $-0,18$ & $+0,17$ \\
\hline Worbung & 2,67 & $-0,20$ & $+0,15$ & $+0,28$ & 2,52 & $-0,12$ & $+0,27$ & $-0,01$ & 2,45 & $-0,15$ & $+0,14$ & $-0,12$ \\
\hline Persōnlicher Verkaut & 1,90 & $+0,04$ & $-0,14$ & $+0,15$ & 1,85 & $+0,13$ & $-0,14$ & $-0,05$ & 2,02 & $+0,01$ & $-0,15$ & $+0,03$ \\
\hline Verkautsforderung & 2,58 & $-0,24$ & $+0,11$ & $+0,46$ & 2,24 & $-0,19$ & $+0,43$ & $+0,11$ & 2,29 & $-0,11$ & $+0,10$ & $+0,23$ \\
\hline Public Relations & 2,57 & $-0,16$ & $+0,31$ & $-0,12$ & 2,45 & $-0,12$ & $+0,13$ & $+0,13$ & 2,66 & $-0,14$ & $+0,31$ & $+0,01$ \\
\hline Sponsoring & 4,45 & $-0,15$ & $+0,22$ & $+0,02$ & 3,84 & $+0,05$ & $+0,04$ & $-0,13$ & 4,13 & $-0,11$ & $+0,15$ & $+0,11$ \\
\hline Absatzwegegestaltung & 2,85 & $-0,27$ & $+0,18$ & $+0,31$ & 2,64 & $-0,13$ & $+0,33$ & $+0,02$ & 2,62 &,- 012 & $+0,28$ & 0,00 \\
\hline Logistik & 3,06 & $-0,26$ & $+0,03$ & $+0,62$ & 2,54 & $-0,16$ & $+0,04$ & $+0,31$ & 2,45 & $-0,13$ & $+0,45$ & $-0,05$ \\
\hline Anzahl der Unternehmen & 104 & 51 & 33 & 20 & 159 & 85 & 33 & 41 & 112 & 62 & 29 & 21 \\
\hline
\end{tabular}

Tab. 20: Mittelwertabweichungen der Marketinginstrumente bei den strategischen High-Tech-Gruppen in den Situationen A, B und C 


\begin{tabular}{|c|c|c|c|c|c|c|c|c|c|c|c|c|c|}
\hline \multirow{2}{*}{\multicolumn{2}{|c|}{\begin{tabular}{|l|} 
Variablen zur Beschleuni- \\
gung von Innovationen
\end{tabular}}} & \multicolumn{4}{|c|}{ Situation $A$} & \multicolumn{4}{|c|}{ Situation B } & \multicolumn{4}{|c|}{ Situation C } \\
\hline & & $\varnothing$ & \begin{tabular}{|l|} 
Diflerenzio- \\
rungsorionit.
\end{tabular} & \begin{tabular}{c|}
$\begin{array}{c}\text { Kostenorien } \\
\text { tierte }\end{array}$ \\
\end{tabular} & $\begin{array}{c}\begin{array}{c}\text { Innovalions } \\
\text { orientiente }\end{array} \\
\end{array}$ & $\varnothing$ & \begin{tabular}{|l|} 
Difllorenzie- \\
rungeorient.
\end{tabular} & $\begin{array}{c}\text { Kostenorien- } \\
\text { tierte }\end{array}$ & 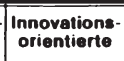 & $\varnothing$ & \begin{tabular}{l|} 
Ditferenzie- \\
rungsorient.
\end{tabular} & $\bar{c} \begin{array}{c}\text { Koslenorien- } \\
\text { tierte }\end{array}$ & $\begin{array}{c}\text { Innovations } \\
\text { orientierte }\end{array}$ \\
\hline \multirow{15}{*}{ 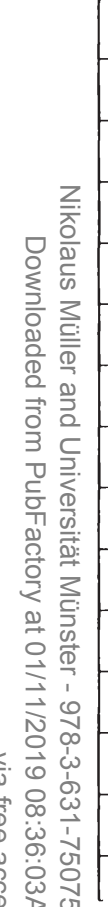 } & Know-how Zukauf & 2,67 & $+0,09$ & $-0,29$ & $+0,20$ & 2,19 & $-0,18$ & $-0,12$ & $+0,27$ & 2,48 & $-0,12$ & $+0,02$ & $+0,33$ \\
\hline & Erhöhter Ressourceneinsatz & 2,41 & $+0,03$ & $+0,12$ & $-0,26$ & 2,30 & $-0,13$ & $+0,27$ & $-0,16$ & 2,30 & $-0,11$ & $+0,26$ & $-0,12$ \\
\hline & Unternehmenskooperation & 2,61 & $+0,27$ & $+0,05$ & $-0,77$ & 2,12 & $+0,12$ & -0.18 & $+0,27$ & 2,57 & $+0,01$ & $-0,27$ & $+0,26$ \\
\hline & Fertigungstiefenreduktion & 2,97 & +0.09 & $-0,20$ & $-0,14$ & 2,58 & $+0,06$ & -0.12 & +0.02 & 2,68 & $+0,09$ & $-0,26$ & $+0,08$ \\
\hline & Just-in-time-Beschaffung & 2,75 & $-0,18$ & $+0,12$ & $+0,31$ & 2,32 & $-0,13$ & $+0,07$ & $+0,12$ & 2,32 & $-0,15$ & $+0,09$ & $+0,30$ \\
\hline & Optimierung Durchlautzeiten & 1,92 & $-0,04$ & $-0,13$ & +0.14 & 1,67 & $+0,03$ & $-0,12$ & +0.06 & 1,62 & $-0,01$ & $-0,12$ & $+0,11$ \\
\hline & Globale Vermarktung & 2,89 & $+0,12$ & $-0,07$ & $+0,09$ & 2,50 & $-0,06$ & $+0,08$ & $-0,07$ & 2,55 & $+0,08$ & $+0,20$ & $-0,45$ \\
\hline & Kooperation mit Pilotkunden & 2,13 & $-0,03$ & $+0,27$ & $-0,30$ & 1,86 & $+0,01$ & $+0,14$ & $-0,14$ & 2,17 & $-0,09$ & $+0,33$ & $-0,12$ \\
\hline & Erhohung Worbedruck & 2,81 & $-0,11$ & $-0,06$ & $+0,23$ & 2,72 & $-0,12$ & $+0,18$ & $+0,09$ & 2,83 & $-0,12$ & $+0,13$ & $+0,03$ \\
\hline & Vertriebskooperationen & 2,64 & $+0,16$ & $-0,09$ & -0.14 & 2,26 & $+0,13$ & $-0,06$ & $+0,04$ & 3,08 & $+0,02$ & $-0,22$ & $-0,39$ \\
\hline & Mitarbeitermotivation & 1,50 & $-0,12$ & $+0,08$ & $-0,11$ & 1,45 & -0.11 & $+0,40$ & $-0,12$ & 1,67 & $-0,16$ & $+0,52$ & $-0,18$ \\
\hline & Interdisziplinâre Teams & 2,12 & $-0,06$ & $-0,03$ & $+0,09$ & 1,99 & $+0,02$ & $-0,07$ & $-0,11$ & 2,28 & $-0,08$ & $+0,54$ & $-0,20$ \\
\hline & Flexibilihat U-Grundsatz & 1,79 & $-0,15$ & $+0,02$ & $+0,10$ & 1,67 & -0.11 & $+0,12$ & $+0,13$ & 1,76 & $-0,11$ & $+0,12$ & $-0,06$ \\
\hline & Zehteffizionz U-Grundsatz & 2,10 & $+0,12$ & $-0,13$ & $+0,01$ & 1,87 & $+0,12$ & $-0,13$ & $+0,03$ & 2,15 & $+0,11$ & $-0,18$ & $-0,12$ \\
\hline & Anzahl der Unternehmen & 104 & 51 & 33 & 20 & 159 & 85 & 33 & 41 & 112 & 62 & 29 & 21 \\
\hline
\end{tabular}

Tab. 21: Mittelwertabweichungen der Instrumente zur Verkürzung der Entwicklungszeiten und Beschleunigung der Vermarktung der strategischen High-Tech-Gruppen in Situationen A, B und C 


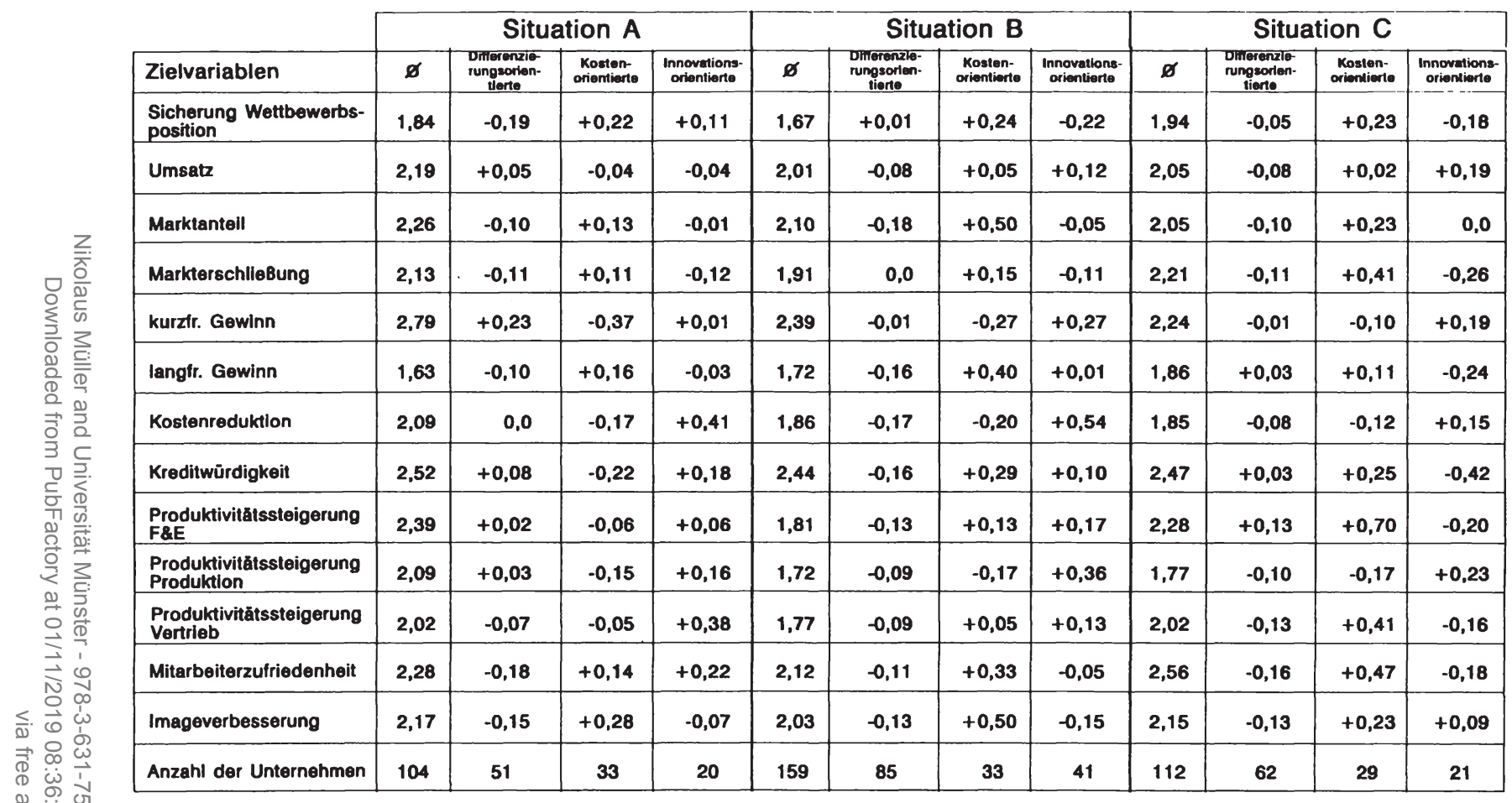

Tab. 22: Mittelwertabweichungen der Unternehmensziele bei den strategischen High-Tech-Gruppen in den Situationen A, B und C 


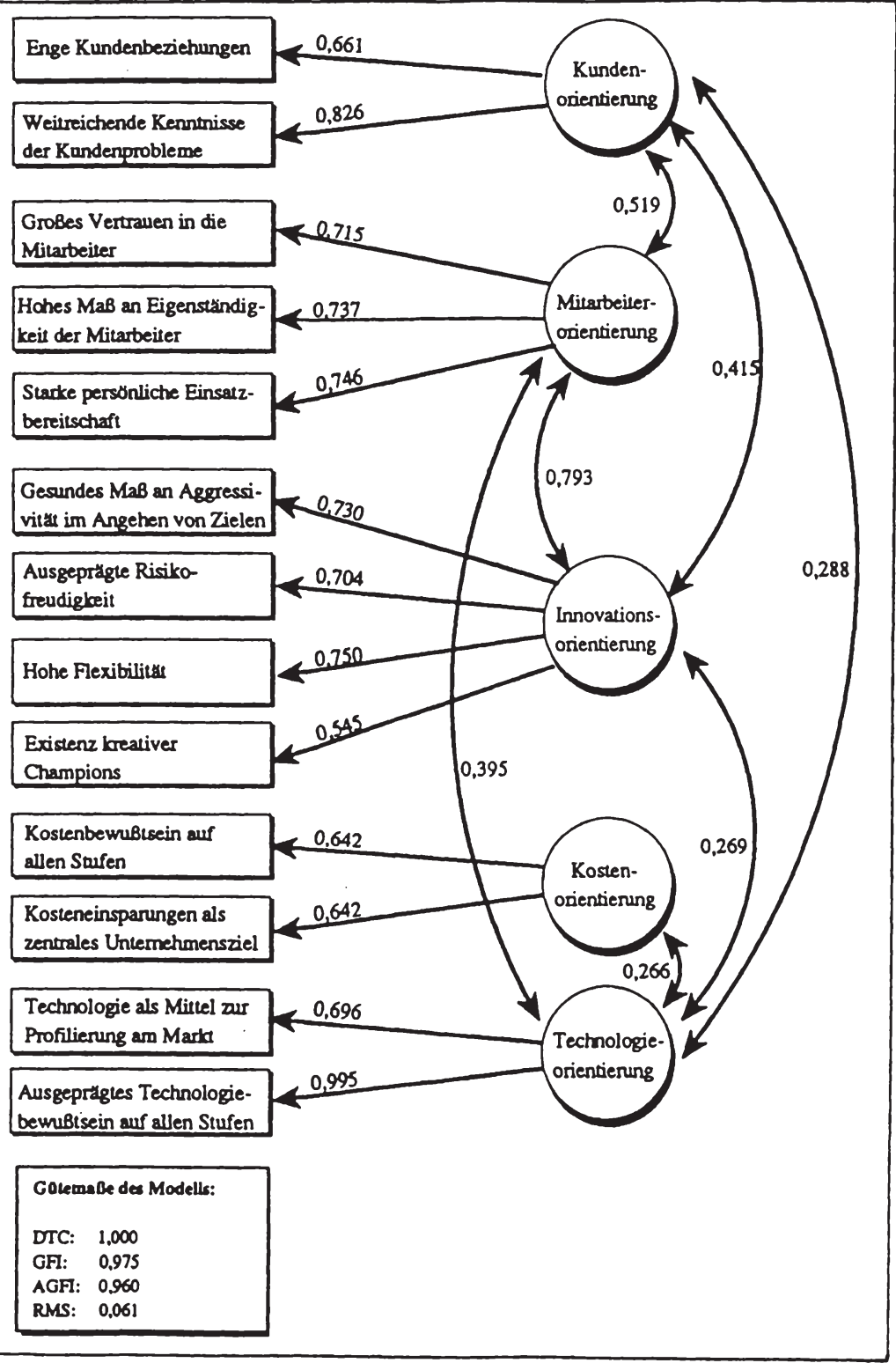

Tab. 23: Ergebnis der konfirmatorischen Faktorenanalyse zu kulturellen Grundorientierungen in High-Tech-Märkten 


\begin{tabular}{|c|c|c|c|c|c|c|c|c|c|c|c|c|}
\hline \multirow[b]{2}{*}{ Kulturvariablen } & \multicolumn{4}{|c|}{ Situation A } & \multicolumn{4}{|c|}{ Situation B } & \multicolumn{4}{|c|}{ Situation C } \\
\hline & $\boldsymbol{E}$ & $\begin{array}{l}\text { Dingrencio- } \\
\text { rungeorien- } \\
\text { tiente }\end{array}$ & $\begin{array}{c}\text { Kostion- } \\
\text { orlentlerie }\end{array}$ & $\begin{array}{l}\text { Imnovations- } \\
\text { orlentlente }\end{array}$ & $\mathscr{6}$ & $\begin{array}{l}\text { Oinerensele- } \\
\text { rungaorion- } \\
\text { tionte }\end{array}$ & $\begin{array}{c}\text { Koslen- } \\
\text { orientierte }\end{array}$ & $\begin{array}{l}\text { Innovations- } \\
\text { orlentlente }\end{array}$ & $\varnothing$ & $\begin{array}{l}\text { Diferenziza- } \\
\text { rungacoien- } \\
\text { therle }\end{array}$ & $\begin{array}{c}\text { Kosten- } \\
\text { orientiente }\end{array}$ & $\begin{array}{l}\text { Innovations- } \\
\text { orientierte }\end{array}$ \\
\hline $\begin{array}{l}\text { Enge Kundenbe- } \\
\text { ziehungen }\end{array}$ & 1,49 & $+0,06$ & $-0,16$ & $+0,12$ & 1,39 & $-0,06$ & $+0,03$ & $+0,10$ & 1,62 & $-0,20$ & $+0,48$ & $-0,05$ \\
\hline $\begin{array}{l}\text { Kenntinls der } \\
\text { Kundenprobleme }\end{array}$ & 1,74 & $-0,11$ & $+0,05$ & $+0,20$ & 1,66 & $-0,02$ & $+0,22$ & $-0,11$ & 1,82 & $-0,13$ & $+0,28$ & $-0,01$ \\
\hline Vertrauen in Mitarbelter & 2,04 & $-0,16$ & $+0,17$ & $+0,13$ & 1,86 & $-0,10$ & $+0,29$ & $-0,03$ & 2,12 & $-0,20$ & $+0,28$ & $+0,17$ \\
\hline $\begin{array}{l}\text { Eigenstalndigkeit der } \\
\text { Mitarbehter }\end{array}$ & 2,25 & $-0,13$ & $+0,11$ & $+0,14$ & 1,83 & 0,0 & $+0,07$ & $-0,05$ & 2,30 & $-0,14$ & $+0,25$ & $+0,08$ \\
\hline $\begin{array}{l}\text { Einsatzbereitschaft der } \\
\text { Mitarbelter }\end{array}$ & 1,99 & $-0,03$ & $-0,05$ & $+0,18$ & 1,74 & $-0,01$ & $-0,01$ & $+0,04$ & 1,98 & $-0,14$ & $+0,12$ & $+0,25$ \\
\hline $\begin{array}{l}\text { Konsequente Ziel- } \\
\text { verfolgung }\end{array}$ & 2,46 & $-0,03$ & $+0,02$ & $+0,04$ & 2,28 & $-0,16$ & $+0,45$ & $-0,01$ & 2,44 & $-0,07$ & $+0,19$ & $+0,19$ \\
\hline Risikofreudigkelt & 3,14 & $+0,06$ & $-0,08$ & $-0,03$ & 2,92 & $-0,10$ & $+0,26$ & $+0,01$ & 3,31 & $-0,07$ & $+0,10$ & $+0,07$ \\
\hline Kreatlvităt & 2,75 & $-0,06$ & +0.25 & $-0,31$ & 2,52 & $-0,11$ & $+0,39$ & $-0,11$ & 2,89 & $-0,04$ & $+0,32$ & $-0,34$ \\
\hline Kostenbewubtsein & 2,95 & $+0,07$ & $-0,19$ & $+0,16$ & 2,60 & $-0,04$ & $+0,01$ & $+0,08$ & 2,74 & $-0,03$ & $+0,05$ & $+0,02$ \\
\hline Kostenorientierung & 2,92 & $+0,10$ & $-0,44$ & $+0,52$ & 2,58 & $-0,05$ & $-0,25$ & $+0,32$ & 2,57 & $-0,07$ & $+0,02$ & $+0,19$ \\
\hline Technologieorientierung & 2,29 & $-0,11$ & $+0,29$ & $-0,18$ & 1,65 & $-0,04$ & $+0,23$ & $-0,10$ & 2,09 & $-0,12$ & $+0,53$ & $-0,38$ \\
\hline TechnologiebewuBtsein & 2,61 & $+0,01$ & $+0,24$ & $-0,44$ & 2,07 & $-0,05$ & $+0,32$ & $-0,17$ & 2,59 & $-0,15$ & $+0,55$ & $-0,30$ \\
\hline Anzahl der Unternehmen & 104 & 51 & 33 & 20 & 159 & 85 & 33 & 41 & 112 & 62 & 29 & 21 \\
\hline
\end{tabular}

Tab. 24: Mittelwertabweichungen der Kulturdimensionen bei den strategischen High-Tech-Gr in den Situationen $\mathbf{A}, \mathbf{B}$ und $\mathbf{C}$ 


\begin{tabular}{|c|c|c|c|c|c|}
\hline & & $\phi$ & 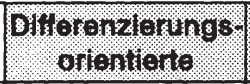 & 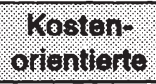 & $\begin{array}{l}\text { Innovations- } \\
\text { orientierte }\end{array}$ \\
\hline \multirow{3}{*}{$\frac{c}{c}$} & Umsatz 1988 & 386 & 479 & 267 & 404 \\
\hline & Umsatz 1989 & 419 & 520 & 284 & 447 \\
\hline & Umsatz 1990 & 457 & 581 & 296 & 482 \\
\hline \multirow{3}{*}{$\frac{6}{5}$} & Umsatz 1988 & 546 & 491 & 511 & 656 \\
\hline & Umsatz 1989 & 602 & 544 & 551 & 724 \\
\hline & Umsazz 1990 & 658 & 591 & 593 & 801 \\
\hline \multirow{3}{*}{$\begin{array}{c}0 \\
\frac{c}{3} \\
\frac{3}{3}\end{array}$} & Umsatz 1988 & 547 & 611 & 308 & 732 \\
\hline & Umsatz 1989 & 604 & 680 & 339 & 792 \\
\hline & Umsatz 1990 & 660 & 750 & 374 & 835 \\
\hline
\end{tabular}

Tab. 25: Durchschnittliche Umsätze der strategischen Gruppen innerhalb der Umfeldsituationen in den Jahren 1988-1990

\begin{tabular}{|c|c|c|c|c|c|c|c|c|c|}
\hline \multirow{3}{*}{$\begin{array}{l}\text { Erfolgs- } \\
\text { variablen }\end{array}$} & \multicolumn{3}{|c|}{ Situation $\mathrm{A}$} & \multicolumn{3}{|c|}{ Situation B } & \multicolumn{3}{|c|}{ Situation C } \\
\hline & \multicolumn{3}{|c|}{ T-Test } & \multicolumn{3}{|c|}{ T-Test } & \multicolumn{3}{|c|}{ T-Test } \\
\hline & $1 / 2$ & $1 / 3$ & & $1 / 2$ & $1 / 3$ & $2 / 3$ & $1 / 2$ & $1 / 3$ & $2 / 3$ \\
\hline $\begin{array}{c}\text { AUmsatz } \\
1988 / 89\end{array}$ & & & 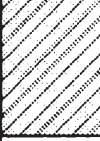 & & & & & & \\
\hline $\begin{array}{c}\Delta \text { Umsatz } \\
1989 / 90\end{array}$ & 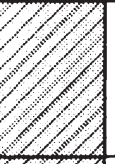 & & & & & & & Eहy, & EFy \\
\hline $\begin{array}{c}\text { MA } \\
\text { Deutschland }\end{array}$ & 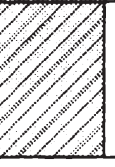 & & & & & [ै/ & & Eeyes & \\
\hline Legende & $\begin{array}{l}=\alpha \leq \\
=\alpha \leq \\
=\text { nicht }\end{array}$ & $\begin{array}{l}0,05 \\
0,1 \\
\text { signi }\end{array}$ & & $\begin{array}{l}1= \\
2= \\
3=\end{array}$ & teno & $\begin{array}{l}\text { rungsorie } \\
\text { antierte } \\
\text { sorientier }\end{array}$ & ierte & & \\
\hline
\end{tabular}

Tab. 26: Ergebnisse der Mittelwertvergleichstests für die absoluten Erfolgsmerkmale der strategischen High-Tech-Gruppen 
Nikolaus Müller and Universität Münster - 978-3-631-75075-9 Downloaded from PubFactory at 01/11/2019 08:36:03AM via free access 


\section{A NHANG III}

\section{Fragebogen}


Nikolaus Müller and Universität Münster - 978-3-631-75075-9 Downloaded from PubFactory at 01/11/2019 08:36:03AM via free access 


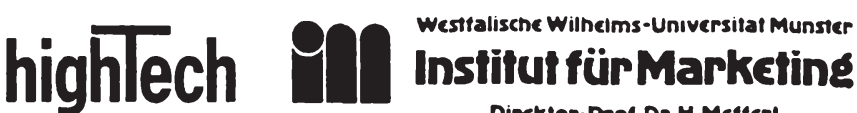 \\ Direktor: Prof.Dr.H.Meffert
}

\section{Vertraulicher Antwortvordruck}

\author{
zur Studie über "High-Tech-Marketıng" in Zusammenarbeıt \\ zwischen dem Wirschaftsmagazın hıghTech \\ und der Unternehmensberatung Maisberger \& Partner GmbH
}

Wissenschaftliche Projektleitung:

Prof. Dr. Heribert Meffert, Prof. Dr. Siegtried Lamnek

\section{FRAGEN ZUM MARKFTING}

1. High-Tech-Markte stellen spezielle Anforderungen an die Unternehmen, die in diesen Markten tatig sind. Kennzeichnen Sie bitte anhand der folgenden Statements die Herausforderungen in den von Ihnen bearbeiteten Märkten!

Es besteht ein hoher Wettbewerbs. druck durch preisaggressive Anbieter.

Es besteht ein intensiver Technolo. giewettbewerb.

Die Kunden sind sehr preisbewuBt.

Es besteht bislang kein sicherer Industriestandard.

Neue technologische Entwicklungen verbessern laufend die Produktqualität.

Das Marktpotential ist bei weitem nicht ausgeschopft.

Neue Anbieter müssen nur geringe Barrieren beım Markteintritt über. winden.

Die Produktlebenszykien haben sich in den letzien Jahren verkürt. Die Entwicklungszeiten für neue Produkte haben sich in den letzten Jahren verlianger.

Es besteht Kaufzurückhaliung bei den porentiellen Nachtragem.

Die Preise im Absatzmarkt sinken laufend.

Der Kostendruck aus dem Beschaf. fungsmarkt schliggt unmittelbar in den Absatzpreisen durch.

\begin{tabular}{|c|c|}
\hline $\begin{array}{l}\text { trifft } \\
\text { sehr zu }\end{array}$ & $\begin{array}{c}\text { trifft gar } \\
\text { nicht zu }\end{array}$ \\
\hline 1 & $\dot{0} \ldots$ \\
\hline
\end{tabular}

$\therefore 0 \cdots 0 \cdots 0 \cdots 0 \cdots 0$

, $0 \cdots 0 \cdots 0 \cdots 0 \cdots 0$

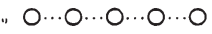
in $0 \cdots 0 \cdots 0 \cdots 0 \cdots 0$ " $0 \cdots 0 \cdots 0 \cdots 0 \cdots 0$ $: 0 \cdots 0 \cdots 0 \cdots 0 \cdots 0$ $\therefore 0 \cdots 0 \cdots 0 \cdots 0 \cdots 0$ $\therefore 0 \cdots 0 \cdots 0 \cdots 0 \cdots 0$ , $0 \cdots 0 \cdots 0 \cdots 0 \cdots 0$ in $0 \cdots 0 \cdots 0 \cdots 0 \cdots 0$ $\because 0 \cdots 0 \cdots 0 \cdots 0 \cdots 0$

2. Wie beurteilen Sie aus Ihrer Kundenkenntnis die folgenden Aussagen?

Unsere Kunden legen zunehmend Wert auf eine einfache Handhabung komplexer Produkte.
Unsere Kunden sind zunehmend bereit. in Schulungen zu investieren.

i $0 \cdots 0 \cdots 0 \cdots 0 \cdots 0$

Die Lernkurve unserer Kunden mit neuen Produkten hat sich verkürzt.

Das Technologie-Know-how unserer Kunden hat sich verbessert.

Die Nutzenerwartung an HighTech-Produkten hat sich deutlich erhöht.

Die Anwendungen unserer Kunden werden zunehmend komplexer.

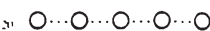

$\therefore \quad 0 \cdots 0 \cdots 0 \cdots 0 \cdots 0$

$\because 0 \cdots 0 \cdots 0 \cdots 0 \cdots 0$

$\because 0 \cdots 0 \cdots 0 \cdots 0 \cdots 0$

3. In High-Tech-Markten treten typische Marketingfehler auf. Welche der folgenden Fehler sind in den won Ihnen bearbeiteten Märkten besonders haufig zu beobachten?

Falsches Timing̣ des Markteintritts.

$$
\begin{aligned}
& \text { besinderv uberhaup } \\
& \text { haufif nicht }
\end{aligned}
$$

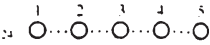$$
\text { Keine am Kundennutzen ausge }
$$$$
\text { richtete Markisegmentierung. }
$$$$
\text { Fehiende Abstimmung zuischen }
$$$$
\text { F\&E. Markeling und vertrieb. }
$$$$
\text { Überbetonung lechnischer Ver- }
$$$$
\text { hilutidargumente. }
$$$$
\text { Zu geringe Kenninisse der Kun- }
$$$$
\text { denbedurtinisse. }
$$

Zu kurze Innovationsziklen mı gering̣en Produkiverbeiserungen.

Uberbetonung aggrewiver Preis. strategien.

Technologiedominante Unierneh. nenskultur zu Listen des Marke. IIṇฺ.

Mangeinde Bereitschafi zur Anpas. sung der Vertriebswege.

Vermachlajssigung der Senicesysteme.

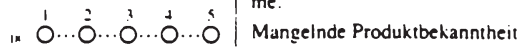
durch falsche Werbestrategie. Fehlende Sensibilitat gegenüber umwelt- und gesellschafispolitischen Herausforderungen. 
4. Wieviel Wert wird derzeit in Ihrem Linternehmen auf die folgenden Lnternehmensziele gelegt?

kurzfristige Gewinnerzielung langfristıge Gewinnerzielung Limsatz.

Marktanteil

Erschließung neuer Märkte

Kosteneinsparung

Produktivitätssteigerung

- in der F\&E

- in der Produktion

- im Venrieb

Imağe(verbesserung )

Mitarbeitermotivation

Sicherung der Wetrbewerbsfähigkeit

Kreditwürdigkeit

5. In welcher Form existieren in Ihrem Unternehmen Marketingpläne und für welchen Zeitraum sind diese gültig?
‘ $\square$ keine Marketingpläne
: $\square$ ausformulierte Pläne
- $\square$ mündlich vereinbarte Pläne
(II!) $\square$ kurzfristig gültig (unter I Jahr)
411: $\square$ mittelfristig gültig ( 1 bis 3 Jahre)
(w.. $\square$ langfristig gültig (mehr als 3 Jahre)

6. Wie oft nutzen Sie die nachfolgenden Informationsquellen. um sich über die Entwicklungen in Ihrem Markt auf dem laufenden zu halten?

Berichie des Außendienstes

Tuggeszeitung̨en

Fachzeitschriften

Wirtschaftspresse

Karnmern/Nerbïnde

Messen/Ausstellungen

Amtliche Statistiken

Datenbanken

Hausbank

Aktive Marktforschung

Mafo-Berichte von Instituten sonstige Quellen

\# $0 \cdots 0 \cdots 0 \cdots 0 \cdots 0$

म: $0 \cdots 0 \cdots 0 \cdots 0 \cdots 0$

4. $0 \cdots 0 \cdots 0 \cdots 0 \cdots 0$

th $0 \cdots 0 \cdots 0 \cdots 0 \cdots 0$

n $0 \cdots 0 \cdots 0 \cdots 0 \cdots 0$

A $0 \cdots 0 \cdots 0 \cdots 0 \cdots 0$

O...'0

? $0 \cdots 0 \cdots 0 \cdots 0 \cdots 0$

s/ $0 \cdots 0 \cdots 0 \cdots 0 \cdots 0$

m $0 \cdots 0 \cdots 0 \cdots 0 \cdots 0$

m $0 \cdots 0 \cdots 0 \cdots 0 \cdots 0$

ni $0 \cdots 0 \cdots 0 \cdots 0 \cdots 0$

: $: 10 \cdots 0 \cdots 0 \cdots 0 \cdots 0$

7. Wie oft setzen Sie die nachfolgenden Analyseinstrumente als Planungsgrundlage fur Ihre Marketingstrategie ein:"

Chancen-Risiken-Analyse

Stärken-Schwächen-Analyse

Lebenszyklusanalyse

Erfahrungskurvenanalyse

Produki-Ponfolio-Analysen

Technologie-Portfolio-Analysen

Wertkettenanalyse

Sonstige:

t: $0 \cdots 0 \cdots 0 \cdots 0 \cdots 0$

(at

nen Produkten bestehende Märkte

ausschöpfen)

Markrentwicklung (bestehende

Produkte in neue Märkte bringen)

Produktentwicklung (neue Produk-

te für bestehende Märkte entwih.

keln)

Diversifikation (neue Produkte für

neue Märkte schaffen)

$$
\begin{aligned}
& \text { immer nis } \\
& n: \hat{O}^{\prime} \cdots \dot{0} \cdots \dot{0} \cdots \dot{0} \cdots
\end{aligned}
$$

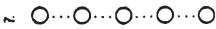

$$
\begin{aligned}
& \text { n } 0 \cdots 0 \cdots 0 \cdots 0 \cdots 0 \\
& \text { m } \mathrm{O} \cdots \mathrm{O} \cdots \mathrm{O} \cdots \mathrm{\cdots} \cdot \mathrm{\cdots} \\
& \text { - }-0 \cdots 0 \cdots 0 \cdots 0 \cdots 0 \\
& \text { n O } \cdots 0 \cdots 0 \cdots 0 \cdots 0 \\
& \text { m } 0 \cdots 0 \cdots 0 \cdots 0 \cdots 0 \\
& \because 0 \cdots 0 \cdots 0 \cdots 0 \cdots 0
\end{aligned}
$$

8. Welche Bedeutung haben in Ihrem Unternehmen die folgenden Strategien der Marktbearbeitung?

$$
\begin{aligned}
& \text { hohe - keine } \\
& \text { Bedeutung Hedeutun: } \\
& \because \text { Oे } \cdots \dot{0} \cdots \dot{0} \cdots+\dot{0} \cdots \dot{0} \\
& \because 0 \cdots 0 \cdots 0 \cdots 0 \cdots 0 \\
& \because \mathrm{O} \cdots \mathrm{O} \cdots \mathrm{O} \cdots \mathrm{O} \cdots \mathrm{O} \\
& { }_{4} .0 \cdots 0 \cdots 0 \cdots 0 \cdots 0
\end{aligned}
$$

9. Welche Bedeutung haben in Ihrem Unternehmen die folgenden Wettbewerbsstrategien?

Erreichung der Technologie-Führerschaft

Preis/Kosten-Führerschaft

Bedeutung keine

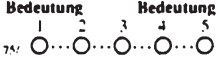

Qualitäts-Führerschaft

n $0 \cdots 0 \cdots 0 \cdots 0 \cdots 0$

Service-Führerschaft

$n, 0 \cdots 0 \cdots 0 \cdots 0 \cdots 0$

${ }_{7 \times}, 0 \cdots 0 \cdots 0 \cdots 0 \cdots 0$

Image-Führerschaft

rथ, $0 \cdots 0 \cdots 0 \cdots 0 \cdots 0$

Nutzen-Führerschaft

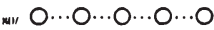

10. Wie ist Ihre derzeitige Positionierung im Wettbewerbsumfeld?

Marktführer

Herausforderer des Marktführers

trim trim sar sehr zu nicht zu

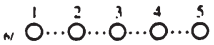

Markımitläufer

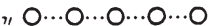

Nischenanbieter

m $0 \cdots 0 \cdots 0 \cdots 0 \cdots 0$

" $0 \cdots 0 \cdots 0 \cdots 0 \cdots 0$

11. In welcher Rolle treten Sie mit Produktinnovationen an den Markt?

als Pionier

als früher Folger

als später Folger

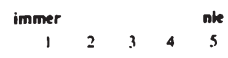

Im $0 \cdots 0 \cdots 0 \cdots 0 \cdots 0$

"1 $0 \cdots 0 \cdots 0 \cdots 0 \cdots 0$

12 $0 \cdots 0 \cdots 0 \cdots 0 \cdots 0$ 
12. Wie wickeln Sie Ihre internationale Geschäftstätigkeit in den für Sie zentralen Markträumen ab?

markte

Lizenzvergabe an ausländische

Parner

Joint-ventures mit ausländischen

Partnem

Auslandsniederlassungen

Tochtergesellschaften

Globale Bearbeitung des Welt-

marktes

Individuelle/differenziene Bearbei-

tung einzelner Laindermärkie

$$
\begin{aligned}
& \text { trim trimt gar }
\end{aligned}
$$

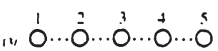

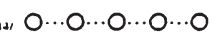

$$
\begin{aligned}
& \text { 11, } 0 \cdots 0 \cdots 0 \cdots 0 \cdots 0 \\
& \text { in } 0 \cdots 0 \cdots 0 \cdots 0 \cdots 0
\end{aligned}
$$

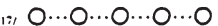

$$
\begin{aligned}
& \text { 1x/ } 0 \cdots 0 \cdots 0 \cdots 0 \cdots 0 \\
& \text { (4) } 0 \cdots 0 \cdots 0 \cdots 0 \cdots 0
\end{aligned}
$$

13. Wie wichtig sind die folgenden Marketinginstrumente in Ihrem Unternehmen?

Presspolitik$$
\text { sehr }
$$
sehr
unwichtig

Konditionen

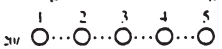

Produkiptlege

Produktinnovation

Benuizerfreundlichkeit

Design

Prindukicualitiat

Sonimentstietie/-breite

Service

Absatzweggegestaltung

Logistik

Werbung:

persönlicher Verkauf

Verkaufisorderung

Orientlichkettsarbeit

Sponoring

14. Welche Trends der Standardisierung bzw. Individualisierung sehen Sie in den folgenden Bereichen?

Technologic

Produhthern (<. B. Hardware)

Service

Binutzeramwendung $1 \%$. B. Sofi"ilre!

0 .

$\therefore 0 \cdots 0 \cdots 0 \cdots 0 \cdots 0$

$\because 0 \cdots 0 \cdots 0 \cdots 0 \cdots 0$

:n $0 \cdots 0 \cdots 0 \cdots 0 \cdots 0$

$\therefore 0 \cdots 0 \cdots 0 \cdots 0 \cdots 0$

$x, 0 \cdots 0 \cdots 0 \cdots 0 \cdots 0$

$\therefore 0 \cdots 0 \cdots 0 \cdots 0 \cdots 0$

n, $0 \cdots 0 \cdots 0 \cdots 0 \cdots 0$

". $0 \cdots 0 \cdots 0 \cdots 0 \cdots 0$

$\because 0 \cdots 0 \cdots 0 \cdots 0 \cdots 0$

" $0 \cdots 0 \cdots 0 \cdots 0 \cdots 0$

4. $0 \cdots 0 \cdots 0 \cdots 0 \cdots 0$

" $0 \cdots 0 \cdots 0 \cdots 0 \cdots 0$

15. Welchen Einfluß haben technologische Standardisierungstrends auf die unterschiedlichen Funktionen in Ihrem Unternehmen?

Forschung \& Entwicklung

hoher
Einnull

« O...

Produktdesign

Beschaffung/Einkauf

Produktion

Technischer Kundendienst

n $0 \cdots 0 \cdots 0 \cdots 0 \cdots 0$

स: $0 \cdots 0 \cdots 0 \cdots 0 \cdots 0$

^. $0 \cdots 0 \cdots 0 \cdots 0 \cdots 0$

n: $0 \cdots 0 \cdots 0 \cdots 0 \cdots 0$

Marketing.

* $0 \cdots 0 \cdots 0 \cdots 0 \cdots 0$

Logistik

m $0 \cdots 0 \cdots 0 \cdots 0 \cdots 0$

16. Für wie wichtig halten Sie die Abstimmung des Marketing mit den folgenden Unternehmensbereichen":

Forschung \& Entwicklung

Sehr
wichtig unwichtig

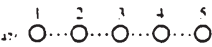

Beschaffung/Einkauf

m $0 \cdots 0 \cdots 0 \cdots 0 \cdots 0$

Produktion

sw $0 \cdots 0 \cdots 0 \cdots 0 \cdots 0$

Technischer Kundendienst

4m $0 \cdots 0 \cdots 0 \cdots 0 \cdots 0$

Controlling

Logistik

Personalwesen

" $0 \cdots 0 \cdots 0 \cdots 0 \cdots 0$

$\because 0 \cdots 0 \cdots 0 \cdots 0 \cdots 0$

«0 $0 \cdots 0 \cdots 0 \cdots 0 \cdots 0$

Finanzierung

w. $0 \cdots 0 \cdots 0 \cdots 0 \cdots 0$

17. Wie wichtig sind die folgenden Mallnahmen zur Verkür. zung der Entwicklungszeiten und zur Beschleunigung der Vermarktung in Ihrem Unternehmen?

Im F\&E-Bereich:

Zukaution Know-how

Erhöhung des Ressourceneinsatzes

Kooperation mit anderen Unter-

nehmen

In der Produktion:

Verringerung der Fertigungstiefe $\quad$, $0 \cdots 0 \cdots 0 \cdots 0 \cdots 0$

Just-in-Time-Beschaffun

Optimierung der Durchlijufzeiten

Im Marketing:

Gilobale Vermirkiung

Konperstion mit Pilotkunden

Erhöhicr Werbedruck

Verriebukooperation

In Organisation und Führung:

Motrutuon der Mitarbetter

Interdisziplinare Team,

Flexibilitat als Untemehmen. grundsacz

Zeiteffizienz als Unternehmensgrundsatz

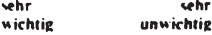

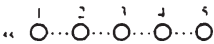

\& $0 \cdots 0 \cdots 0 \cdots 0 \cdots 0$

.. $0 \cdots 0 \cdots 0 \cdots 0 \cdots 0$

, $0 \cdots 0 \cdots 0 \cdots 0 \cdots 0$

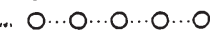

. $0 \cdots 0 \cdots 0 \cdots 0 \cdots 0$

$\therefore 0 \cdots 0 \cdots 0 \cdots 0 \cdots 0$

onomomono

« $0 \cdots 0 \cdots 0 \cdots 0 \cdots 0$

. $0 \cdots 0 \cdots 0 \cdots 0 \cdots 0$

- $0 \cdots 0 \cdots 0 \cdots 0 \cdots 0$

$\therefore 0 \cdots 0 \cdots 0 \cdots 0 \cdots 0$

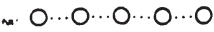


18. Wodurch wird Ihrer Meinung nach der Entscheidungsund Handlungsspielraum der Marketingverantwortlichen eingeschränkt?

Finanzielle Beschränkungen

Personelle Engpässe

Mangelndes Marketingverständnis im Gesamtunternehmen

Zu spate Beteiligung des Marketing am Prozeß der Produktentwicklung
5. Schätzen Sie bitte den Marktanteil des Mlarktführers bzw. der drei Hauptanbieter!
icin Marktführer:
-ri
1\%1. Drei Hauptanbieter: _ri

6. Wie hoch schätzen Sie das Marktwachstum fur die von Innen angebotenen Produkte in den folgenden .larkten?

$$
\begin{aligned}
& \text { 11.:" _ _' Deutschland } \\
& \therefore-: \text { _r Osteuropat } \\
& \because:-\because \text { _ } ; E G \\
& \therefore \text {-in Yri Südostasien } \\
& \because \because \text { _ C LISA }
\end{aligned}
$$

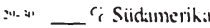

\section{Der Unternehmensbereich}

1. Welchem der folgenden High-Tech-Märkte ist Ihr Linternehmen am ehesten zuzuordnen? (Bitte nur eine Nennung!)

$$
\begin{aligned}
& \square \text { Mikroelektronik } \\
& \text { : } \square \text { Optoclektronik/Lasertechnik } \\
& \text { : } \square \text { Softwareentwicklung } \\
& \text { : } \square \text { Biotechnologie } \\
& \text {. } \square \text { Automatisierungstechnik } \\
& \text {. } \square \text { Nachrichtentechnik } \\
& \text { - } \square \text { Büroautomatisierungstechnik } \\
& \text {. } \square \text { verfahrenstechnik } \\
& \text { " } \square \text { Energietechnik } \\
& \text { ". } \square \text { Umwelttechnik } \\
& \text {. } \square \text { Sonstige: }
\end{aligned}
$$

2. Seit wann ist Ihr Unternehmen in diesem Markt aktiv:?

$$
\text { ain seit } 19
$$

3. Wie würden Sie den Markt kennzeichnen. auf den Ihr Unternehmen schwerpunktmäßig tätig ist?

$$
\begin{aligned}
\because & \square \text { schnell wachsend } \\
& =\square \text { wachsend } \\
& \square \text { stagnierend } \\
+ & \square \text { schrumpfend } \\
& \square \text { schnell schrumpfend }
\end{aligned}
$$

4. Wie schätzen Sie die Produktlebenszeit Ihrer Produkte durchschnittlich ein?

$$
\begin{aligned}
& \text { a) In den letzten } 2 \text { bis } 3 \text { Jahren: } \\
& \text { b) Heute. } \\
& \text { c) In den nächsten } 2 \text { bis } 3 \text { Jahren: } \\
& \text { :- -1. _. Monate }
\end{aligned}
$$

7. Bitte geben Sie an. inwieweit die folgenden Statements fur Ihr Linternehmen zutreffen.

Enge Kundenteziehungen.

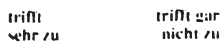
$\therefore \stackrel{1}{0} \cdots \dot{0} \cdots \dot{0} \cdots \dot{0} \cdots$

Weitreichende Kenntnisse der Kundenprobleme.

Grobes Vertrauen in die Mitarheiter.

Hohes MaB an Eigenstindigheit der Mit:urbeiter.

Starke persönliche Einsatzbereitschatt.

Gesundes Maß an Aggressivitit im Angehen von Zielen.

Ausgeprigtc Risikofreudigheit.

Hohe Flexibilitit.

Existenz kreativer Ch:mpions.

Kostenbewußtsein auf allen Stufen.

Kosteneinsparung als zentrales U/nternehmensziel.

Technologie als Mittel zur Profilierung im Markt.

Ausgeprigtes Technologiebewußtsein aut allen Stuten.

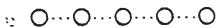$$
: 0 \cdots 0 \cdots 0 \cdots 0 \cdots 0
$$$$
: 0 \cdots 0 \cdots 0 \cdots 0 \cdots 0
$$$$
\therefore 0 \cdots 0 \cdots 0 \cdots 0 \cdots 0
$$$$
\therefore 0 \cdots 0 \cdots 0 \cdots 0 \cdots 0
$$$$
\therefore 0 \cdots 0 \cdots 0 \cdots 0 \cdots 0
$$$$
\therefore 0 \cdots 0 \cdots 0 \cdots 0 \cdots 0
$$$$
\therefore 0 \cdots 0 \cdots 0 \cdots 0 \cdots 0
$$

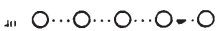$$
\text { s. } 0 \cdots 0 \cdots 0 \cdots 0 \cdots 0
$$$$
\therefore 0 \cdots 0 \cdots 0 \cdots 0 \cdots 0
$$$$
\text { ^: } 0 \cdots 0 \cdots 0 \cdots 0 \cdots 0
$$

8. Wie viele Beschäftigte hat Ihr Unternehmen etwa?

د土d”, zur Zeit

w..s) Plan für 1991

9. Wie viele Mitarbeiter sind in Ihrem Unternehmen mit Marketingaufgaben befaßt?

$\sin x$ Mitarbeiter

s4a das sind __ der Gesamtbeschiaftigten

10. Wie hoch war/ist der (iesamtumsatz Ihres Unternehmens in Millionen DM?

A1-nes 1988:

м⿻- - 19: 1989:

11-3) 1990:

7n-mir Plan 1991: 
11. Können Sie den prozentualen Anteil Ihres Unternehmens am Gesamtumsatz in den jeweiligen Märkten angeben?

".- Deutuchland: _.ir

-.. Oveuropa:

Ci"

w. FC:

-....'

$\therefore$ : Südoslasien:

: 4 i" LSt:

… Siidameriha:

${ }^{\prime \prime \prime}$

12. Wie hoch int das Marketinghudget Ihres Lnternehmens in Prozent des Linsattzes:?

… E E (.). 'i

13. Wic hat es sich in den letzten 3.Jahren entwickelt?

$$
\begin{aligned}
& \text {.... E entall } \\
& \text {, } \square \text { velhr viel hioher } \\
& \text { - } \square \text { hioher } \\
& \text { : } \square \text { gleich hoch } \\
& \text { - }[\text { nicdriger } \\
& \text { - } \square \text { xhr vicl medriger }
\end{aligned}
$$

14. Wie wird sich der fitat in den nächsten Jahren sorausichtlich entwickeln?

$$
\begin{aligned}
& \therefore \square \text { entfillı } \\
& \text { - D varh veigend } \\
& \text { : E' ell a veigend } \\
& \text { [ gleshbleitend } \\
& \text { - } \square \text { erwar vinhend } \\
& \text { - } \square \text { vark vinkend }
\end{aligned}
$$

15. Welches Ansehen hat das Marketing nach Ihrer Einschätang in Ihrem Unternehmen:

$$
\begin{aligned}
& : \because \square \text { enttillt } \\
& \text { - } \square \text { is wr nedrig } \\
& \text { : } E \text { nectrin: } \\
& \text { Emittel } \\
& \text {. Ihexh } \\
& \text { - E vehr hoxh }
\end{aligned}
$$

16. Auf welcher hierarchischen Ebene sind Sie in das Unternehmen eingegliedert?

: $\square$ Geschäftstühnung/Vorstand (1. Ebene)

: $\square$ Bereichsleiter (2. Ebene)

: $\square$ Abteilungsietier (3. Ebene)

. $\square$ Gruppenleiter (t. Ebene)

17. Welche Funktion üben Sie zur Zeit in Ihrem Lnternehmen aus?

$\therefore:$

\section{SOZIALSTATISTIK}

\section{Sind Sie}

$$
\begin{aligned}
\text { :nt } \square \text { miinnlich } \\
: \square \text { weiblich }
\end{aligned}
$$

2. Wann sind Sie geboren?

$$
\because 19
$$

3. Wie hoch ist Ihr jährliches Bruttoeinkommen aus Ihrer beruflichen Tätigkeit incl. aller Zulagen?

$\therefore \square$ unter 80000 DM

: $\square 0000$ bis unter 100000 DM

: $\square$ 100000 bis unter 120000 DM

- $\square$ 120000 bis unter 150000 DM

$\therefore 1.50000$ bis unter $180000 \mathrm{DM}$

- $\square$ 180000 bis unter 200000 DM

- $\square$ darüber —DM

4. Seit wann sind Sie berufstätig?

4..11 Seit 19

5. Seit wann bekleiden Sie Ihre jetzige berufliche Position? 1:-11 Seit 19

6. Auf welchem Weg haben Sie Ihre heutige Position erreicht?

: $\square$ aus dem Marketingbereich Ihres Unternehmens

: $\square$ aus einem anderen Bereich Ihres Unternehmens

: $\square$ aus den Marketingbereich eines anderen Unterneh. mens

, $\square$ aus einem anderen Bereich eines anderen Unternehmens

- $\square$ aus

7. Weiche Ausbildung haben Sie absolviert?

w., $\square$ Lehre mit anschließender Berufspraxis

: $\square$ Studium mit anschliebender Berufspraxis Bei Studium bilte Fachrichtung angeben: 
8. Ihre berufliche Zuständigkeit/Verantwortung erstreckt sich auf

in E das Gesamtuntemehmen

: Eeinen Teilbereich des Untemehmens

: - - ein Produkt / eine spezielle Dienstleistung des Unter. nehmens

9. Welche der folgenden Publikation zählen zu Ihrer Lektüre?

Süddeutsche Zeitung

Frankfurter Allgemeine Zeitung

Die Welt

Handelsblatl

Blick durch die Wirtschaft

Capital

Impulse

hightech

Manager Magazin

Absatzwirtschaft

Der Spiegel

Sonstige: regeimäfig

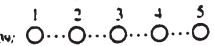

n $0 \cdots 0 \cdots 0 \cdots 0 \cdots 0$

\# $0 \cdots 0 \cdots 0 \cdots 0 \cdots 0$

$\therefore 0 \cdots 0 \cdots 0 \cdots 0 \cdots$

n $0 \cdots 0 \cdots 0 \cdots 0 \cdots 0$

य $0 \cdots 0 \cdots 0 \cdots 0 \cdots 0$

n. $0 \cdots 0 \cdots 0 \cdots 0 \cdots 0$

th $0 \cdots 0 \cdots 0 \cdots 0 \cdots 0$

म $0 \cdots 0 \cdots 0 \cdots 0 \cdots 0$

sm $0 \cdots 0 \cdots 0 \cdots 0 \cdots 0$

sw $0 \cdots 0 \cdots 0 \cdots 0 \cdots 0$

* $0 \cdots 0 \cdots 0 \cdots 0 \cdots 0$

Sollten wir Ihrer Meinung nach wichtige Dinge im Fragebo. gen übersehen haben. so nennen Sie uns diese bitte:

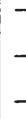

Bei Rückfragen: Tel. (089) 291334

Vielen Dank für Ihre Mitarbeit!

10. Nennen Sie zum Abschluß die aus Ihrer Perspektive wesentlichen Herausforderungen für das High-Tech-Marketing in den Mer Jahren. 


\section{Literaturverzeichnis}

Aaby, N.E., McGann, A.F., Corporate strategy and the role of navigational marketing, in: European Journal of Marketing, Vol. 23 (1989), No. 10, S. 18-31

Aaker, D.A., Strategic Market Management, New York u.a. 1984

Aaker, D.A., Day, G.S., Marketing Research, 3. Aufl., New York u.a. 1986

Aaker, D.A., Kriterien zur Identifikation dauerhafter Wettbewerbsvorteile, in: Wettbewerbsvorteile und Wettbewerbsfähigkeit, Hrsg.: Simon, H., Stuttgart 1988, S. 37-46

Abell, D.F., Defining the business, Englewood Cliffs, N.J. 1980

Albach, H., Strategische Allianzen, strategische Gruppen und strategische Familien, in: ZfB, 1992, Nr. 6, S. 663-670

Albach, H., De Pay, D., Rojas, R., Der Innovationsprozeß bei kulturspezifisch unterschiedlich innovationsfreudigen Konsumenten, in: Innovationsmanagement, Theorie und Praxis im Kulturvergleich, Hrsg.: Albach, H., Wiesbaden 1990, S. 109-129

Albers, S., Eggert, K., Kundennähe - Strategie oder Schlagwort, in: Marketing ZFP, 1988, Nr. 1, S. 5-16

Alpert, F.H., Kamins, M.A., Graham, J.L., An examination of reseller buyer attitudes toward order of brand entry, in: Journal of Marketing, Vol. 56 (1992), S. 25-37

Amel, D.F., Rhoades, St.A., Strategic groups in banking, in: The Review of Economics and Statistics, 1988, No. 4, S. 685-689

Ansoff, H.I., Management-Strategie, München 1966

Ansoff, H.I., Die Bewältigung von Überraschungen und Diskontinuitäten durch die Unternehmensführung - Strategische Reaktionen auf schwache Signale, in: Planung und Kontrolle, Hrsg.: Steinmann, H., München 1981, S. 233-264

Ansoff, H.I., Managing discontinuous strategic change: The learning action approach, in: Understanding and managing strategic change, Hrsg.: Ansoff, H.I., Bosman, A., Storm, P.M., Amsterdam, New York, Oxford 1982, S. 1-31

Ansoff, H.I., Implanting strategic management, Englewood Cliffs, N.J. 1984

Arbeitsgruppe der Industrie des IT-Gesprächskreises "Mikroelektronik", Konsenspapier der Industrie zum Stand der Mikroelektronik in Deutschland mit Vorschlägen zur Sicherung der Wettbewerbsfähigkeit der deutschen Halbleiterunternehmen, deren Kunden und Zulieferer, o.O. 1993, o.S.

Assmus, G., Farley, J.U., Lehmann, D.R., How advertising affects sales: Meta analysis of econometric results, in: Journal of Marketing Research, 1984, S. 65-74

Baaken, Th., Technologie-Marketing, in: Investitionsgütermarketing, Hrsg.: Kliche, M., Wiesbaden 1990, S. 289-309

Baaken, Th., Qualifizierung des Kunden als integrierte Aufgabe im Technologie-Marketing, in: Technologie-Marketing, Hrsg.: Töpfer, A., Sommerlatte, T., Landsberg a.L. 1991, S. 201-220 
Baaken, Th., Launen, M., Software-Marketing, München 1993

Backhaus, K., Die Abgrenzung des relevanten Marktes vor dem Hintergrund kartellrechtlicher Mißbrauchsaufsicht, Arbeitspapier Nr. 18 des Instituts für Unternehmensführung und Untermehmensforschung, Bochum 1979

Backhaus, K., Die Macht der Allianz, in: asw, 1987, Nr. 11, S. 122-130

Backhaus, K., Strategien auf sich verändernden Weltmärkten - Chancen und Risiken, in: DBW, 1989 , Nr. 4, S. 465-481

Backhaus, K., Auswirkungen kurzer Lebenszyklen bei High-Tech-Produkten, in: Thexis, 1991, Nr. 6, S. 11-13

Backhaus, K., Investitionsgütermarketing, 3. Aufl., München 1992

Backhaus, K., Systemgeschäfte verändern Marktprozesse, in: asw, 1992, Nr. 8, S. 60-63

Backhaus, K., Was heißt Investitionsgütermarketing?, Arbeitspapier Nr. 15 des Betriebswirtschaftlichen Instituts für Anlagen und Systemtechnologien, Münster 1992

Backhaus, K., et al., Multivariate Analysemethoden, 7. Aufl., Berlin u.a. 1993

Backhaus, K., Meyer, M., Strategische Allianzen und strategische Netzwerke, in: WiSt, 1993, Nr. 7, S. 330-334

Backhaus, K., Piltz, K., Strategische Allianzen, in: ZfbF, 1989, Sonderheft 27, S. 1-11

Backhaus, K., Späth, G.-M., Applikationen prägen die zum Einsatz kommende Technik: Hochgeschwindigkeits-Netze sind aus Anwendersicht unumgänglich, in: Computerwoche, 1993, Nr. 6, S. 23-24

Backhaus, K., Weiber, R., Systemtechnologien - Herausforderung des Investitionsgütermarketing, in: Harvard Manager, 1987, Nr. 4, S. 70-80

Backhaus, K., Weiber, R., Technologieintegration und Marketing, Arbeitspapier Nr. 10 des Betriebswirtschaftlichen Instituts für Anlagen und Systemtechnologien, Münster 1988

Backhaus, K., Weiss, P.A., Kompetenz - Die entscheidende Dimension im Marketing, in: Harvard Manager, 1989, Nr. 3, S. 107-114

Bahrami, H., Evans, St., Stratocracy in high-technology firms, in: California Management Review, Vol. 30 (1987), S. 51-65

Bain, J.S., Industrial organization, 2. Aufl., New York 1968

Baird, I.S., Sudharshan, D., Strategic groups: A three mode factor analysis of some measures of financial risk, BEBR, Faculty Working Paper No. 931, College of Commerce and Business Administration, University of Illinois at Urbana-Champaign, February 1983

Baird, I.S., Sudharshan, D., Thomas, H., Addressing temporal change in strategic groups analysis: A three-mode factor analysis approach, in: Journal of Management, Vol. 14 (1988), No. 3, S. 425-439

Barnes, J.G., Glynn, W.J., The customer wants service: Why technology is no longer enough, in: Journal of Marketing Management, Vol. 9 (1993), S. 43-53 
Barney, J.B., Hoskisson, R.E., Strategic groups: Untested assertions and research proposals, in: Managerial and Decision Economics, Vol. 11 (1990), S. 187-198

Baubin, Th., Effiziente Wachstumsstrategien der informationstechnischen Industrie, Diss., Vallendar 1990

Bauche, K., Segmentierung von Kundendienstleistungen auf investiven Märkten, Frankfurt a.M. u.a. 1994

Bauer, F., Datenanalyse mit SPSS, Berlin u.a. 1984

Bauer, H.H., Marktabgrenzung: Konzeption und Problematik von Ansätzen und Methoden zur Abgrenzung und Strukturierung von Märkten unter besonderer Berücksichtigung von marketingtheoretischen Verfahren, Berlin 1989

Bauer, H.H., Unternehmensstrategie und Strategische Gruppen, in: Unternehmensdynamik, Hrsg.: Kistner, K.-P., Schmidt, R., Wiesbaden 1991, S. 389-416

Baum, H., Der relevante Markt als Problem der Wettbewerbspolitik, in: Wirtschaft und Wettbewerb, 1980, Nr. 6, S. 401-407

Becker, J., Marketing-Konzeption, Grundlagen des strategischen Marketing-Management, 5. Aufl., München 1992

Beckmann, P., Die Abgrenzung des relevanten Marktes im Gesetz gegen Wettbewerbsbeschränkungen, Bad Homburg 1968

Beckurts, K.H., Chancen für einen zweiten Aufbruch, in: Manager Magazin, 1984, Nr. 9, S. 154-167

Bender, H.O., High-Tech-Marketing, in: Industrial marketing - A German - American perspective, Hrsg.: Backhaus, K., Wilson, D.T., Berlin u.a. 1986, S. 191-224

Bender, H.O., Das Marketing von Technologieprodukten, in: asw, 1988, Nr. 8, S. 116-121

Benkenstein, M., F\&E und Marketing, Wiesbaden 1987

Benkenstein, M., Modelle technologischer Entwicklungen als Grundlage für das Technologiemanagement, in: DBW, 1989, Nr. 4, S. 497-509

Benkenstein, M., High Tech-Marketing, in: DBW, 1990, Nr. 3, S. 397-398

Benkenstein, M., Strategisches Marketing-Management in High-Tech-Branchen, in: Thexis, 1992, Nr. 1, S. 8-14

Benkenstein, M., Die Reduktion der Fertigungstiefe als betriebswirtschaftliches Entscheidungsproblem, unver. Habil., Münster 1992

Benkenstein, M., Integriertes Innovationsmanagement - Ansatzpunkte zum "lean innovation", in: Marktforschung und Management, 1993, Nr. 1, S. 21-25

Benkenstein, M., Technikfolgenabschätzung, in: DBW, 1993, Nr. 3, S. 423-424

Benkenstein, M., Innovationsmanagement in High-Tech-Branchen, Freiräume für Pioniere, in: asw, 1993, Nr. 6, S. 102-107 
Benkenstein, M., Henke, N., Vertikale Integration - Eine transaktionskostentheoretische Interpretation, Arbeitspapier Nr. 39 des Instituts für Marketing, Hrsg.: Meffert, H., Münster 1990

Berekoven, L., Eckert, W., Ellenrieder, P., Marktforschung, Methodische Grundlagen und praktische Anwendung, 4. Aufl., Wiesbaden 1989

Bergs, S., Optimalität bei Cluster-Analysen, Diss., Münster 1981

Beuthner, A., Gerangel um Massenmarkt und Wettlauf der Systeme: Nach acht Jahren Kampf immer noch kein Sieger, in: Computer Zeitung, 1993, Nr. 32 + 33, S. 17

Beuttel, W., Marketingstrategien in schnell wachsenden Märkten, in: Handbuch Strategisches Marketing, Hrsg.: Wieselhuber, N., Töpfer, A., Landsberg a.L. 1984, S. 308-318

Birkelbach, R., Strategische Geschäftsfeldplanung im Versicherungssektor, in: Marketing ZFP, 1988, Nr. 3, S. 231-239

Birkelbach, R., Qualitätsmanagement in Dienstleistungscentern, Frankfurt a.M. u.a. 1993

Bittner, L., Ursachen und Folgen der steigenden Bedeutung des DV-Service, in: SoftwareMarketing, Hrsg.: Wimmer, F., Bittner, L., Wiesbaden 1992, S. 159-189

Bleicher, K., Zum Verhältnis von Kulturen und Strategien der Unternehmung, in: Organisationskultur: Phänomen - Philosophie - Technologie, Hrsg.: Dülfer, E., Stuttgart 1988, S. $95-112$

Bleicher, K., Chancen für Europas Zukunft, Wiesbaden 1989

Bleicher, K., Organisation: Strategien, Strukturen, Kulturen, 2. Aufl., Wiesbaden 1990

Bleymüller, J., Multivariate Analyse für Wirtschaftswissenschaftler, unver. Manuskript, Münster 1989

Bleymüller, J., Gehlert, G., Gülicher, H., Statistik für Wirtschaftswissenschaftler, 5. Aufl., München 1988

BMFT (Hrsg.), Deutscher Delphi-Bericht zur Entwicklung von Wissenschaft und Technik, Bonn 1993

Böbel, I., Wettbewerb und Industriestruktur, Berlin u.a. 1984

Böcker, F., Marketing, 5. Aufl., Stuttgart 1994

Boeker, W.P., Strategic change - The effects of founding and history, in: Academy of Management Journal, Vol. 32 (1989), No. 3, S. 489-515

Böndel, B., Innovationsklima: Ära der Systeme, in: Wirtschaftswoche, 1994, Nr. 3, S. 70-72

Bogner, W.C., Thomas, H., The role of competitive groups in strategy formulation: A dynamic integration of two competiting models, in: Journal of Management Studies, Vol. 30 (1993), No. 1, S. 51-67

Bolz, J., Wettbewerbsorientierte Standardisierung der internationalen Marktbearbeitung, Darmstadt 1992

Bourgeois, L.J., Strategy and environment - A conceptional integration, in: The Academy of Management Review, Vol. 5 (1980), No. 1, S. 25-39 
Bourgeois, L.J., Eisenhardt, K.M., Strategic decision processes in high velocity environments: Four cases in the microcomputer industry, in: Management Science, Vol. 34 (1988), No. 7, S. 816-835

Braunschweig, E., Typen als Zielgruppen-Kriterien, in: "Typologien", Hrsg.: Heinrich Bauer Stiftung, Velbert 1975, S. 7-21

Brezski, E., Konkurrenzforschung im Marketing, Wiesbaden 1993

Brockhoff, K., Forschung und Entwicklung, 2. Aufl., München, Wien 1989

Brogsitter, B., Kundennutzen - Herzstück der ganzheitlichen Firmenstrategie, in: Harvard Manager, 1988, Nr. 4, S. 113-119

Bronder, Ch., Kooperationsmanagement, Unternehmensdynamik durch strategische Allianzen, Frankfurt a.M., New York 1993

Brosius, G., SPSS/PC+ Advanced Statistics und Tables, Hamburg, New York 1989

Bruhn, M., Das soziale Bewußtsein von Konsumenten, Wiesbaden 1978

Bruhn, M., Sponsoring als Instrument der Unternehmenskommunikation, Erscheinungsformen, Planungskonzepte und Integrationsaspekte, in: Sponsoring, Dokumentationspapier Nr. 55 der Wissenschaftlichen Gesellschaft für Marketing und Unternehmensführung e.V., Hrsg.: Meffert H., Wagner, H., Münster 1989

Brunken, A., Wettbewerbsstrategien in der mittelständischen Brauwirtschaft, Diss., Berlin 1991

Bühner, R., Technologieorientierung als Wettbewerbsstrategie, in: ZfbF, 1988, Nr. 5, S. 387 406

Bühner, R., Betriebswirtschaftliche Organisationslehre, 4. Aufl., München, Wien 1991

Büker, B., Qualitätsbeurteilung investiver Dienstleistungen, Operationalisierungsansätze an einem Beispiel zentraler EDV-Dienste, Frankfurt a.M. u.a. 1991

Bullinger, H.-J., Wasserloos, G., Der Wettbewerbsfaktor Zeit muß konsequent genutzt werden, Die Stellschraube anziehen, in: Computerwoche vom 12.04.91, S. 10-11

Buschmann, E., et al., Der Software-Markt in der Bundesrepublik Deutschland, Hrsg.: Gesellschaft für Mathematik und Datenverarbeitung, GMD-Studie Nr. 167, St. Augustin 1989

Buzzell, R.D., Gale, B.T., Das PIMS-Programm, Strategien und Unternehmenserfolg, Wiesbaden 1989

Carpenter, G.S., Nakamoto, K., Competitive strategies for late entry into a market with a dominant brand, in: Management Science, Vol. 36 (1990), No. 10, S. 1268-1278

Carroll, Ch., Lewis, P., Thomas, H., Developing competitive strategies in retailing, in: Long Range Planning, Vol. 25 (1992), No. 2, S. $81-88$

Caves, R.E., Ghemawat, P., Identifying mobility barriers, in: Strategic Management Journal, Vol. 13 (1992), S. 1-12 
Caves, R.E., Porter, M., From entry barriers to mobility barriers: Conjectural decisions and contrived deterrence to new competition, in: Quarterly Journal of Economics, Vol. 91 (1977), S. 241-261

Caves, R.E., Pugel, Th., Intra-Industry differences in conduct and performance: Viable strategies in U.S. manufacturing industries, New York 1980

Chandler, A.D. Jr., Strategy and structure, Chapters in the history of industrial enterprise, Cambridge, Mass., London 1962

Conant, J.S., Mokwa, M.P., Varadarajan, R.P., Strategic types, distinctive marketing competencies and organizational performance: a multiple measures-based study, in: Strategic Management Journal, Vol. 11 (1990), S. 365-383

Cool, K.O., Schendel, D., Strategic group formation and performance: The case of the U.S. Pharmaceutical Industry, 1963-1982, in: Management Science, Vol. 33 (1987), No. 9, S. $1102-1124$

Cool, K.O., Schendel, D., Performance differences among strategic group members, in: Strategic Management Journal, Vol. 9 (1988), S. 207-223

Cool, K.O., Dierickx, I., Rivalery, Strategic Groups and firm profitability, in: Strategic Management Journal, Vol. 14 (1993), S. 47-59

Cooper, R.G., Project new products: Factors in new product success, in: European Journal of Marketing, 1980, No. 5-6, S. 277-292

Cooper, R.G., New product strategies: What distinguishes the top performers, in: Journal of Product Innovation Management, 1984, No. 2, S. 151-164

Cooper, R.G., Overall corporate strategies for new product programms, in: Industrial Marketing Management, Vol. 14 (1985), S. 179-193

Cooper, R.G., Predevelopment activities determine new product success, in: Industrial Marketing Management, Vol. 17 (1988), S. 237-247

Cox, H., Hübner, H., Entwicklung und Stand der wettbewerbstheoretischen Diskussion, in: Handbuch des Wettbewerbs, Hrsg.: Cox, H., Jens, U., Markert, K., München 1981, S. 12-37

Crawford, J.D., New products management, Homewood, Ill. 1983

Czepiel, J.A., Competitive marketing strategy, Englewood Cliffs, N.J. 1992

Davidow, W.H., Marketing High Technology, An Insider's View, New York, London 1986

Davis, R.T., Smith, F.G., Marketing in emerging companies, Reading, Mass. 1984

Day, G.S., Wensley, R., Assessing advantage: A framework for diagnosing competitive superiority, in: Journal of Marketing, Vol. 52 (1988), No. 2, S. 1-20

De Pay, D., Kulturspezifische Determinanten der Organisation von Innovationsprozessen, in: Innovationsmanagement: Theorie und Praxis im Kulturvergleich, Hrsg.: Albach, H., Wiesbaden 1990, S. 131-175

Degens, P.O., Hermes, H.J., Opitz, O. (Hrsg.), Die Klassifikation und ihr Umfeld, Frankfurt a.M. 1986 
Dellmann, K., Einflußfaktoren der Erfolgsdynamik, in: Unternehmensdynamik, Hrsg.: Kistner, K.-P., Schmidt, R., Wiesbaden 1991, S. 419-442

Deshpandé, R., Parasuraman, A., Linking corporate culture to strategic planning, in: Business Horizons, 1986, No. 3, S. 28-37

Dess, G.G., Davis, P.S., Porter's (1980) generic strategies as determinants of strategic group membership and organizational performance, in: Academy of Management Journal, Vol. 27 (1984), No. 3, S. 467-488

Deutsch, Chr., Reiner Selbstmord, in: Wirtschaftswoche, 1993, Nr. 21, S. 58-60

Dichtl, E., Produktauslagerung und Fertigungstiefe als Determinanten der Wertschöpfung, in: Marketing-Schnittstellen: Herausforderungen für das Management, Hrsg.: Specht, G., Silberer, G., Engelhardt, W.H., Stuttgart 1989, S. 87-102

Diller, H., Preisbaukästen als preispolitische Option, in: WiSt, 1993, Nr. 6, S. 270-275

Diller, H., Lücking, M.A., Kaffenberger, A., Das "Schicksal" von Marktfuihrern, Eine empirische Analyse in 29 Märkten für technische Gebrauchsgüter, Arbeitspapier Nr. 26 des Lehrstuhls für Marketing, Erlangen-Nürnberg 1993

Domsch, M., Gerpott, T., Akzeptanz von Zeitkontrollen in der industriellen Forschung und Entwicklung, in: ZfbF, 1988, Sonderheft Nr. 23, S. 86-111

Douglas, S.P., Rhee, D.K., Examining generic competitive strategy types in U.S. and European markets, in: Journal of International Business Studies, Vol. 20 (1989), No. 3, S. 437-463

Doyle, P., Holley, G.J., Strategic orientation and corporate performance, in: International Journal of Research in Marketing, Vol. 9 (1992), S. 59-73

Drexel, G., Strategisches Marketing in der Praxis, in: Die Unternehmung, 1984, Nr. 2, S. 101 119

Drazin, R., Kazanjian, R.K., A reanalysis of Miller and Friesen's life cycle data, in: Strategic Management Journal, 1990, No. 4, S. 319-325

Drazin, R., Van de Ven, A., Alternative forms of fit in contingency theory, in: Administrative Science Quarterly, Vol. 30 (1985), No. 4, S. 514-559

Drucker, P., The age of discontinuity, New York 1968

Dunn, D.T., Jr., Friar, J.H., Thomas, C.A., An approach to selling high-tech solutions, in: Industrial Marketing Management, Vol. 20 (1991), S. 149-159

Dussauge, P., Hart, St., Ramanantsoa, B., Strategic technology management, Chichester u.a. 1992

Dvir, D., Segev, E., Shenhar, A., Research notes and communications - Technology's varying impact on the success of strategic business units within the miles and snow typology, in: Strategic Management Journal, Vol. 14 (1993), S. 155-161

Dziuban, C.D., Shikey, E.C., When is a correlation matrix appropriate for factor analysis?, in: Psychological Bulletin, Vol. 81 (1974), S. 358-361 
Easingwood, Ch., Beard, Ch., High technology launch strategies in the U.K., in: Industrial Marketing Management, Vol. 18 (1989), S. 125-138

Eisele, R., Industriemärkte im Wandel - Auswirkungen auf die Unternehmensführung, in: Marketing-Schnittstellen: Herausforderungen für das Management, Hrsg.: Specht, G., Silberer, G., Engelhardt, W.H., Stuttgart 1989, S. 327-334

Engelhardt, W.H., Herausforderungen des Total Quality Management aus wettbewerbsorientierter Sicht, in: Kosten, Qualität und Zeit als Wettbewerbsvorteile, Was bringt Total Quality Management?, Dokumentationspapier Nr. 75 der Wissenschaftlichen Gesellschaft für Marketing und Unternehmensführung e.V., Hrsg.: Meffert H., Wagner, H., Backhaus, K., Münster 1992, S. 21-32

Englert, W.H., Marketing von Standard-Anwendungssoftware, München 1977

EPA (Hrsg.), Technologieprognose für das Jahr 2010, Tokio 1992

Ewald, A., Organisation des strategischen Technologie-Managements, Stufenkonzept zur Implementierung einer integrierten Technologie- und Marktplanung, Berlin 1989

Faselow, K.-H., Stedler, H., Venture Capital in Deutschland, in: Die Bank, 1988, S. 555-560

Faulkner, D., Bowman, C., Generic strategies and congruent organizational structures: Some suggestions, in: European Management Journal, Vol. 10 (1992), S. 494-498

Faust, K., Technologische Wettbewerbsposition im Licht der Ifo-Patentstatistik, in: IfoSchnelldienst, 1992, Nr. 32, S. 11-20

Felser, G., Kein Fall für Hacker, Gefährlicher Computermüll: Gifte von Arsen bis Zinn, in: Natur und Umwelt, 1994, Nr. 2, S. 16-19

Fiegenbaum, A., Primeaux, W.J., Strategic groups and mobility barriers: The level of struggle in an industry, BEBR, Faculty Working Paper No. 984, College of Commerce and Business Administration, University of Illinois at Urbana-Champaign, November 1983

Fiegenbaum, A., Primeaux, W.J., An empirical examination of the dynamics of strategic groups, BEBR, Faculty Working Paper No. 1108, College of Commerce and Business Administration, University of Ilinois at Urbana-Champaign, January 1985

Fiegenbaum, A., Thomas, H., Strategic Groups and Performance: The U.S. Insurance Industry, 1970-84, in: Strategic Management Journal, Vol. 11 (1990), S. 197-215

Fischer, G., Schwarzer, U., Außer Atem, in: Manager Magazin, 1992, Nr. 11, S. 242-253

Flaherty, M.T., Market-Share, technology leadership and competition in international semiconductor markets, in: Research on technological innovation, management and policy, Hrsg.: Rosenbloom, R.S., Greenwich, Conn. 1983, S. 69-102

Fraunhofer Institut für Systemtechnik und Innovationsforschung (Hrsg.), Technologie am Beginn des 21. Jahrhunderts, Karlsruhe 1993

Frese, E., Grundlagen der Organisation, Konzepte - Prinzipien - Strukturen, 5. Aufl., Wiesbaden 1993

Freter, H., Marktsegmentierung, Stuttgart u.a. 1983

Freeman, E.R., Stratecic management, A stakeholder approach, Boston u.a. 1984 
Fritz, W., Warentest und Konsumgüter-Marketing, Wiesbaden 1984

Fritz, W., Marketing - ein Schlüsselfaktor des Unternehmenserfolges?, in: Marketing ZFP, 1990, Nr. 2, S. 91-109

Fritz, W., Ansätze der Wettbewerbstheorie aus Sicht der Marketingwissenschaft, in: DBW, 1990, Nr. 4, S. 491-512

Fritz, W., Marktorientierte Unternehmensführung und Unternehmenserfolg, Stuttgart 1992

Fritz, W., Marktorientierte Unternehmensfuihrung und Unternehmenserfolg, in: Marketing ZFP, 1993, Nr. 4, S. 237-246

Fritz, W., et al., Unternehmensziele in Industrie und Handel, in: DBW, 1988, Nr. 4., S. 375394

Fröhling, O., Dynamisches Kostenmanagement, Konzeptionelle Grundlagen und praktische Umsetzung im Rahmen eines strategischen Kosten- und Erfolgs-Controlling, München 1994

Frohmann, A.L., Technologie als Waffe im Wettbewerb, in: Harvard Manager, 1984, Nr. 4, S. 62-69

Fronhoff, B., Die Gestaltung von Marketingstrategien, Köln 1986

Fry, L.W., Smith, D.A., Congruence, contingency and theory building, in: Academy of Management Review, Vol. 12 (1987), No. 1, S. 117-132

Fulmer, W., Fulmer, R., Strategic group technique - involving managers in strategic planning, in: Long Range Planning, Vol. 23 (1990), No. 2, S. 79-84

Gabele, E., Einführung in die Geschäftsfeldorganisation, Tübingen 1981

Gabele, E., Kretschmer, H., Unternehmensgrundsätze, Zürich 1986

Gahl, A., Die Konzeption strategischer Allianzen, Berlin 1991

Gaitanides, M. Vertikale und horizontale Erfolgsquellen im Branchenvergleich - Befunde zweier empirischer Studien, in: Zeitschrift für Planung, 1991, Nr. 2, S. 87-103

Gaitanides, M., Westphal, J., Strategische Gruppen und Unternehmenserfolg - Ergebnisse einer empirischen Studie, in: Zeitschrift für Planung, 1991, Nr. 3, S. 247-265

Galbraith, C., Schendel, D., An empirical analysis of strategy types, in: Strategic Management Journal, Vol. 4 (1983), S. 153-173

Garud, R., Kumaraswamy, A., Changing competitive dynamics in network industries: An exploration of sun microsystems' open systems strategy, in: Strategic Management Journal, Vol. 14 (1993), S. 351-369

Garvin, D.A., Die acht Dimensionen der Produktqualität, in: Harvard Manager, 1988, Nr. 3, S. 66-74

Geroski, P., Gilbert, R., Jacquemin, A., Barriers to entry and strategic competition, Chur 1990

Gerpott, T., Wittkemper, G., Verkürzung der Produktentwicklungszeiten, in: Integriertes Technologie- und Innovationsmanagement, Konzepte zur Stärkung der Wettbewerbs- 
kraft von High-Tech-Unternehmen, Hrsg.: Booz, Allen \& Hamilton, Berlin 1991, S. 119-145

Gerstenberger, W., Zur Wettbewerbsposition der deutschen Industrie im High-Tech-Bereich, in: Ifo-Schnelldienst, 1992, Nr. 13, S. 14-23

Ghemawat, P., Dauerhafte Wettbewerbsvorteile aufbauen, in: Harvard Manager, 1987, Nr. 2, S. $104-108$

Gierl, H., Kotzbauer, N., Der Einfluß des F\&E-Aufwandes auf den wirtschaftlichen Erfolg von Industrieunternehmen, in: ZfbF, 1992, Nr. 11, S. 974-989

Görgen, W., Strategische Wettbewerbsforschung, Bergisch Gladbach, Köln 1992

Görgen, W., Van Kerkom, K., Der Wechsel der Wettbewerbsstrategie - Eine kritische Analyse der Bestimmungsfaktoren und Maßnahmen, Arbeitspapier des Instituts für Markt- und Distributionsforschung, Köln 1991

Gottschlich, W., Strategische Führung in mittleren Unternehmen, Frankfurt a.M. 1989

Green, K., Creating demand for biotechnology, in: Technological change and company strategies, Hrsg.: Coombs, R., Saviotti, P., Walsh, V., London u.a. 1992, S. 164-184

Greening, T., Diversification, strategic groups and the structure-conduct-performance relationship, in: Review of Economics and Statistics, Vol. 62 (1980), S. 475-477

Große-Oetringhaus, W., Mega- und Supratechnologien: Kundenorientierte Wege zum Verständnis und zur Gestaltung des Technologiewandels, in: Technologie-Marketing, Hrsg.: Töpfer, A., Sommerlatte, T., Landsberg a.L. 1991, S. 113-140

Große-Oetringhaus, W., Internationale Marketingstrategien von Groß-Unternehmen in den Investitionsgüter- und High-Tech-Märkten, in: Investitionsgüter- und High-TechMarketing, Hrsg.: Hofmaier, R., Landsberg a.L. 1992, S. 25-44

Habib, M.M., Victor, B., Strategy, structure and performance of U.S. Manufacturing and Service MNCs: A comparative analysis, in: Strategic Management Journal, Vol. 12 (1991), S. 589-606

Haedrich, G., Entwicklung von Marketingstrategien, in: Marketing ZFP, 1983, Nr. 3, S. 175180

Haedrich, G., Tomczak, Th., Strategische Markenführung, Berlin, Stuttgart 1990

Hall, R., The strategic analysis of intangible resources, in: Strategic Management Journal, Vol. 13 (1992), S. 135-144

Hambrick, D.C., Some tests of the effectiveness and functional attributes of miles and snow's strategic types, in: Academy of Management Journal, Vol. 26 (1993), No. 1, S. 5-26

Hammann, P., Erichson, B., Marktforschung, 2. Aufl., Stuttgart, New York 1990

Hannig, U., Die Entwicklung wettbewerbsorientierter Marketingstrategien auf Basis des Konzeptes der strategischen Gruppen, Frankfurt a.M. u.a. 1993

Hanser, P., Marketing-Outsourcing, Schlankheitskur mit Risiko, in: asw, 1993, Nr. 8, S. 34-39

Harrigan, K.R., An application of clustering for strategic group analysis, in: Strategic Management Journal, Vol. 6 (1985), S. 55-73 
Hartung, J., Statistik, München, Wien 1989

Hatten, K.J., Strategic models in the brewing industry, Diss., Purdue University 1974

Hatten, K.J., Hatten, M.L., Strategic groups, asymmetrical mobility barriers and contestability, in: Strategic Management Journal, Vol. 8 (1987), S. 329-342

Hatten, K.J., Schendel, D.E., Heterogenity within industry, in: Journal of Industrial Economics, Vol. 26 (1977), No. 2, S. 97-113

Hatten, K.J., Schendel, D.E., Cooper, A.C., A strategic model of the U.S. brewing industry: 1952-1971, in: Academy of Management Journal, Vol. 21 (1978), No. 4, S. 592-610

Hauschildt, J., Innovationsstrategien und ihre organisatorischen Konsequenzen, in: Strategieentwicklung, Hrsg.: Riekhof, H.-Ch., Stuttgart 1989, S. 255-270

Hawes, J.M., Crittenden, W.F., A taxonomy of competitive retailing strategies, in: Strategic Management Journal, Vol. 5 (1984), S. 275-287

Hax, A.C., Majluf, N.S., Strategisches Management, Ein integratives Konzept aus dem MIT, Frankfurt a.M., New York 1991

Heinemann, G., Betriebstypenprofilierung und Erlebnishandel, Wiesbaden 1989

Heinen, E., Das Zielsystem der Unternehmung, Wiesbaden 1966

Heinen, E., Grundlagen betriebswirtschaftlicher Entscheidungen, Das Zielsystem der Unternehmung, 3. Aufl., Wiesbaden 1976

Heinen, E., Einführung in die Betriebswirtschaftslehre, 9. Aufl., Wiesbaden 1986

Heinen, E., Unternehmenskultur als Gegenstand der Betriebswirtschaftslehre, in: Unternehmenskultur, Perspektiven für Wissenschaft und Praxis, Hrsg.: Heinen, E., München, Wien 1987 , S. $1-48$

Henkel, H.O., Serviceleistungen sind eigenständige Produkte, in: FAZ vom 07.07.1993, S. B6

Henzler, H., Europreneurs: Europas Unternehmen melden sich zurück, Frankfurt a.M. 1992

Herbert, Th., Deresky, H., Generic strategie's: An empirical investigation of typology validity and strategy content, in: Strategic Management Journal, Vol. 8 (1987), S. 135-147

Herdzina, K., Wirtschaftliches Wachstum, Strukturwandel und Wettbewerb, Berlin 1981

Hergert, M., Causes and consequences of strategic grouping in U.S. Manufacturing Industries, in: International Studies of Management and Organization, Vol. 18 (1987), No. 1, S. 26-49

Herzig, H., Outsourcing - Chancen und Grenzen, in: Lean Marketing, Hrsg.: Geyer, D., Bauer, A., Landsberg a.L. 1993, S. 167-187

HeB, G., Marktsignale und Wettbewerbsstrategie, Stuttgart 1991

Heß, G., Kampf um den Standard!, Erfolgreiche und gescheiterte Standardisierungsprozesse Fallstudien aus der Praxis, Stuttgart 1993

Hildebrandt, L., Wettbewerbssituation und Unternehmenserfolg, Empirische Analysen, in: ZfB, 1992, Nr. 10, S. 1069-1084 
Hilker, J., Marketingimplementierung, Konzeptionelle Analysen und Umsetzung am Beispiel ostdeutscher Unternehmen, Diss., Münster 1993

Hill, C., Differentiation versus low cost or differentiation and low cost: A contingency framework, in: Academy of Management Review, Vol. 13 (1988), S. 401-412

Hinterhuber, H., Wettbewerbsstrategie, 2. Aufl., Berlin, New York 1990

Hinterhuber, H., Kirchebner, M., Die Analyse strategischer Gruppen von Unternehmungen, in: ZfB, 1983, Nr. 9, S. 854-868

Höft, U., Lebenszykluskonzepte, Grundlage für das strategische Marketing- und Technologiemanagement, Berlin 1992

Hofer, C.W., Schendel, D., Strategy Formulation: Analytical Concepts, St. Paul 1978

Hoffmann, K., Wolff, V., Zur Systematik von Absatzstrategien als Grundlage langfristig wirkender Entscheidungen im Absatzbereich, in: Jahrbuch der Absatz- und Verbrauchsforschung, 1977, S. 161-175

Hofmaier, R., Das "Magische Viereck" strategischen I+T-Marketings als Basis eines ganzheitlichen I+T-Marketingansatzes, in: Investitionsgüter- und High-Tech-Marketing, Hrsg.: Hofmaier, R., Landsberg a.L. 1992, S. 73-93

Hofmaier, R., Ein systematischer I+T-Marketing-Gesamtansatz - Vom klassischen Verkaufszum Produktmanagement hin zum "integrierten" Segment- und Erfolgspotentialmanagement, in: Investitionsgüter- und High-Tech-Marketing, Hrsg.: Hofmaier, R., Landsberg a.L. 1992, S. 141-172

Holley, G.J., Lynch, J.E., Jobber, D., Generic marketing strategies, in: International Journal of Research in Marketing, Vol. 9 (1992), S. 75-89

Homburg, Ch., Wettbewerbsanalyse mit dem Konzept der strategischen Gruppen mit einem Beispiel aus dem Maschinenbau, in: Marktforschung \& Management, 1992, Nr. 2, S. 83-87

Homburg, Ch., Sütterlin, St., Strategische Gruppen: Ein Survey, in: ZfB, 1992, Nr. 6, S. 635662

Horst, B., Ein mehrdimensionaler Ansatz zur Segmentierung von Investitionsgütermärkten, Diss., Köln 1988

Howell, R.D., Frazier, G.L., Business definition and performance, in: Journal of Marketing, Vol. 47 (1983), S. 59-67

Hrebiniak, L.G., Joyce, W.F., Organizational adaption: strategic choice and environmental determinism, in: Administrative Science Quarterly, September 1985, S. 336-349

Hunt, M.S., Competition in the major home appliance industry, Diss., Harvard University 1972

Hurry, D., Miller, A.T., Bowmann, E.H., Calls on high-technology: Japanese exploration of venture capital investments in the united states, in: Strategic Management Journal, Vol. 13 (1992), S. 85-101

Illing, A., Konkurrenzanalyse mit Hilfe multivariater Klassifikation, Frankfurt a.M. 1980

ISI (Hrsg.), Technologie am Beginn des 21. Jahrhunderts, Karlsruhe 1993 
Itami, H., Numagami, T., Dynamic interaction between strategy and technology, in: Strategic Management Journal, Vol. 13 (1992), S. 119-135

Jacobs, F., Produktinnovation bei komplexer Technologie, Organisationstheoretische Analyse der Bedingungen und Prozesse von Produktinnovationen auf der Grundlage einer empirischen Untersuchung der Branche "Bürokommunikation", Frankfurt a.M. u.a. 1988

Jain, S.C., Marketing planning and strategy, Cincinnati, OH 1985

Jehle, E., Unternehmung und gesellschaftliche Umwelt, Stuttgart 1980

Joas, A., Konkurrenzforschung als Erfolgspotential im strategischen Marketing, Augsburg 1990

Jörg, G., Die Abstimmung zwischen Zielgruppenkampagnen und Marktkommunikation am Beispiel eines ausgewählten Informationstechnologie-Anbieters, in: Investitionsgüterund High-Tech-Marketing, Hrsg.: Hofmaier, R., Landsberg a.L. 1992, S. 407-417

Kaas, K.P., Marketing-Mix, in: Vahlens Großes Marketinglexikon, Hrsg.: Diller, H., München 1992, S. $682-686$

Kaiser, H.F., A second generation little jiffy, in: Psychometrica, Vol. 35 (1970), S. 401-415

Kaiser, H.F., Rice, J., Mark IV, in: Educational and Psychological Measurement, Vol. 34 (1974), S. 111-117

Kaluza, B., Klenter, G., Zeit als strategischer Erfolgsfaktor von Industrieunternehmen, Teil II: Erfolgskritische Komponenten des strategischen Erfolgsfaktors Zeit, Diskussionsbeitrag Nr. 176 des Fachbereichs Wirtschaftswissenschaft, Duisburg 1993

Karnani, A., Generic competitive strategies - an analytical approach, in: Strategic Management Journal, Vol. 5 (1984), No. 4, S. 367-380

Kaufer, E., Industrieökonomik, München 1980

Kerin, R.A., Varadarajan, P.R., Peterson, R.A., First-mover advantage: A synthesis, conceptual framework and research propositions, in: Journal of Marketing, Vol. 56 (1992), S. 33-52

Kern, W., Hagemeister, St., Konzeption und Problematik der Clusteranalyse bei betriebswirtschaftlichen Anwendungen, in: WISU, 1986, Nr. 2, S. 29-36

Kieser, A., Unternehmenskultur und Innovation, in: Unternehmenskultur und Unternehmensführung, Dokumentationspapier Nr. 39 der Wissenschaftlichen Gesellschaft für Marketing und Unternehmensfuihrung e.V., Hrsg.: Meffert H., Wagner, H., Münster 1987, S. 20-26

Kieser, A., Der situative Ansatz, in: Organisationstheorien, Hrsg.: Kieser, A., Stuttgart, Berlin, Köln 1993, S. 161-191

Kieser, A., Kubicek, H., Organisation, 3. Aufl., Berlin, New York 1993

Kim, L., Lim, Y., Environment, generic strategies and performance in rapidly developing country: a taxonomic approach, in: Academy of Management Journal, Vol. 31 (1988), S. $802-827$ 
Kirchgeorg, M., Ökologieorientiertes Unternehmensverhalten, Wiesbaden 1990

Kleinaltenkamp, M., Der Einfluß der Normung und Standardisierung auf die Diffusion technischer Innovationen, in: "Neue Informationstechnologien von CIM-Systemen auf der Basis teilautonomer flexibler Fertigungsstrukturen", Arbeitspapier Nr. 187 des SFB, Bochum 1990

Kloock, J., Sabel, H., Schuhmann, W., Die Erfahrungskurve in der Unternehmenspolitik, in: ZfB, 1987, Ergänzungsheft Nr. 2, S. 3-51

Knoblich, H., Die typologische Methode in der Betriebswirtschaftslehre, in: WiSt, 1972, Nr. 4, S. 141-147

Knorr, J., High-Tech-Marketing in der Mikroelektronik: Erfahrungen aus dem Mega-Projekt, in: Technologie-Marketing, Hrsg.: Töpfer, A., Sommerlatte, T., Landsberg a.L. 1991, S. $325-343$

Kodama, F., Technologiefusion - der Weg zum Erfolg auf Zukunftsmärkten, in: Harvard Business Manager, 1993, Nr. 1, S. 41-48

Köhler, R., Beiträge zum Marketing-Management, 2. Aufl., Stuttgart 1991

Kolbe, C., Eintrittsbarrieren und die Eintrittsfähigkeit potentieller Konkurrenten, Göttingen 1991

Kopp, M., Hypothesenformulierung in der Absatzforschung, Berlin 1972

Kotler, Ph., Bliemel, F., Marketing-Management, 7. Aufl., Stuttgart 1992

Kreikebaum, H., Strategische Unternehmensplanung, 3. Aufl., Stuttgart u.a. 1989

Kreilkamp, E., Strategisches Management und Marketing, Berlin, New York 1987

Kroeber-Riel, W., Konsumentenverhalten, 5. Aufl., München 1992

Krups, M., Marketing innovativer Dienstleistungen am Beispiel elektronischer Wirtschaftsinformationsdienste, Frankfurt a.M. u.a. 1985

Kubicek, H., Heuristischer Bezugsrahmen und heuristisch angelegte Forschungsdesigns als Elemente einer Konstruktionsstrategie empirischer Forschung, in: Empirische und handlungstheoretische Forschungskonzeption in der Betriebswirtschaftslehre, Hrsg.: Köhler, R., Stuttgart 1977, S. 3-36

Kühn, R., Methodische Überlegungen zum Umgang mit der Kundenorientierung im Marketing-Management, in: Harvard Manager, 1987, Nr. 3, S. 28-36

Kumar, N., Mobility barriers and profitability of multinational and local enterprises in Indian manufacturing, in: The Journal of Industrial Economics, Vol. 38 (1990), No. 4, S. 449463

Kupsch, P., Unternehmensziele, Stuttgart, New York 1979

Läbe, M., Stolpmann, F., Dienst am Kunden total?, Wie die Investitionsgüterindustrie mit dem Service umgeht, in: asw, Sondernummer Oktober 1993, S. 22-34

Lahti, A., Strategy and performance of a firm: An empirical investigation in the Knitwear Industry in Finland in 1969-81, Helsinki 1983 
Lambkin, M., Pioneering new markets: A comparison of market share winners and losers, in: International Journal of Research in Marketing, Vol. 9 (1992), S. 5-22

Laux, H., Liermann, F., Grundlagen der Organisation, Die Steuerung von Entscheidungen als Grundproblem der Betriebswirtschaftslehre, 3. Aufl., Berlin u.a. 1993

Lawless, M.W., Finch, L.K., Choice and determinism: A test of Hrebiniak and Joyce's framework on strategy-environment fit, in: Strategic Management Journal, Vol. 10 (1989), S. $351-365$

Leder, M., Innovationsmanagement - Ein Überblick, in: Innovationsmanagement, Theorie und Praxis im Kulturvergleich, Hrsg.: Albach, H., Wiesbaden 1990, S. 1-54

Lehnert, St., Die Bedeutung von Kontingenzansätzen für das Strategische Management, Frankfurt, Bern, New York 1983

Leibfried, K., McNair, C.J., Benchmarking, New York 1992

Lewis, P., Thomas, H., The linkage between strategy, strategic groups and performance in the U.K. Retail Grocery Industry, in: Strategic Management Journal, Vol. 11 (1990), S. 385-397

Lilien, G.L., Yoon, E., Determinants of new industrial product performance: A strategy reexamination of the empirical literature, in: IEEE Transactions on Engineering Management, Vol. 36 (1989), No. 1, S. 3-10

Lilien, G.L., Yoon, E., The timing of competitive market entry: An exploratory study of new industrial products, in: Management Journal, Vol. 36 (1990), S. 568-585

Link, J., Überlegen mit starken Produkten, in: asw, Sondernummer Oktober 1993, S. 188-197

Link, U., Strategische Konkurrenzanalyse im Konsumgütermarketing, Idstein 1988

Little, A.D. (Hrsg.), The strategic management of technology, European Management Forum, Davos 1981

Little, A.D. (Hrsg.), Management erfolgreicher Produkte, Wiesbaden 1994

Luchs, R., Successful businesses compete on quality - not costs, in: Long Range Planning, Vol. 19 (1986), No. 1, S. 12-17

MacInnis, M., Heslop, L.A., Market planning in a high-tech environment, in: Industrial Marketing Management, Vol. 19 (1990), S. 107-116

Mager, H.J., Siegberg, U., Erfolgsfaktor "Technologie-Innovation" bei der Entwicklung medizintechnischer Großgeräte, in: Technologie-Marketing, Hrsg.: Töpfer, A., Sommerlatte, T., Landsberg a.L. 1991, S. 481-500

Maidique, M.A., Hayes, R.H., The art of high-technology management, in: Sloan Management Review, Vol. 26 (1984), S. 17-31

Maidique, M.A., Patch, P., Corporate strategy and technological policy, in: Readings in the management of innovation, Hrsg.: Tushman, M.L., Moore, W.L., Cambridge 1982, S. 273-285

Maidique, M.A., Zirger, B.J., A study of success and failure in product innovation: The case of U.S. electronics industry, in: IEEE, Stanford University, 1984, No. 4, S. 192-203 
Manns, J.R., Produktinnovationen als Ergebnis der Koordination von F\&E und Marketing, Ursachen, Auswirkungen und Lösungsmöglichkeiten, dargestellt am Beispiel eines Unternehmens der mobilen Kommunikation, Ludwigsburg, Berlin 1992

Maringer, A., Preisverfall mikroelektronischer Bauelemente am Beispiel DRAM-Speicherbauelemente, in: ZfbF, 1990, Nr. 4, S. 423-439

Marone, J.G., Winning in high-tech markets, the role of general management, Boston, Mass. 1993

Marshall, A., Principles of economics, 8. Aufl., London 1966

Mascarenhas, B., Strategic group dynamics, in: Academy of Management Journal, Vol. 32 (1989), No. 2, S. 333-352

Mason, E.S., Economic concentration and the monopoly problem, Cambridge 1957

Mauthe, K.D., Strategische Analyse, München 1984

Mayndel, E., Technologie-Akzeptanz im Unternehmen: Mitarbeiter für neue Informationstechnologien gewinnen, Wiesbaden 1987

McArthur, A.W., Nystrom, P.C., Environmental dynamism, complexity and munificence as moderators of strategy-performance relationships, in: Journal of Business Research, Vol. 23 (1991), S. 349-361

McGee, J., Strategic groups: A bridge between industry structure and strategic management?, in: Strategic marketing and management, Hrsg.: Thomas, H., Gardner, D., Chichester u.a. 1985 , S. 293-313

McGee, J., Thomas, H., Strategic groups: A useful linkage between industry structure and strategic management?, BEBR, Faculty Working Paper No. 1100, College of Commerce and Business Administration, University of Illinois at Urbana-Champaign, December 1984

McGee, J., Thomas, H., Strategic groups: Theory, research and taxonomy, in: Strategic Management Journal, Vol. 7 (1986), S. 141-160

McGee, J., Thomas, H., Research notes and communications - Strategic groups: A further comment, in: Strategic Management Journal, Vol. 10 (1989), S. 105-107

McKenna, R., Market positioning in high technology, in: California Management Review, Vol. 27 (1985), No. 3, S. 82-108

McKenna, R., Relationship marketing, Successful strategies for the age of the customers, New York u.a. 1991

McKenna, R., Borrus, M., Cohen, S., Industrial policy and international competition in high technology, Part I: Blocking capital formation, in: California Management Review, 1984, No. 2, S. $15-27$

McKelvey, B., Organizational systematics: Taxonomic lessons from biology, in: Management Science, Vol. 24 (1978), No. 13, S. 1428-1440

McNannee, P., McHugh, M., Mapping competitive groups in the clothing industry, in: Long Range Planning, Vol. 22 (1989), S. 89-97 
Meffert, H., Die Flexibilität in betriebswirtschaftichen Entscheidungen, unver. Habil., München 1968

Meffert, H., Zum Problem der betriebswirtschaftlichen Flexibilität, in: ZfB, 1969, S. 779-800

Meffert, H., Unternehmensziele, in: Jahrbuch des Marketing, Hrsg.: Schöttle, K.M., Essen 1971 , S. 22-34

Meffert, H., Marketing als Führungsaufgabe, Aspekte des Investitionsgütermarketing, in: Datascope, 1972, Nr. 7, S. 3-13

Meffert, H., Industriewerbung, in: Technische Mitteilungen, 1972, Nr. 4, S. 155-159

Meffert, H., Interpretation und Aussagewert des Lebenszyklus-Konzeptes, in: Neuere Ansätze der Marketingtheorie, Hrsg.: Hammann, P., Kroeber-Riel, W., Meyer, C.W., Berlin 1974 , S. 85-134

Meffert, H., Die Durchsetzung von Innovationen in der Unternehmung und im Markt, in: ZfB, 1976, Nr. 2, S. 77-100

Meffert, H., Marketing, Einführung in die Absatzpolitik, 6. Aufl., Wiesbaden 1982

Meffert, H., Marketing und neue Medien, Stuttgart 1985

Meffert, H., Marketing-Flexibilität als Erfolgsfaktor der Unternehmung, in: Thexis, 1985, Nr. 4 , S. $8-13$

Meffert, H., Zur Bedeutung von Konkurrenzstrategien im Marketing, in: Marketing ZFP, 1985, Nr.1, S. 13-19

Meffert, H., Marketing, Grundlagen der Absatzpolitik, 7. Aufl., Wiesbaden 1986

Meffert, H., Marketing und strategische Unternehmensfuihrung - ein wettbewerbsorientierter Kontingenzansatz, in: Strategische Unternehmensplanung, Hrsg.: Hahn, D., Taylor, B., Wien 1986, S. 664-683

Meffert, H., Marktein- und -austrittsbarrieren, in: DBW, 1987, Nr. 5, S. 629-630

Meffert, H., Kundendienstpolitik, Eine Bestandsaufnahme zu einem komplexen Marketinginstrument, in: Marketing ZFP, 1987, Nr. 2, S. 93-102

Meffert, H., Strategische Unternehmensführung und Marketing, Wiesbaden 1988

Meffert, H., Marktanalyse, in: Handwörterbuch der Planung, Hrsg.: Szyperski, N., Stuttgart 1989, Sp. 1020-1030

Meffert, H., Marketingstrategien in unterschiedlichen Marktsituationen, in: Handbuch des Marketing, Hrsg.: Bruhn, M., München 1989, S. 277-306

Meffert, H., Die Wertkette als Instrument einer integrierten Unternehmensplanung, in: Der Integrationsgedanke in der Betriebswirtschaftslehre, Hrsg.: Delfmann, W., Wiesbaden 1989, S. 256-277

Meffert, H., Klassische Funktionenlehre und marktorientierte Führung, Integrationsperspektiven aus Sicht des Marketing, in: Integration und Flexibilität, Eine Herausforderung an die allgemeine Betriebswirtschaftslehre, Hrsg.: Adam, D., Backhaus, K., Meffert, H., Wagner, H., Münster 1989, S. 373-408 
Meffert, H., Entwicklungslinien des Marketing, Akzente der marktorientierten Unternehmensführung in den 90er Jahren, in: Jahrbuch Marketing, Hrsg.: Schöttle, K.M., 5. Aufl., Wiesbaden 1990, S. 12-21

Meffert, H., Marktorientierte Unternehmensführung und Direct Marketing, in: Handbuch Direct Marketing, Hrsg.: Dallmer, H., 6. Aufl., Wiesbaden 1990, S. 31-49

Meffert, H., Programmierte Flops im Technologiemarketing, in: HighTech, 1990, Nr. 9, S. 56

Meffert, H., Erfolgreiches Marketing in den neunziger Jahren, in: Marketing im Umbruch Chancen und Gefahren für Unternehmer, Zürich 1991, S. 7-43

Meffert, H., Integrierte Marktkommunikation, unver. Skript, Münster 1991

Meffert, H., Marketingforschung und Käuferverhalten, 2. Aufl., Wiesbaden 1992

Meffert, H., Marketing-Theorie, in: Vahlens Großes Marketinglexikon, Hrsg.: Diller, H., München 1992, S. 698-702

Meffert, H., Konsumgütermarketing, in: Handwörterbuch der Betriebswirtschaftslehre, 5. Aufl., Band II, Hrsg.: Wittmann, W., et al., Stuttgart 1992, Sp. 2241-2255

Meffert, H., Strategien zur Profilierung von Marken, in: Marke und Markenartikel als Instrumente des Wettbewerbs, Hrsg.: Dichtl, E., Eggers, W., München 1992, S. 129-156

Meffert, H., Marktorientierte Führung von Dienstleistungsunternehmen - Neuere Entwicklungen in Theorie und Praxis, Arbeitspapier Nr. 78 der Wissenschaftlichen Gesellschaft für Marketing und Unternehmensführung e.V., Hrsg.: Meffert H., Wagner, H., Backhaus, K., Münster 1993

Meffert, H., Preispolitik, in: Handwörterbuch des Rechnungswesens, 3. Aufl., Hrsg.: Chmielewicz, K., Schweitzer, M., Stuttgart 1993, Sp. 1583-1591

Meffert, H., Marketing-Management, Analyse, Strategie, Implementierung, Wiesbaden 1994

Meffert, H., Erfolgreiches Marketing in der Rezession, Strategien und Maßnahmen in engeren Märkten, Wien 1994

Meffert, H., Dienstleistungsmarketing, in: Handwörterbuch des Marketing, Hrsg.: Köhler, R., Tietz, B., Zentes, J., 2. Aufl., Stuttgart 1994 (im Druck)

Meffert, H., Benkenstein, M., Wertkette, in: DBW, 1989, Nr. 6, S. 785-787

Meffert, H., Bolz, J., Globale Wettbewerbsstrategien und länderübergreifende Integration Status und Entwicklungsperspektiven, Arbeitspapier Nr. 70 der Wissenschaftlichen Gesellschaft für Marketing und Unternehmensführung e.V., Hrsg.: Meffert H., Wagner, H., Backhaus, K., Münster 1992

Meffert, H., Hafner, K., Unternehmenskultur und marktorientierte Unternehmensführung, Bestandsaufnahme und Wirkungsanalyse, Arbeitspapier Nr. 35 der Wissenschaftlichen Gesellschaft für Marketing und Unternehmensführung e.V., Hrsg.: Meffert H., Wagner, H., Münster 1987

Meffert, H., Hafner, K., Unternehmenskultur praxistauglich?, in: asw, Sondernummer Oktober 1988 , S. 22-35 
Meffert, H., Hafner, K., Poggenpohl, M., Unternehmenskultur und Unternehmensführung, Ergebnisse einer empirischen Untersuchung, Arbeitspapier Nr. 43 der Wissenschaftlichen Gesellschaft für Marketing und Unternehmensführung e.V., Hrsg.: Meffert H., Wagner, H., Münster 1988

Meffert, H., Heinemann, G., Strategische Gruppen im Handel, Eine empirische Analyse am Beispiel des textilen Einzelhandels, in: Handelsforschung 1989, Hrsg.: Trommsdorff, V., Wiesbaden 1989, S. 119-133

Meffert, H., Heinemann, G., Operationalisierung des Imagetransfers, in: Marketing ZFP, 1990, Nr. 1, S. 5-10

Meffert, H., Kirchgeorg, M., Umweltschutz als Unternehmensziel, in: Marketing-Schnittstellen: Herausforderungen für das Management, Hrsg.: Specht, G., Silberer, G., Engelhardt, W.H., Stuttgart 1989, S. 179-199

Meffert, H., Kirchgeorg, M., Marktorientiertes Umweltmanagement, Stuttgart 1992

Meffert, H., Lamnek, S., Maisberger, P., High-Tech-Marketing, Branchenspezifische Trends und Strategien für die 90er Jahre, Düsseldorf 1991

Meffert, H., Remmerbach, K.-U., Marketingstrategien in jungen Märkten, in: DBW, 1988, Nr. 3, S. 331-346

Meffert, J., Standards als Integrationsinstrument in der Computer- und Kommunikationsindustrie, Konstanz 1994

Meldrum, M.J., Millmann, A.F., Ten risks in marketing high-technology products, in: Industrial Marketing Management, Vol. 20 (1991), S. 43-50

Merkle, E., Technologiemarketing: Ein Ansatz zur Bewältigung des technologischen Wandels, in: Marketing ZFP, 1984, Nr. 1, S. 5-14

Meyer, H., Noch einmal und zum letzten Mal: Was mißt der LPC Fiedlers?, in: DBW, 1982 , Nr. 3, S. 427-439

Meyer, J., Qualität als strategische Wettbewerbswaffe, in: Wettbewerbsvorteile und Wettbewerbsfähigkeit, Hrsg. Simon, H., Stuttgart 1988, S. 73-88

Meyer, N., Hebelpunkte des strategischen Entwicklungsmanagement, in: Technologie-Marketing, Hrsg.: Töpfer, A., Sommerlatte, T., Landsberg a.L. 1991, S. 88-110

Michel, K., Technologie im strategischen Management, 2. Aufl., Berlin 1991

Middelhoff, Th., Walters, M., Akzeptanz neuer Medien - Eine empirische Analyse aus Unternehmenssicht, Arbeitspapier Nr. 27 des Instituts für Marketing der Universität Münster, Hrsg.: Meffert, H., Münster 1981

Miles, R.E., Snow, Ch.C., Organizational strategy, structure and process, New York 1978

Miles, R.E., Snow, Ch.C., Fit, failure and the hall of fame, in: California Management Review, Vol. 26 (1984), No. 3, S. 10-28

Miles, R.E., Snow, Ch.C., Sharfman, M.P., Industry variety and performance, in: Strategic Management Journal, Vol. 14 (1993), S. 163-177 
Miller, A., A taxonomy of technological settings, with related strategies and performance levels, in: Strategic Management Journal, Vol. 9 (1988), S. 239-254

Miller, A., Gartner, W.B., Wilson, R., Entry order, Market share and competitive advantage: A study of their relationships in new corporate ventures, in: Journal of Business Venturing, Vol. 4 (1989), S. 197-209

Miller, D., Relating Porter's business strategies to environment and structure, in: Academy of Management Journal, Vol. 31 (1988), No. 2, S. 280-308

Miller, D., The genesis of configuration, in: Academy of Management Review, Vol. 12 (1987), No. 4, S. 686-701

Miller, D., The generic strategy trap, in: The Journal of Business Strategy, 1992, No. 1, S. 3741

Milling, P., Schwierigkeiten bei der Umsetzung des neuen Wissens, Serie: Standort Deutschland (2), in: BddW, 1993, Nr. 147, S. 7

Milling, P., Forschung und Innovation in der Wirtschaft, in: Standort Bundesrepublik Deutschland, Die Wettbewerbsbedingungen auf dem Prüfstand, Hrsg.: Dichtl, E., Frankfurt a.M. 1994

Minderlein, M., Markteintrittsbarrieren und Unternehmensstrategie, Wiesbaden 1989

Minderlein, M., Markteintrittsbarrieren und strategische Verhaltensweisen, in: ZfB, 1990, Nr. 2, S. $155-178$

Mitchell, W., Dual clocks: Entry order influence on incumbent and newcomer market share and survival when specialized assets retain their value, in: Strategic Management Journal, 1991, No. 1, S. 85-100

Moriarty, R.T., Kosnik, Th.J., High-Tech marketing: Concepts, continuity and change, in: Sloan Management Review, 1989, S. 7-17

Muchna, C., Strategische Marketing-Früherkennung auf Investitionsgütermärkten, Wiesbaden 1988

Müller, W., Planung von Marketing-Strategien, Die Entwicklung von Marketing-Strategien im strategischen Marketing-Planungsprozeß, Frankfurt a.M., Bern, New York 1986

Müller, W., Service-"Tuning" als Wettbewerbsvorteils-Konzept im Marketing von KfzZulieferunternehmen und Herstellern, in: Investitionsgüter- und High-Tech-Marketing, Hrsg.: Hofmaier, R., Landsberg a.L. 1992, S. 515-554

Murray, J.A., A contingency view of Porter's generic strategies, in: The Academy of Management Review, Vol. 13 (1988), No. 2, S. 390-400

Nayyar, P., Research notes and communications strategic groups: A comment, in: Strategic Management Journal, Vol. 10 (1989), S. 101-103

Neugebauer, U., Das Software-Unternehmen, Empirische Untersuchungen des Unternehmensverhaltens und der Faktoren des Unternehmenserfolges, GMD-Bericht Nr. 157, München, Wien 1986

Newman, H.M., Strategic groups and the structure-performance relationship: a study with respect to the chemical process industry, Diss., Harvard University 1973 
Newman, H.M., Strategic groups and the structure-performance relationships, in: The Review of Economics and Statistics, Vol. 60 (1978), S. 417-427

Niebauer, F.F., Das PIMS-Programm und Portfolio-Management, in: Strategische Unternehmensplanung, Hrsg.: Hahn, D., Taylor, B., Würzburg, Wien 1983, S. 135-162

Niemeier, J., Wettbewerbsumwelt und interne Konfiguration, Frankfurt a.M., Bern, New York 1986

Nieschlag, R., Dichtl, E., Hörschgen, H., Marketing, 16. Aufl., Berlin 1991

Nyström, H., Technological and market innovation, strategies for product and company development, Chichester u.a. 1990

o.V., Who's excellent now?, in: Business Week, 1984, Nr. 11, S. 46-55

o.V., High Tech, High tech can be anywhere you define it, in: Wall Street Journal, 4th of January 1984, S. 3

o.V., Hochgeschwindigkeits-Netze sind aus Anwendersicht unumgänglich, in: Computerwoche, 1993, Nr. 6, S. 23-24

o.V., Zeitungen in Amerika experimentieren mit elektronischen Medien, in: FAZ vom 03.08.1993, S. 14

o.V., Der unendlich schrumpfende Transistor, in: Computer Zeitung vom 12.08.1993, S. 19

o.V., Innovation: Wettlauf der Erfinder, in: Focus, 1993, Nr. 13, S. 184-188

o.V., Der rasche Siegeszug der glitzernden Scheibe, in: Werben \& Verkaufen, 1993, Nr. 39, S. 56-58

o.V., Studie: Technologie-Akzeptanz, in: asw, 1993, Nr. 11, S. 26

o.V., Jeder Zweite hat Angst vor neuer Technik, in: PC-Welt, 1993, Nr. 12, S. 15

o.V., Komplexe Systeme erfordern mehr Beratung, in: Computer Zeitung, 1993, Nr. 48, S. 21

o.V., Technologie, Gebremste Patentaktivität, in: Außen-Wirtschaft, 1994, Nr. 3, S. 22-24

o.V., Kaum Gewinne im Einkaufstempel: Vobis-Kunden wollen mehr Beratung, in: Werben \& Verkaufen, 1994, Nr. 6, S. 12

o.V., PC-Software-Umsatz in Europa, in: Computerwoche, 1994, Nr. 1, S. 48

o.V., PC-Markt wächst weltweit um 16 Prozent, in: Inside Multimedia, 1994, Nr. 4, S. 9.

Oates, J., An economic theory of firm, London 1982

Ohlsen, G., Marketing-Strategien in stagnierenden Märkten, Wiesbaden 1985

Olschewski, J., Das I+T-Unternehmen in der Verantwortung gegenüber der Öffentlichkeit Neue Marketingstrategien unter Berücksichtigung von Recycling, Ergonomie und Sicherheit, in: Investitionsgüter- und High-Tech-Marketing, Hrsg.: Hofmaier, R., Landsberg a.L. 1992, S. 579-588

O'Neil, P., Proprietäre Systeme und isolierte PC-Stationen als Herausforderung: Im Büro bleibt für den Generalunternehmer noch viel zu tun, in: Computer Zeitung, 1993, Nr. 29, S. 19 
Oster, Sh., Intraindustry structure and the ease of strategic change, in: Review of Economics and Statistics, Vol. 64 (1982), No. 3, S. 376-384

Oster, Sh., Modern competitive analysis, New York, Oxford 1990

Ostmeier, H., Ökologieorientierte Produktinnovationen, Frankfurt a.M. u.a. 1990

Patt, P.J., Strategische Erfolgsfaktoren im Einzelhandel, Frankfurt a.M. u.a. 1988

Pegels, C.C., Sekar, Ch., Determining strategic groups using multidimensional scaling, in: Interfaces, Vol. 19 (1989), S. 47-57

Perillieux, R., Der Zeitfaktor im strategischen Technologie-Management, Berlin 1987

Perillieux, R., Strategisches Timing von F\&E und Markteintritt bei innovativen Produkten, in: Integriertes Technologie- und Innovationsmanagement, Konzepte zur Stärkung der Wettbewerbskraft von High-Tech-Unternehmen, Hrsg.: Booz, Allen \& Hamilton, Berlin 1991, S. 21-48

Perillieux, R., Wittkemper, G., Ziele und Module eines integrierten Technologie- und Innovationsmanagements, in: Integriertes Technologie- und Innovationsmanagement, Konzepte zur Stärkung der Wettbewerbskraft von High-Tech-Unternehmen, Hrsg.: Booz, Allen \& Hamilton, Berlin 1991, S. 13-20

Pernicky, R., Schneller werden, in: Management der Hochleistungsorganisation, Hrsg.: Little, A.D., Wiesbaden 1989, S. 61-71

Perry, M., Bass, F.M., When to lead or follow?, It depends, in: Marketing Letters, 1990, No. 1, S. 187-198

Peters, Th., Strategy follows structure: Developing distinctive skills, in: California Management Review, Vol. 26 (1984), No. 3, S. 111-125

Peters, Th., Waterman, R., Auf der Suche nach Spitzenleistungen, Landsberg a.L. 1984

Pinkwart, A., Chaos und Unternehmenskrise, Wiesbaden 1992

Pfeiffer, St., Technologie-Frühaufklärung, Hamburg 1992

Popper, E.T., Buskirk, B.D., Technology life cycles in industrial markets, in: Industrial Marketing Management, Vol. 21 (1992), S. 23-31

Porter, M., Consumer behavior, retailer power and manufacturing strategy in consumer goods industries, Diss., Harvard University 1973

Porter, M., The structure within industries and companies performance, in: Review of Economics and Statistics, Vol. 61 (1979), S. 214-227

Porter, M., The contribution of industrial organization to strategic management, in: Academy of Management Review, Vol. 6 (1981), No. 4, S. 609-620

Porter, M., The technological dimensions of competitive strategy, in: Research on technological innovation, management and policy, Hrsg.: Rosenbloom, R.S., Greenwich, Conn. 1983, S. 1-33

Porter, M., Wettbewerbsstrategie, Frankfurt a.M., New York 1988

Porter, M., Wettbewerbsvorteile, Frankfurt a.M., New York 1989 
Posselt, Th., Mobilitätsverhalten von Unternehmen, Wiesbaden 1993

Poth, L.G., Erfolgsfaktoren von Marktfuihrern, Ergebnisse einer Studie im Fachbereich Wirtschaft der Fachhochschule Düsseldorf, in: Apitz, K., et al., Erfolgsfaktoren von Marktführern, Landsberg a.L. 1989, S. 195-249

Powell, Th.C., Organizational alignment as competitive advantage, in: Strategic Management Journal, Vol. 13 (1992), S. 119-134

Preisl, A., Unternehmenskultur kontra Effektivität, in: WISU, 1990, Nr. 10, S. 543-544

Preiß, F.J., Strategische Erfolgsfaktoren im Software-Marketing, Frankfurt a.M. u.a. 1991

Preiß, F.J., Strategische Erfolgsfaktoren im Software-Marketing, in: Software-Marketing, Hrsg.: Wimmer, F., Bittner, L., Wiesbaden 1992, S. 45-69

Primeaux, W.J., A method for determining strategic groups and life cycle stages of an industry, BEBR, Faculty Working Paper No. 960, College of Commerce and Business Administration, University of Illinois at Urbana-Champaign, June 1983

Primeaux, W.J., The interdependence of the life cycle and strategic group concepts: Theory and evidence, BEBR, Faculty Working Paper No. 961, College of Commerce and Business Administration, University of Ilinois at Urbana-Champaign, June 1983

Pues, C., Markterschließungsstrategien bundesdeutscher Unternehmen in Osteuropa, Eine Analyse der Ausgestaltungsformen und Einflußfaktoren auf empirischer Grundlage, Diss., Münster 1994

Pümpin, C., Management strategischer Erfolgspositionen, Bern, Stuttgart 1982

Pümpin, C., Bedeutung der Unternehmenskultur für die Unternehmensführung, in: Unternehmenskultur und Unternehmensführung, Dokumentationspapier Nr. 39 der Wissenschaftlichen Gesellschaft für Marketing und Unternehmensführung e.V., Hrsg.: Meffert H., Wagner, H., Münster 1987, S. 4-19

Pümpin, C., Wüthrich, H.A., Die Marketing-Kultur als betrieblicher Wert von strategischer Bedeutung, in: Thexis, 1985, Nr. 4, S. 22-25

Pümpin, C., Koller, H.P., Die Bedeutung der Unternehmenskultur für die Unternehmensstrategie, in: Die Unternehmenskultur: ihre Grundlagen und ihre Bedeutung für die Führung der Unternehmung, Hrsg.: Lattmann, Ch., Heidelberg 1990, S. 303-317

Raffée, H., Fritz, W., Dimensionen und Konsistenz der Führungskonzeption von Industrieunternehmen - Ergebnisse einer empirischen Untersuchung, in: ZfbF, 1992, Nr. 4, S. 307-322

Raffée, H., Effenberger, J., Fritz, W., Strategieprofile als Faktoren des Unternehmenserfolges, Arbeitspapier Nr. 93 des Instituts für Marketing, Hrsg.: Dichtl, E., Geist, M., Raffée, H., Mannheim 1992

Raffée, H., Wiedmann, K.-P., Marketingumwelt 2000, in: Marketing 2000, Perspektiven zwischen Theorie und Praxis, Hrsg.: Schwarz, Chr., Wiesbaden 1987, S. 185-209

Ramsler, M., Strategic groups and foreign market entry in global banking competition, Diss., Harvard University 1982

Rasche, Chr., Kernkompetenzen, in: DBW, 1993, Nr. 3, S. 425-427 
Reger, R.K., Huff, A.S., Strategic groups: A cognitive perspective, in: Strategic Management Journal, Vol. 14 (1993), S. 103-124

Reinecke, S., Rezession und Strukturwandel in der Informationstechnologie-Branche: Problemanalyse und Erfolgsansätze, Forschungskolloquium IfM-FAH, Münster 1994, S. $1-29$

Remmerbach, K.-U., Markteintrittsentscheidungen, Wiesbaden 1988

Remmerbach, K.-U., Value Added Marketing, in: Marktforschung \& Management, 1990, Nr. 2, S. 13-21

Rexroad, R.A., High Technology Marketing Management, New York 1983

Rieger, G., Die kommunikative Gestaltung des "Markenauftritts" von I+T-Produkten, in: Investitionsgüter- und High-Tech-Marketing, Hrsg.: Hofmaier, R., Landsberg a.L. 1992, S. 419-439

Riggs, B., Der Personalcomputer der Zukunft soll Strom sparen und "mitdenken": Amerikaner fordern von Herstellern bedienungsfreundlichere PC's, in: Computer Zeitung, 1993, Nr. 48, S. 21

Roberts, E.B., Berry, Ch., Entering new business: Selecting strategies for success, in: The Best of MIT's Sloan Management Review, Cambridge, Mass. 1986, S. 57-71

Roberts, E.B., Entrepreneurs in high technology: Lessons from MIT and beyond, New York u.a. 1991

Robinson, W.T., Sources of market pioneer advantages: The case of industrial goods industries, in: Journal of Marketing Research, Vol. 25 (1988), S. 87-94

Robinson, W.T., Fornell, C., Market pioneering and sustainable market share advantages, The PIMS-Letter on Business Strategy, No. 39, Strategic Planning Institute, Cambridge, Mass. 1986

Robinson, W.T., Fornell, C., Sullivan, M., Are market pioneers intrinsically stronger than later entrants?, in: Strategic Management Journal, Vol. 13 (1992), S. 609-624

Robinson, R.B., Pearce, J.A., Planned patterns of strategic behavior and their relationship to business-unit performance, in: Strategic Management Journal, Vol. 9 (1988), S. 43-60

Römer, E., Konkurrenzforschung, Informationsgrundlagen der Wettbewerbsstrategie, in: ZfB, 1988, Nr. 4, S. 481-501

Rößl, D., Ein Stufenplan zur Marktabgrenzung, Wien 1986

Rößl, D., Die Entwicklung eines Bezugsrahmens und seine Stellung im Forschungsprozeß, in: Journal für Betriebswirtschaft, 1990, Nr. 2, S. 99-110

Rohleder, P.J., Unternehmens- und Geschäftsfeldstrategien, in: Marketing, Hrsg.: Fischer, G., Landsberg a.L. 1992, S. 1-19

Rominski, D., Wie kommen Computerhersteller über den Berg?, Dynamik aus neuer Offenheit, in: Absatzwirtschaft, 1992, Nr. 3, S. 30-39

Rothwell, R., The characteristics of successful innovators and technically progressive firms, in: R+D Management, 1977, No. 3, S. 191-306 
Rüdiger, B., Schlüsseltechnologien: Die Herausforderungen für die 90er Jahre, in: Technologie-Marketing, Hrsg.: Töpfer, A., Sommerlatte, T., Landsberg a.L. 1991, S. 35-52

Saad, K.N., Roussel, Ph.A., Tiby, C., Management der F\&E-Strategie, Hrsg.: Little, A.D., Wiesbaden 1991.

Samli, A.C., Wills, J.R., Strategies for marketing computers and related products, in: Industrial Marketing Management, Vol. 15 (1986), S. 23-32

Samli, A.C., Wills, J.R., Global computer marketing strategies, in: Industrial Marketing Management, Vol. 18 (1989), S. 271-279

Savage, G.T., et al., Strategies for assessing and managing organizational stakeholders, in: Academy of Management, Vol. 5 (1991), S. 61-75

Schaible, J., Hönig, A., High-Tech-Marketing in der Praxis, München 1991

Schamann, H.-D., Qualifizierung der Mitarbeiter als Bestandteil eines erfolgreichen Technologie-Management, in: Technologie-Marketing, Hrsg.: Töpfer, A., Sommerlatte, T., Landsberg a.L. 1991, S. 221-233

Scherer, F.M., Industrial market structure and economic performance, 2. Aufl., Chicago 1980

Schewe, G., Imitationsmanagement, Nachahmung als Option des Technologiemanagements, Stuttgart 1992

Schewe, G., Die Innovation im Wettbewerb, in: ZfB, 1992, Nr. 9, S. 967-988

Schildhauer, Th., Strategisches Software-Marketing, Übersicht und Bewertung, Wiesbaden 1992

Schmelzer, H.J., Buttermilch, K.-H., Reduzierung der Entwicklungszeiten in der Produktentwicklung als ganzheitliches Problem, in: ZfbF, 1988, Sonderheft Nr. 23, S. 43-73

Schmoch, U., Schnöring, Th., Wie steht es um die Wettbewerbsposition der Telekommunikationsgeräteindustrie in Europa?, Diskussionsbeitrag Nr. 104 des Wissenschaftlichen Instituts für Kommunikationsdienste, Bad Honnef 1993

Schnaars, St. P., When entering growth markets, are pioneers better than poachers?, in: Business Horizons, Vol. 29 (1986), No. 3-4, S. 27-36

Schneider, D.J., Müller, M.E., Durch Koordination zu marktgerechten Innovationen - Schnittstellengestaltung zwischen F\&E und Marketing, in: Marktforschung und Management, 1993, Nr. 1, S. 6-13

Scholz, Ch., Strategisches Management, Ein integrativer Ansatz, Berlin, New York 1987

Scholz, Ch., Corporate culture and strategy, The problem of strategic fit, in: Long Range Planning, Vol. 20 (1987), S. 78-87

Scholz, Ch., Organisationskultur, Zwischen Schein und Wirklichkeit, in: ZfbF, 1988, Nr. 3, S. 243-272

Scholz, Ch., Organisationskultur: die 4 Erfolgsprinzipien, Wiesbaden 1990

Schrape, K., Medienmärkte im Umbruch?, in: Planung und Analyse, 1994, Nr. 1, S. 19-21

Schreyögg, G., Umwelt, Technologie und Organisationsstruktur, Bern, Stuttgart 1978 
Schreyögg, G., Unternehmensstrategie, Berlin, New York 1984

Schürmann, U., Erfolgsfaktoren der Werbung im Produktlebenszyklus, Frankfurt a.M. u.a. 1992

Schuster, J., Management compensation in high technology companies, Lexington, Mass. 1984

Schuster, L., Widmer, A.W., Theorie und Praxis der Unternehmenskultur, in: ZfO, 1984, Nr. 8, S. 489-493

Schwalbach, J., Marktanteil und Unternehmensgewinn, in: ZfB, 1988, Nr. 4, S. 535-550

Seitz, K., Die japanische-amerikanische Herausforderung, Deutschlands HochtechnologieIndustrien kämpfen ums Überleben, München 1991

Segev, E., Strategy, Strategy-Making and performance in a business game, in: Strategic Management Journal, Vol. 8 (1987), S. 565-577

Servatius, H.-G., Methodik des strategischen Technologie-Managements, Grundlage für erfolgreiche Innovationen, Berlin 1985

Servatius, H.-G., Schwerpunkte und Methoden des Management von Technologien, in: Technologie-Marketing, Hrsg.: Töpfer, A., Sommerlatte T., Landsberg a.L. 1991, S. 55-86

Shanklin, W.L., Ryans, J.K., Essentials of marketing high technology, Lexington, Mass., Toronto 1987

Sheperd, W.G., The elements of market structure, in: Review of Economics and Statistics, Vol. 54 (1972), No. 1, S. 25-37

Sheperd, W.G., The economics of industrial organization, Englewood Cliffs, N.J. 1979

Siefke, A., Die Eignung der Faktorenanalyse zur Beurteilung des Evoked Set, unver. Diplomarbeit, Münster 1992

Simon, H., Goodwill und Marketingstrategie, Wiesbaden 1985

Simon, H., Management strategischer Wettbewerbsvorteile, in: Wettbewerbsvorteile und Wettbewerbsfähigkeit, Hrsg.: Simon, H., Stuttgart 1988, S. 1-17

Simon, H., Management strategischer Wettbewerbsvorteile, in: ZfB, 1988, S. 461-480

Simon, H., Die Zeit als strategischer Erfolgsfaktor, in: Strategieentwicklung, Hrsg.: Riekhof, H.-Ch., Stuttgart 1989, S. 47-75

Simon, H., Preisbündelung, in: ZfB, 1992, Nr. 11, S. 1213-1235

Simon, H., Preismanagement, 2. Aufl., Wiesbaden 1992

Slater, St.F., Narver, J.C., Does competitive environment moderate the market orientationperformance relationship?, in: Journal of Marketing, Vol. 58 (1994), S. 46-55

Sommerlatte, T., Deschamps, J.-Ph., Der strategische Einsatz von Technologien, in: Management im Zeitalter der strategischen Führung, Hrsg.: Little, A.D., Wiesbaden 1985, S. 978

Sommerlatte, T., Töpfer, A., Die Integration von Technologie und Marketing als strategischer Erfolgsfaktor: Übersicht und Einordnung der Beiträge, in: Technologie-Marketing, Hrsg.: Töpfer, A., Sommerlatte T., Landsberg a.L. 1991, S. 11-33 
Specht, G., Technologiemanagement: Grundgedanken zum Gegenstand und zugleich Sammelrezension, in: DBW, 1992, Nr. 4, S. 547-566

Specht, G., Perillieux, R., Erfolgsfaktoren technischer Führer- und Folgerpositionen auf Investitionsgütermärkten, in: $\mathrm{ZfbF}, 1988$, S. 204-226

Specht, G., Zörgiebel, W., Technologieorientierte Wettbewerbsstrategien, in: Strategisches Marketing, Hrsg.: Raffée, H., Wiedmann, K.-P., Stuttgart 1985, S. 491-517

Spital, F., Gaining market share advantage in the semiconductor industry by lead time in innovation, in: Research on technological innovation, management and policy, Hrsg.: Rosenbloom, R.S., Greenwich, Conn. 1983, S. 55-67

Srinivasan, K., Pioneering versus early following in new product markets, Diss., University of California, Los Angeles 1990

Staehle, W.H., Management, 5. Aufl., München 1990

Staerkle, R., Wechselwirkungen zwischen Unternehmenskultur und Organisationsstruktur, in: Integriertes Management, Hrsg.: Probst, G.J., Siegwart, H., Bern, Stuttgart 1985, S. 529-553.

Steffenhagen, H., Der Strategiebegriff in der Marketingplanung, Arbeitspapier Nr. 29 des Instituts für Marketing der Universität Münster, Hrsg.: Meffert, H., Münster 1982

Steinbacher, F., Kultur, Begriff, Theorie, Funktion, Stuttgart 1976

Steinmann, H., Heß, G., Die Rolle von Marktsignalen bei der Etablierung von Kompatibilitätsstandards im Rahmen der Wettbewerbsstrategie, in: DBW, 1993, S. 167-185

Steinmann, H., Schreyögg, G., Management, Stuttgart 1990

Stewart, D.W., The application and misapplication of factor analysis in marketing research, in: Journal of Marketing Research, Vol. 18 (1981), S. 51-62

Stratz, R., Erfolgsfaktoren im PC-Handel und Möglichkeiten der Zusammenarbeit mit Herstellern, in: Investitionsgüter- und High-Tech-Marketing, Hrsg.: Hofmaier, R., Landsberg a.L. 1992, S. 555-564

Strothmann, K.-H., et al., Integrationspolitik und Technologiebeobachtung im Innovationsmarketing, Würzburg 1988

Tebbe, K., Die Organisation von Produktinnovationsprozessen, Stuttgart 1990

Thomas, H., Gardner, D., Introduction to conference papers on competitive strategy and strategic competition, in: Strategic marketing and management, Hrsg.: Thomas, H., Gardner, D., Chichester u.a. 1985, S. 267-272

Thomas, H., Venkatraman, N., Research on strategic groups: Progress and prognosis, in: Journal of Management Studies, Vol. 25 (1988), No. 6, S. 537-555

Töpfer, A., Marketing für Start-up-Geschäfte mit Technologieprodukten, in: TechnologieMarketing, Hrsg.: Töpfer, A., Sommerlatte, T., Landsberg a.L. 1991, S. 163-200

Tremblay, V.J., Strategic groups and the demand for beer, in: Journal of Industrial Economics, Vol. 34 (1985), No. 2, S. 183-198 
Trommsdorff, V., Erfolgsfaktorenforschung, Produktinnovationen und Schnittstelle Marketing - F\&E, Arbeitspapier des Instituts für Betriebswirtschaftslehre, Berlin 1990

Trommsdorff, V., Schuster, H., Strategie- und Imageplanung für junge Technologiefirmen, in: asw, 1985, Nr. 9, S. 116-121

Türk, K., Neuere Entwicklungen in der Organisationsforschung, Stuttgart 1989

Tylecote, A., Demirag, I., Short-Termism: Culture and structures as factors in technological innovation, in: Technological change and company strategies, Hrsg.: Coombs, R., Saviotti, P., Walsh, V., London u.a. 1992, S. 201-225

Ulrich, P., Fluri, E., Management, 3. Aufl., Bern, Stuttgart 1984

Urban, G.L., et al., Market share rewards to pioneering brands: An empirical analysis and strategic implications, in: Management Science, Vol. 32 (1986), S. 645-659

Van den Abele, P., Christiaens, I., Strategies of belgian high-tech firms, in: Industrial Marketing Management, Vol. 15 (1986), S. 299-308

Varadarajan, P.R., A Two-factor classification of competitive strategy variables, in: Strategic Management Journal, Vol. 6 (1985), S. 357-375

Venkatesan, R., Make or Buy: Die Stärken des Endproduktes schützen, in: Harvard Business Manager, 1993, Nr. 2, S. 98-108

Venkatraman, N., Camillus, J.C., Exploring the concept of "Fit" in strategic management, in: Academy of Management Review, 1984, S. 513-525

Vidal, M., Wettbewerbsstrategien für Pionierunternehmen, Wiesbaden 1993

Vogel, F., Probleme und Verfahren der numerischen Klassifikation, Göttingen 1975

Voigt, S., Strategische Allianzen, Modisches Schlagwort oder Antwort auf globale Herausforderungen?, in: WiSt, 1993, Nr. 5, S. 246-249

Vollmer, M., Anwendungsarchitekturen bestimmen die Lösungswelt: Trend zur Standardisierung beeinflußt Softwareangebot, in: Computer Zeitung, 1993, Nr. 45, S. 17

Walters, M., Marktwiderstände und Marketingplanung, Wiesbaden 1984

Waterman, R.H., The seven elements of strategic fit, in: Journal of Business Strategy, 1982, S. 69-72

Watson, G.H., The Benchmarking Workbook, Cambridge, Norwalk 1992

Weber, M., Unternehmerische Marktstrategien bei dynamischem Wettbewerb, Diss., Münster 1992

Wegwart, J., Preis- und Kontrahierungspolitik im Kundendienst unter besonderer Berücksichtigung von Wartungs- oder Call-Service, in: Kundendienst-Management, Hrsg.: Meffert, H., Frankfurt a.M., Bern 1982, S. 113-123

Weiber, R., Faktorenanalyse, Eine anwendungsorientierte computergestützte Einführung mit Übungen, St. Gallen 1984

Weiber, R., Die Diffusion von Kritische Masse-Systemen, Wiesbaden 1992 
Weiber, R., Leapfrogging-Behavior: Herausforderung für das Marketing-Management neuer Technologien, Beitrag zur Hochschullehrertagung, Stuttgart 1993, o.S.

Weiss, P., Die Kompetenz von Systemanbietern: Ein neuer Ansatz im Marketing für Systemtechnologien, Berlin 1992

Westphal, J., Vertikale Wettbewerbsstrategien in der Konsumgüterindustrie, Wiesbaden 1991

Wever, U., Unternehmeskultur in der Praxis, Frankfurt a.M., New York 1989

Wieandt, A., Zum Marketing von Innovationen, in: Marketing ZFP, 1994, Nr. 1, S. 21-30

Wieselhuber, N., Strategische Marketingplanung, in: Strategisches Marketing, Hrsg.: Wieselhuber, N., Töpfer, A., Landsberg a.L. 1984, S. 67-86

Wieselhuber, N., Erschließung von neuen Wachstumsquellen durch Diversifikation, in: Handbuch Strategisches Marketing, Hrsg.: Wieselhuber, N., Töpfer, A., Landsberg a.L. 1984, S. 426-440.

Willumeit, K., Heike, G., Strategische CI-Entwicklung für Unternehmen mit Technologieprodukten am Beispiel der SIHI-Gruppe, in: Technologie-Marketing, Hrsg.: Töpfer, A., Sommerlatte, T., Landsberg a.L. 1991, S. 255-271

Witte, A., Integrierte Qualitätssteuerung im Total Quality Management, Münster, Hamburg 1993

Wittek, B., Strategien auf Kundennutzen aufbauen, in: Wettbewerbsvorteile und Wettbewerbsfähigkeit, Hrsg. Simon, H., Stuttgart 1988, S. 66-72

Wolfrum, B., Strategisches Technologiemanagement, Wiesbaden 1991

Wolfrum, B., Strategische Gruppen, in: DBW, 1992, Nr. 3, S. 415-417

Wolfrum, B., Technologiestrategien im strategischen Management, in: Marketing ZFP, 1992, Nr. 1, S. 23-36

Wright, P., Research notes and communications - A refinement of Porter's strategies, in: Strategic Management Journal, Vol. 8 (1987), S. 93-101

Wübben, J., Die Unternehmenskultur als Bestimmungsfaktor marktorientierter Unternehmensstrategien, unver. Diplomarbeit, Münster 1991

Wüthrich, H.A., Neuland des strategischen Denkens, Wiesbaden 1991

Yeager, R.L., Strategy development: Engineering the customer-oriented marketing-strategy, in: Business Marketing, 1985, S. 61-72

Yip, G.S., Barriers to entry, Lexington, Mass., Toronto 1982

Zabel, S., High-Tech-Produkte brauchen ein spezielles Marketing, in: BddW, 1992, Nr. 161, S. 1

Zahn, E., Innovations- und Technologiemanagement, in: Technologie- und Innovationsmanagement, Hrsg.: Zahn, E., Berlin, München 1986, S. 9-48

Zahn, E., Neue Produktionstechnologien: Potentiale für Wettbewerbsvorteile, in: Strategieentwicklung, Hrsg.: Riekhof, H.-Ch., Stuttgart 1989, S. 153-189 
Zahn, E., Innovation und Wettbewerb, in: Innovations- und Technologie-Management, Hrsg.: Müller-Böling, D., Seibt, D., Winand, U., Stuttgart 1991, S. 115-133

Zerr, K., Jugel, St., Dienstleistungen als strategisches Element eines Technologie-Marketing, Arbeitspapier Nr. 68 des Instituts für Marketing, Hrsg.: Dichtl, E., Geist, M., Raffée, H., Mannheim 1989

Zimmermann, A., High-Tech-Marketing, in: Thexis, 1987, Nr. 1, S. 17-18

Zöllner, W.A., Strategische Absatzmarktplanung, Berlin u.a. 1990

Zörgiebel, W.W., Technologie in der Wettbewerbsstrategie, Berlin 1983 


\section{SCHRIFTEN ZUM MARKETING}

Band 1 Friedrich Wehrle: Strategische Marketingplanung in Warenhäusern. Anwendung der Portfolio-Methode. 1981. 2. Auflage. 1984.

Band 2 Jürgen Althans: Die Übertragbarkeit von Werbekonzeptionen auf internationale Märkte. Analyse und Exploration auf der Grundlage einer Befragung bei europaweit tätigen Werbeagenturen. 1982 .

Band 3 Günter Kimmeskamp: Die Rollenbeurteilung von Handelsvertretungen. Eine empirische Untersuchung zur Einschätzung des Dienstleistungsangebotes durch Industrie und Hąndel. 1982.

Band 4 Manfred Bruhn: Konsumentenzufriedenheit und Beschwerden. Erklärungsansätze und Ergebnisse einer empirischen Untersuchung in ausgewählten Konsumbereichen. 1982.

Band 5 Heribert Meffert (Hrsg.): Kundendienst-Management. Entwicklungsstand und Entscheidungsprobleme der Kundendienstpolitik. 1982.

Band 6 Ralf Becker: Die Beurteilung von Handelsvertretern und Reisenden durch Hersteller und Kunden. Eine empirische Untersuchung zum Vergleich der Funktionen und Leistungen. 1982.

Band 7 Gerd Schnetkamp: Einstellungen und Involvement als Bestimmungsfaktoren des sozialen Verhaltens. Eine empirische Analyse am Beispiel der Organspendebereitschaft in der Bundesrepublik Deutschland. 1982.

Band 8 Stephan Bentz: Kennzahlensysteme zur Erfolgskontrolle des Verkaufs und der MarketingLogistik. Entwicklung und Anwendung in der Konsumgüterindustrie. 1983.

Band 9 Jan Honsel: Das Kaufverhalten im Antiquitätenmarkt. Eine empirische Analyse der Kaufmotive, ihrer Bestimmungsfaktoren und Verhaltenswirkungen. 1984.

\section{SCHRIFTEN ZU MARKETING UND MANAGEMENT}

Band 10 Matthias Krups: Marketing innovativer Dienstleistungen am Beispiel elektronischer Wirtschaftsinformationsdienste. 1985.

Band 11 Bernd Faehsler: Emotionale Grundhaltungen als Einflußfaktoren des Käuferverhaltens. Eine empirische Analyse der Beziehungen zwischen emotionalen Grundhaltungen und ausgewählten Konsumstrukturen. 1986.

Band 12 Ernst-Otto Thiesing: Strategische Marketingplanung in filialisierten Universalbanken. Integrierte Filial- und Kundengruppenstrategien auf der Grundlage erfolgsbeeinflussender Schlüsselfaktoren. 1986.

Band 13 Rainer Landwehr: Standardisierung der internationalen Werbeplanung. Eine Untersuchung der Prozeßstandardisierung am Beispiel der Werbebudgetierung im Automobilmarkt. 1988.

Band 14 Paul-Josef Patt: Strategische Erfolgsfaktoren im Einzelhandel. Eine empirische Analyse am Beispiel des Bekleidungsfachhandels. 1988. 2. Auflage. 1990.

Band 15 Elisabeth Tolle: Der Einfluß ablenkender Tätigkeiten auf die Werbewirkung. Bestimmungsfaktoren der Art und Höhe von Ablenkungseffekten bei Rundfunkspots. 1988.

Band 16 Hanns Ostmeier: Ökologieorientierte Produktinnovationen. Eine empirische Analyse unter besonderer Berücksichtigung ihrer Erfolgseinschätzung. 1990.

Band 17 Bernd Büker: Qualitätsbeurteilung investiver Dienstleistungen. Operationalisierungsansätze an einem empirischen Beispiel zentraler EDV-Dienste. 1991.

Band 18 Kerstin Ch. Monhemius: Umweltbewußtes Kaufverhalten von Konsumenten. Ein Beitrag zur Operationalisierung, Erklärung und Typologie des Verhaltens in der Kaufsituation. 1993. 
Band 19 Uwe Schürmann: Erfolgsfaktoren der Werbung im Produktlebenszyklus. Ein Beitrag zur Werbewirkungsforschung. 1993.

Band 20 Ralf Birkelbach: Qualitätsmanagement in Dienstleistungscentern. Konzeption und typenspezifische Ausgestaltung unter besonderer Berücksichtigung von Verkehrsflughäfen. 1993.

Band 21 Simone Frömbling. Zielgruppenmarketing im Fremdenverkehr von Regionen. Ein Beitrag zur Marktsegmentierung auf der Grundlage von Werten, Motiven und Einstellungen. 1993.

Band 22 Marcus Poggenpohl: Verbundanalyse im Einzelhandel auf der Grundlage von Kundenkarteninformationen. Eine empirische Untersuchung von Verbundbeziehungen zwischen Abteilungen. 1994.

Band 23 Kai Bauche: Segmentierung von Kundendienstleistungen auf investiven Märkten. Dargestellt am Beispiel von Personal Computern. 1994.

Band 24 Ewald Werthmöller: Räumliche Identität als Aufgabenfeld des Städte- und Regionenmarketing. Ein Beitrag zur Fundierung des Placemarketing. 1995.

Band 25 Nicolaus Müller: Marketingstrategien in High-Tech-Märkten. Typologisierung, Ausgestaltungsformen und Einflußfaktoren auf der Grundlage strategischer Gruppen. 1995. 